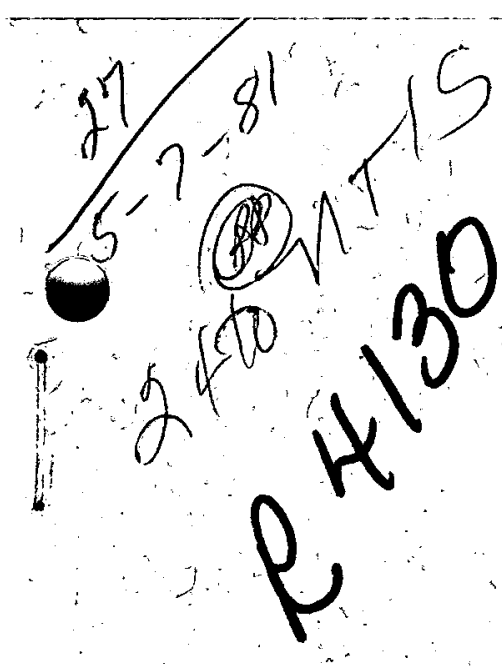

UCID; 18870

\title{
CALCÜLATED NUCLIDE COMPOSITIONS AND GAMMA-RAY EXPOSURE RATES FOR FALLOUT FROM THE HARRY, SMOKY, AND ANNIE EVENTS
}

Harry G. Hicks

March 3, 1981

This is an informal report intended primarily for internal or limited external distribution. The opinions and conclusions stated are those of the author and may or may not be those of the Laboratory.

Work performed under the auspices of the U.S. Department of Energy by the Lawrence Livermore Laboratory under Contract W-7405-Eng-48. 


\section{DISCLAIMER}

This report was prepared as an account of work sponsored by an agency of the United States Government. Neither the United States Government nor any agency Thereof, nor any of their employees, makes any warranty, express or implied, or assumes any legal liability or responsibility for the accuracy, completeness, or usefulness of any information, apparatus, product, or process disclosed, or represents that its use would not infringe privately owned rights. Reference herein to any specific commercial product, process, or service by trade name, trademark, manufacturer, or otherwise does not necessarily constitute or imply its endorsement, recommendation, or favoring by the United States Government or any agency thereof. The views and opinions of authors expressed herein do not necessarily state or reflect those of the United States Government or any agency thereof. 


\section{DISCLAIMER}

Portions of this document may be illegible in electronic image products. Images are produced from the best available original document. 
CALCULATED NUCLIDE COMPOSITIONS AND GAMMA-RAY EXPOSURE RATES FOR FALLOUT FROM THE HARRY, SMOKY AND ANNIE EVENTS

Harry G. Hicks

The purpose of the report is to document the results of computer calculations of the nuclide composition and associated external gamma-ray exposure rates for fallout from the HARRY, SMOKY and ANNIE Events.

The following data are put into the calculation via cards:

Shot name

Fission yield $(k t)^{l}$

Fission split?

Neutron-induced nuclides, atoms per fission 2

The following data are put into the calculation via teletype:

Fraction of refractories present

Relaxation length*

$\mathrm{mR} / \mathrm{hr}$ at $\mathrm{H}+12 \mathrm{hrs}$

The $233 \mathrm{U}, 235 \mathrm{U}, 238 \mathrm{U}$, and $239,240 \mathrm{pu}$ data are omitted primarily to keep the output unclassified. Furthermore, the natural uranium content of the soil is about one million times that received from fallout, and at least half of the plutonium in Nevada and Utah soils comes from worldwide fallout. 4

The fission product distribution is calculated for each event with the appropriate neutron spectrum and the fractions of fissions due to each fissionable material. 5 Some of the calculations are made with specified fractions of the refractory nuclides deleted to simulate fractionation effects. Surface roughness effects on the gamma-ray exposure rates one meter above ground level are simulated by using the values of $(\mathrm{mR} / \mathrm{hr}) /\left(\mu \mathrm{Ci} / \mathrm{m}^{2}\right)$ for a relaxation length of $0.16 \mathrm{gm} / \mathrm{cm}^{2}$.

The total number of microcuries per square meter and the gamma-ray exposure: rates (mR/hr, 1 meter above ground level) are calculated for 152 fission products and 25 neutron-induced nuclides. The decay of each nuclide is followed from zero time to 50 years postshot and the sum of $\mu \mathrm{Ci} / \mathrm{m}^{2}$ and $\mathrm{mR} / \mathrm{hr}$ calculated at each decay interval. Each value is then divided by the value of the total mR/hr at $\mathrm{H}+12$ hours, normalizing each value and sum to values consistent with a gamma-ray exposure rate of $1 \mathrm{mR} / \mathrm{hr}$ at $\mathrm{H+1} 2$ hours. The data in the Appendices are these normalized values. For actual exposure or depcsition calculations, these normalized values can be scaled by multiplying by the exposure rate taken from the fallout pattern, $\mathrm{mR} / \mathrm{hr}$ at $\mathrm{H}+12$ hours.

*According to Beck, 3 the concentration of fallout varies exponentially with soil depth, $z ; C=C_{0} e^{-\alpha z}$. He defines relaxation length as $1 / \alpha$. 
Since fallout patterns are produced from gamma-ray exposure rates at $\mathrm{H}+12$ hours, gamma-ray exposure rates and the composition and concentration of fallout from arrival time to 50 years postshot can be calculated with an assumption about fractionation behavior (fraction of refractory nuclides present).

The appended output has 30 decay intervals, 10 from 1 to 21 hours, 10 from 1 to 300 days, and 10 from 1 to 50 years. For each of these intervals and for zero time, there are values of $\mu \mathrm{Ci} / \mathrm{m}^{2}$ (Appendices 1, 4, and 7) and $\mathrm{mR} / \mathrm{hr}$ (Appendices 2, 5, and 8) for each nuclide present, and the total.

The computer code also generates an input file for a second code that fits by the method of least squares the total $\mu \mathrm{Ci} / \mathrm{m}^{2}$ and $\mathrm{mR} / \mathrm{hr}$ values to the following expression:

$$
\sum_{i=1}^{11} a_{j} e^{-\lambda_{j} t}
$$

The program uses a set of input values of $\lambda_{i}$ to give a least squares fit for the $11 \mathrm{a}_{j}$ 's. The output appears in Appendices 3,6, and 9. 


\section{REFERENCES}

1. Announced United States Nuclear Tests, July, 1945, through December, 1979. NV0-209, January, 1980.

2. H. G. Hicks, Radiochemical Data Collected on Events Releasing Radioactivity from the Nevada Testing Complex, UCRL-52934, in publication.

3. H. L. Beck, private communication.

4. E. Hardy, Plutonium in So il Northeast of the Nevada Test Site, HASL-306, pg. I-51, July 1, 1976.

5. D. R. Nethaway and G. W. Barton, A Compilation of Fission Product Yields in Use at the Lawrence Livermore Laboratory, UCRL-51458, October, 1973.

6. H. L. Beck, Exposure Rate Conversion Factors for Radionuclides Deposited on the Ground, EML-378, July, 1980. 


\section{APPENDIX 1 \\ HARRY EVENT}

\section{Microcuries per Square Meter}

Fraction of refractories present $=1.0$

Page

Total $\mathrm{mR} / \mathrm{hr}$ and Total $\mu \mathrm{Ci} / \mathrm{m}^{2}$

$1-1$

Individual nuclide $\mu \mathrm{Ci} / \mathrm{m}^{2}$

$1-2$

Fraction of refractories present $=0.5$

Total $\mathrm{mR} / \mathrm{hr}$ and Total $\mu \mathrm{Ci} / \mathrm{m}^{2}$

$1-12$

Individual nuclide $\mu \mathrm{Ci} / \mathrm{m}^{2}$

$1-13$

Fraction of refractories present $=0.1$

Total $\mathrm{mR} / \mathrm{hr}$ and Total $\mu \mathrm{Ci} / \mathrm{m}^{2}$

$1-23$

Individual nuclide $\mu \mathrm{Ci} / \mathrm{m}^{2}$

$1-24$ 


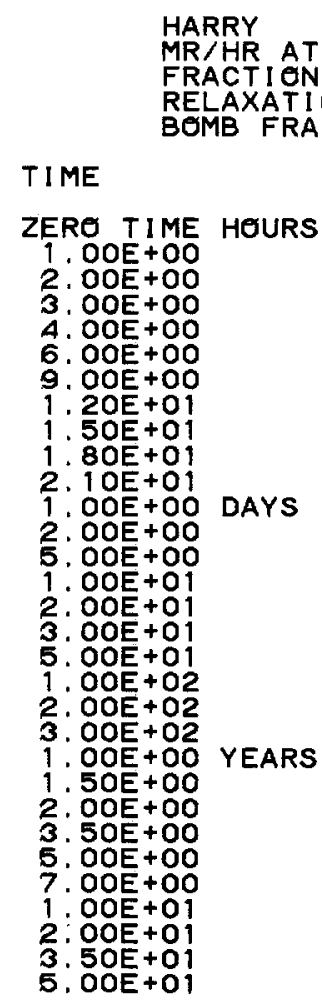

HARRY

MRACTION OF REFRACTORIES 'PRESENT a 1,000

RELAXATI ON LENGTH $=0.96$ GM/SQCM
SO

T I ME

$M R / H R$

MICROCURIES/SQ METER

1. $03 E+0$

3. $11 E+01$

$6.85 E+00$

4. $38 \mathrm{E}+00$

2. $35 \mathrm{E}+00$

OOE + 00

$7.85 \mathrm{E}-0$

6. $45 \mathrm{E}-\mathrm{O}$

$4.62 \mathrm{E}-\mathrm{O}$

2. $12 E-0$

3. $47 \mathrm{E}-02$

1. $40 \mathrm{OE}-\mathrm{O} 2$

8. 32E-03

$1.63 \mathrm{E}-03$

5. $98 \mathrm{E}-04$

$1.30 E-04$

. $58 \mathrm{E}-05$

1. $.07 E-05$

8. $28 E-06$

. $17 E-06$

4. $61 E-06$

3. $22 E-06$

1. $22 \mathrm{E}+04$

9. $17 \mathrm{E}+02$

4. $46 \mathrm{E}+02$

2. $80 \mathrm{E}+02$

$1.55 E+02$

$1.06 \mathrm{E}+02$

9. $18 \mathrm{E}+01$

3. $97 \mathrm{E}+01$

$5.51 E+\infty 0$

1. $98 \mathrm{E}+00$

6.88E-01

1.

$5.87 \mathrm{E}-02$

4. $25 \mathrm{E}-02$

$1.62 \mathrm{~V}-\mathrm{O}$

$7.37 \mathrm{E}-03$

3. $24 \mathrm{E}-03$

$1.22 E-03$ 
MI CROCURIES/SQ METER

HARRY

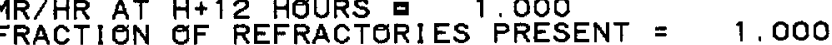

RELAXATI ON LENGTH $=0.16$ GM/SQCM
BOMB FRACTI ON PER SQ. METER $=1.404 E-13$

DEBRIS DECAY FROM 1 TO 21 HOURS

ZERO TIME 1. OOE+DO $2.00 E+00 \quad 3.00 E+00 \quad 4.00 E+00 \quad 6.00 E+00 \quad 9.00 E+00 \quad 1.20 E+01 \quad 1.50 E+01 \quad 1.80 E+012.10 E+01$

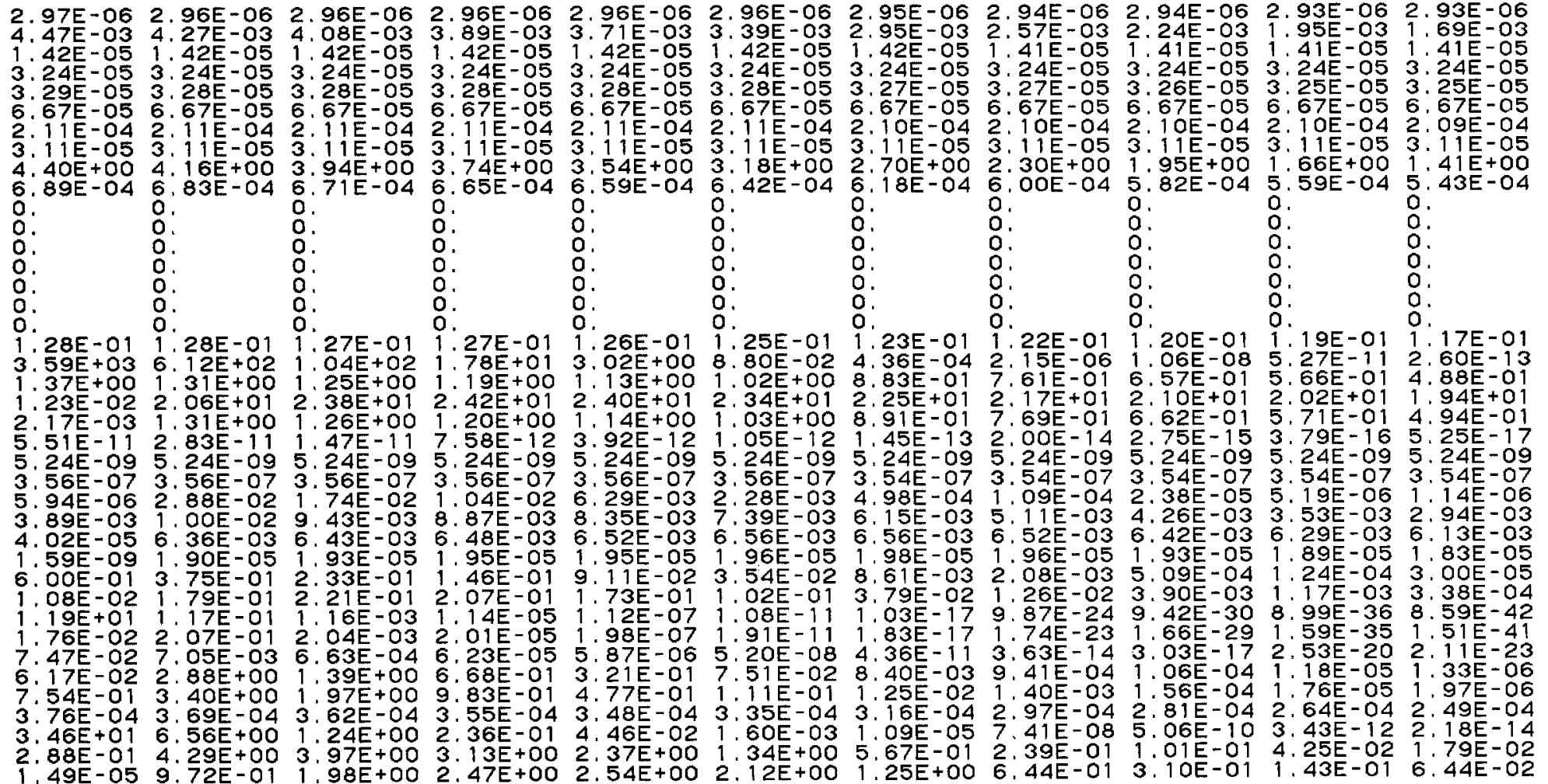




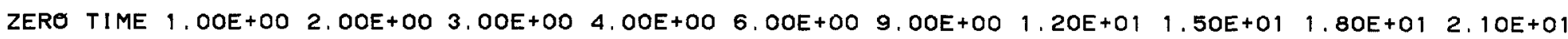

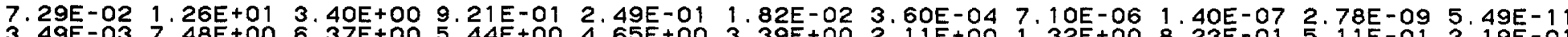

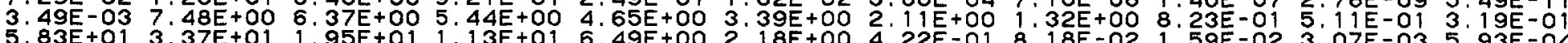

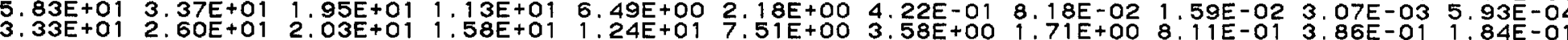

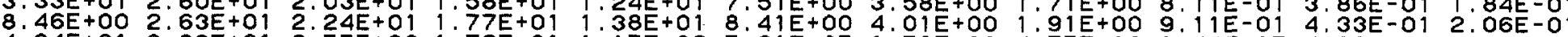

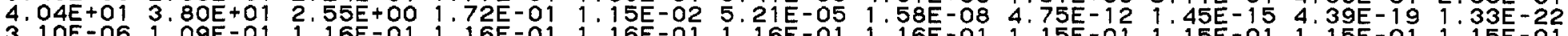

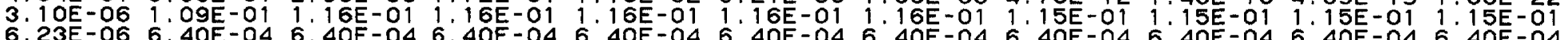

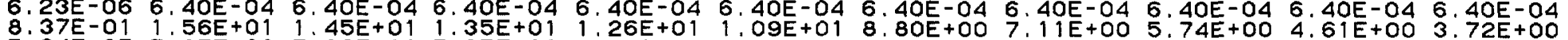
$5.64 E-05 \quad 5.37 E+007.32 E+007.85 E+007,74 E+00-55 E+005.65 E+004.58 E+003.79 E+002.68 E+002.40+00$

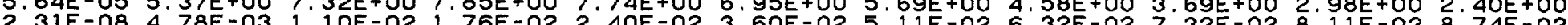

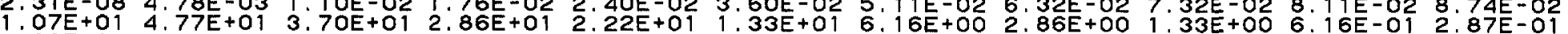
4.93E-01 1.:00E+01 1.58E+01 1.87E+01 1.99E+01 1.89E+01 1.45E+01 9.89E+00 6.36E+00 3.92E+00 2.36E+00

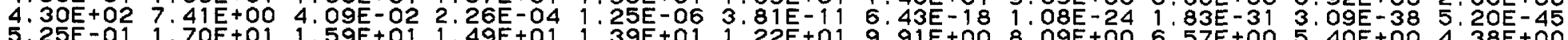
$5.25 E-011.70 E+01$ 1.59E+01 $1.49 E+011.39 E+011.22 E+01 \quad 9.91 E+00 \quad 8.09 E+006.57 E+00 \quad 5.40 E+00 \quad 4.38 E+00$

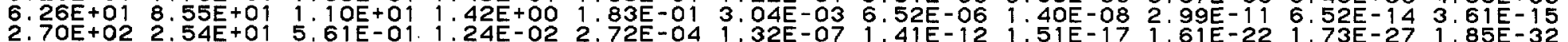

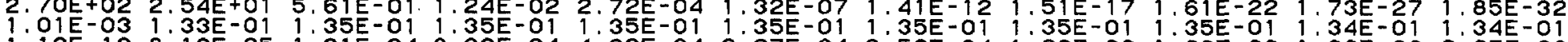
$1: 13 E-10$ 8.19E-05 1.91E-04 3.00E-04 4.09E-04 6.27E-04 9.52E-04 $1.28 E-03$ 1.60E-03 1.93E-03 2.25E-03

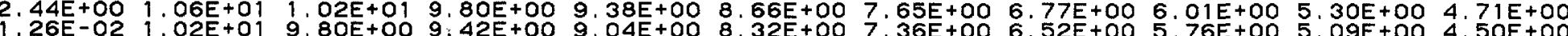

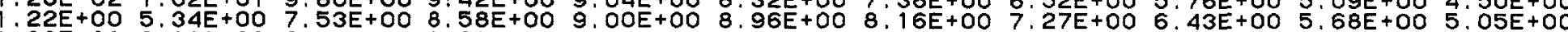
$1.38 \mathrm{E}+01$ 6. $13 \mathrm{E}+00 \mathrm{2}$ 2.71E+00 1.20E+00 5.31E-01 1.04E-01 8.99E-03 7.76E-04 6.76E-05 5.86E-06 5.04E-07

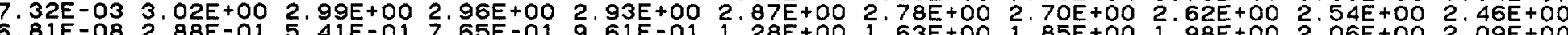

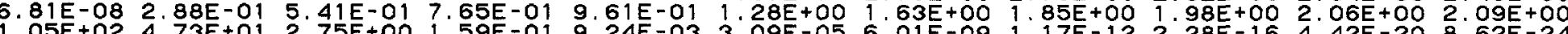
$1.05 E+024.73 E+012.75$

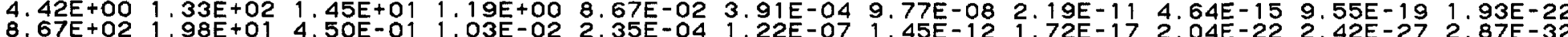

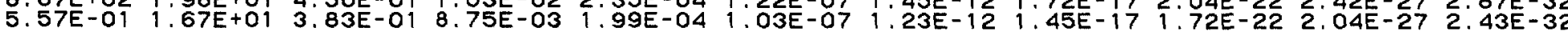

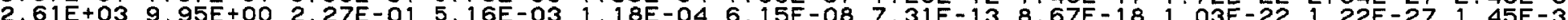

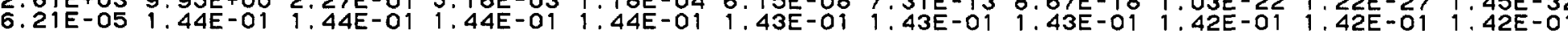

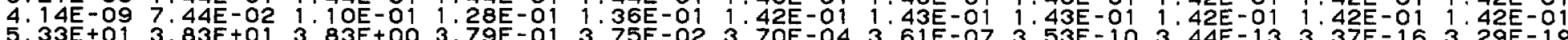

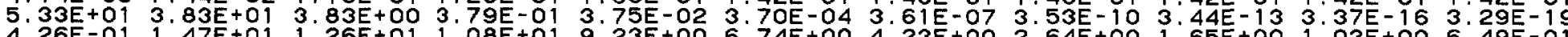

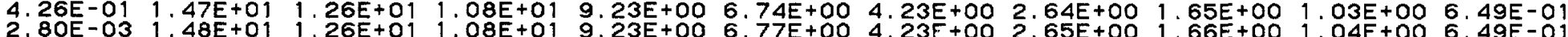

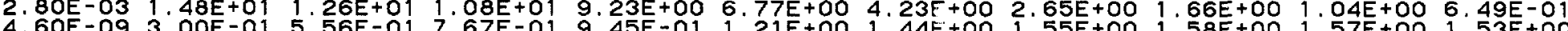
4.60E-09 3.00E-01 5.50E

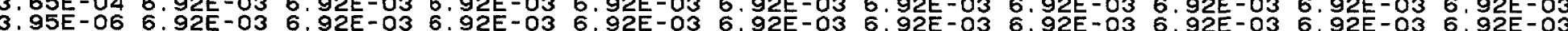

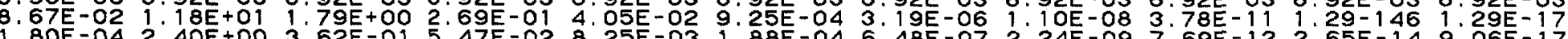


HARRY MR/HR AT H+12 HOURS =
FRACTION OF REFRACTORIES PRESENT = 1.000
RELAXATION LENGTH $=0.16$ GM SQCM DEBRIS DECAY FROM 1 TO 21 HOURS

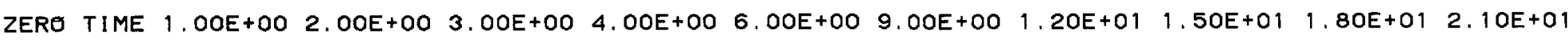

PD109

AG109M

AG 111 M

$A G 111$

PDI 12

AG 112

AG 113

CD1 $15 \mathrm{M}$

CD 115

IN115M

IN 117 M

CD 118

INi 18

INI $19 \mathrm{M}$

INI19

SN121

SN123

SN125

SB 125

SN127

TE127

SN128

SB 128

SN129M

SN129

TE $129 \mathrm{M}$

TE 129

SB 130

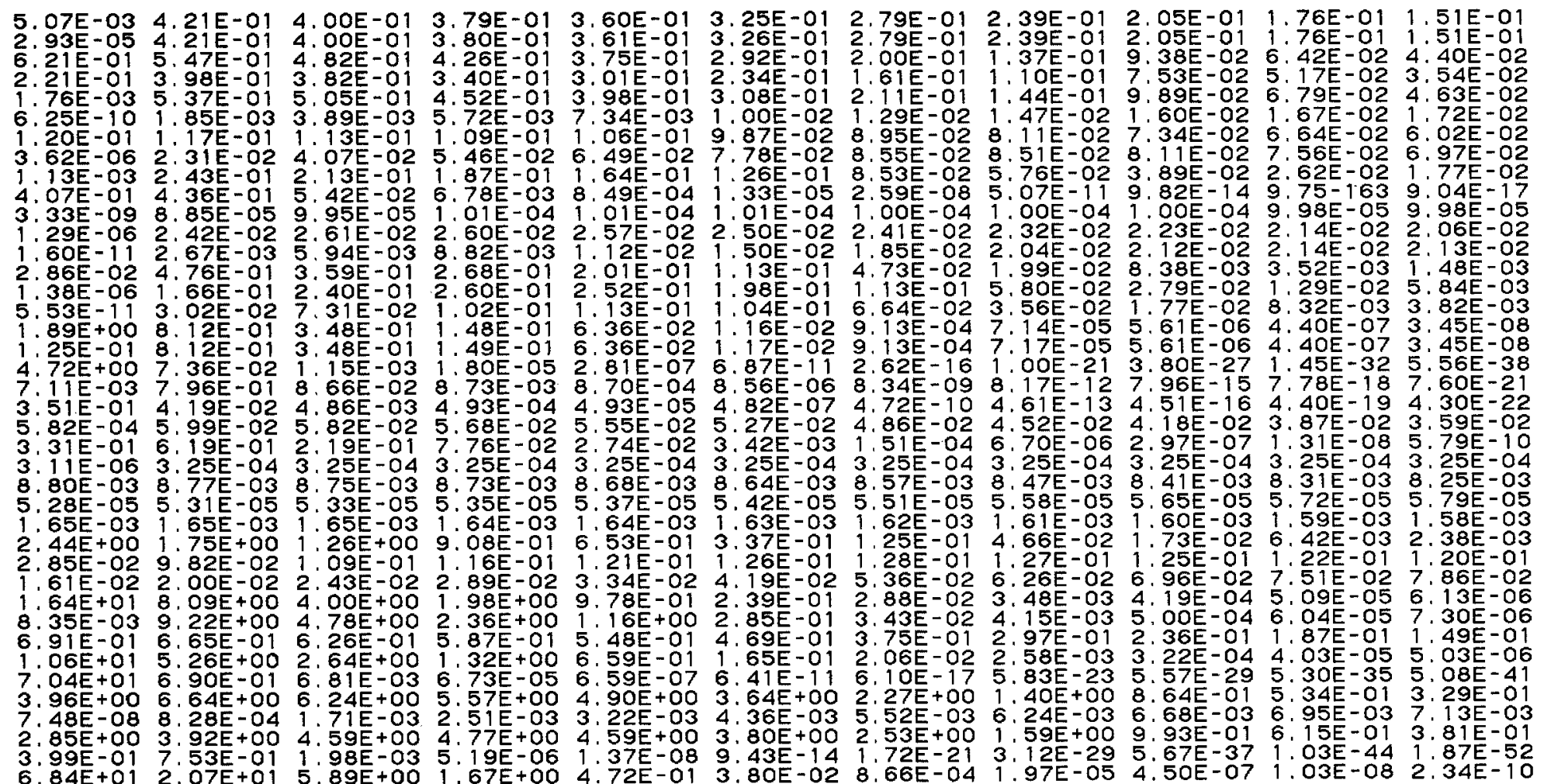


HARRY
MRRAR AT H+12 HOURS MICROCURIES/SQ METER
FRACTION OF REFRACTORIES POOO PRENT = 1.000 RELAXATION LENGTH $=0.16$ GM/SQCM
BOMB FRACTION PER SQ. METER $1,404 E-13$

DEBRIS DECAY FROM 1 TO 21 HOURS

ZERE TIME $1.00 E+00 \quad 2.00 E+00 \quad 3.00 E+00 \quad 4.00 E+00 \quad 6.00 E+00 \quad 9.00 E+00 \quad 1.20 E+01 \quad 1.50 E+01 \quad 1.80 E+01 \quad 2.10 E+01$

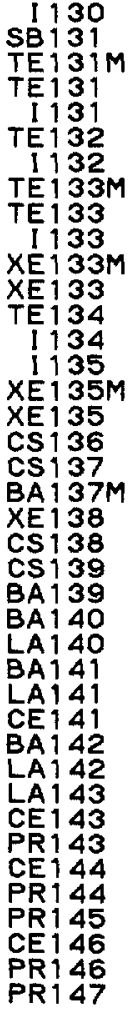

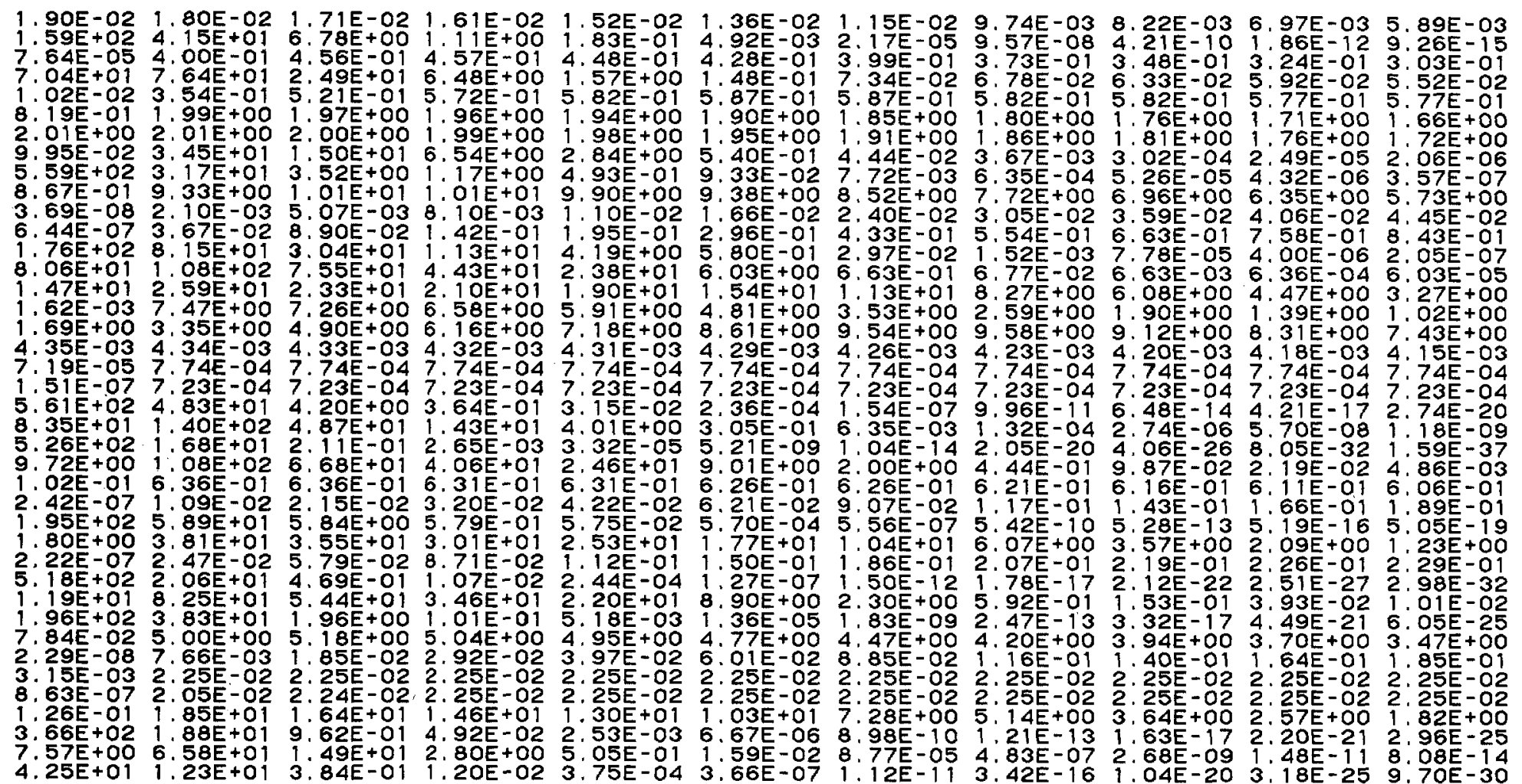


HARRY AT MICRECURIES/SQ METER MRACTION OF REFRACTORI ES PRESENT $=1.000$ RELAXATION LENGTH $=0.16$ GM SOCM
BOMB FRACTI ON PER SQ. METER $=1.404 E-13$ DEBRIS DECAY FROM 1 TO 21 HOURS

ZERO TIME $1.00 E+00 \quad 2.00 E+00 \quad 3.00 E+00 \quad 4.00 E+00 \quad 6.00 E+00 \quad 9.00 E+00 \quad 1.20 E+01 \quad 1.50 E+01 \quad 1.80 E+01 \quad 2.10 E+01$

ND147
ND149
PM149
PM150
ND151
PM151
PM152
SM153
SM155
EU155
SM156
EU156
EU157
EU158
EU159
GD159
TB161

TETAL

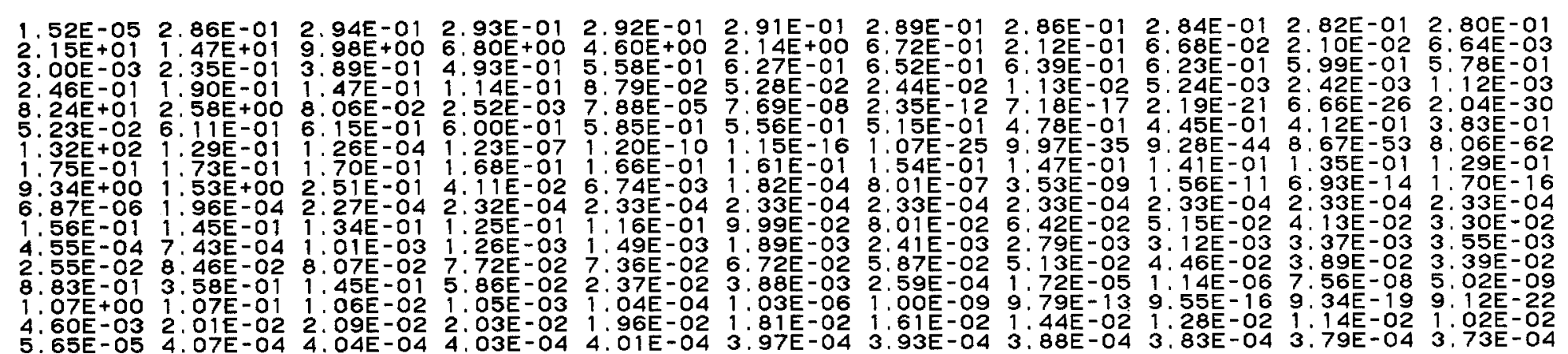

$\begin{array}{lllllll}1.22 E+04 & 2.54 E+03 & 9.17 E+02 & 5.46 E+02 \quad 4.04 E+02 & 2.80 E+02 \quad 1.98 E+02 \quad 1.55 E+02 & 1.26 E+02 \quad 1.06 E+02 \quad 9.18 E+01\end{array}$ 
DEBRIS DECAY FROM I TO 300 DAYS

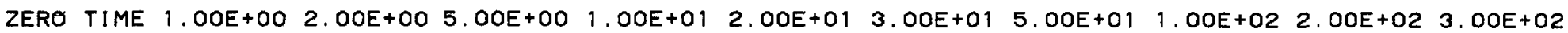

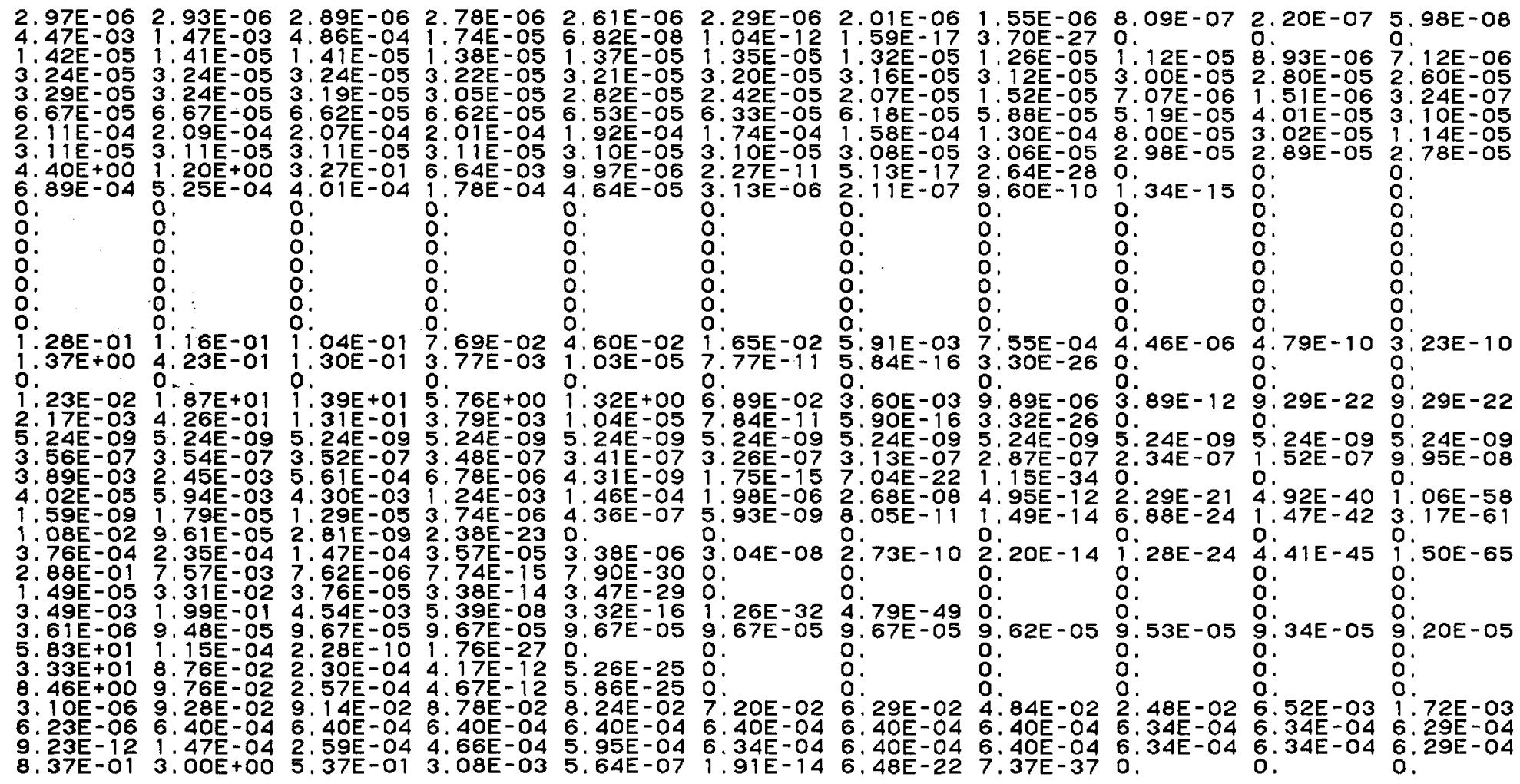


DEBRIS DECAY FROM 1 TO 300 DAYS

ZERO TIME 1, OOE+OO $2 ., 00 E+00 \quad 5.00 E+00 \quad 1.00 E+01 \quad 2,00 E+01 \quad 3.00 E+01 \quad 5.00 E+01 \quad 1.00 E+02 \quad 2.00 E+02 \quad 3.00 E+02$

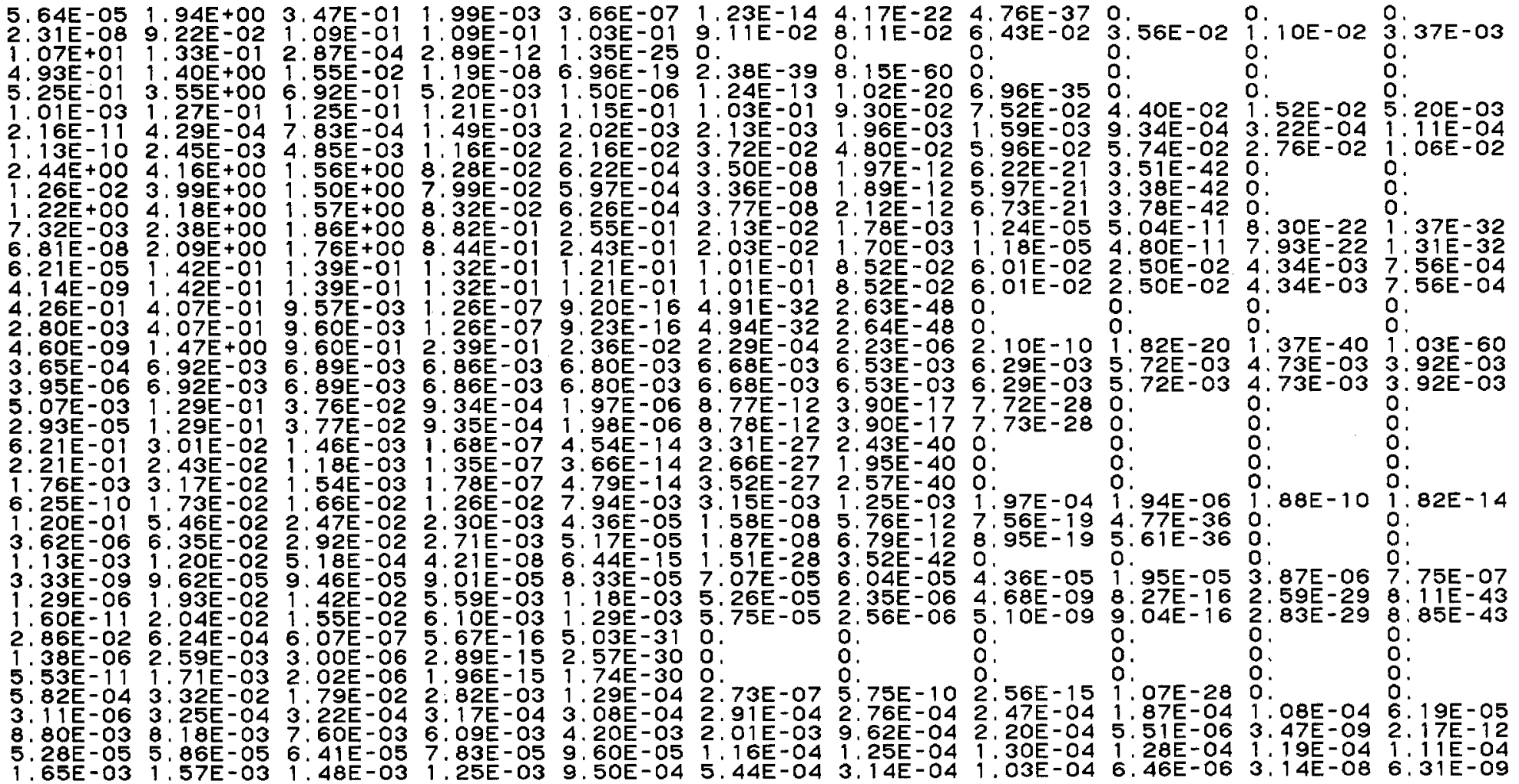

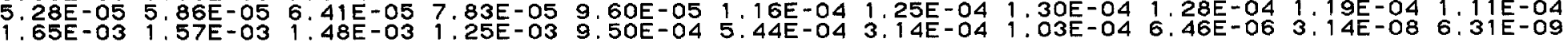


HARRY
MR/HR AT H+12 HOURS MI CROCURIES/SQ METER
FRACTION OF REFRACTORIES PRESENT $=1.000$

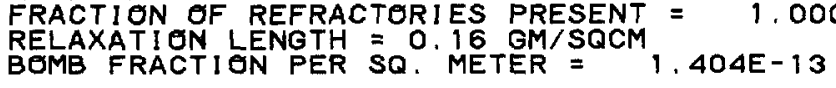

DEBRIS DECAY FROM 1 TO 300 DAYS

ZERO TIME 1.0OE+00 $2.00 E+00 \quad 5.00 E+00 \quad 1.00 E+01 \quad 2.00 E+01 \quad 3.00 E+01 \quad 5.00 E+01 \quad 1.00 E+02 \quad 2.00 E+02 \quad 3.00 E+02$

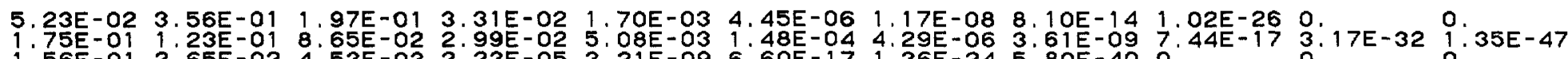

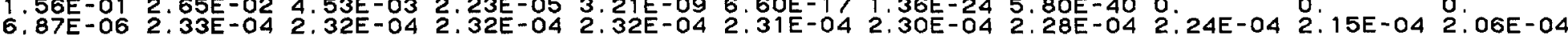
$4.55 E-043.70 E-034.10 E-033.68 E-032.92 E-031.84 E-03$ 1. $16 E-034.59 E-044.55 E-054.48 E-074.42 E-09$

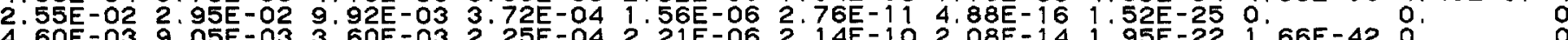

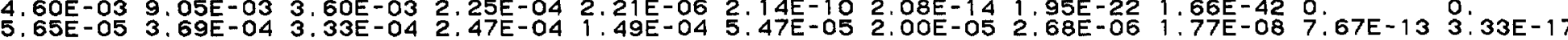

TUTAL

$2.70 E+02 \quad 7.98 E+01 \quad 3.97 E+01 \quad 1.45 E+01 \quad 5.51 E+00 \quad 1.98 E+00 \quad 1.22 E+00 \quad 6.88 E-01 \quad 3.10 E-01 \quad 1.17 E-01 \quad 5.87 E-02$

PAGE $1-10$ 
PAgE $1-12$

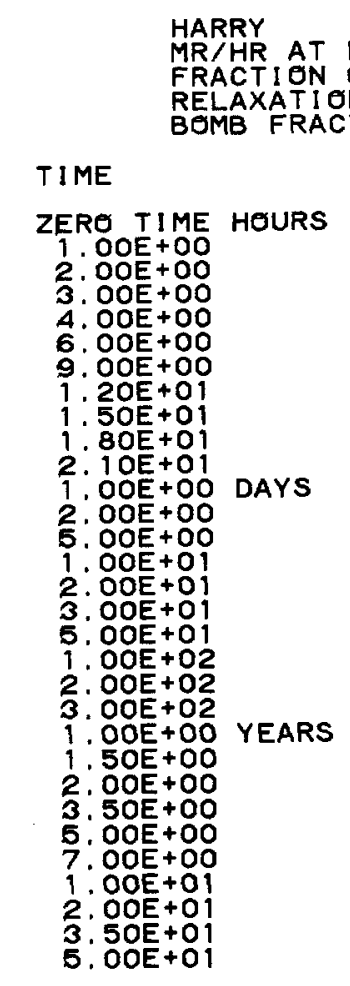
$1.08 \mathrm{E}+02$
1. $35 \mathrm{E}+\mathrm{Q} 1$
$7.21 E+00$
$4.47 E+00$
1. $33 \mathrm{E}+00$
1. $80 \mathrm{OE}+00$
6. $42 \mathrm{E}-\mathrm{O}$
5. $38 \mathrm{E}-01$
2. OSE-O1
3. $72 \mathrm{E}-02$
$8.41 \mathrm{E}-03$
3. $79 \mathrm{E}-03$
4.21E-04
1. $65 \mathrm{E}-04$
3. $31 \mathrm{-O5}$
2. $08 \mathrm{BE}-05$
$1:$ OOE-O5
$8.63 E-06$
$7.67 E-06$
5. $84 E-06$
$4.11 \mathrm{E}-06$
2. $90 \mathrm{E}-06$

.000

ES PRESENT a 0.500

1.798E-13

MICROCURIES/SO METER

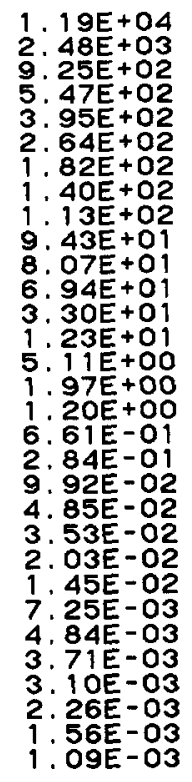


DEBRIS DECAY FROM 1 TO 21 HEURS

ZERO TIME $1.00 E+00 \quad 2.00 E+00 \quad 3.00 E+00 \quad 4.00 E+00 \quad 6.00 E+00 \quad 9.00 E+00 \quad 1.20 E+01 \quad 1.50 E+01 \quad 1.80 E+01 \quad 2.10 E+01$

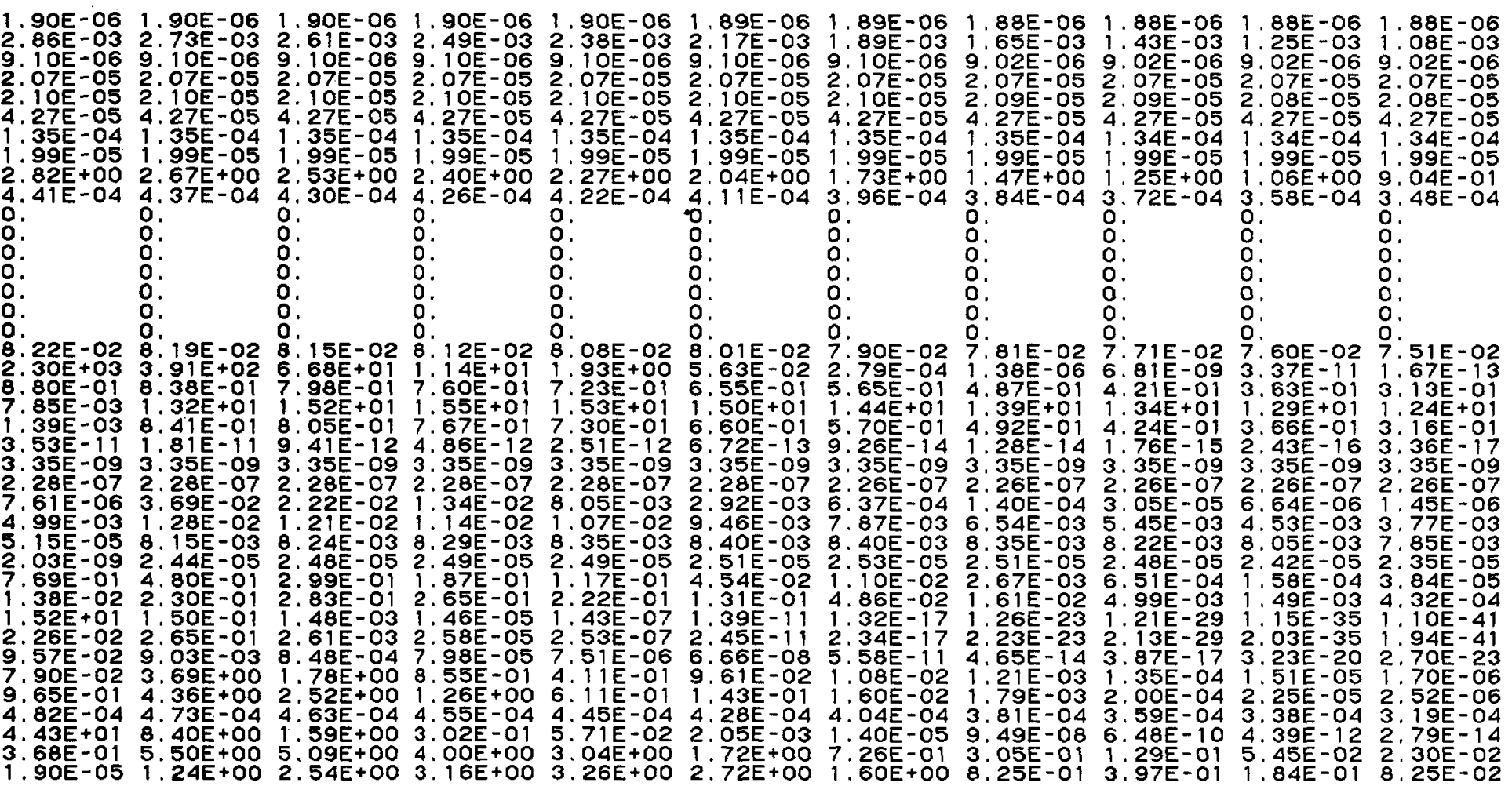


HARRY MR/HR AT H+12 HOURS = MICGROCURIES/SQ METER

MR $/$ HR AT H+12 HOURS $=$ MT 1 ' OOO
FRACTI ON OF REFRACTORIES PRESENT $=0.500$

RELAXATION LENGTH a O. 16 GM/SQCM $1.798 E-13$
BOMB FRACTION PER SQ. METER $=1.798-13$

DEBRIS DECAY FROM 1 TO 21 HOURS

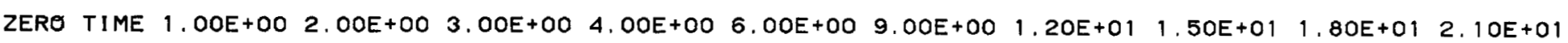

KR 84

KR $85 M$

KR 87

RB 88

RB 89

SR 89

SR 91

$Y$ 9 1

SR 92

SR 92

$Y 93$

Y 94

$\begin{array}{r}Y \\ \text { ZR } 95 \\ \hline\end{array}$

NB 95

NB $97 M$

NB 97

$\begin{array}{ll}\mathrm{NB} & 98 \\ \mathrm{MO} & 99\end{array}$

TC 99M

TClO1

Mc102M

TC102

RH103M

RH1O3M

Ruios

RH1 05M

RH105

RH 106

$\mathrm{RH} 107$
$\mathrm{PD} 107 \mathrm{M}$

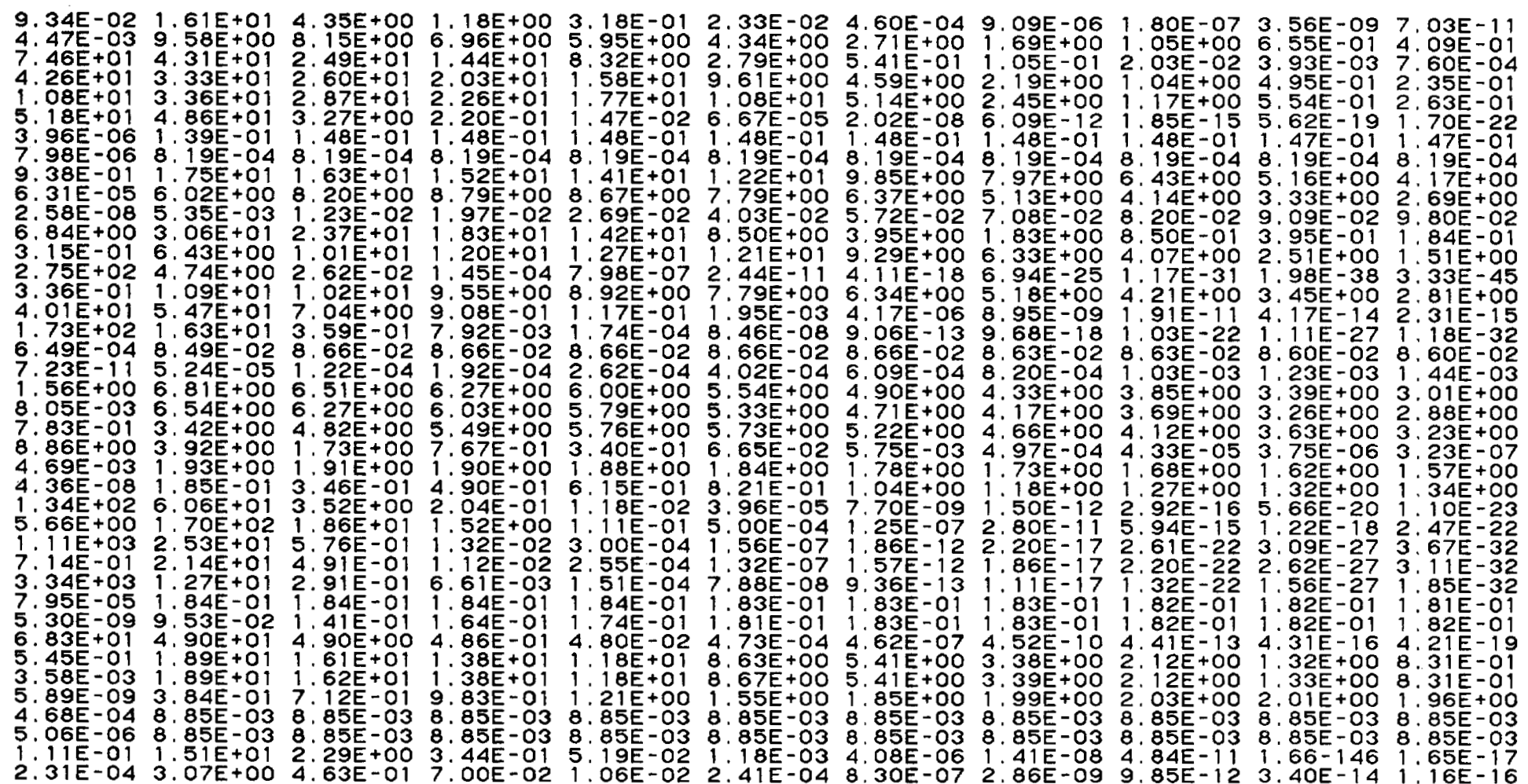




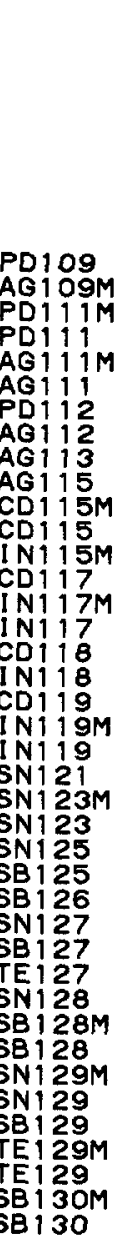

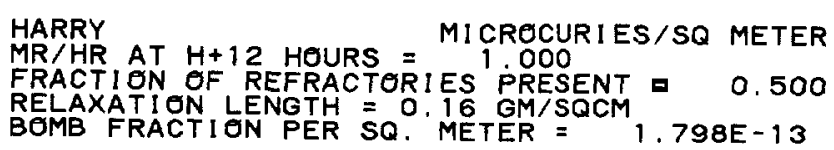

DEBRIS DECAY FROM 1 TO 21 HOURS

ZERO TIME $1.00 E+002.00 E+00 \quad 3.00 E+00 \quad 4.00 E+00 \quad 6.00 E+00 \quad 9.00 E+00 \quad 1.20 E+01 \quad 1.50 E+01 \quad 1.80 E+01 \quad 2.10 E+01$

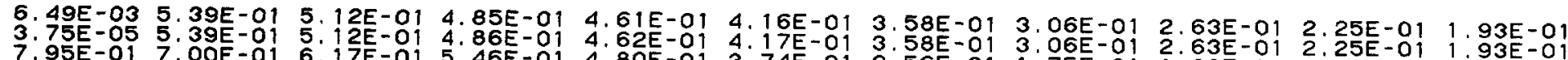

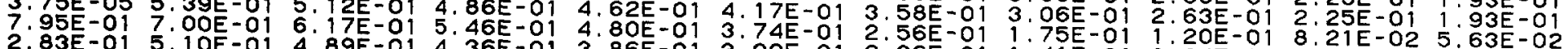

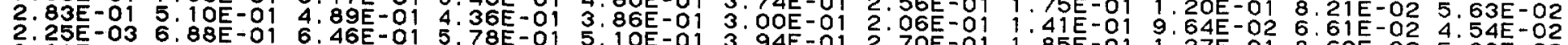

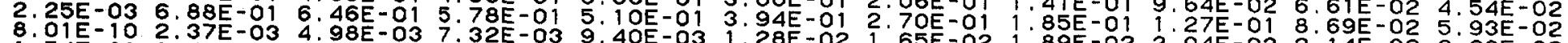

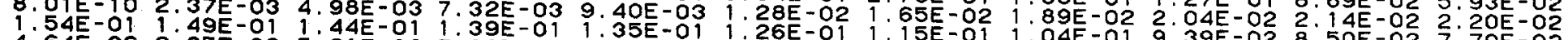

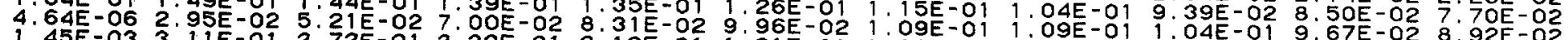

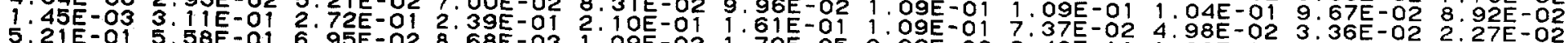

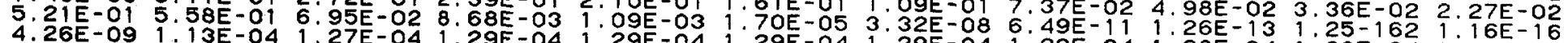

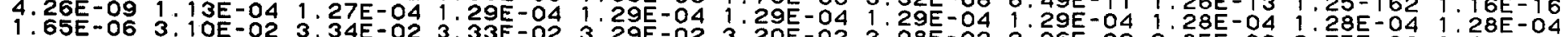

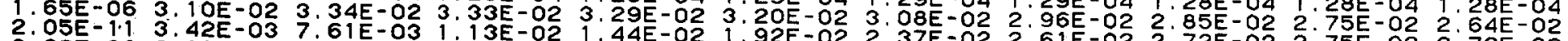

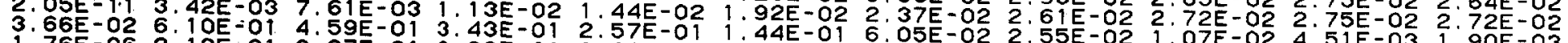

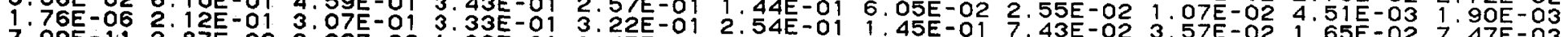

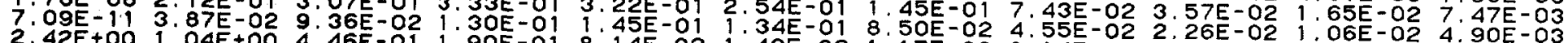

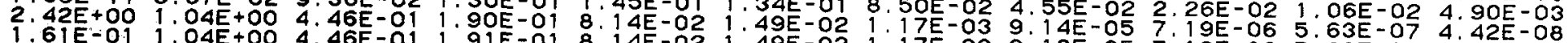

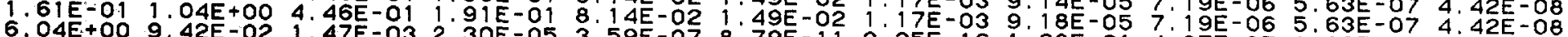

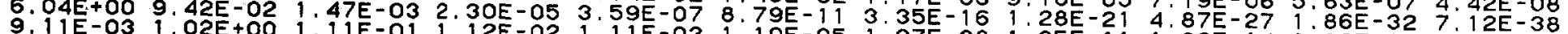

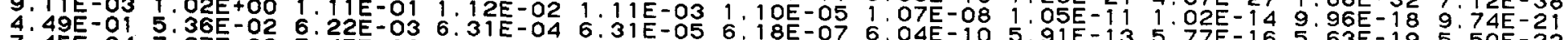

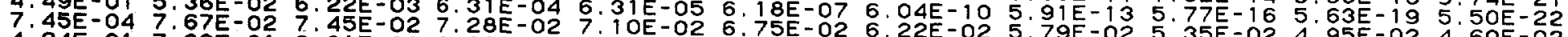
4.24E-01 7:92E-01 2.81E-01 9:93E-02 3.51E-02 4.38E-03 $1.92 E-02$ 5.79E-02 5. 35E-02 4.95E-02 4.60E-02

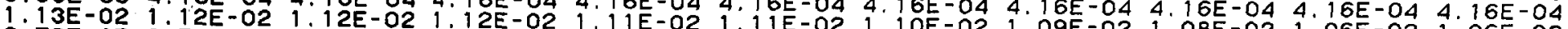

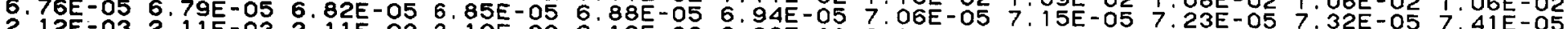
3. $12 E-0332.11 E-032.11 E-032.10 E-03 \quad 2.10 E-03$ 2.09E-03 2.07E-03 2.06E-03 2.04E-03 2.03E-03 $2.02 E-03$

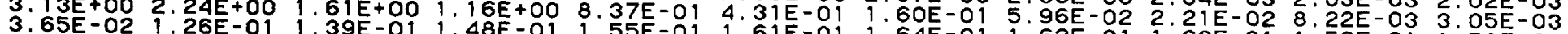

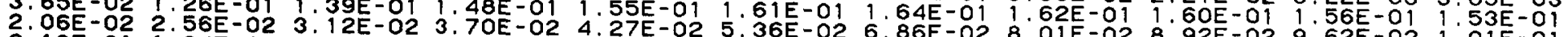

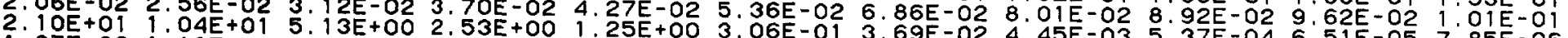

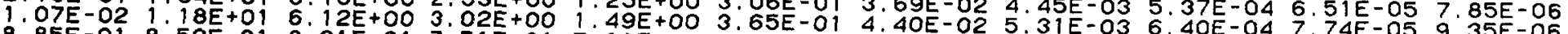
8.85E-01 8.52E-01 8.01E-01 7.51E-01 7.01E-01 6.01E-01 4.80E-01 3.31E-03 6. $40 E-04$ 7.74E-05 9.35E-06

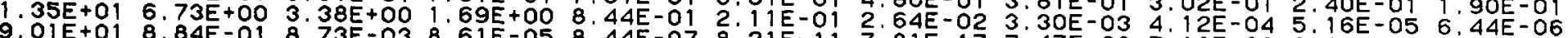
9.01E+01 8.84E-01 8.73E-03 8.61E-05 8.44E-07 8.21E-11 7.81E-17 7.47E-23 7. 13E-29 6.79E-35 6. 50 E -41

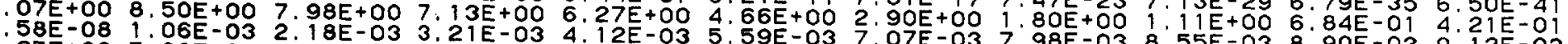
$\begin{array}{llllll} & \end{array}$ $\begin{array}{lllll}5.11 E-01 & 9.64 E-01 & 2 & 53 E-03 & 6\end{array}$ 
HARRY AT H+12 HOURS $=$ MICROCURIES/SQ METER FRACTI ON OF REFRACTORI ES PRESENT

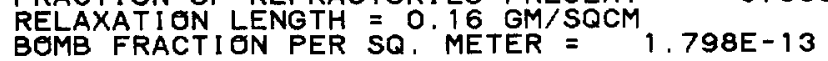

DEBRIS DECAY FROM 1 TO 21 HOURS

ZERO TIME 1.OOE+OO 2. $2.00 E+00 \quad 3.00 E+00 \quad 4.00 E+00 \quad 6.00 E+00 \quad 9.00 E+00 \quad 1.20 E+01 \quad 1.50 E+01 \quad 1.80 E+012.10 E+01$

SB́ 130

TE131M

I 131

TEI32

TE $133 \mathrm{M}$

TE 133

XE133M

XE 133

I 34

XE135M

XE 135

CS137

BA137M

XE

CS139

BA139

BA140

BA 140

CA 141

BA142

LA 142

LA 143

CE 143

PR 144

PR 145

CE 1 46

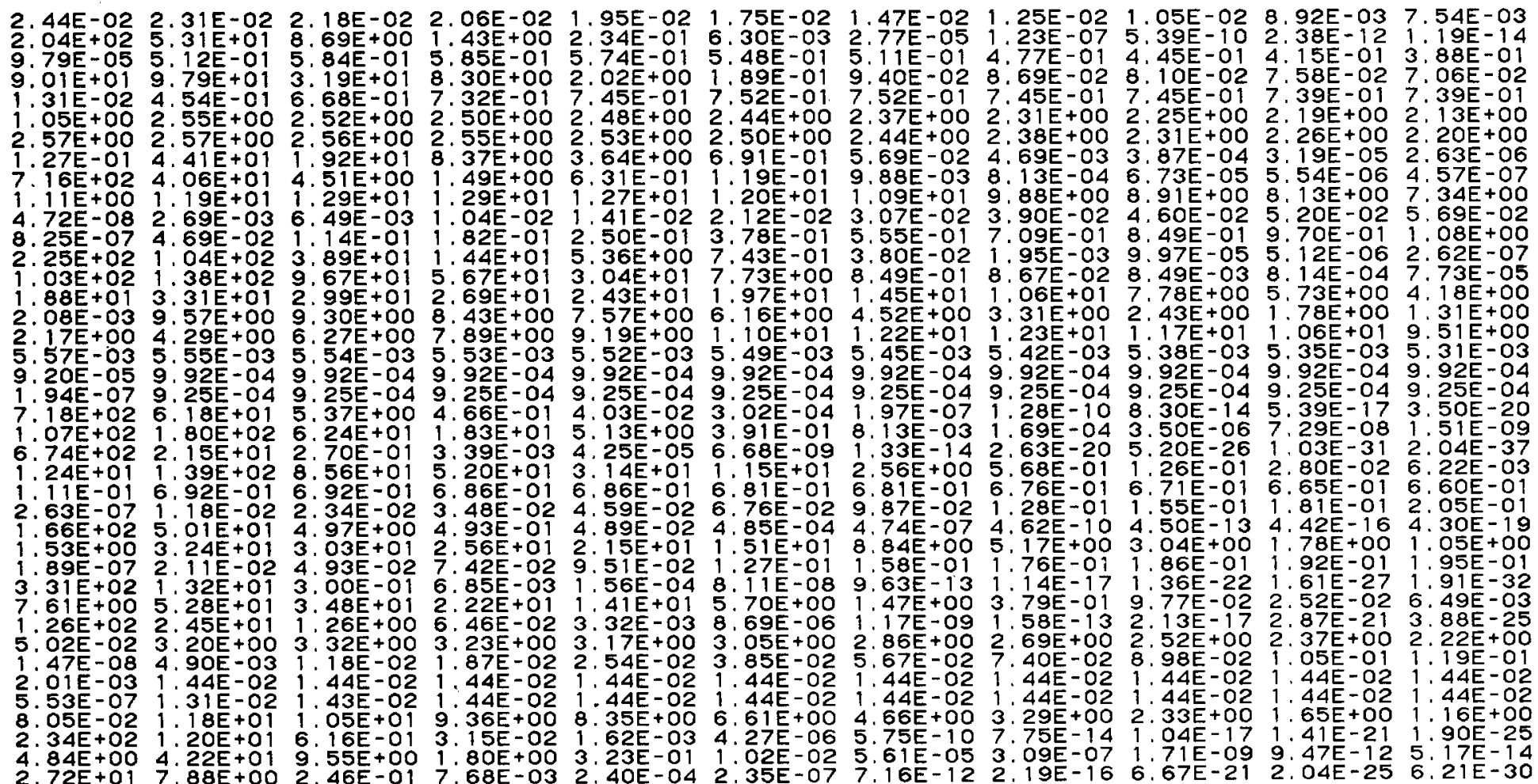


MARRY MR H H H 12 HOURS $=$ MICROCURIES/SQ METER FRACTION OF REFRACTORIES 'PRESENT $=0.500$ RELAXATION LENGTH $=$ O. 16 GM SOCM $=$
BOMB FRACTION PER SQ. METER $=1.798 E-13$

DEBRIS DECAY FROM 1 TO 21 HOURS

ZERO TIME $1.00 E+00 \quad 2.00 E+00 \quad 3.00 E+00 \quad 4.00 E+00 \quad 6.00 E+00 \quad 9.00 E+00 \quad 1.20 E+01 \quad 1.50 E+01 \quad 1.80 E+01 \quad 2.10 E+01$

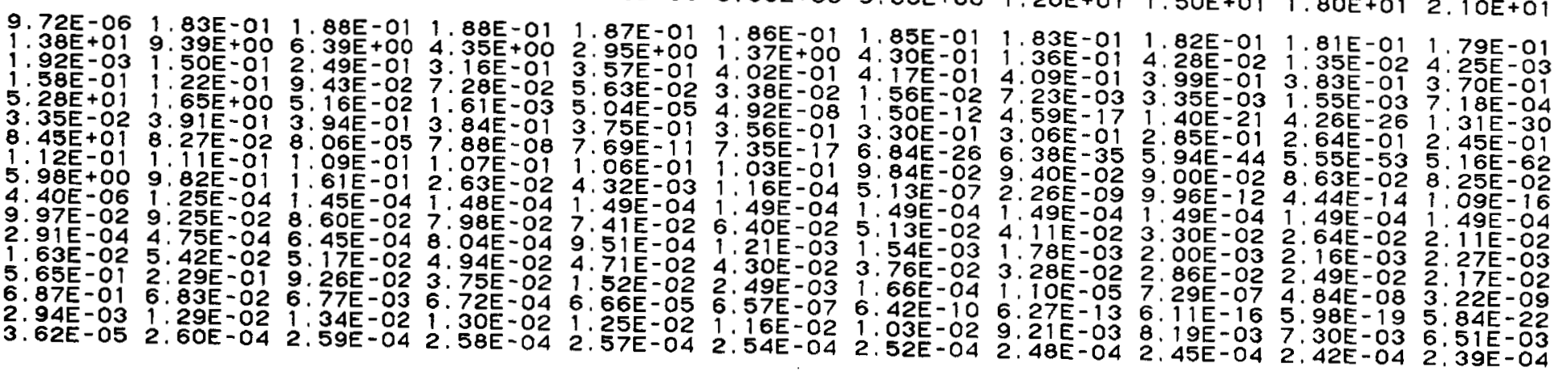

TOTAL

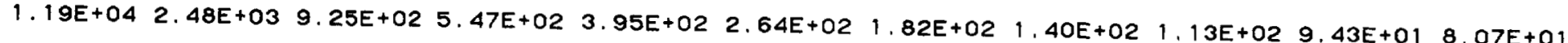


DEBRIS DECAY FROM 1 TO 300 DAYS

ZERO TIME 1. OOE+OO 2. 20OE+OO $5.00 E+00 \quad 1.00 E+01 \quad 2.00 E+01 \quad 3.00 E+01 \quad 5.00 E+01 \quad 1.00 E+02 \quad 2.00 E+02 \quad 3.00 E+.02$

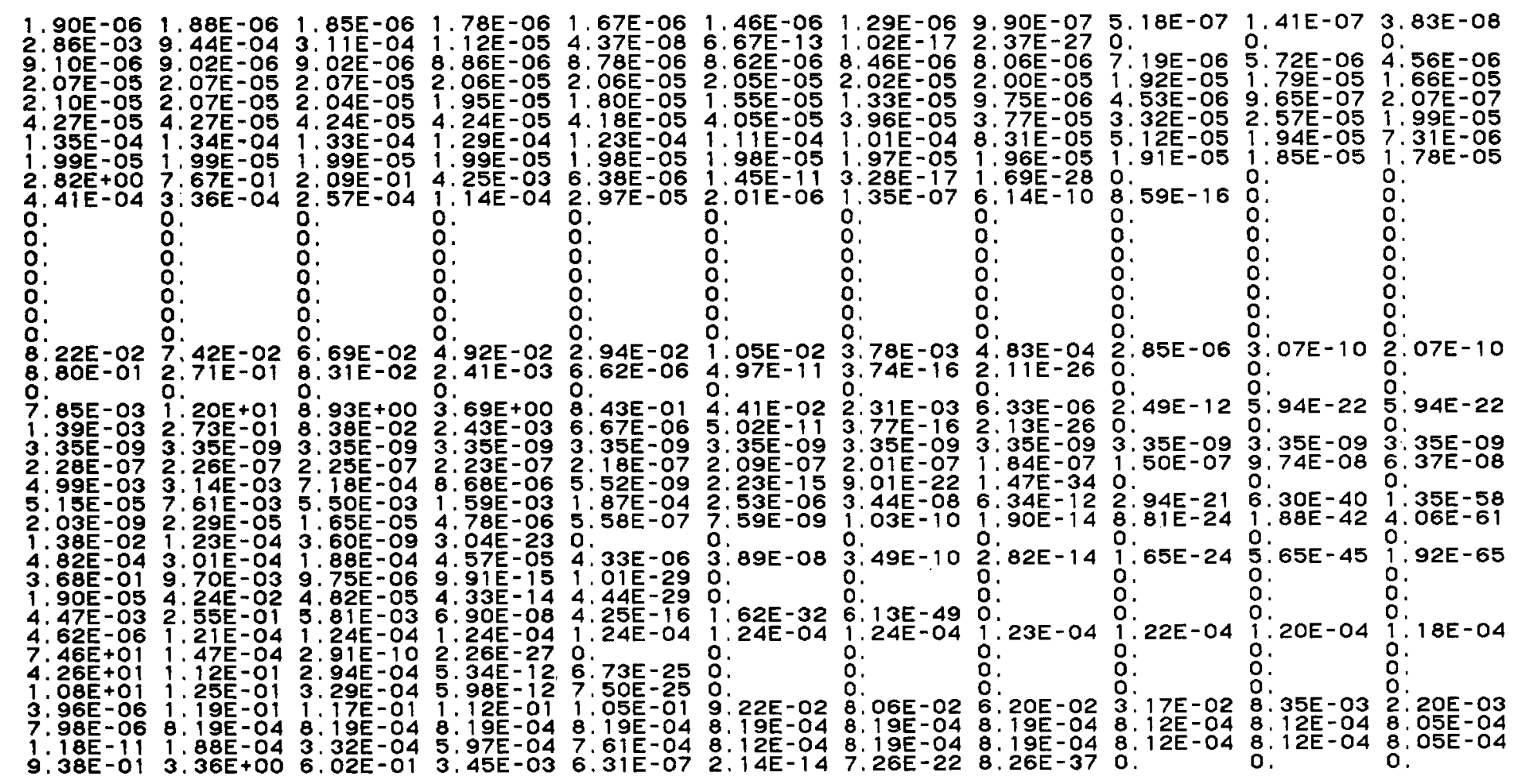


Page $1-19$

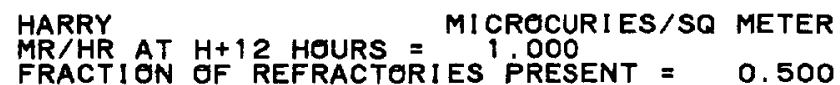

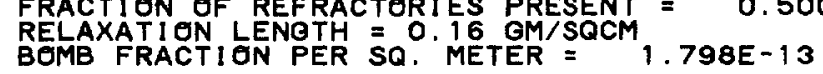

DEBRIS DECAY FROM 1 TO 300 DAYS

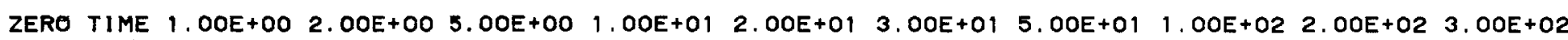

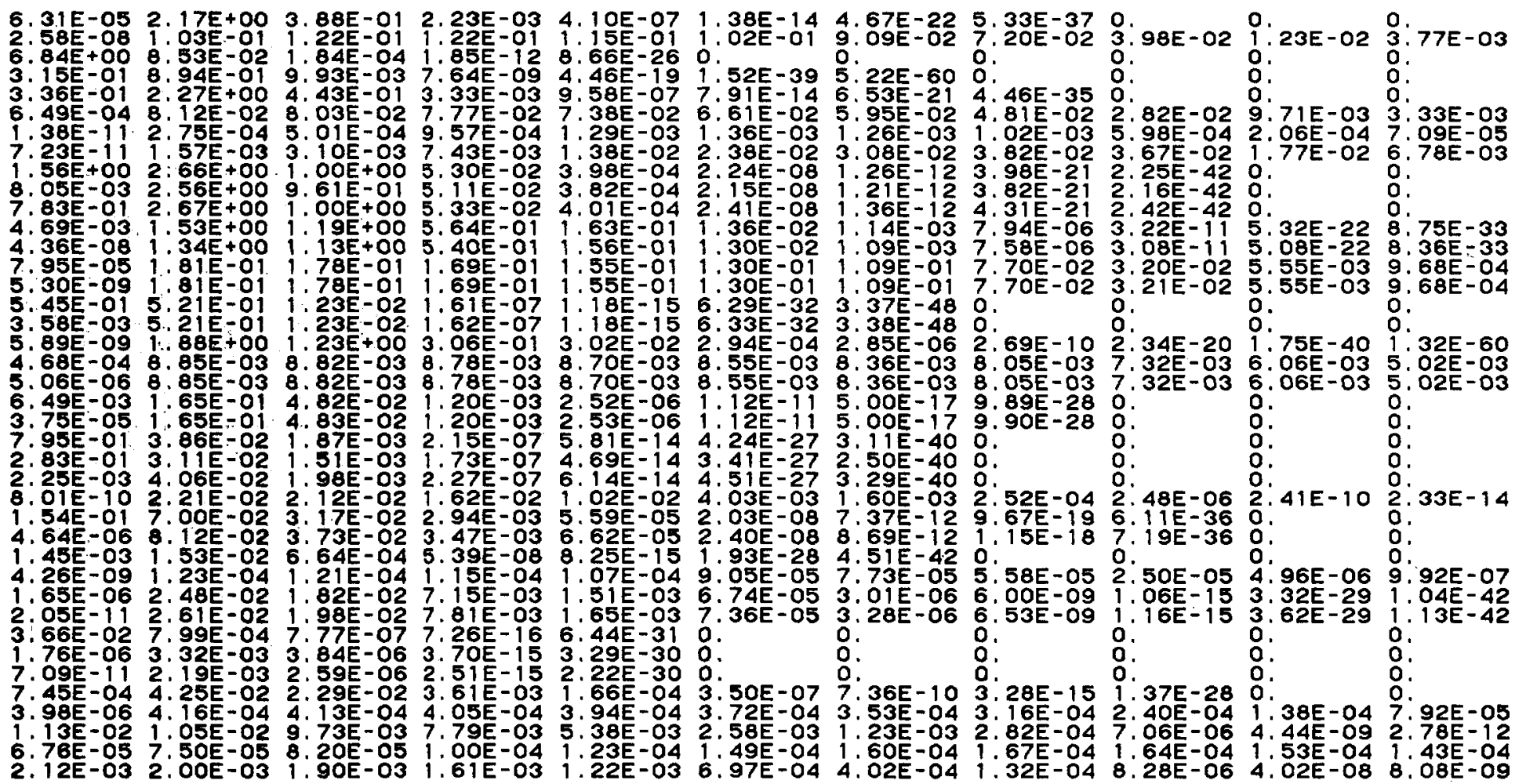


HARRY AT HICROCURIES/SO METER

MR H HR AT H+12 HOURS = MT 1 OOO RELAXATION LENGTH $=0.16$ GM/SQCM
BOMB FRACTI ON PER SQ. METER $=1.798 E-13$

DEBRIS DECAY FROM 1 TO 300 DAYS

ZERO TIME 1. $1.00 E+00 \quad 2.00 E+00 \quad 5.00 E+00 \quad 1.00 E+01 \quad 2.00 E+01 \quad 3.00 E+01 \quad 5.00 E+01 \quad 1.00 E+02 \quad 2.00 E+02 \quad 3.00 E+02$

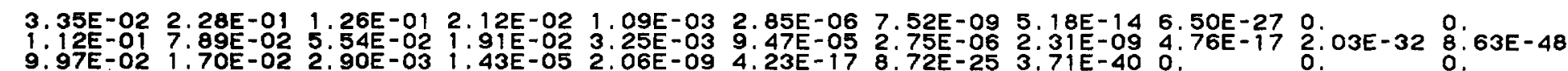

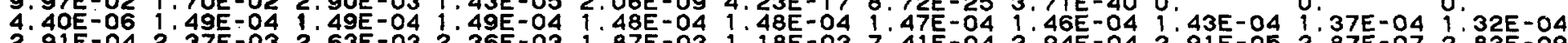
2. $91 E-042.37 E-032.63 E-032.36 E-031.87 E-031: 18 E-03$ 7. $41 E-042.94 E-042.91 E-052.87 E-072.83 E-09$

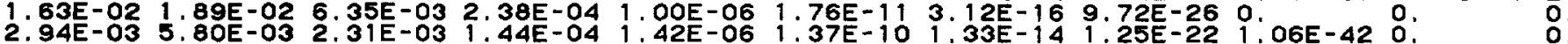

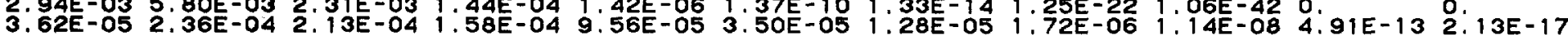

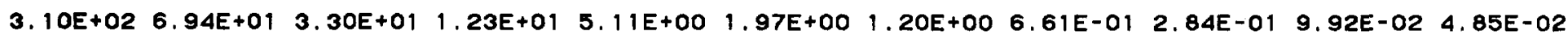

PAGE $|-2|$

TOTAL 


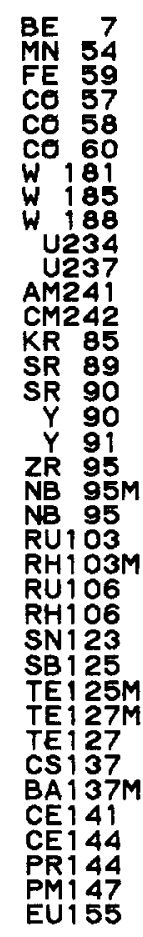

TOTAL

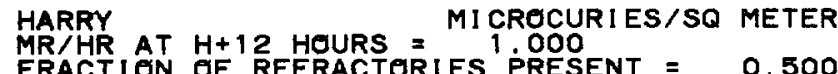

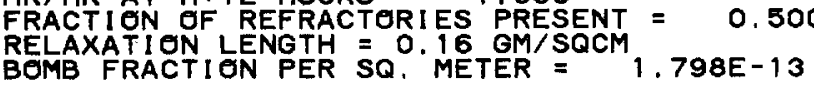

DEBRIS DECAY FROM 1 TO 50 YEARS

ZERO TIME 1. DOE+OO 1.5OE+OO 2.0OE+OO 3.5OE+OO 5. OOE+OO 7. OOE+OO $1.00 E+01 \quad 2.00 E+01 \quad 3.50 E+01 \quad 5.00 E+01$

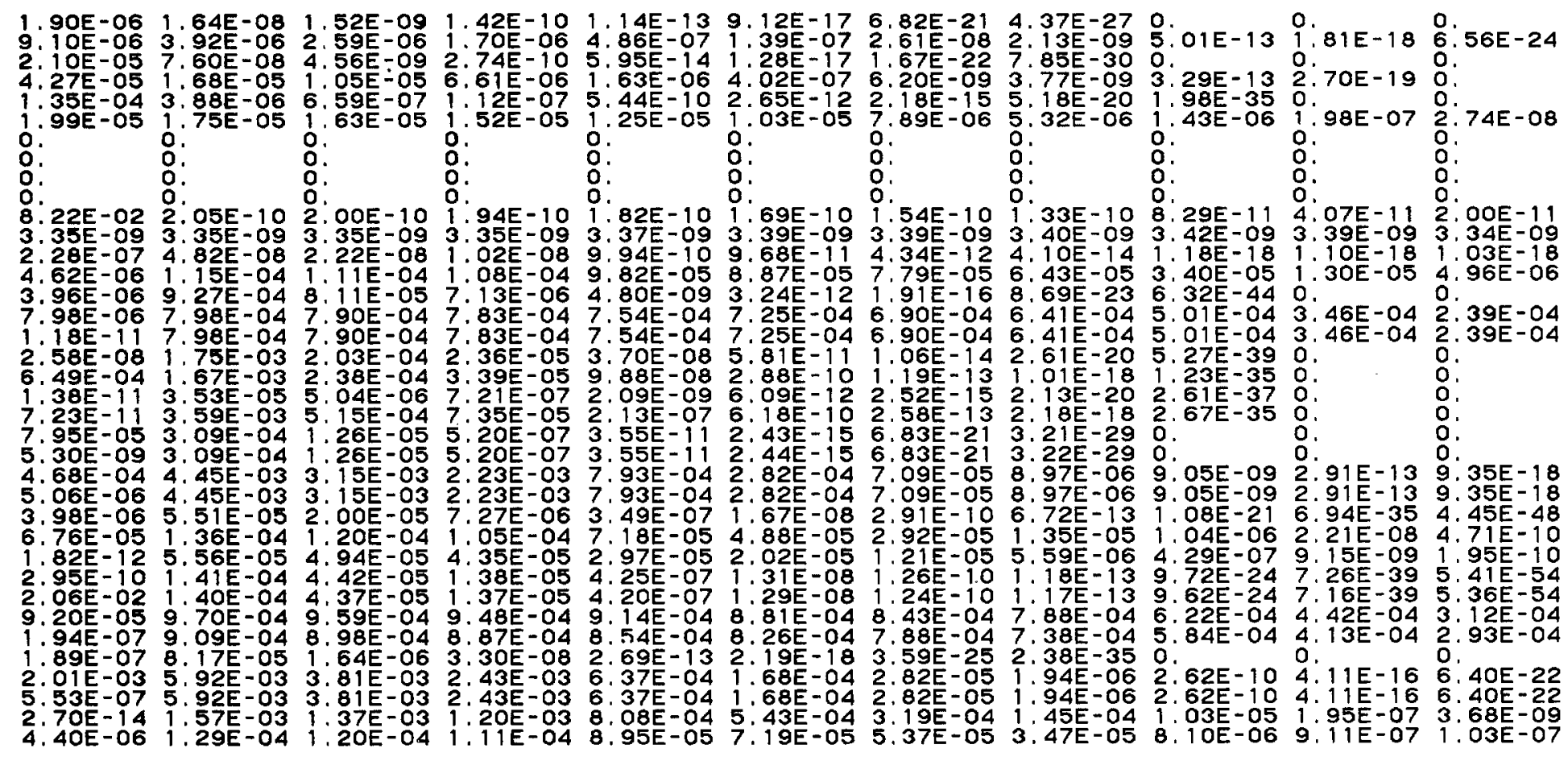

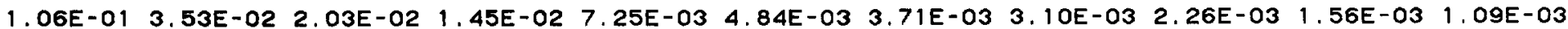


PAGE $1-23$

\begin{tabular}{|c|c|c|c|}
\hline \multicolumn{4}{|c|}{ 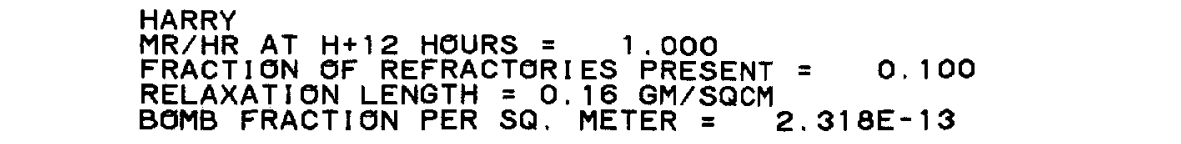 } \\
\hline TIME & & $M R / H R$ & MICROCURIES/SO METER \\
\hline 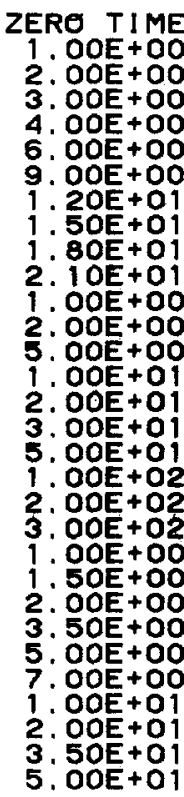 & HOURS & 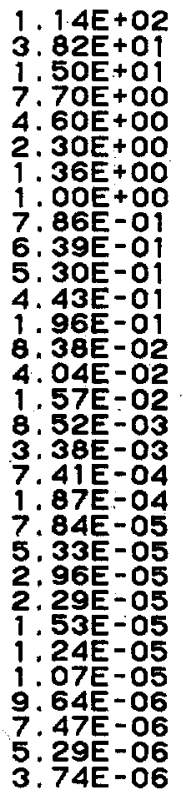 & 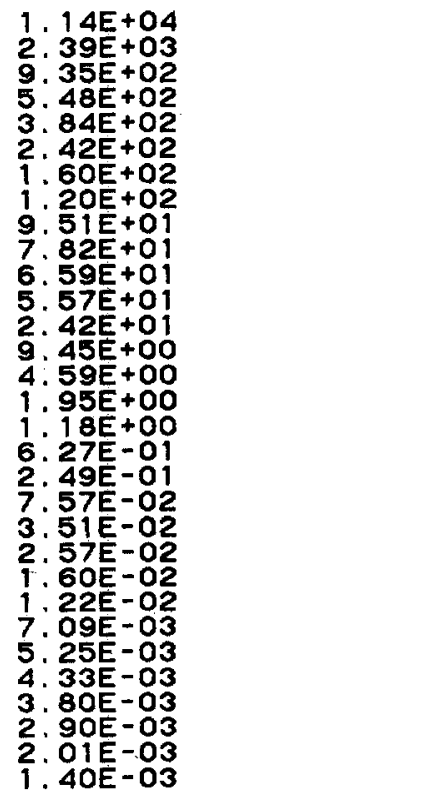 \\
\hline
\end{tabular}


DEBRIS DECAY FROM 1 TO 21 HOURS

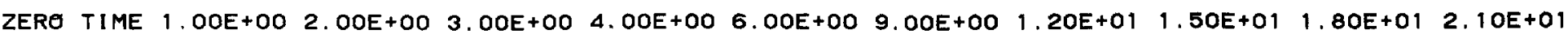

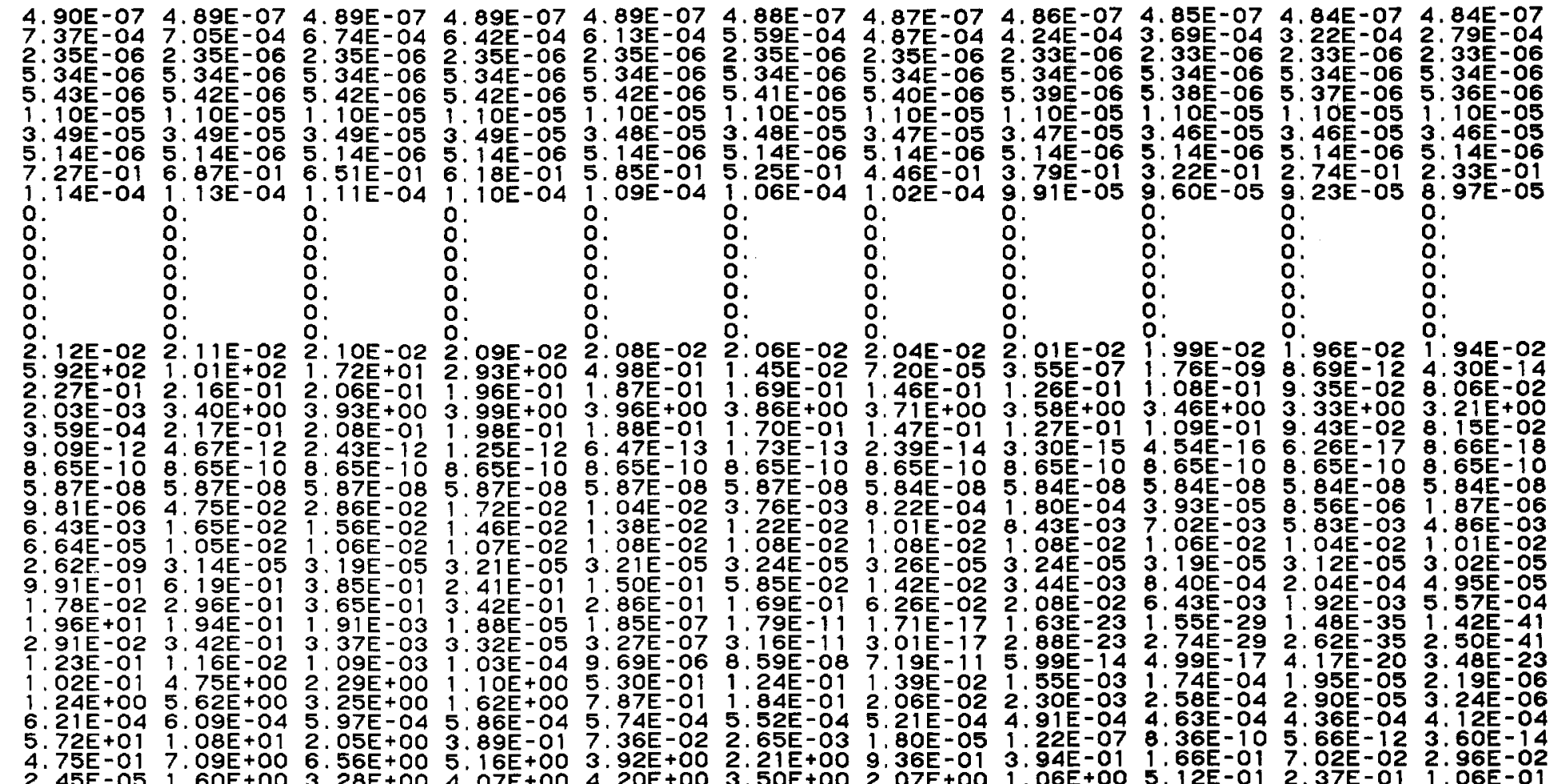

4.75E-01 7.09E+00 6.56E+00 5.16E+00 3.92E+00 2.21E+00 9.36E-01 3.94E-01 1.66E-012.02E-02 2.96E-02 
FRACTION OF REFRACTORI ES PRESENT = 0.100

RELAXATION LENGTH $=0.16$ GM SOCM $=0.100$
BOMB FRACTION PER SQ. METER $=2.318 E-13$

DEBRIS DECAY FROM 1 TO 21 HOURS

ZERO TIME 1. DOE+OO 2. OOE+OO $3.00 E+00 \quad 4.00 E+00 \quad 6.00 E+D 0 \quad 9.00 E+00 \quad 1.20 E+01 \quad 1.50 E+01 \quad 1.80 E+012.10 E+01$ $1.20 E-012.08 E+015$

$.61 E+001.52 E+00 \quad 4.10 E-01 \quad 3.00 E-02 \quad 5.94 E-04 \quad 1.17 E-05$ 2.32E-07 $4.59 E-092.05 E-11$

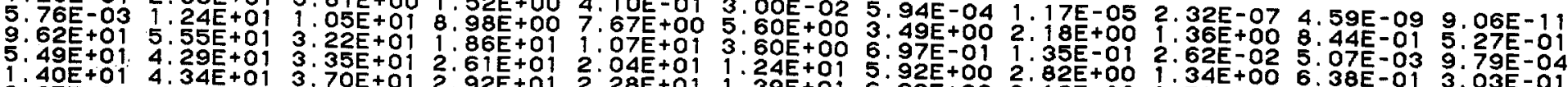

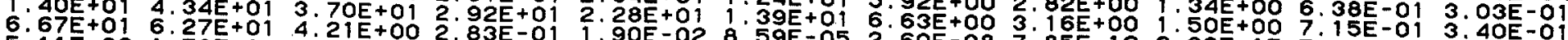
5.11E-06 1.79E-01 1.91E-01 1.91E-01 1.91E-01 1.99E-05 2.60E-08 7.85E-12 2.39E-15 7.24E-19 2.20E-22 1.03E-05 1.06E-03 1.06E-03 1.06E-03 1.06E-03 1.06E-03 1.91E-01 1.91E-01 1.91E-01 $1.90 E-011.90 E-01$

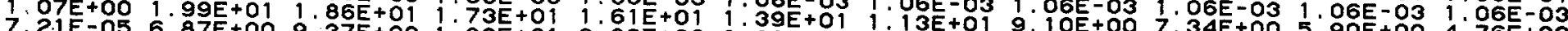

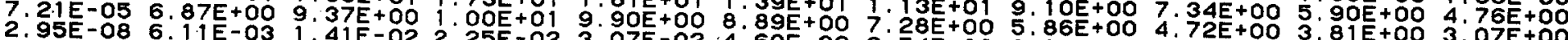
$1.76 E+007.88 E+006.41 E-022.25 E-02$ 3.07E-02 $4.60 E-026.54 E-028.09 E-029.37 E-021.81 E+003.07 E+00$ 8.13E-02 1. 66E+00 2.60E+O0 $3.73 E+00$ 3.6E+00 $2.19 E+001.02 E+004.73 E-012.19 E-011.02 E-014.73 E-02$ $7.10 E+01,22 E+00$ 6. $75 E-033.03 E+003.28 E+003.13 E+002.40 E+001.63 E+001.05 E+006.47 E-013.03 E-02$ 8. $66 E-022.80 E+002.63 E+002.46 E+002.30 E+002.29 E-121.06 E-181.79 E-25$ 3.02E-32 5.09E-39 8.58E-46 $1.03 E+011.41 E+011.82 E+002.34 E-013.02 E-025.02 E-041.64 E+001.34 E+001.08 E+008.91 E-017.24 E-01$ 4. $46 E+01$ 4. 20E+00 9.25E-02 2.04E-03 4.49E-05 2.18E-08 2.34E-13 2.31E-09 4.93E-12 $1.08 E-145.96 E-16$

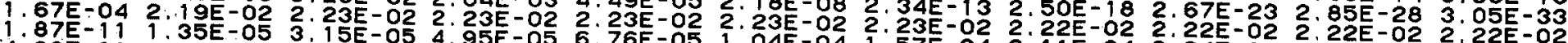

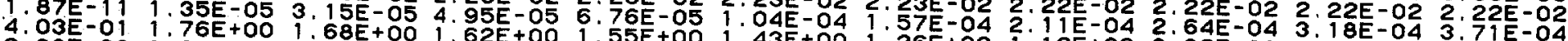

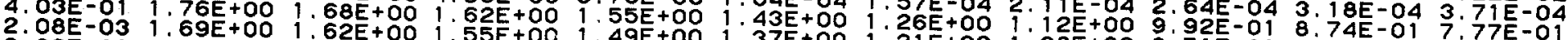
2.02E-01 $8.81 E-01 \quad 1.24 E+001.42 E+001.49 E+001.37 E+001.21 E+001.08 E+009.51 E-018.40 E-017.43 E-01$ 2.29E+00 1.01E+00 4.47E-01 1.98E-01 8.77E-02 1:.72E-02 1.35E+00 1.20E+00 1.06E+00 9.37E-01 8.33E-01 . 1 E - 03 4.99E-01 4.94E-01 4.89E-01 4.84E-01 4.74E-01 4.60E-01 4. $45 E-04$. $12 E-059.66 E-078.32 E-08$ $.12 E-08$ 4.76E-02 $8.93 E-021.26 E-01 \quad 1.59 E-012.12 E-012.69 E-01 \quad 4.45 E-014.32 E-014.19 E-014.06 E-01$ $30 E+002.01+01.53 E-025.11 E-05$ 9.93E-09 $1.93 E-12 \quad 3.76 E-163.40 E-013.45 E-01$

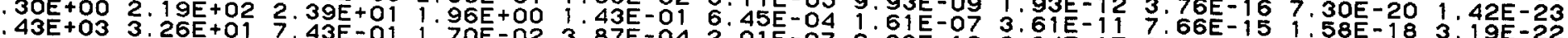

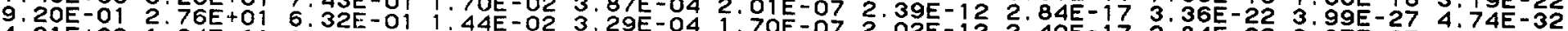
4. 31E+03 1.64E+01 3.75E-01 8.52E-03 1.

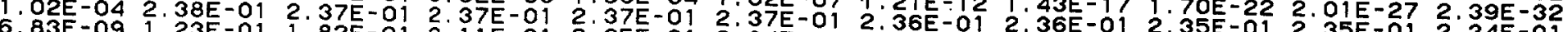
6.83E-09 1.23E-01 1.82E-01 2.11E-01 2.25E-01 2.34E-01 2.36E-01 2.36E-01 2.35E-01 $2.35 \mathrm{E}-012.34 \mathrm{E}-01$

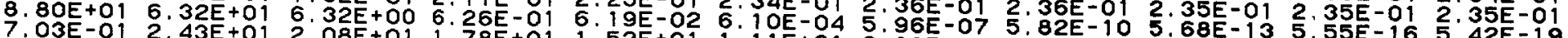
$4.62 E-03 \quad 2.43 E+0112.08 E+011.78 E+011.52 E+011.11 E+016.98 E+00 \quad 4.36 E+002.73 E+00 \quad 1.71 E+00 \quad 1.42 E-19$

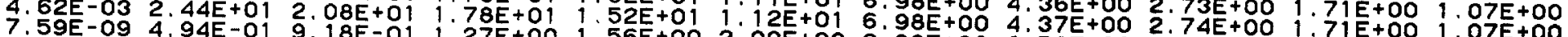
$6.03 E-041.14 E-021.14 E-021.27 E+001.56 E+002.00 E+002.38 E+002.56 E+002.62 E+002.59 E+002.57 E+00$

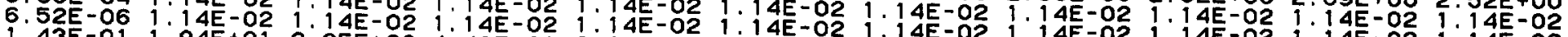

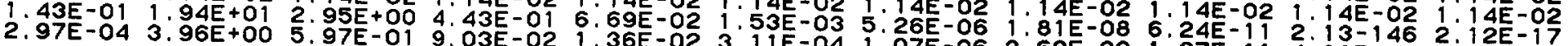


DEBRIS DECAY FROM 1 TO 21 HOURS

PDiog AG109M PDI 1 M AGII PDI1 AG112 AGI 15 CDII5 IN1 $15 M$ IN117M CDI 178 IN118 CDI19 INiT SNI21 SN123 SN125

SBi

SN127

SB127

SN128 SB128M SB128 SNi SB129 TE129M TE129 SB130

ZERO TIME $1.00 E+00 \quad 2.00 E+00 \quad 3.00 E+00 \quad 4.00 E+00 \quad 6.00 E+00 \quad 9.00 E+00 \quad 1.20 E+01 \quad 1.50 E+01 \quad 1.80 E+012.10 E+01$

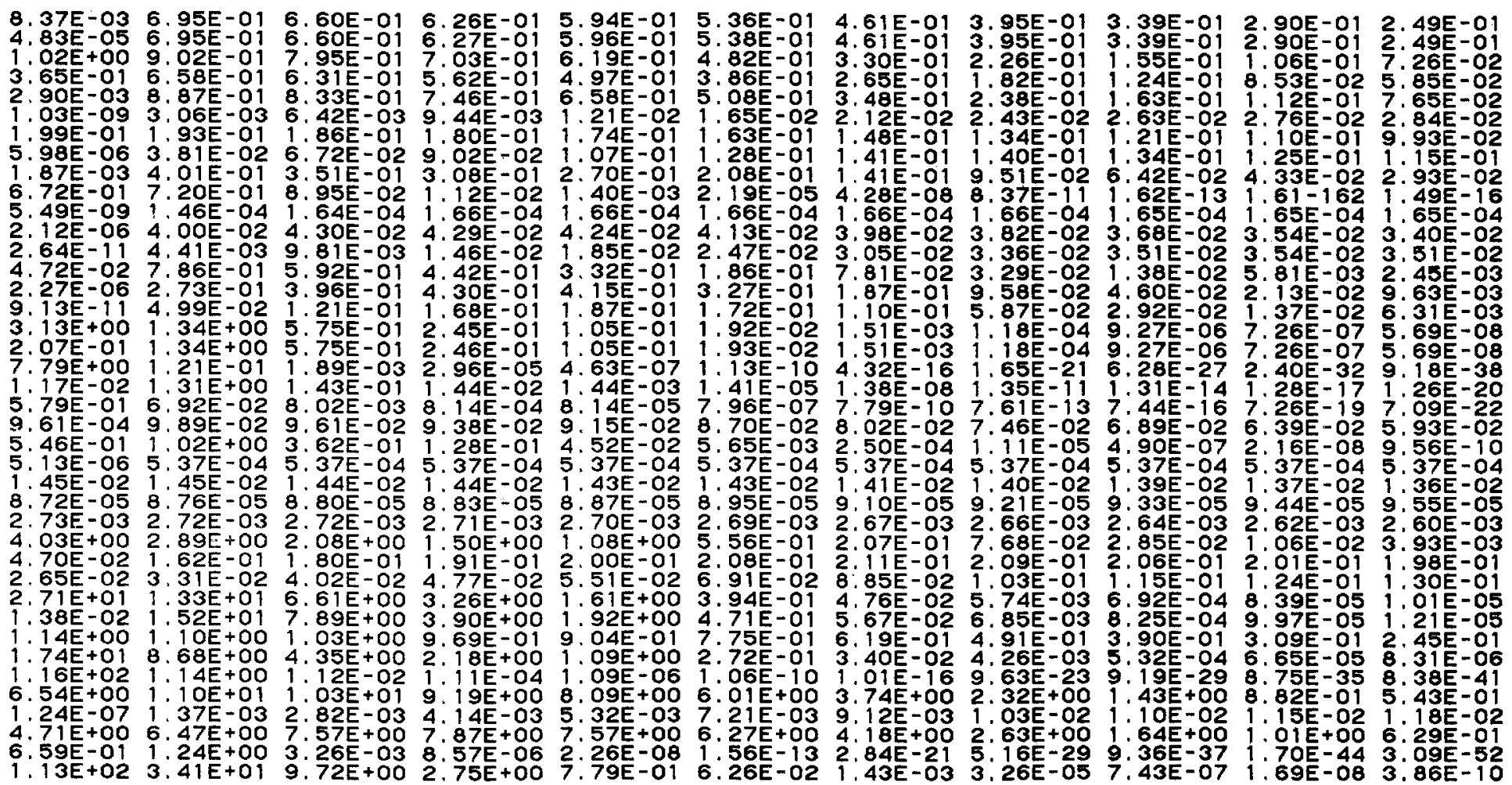


HARRY MICROCURIES/SQ METER

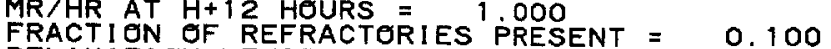
RELAXATION LENGTH $=0.16 \mathrm{GM} / \mathrm{SQCM}$.
BOMB FRACTION PER SQ. METER = 2.318E-13

DEBRIS DECAY FREM 1 TO 21 HOURS

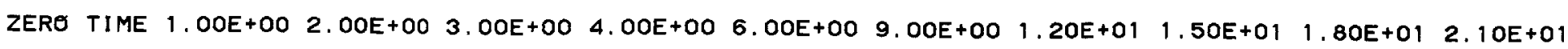

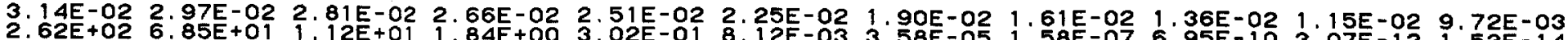

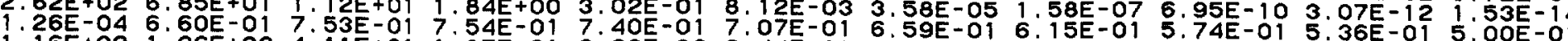

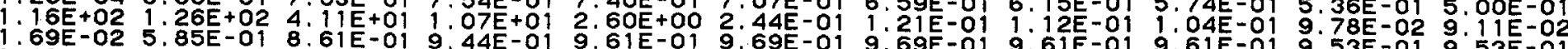
$1.69 E-025.85 E-01$ 8.61E-01 9.44E-01 9.61E-01 9.69E-01 9.69E-01 9.61E-01 9.61E-01 9.53E-01 9.53E-01

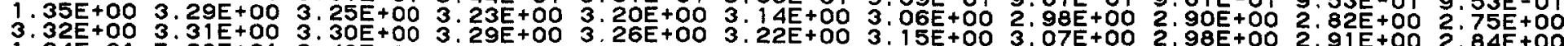

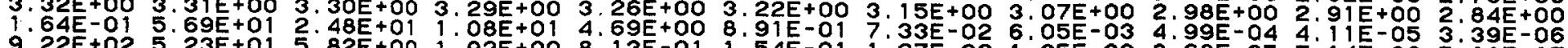

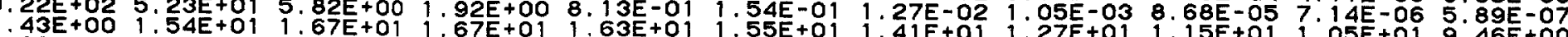

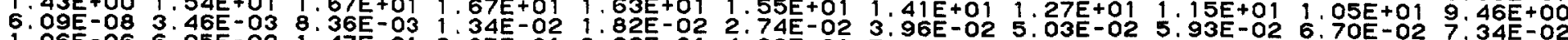

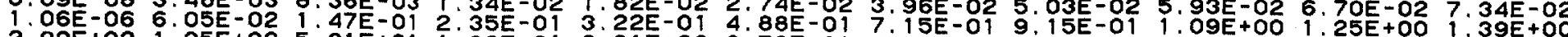

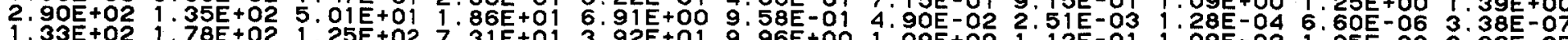

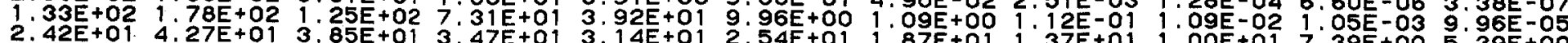

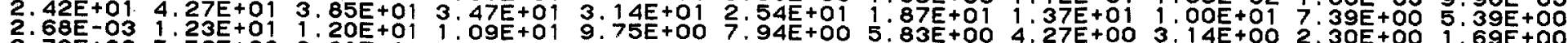

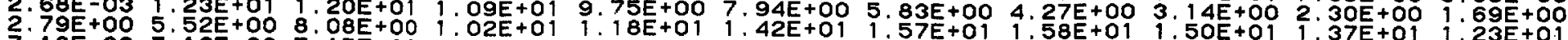

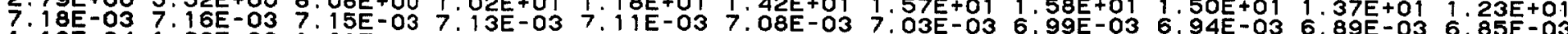

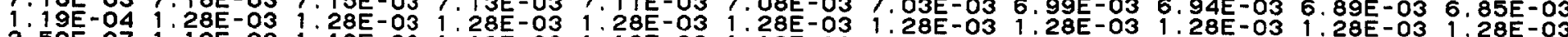

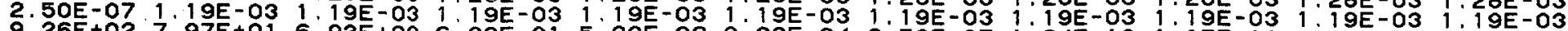

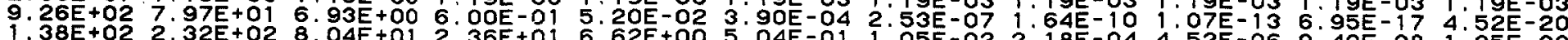

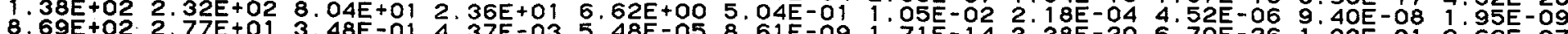

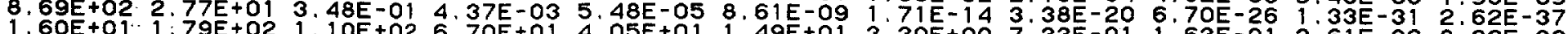
23E-01 $7.66 E-017.66 E-017.60 E-017.60 E-017.54 E-013.30 E+007.33 E-017.63 E-013.61 E-028.02 E-03$

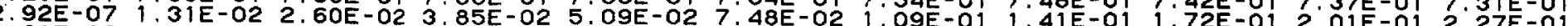

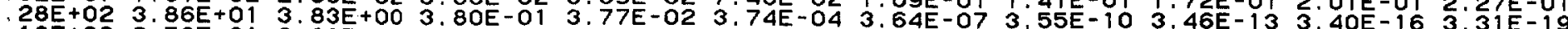
$.18 \mathrm{E}+002.50 \mathrm{E}+012.33 \mathrm{E}+011.97 \mathrm{E}+011.66 \mathrm{E}+011.16 \mathrm{E}+016.80 \mathrm{E}+003.98 \mathrm{E}+002.34 \mathrm{E}+001.37 \mathrm{E}+00 \mathrm{3} .05 \mathrm{E}-01$

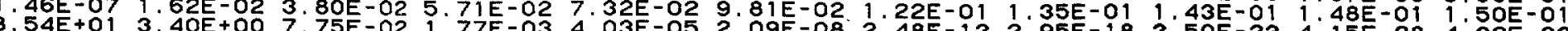

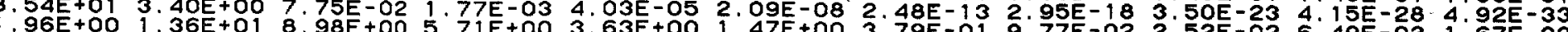
24E+01 $6.32 E+003.24 E-011.67 E-02 ~ 8.55 E-042.47 E 0 ~ 3.79 E-019.77=022.32 E-026.49 E-031.67 E-03$

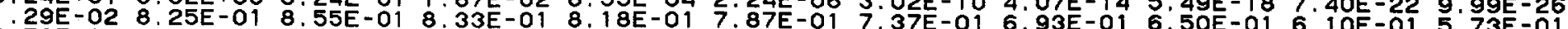
3.79E-09 1.26E-03 3.05E-03 4.82E-03 6.56E-03 9.92E-03 1. 46E-02 $1.91 E-02 \quad 2.32 E-02 \quad 2.70 E-023.065-02$

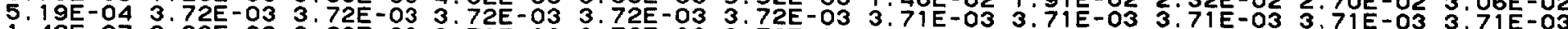
$1.42 \mathrm{E}-07$ 3. $39 \mathrm{E}-03$ 3. $09 \mathrm{E}-03$ 3.71E-03 3.72E-03 3.72E-03 3.72E-03 3.72E-03 3.71E-03 3.71E-03 3.71E-03 2. 01 . 作

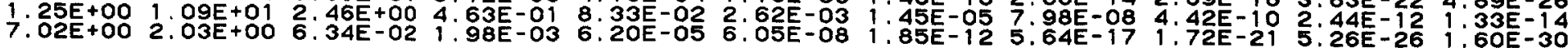


HARRY AT H+12 HEURS = MICROCURIES/SO METER FRACTION OF REFRACTORIES PRESENT $=0.100$

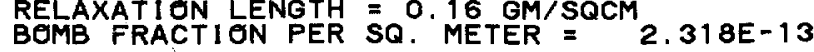

DEBRIS DECAY FROM 1 TO 21 HOURS

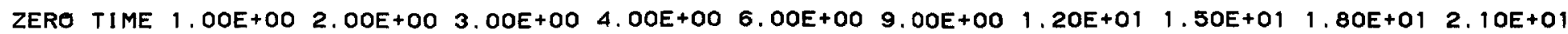

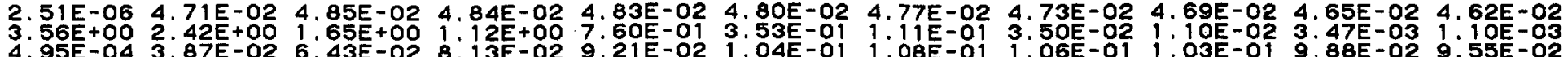

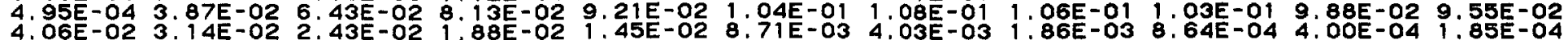
4. $06-02$ 3. 8.63E-03 1.01E-01 T O1E-01 $9.90 E-02$ 9.66E-02 $9.17 E-02$ 8.51E-02 7.90E-02 7.35E-02 6.80E-02 6.32E-02

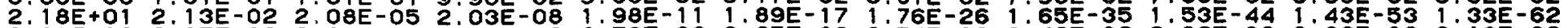
2. $90 \mathrm{E}-02$ 2.85E-02 $2.81 \mathrm{E}-02$ 2.77E-02 2.73E-02 2.65E-02 2.54E-02 $2.42 E-022.32 E-022.22 E-022.13 E-02$

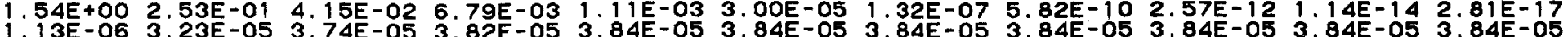
1. $13 E-06$ 3.23E-05 3.74E-05 3.82E-05 3.84E-05 3.84E-05 3.84E-05 3.84E-05 3.84E-05 3.84E-05 3.84E-05 \begin{tabular}{l}
$2.57 E-02$ \\
$7.51 .39 E-02$ \\
\hline
\end{tabular} $4.21 E-031.40 E-02$ 1:33E-02 1.27E-02 1.21E-02 1:11E-02 9.69E-03 8.46E-03 7.37E-03 6.41E-03 5.59E-03 .46E-01 $5.91 E-02$ 2.39E-02 9.68E-03 3.92E-03 6.41E-04 4.27E-05 2.83E-06 1.88E-07 1.25E-08 8.29E- 10

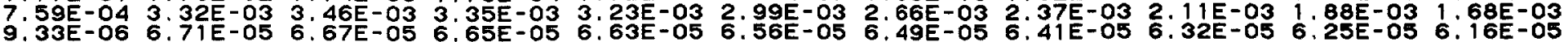


HARRY MICROCURIES/SQ METER

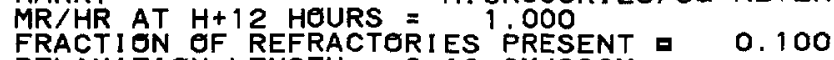
RELAXATION LENGTH $=0.16$ GM/SQCM $2.318 E-13$

DEBRIS DECAY FROM 1 TO 300 DAYS

ZERO TIME 1. OOE+OO $2.00 E+00 \quad 5.00 E+00 \quad 1.00 E+01 \quad 2.00 E+01 \quad 3.00 E+01 \quad 5.00 E+01 \quad 1.00 E+02 \quad 2.00 E+02 \quad 3.00 E+02$

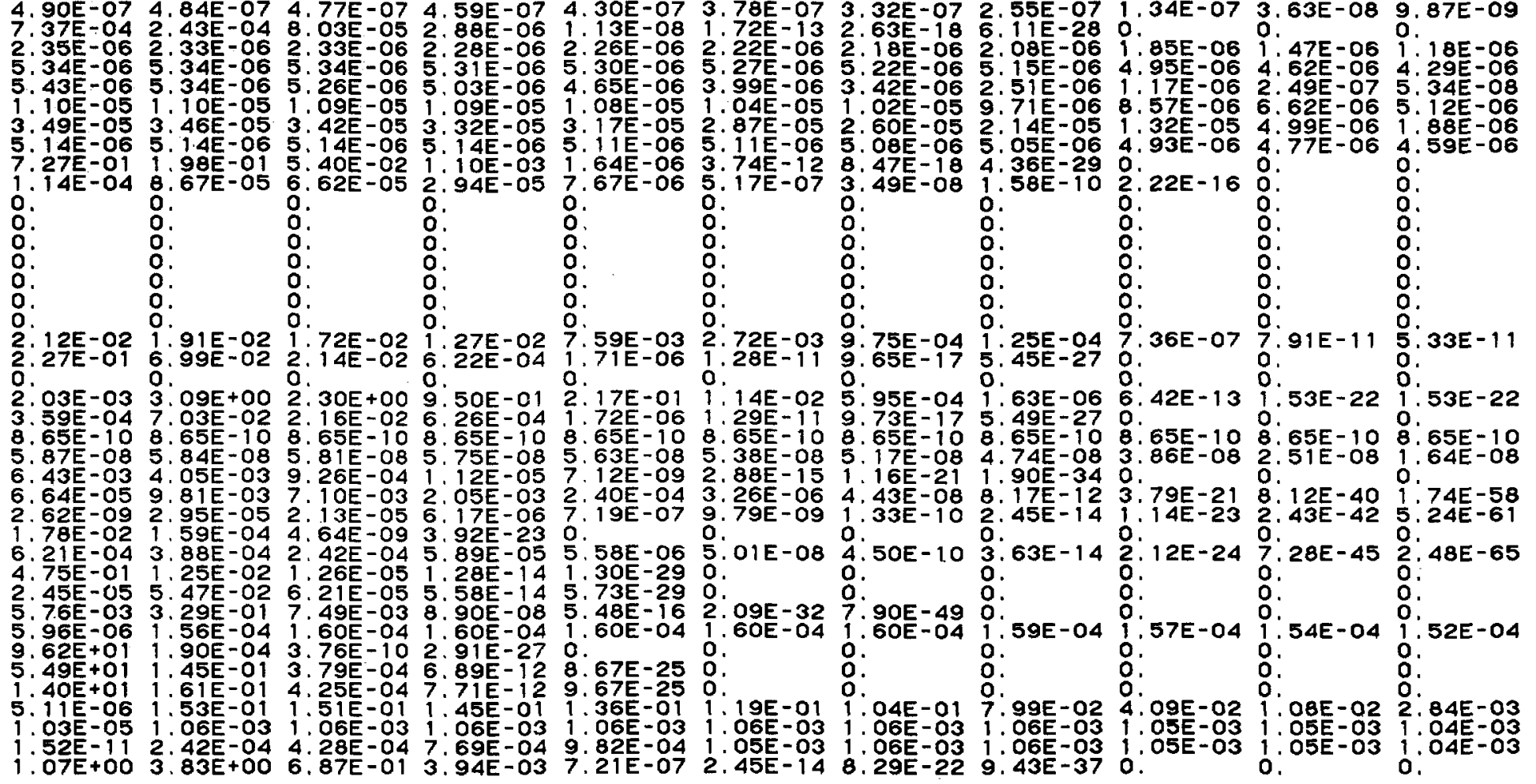


HARRY

MICROCURIES/SQ METER

MACTION OF REFRACTORIES PRESENT = 0.100

RELAXATI ON LENGTH $=0$. 16 GM/SQCM $2.318 E-13$

DEBRIS DECAY FROM 1 TO 300 DAYS

ZERO TIME 1. OOE+OO 2.0OE+OO $5.00 E+00 \quad 1.00 E+01 \quad 2.00 E+01 \quad 3.00 E+01 \quad 5.00 E+01 \quad 1.00 E+02 \quad 2.00 E+023.00 E+02$

\begin{tabular}{|c|c|c|c|c|c|c|c|c|c|c|}
\hline 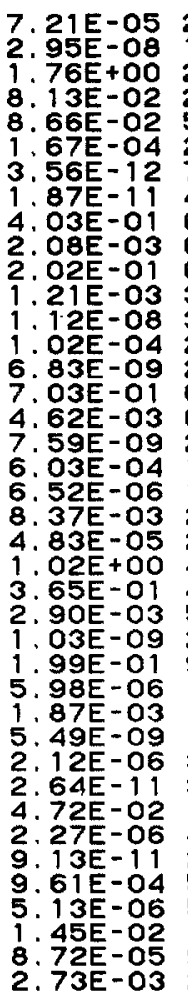 & $\begin{array}{l}2.48 \mathrm{E}+000 \\
1.18 \mathrm{E}-01 \\
2.20 \mathrm{E}-02 \\
2.31 \mathrm{E}-01 \\
5.85 \mathrm{E}-01 \\
2.09 \mathrm{E}-02 \\
7.09 \mathrm{E}-05 \\
4.05 \mathrm{E}-04 \\
6.86 \mathrm{E}-01 \\
6.59 \mathrm{E}-01 \\
6.89 \mathrm{E}-01 \\
3.93 \mathrm{E}-01 \\
3.46 \mathrm{E}-01 \\
2.34 \mathrm{E}-01\end{array}$ & $\begin{array}{l}4.43 \mathrm{E}-01 \\
1.39 \mathrm{E}-01 \\
4.74 \mathrm{E}-05 \\
2.56 \mathrm{E}-03 \\
1.14 \mathrm{E}-01 \\
2.07 \mathrm{E}-02 \\
1.29 \mathrm{E}-04 \\
8.00 \mathrm{E}-04 \\
2.58 \mathrm{E}-01 \\
2.48 \mathrm{E}-01 \\
2.59 \mathrm{E}-\mathrm{O} 1 \\
3.07 \mathrm{E}-01 \\
2.91 \mathrm{E}-01 \\
2.29 \mathrm{E}-\mathrm{O} 1 \\
2.30 \mathrm{E}-01 \\
1.58 \mathrm{E}-02 \\
1.58 \mathrm{E}-02 \\
1.58 \mathrm{E}+00\end{array}$ & $\begin{array}{l}2.5 \\
1.3 \\
4.7 \\
1.9 \\
8.5 \\
2.0 \\
2.4 \\
1.9 \\
1.3 \\
1.3 \\
1.3 \\
1.4 \\
1.3 \\
2.1 \\
2.1 \\
2.0\end{array}$ & $\begin{array}{l}E-01 \\
E-26 \\
E-19 \\
E-07 \\
E-02 \\
E-04 \\
-03 \\
E-04 \\
E-05 \\
E-04 \\
E-02 \\
E-02 \\
E-01 \\
E-01 \\
E-15 \\
E-15\end{array}$ & $\begin{array}{l}1.17 E-01 \\
0 . \\
3.93 E-40 \\
2.04 E-14 \\
1.70 E-02 \\
3.51 E-04 \\
6.15 E-03 \\
5.78 E-09 \\
5.55 E-09 \\
6.23 E-09 \\
3.52 E-03 \\
3.36 E-03 \\
1.68 E-O 1 \\
1.68 E-01 \\
8.10 E-32 \\
8.16 E-32 \\
3.79 E-04 \\
1.10 E-O 2 \\
1.10 E-O 2 \\
1.45 E-11\end{array}$ & $\begin{array}{l}1.04 E-01 \\
0 . \\
1.35 E-60 \\
1.68 E-21 \\
1.53 E-02 \\
3.24 E-04 \\
7.93 E-03 \\
3.25 E-13 \\
3.12 E-13 \\
3.50 E-13 \\
2.93 E-04 \\
2.80 E-04 \\
1.41 E-01 \\
1.41 E-01 \\
4.34 E-48 \\
4.35 E-48 \\
3.68 E-06 \\
1.08 E-02 \\
1.08 E-02 \\
6.44 E-17 \\
6.44 E-17 \\
4.01 E-40 \\
3.22 E-40 \\
4.24 E-40 \\
2.06 E-03 \\
9.511 E-12 \\
1.12 E-11\end{array}$ & $\begin{array}{l}02 \\
\\
35 \\
02 \\
04 \\
03 \\
21 \\
22 \\
21 \\
06 \\
06 \\
02 \\
02\end{array}$ & 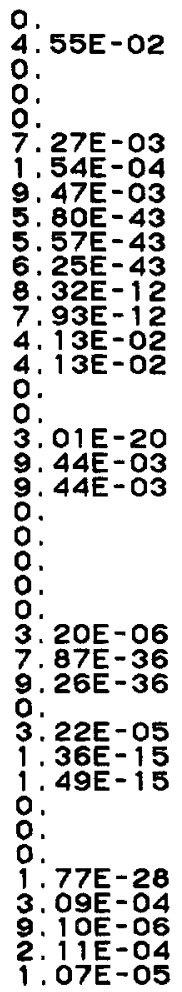 & $\begin{array}{l}1.4 \\
0.4 \\
0 . \\
0 . \\
2.5 \\
5.3 \\
4.5\end{array}$ & $\begin{array}{l}4 . \\
0 . \\
0 . \\
0: \\
8 . \\
1 . \\
1: \\
0 . \\
0 . \\
0 . \\
2 . \\
2 . \\
1 . \\
1 . \\
0 .\end{array}$ \\
\hline
\end{tabular}


MARRY MR

MRAHR AT H+12 HOURS $=$ MTI OOOO

RELAXATION LENGTH $=0.16 \mathrm{GM} / \mathrm{SQCM}$
BOMB FRACTION PER SQ. METER $=2.318 \mathrm{E}-13$

DEBRIS DECAY FROM 1 TO 300 DAYS

ZERO TIME $1.00 E+00 \quad 2.00 E+00 \quad 5.00 E+001.00 E+01 \quad 2.00 E+01 \quad 3.00 E+01 \quad 5.00 E+01 \quad 1.00 E+02 \quad 2.00 E+02 \quad 3.00 E+02$

SN127

TEI 27M

$\mathrm{SB} 128$

TE129M

TE1 29

TEI3IM

I131

XEI3IM

TE132

1
$X$

XE133M

I 35

XE $135 \mathrm{M}$

CS136

CS137

BA139

BA 40

A 4

CEI 14

CA142

PR143

CEI 44

PR144

NDI4

ND1 49

PM149

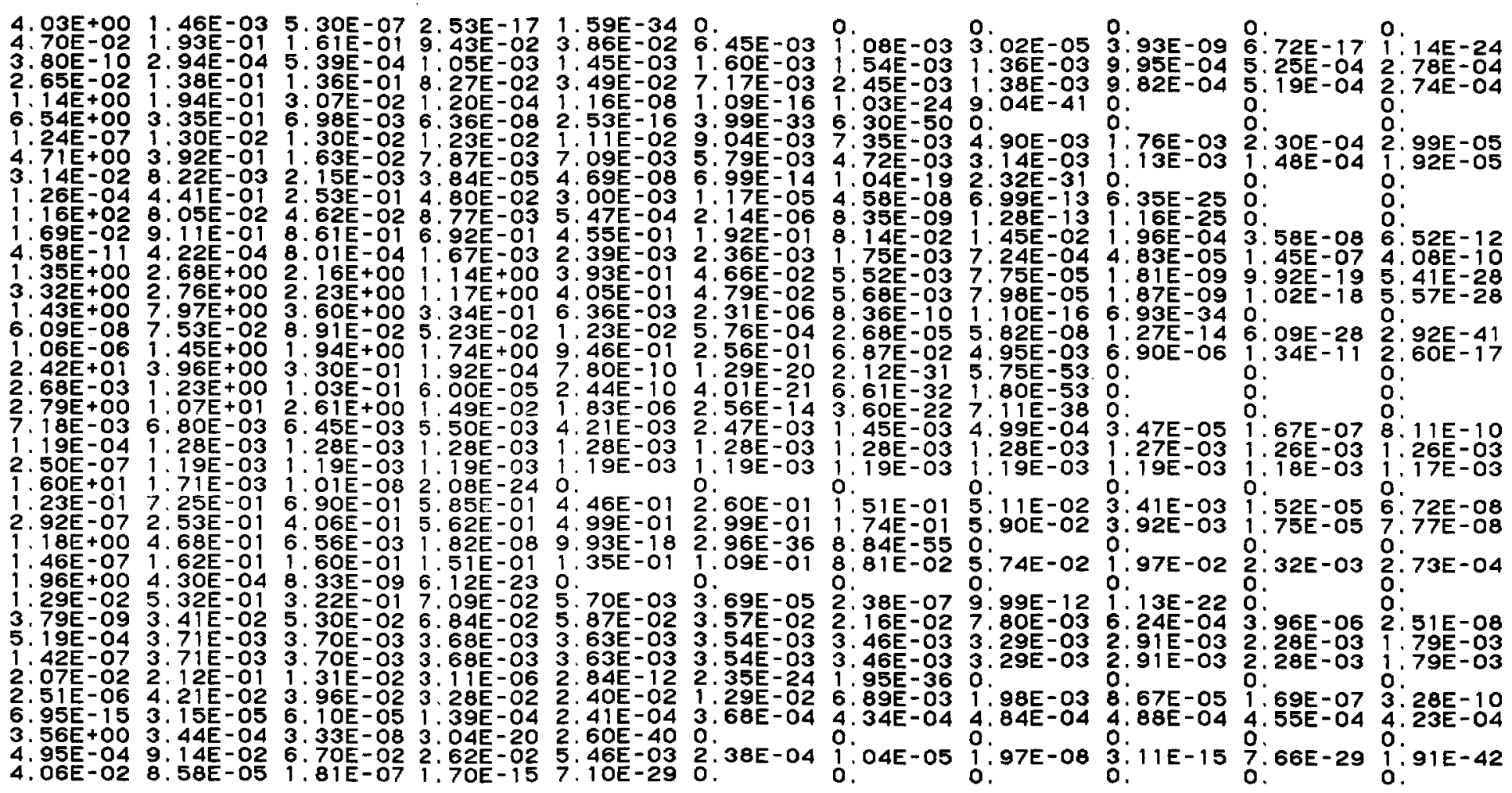




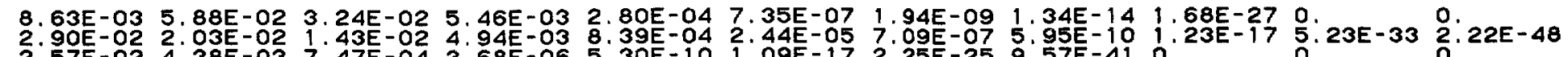
2.57E-02 4.38E-03 7.47E-04 3.63E-06 5.30E-05 3.81E-05 3.79E-05 3.76E-05 3.69E-05 3.54E-05 3.41E-05

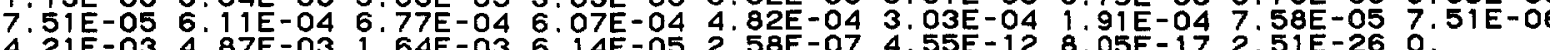

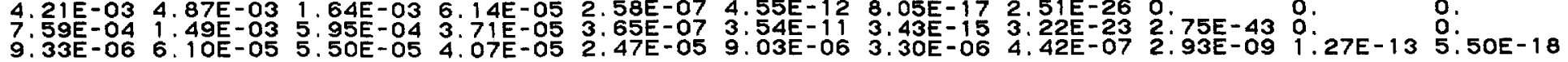

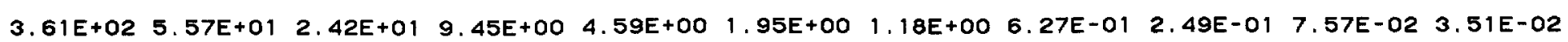

TOTAL 
HARRY $M$ MR

MRAHR AT H+12 HOURS $=$ MI 1 OOO

RELAXATION LENGTH $=$ O. 16 GM SQCM $=$
BOMB FRACTION PER SQ. METER $=2.318 E-13$

DEBRIS DECAY FROM 1 TO 50 YEARS

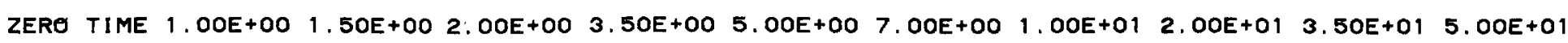

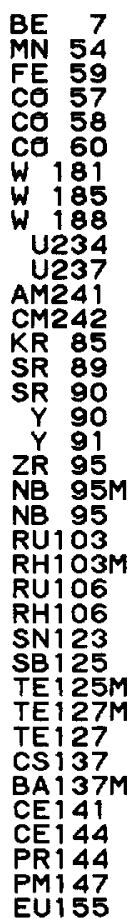

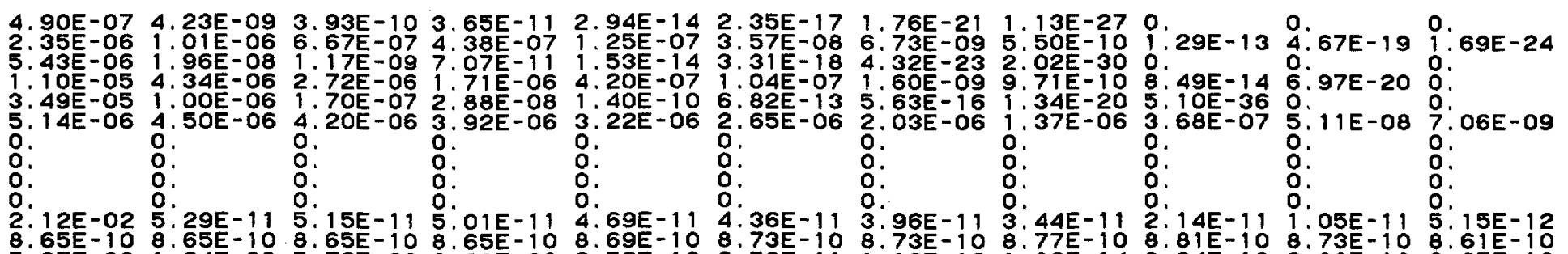

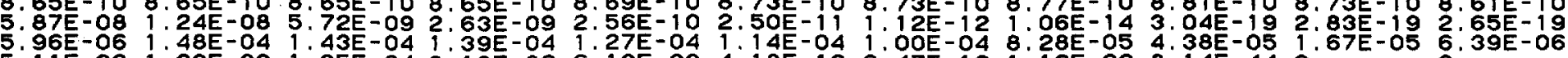

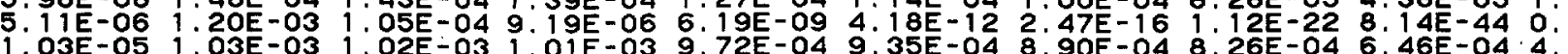

1.03E-05 1.03E-03 1.02E-03 1.01E-03 9.72E-04 9.35E-04 8.90E-04 8.26E-04 6. $46 E-04.4 .46 E-04$ 3.09E-04 1.52E-11 1.03E-03 1.02E-03 $1.01 E-03$ 9.72E-04 9.35E

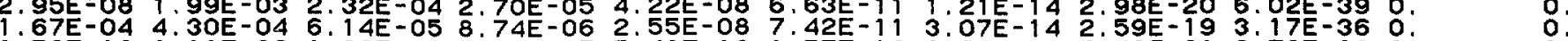

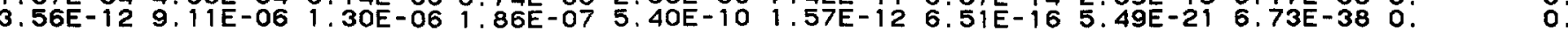
$87 E-11925 E-04$ (3)

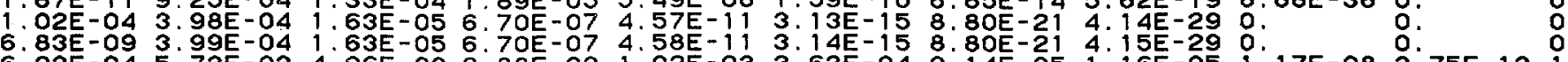

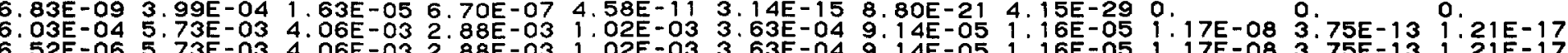

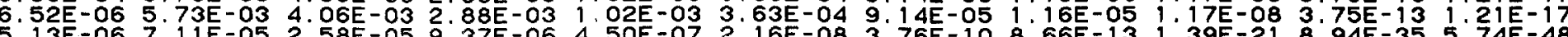
8. 72

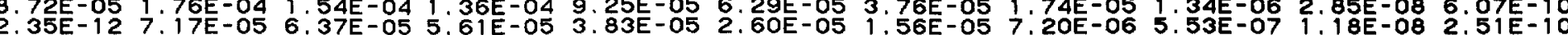
3.80E-10 1.82E-04 5.70E-05 1.78E-05 5.48E-07 1.69E-08 $1.62 E-10$ 1.52E-13 $1.25 E-23$ 9.36E-39 $6.97 E-54$ 2.65E-02 1.80E-04 5.63E-05 $1.76 E-05$ 5.42E-07 $1.67 E-08$ 1.60E-10 $1.51 E-13$ 1.24E-23 9.23E-39 6.91E-54

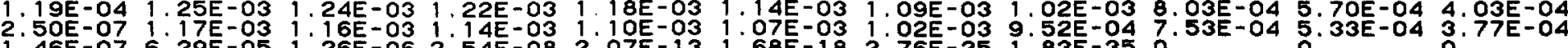

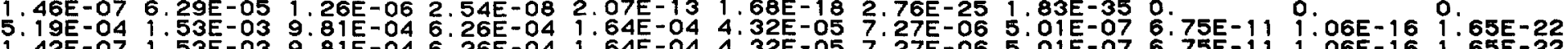
$1.42 E-07$ 1.53E-03 9.81E-04 6.26E-04 1.64E-04 4.32E-05 7.27E-06 $5.01 E-07$ 6.75E-11 $1.06 E-16 \quad 1.65 E-22$

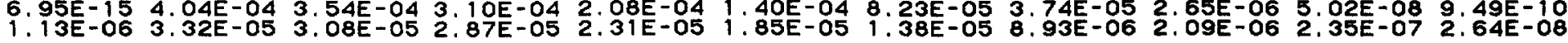

TOTAL

4.94E-02 2.57E-02 $1.60 E-02 \quad 1.22 E-02 \quad 7.09 E-03 \quad 5.25 E-03 \quad 4.33 E-03 \quad 3.80 E-03 \quad 2.90 E-03 \quad 2.01 E-03 \quad 1.40 E-03$ 


\section{APPENDIX 2}

\section{HARRY EVENT}

\section{$\mathrm{mR} / \mathrm{hr}$}

$\begin{array}{lr}\text { Fraction of refractories present }=1.0 & \frac{\text { Page }}{2-1} \\ \text { Fraction of refractories present }=0.5 & 2-11 \\ \text { Fraction of refractories present }=0.1 & 2-21\end{array}$




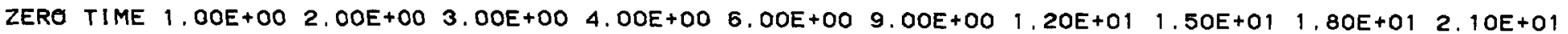

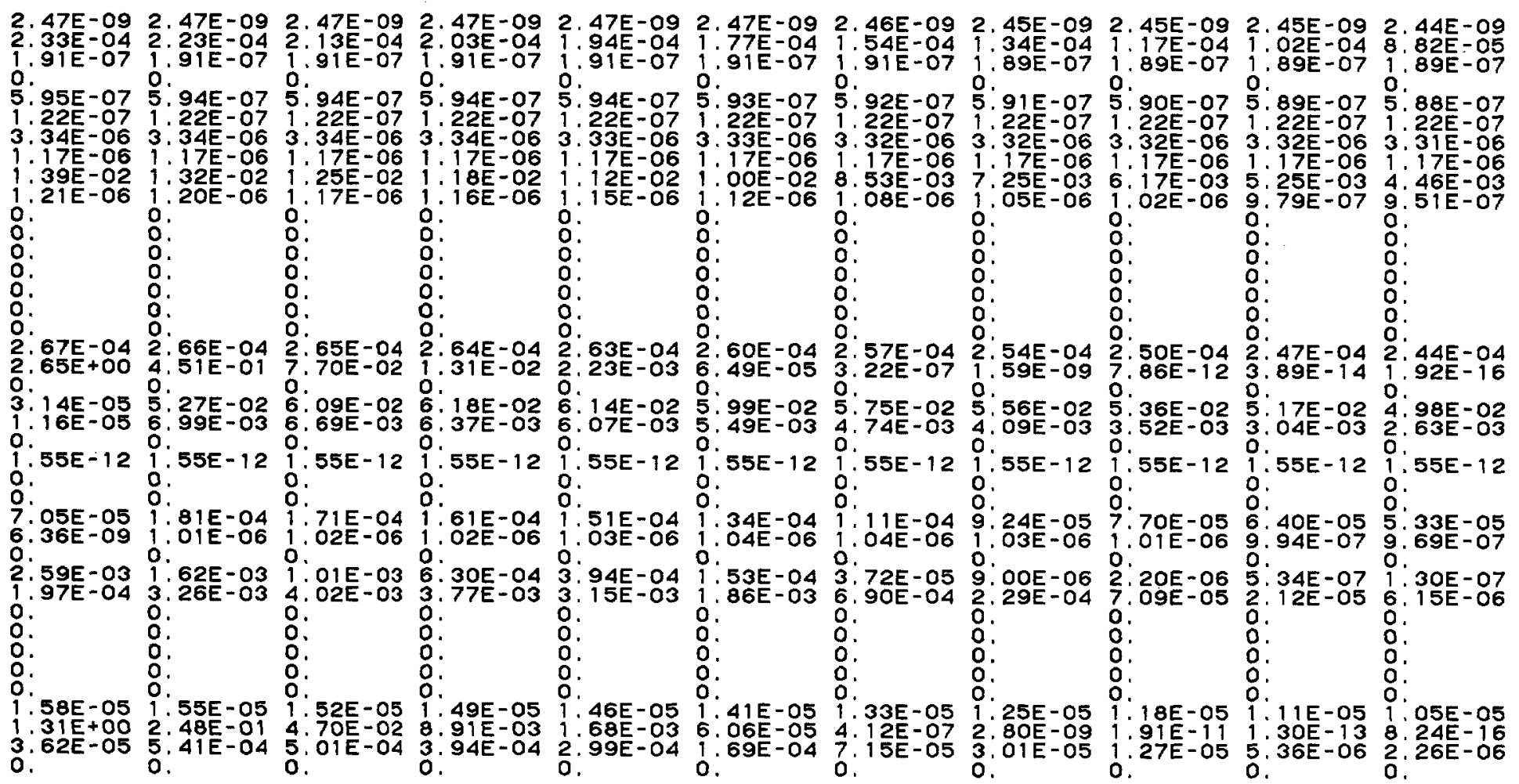


HARRY MR $H+12$ HOURS = MR/HR FRACTION OF REFRACTORIES PRESENT $=1,000$

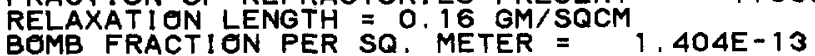

DEBRIS DECAY FROM 1 TO 21 HOURS

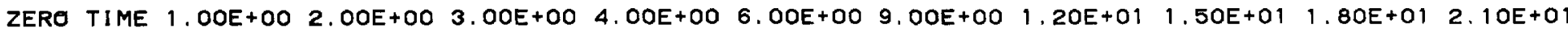

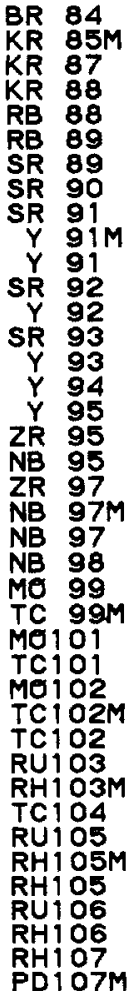

\begin{tabular}{|c|c|c|c|c|c|c|c|c|c|c|}
\hline $\begin{array}{l}65 E-02 \\
22 E+00\end{array}$ & $\begin{array}{c}38 E-01 \\
15 E+00\end{array}$ & $\begin{array}{l}03 E-01 \\
74 E-02 \\
60 E-01 \\
47 E-02\end{array}$ & $\begin{array}{l}\text { o. } \\
0: \\
0 . \\
1: 60 E-01 \\
5: 20 E-03 \\
0 . \\
0 . \\
1: 49 E-01 \\
6.94 E-02\end{array}$ & $\begin{array}{l}\text { o: } \\
0 . \\
1.25 E-01 \\
3.48 E-04 \\
0 . \\
0: \\
1.38 E-01\end{array}$ & $\begin{array}{l}4.44 E-04 \\
0 . \\
0 . \\
0 . \\
7.60 E-02 \\
1.58 E-06 \\
0 . \\
0 . \\
1.20 E-01 \\
6.15 E-02\end{array}$ & $\begin{array}{l}8.77 E-06 \\
0 . \\
0 . \\
0 . \\
3.63 E-02 \\
4.77 E-10 \\
0 . \\
0 . \\
9.68 E-02 \\
5.03 E-02\end{array}$ & $\begin{array}{l}73 E-01 \\
73 E-02 \\
44 E-13 \\
82 E-02 \\
05 E-02\end{array}$ & $\begin{array}{l}\text { O: } 43 E-19 \\
0: \\
0 . \\
8.23 E-03 \\
4: 39 E-17 \\
0 . \\
0 . \\
\text { 6: } 32 E-02 \\
3\end{array}$ & $\begin{array}{r}11 \\
-03 \\
-20\end{array}$ & 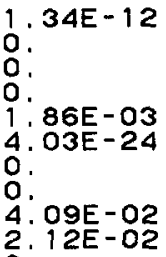 \\
\hline $\begin{array}{l}93 E-03 \\
51 E+01 \\
87 E-04 \\
51 E-01 \\
75 E+00 \\
22 E-05 \\
40 E-12 \\
77 E-03\end{array}$ & $\begin{array}{l}9.45 E-01 \\
3.93 E-02 \\
2.59 E-01 \\
2.22 E-02 \\
1.03 E+00 \\
4.48 E=01 \\
1.59 E-03 \\
1.01 E-06 \\
2.95 E-02 \\
1.22 E-01 \\
5.77 E-02\end{array}$ & $\begin{array}{l}6.16 E-02 \\
1.43 E-03 \\
2.09 E-02 \\
1.32 E-01 \\
9.87 E-03 \\
1.62 E-03 \\
2.37 E-06 \\
2.82 E-02 \\
1.17 E-01 \\
8.13 E-02\end{array}$ & $\begin{array}{l}7.33 \bar{E}-02 \\
7.91 \mathrm{E}-06 \\
1.95 \mathrm{E}-02 \\
1.70 \mathrm{E}-02 \\
2.18 \mathrm{E}-04 \\
1.62 \mathrm{E}-03 \\
3.72 \mathrm{E}-06 \\
2.71 \mathrm{E}-02 \\
1.12 \mathrm{E}-01\end{array}$ & $\begin{array}{l}7.78 \mathrm{E}-02 \\
4.36 \mathrm{E}-08 \\
1.82 \mathrm{E}-02 \\
2.20 \mathrm{E}-03 \\
4.79 \mathrm{E}-06 \\
1.62 \mathrm{E}-03 \\
5.07 \mathrm{E}-06 \\
2.60 \mathrm{E}-02 \\
1.08 \mathrm{E}-01\end{array}$ & $\begin{array}{l}3 \mathrm{E}-01 \\
11 \mathrm{E}-02 \\
33 \mathrm{E}-12 \\
39 \mathrm{E}-02 \\
35 \mathrm{E}-05 \\
33 \mathrm{E}-09 \\
32 \mathrm{E}-03 \\
8 \mathrm{E}-06 \\
4 \mathrm{E}-02 \\
31 \mathrm{E}-02\end{array}$ & $\begin{array}{l}22 E-01 \\
38 E-02 \\
35 E-19 \\
30 E-02 \\
32 E-08 \\
49 E-14 \\
32 E-03 \\
8 E-05 \\
2 E-02 \\
76 E-02\end{array}$ & $\begin{array}{l}E-02 \\
E-02 \\
E-26 \\
E-02 \\
E-10 \\
E-19 \\
E-03 \\
E-05 \\
E-02 \\
E-02\end{array}$ & $\begin{array}{l}-02 \\
-02 \\
-03 \\
-33 \\
-03 \\
-13 \\
-24 \\
-03 \\
-05 \\
-02 \\
-02 \\
-02\end{array}$ & $\begin{array}{l}2 \\
2 \\
9 \\
3 \\
3 \\
6 \\
9 \\
3 \\
5 \\
2 \\
2 \\
2\end{array}$ & $\begin{array}{l}3 E-03 \\
3 E-03 \\
2 E-46 \\
4 E-03 \\
4 E-17 \\
5 E-34 \\
1 E-03 \\
9 E-05 \\
0 E-02\end{array}$ \\
\hline 4 & $\begin{array}{l}\text { E-04 } \\
\text { E+0. } \\
\text { E-01 } \\
\text { E-01 }\end{array}$ & $\begin{array}{l}-03 \\
-03 \\
-02\end{array}$ & $\begin{array}{l}-03 \\
-03 \\
-03\end{array}$ & 03 & -03 & 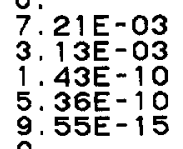 & $\begin{array}{l}03 \\
03 \\
14 \\
13\end{array}$ & $\begin{array}{l}03 \\
03 \\
18 \\
17 \\
24\end{array}$ & & \\
\hline & & & & & & & & & & \\
\hline 3 & 83 & 01 & 01 & $\begin{array}{l}1 \\
03\end{array}$ & 3 & $\overline{3}$ & & & & \\
\hline & 5 & 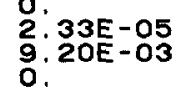 & 3 & 0 & .7 & 0 & & 95 & 4 & \\
\hline
\end{tabular}


MARRY

MRACTION OF REFRACTORIES PRESENT $=1.000$

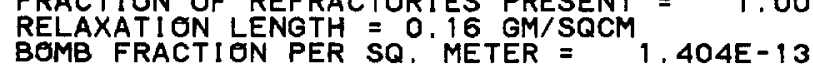

DEBRIS DECAY FROM 1 TO 21 HOURS

ZERO TIME 1. OOE+00 2. $200 E+00 \quad 3.00 E+00 \quad 4.00 E+00 \quad 6.00 E+00 \quad 9.00 E+00 \quad 1.20 E+01 \quad 1.50 E+01 \quad 1.80 E+01 \quad 2.10 E+01$

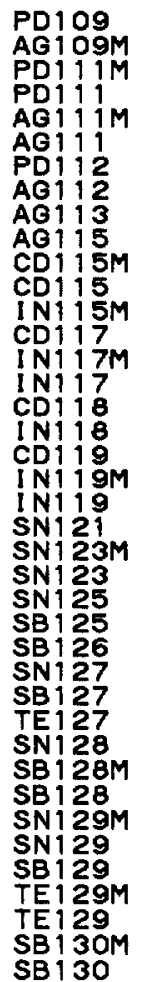

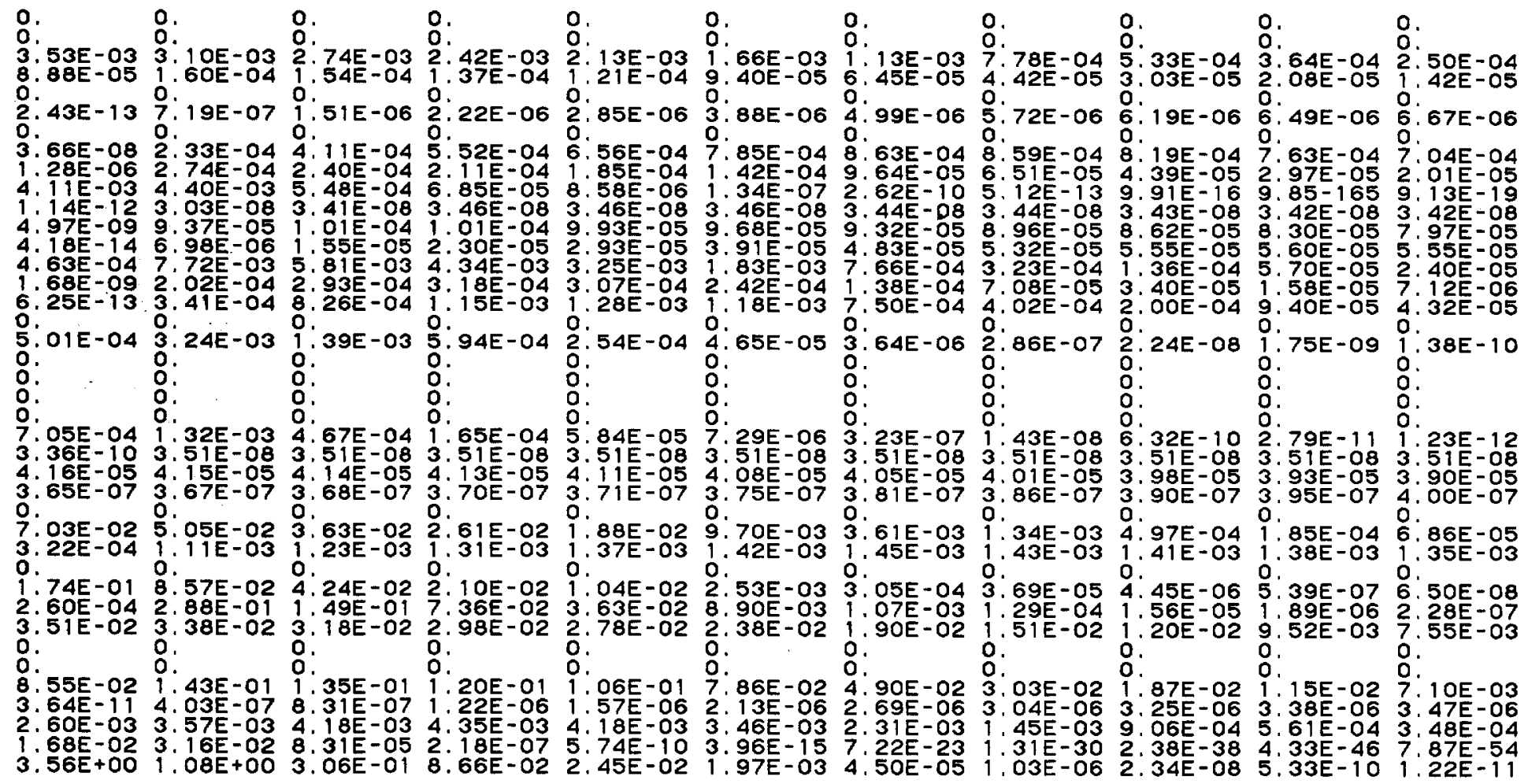


HARRY
MR/HR AT H+12 HOURS MR/HR
FRACTION OOF REFRACTORIES PREO
RELAXATION $M R / H R$

RELAXATION LENGTH $=0.16$ GM/SQCM $1.404 E-13$

DEBRIS DECAY FROM I TO 21 HOURS

ZERO TIME 1. OOE+OO 2. OOE+OO 3. OOE+OO 4. OOE+OO $\quad 6.00 E+00 \quad 9.00 E+00 \quad 1.20 E+01 \quad 1.50 E+01 \quad 1.80 E+01 \quad 2.10 E+01$

6.68E-04 6.32E-04 5.98E-04 5.65E-04 5.35E-04 4.78E-04 4.04E-04 3.42E-04 2.89E-04 2.45E-04 $2.07 E-04$

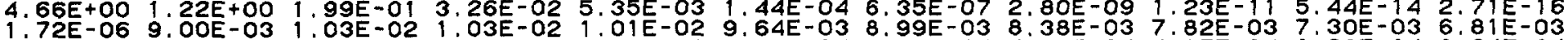
$4.64 E-015.04 E-01 ; .64 E-0144 E-02$ 1 $04 E-02$ 9.74E-04 4.84E-04 $4.47 E-044.17 E-043.90 E-043.64 E-04$ 6. $46 \mathrm{E}-052.24 \mathrm{E}-03$ 3. $30 \mathrm{E}-03$ 3. $02 \mathrm{E}-03$ 3.68E-03 3.71E-03 3.71E-03 3.68E-03 3.68E-03 $3.65 \mathrm{E}-03 \quad 3.65 \mathrm{E}-03$ 2.77E-03 $6.73 E-03 \quad 6.66 \mathrm{E}-03$ 6.61E-03 6.55E-03 6.43E-03 6.26E-03 6.09E-03 5.94E-03 5.77E-03 5.62E-03 7.36E-02 7.34E-02 7.32E-02 7.29E-02 7.23E-02 7. $14 \mathrm{E}-02$ 6.98E-02 6.80E-02 6.62E-02 6.45E-02 6.29E-02 $3.58 E-03 \quad 1.24 E+005.40 E-012.35 E-01 \quad 1.02 E-011.94 E-02 \quad 1.60 E-03 \quad 1.32 E-04 \quad 1.09 E-058.97 E-077.40 E-08$

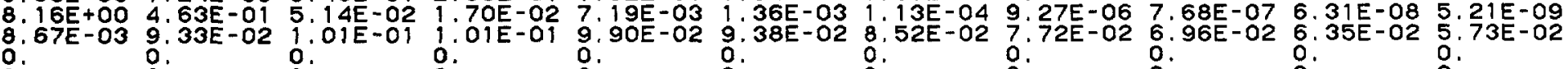
0 .

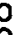
:. 0. o. O. 0 .

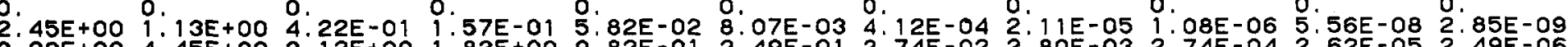

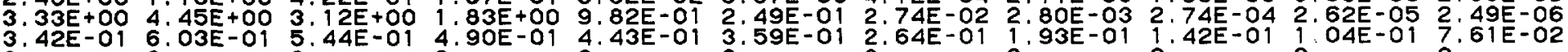

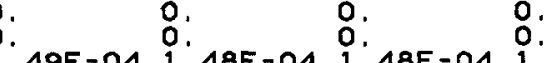
1. $49 \mathrm{E}-04$ 1: $48 \mathrm{E}-04$ 1: $48 \mathrm{E}-04$ 1: $48 \mathrm{E}-04$ i. : :. . : 0 . O $\begin{array}{lll}0 . & 0 & 0 \\ 0 & 0 & 0\end{array}$ 0. 0 0 . 0 . 7. $19 \mathrm{E}-067$ $19 E-06$ ? $19 E-067$ $1.44 \mathrm{E}-04$ .83E+00 4.76E+00 1.65E+00 4.85E-01 . $36-01$ 1. $04 E-022.15 E-044^{\circ}, 47 E-06$ . $.43 E-04$ 1:42E-04

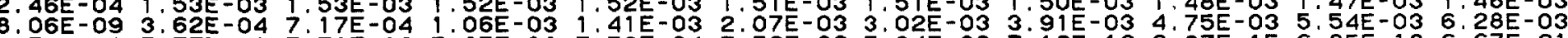

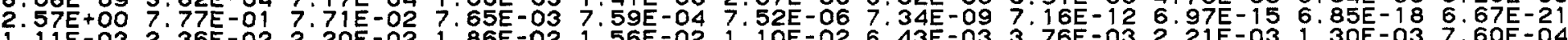
$2.42 E-102.36=-052.31 E-05$ 9. $50 E-05$, $22 E-04, .63 E-042.03 E-042.25 E-042.38 E-042.46 E-042.50 E-04$

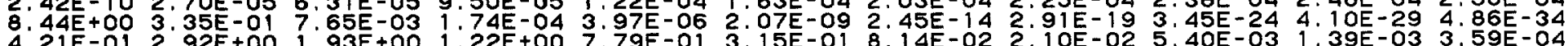

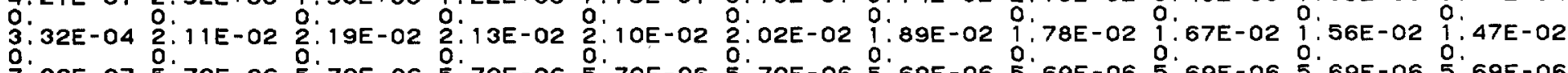

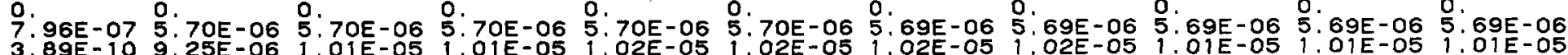
3.89E-10 9.25E-06 1.01E-05 1.01E-05 1.02E-05 1.02E-05 1.02E-05 1.02E-05 1.01E-05 $1.01 E-05$ 1.01E-05

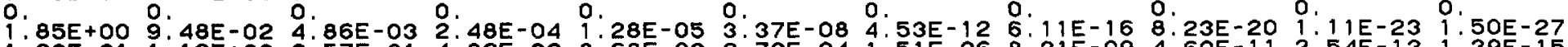

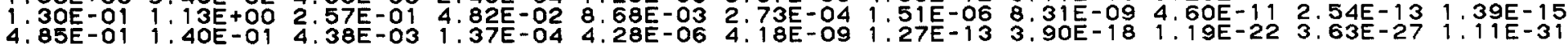




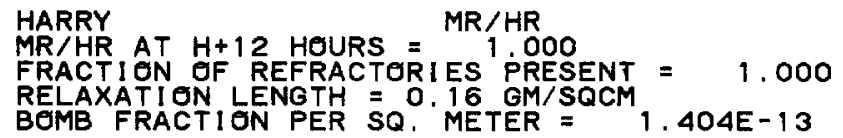

ZERO TIME 1. DOE+OO 2. $2.00 E+003.00 E+00 \quad 4.00 E+00 \quad 6.00 E+00 \quad 9.00 E+001.20 E+01 \quad 1.50 E+01 \quad 1.80 E+01 \quad 2.10 E+01$

3.37E-08 6.34E-04 6.52E-04 6.51E-04 6.49E-04 6.46E-04 6.41E-04 6.36E-04 6.31E-04 6.26E-04 6.21E-04

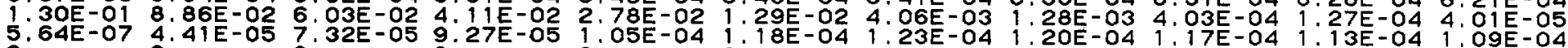

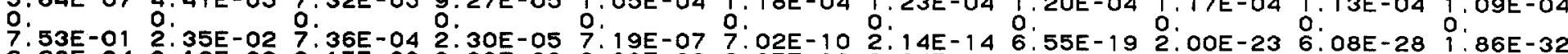

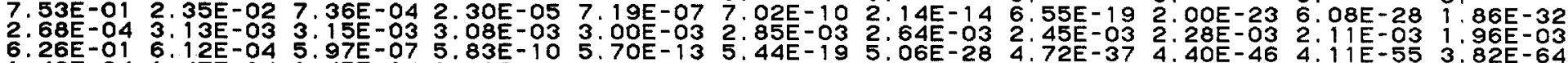

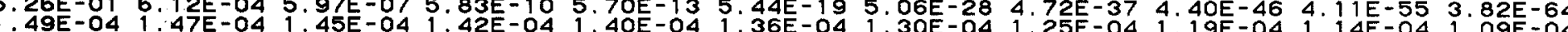

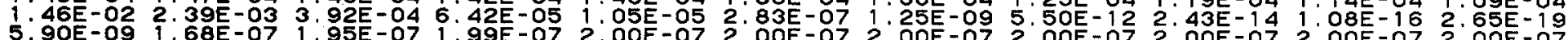

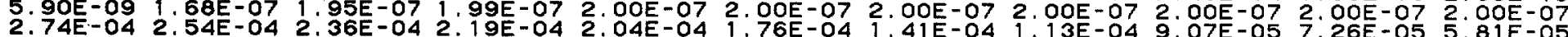

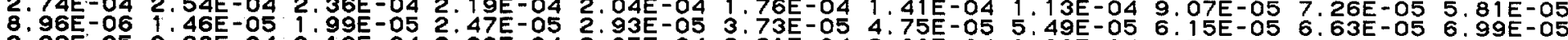

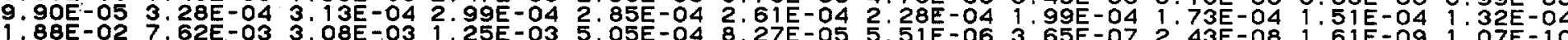
4:82E-03 4:79E-04 4:75E-05 4:71E-06 4:67E-07 4:61E-09 4:50E-12 4.39E-15 4: $29 \mathrm{E}-18$ 4. $19 \mathrm{E}-21$ 4: $10 \mathrm{E}-24$

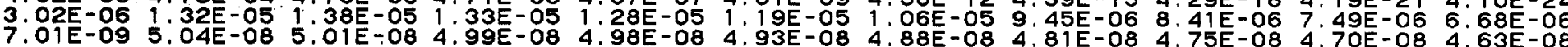

TOTAL

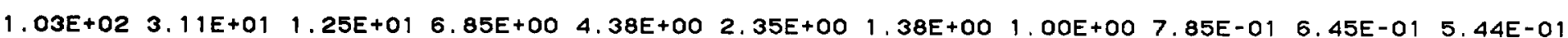


DEBRIS DECAY FROM 1 TO 300 DAYS

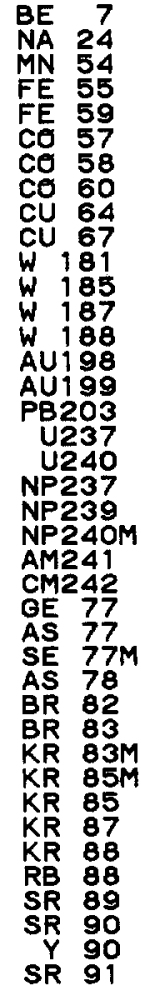

ZERO TIME 1. OOE+OO 2. $2.00 E+00 \quad 5.00 E+00 \quad 1.00 E+01 \quad 2.00 E+01 \quad 3.00 E+01 \quad 5.00 E+01 \quad 1.00 E+02 \quad 2.00 E+02 \quad 3.00 E+02$ 2. $47 \mathrm{E}-09$ 2.44E-09 2.41E-09 2.32E-09 2.17E-09 $1.91 \mathrm{E}-09 \quad 1.68 \mathrm{E}-09 \quad 1.29 \mathrm{E}-09 \quad 6.75 \mathrm{E}-10 \quad 1.83 \mathrm{E}-10 \quad 4.98 \mathrm{E}-11$

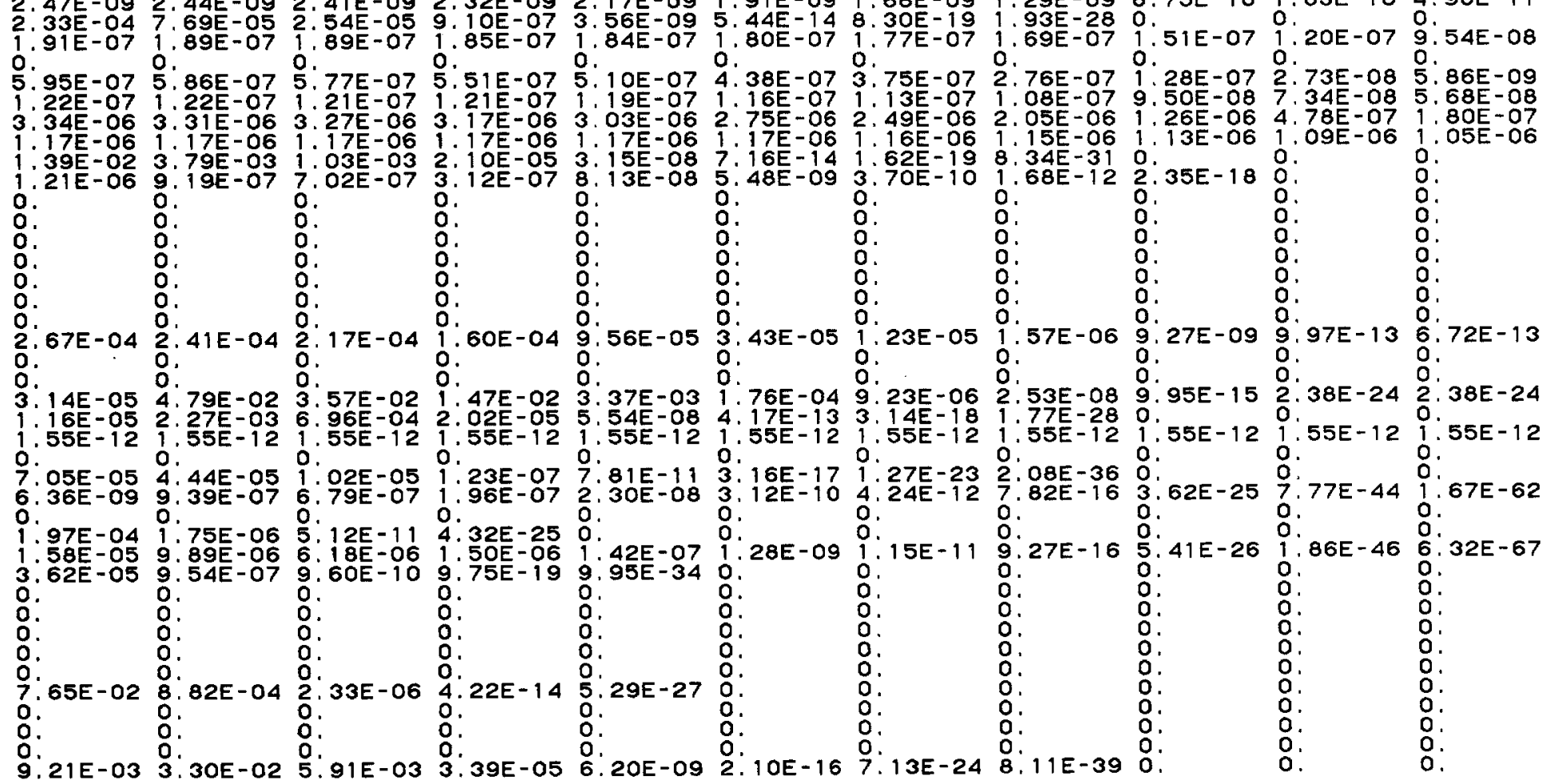


DEBRIS DECAY FROM 1 TO 300 DAYS

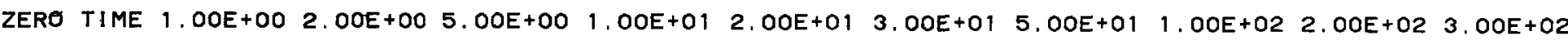
4. 98E-07 1.71E-02 3.06E-03 1.76E-05 3.23E-09 1.09E-16 3.69E-24 4.21E-39 0

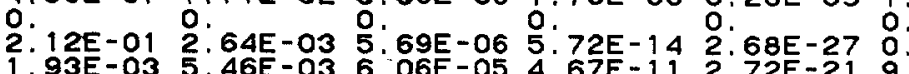

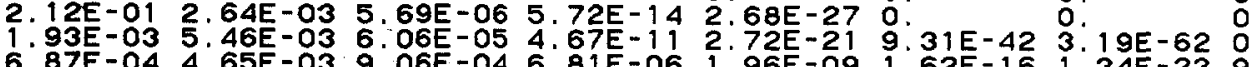

$6.87 E-04 \quad 4.65 E-039.06 E-046.81 E-06 \quad 1.96 E-09$ 1.62E-16 $1.34 E-23 \quad 9.12 E-38$ :

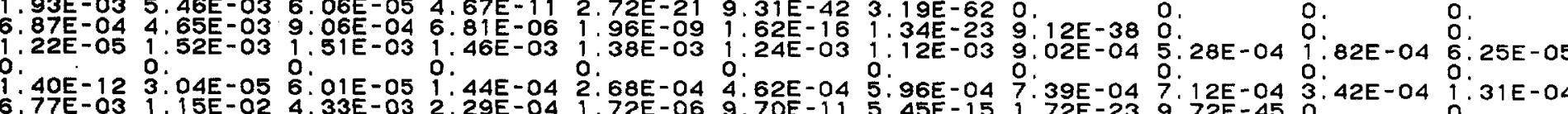

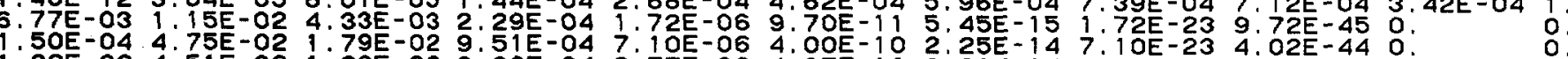

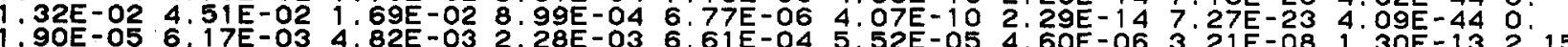

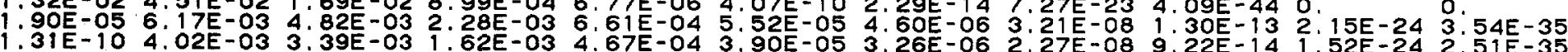

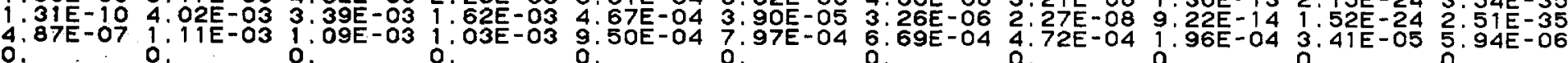

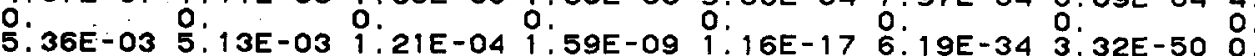

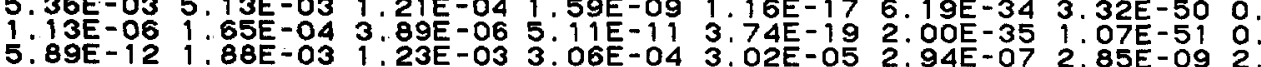

$-092,69 E-13$ : $233 E-23$

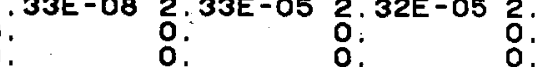
( 2. $29 \mathrm{E}-052$.

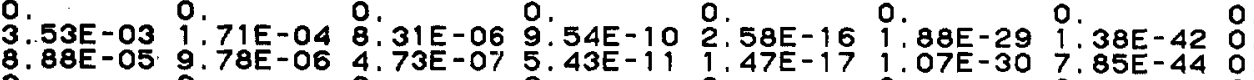
2. $43 E-13$ 6:.70E-06 6: 43E-06 4.90E-06 3.08E-06 $1.22 E-06$ 4. 85E-07 7.65E-08

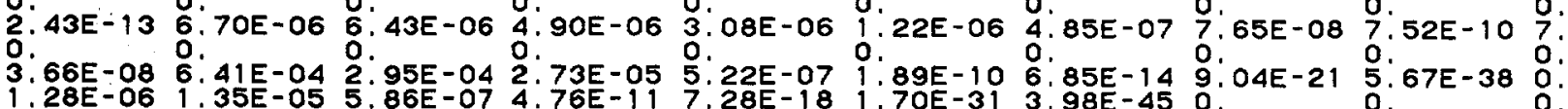

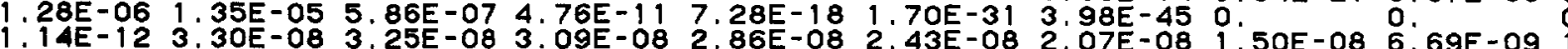

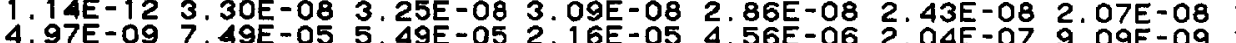

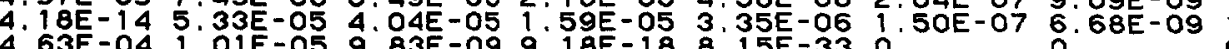
$1.68 \mathrm{E}-09$ 3.16E-06 3:66E-09 $3: 53 \mathrm{E}-183.13 \mathrm{~B}-33 \mathrm{O}$ 6.25E-13 1. $93 E-05$ 2. $29 E-082.21 E-17$ 1:96E-32 :

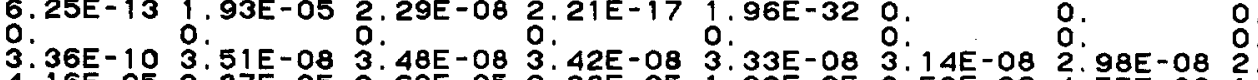

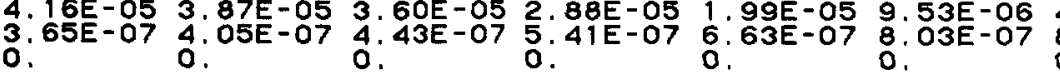

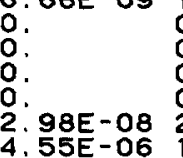
5OE-08 6. 69E-09 1.33E-09 2.66E-10

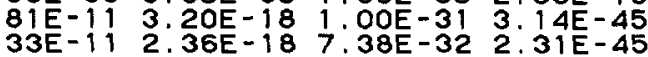
0
0 $\cos 0$ o. \begin{tabular}{ll}
$.04 \mathrm{E}-06$ & 2 \\
$9.01 \mathrm{E}-07$ & 8 \\
\hline
\end{tabular}

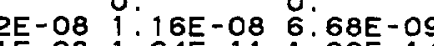
$\begin{array}{lll}61 E-08 & 1 \cdot 64 E-11 & 1: 03 E-1 \\ 84 E-07 & 8.25 E-07 & 7: 70 E-07\end{array}$ 
HARRY MR $H+12$ HEURS $=$ MR/HR 1 HOO

FRACTION OF REFRACTERIES PRESENT $=1.000$

RELAXATION LENGTH $=0.16 \mathrm{GM} / \mathrm{SQCM}$
BOMB FRACTION PER SQ. METER $=1.404 \mathrm{E}-13$

DEBRIS DECAY FROM 1 TO 300 DAYS

ZERO TIME $1.00 E+002.00 E+00 \quad 5.00 E+00 \quad 1.00 E+01 \quad 2.00 E+01 \quad 3.00 E+01 \quad 5.00 E+01 \quad 1.00 E+02 \quad 2.00 E+02 \quad 3.00 E+02$ 
MARRY

FRACTION OF REFRACTORIES PRESENT $=1.000$

RELAXATION LENGTH $=0.16$ OM/SOCM $1,404 E-13$

DEBRIS DECAY FROM 1 TO 300 DAYS

ZERE TIME 1. OOE+OO $2.00 E+00 \quad 5.00 E+00 \quad 1.00 E+01 \quad 2.00 E+01 \quad 3.00 E+01 \quad 5.00 E+01 \quad 1.00 E+02 \quad 2.00 E+02 \quad 3.00 E+02$

PM151

SM156

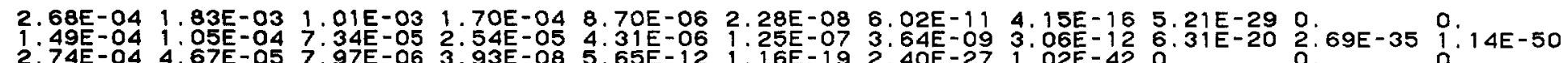

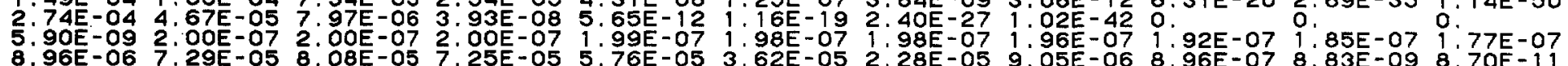

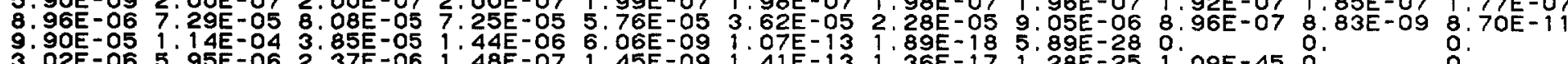

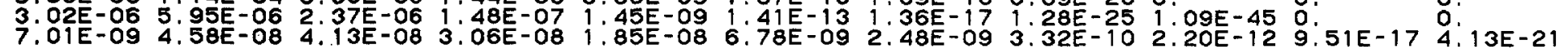

CDi5g

TOTAL

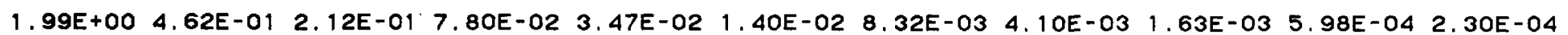


MARRY
MRR ARR AT H+12 HOURS $=$ MR/HR
FRACTION OF REFRACTERIES PRESENT $=1.000$

RELAXATION LENGTH DO 1 . 16 GM SQSQCM $=1.000$

DEBRIS DECAY FROM 1 TO 50 YEARS

ZERO TIME $1.00 E+00 \quad 1.50 E+00 \quad 2.00 E+00 \quad 3.50 E+00 \quad 5.00 E+00 \quad 7.00 E+00 \quad 1.00 E+01 \quad 2.00 E+01 \quad 3.50 E+01 \quad 5.00 E+01$

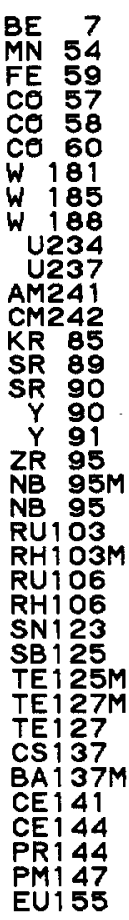

TETAL

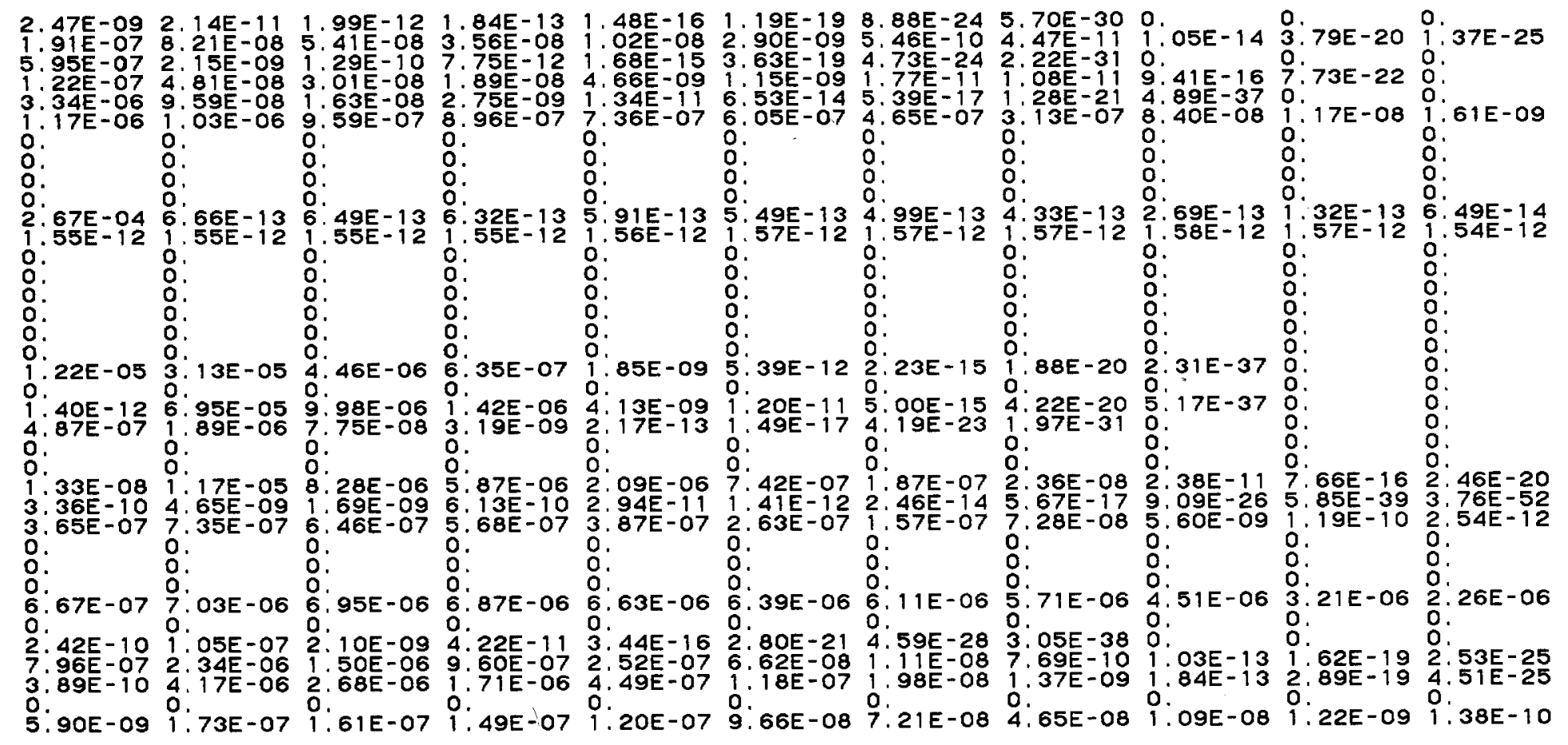

2.87E-04 1.30E-04 3.58E-05 1.91E-05 1.07E-05 8.28E-06 7.02E-06 6.17E-06 4.61E-06 3.22E-06 2.27E-06 
PAge $2-11$

HARRY
MRAHR AT H+12 HEURS $=$ MR/HR
FRACTION OF REFRACTORIES PRESENT $=0.500$

RELAXATION LENOTH $=0$. 16 GM SQCM $1.798 E-13$

DEBRIS DECAY FROM 1 TO 21 HOURS

ZERO TIME $1.00 E+00 \quad 2.00 E+00 \quad 3.00 E+00 \quad 4.00 E+00 \quad 6.00 E+00 \quad 9.00 E+00 \quad 1.20 E+01 \quad 1.50 E+01 \quad 1.80 E+01 \quad 2.10 E+01$

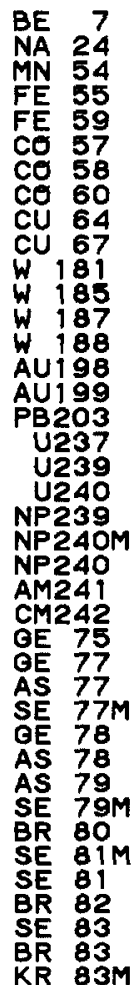

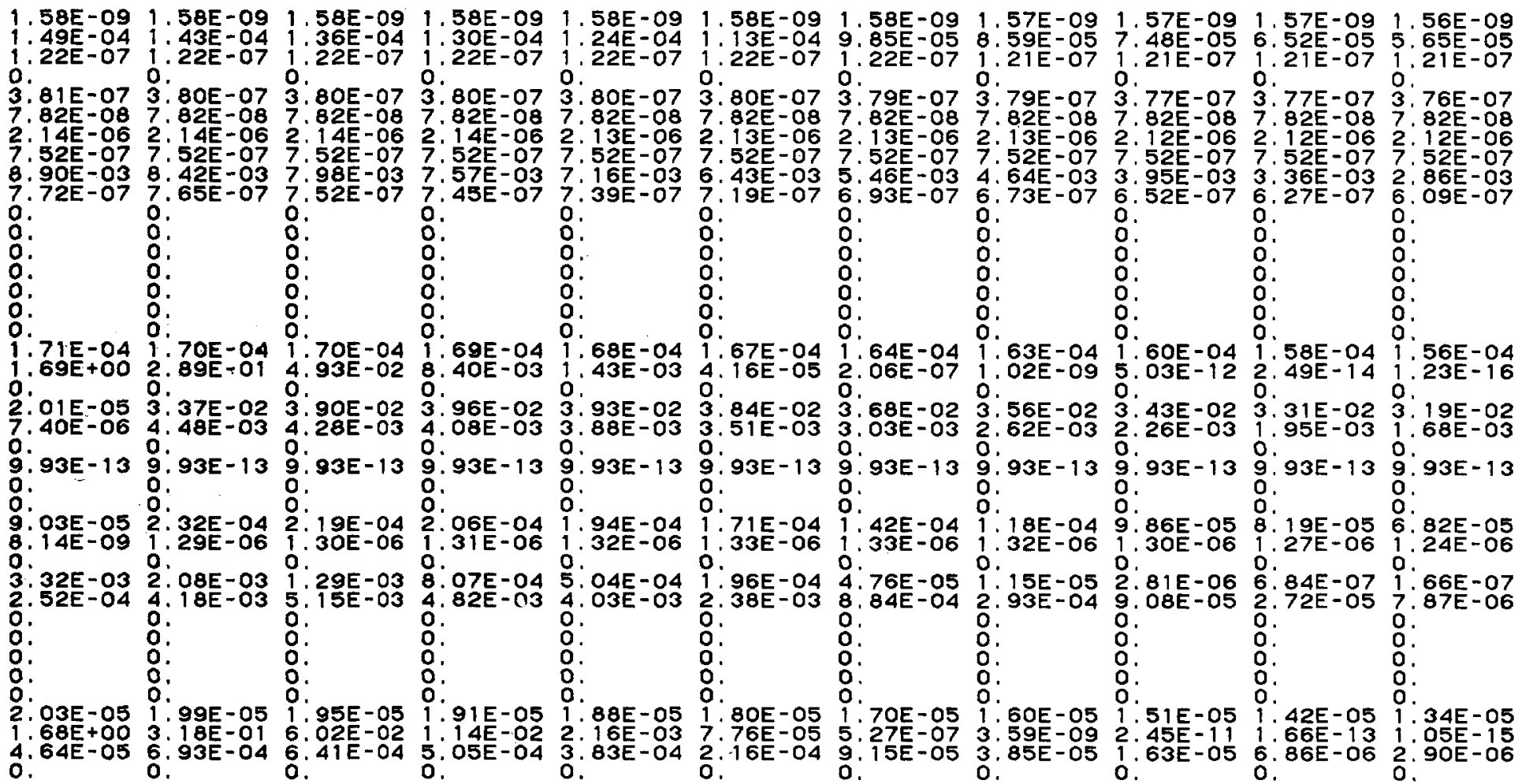


HARRY FRACTI ON OF REFRACTORIES PRESENT $=0.500$ RELAXATION LENGTH = O. 16 GM/SQCM $1.798 E-13$

DEBRIS DECAY FROM 1 TO 21 HEURS

ZERO TIME 1.OOE+OO 2. $2.00 E+00 \quad 3.00 E+00 \quad 4.00 E+00 \quad 6.00 E+00 \quad 9.00 E+00 \quad 1.20 E+01 \quad 1.50 E+01 \quad 1.80 E+01 \quad 2,10 E+01$

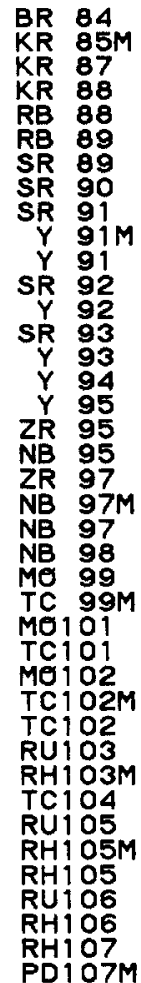

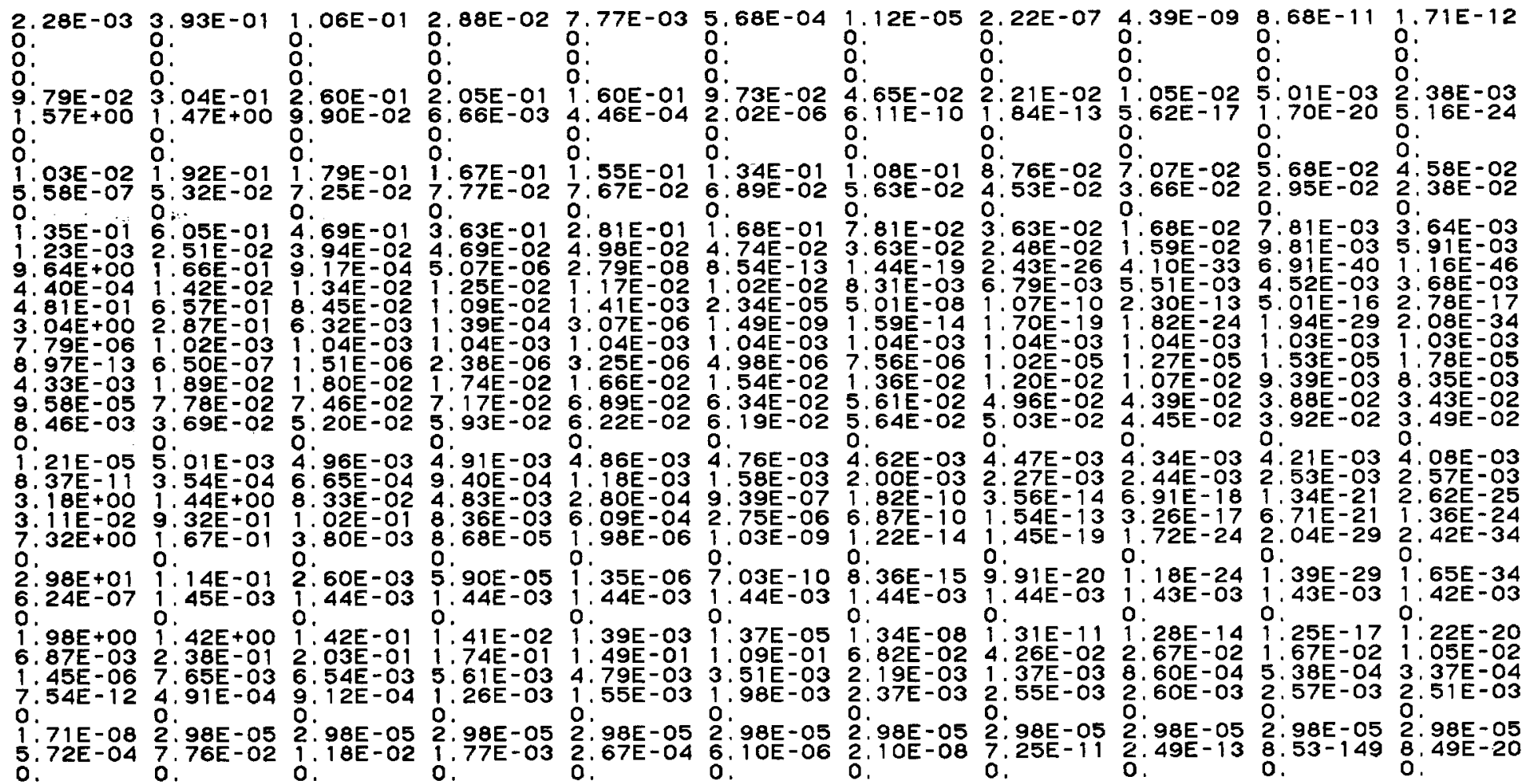


PAgE $2-13$

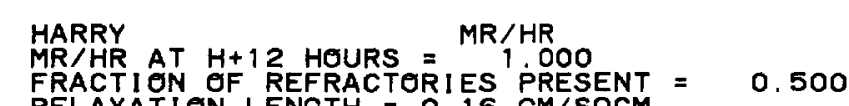

HARRY MR/HR AT $H+12$ HOURS $=$ MR/HR 1 HOOO FRACTION OF REFRACTORIES PRESENT $=0.500$ RELAXATION LENGTH $=0.16$ GM/SOCM $1.798 E-13$

DEBRIS DECAY FROM 1 TO 21 HOURS

ZERO TIME $1.00 E+00 \quad 2.00 E+00 \quad 3.00 E+00 \quad 4.00 E+00 \quad 6.00 E+00 \quad 9.00 E+00 \quad 1.20 E+01 \quad 1.50 E+01 \quad 1.80 E+01 \quad 2.10 E+01$

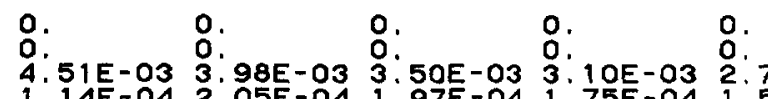

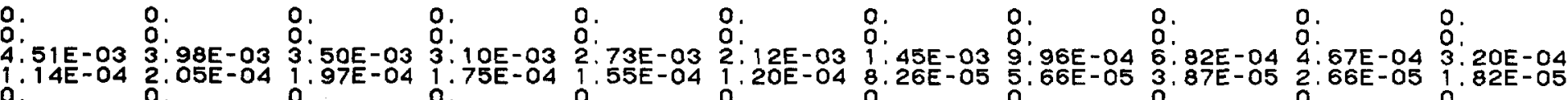
0. 0.

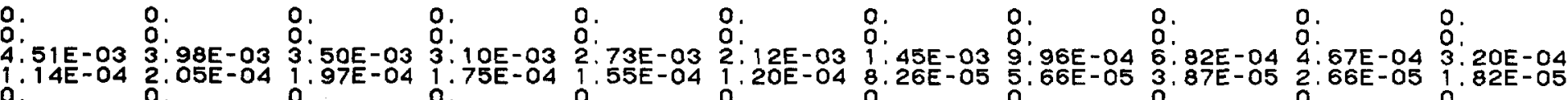

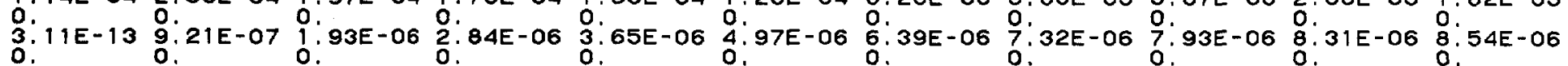

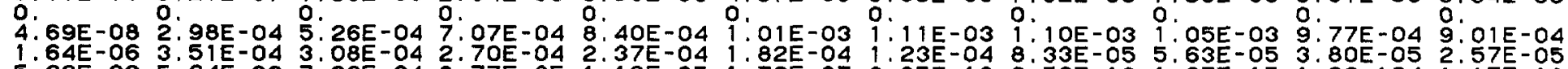
$1.64 E-06$
$5.26 E-03$
$1.51 E-04$ 1:46E-12 3.89E-08 4.37E-08 4.42E-08 4.42E-08 4.42E-08 4.41E-08 4.41E-08 4. 40E-08 4.38E-08 4.38E-08 $5.35 E-14$ 8.94E-06 $1.99 E-05$ 2.95E-05 $3.76 E-05$ 5.01E-05 $6.18 E-05$ 6.81E-05 $7.10 E-05$ 7.17E-05 $7.11 E-05$ $5.93 E-049.88 E-037.44 E-03$ 5.56E-03 $4.17 E-03 \quad 2.34 E-03 \quad 9.81 E-04 \quad 4.13 E-04 \quad 1.74 E-04$ 7.30E-05 3.07E-05

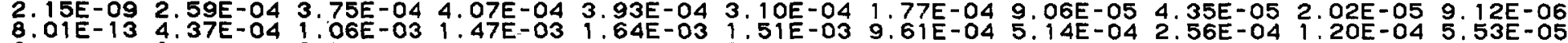

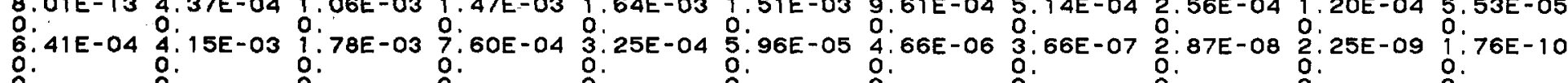
$\begin{array}{llll}0 . & 0 & 0 & 0 \\ 0.0 & 0 & 0 & 0\end{array}$

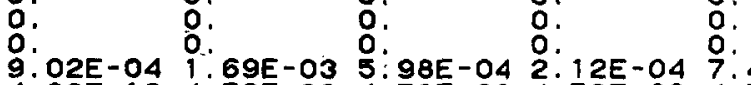
: : 0 :

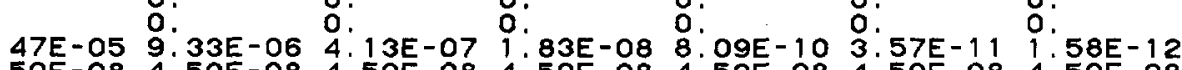
:.'

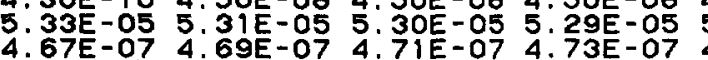

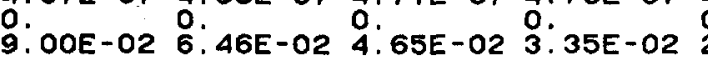
D. : 0.0 :

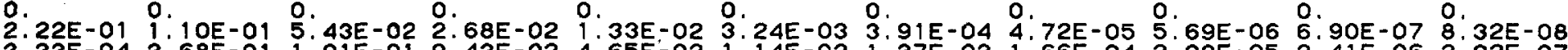

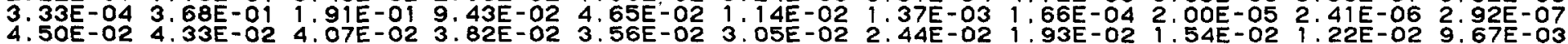
4.50E-02 4. 33E-02 $4.07 E-02$ 3. $82 E-02$ 3. 0. . $\therefore$ : 0. :. 1.10 -01 1.84E-01 1.72E-01 1.54E-01 1.36E-01 1.01E-01 6.27E-02 3..88E-02 2.39E-02 1. $48 E-02$ 9:09E-03

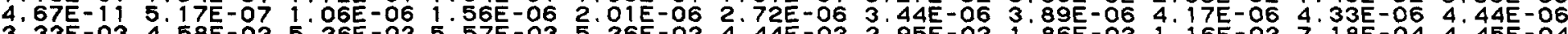

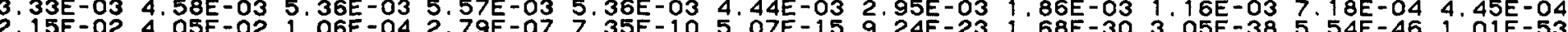

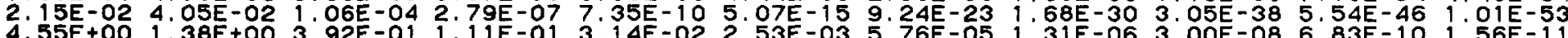


MARRY MR A AT $H+12$ HOURS $=$ MR/HR

MR HR AT H+12 HOURS I

ROMAXATION LENGTH $=0$. 16 GM S SQCM $1.798 E-13$

DEBRIS DECAY FROM 1 TO 21 HOURS

ZERO TIME $1.00 E+00 \quad 2.00 E+00 \quad 3.00 E+00 \quad 4.00 E+00 \quad 6.00 E+00 \quad 9.00 E+00 \quad 1.20 E+01 \quad 1.50 E+011.80 E+01 \quad 2.10 E+01$

\begin{aligned} 1130 \\ $\sin 131 \\$\hline 3\end{aligned}

TE $T$ I I M

1131

TEI 32

TEI $33 \mathrm{M}$

TE

XE

XE 133

TEI34

II 35

XEI35

CS136

BA137M

CS138

CS139

BA139

BAA

LA141

CE 141

BA1 42

LA 142

CEE 43

CEI44

PRT 144

CE: 46

PRI47

8.56E-04 8.09E-04 7.66E-04 7.24E-04 6.85E-04 6.13E-04 5.17E-04 4.38E-04 3.70E-04 3.13E-04 2.65E-04

5.96E+00 1.56E+00 2.54E-01 4.18E-02 6.86E-03 1.85E-04 8. $13 E-073.59 E-09$ 1.58E-11 $6.97 E-14$ 3.48E-16

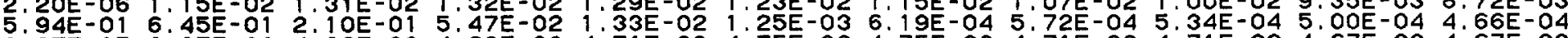

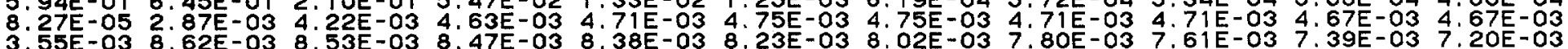

3.55E-03 8.62E-03 8.53E-03 8.47E-03 8.38E-03 8.23E-03 8.02E-03 7.80E-03 7.61E-03 7.39E-03 7.20E-03

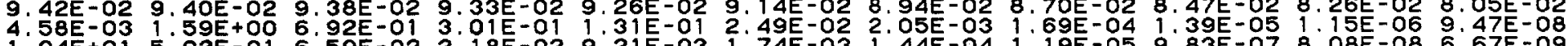

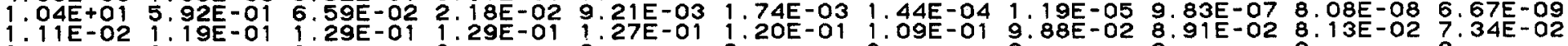

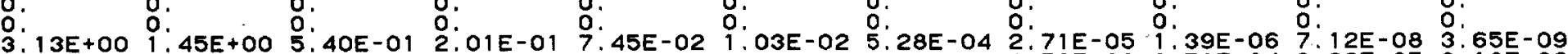

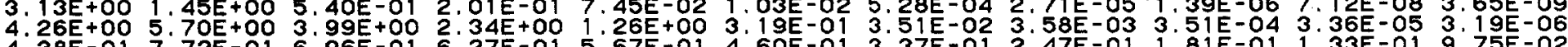

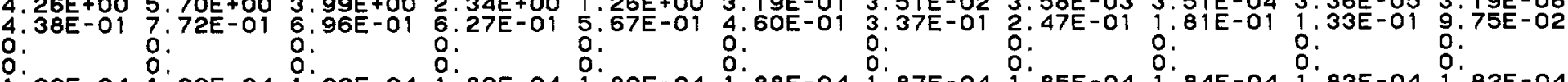
:

1.9OE-04 1.9OE-04 1.9OE-04 1.89E-04 $1.89 E-041.88 E-041.87 E-04$ o. -06 $9.20 \mathrm{E}-06 \mathrm{~g}$

$85 E-041.84 E-04 \quad 1.83 E-04 \quad 1.82 E-04$ $\begin{array}{ll} & \\ 0\end{array}$ 7.11E-03 7.92E-02 $4.89 E-02$ 2.97E-02 1.8OE-02 6.59E-03 1.46E-03 3.25E-04 7.22E-05 $1.60 E-05$ 3.55E-06 $2.67 E-04 \quad 1.67 E-03 \quad 1.67 E-03 \quad 1.65 E-03 \quad 1.65 E-03 \quad 1.64 E-03 \quad 1.64 E-03 \quad 1.63 E-031.62 E-031.60 E-031.59 E-03$ 2. 7 TE-09 3.94E-04 7.86E-02 6.51E-03 6.46E-04 6.41E-06 6.25E-09 6.09E-12 5.94E-15 5.83E-18 5.68E-21 $9.48 E-042.01 E-02$ 1.87E-02 1.59E

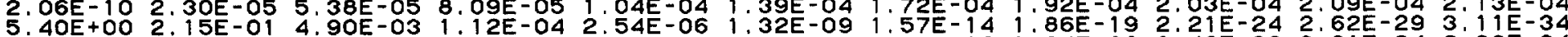

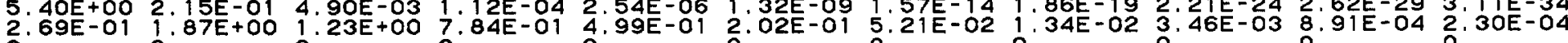

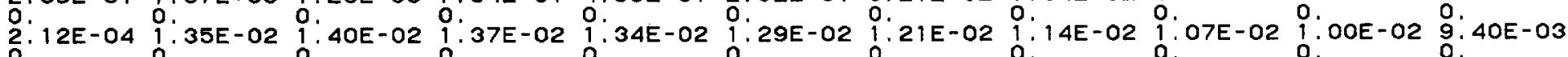

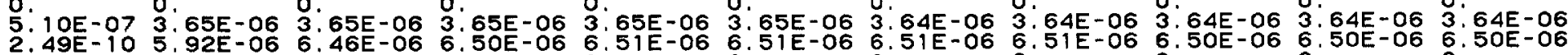

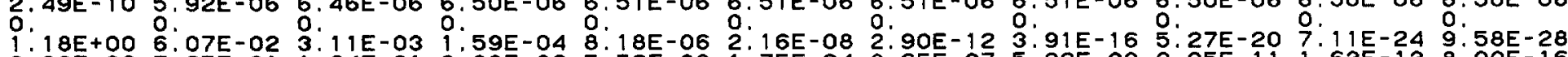

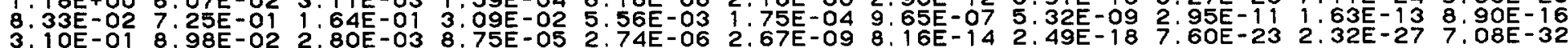


MARRY

FRACTIEN OF REFRACTORIES PRESENT $=0.500$

RELAXATION LENGTH $=0.166$ GMSSSCM $1.798 E-13$

DEBRIS DECAY FROM 1 TO 21 HOURS

ZERO TIME 1. OOE+OO 2. OOE+OO 3.OOE+OO $\quad 4.00 E+00 \quad 6.00 E+00 \quad 9.00 E+00 \quad 1.20 E+01 \quad 1.50 E+01 \quad 1.80 E+01 \quad 2.10 E+01$

ND1 147

PM149

ND151

PM152

SM153

SM155

SM156

EU156

EU158

ED159

2. 16 E-08 4.06E-04 4.17E-04 4.17E-04 4.15E-04 4. 14E-04 4.10E-04 4.07E-04 4.04E-04 4.01E-04 3.98E-04

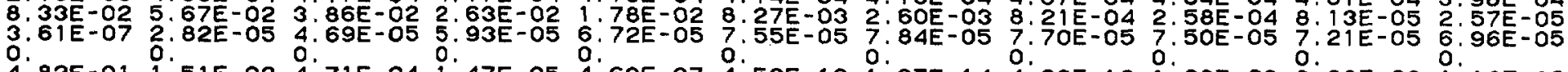

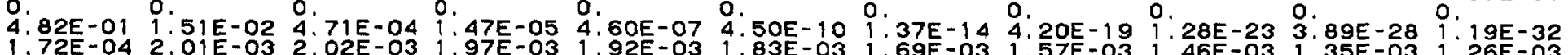

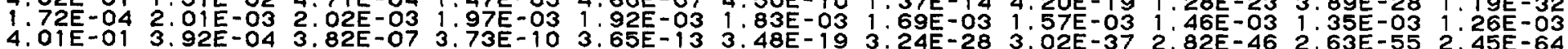
9.52E-05 9.38E-05 9.25E-05 9.11E-05 8.99E-05 8.72E-05 8.35E-05 7.97E-O5 7.64E-O5 7.31E-05 6.99E-05

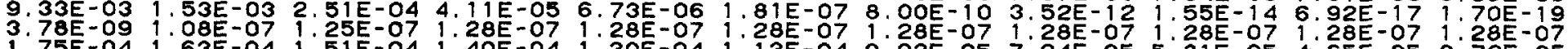

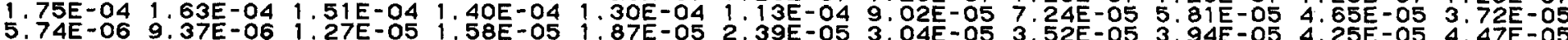
6.34E-05 2.10E-04 2.01E-04 1.92E-04 1.83E-04 1.67E-04 1.46E-04 1.27E-04 1. $11 E-04$ 9.65E-05 8.42E-05

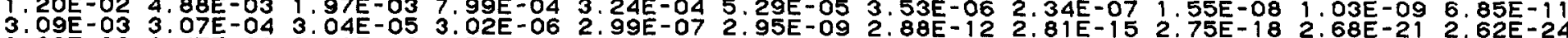

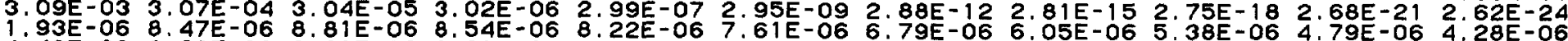

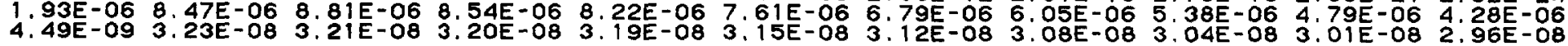

TOTAL

$1.08 E+023.41 E+01 \quad 1.35 E+01 \quad 7.21 E+00 \quad 4.47 E+00 \quad 2.33 E+00 \quad 1.37 E+00 \quad 1.00 E+00 \quad 7.86 E-01 \quad 6.42 E-01 \quad 5.38 E-01$ 
MARRY

FRACTION OF REFRACTORIES PRESENT $=0.500$

RELAXATION LENGTH $=0$. 16 GM SOCM $1.798 E-13$

DEBRIS DECAY FROM 1 TO 300 DAYS

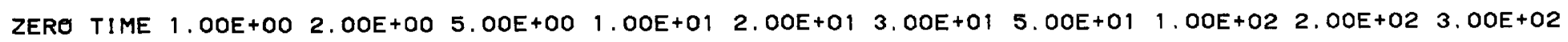


HARRY MR $H+12$ HOURS $=$ MR/HR

FRACTION OF REFRACTORIES PRESENT $=0.500$

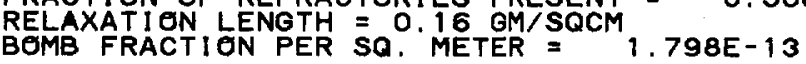

DEBRIS DECAY FROM 1 TO 300 DAYS

ZERO TIME $1.00 E+002.00 E+00 \quad 5.00 E+00 \quad 1.00 E+01 \quad 2.00 E+01 \quad 3.00 E+01 \quad 5.00 E+01 \quad 1.00 E+02 \quad 2.00 E+02 \quad 3.00 E+02$

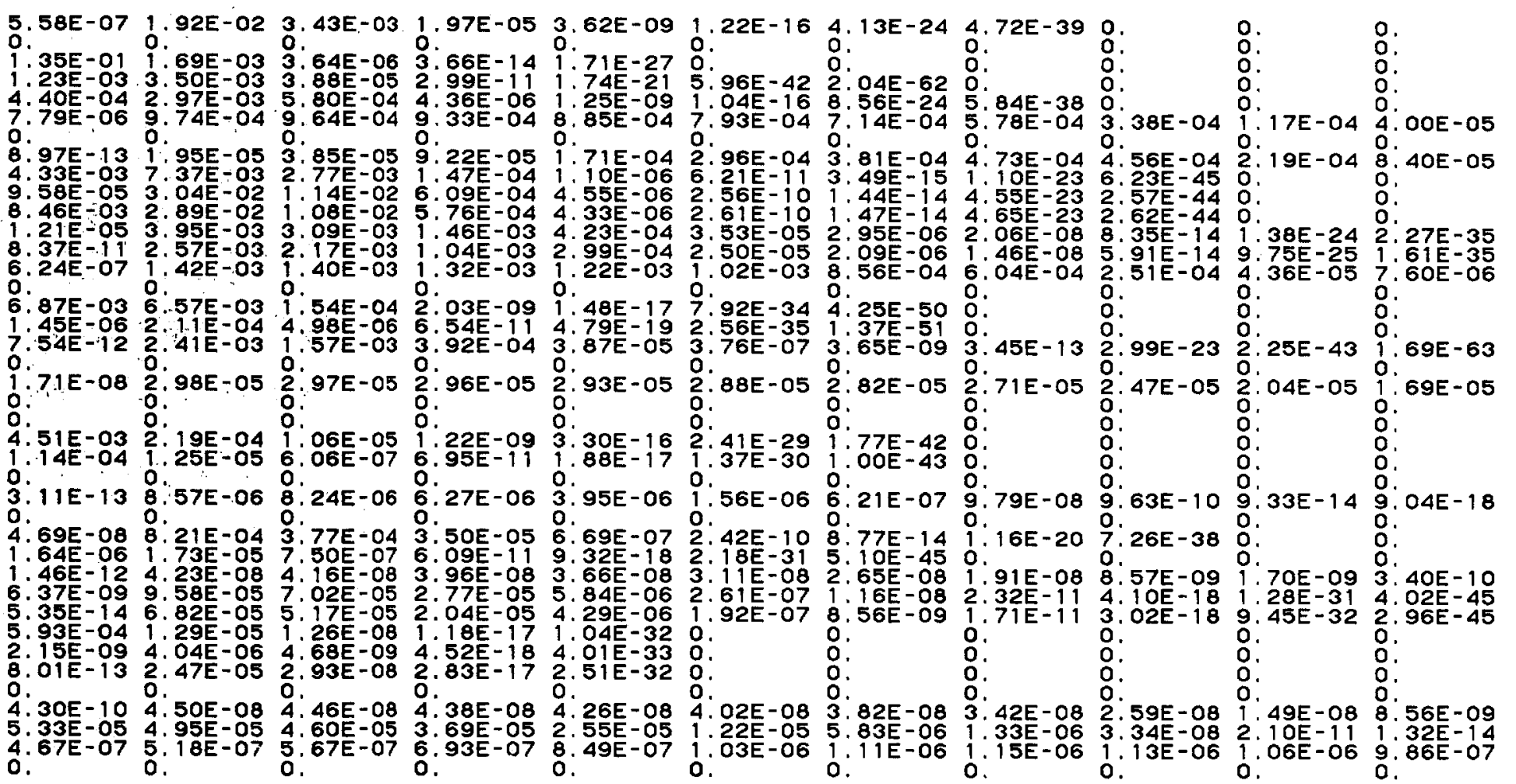


HARRY $A T H+12$ HOURS $=$ MR/HR

FRACTION OF REFRACTORIES PRESENT $=0.500$

RELAXATI ON LENGTH $=0.16$ GM/SQCM $1.798 E-13$

DEBRIS DECAY FROM 1 TO 300 DAYS

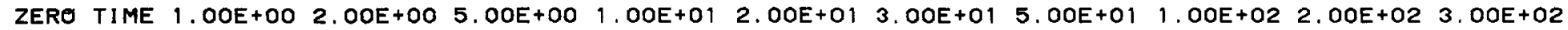

SN127

TE1 27M

TE127

SB129.

TE129

TE $31 \mathrm{M}$

TEI31

XEI3IM

TE 132

X1133

XE $X$ I3

XE135M

XE 35

CS136

BA137M

BA 40

CA 41

LA 42

CER 143

PRI 44

PR 145

PM1 47

PM1 49

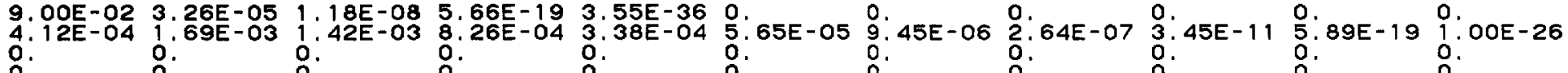

$\begin{array}{lll}0.0 & 0 \\ 0 & 0 & 0\end{array}$

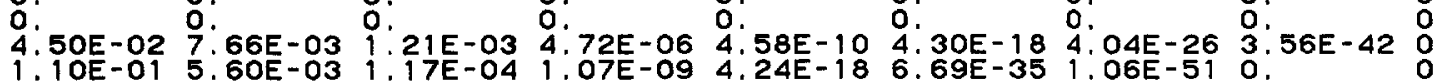

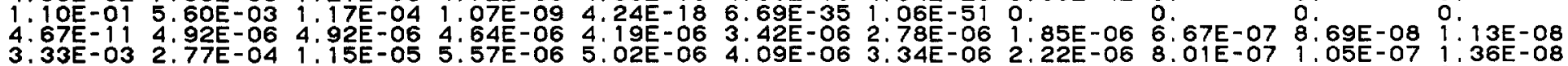

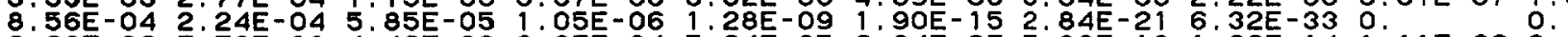

2. $20 \mathrm{E}-06 \quad 7.70 \mathrm{E}-03 \quad 4.42 \mathrm{E}-03 \quad 8.37 \mathrm{E}-04 \quad 5.24 \mathrm{E}-05$ 2.04E-07 7.99E-10 1.22E-14 1.11E-26 0.

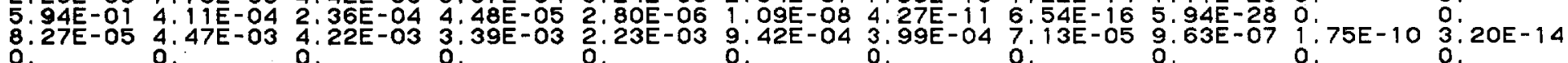

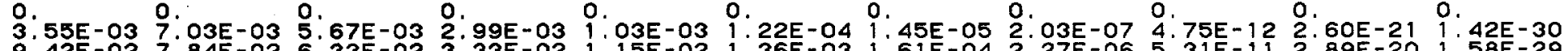

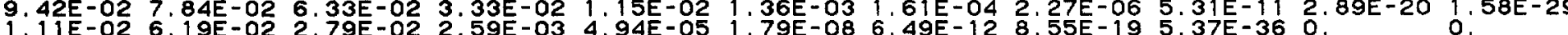

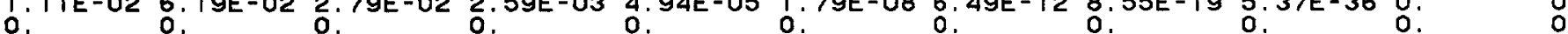

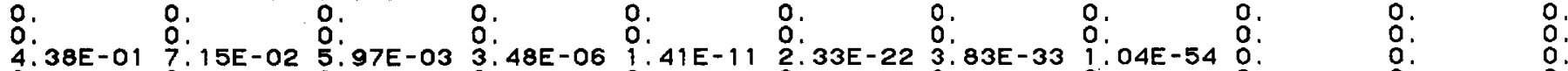

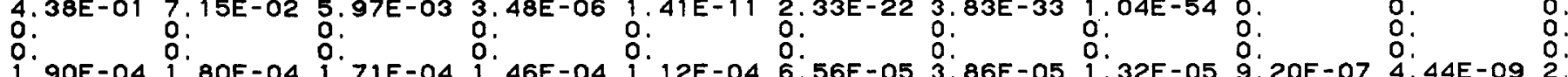

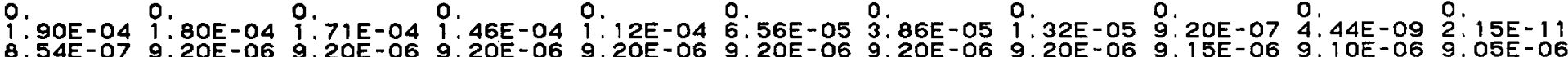

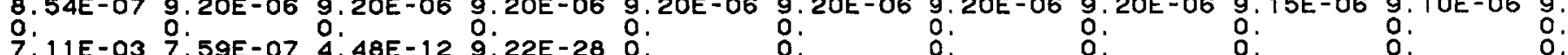

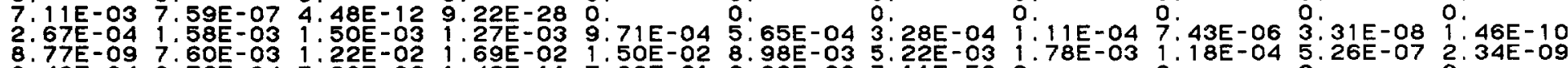

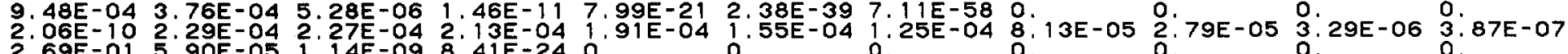

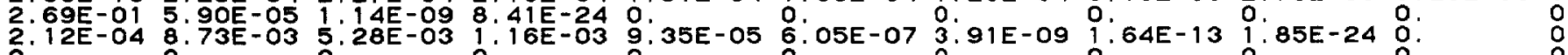

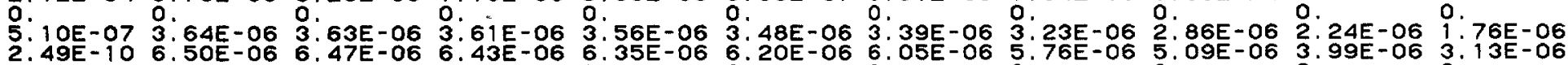

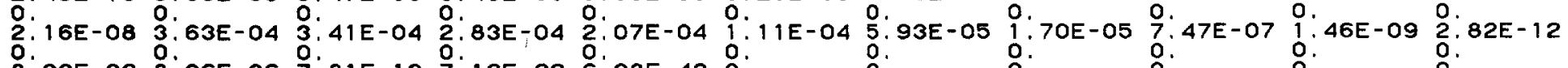

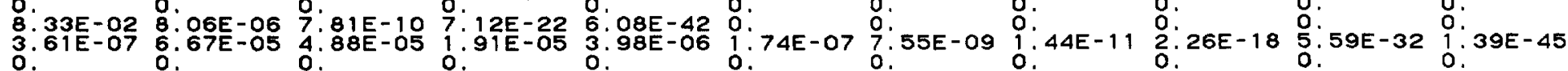


MARRY

FRACTION OF REFRACTORIES PRESENT = 0.500

RELAXATION LENGTH $=0.16$ GM/SQCM $1.798 E-13$

BOMB FRACTION PER SQ. METER =

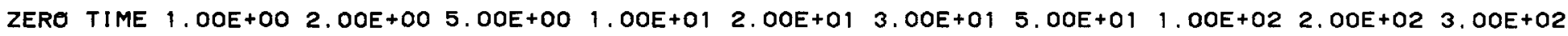

$\begin{array}{lllllllllllll}1.72 E-04 & 1.17 E-03 & 6.45 E-04 & 1.09 E-04 & 5.57 E-06 & 1.46 E-08 & 3.86 E-11 & 2.66 E-16 & 3.34 E-29 & 0.1 \\ 9.52 E-05 & 6.69 E-05 & 4.70 E-05 & 1.62 E-05 & 2.76 E-06 & 8.03 E-08 & 2.33 E-09 & 1.96 E-12 & 4.04 E-20 & 1.72 E-35 & 0.31 .31 E-51\end{array}$

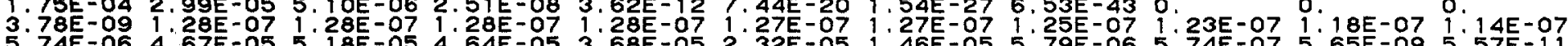
$5.74 E-06 \quad 4.67 E-05$ 5.18E-05 4.64E-05 3.68E-05 2.32E-05 1.46E-05 5.79E-06 5.74E-07 5.65E-09 5.57E-11

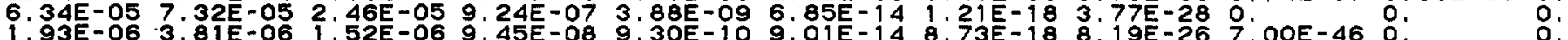

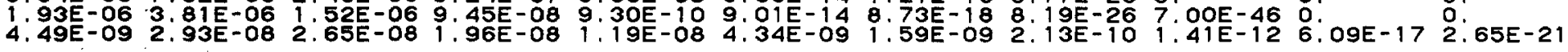

GDi59

$\begin{array}{lllllllllll}2.03 E+00 & 4.54 E-01 & 2.05 E-01 & 8.05 E-02 & 3.72 E-02 & 1.48 E-02 & 8.41 E-03 & 3.79 E-03 & 1.25 E-03 & 4.21 E-04 & 1.65 E-04\end{array}$

TOTAL 


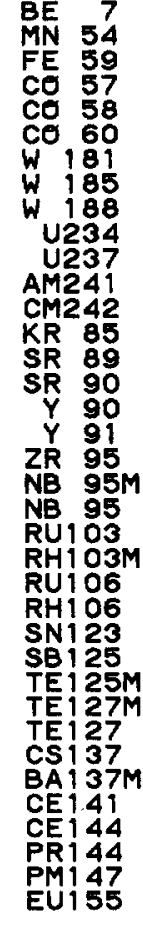

TOTAL
HARRY

$\mathrm{MR} / \mathrm{HR}$

FRACTION GF REFRACTORIES PRESENT $=0.500$

RELAXATION LENGTH $=0.16$ GM/SOCM $1.798 E-13$

DEBRIS DECAY FROM 1 TO 50 YEARS

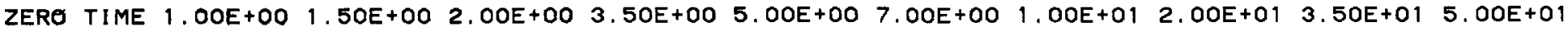

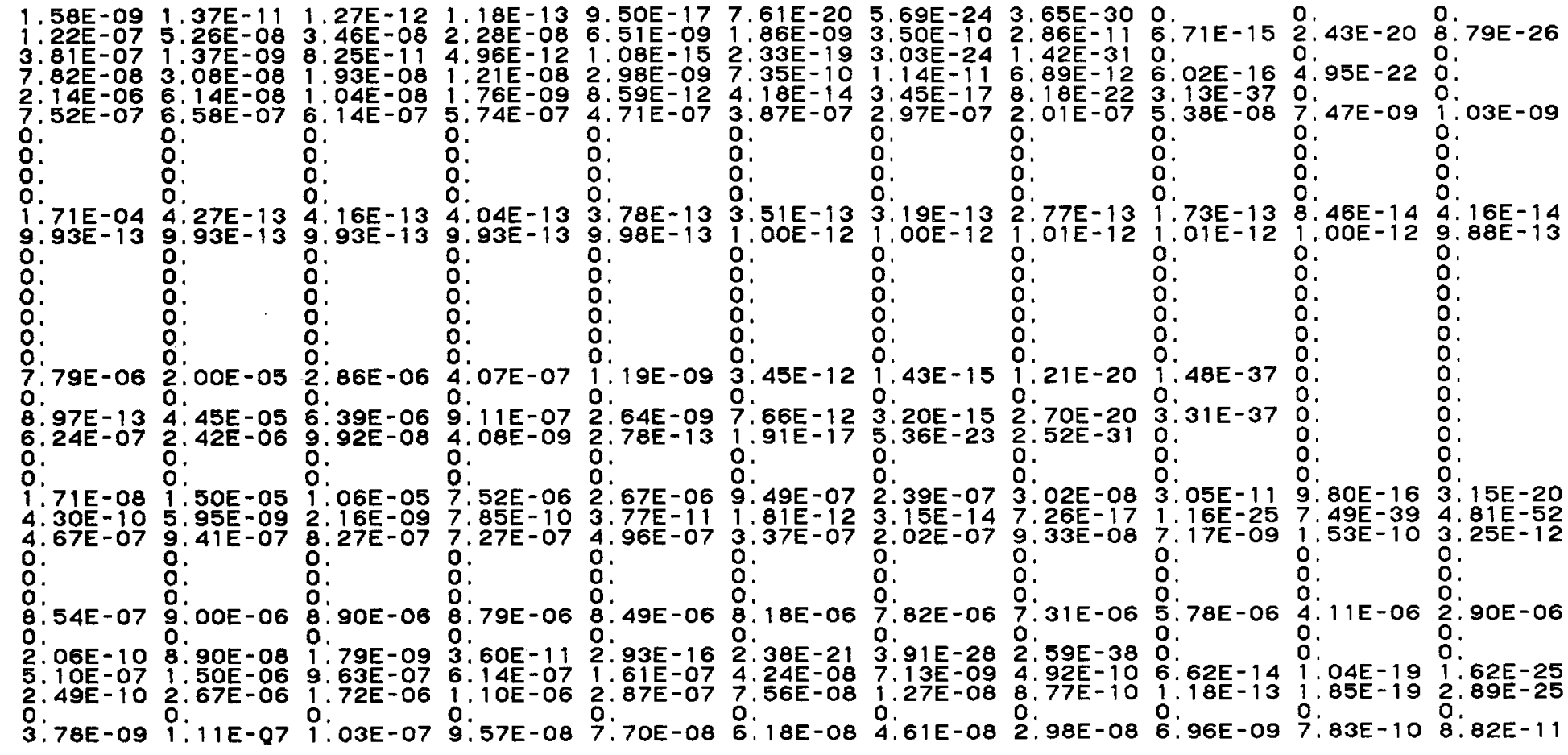

1.85E-04 9.70E-05 3.31E-05 2.08E-05 1.27E-05 1.00E-05 8.63E-06 7.67E-06 5.84E-06 4.11E-06 2.90E-06 
MARRY AT $H+12$ HOURS $=$ MR/HR

FRACTION OF REFRACTERIES PRESENT - 0.100

RELAXATION LENGTH $=0.16$ GM SQCM $2.318 E-13$

DEBRIS DECAY FROM 1 TO 21 HOURS

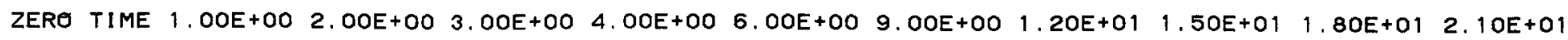

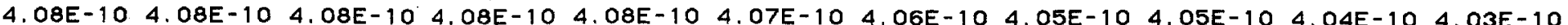

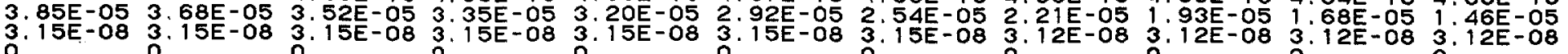

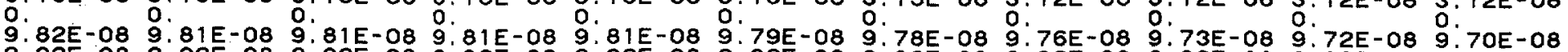

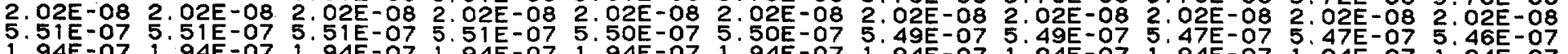

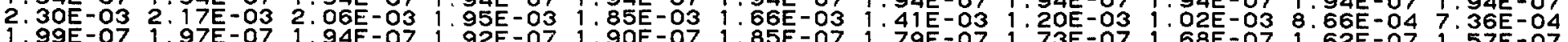

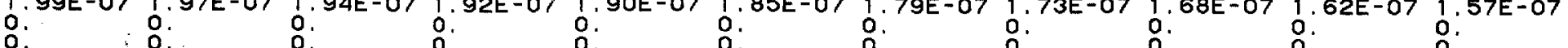

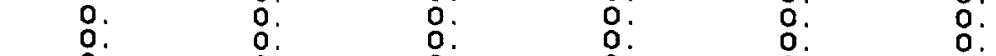
$\begin{array}{llll}0 . & 0 & 0 & 0 \\ 0.0 & 0 & 0 & 0 \\ 0 & 0 & 0 & 0\end{array}$

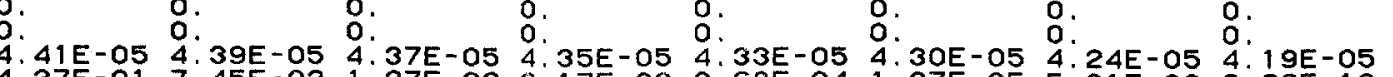

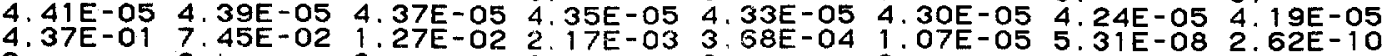

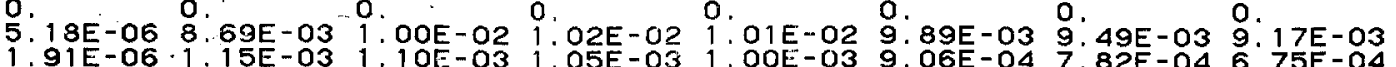
1.91E-06 $1.1 .15 E-03 \quad 1.10 E-03 \quad 1.05 E-03 \quad 1.00 E-03 \quad 9.06 E-04$ 7.82E-04 6.75E-04

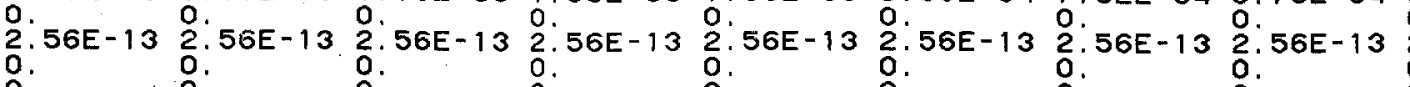

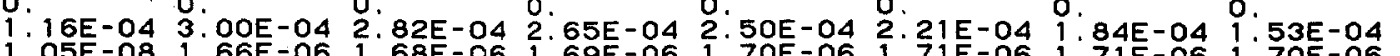
1.05E-08 1.66E-06 1.68E-06 1.69E-06 1:70E-06 1:.71E-06 1:71E-06 1:70E-06

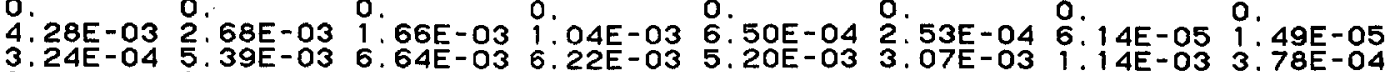
0 : : ०.: $\quad 0 . \quad 0$.

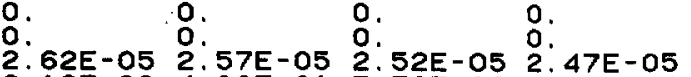
$\begin{array}{ll}0 . & 0 \\ \circ: & 0 \\ 0 & 0\end{array}$ 5. $98 \mathrm{E}-05$ 8. 03 . 0 . 
HARRY MR $\mathrm{H}+12$ HOURS $=$ MR/HR

FRACTION OF REFRACTORIES PRESENT a 0.100

RELAXATION LENGTH $=0.16$ GM/SQCM $2.318 E-13$

DEBRIS DECAY FROM 1 TO 21 HOURS

ZERO TIME $1.00 E+00 \quad 2.00 E+00 \quad 3.00 E+00 \quad 4.00 E+00 \quad 6.00 E+00 \quad 9.00 E+00 \quad 1.20 E+01 \quad 1.50 E+01 \quad 1.80 E+01 \quad 2.10 E+01$

BR 84

KR $85 \mathrm{MM}$

$\begin{array}{ll}K R & 88 \\ R B & 88\end{array}$

RB 89

SR 89

SR $91 \mathrm{M}$

$\begin{array}{rr}Y & 91 \\ \text { SR } & 92\end{array}$

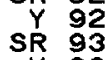

$Y 93$

$\begin{array}{rr}Y & 95 \\ Z R & 95 \\ Z R & 97\end{array}$

NB 95

ZR 97

NB 97

$\mathrm{NB} 98$

MC $99 \mathrm{M}$

Mㄷำ

Mo102

TC102

RH1O3M

RU105

RH105M

RH105

RU106

RH106
RH107
PDiO7M

2. 94E-03 5.07E-01 1.37E-01 3.71E-02 1.00E-02 7.33E-04 1. 45E-05 2.86E-07 5.66E-09 1.12E-10 2.21E-12

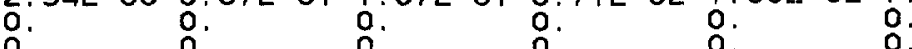

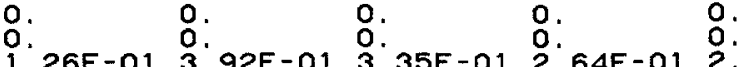

1. $26 E-013.92 E-013: 35 E-012: 64 E-012.06 E-01$

O.

$25 E-015$

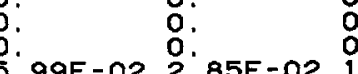

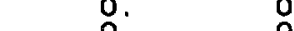

O.: $\quad$ o.

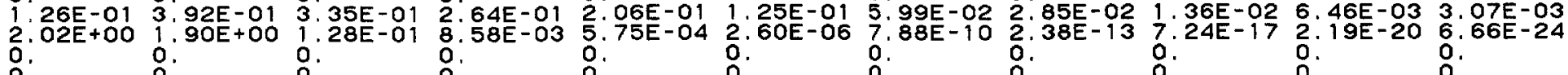

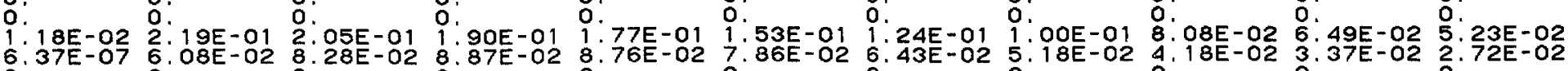

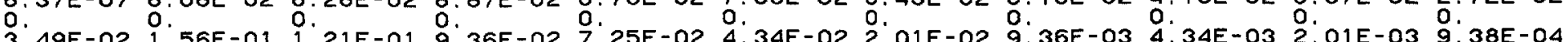

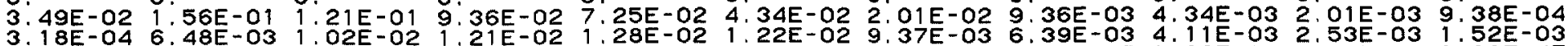

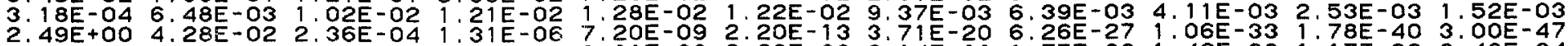

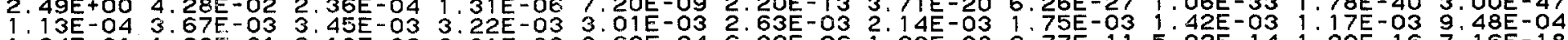

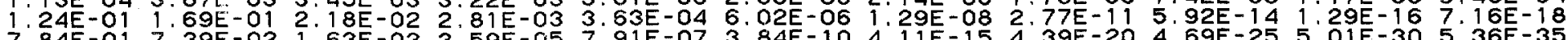

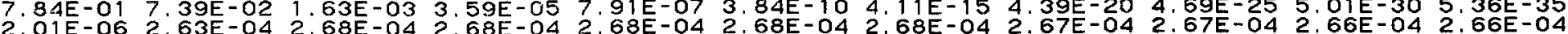

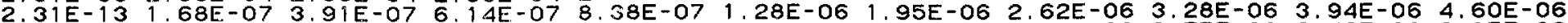

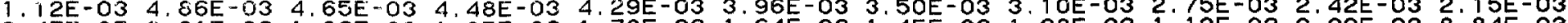

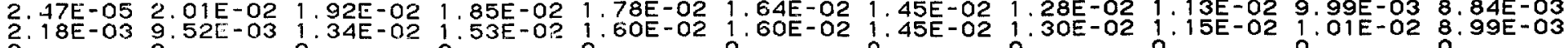

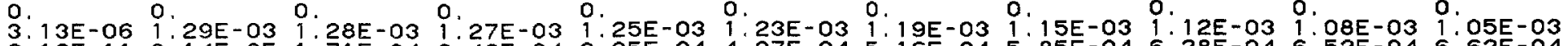

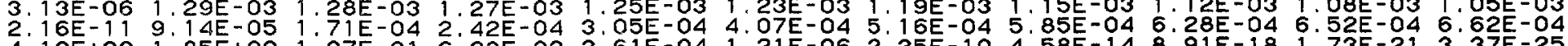
4. $10 \mathrm{E}+00 \quad 1.85 \mathrm{E}+00$ 1.07E-01 6.23E-03 3.61E-04 1.21E-06 2.35E-10 4.58E-14 8.91E-18 $1: 73 \mathrm{E}-213.37 \mathrm{E}-25$

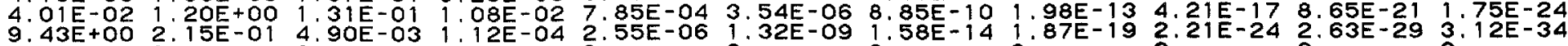

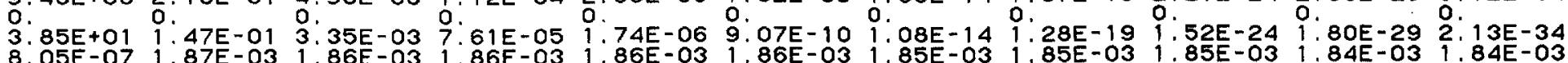

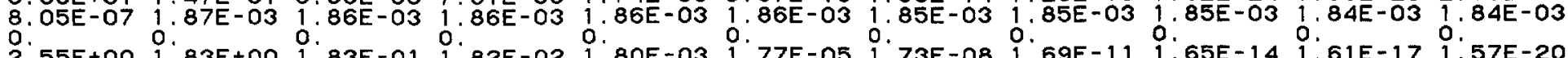

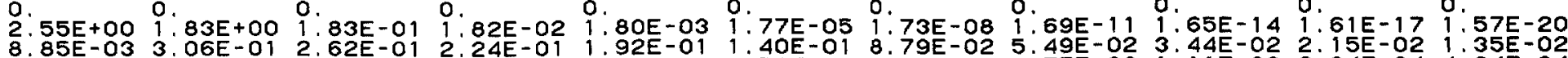

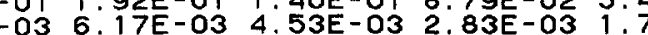

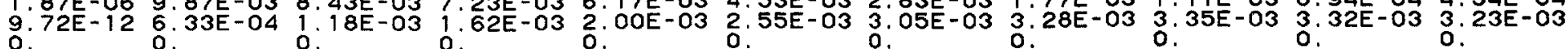

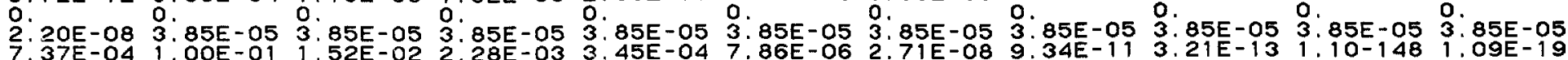

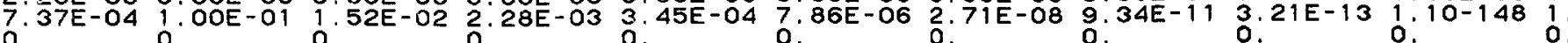
O9E-19 
FRACTION OF REFRACTORIES PRESENT $=0.100$

RELAXATIEN LENGTH $=0.16$ GM/SQCM $=18.100$

DEBRIS DECAY FROM 1 TO 21 HOURS

ZERO TIME 1.0OE+00 2.00E+00 3. $3.00 E+00 \quad 4.00 E+00 \quad 6.00 E+00 \quad 9.00 E+00 \quad 1.20 E+01 \quad 1.50 E+01 \quad 1.80 E+012.10 E+01$

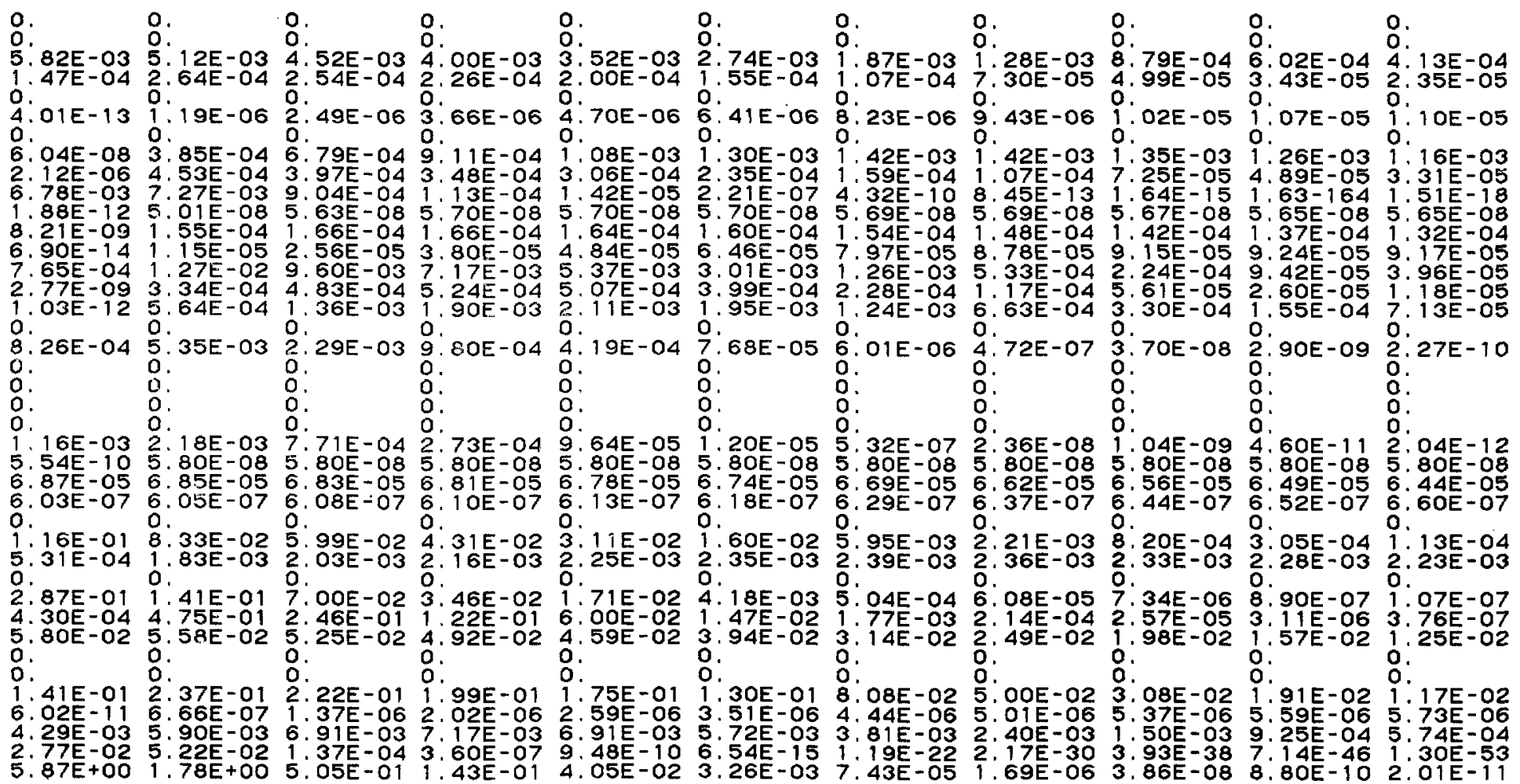


HARRY AT H+12 HOURS $=$ MR/HR

FRACTION OF REFRACTORIES PRESENT $=0.100$

RELAXATION LENGTH $=0.16$ GM/SQCM $2.318 E-13$

DEBRIS DECAY FREM 1 TO 21 HOURS

ZERO TIME $1.00 E+002.00 E+00 \quad 3.00 E+00 \quad 4.00 E+00 \quad 6.00 E+00 \quad 9.00 E+00 \quad 1.20 E+01 \quad 1.50 E+01 \quad 1.80 E+012.10 E+01$

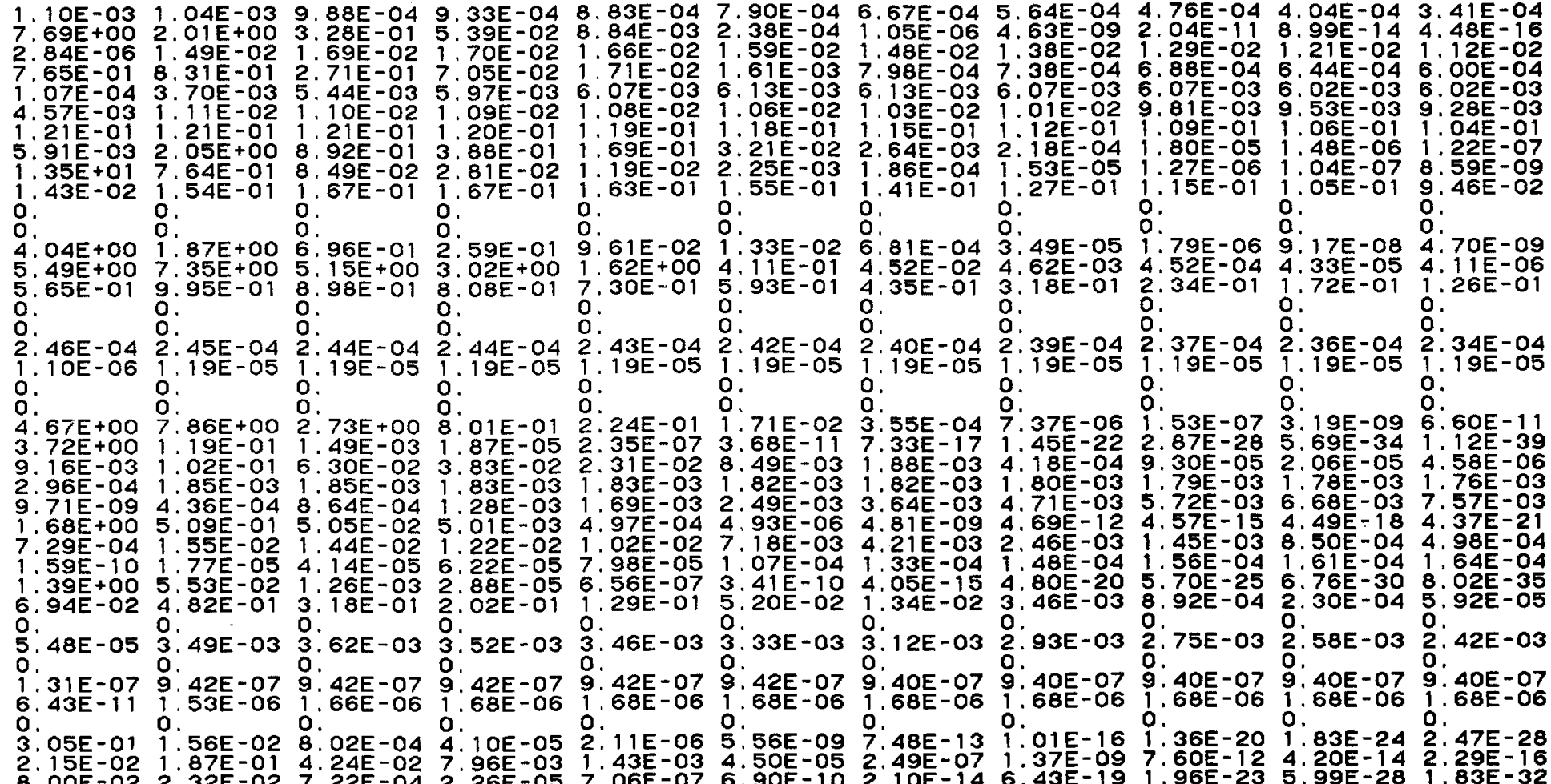

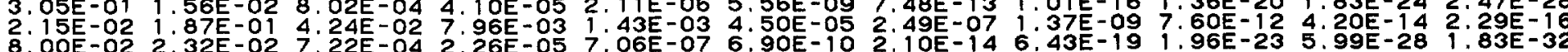


HARRY MR $H+12$ HOURS $=$ MR/HR

FRACTION OF REFRACTORIES PRESENT $=0.100$

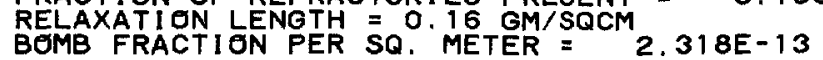

DEBRIS DECAY FROM 1 TO 21 HOURS

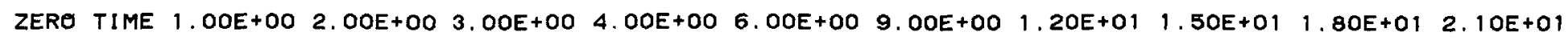

ND1 47
ND149
PM149
PM150
ND151
PM151
PM152
SMI53
SM155
EU155
SM156
EU156
EU157
EU158
EU159
GDi59
TB161

TOTAL

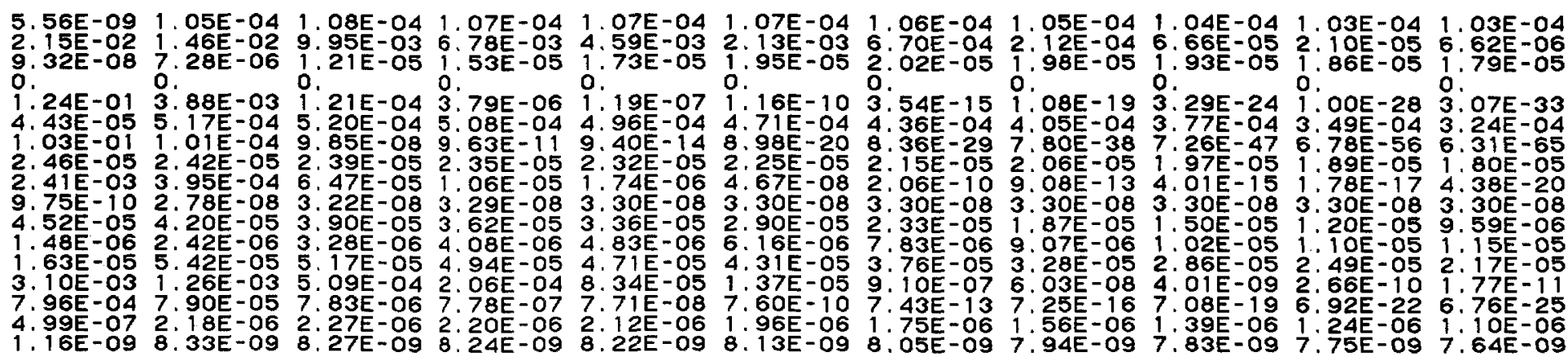

$\begin{array}{llllllllll}1.14 E+02 & 3.82 E+01 & 1.50 E+01 & 7.70 E+00 & 4.60 E+00 & 2.30 E+00 & 1.36 E+00 & 1.00 E+00 & 7.86 E-01 & 6.39 E-01 \quad 5.30 E-01\end{array}$ 

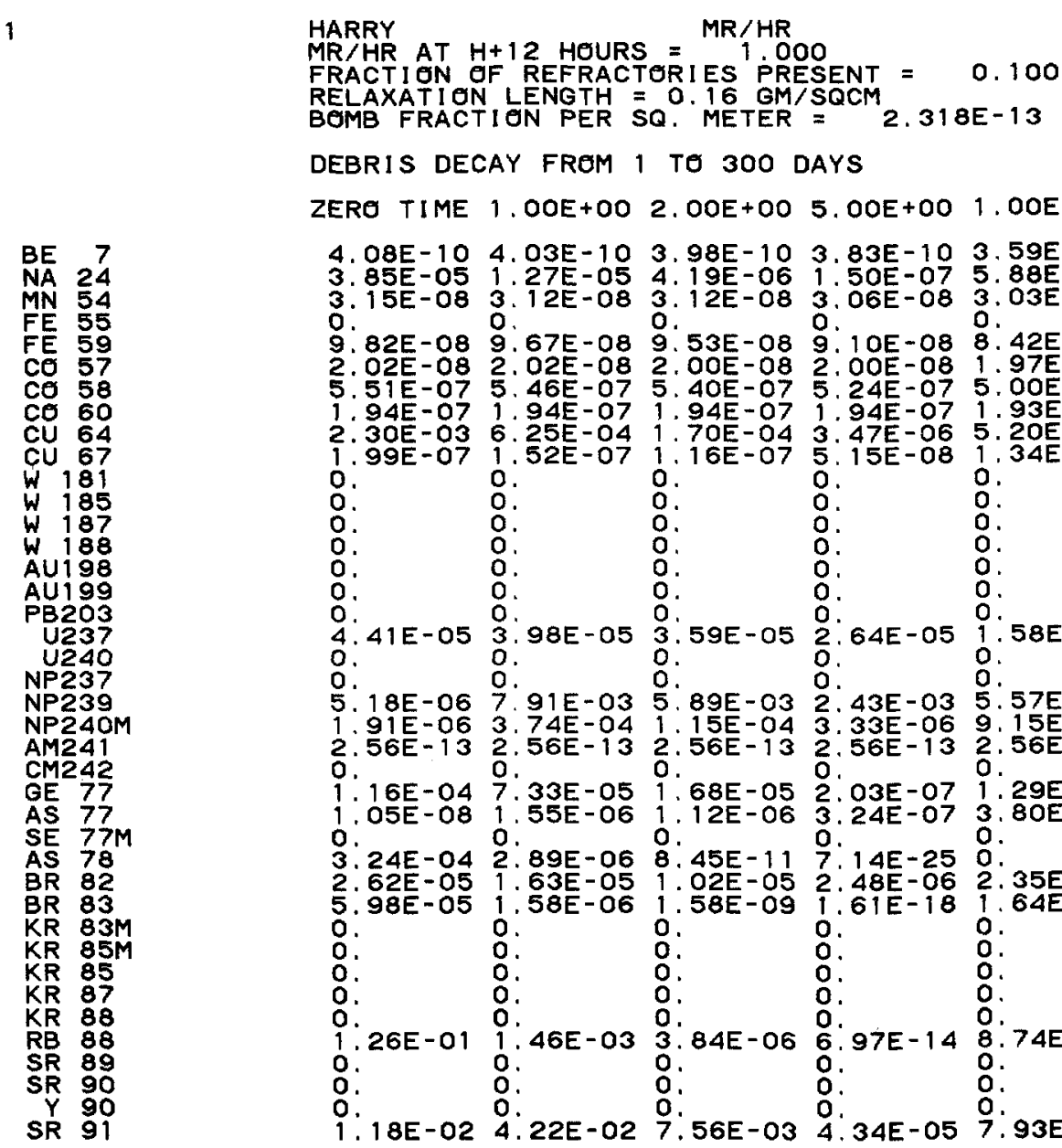

ZERO TIME 1. OOE+OO $2.00 E+00 \quad 5.00 E+00 \quad 1.00 E+01 \quad 2.00 E+01 \quad 3.00 E+01 \quad 5.00 E+01 \quad 1.00 E+02 \quad 2.00 E+02 \quad 3.00 E+02$

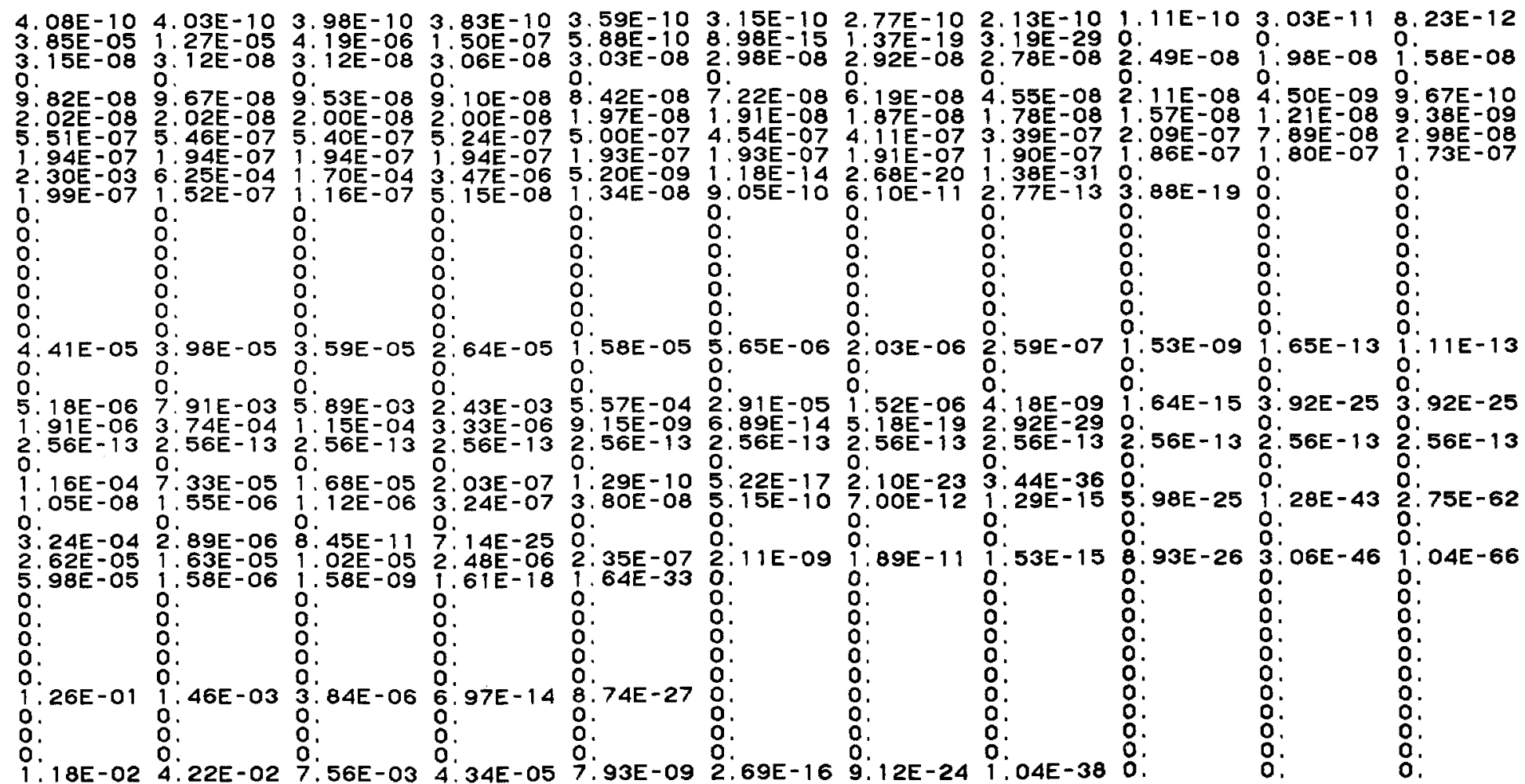


HARRY
MR/HR AT H+12 HOURS $=$ MR/HR
HOOO

FRACTI ON OF REFRACTORIES PRESENT $=0.100$

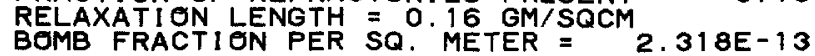

DEBRIS DECAY FROM 1 TO 300 DAYS

ZERO TIME $1.00 E+00 \quad 2.00 E+00 \quad 5.00 E+00 \quad 1.00 E+01 \quad 2.00 E+01 \quad 3.00 E+01 \quad 5.00 E+01 \quad 1.00 E+02 \quad 2.00 E+02 \quad 3.00 E+02$

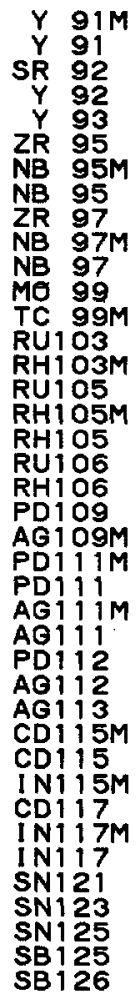

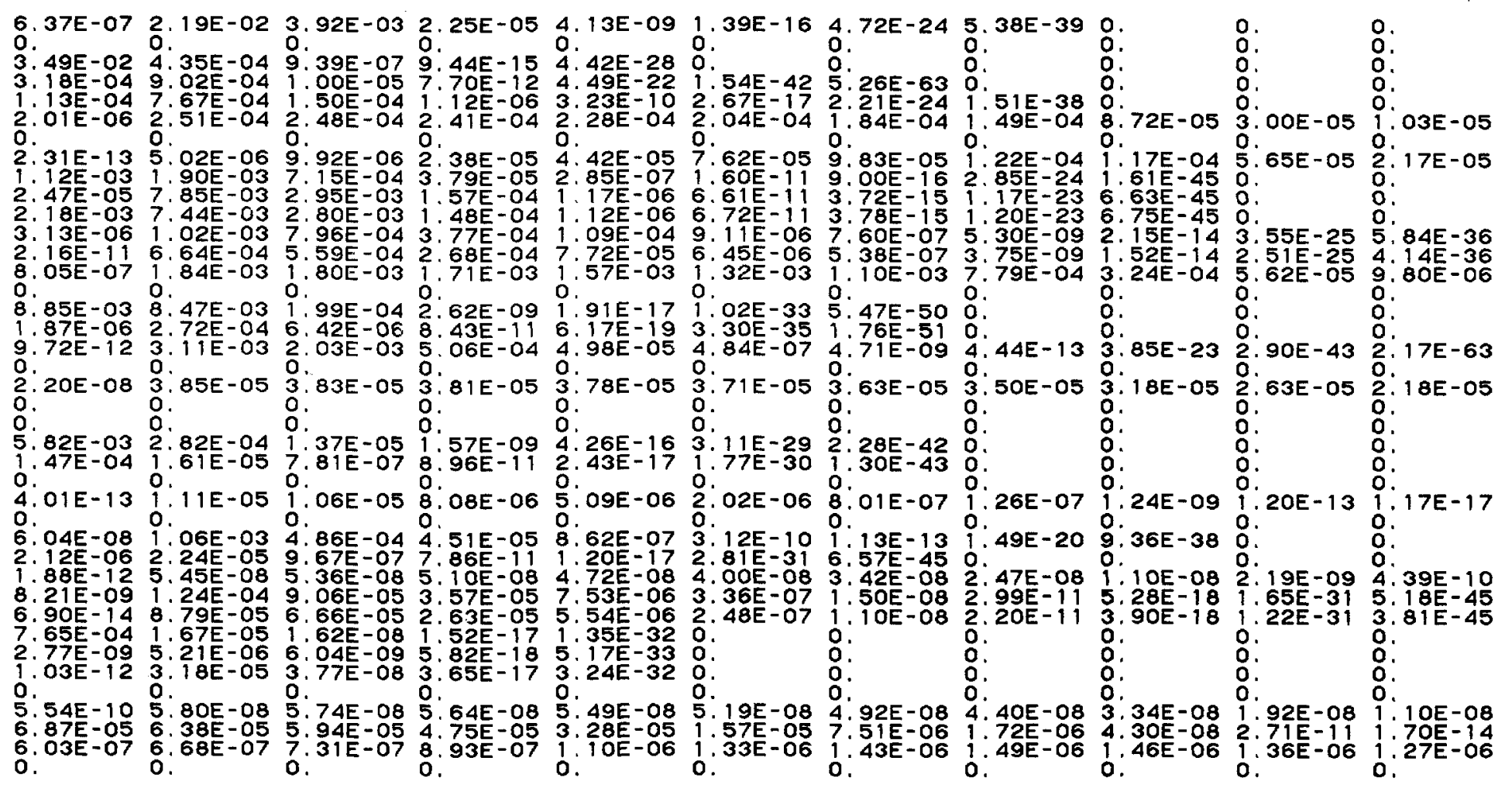


HARRY

$M R / H R$

FRACTION OF REFRACTORIES PRESENT = 0.100

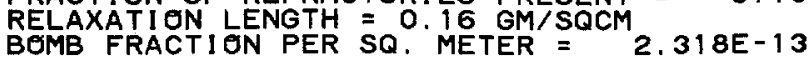

DEBRIS DECAY FROM 1 TO 300 DAYS

ZERO TIME 1. DOE+OO 2. OOE+OO $5.00 E+00 \quad 1.00 E+01 \quad 2.00 E+01 \quad 3.00 E+01 \quad 5.00 E+01 \quad 1.00 E+02 \quad 2.00 E+02 \quad 3.00 E+02$

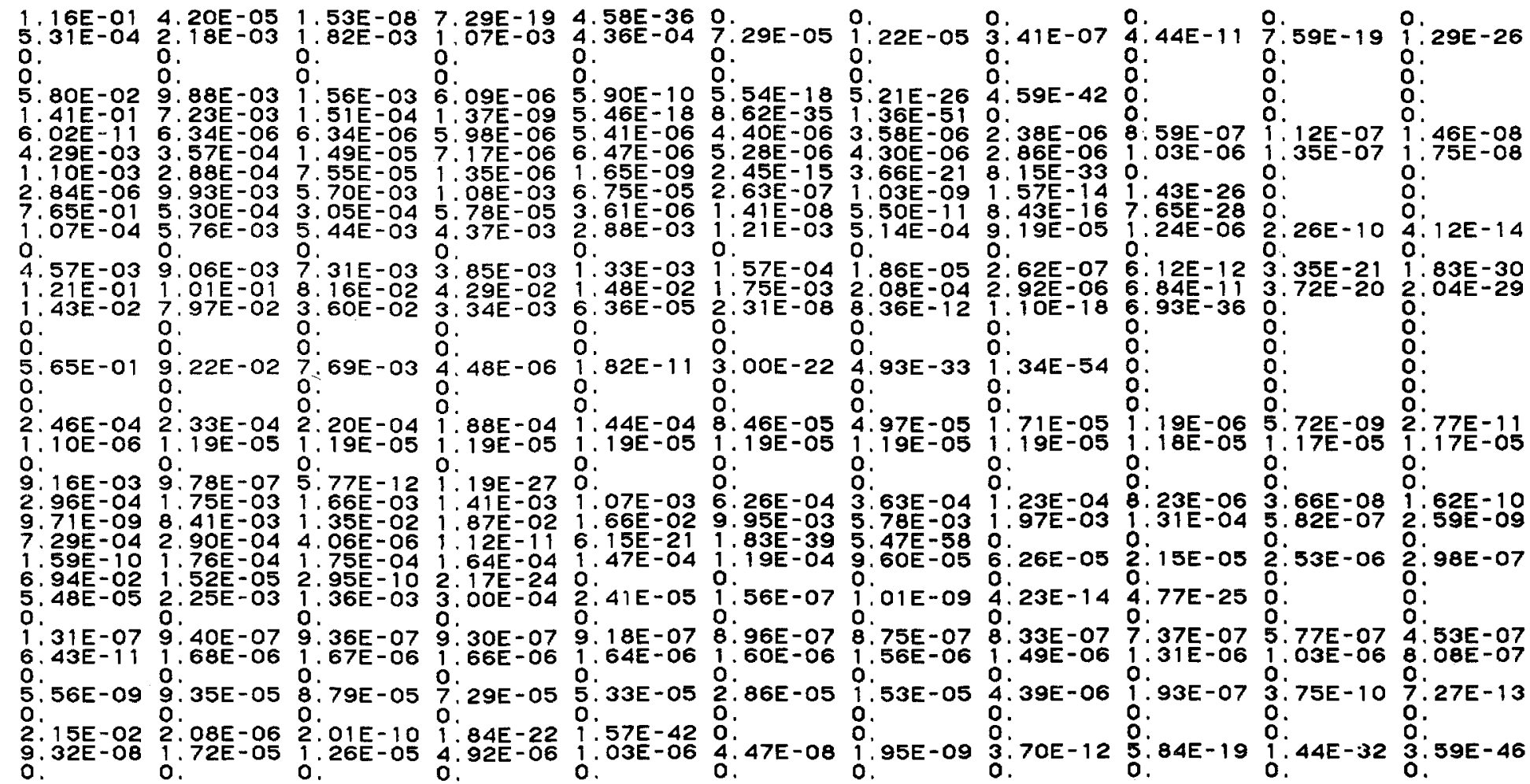


HARRY
MR/HR AT $H+12$ HOURS $=M R / H R$
1.000

FRACTION OF REFRACTORIES PRESENT = 0.100

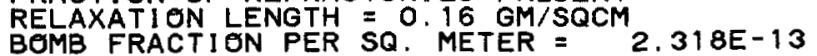

DEBRIS DECAY FROM 1 TO 300 DAYS

ZERO TIME $1.00 E+002.00 E+00 \quad 5.00 E+00 \quad 1.00 E+01 \quad 2.00 E+01 \quad 3.00 E+01 \quad 5.00 E+01 \quad 1.00 E+02 \quad 2.00 E+02 \quad 3.00 E+02$

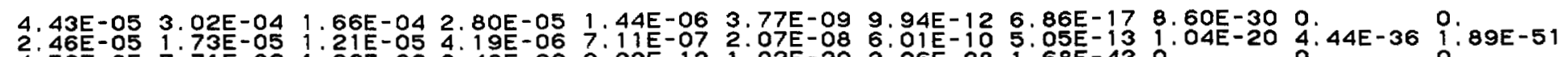

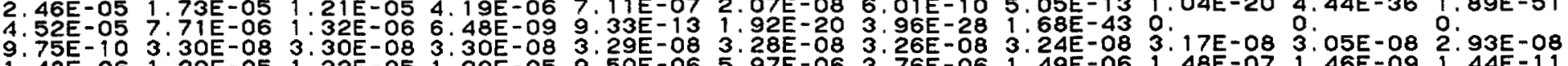
1.48E-06 $1.20 E-05$ 1.33E-05 1.20E-05 9.50E-06 5.97E-06 3.76E-06 1. $49 E-06$ 1. $48 E-07$ 1.46E-09 $1: 44 E-11$

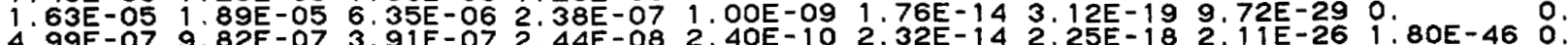

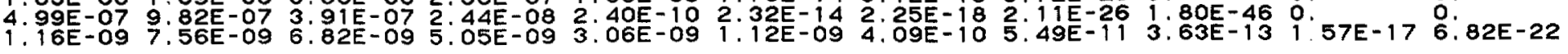


DEBRIS DECAY FROM 1 TO 50 YEARS

ZERO TIME 1.0OE+OO 1.5OE+OO 2.00E+OO 3.5OE+O0 $5.00 E+00 \quad 7.00 E+00 \quad 1.00 E+01 \quad 2.00 E+013.50 E+01 \quad 5.00 E+01$

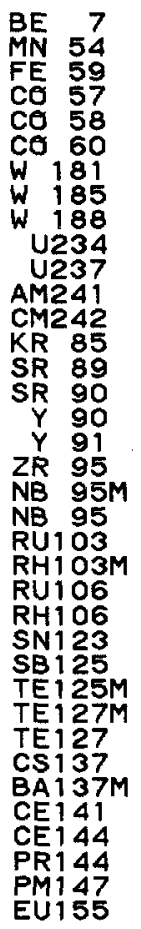

TOTAL

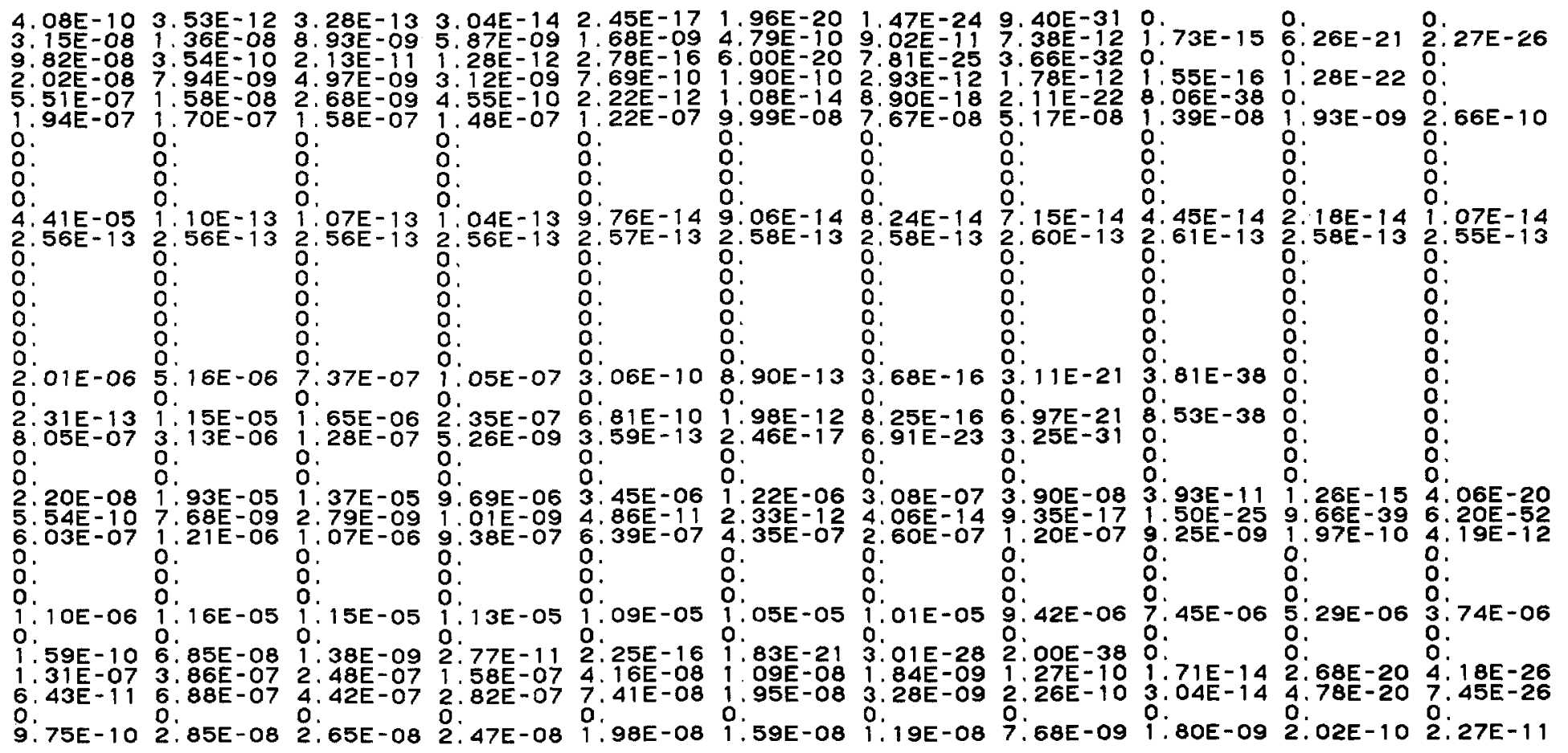

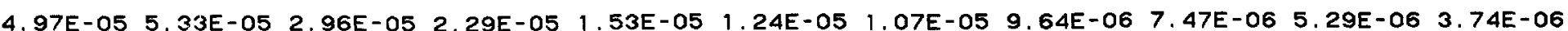




\section{APPENDIX 3}

HARRY EVENT

\section{Fit To Sum of Eleven Exponentials}

The calculated values are fit to the function

$$
\sum_{i=1}^{11} a_{i} e^{-\lambda_{j} t}
$$

In the first table the $a_{i}$ 's are the variables and are designated by an odd number in the column under " $K$ "; the $i$ 's are fixed parameters so designated. The quality of fit to each point is shown in the second table.

$\underline{\text { Page }}$

Fraction refractories present $=1.0$

$3-1$

Fraction refractories present $=0.5$

Fraction refractories present $=0.1$ 
HARRY

CI/SQ KM, 1.O REFRACTORIES PRESENT

PAge 3-1

STND 2. DEV

$4.3358 E+02$

1. $3777 E+02$

$8627 \mathrm{E}+01$

6. $9050 E+00$

1. $3221 \mathrm{E}+00$

1 , $8037 \mathrm{E}-01$

3668E-02

3. $5370 E-03$

9. $6805 E-04$

4. $9096 E-05$

0:

FIXED PARAMETER

FIXED PARAMETER

FIXED PARAMETER

FIXED PARAMETER

FIXED PARAMETER

FIXED PARAMETER

FIXED PARAMETER

FIXED PARAMETER

FIXED PARAMETER

FIXED PARAMETER

FI XED PARAMETER
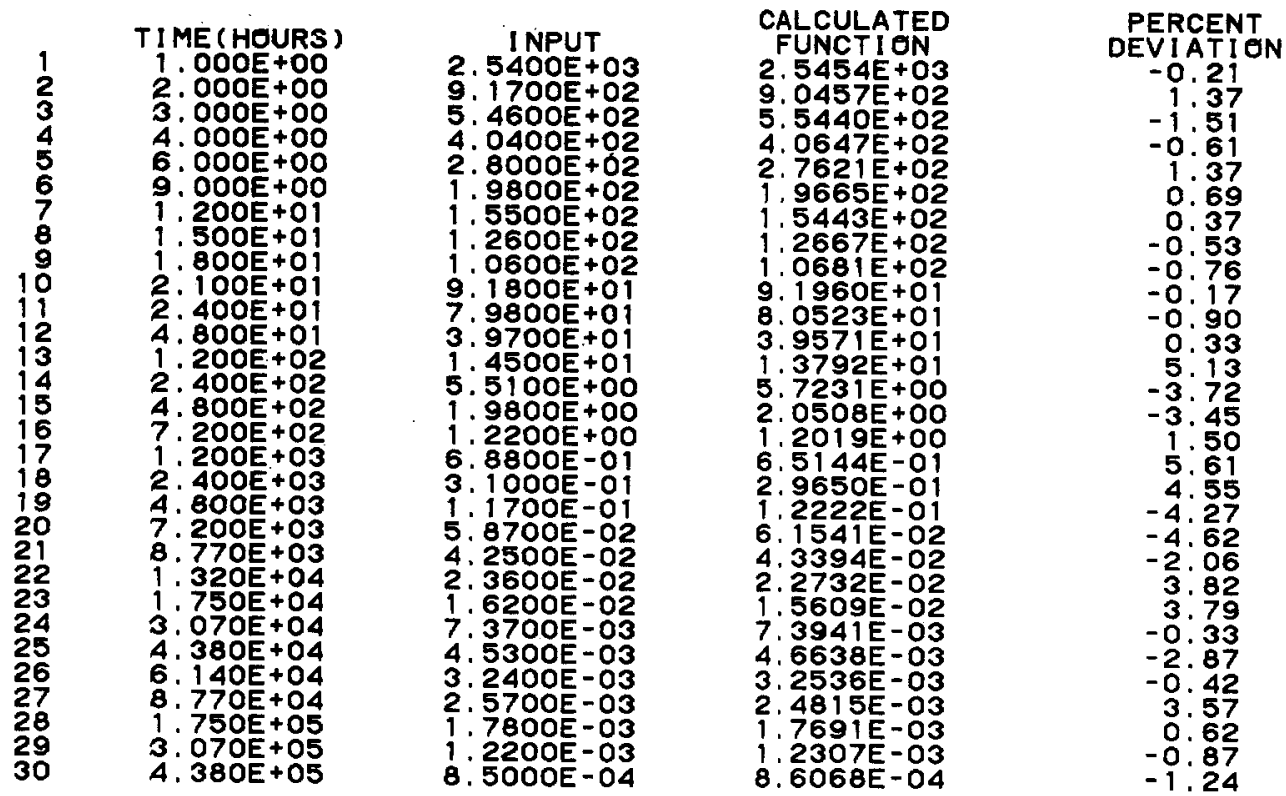
FINAL VALUE $1.5038 E+04$ 2. $2799 E+03$ 8. $8130 E-01$ 3. $3717 E+02$ $3.8960 E-01$ 2. $6954 \mathrm{E}+02$ $7.7103 E+01$ 3. $4400 \mathrm{E}-02$ . 8. $8070 \mathrm{E}-03$ . $6921 \mathrm{t}+0$ 2. $3380 \mathrm{OE}-03$ ( $4.4040 E-04$ 1150E-04 $1.1667 E-02$ $4.5780 \mathrm{E}-05$ 2. $7300 \mathrm{E}-06$

STND DEV 5. $0863 E+02$ I. $5624 E+02$ 3. $0801 E+01$ 7. $3395 E+00$ 1. $4407 E+00$ 2. $0939 E-01$ 2. $6002 E-02$ 4. $1092 E-03$ i. 2486E-03 7. $4703 E-05$ o. $4703 E-05$

FIXED PARAMETER

FIXED PARAMETER FIXED PARAMETER FIXED PARAMETER FIXED PARAMETER FIXED PARAMETER FIXED PARAMETER FIXED PARAMETER FIXED PARAMETER FIXED PARAMETER FIXED PARAMETER
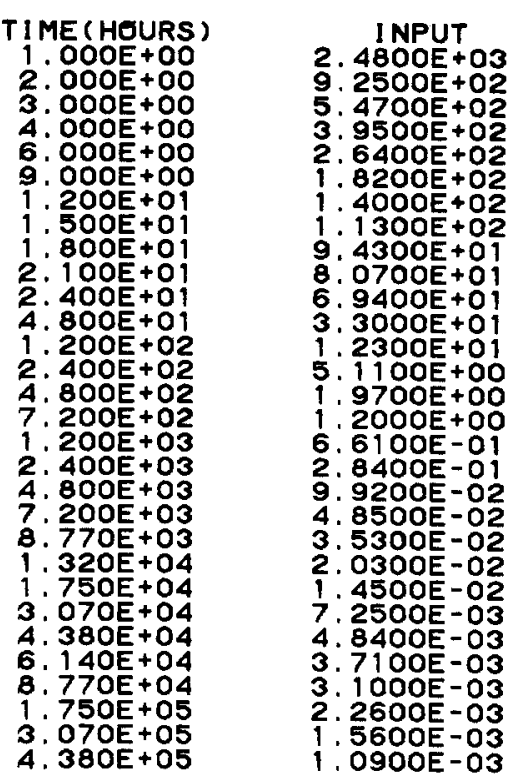

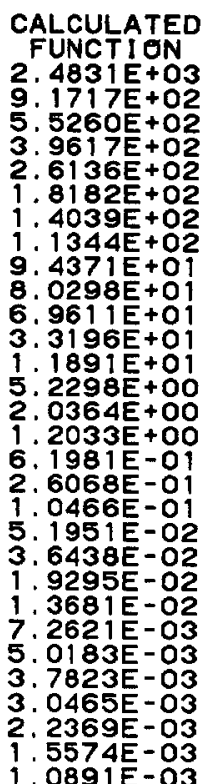

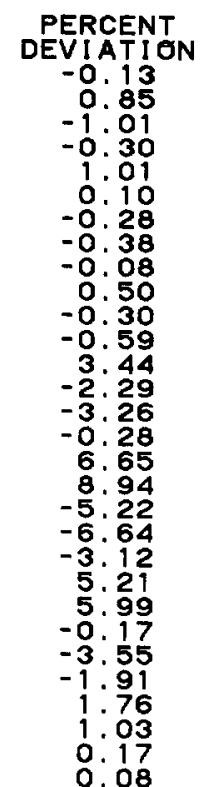



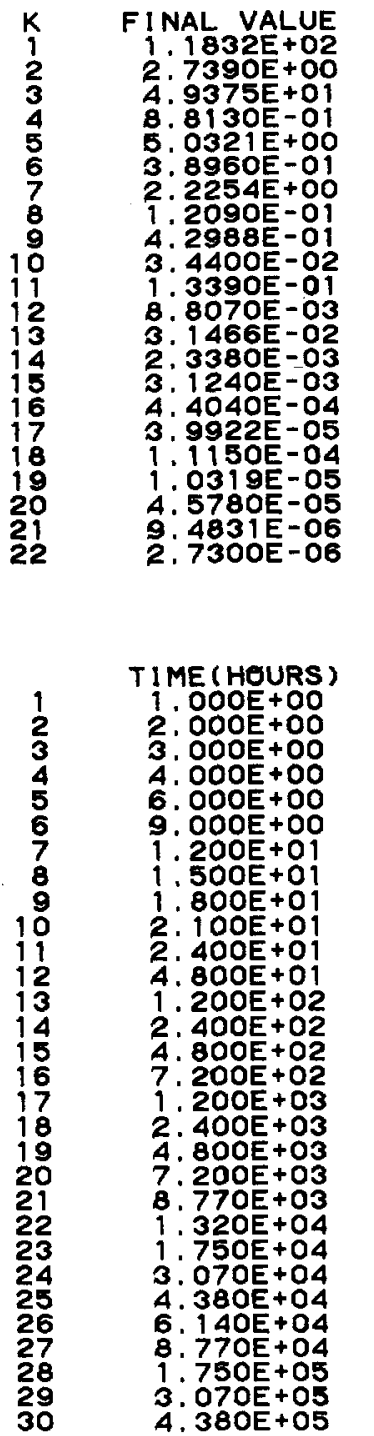

STND DEV.

O. $2769 \mathrm{E}+00$

3. 2256E- 01

4. $9824 E-02$

i. $1227 \mathrm{E}-02$

2. 1974E-03

2. 9939E-04

2. $1067 E-05$

O. 9943E-06

6. $0543 E-07$

4. $3605 E-08$

MR/HR, 0.5 REFRACTURIES PRESENT $0.16 \mathrm{GM} / \mathrm{SQCM}$

FIXED PARAMETER

FIXED PARAMETER

FIXED PARAMETER

FIXED PARAMETER

FIXED PARAMETER

FIXED PARAMETER

FIXED PARAMETER

FIXED PARAMETER

FIXED PARAMETER

FIXED PARAMETER

FIXED PARAMETER

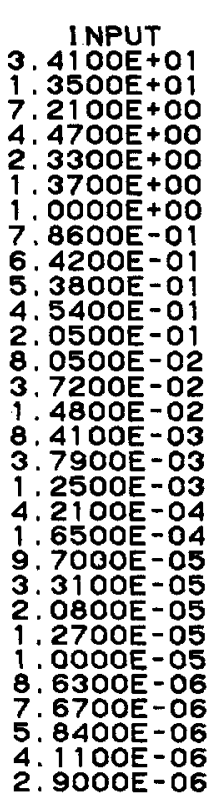

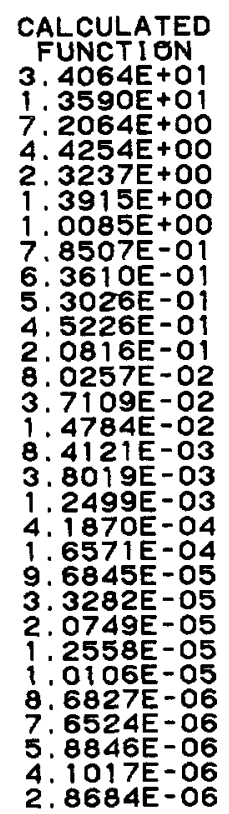

PERCENT
DEVIATION
0.11
-0.67
0.05
1.01
0.27
-1.54
-0.84
0.12
0.93
1.46
0.39
-1.52
0.30
0.25
0.11
-0.03
-0.31
0.01
0.55
-0.43
0.16
-0.55
0.24
1.13
-1.05
-0.61
0.23
-0.76
0.20
1.10


FIXED PARAMETER FIXED PARAMETER 2. $1066 \mathrm{E}+02$ 3. $7933 E+01$ $8.8532 \mathrm{E}+00$ i. $8202 E+00$ 2. $9453 E-01$ 3. $3153 E-02$ 5: $7051 E-03$ O. $9589 \mathrm{E}-03$ o. i. $4106 \mathrm{E}-04$

FIXED PARAMETER FIXED PARAMETER FIXED PARAMETER FI XED PARAMETER FIXED PARAMETER FIXED PARAMETER FIXED PARAMETER FIXED PARAMETER FIXED PARAMETER

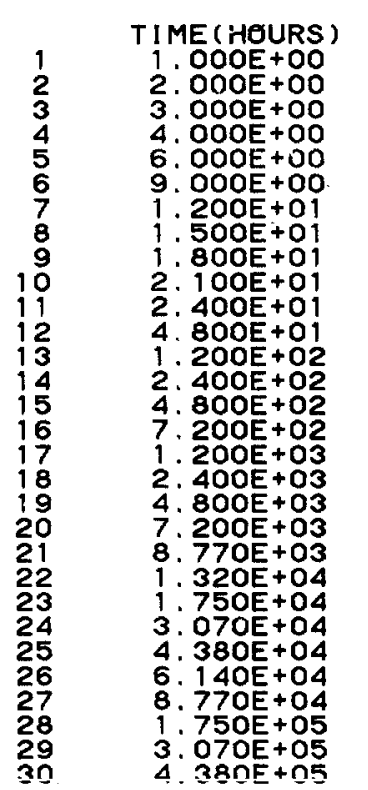

\begin{tabular}{|c|c|}
\hline 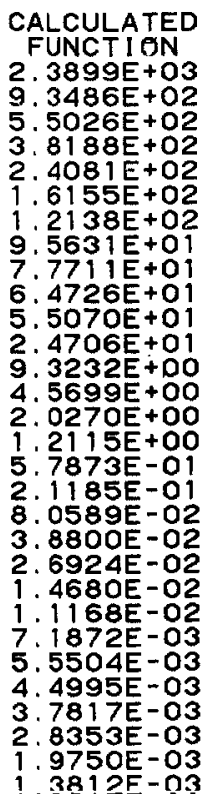 & $\begin{array}{c}\text { PERCENT } \\
\text { DEVIATION } \\
0.00 \\
0.02 \\
-0.41 \\
0.55 \\
0.49 \\
-0.96 \\
-1.14 \\
-0.56 \\
0.63 \\
1.81 \\
1.14 \\
-2.05 \\
1.36 \\
0.44 \\
-3.80 \\
-2.60 \\
8.34 \\
17.54 \\
-6.07 \\
-9.54 \\
-4.55 \\
9.00 \\
9.24 \\
-1.35 \\
-5.41 \\
-3.77 \\
0.48 \\
2.28 \\
1.77 \\
1.36\end{array}$ \\
\hline
\end{tabular}




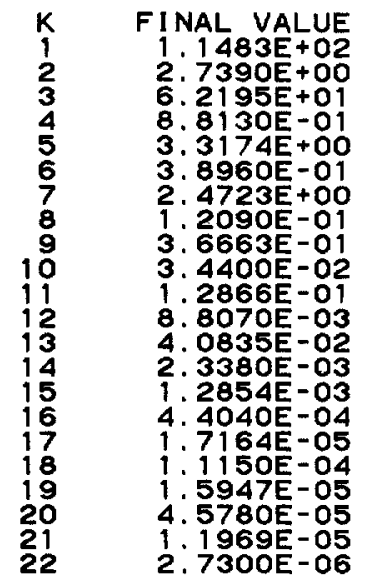

STND. DEV

. $16284 E+01$

1. $7434 \mathrm{E}+00$

2. $6372 E-01$

$5.9711 E-02$

$1.2101 E-02$

1. $5311 \mathrm{E}-03$

6. $8975 E-05$

1. 0544E-05

3. $7226 \mathrm{E}-06$

:. $9507 E-07$

FIXED PARAMETER

FIXED PARAMETER

FIXED PARAMETER

FIXED PARAMETER

FIXED PARAMETER

FIXED PARAMETER

FIXED PARAMETER

FIXED PARAMETER

FIXED PARAMETER

FIXED PARAMETER

FIXED PARAMETER

CALCULATED
FUNCTION
$3.8148 \mathrm{E}+01$
$1.5126 \mathrm{O}+01$
$7.7010 \mathrm{O}+00$
$4.5415 \mathrm{E}+00$
$2.2934 \mathrm{O}+00$
$1.3839 \mathrm{O}+00$
$1.0114 \mathrm{E}+00$
$7.8525 \mathrm{E}-01$
$6.3119 \mathrm{E}-01$
$5.2129 \mathrm{E}-01$
$4.4075 \mathrm{E}-01$
$1.9990 \mathrm{E}-01$
$8.2735 \mathrm{E}-02$
$4.0137 \mathrm{E}-02$
$1.6255 \mathrm{E}-02$
$8.7911 \mathrm{E}-03$
$3.2724 \mathrm{E}-03$
$6.3531 \mathrm{E}-04$
$1.9044 \mathrm{E}-04$
$8.4840 \mathrm{E}-05$
$5.5833 \mathrm{E}-05$
$2.8039 \mathrm{E}-05$
$2.1585 \mathrm{E}-05$
$1.5479 \mathrm{E}-05$
$1.2897 \mathrm{E}-05$
$1.1099 \mathrm{E}-05$
$9.7093 \mathrm{E}-06$
$7.4282 \mathrm{E}-06$
$5.1769 \mathrm{E}-06$
$3.6204 \mathrm{E}-06$
INPUT
. 5000 +O 1
. 7000 +00
2. $3000 \mathrm{E}+\mathrm{OO}$
$3600 \mathrm{E}+00$
8600 - 0
. 3900 -O
$4300=0$
. $9600 E-01$
04O0E-02
$5700 E-02$
38OOE-03
$1.8700 \mathrm{O}-04$
$8400 E-05$
$9600-05$
5300E-05
O700E-05
. $6400 \mathrm{O}-06$
$5.2900 E-06$

PERCENT
DEVIATION
0.14
-0.84
-0.01
1.29
0.29
-1.73
-1.13
0.10
1.24
1.67
0.51
-1.95
1.29
0.66
-3.42
-3.08
3.29
16.64
-1.80
-7.59
-4.54
5.57
5.09
-1.16
-3.85
-3.60
-0.71
0.56
2.18
3.30

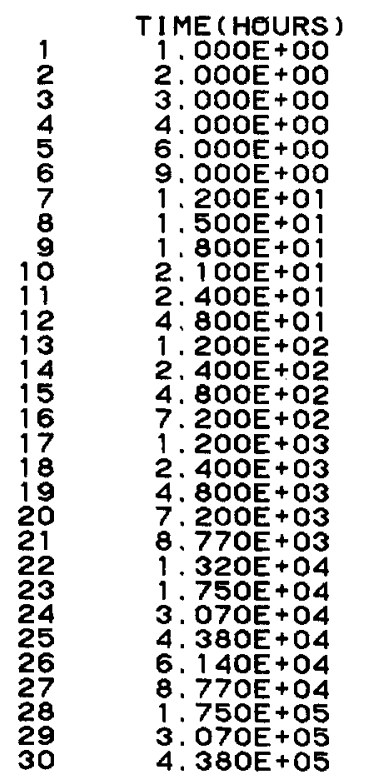




\section{APPENDIX 4}

SMOKY EVENT

\section{Microcuries per Square Meter}

Fraction of refractories present $=1.0$

Total $\mathrm{mR} / \mathrm{hr}$ and Total $\mu \mathrm{Ci} / \mathrm{m}^{2}$

4-1

Individual nuclide $\mu \mathrm{Ci} / \mathrm{m}^{2}$

$4-2$

Fraction of refractories present $=0.5$

Total $\mathrm{mR} / \mathrm{hr}$ and Total $\mu \mathrm{Ci} / \mathrm{m}^{2}$

Individual nuclide $\mu \mathrm{Ci} / \mathrm{m}^{2}$

Fraction of refractories present $=0.1$

Total $\mathrm{mR} / \mathrm{hr}$ and Total $\mu \mathrm{Ci} / \mathrm{m}^{2}$

$4-23$

Individual nuclide $\mu \mathrm{Ci} / \mathrm{m}^{2}$

$4-24$ 


\begin{tabular}{|c|c|c|}
\hline \multicolumn{3}{|c|}{ 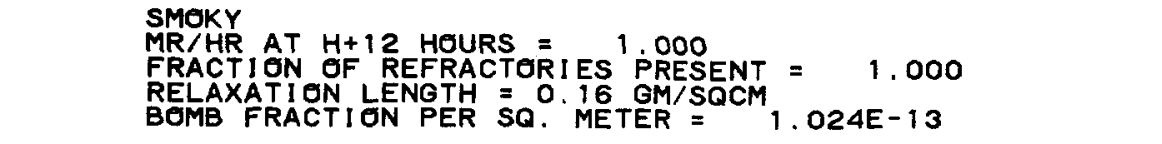 } \\
\hline TIME & MR/HR & MICROCURIES/SQ METER \\
\hline 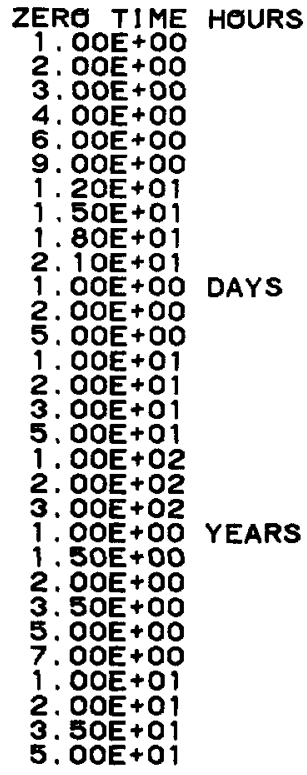 & 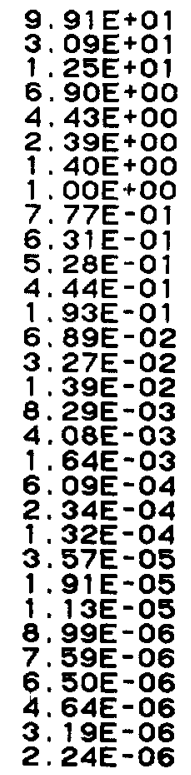 & 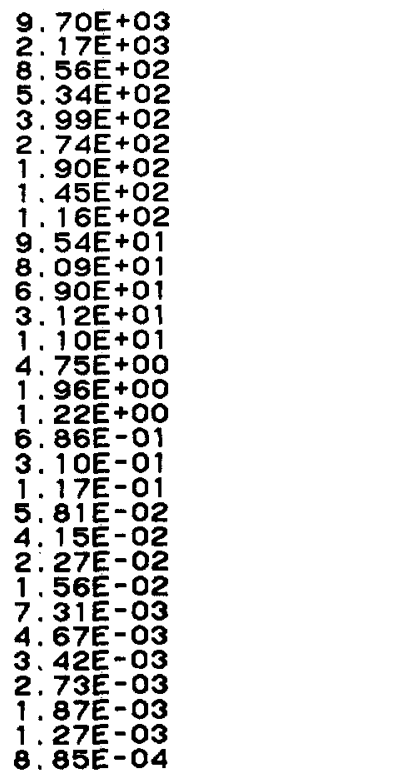 \\
\hline
\end{tabular}

page $4-1$ 
DEBRIS DECAY FROM 1 TO 21 HOURS

ZERO TIME $1.00 E+00 \quad 2,00 E+00 \quad 3.00 E+00 \quad 4.00 E+00 \quad 6.00 E+00 \quad 9.00 E+00 \quad 1.20 E+01 \quad 1.50 E+01 \quad 1.80 E+01 \quad 2.10 E+01$

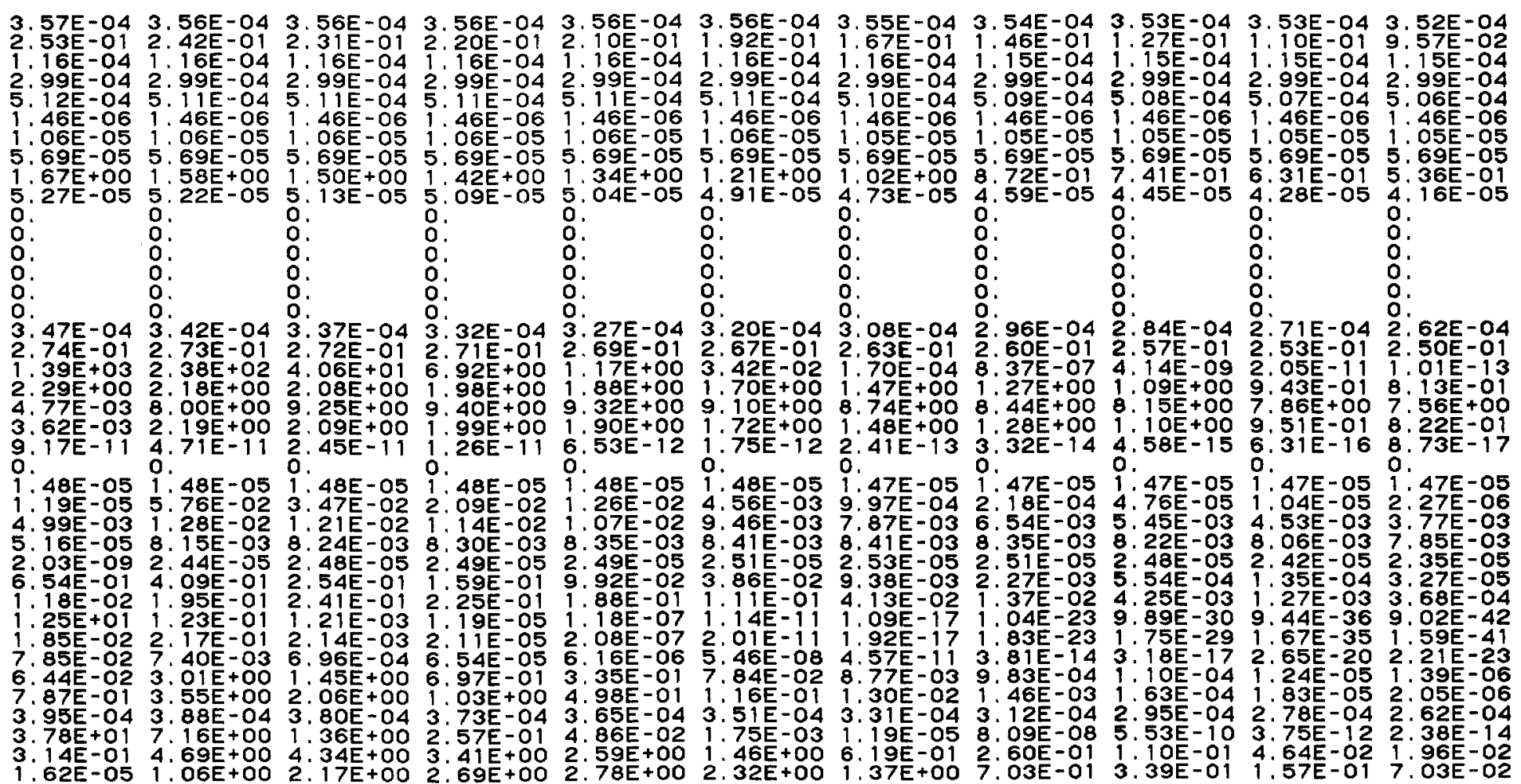




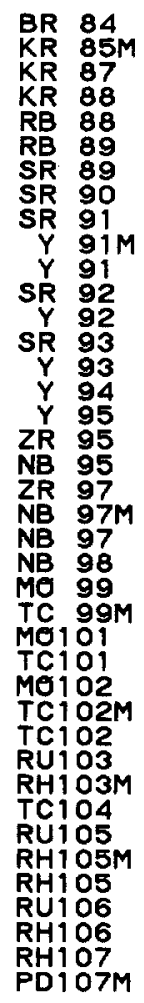

MRACTION OF REFRACTORIES PRESENT = 1.000 BOMB FRACTION PER SO. METER $=1.024 E-13$

DEBRIS DECAY FROM 1 TO 21 HUURS

ZERO TIME 1. $1.00 E+O O \quad 2.00 E+O 0 \quad 3.00 E+00 \quad 4.00 E+00 \quad 6.00 E+00 \quad 9.00 E+00 \quad 1.20 E+01 \quad 1.50 E+01 \quad 1.80 E+012.10 E+01$

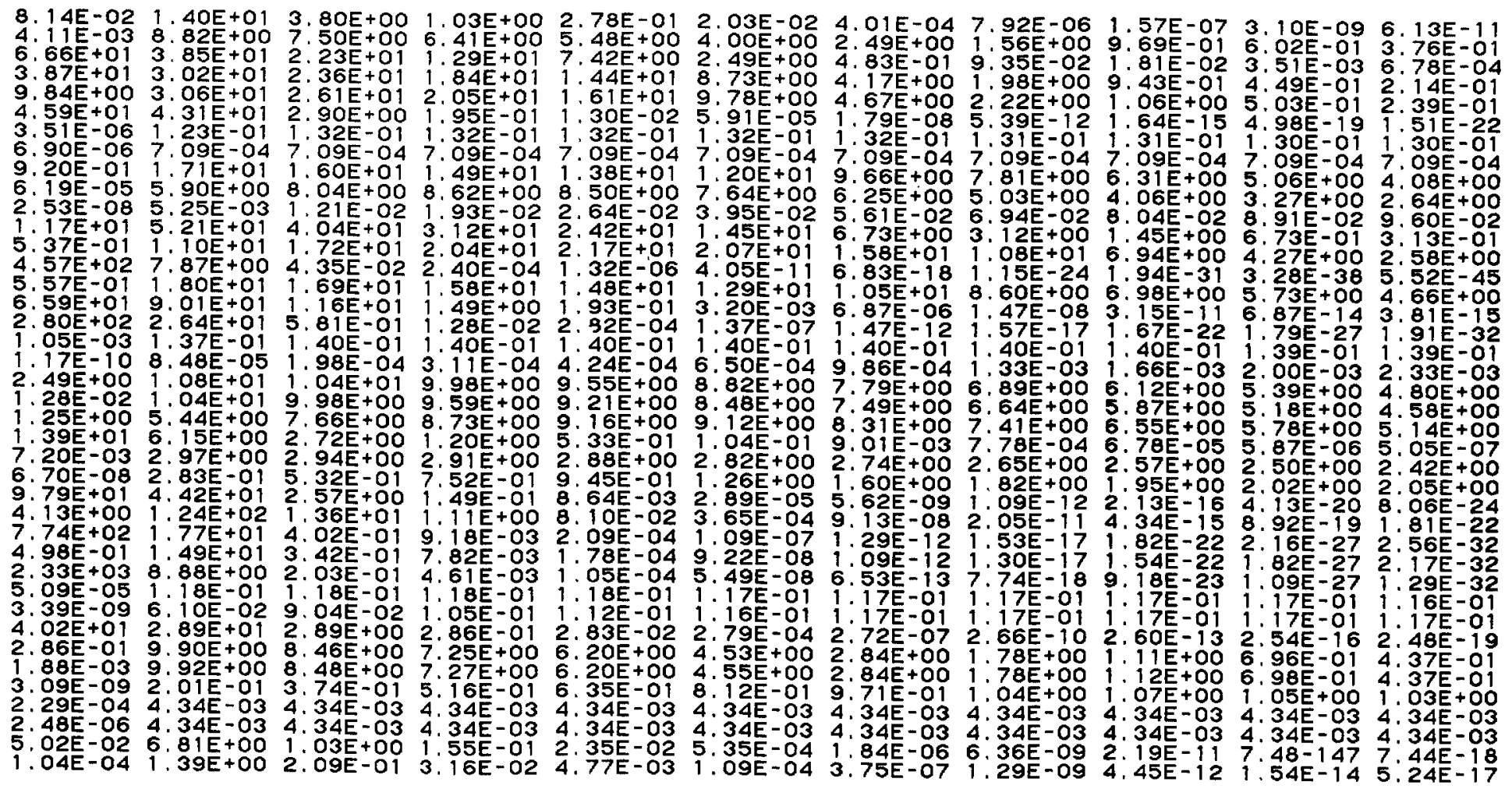


MICROCURIES/SQ METER

DEBRIS DECAY FREM 1 TO 21 HOURS

ZERO TIME $1.00 E+002.00 E+00 \quad 3.00 E+00 \quad 4.00 E+00 \quad 6.00 E+00 \quad 9.00 E+00 \quad 1.20 E+01 \quad 1.50 E+01 \quad 1.80 E+01 \quad 2.10 E+01$

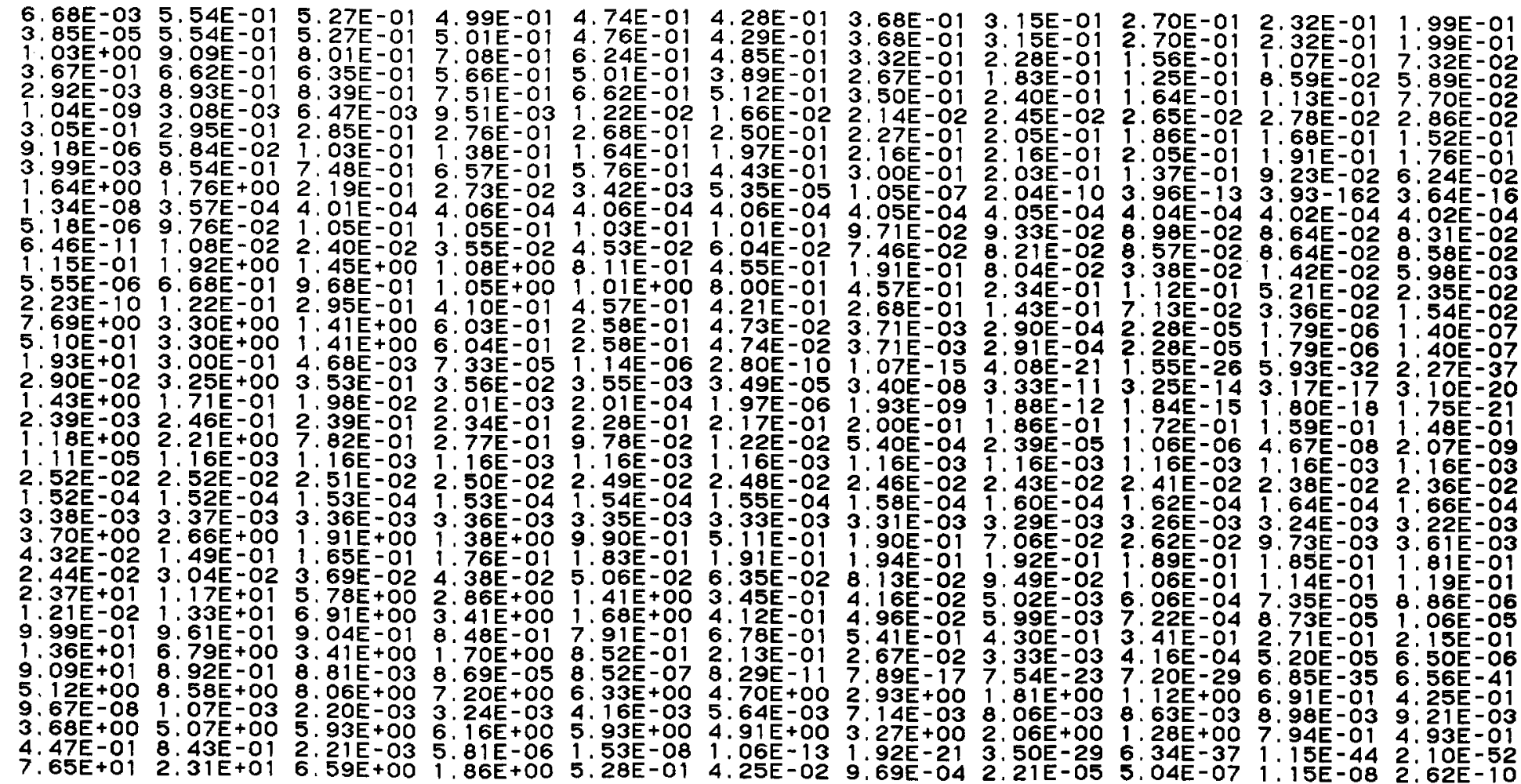


MR/HR AT H+12 HOURS $=$ MICROOORIES/SQ METER

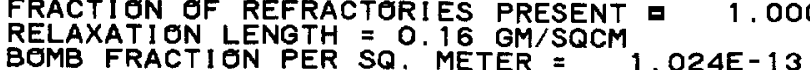

DEBRIS DECAY FROM 1 TO 21 HOURS

ZERO TIME $1.00 E+00 \quad 2.00 E+003.00 E+00 \quad 4.00 E+00 \quad 6.00 E+00 \quad 9.00 E+00 \quad 1.20 E+01 \quad 1.50 E+01 \quad 1.80 E+012.10 E+01$

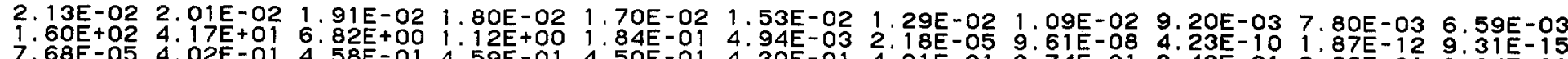

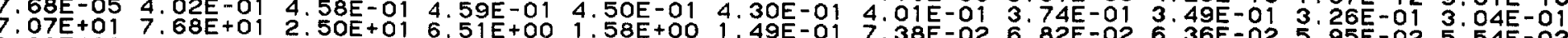
1.03E-02 3.56E-01 5.24E-01 5.75E-01 5.85E-01 5.90E-01 5.38E-02 6.82E-02 6.36E-02 5.95E-02 5.54E-02

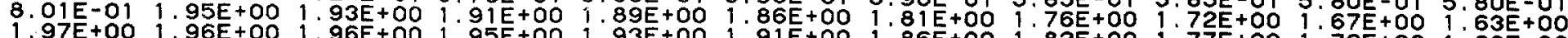

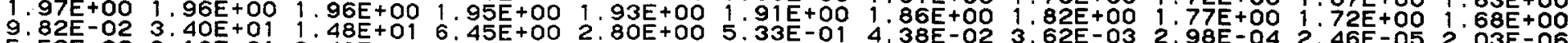

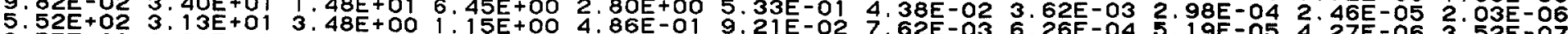

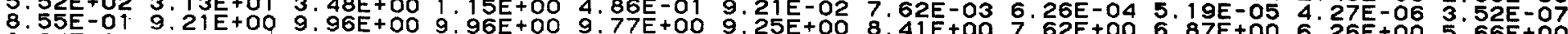

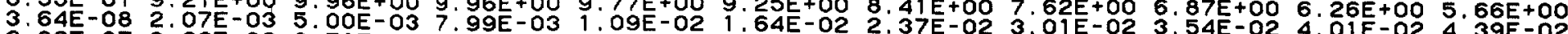

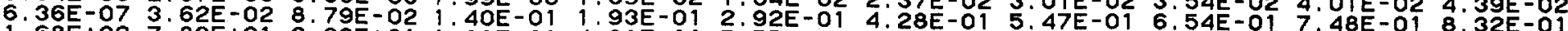

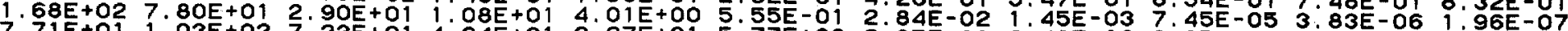

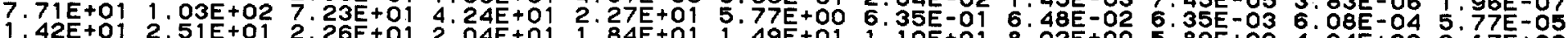

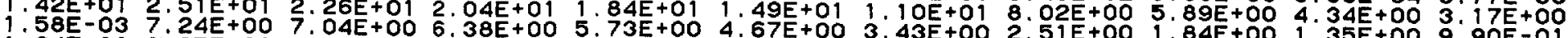

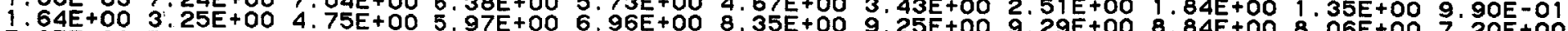

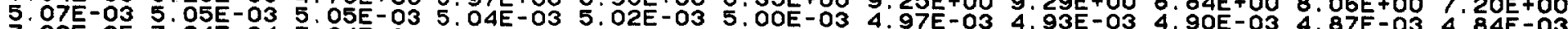
7.09E-05 7.64E-04 7.64E-04 7.64E-04 7.64E-04 7.64E-04 7.64E-04 7.64E-04 7.64E-03 4.87E-03 4.84E-03 1. $49 E-07$ 7.13E-04 7.13E-04 7.13E-04 7.13E-04 7.13E-04 7.13E-04 7.13E-04 7.13E-04 7.13E-04 7. 13 E-04 5.72E+02 4.93E+01 4.28E+00 3.71E-01 3.21E-02 2.41E-04 1.57E-07 1.02E-10 6.61E-14 4.30E-17 2:79E-20

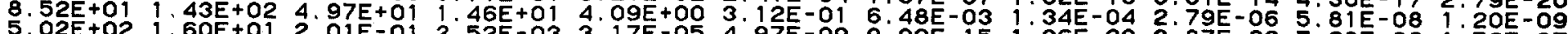

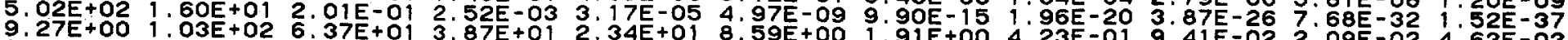

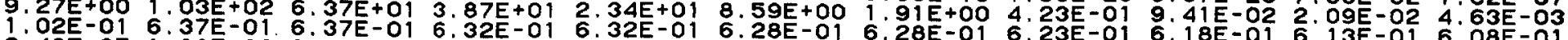

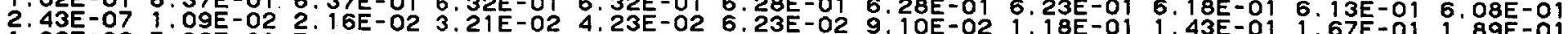

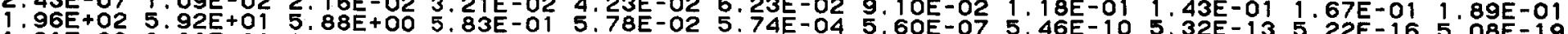

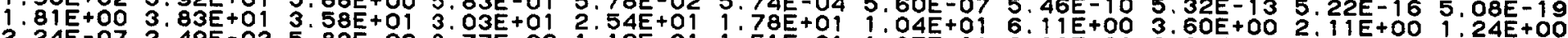

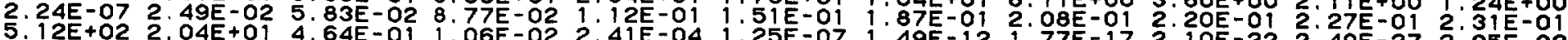

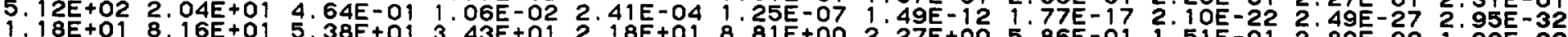

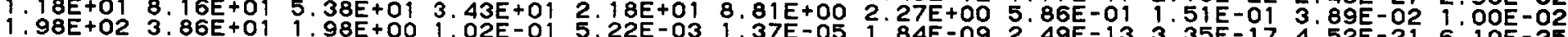

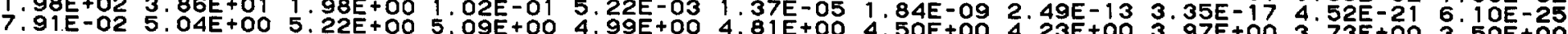

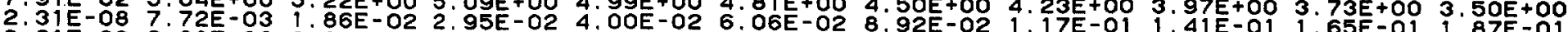

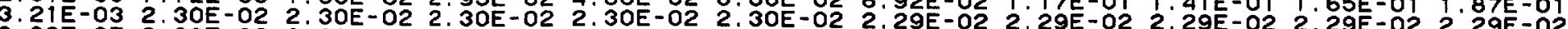

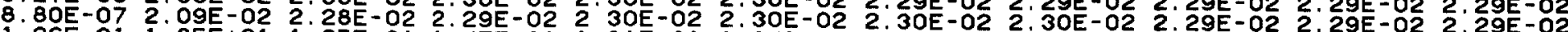

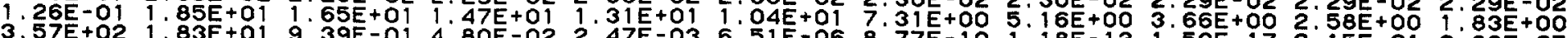
3.50

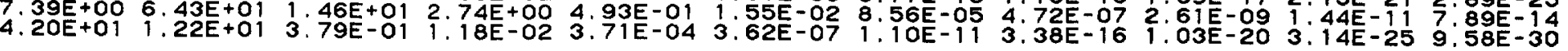


DEBRIS DECAY FROM 1 TO 21 HOURS

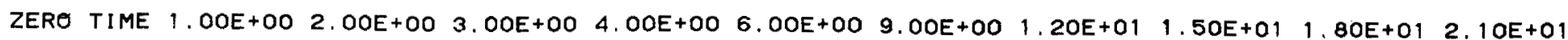

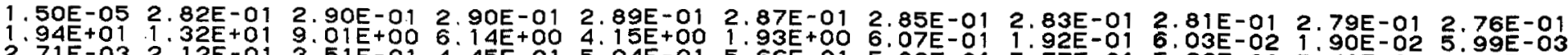
2.71E-03 2.12E-01 3.51E-01 4.45E-01 5.04E-01 5.66E-01 5.88E-01 5.77E-01 5.52E-01 5.40E-01 5. 22E-01 2.22E-01 1.71E-01 1.33E-01 1. $02 E-01$ 7.91E-02 4.75E-02 2.20E-02 1.02E-02 4.71E-03 2. $18 E-031$ 1.01E-03

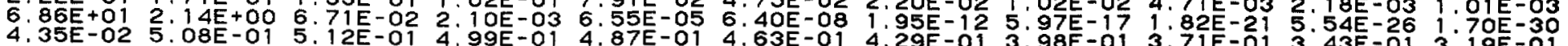

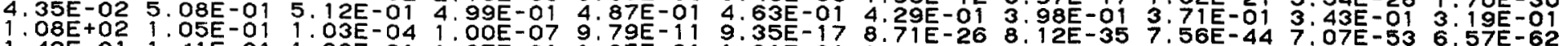

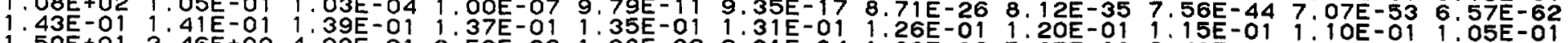

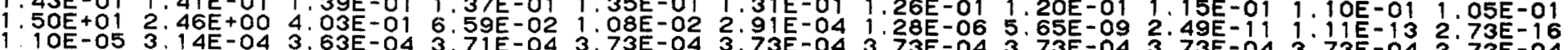
9. $15 \mathrm{E}-02$ 8. $49 \mathrm{E}-02$ 7.89E-02 7.33E-02 6.80E-02 5.87E-02 4.71E-02 3.77E-02 3. 03 E-02 $2.42 \mathrm{E}-02$. $94 \mathrm{E}-02$

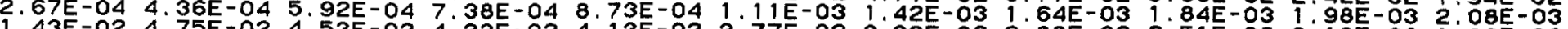
1. $43 E-02$ 4.75E-02 4.53E-02 4.33E-02 $4.13 E-02$ 3.77E-02 3.30E-02 2.88E-02 2.51E-02 $2.18 E-02 \quad 1.90 E-02$ 3.39E 1.37 .

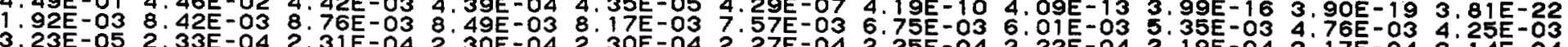


SMOKY

MRAHR AT H+12 HOURS $={ }^{H}{ }^{1}{ }^{1}$ OORACTION OF REFRACTERIES PRESENT $=1.000$

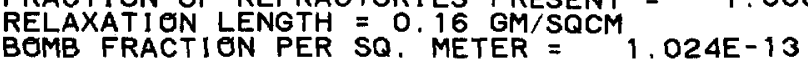

DEBRIS DECAY FROM 1 TO 300 DAYS

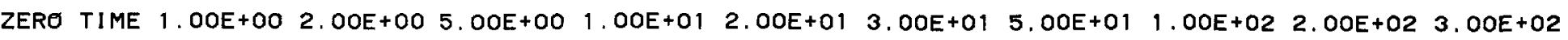

3.57E-04 3.52E-04 3.48E-04 3.35E-04 3.13E-04 2.75E-04 2.42E-04 1.86E-04 9.73E-05 2.64E-05 7.19E-06 1.16E-04 1.15E-04 1.15E-04 1.12E-04 1.11E-04 1.09E-04 1.07E-04 1.02E-04 9.13E-05 $7.26 E-05$ 5. $79 E-05$

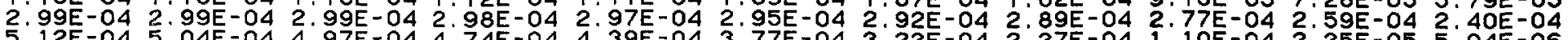

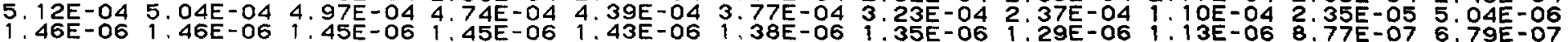

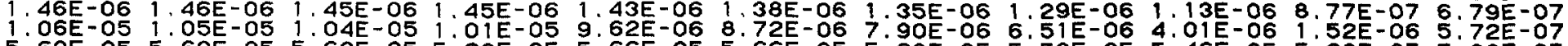
5.69E-05 5.69E-05 5.69E-05 5.69E-05 5.66E-05 5.66E-05 5.63E-05 5.59E-05 5.46E-05 5.29E-05 5.09E-05

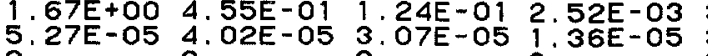

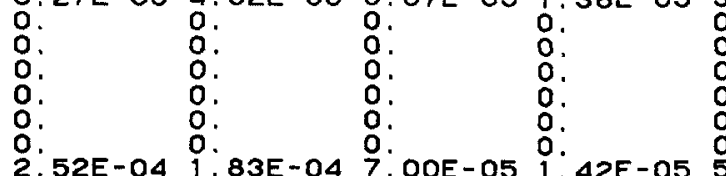

52E-04 1.83E-04 7.00E-05 1:42E-05 5.84E-07 2. 39E-08 $4.02 E-11$ 0.70E-18

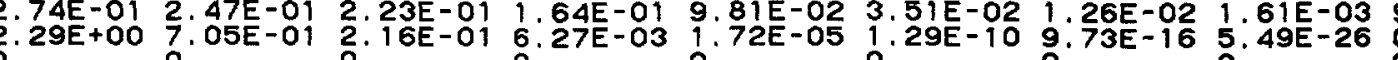

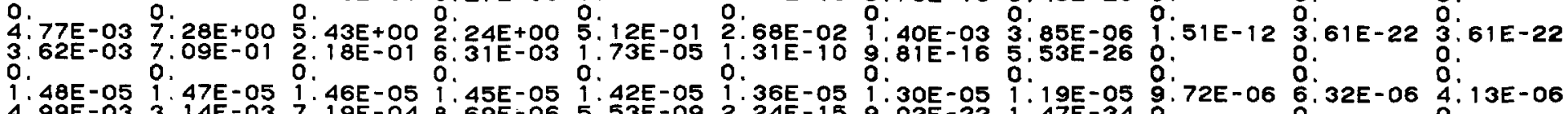

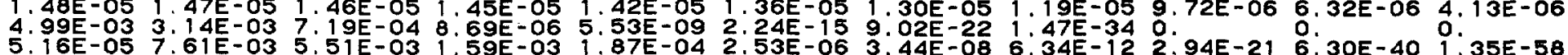
2.03E-09 2.29E-05 1.65E-05 4.79E-06 5.58E-07 7:59E-09 1:03E-10 1.90E-14 8.81E-24 1.88E-42 4.07E-61

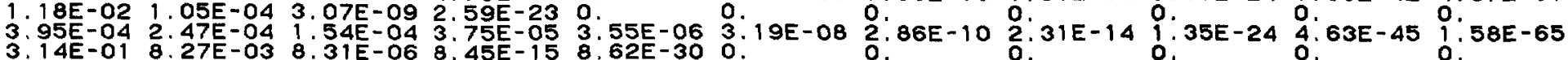

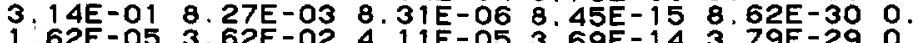

$1.49 E-325.64 E-49$ 4.26E-06 1.12E-04 1.14E-04 1.14E-04 1.14E-04 1.14E-04 1.14E-04 3.66E+01 1.32E-04 2.60E-10 2.02E-27 : $111 E-25:$ 9.84E+00 1.13E-01 2:99E-04 5.43E-12 6.81E-25 0. .51E-06 1.05E-01 1.04E-01 9.97E-02 9.35E-02 8.17E-02

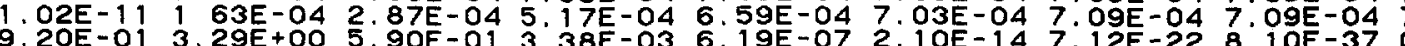

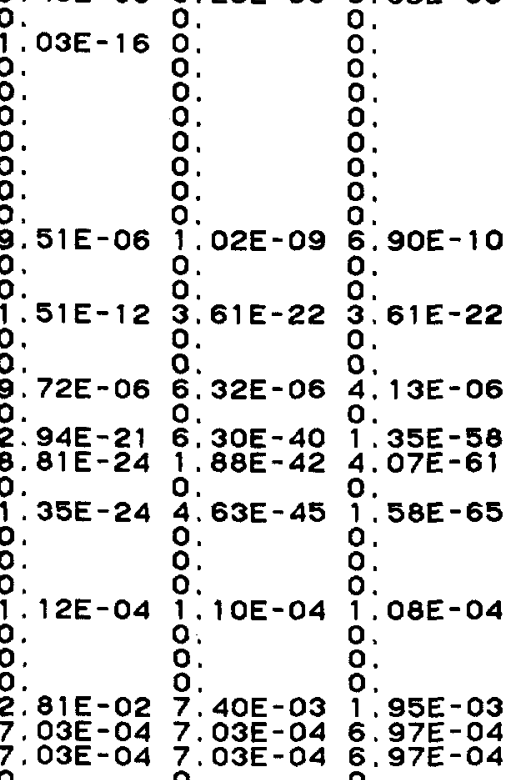


SMOKY

MICROCURIES/SQ METER

FRACTION OF REFRACTERI ISS PRESENT $=1,000$

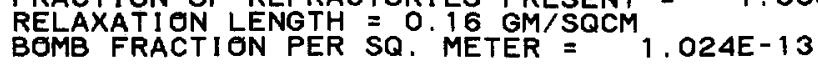

DEBRIS DECAY FREM 1 TO 300 DAYS

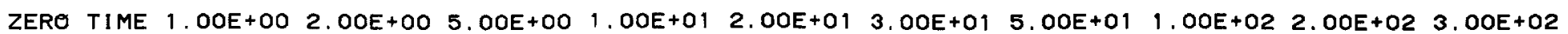

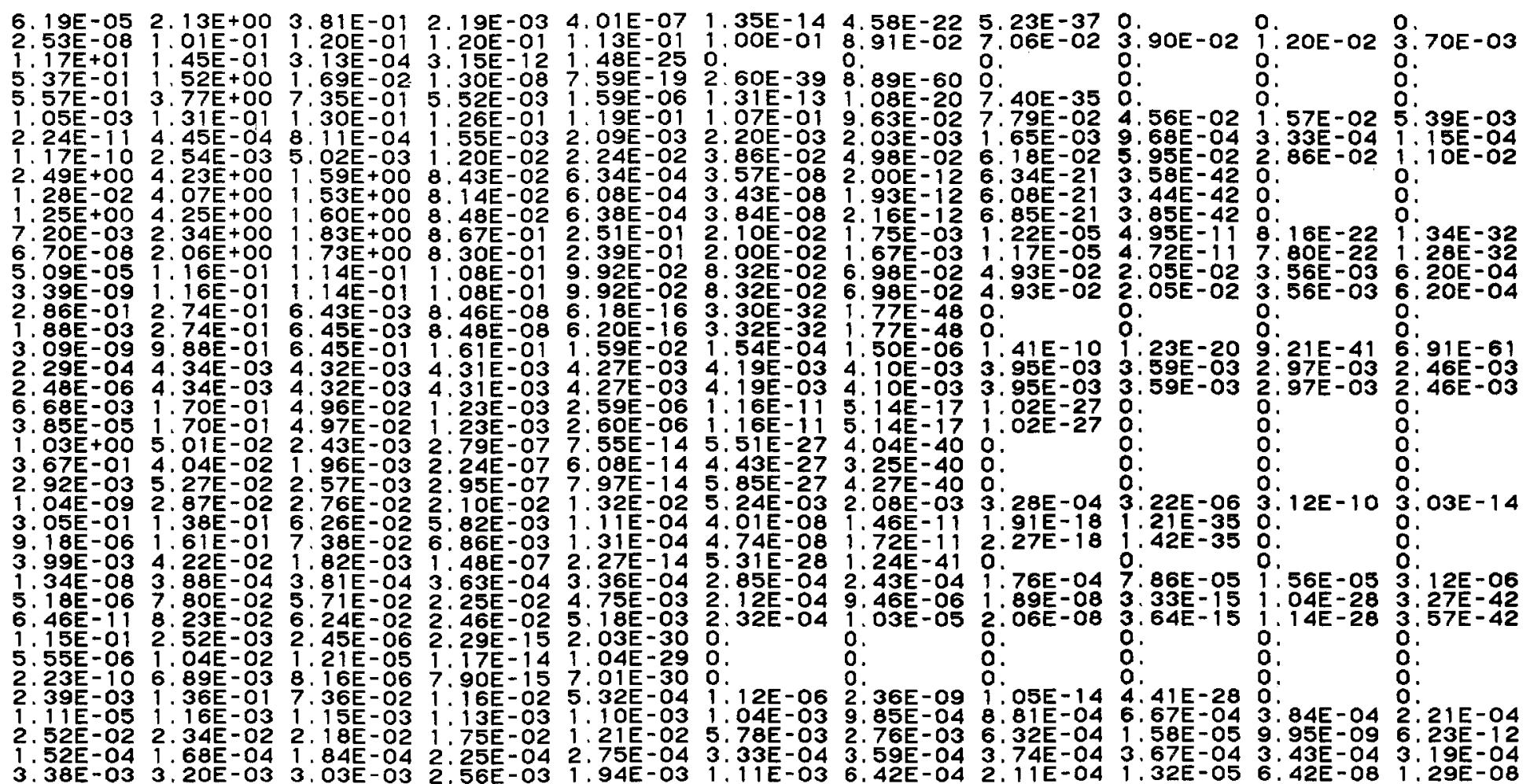


SMEKY

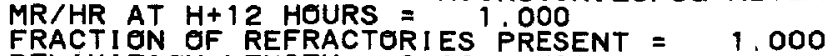

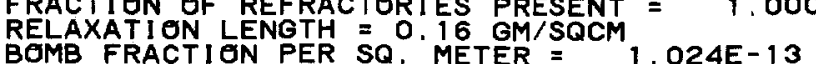

DEBRIS DECAY FROM 1 TO 300 DAYS

ZERO TIME $1.00 E+002.00 E+00 \quad 5.00 E+001.00 E+01 \quad 2.00 E+01 \quad 3.00 E+01 \quad 5.00 E+01 \quad 1.00 E+02 \quad 2.00 E+02 \quad 3.00 E+02$

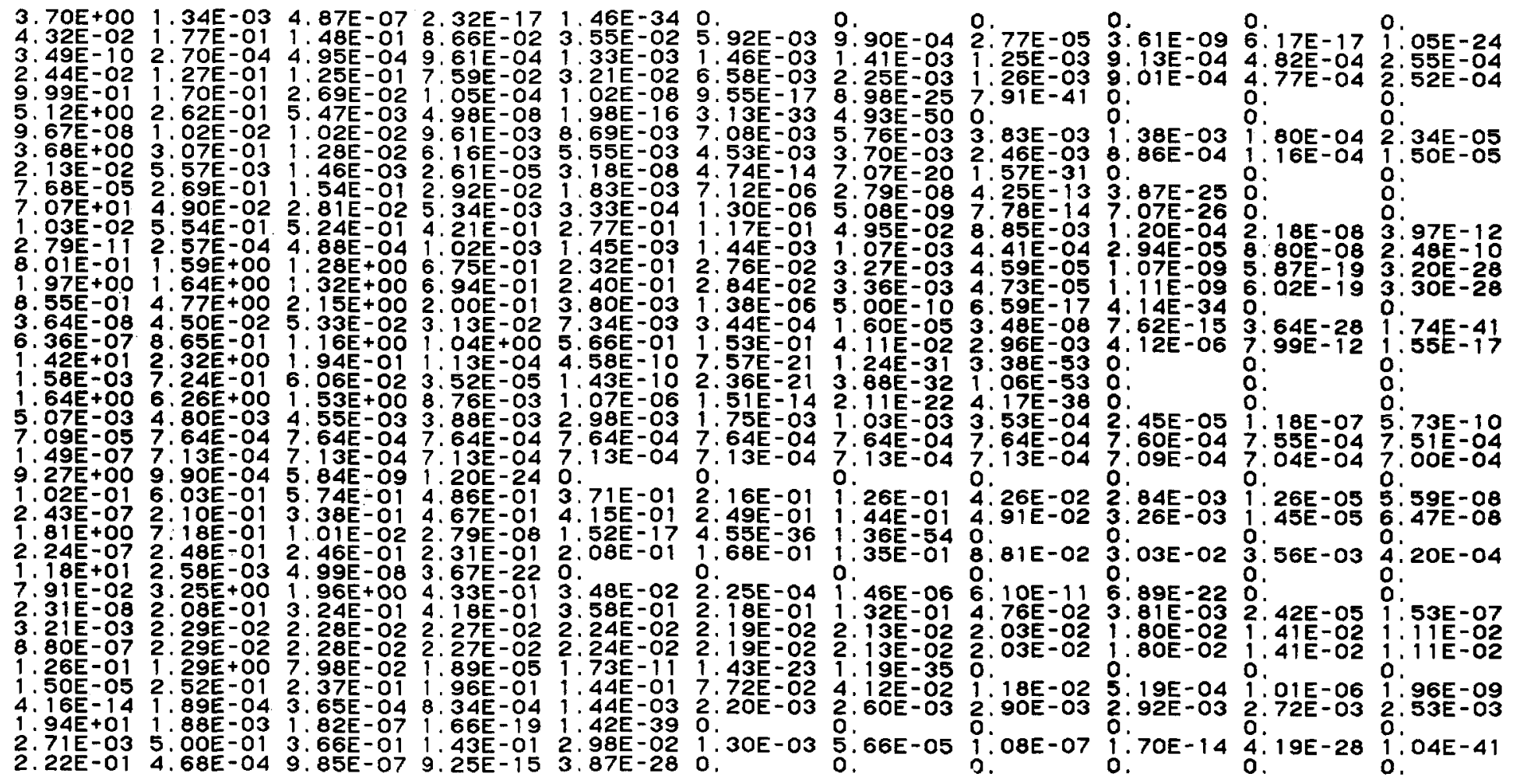


SMOKY

FRACTION OF REFRACTERIES PRESENT $=1.000$

RELAXATION LENGTH $=0.16$ GM/SQCM $1.024 E-13$

DEBRIS DECAY FROM 1 TO 300 DAYS

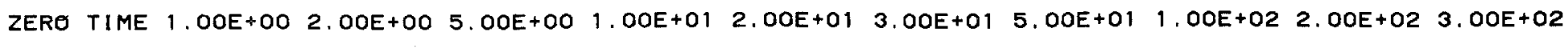

PM151

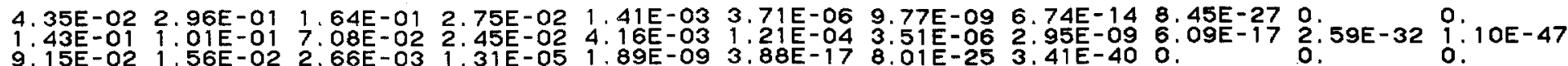

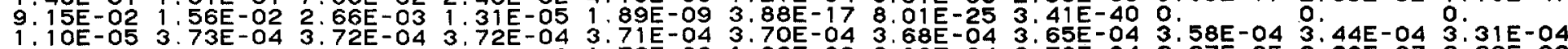

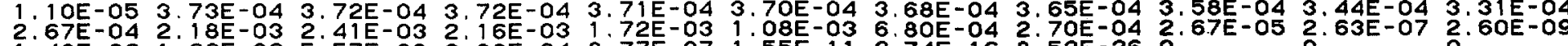

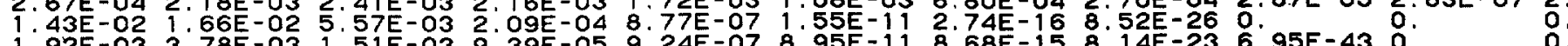

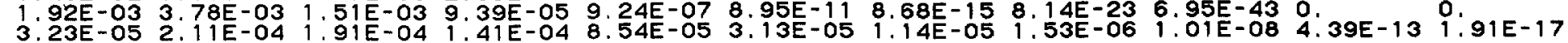

GD159

2. 87E+02 $6.90 E+01 \quad 3.12 E+01 \quad 1.10 E+01 \quad 4.75 E+00 \quad 1.96 E+00 \quad 1.22 E+00 \quad 6.86 E-01 \quad 3.10 E-01 \quad 1.17 E-01 \quad 5.81 E-02$

TOTAL 
SMOKY MI CROCURIES/SQ METER FRACTION OF REFRACTORIES 'PRESENT $=1.000$ RELAXATION LENGTH $=0.16$ GM/SQCM
BOMB FRACTION PER SQ. METER $=1.024 E-13$ DEBRIS DECAY FROM 1 TO 50 YEARS

ZERO TIME 1. OOE+OO $1.50 E+00 \quad 2.00 E+00 \quad 3.50 E+00 \quad 5.00 E+00 \quad 7.00 E+00 \quad 1.00 E+01 \quad 2.00 E+01 \quad 3.50 E+01 \quad 5.00 E+01$

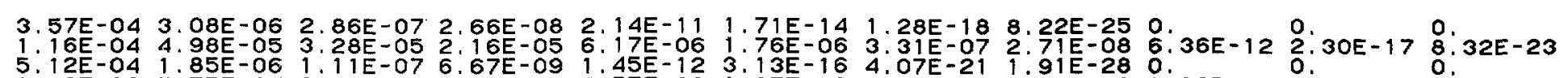

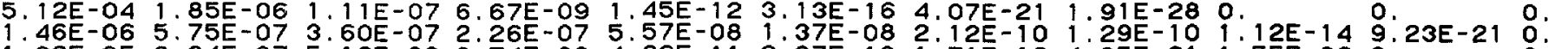

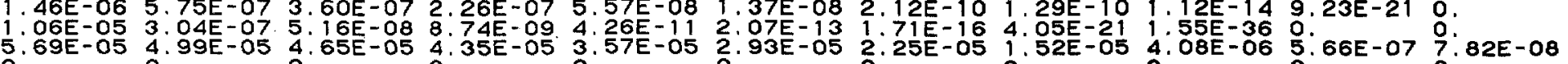
o. $69 \mathrm{E}-05$

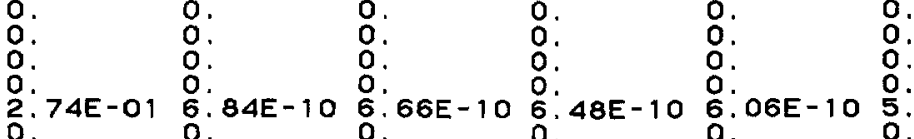

1. $48 \mathrm{E}-05$ 3., $13 \mathrm{E}-06$ O. $44 \mathrm{E}-06$ 6. $62 \mathrm{E}-07$. . $6 E-06$ 1. $06 \mathrm{E}-04$ 1. $44 \mathrm{E}-06$ 6.62E-07 $6.45 \mathrm{E}-08$

3. 51E-06 8.22E-04 7.19E-05 6.32E-06 4.25E-09

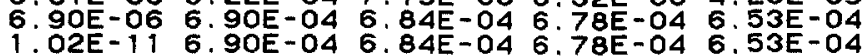

. $22 \mathrm{E}-0 \mathrm{6}$. $90 \mathrm{E}-04 \mathrm{6}$. $84 \mathrm{E}-046.78 \mathrm{E}-04$

$1.05 E-03 \quad 2.70 E-03 \quad 3.85 E-045.39 E-05$ 3. $63 E-08$

2.24E-11 5.72E-05 8. $16 E-061$. $17 E-06$

$1.17 \mathrm{E}-105.81 \mathrm{E}-03$ 8. $34 \mathrm{E}-0491 \mathrm{9E}-04$

3. $39 E-0991.98 E-04$ 8. $09 E-063.33 E-07$ 2. $327 E-11$

$2.48 E-06$
1.11
1

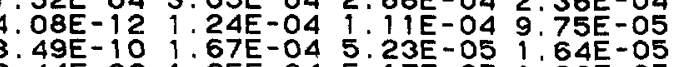

. 0

$2.44 \mathrm{E}-02,65 \mathrm{E}-04$

.

2. $24 \mathrm{E}-07$ 9.65E-05 $1.94 \mathrm{E}-06 \mathrm{3}$ 3.90E-08

O.

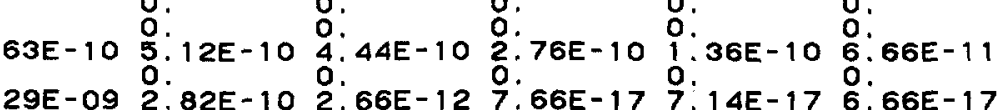
16E-05 7.17E-05 5.91E-05 3.13E-17 7.14E-17 $6.66 E-17$ . . 69E-11 $1.94 E-142.56 E-20$ 5.17E-39 0.99E-04 $66 E-101.93 E-13$ 1.63E-18 $1.99 E-35 \% . \quad 0$ OOE-09 $4.18 E-13$ 3.53E-18 4. $32 E-35$ O 56E-15 4. 37E-21 2. $06 E-29$ 0.

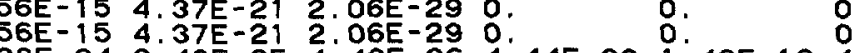
38E-04 3. 48E-05 4. 40E-06 $4.44 E-09$ 1. $43 E-134.59 E-18$

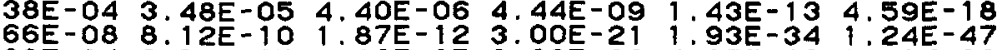
O9E-04 6.53E-05 3.02E-05 2.33E-06 4.95E-08 1.05E-09

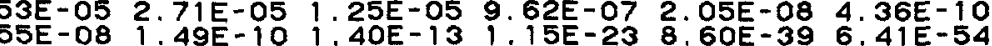

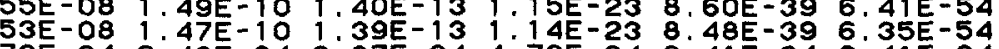

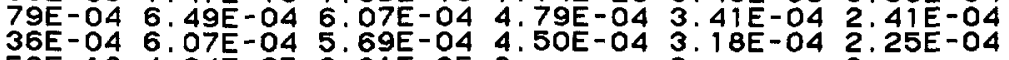

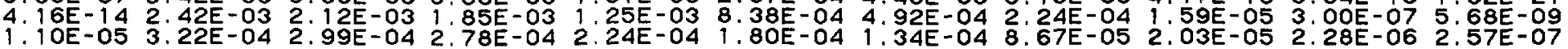

TOTAL

3.04E-01 4.15E-02 2.27E-02 1.56E-02 7.31E-03 4.67E-03 3.42E-03 2.73E-03 $1.87 E-03 \quad 1.27 E-03 \quad 8.85 E-04$ 
SMOKY

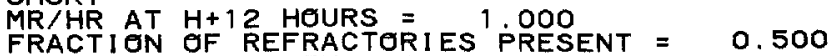

RELAXATION LENGTH $=0.16 \mathrm{GM} / \mathrm{SQCM}$
BOMB FRACTION PER SQ. METER $=1.299 \mathrm{E}-13$

TIME

$\mathrm{MR} / \mathrm{HR}$

MICROCURIES/SQ METER

ZERO TIME HOURS

2.:OOE+OO

1. $02 \mathrm{E}+\mathrm{O} 2$

$1.35 E+01$

$7.21 \mathrm{E}+00$

$4: 00$ + +00

. $.00 E+00$

$20 \mathrm{O}+01$

$80 \mathrm{O}+01$

$1: 100+01$ DAYS

2. OOE +OO

$1.00 \mathrm{O}+01$

2. 00 +o1

3. $O 0 \mathrm{E}+\mathrm{a} 1$

5. $00 \mathrm{OE}+01$

2. $00 \mathrm{E}+02$

1.00E+OO YEARS

$1: 50 \mathrm{D}+\infty 0$

2. OOE+OO

5. $00 \mathrm{OOOO}$

$7.0 O E+D O$

1. OOE+O1

3. $50 \mathrm{OE}+01$

$3.50 E+01$
$5.00 E+01$

4. $49 \mathrm{E}+00$

$38 \mathrm{E}+00$

$1.38 \mathrm{E}+00$

7. $79 \mathrm{EE}-01$

6. 32E-OI

4.41 - 1

1. 91 E-인

$3.55 \mathrm{E}-\mathrm{O} 2$

$1.45 E-03$

3. $69 \mathrm{E}-03$

$1.22 E-03$

4. $18 \mathrm{E}-04$

1. $63 \mathrm{E}-04$

3.15E-05

$.29 \mathrm{E}-05$

. $05 \mathrm{E}-05$

.

.85E-06

.78E-

$4.03 E-06$
$2.84 E-06$
9. $96 \mathrm{E}+03$
$85 \mathrm{E}+02$
$\mathrm{~B}+\mathrm{O} 2$
. $91 \mathrm{E}+02$
$1.76 E+02$
$1: 06 \mathrm{E}+02$
8. $32 E+01$
6. $21 E+01$
$99 \mathrm{E}+00$
4. $59 \mathrm{E}+\infty$
$18 \mathrm{E}+00$
6. $48 \mathrm{E}-01$
.71E-02
4. 32 E -02
$1.87 E-02$
$1.34 \mathrm{E}-02$
$4.92 E-03$
$3.88 \mathrm{E}-03$
3. $25 \mathrm{E}-03$
$1.61 \mathrm{E}-03$
1 . $12 \mathrm{E}-03$ 
SMOKY MR/HR AT H+12 HOURS ${ }_{\text {FRACT ION OF REFRACTORIES }}^{1}$ PRESENT $=0.500$

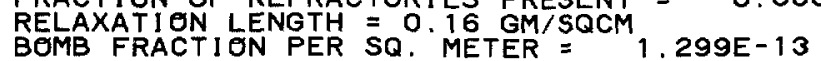

DEBRIS DECAY FROM 1 TO 21 HOURS

ZERO TIME $1.00 E+00 \quad 2.00 E+00 \quad 3.00 E+00 \quad 4.00 E+00 \quad 6.00 E+00 \quad 9.00 E+00 \quad 1.20 E+01 \quad 1.50 E+01 \quad 1.80 E+01 \quad 2.10 E+01$

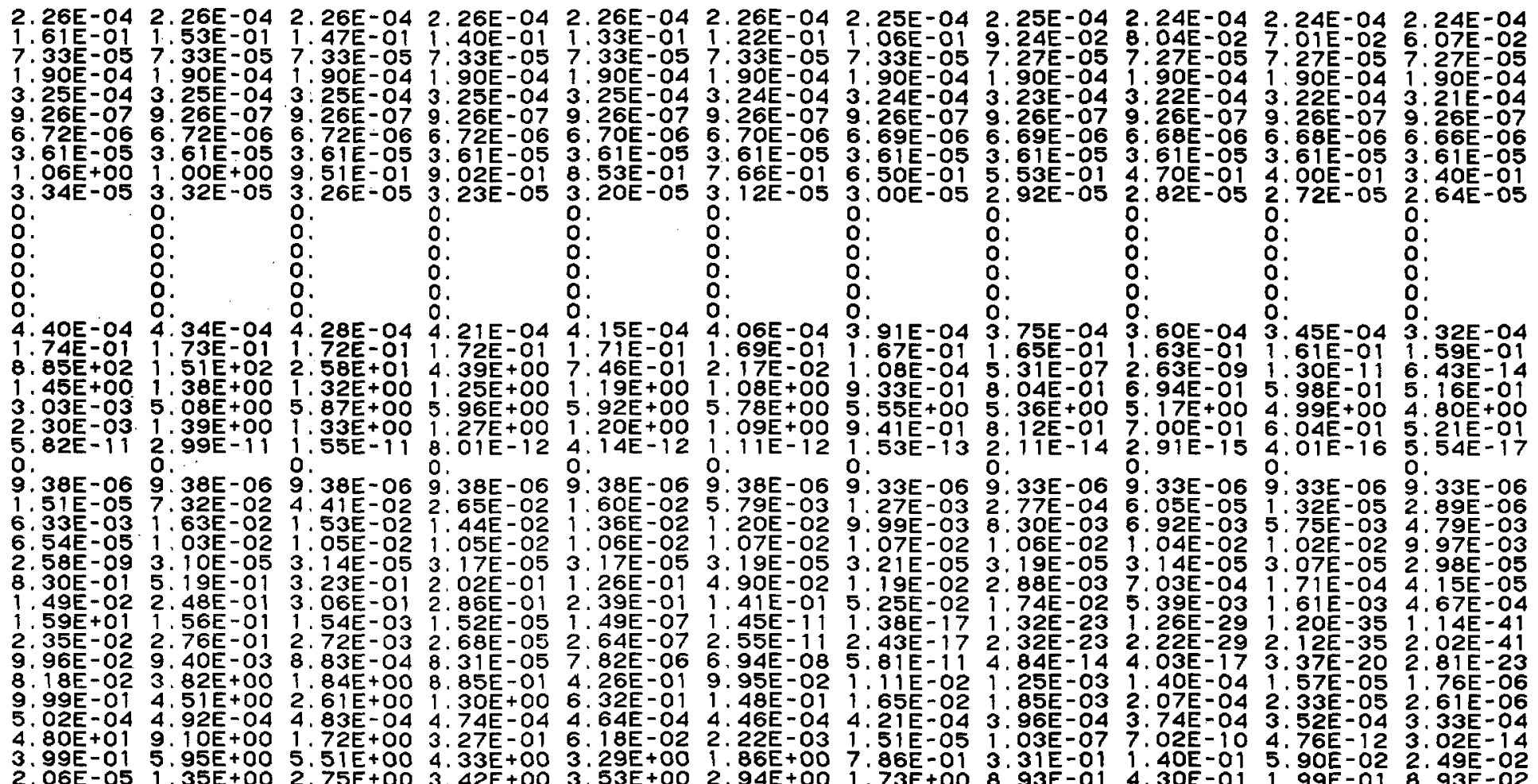


SMOKY MICROCURIES/SQ METER

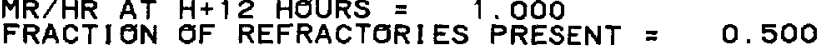

RELAXATI ON LENGTH $=0.16 \mathrm{GM} / \mathrm{SOCM}, 1.299 \mathrm{E}-13$

DEBRIS DECAY FROM 1 TO 21 HOURS

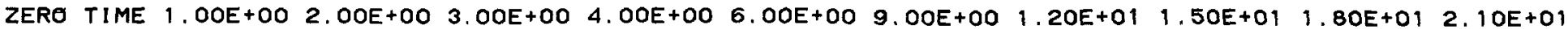

8. $48 E-03 \quad 7.04 E-01 \quad 6.69 E-01$ 6.34E-01 6.02E-01 5.43E-01 4.67E-01 4.00E-01 3.43E-01 2.94E-01 2.53E-01

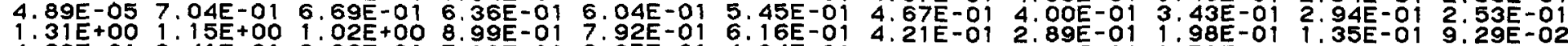
4.66E-01 8.41E-01 8.06E-01 7.99E-01 6.32E-01 6.16E-01 4.21E-0 2.89E-01 1.98E-01 1.35E-O1 9.29E-02

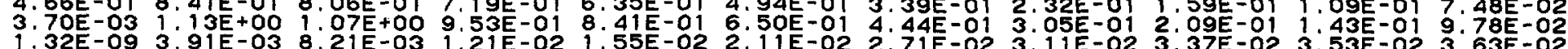
3.87E-01 3.75E-01 3.62E-01 3.50E-01 3.40E-01 3.17E-01 2.88E-01 2.61E-01 2.36E-01 2.13E-01 1. $93 E-01$ $1.17 E-05$ 7.42E-02 $1.31 E-01$ 1.76E-01 2.09E-01 2.50E-01 2.75E-01 2.74E-01 2.61E-01 2.43E-01 2.24E-01

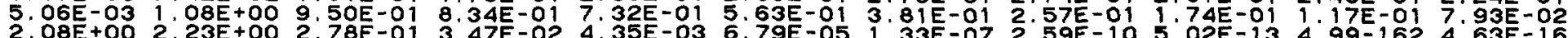

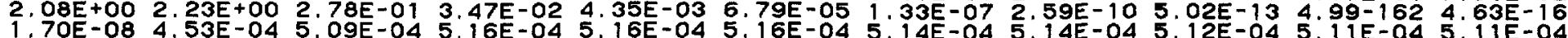
$6.58 E-061.24 E-011.33 E-01,16 E-194$

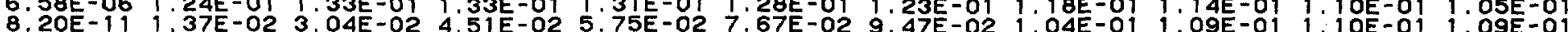
$1.47 E-012.44 E+001.84 E+001.37 E+001$. O3E+00 5.77E-01 2.42E-01, 02E-01 4.29E-02 $1.80 E-027.59 E-03$ 7.04E-06 8.49E-01 1.23E+00 1.33E+00 1.29E+00 1.02E+00 5.81E-01 2.97E-01 1.43E-01 6.61E-02 2.99E-02

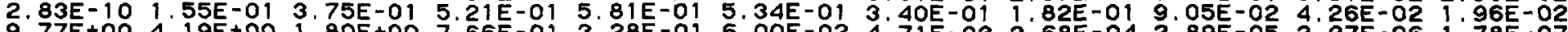
9.77E+00 4.19E+00 $1.80 E+00$ 7.66E-01 3.28E-O1 6.00E-02 4.71E-03 3.68E-04 2.89E-05 2.27E-06 $1.78 E-07$

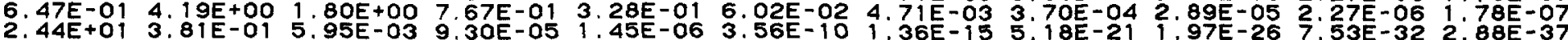

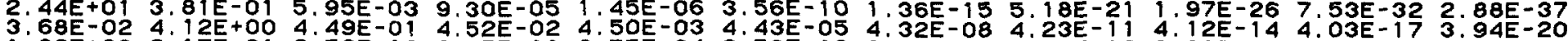
$1.82 E+002$ 2. $17 E-012.52 E-02$ 2.55E-03 2.55E-04 2.5OE-06 2.44E-09 2.39E-12 2.33E-15 2.28E-18 $2.23 E-21$ 3.04E-03 3. $13 \mathrm{E}-01$ 3.04E-01 2.97E-01 2.89E-01 2.75E-01 2.54E-01 2.36E-01 2.18E-01 2.02E-01 $1.88 E-01$ $1.50 E+002.80 E+009.93 E-013.51 E-011.24 E-011.55 E-026.86 E-043.04 E-051.34 E-06 \quad 5.93 E-082.62 E-09$ $1.41 E-051.47 E-03 \quad 1.47 E-03 \quad 1.47 E-03 \quad 1.47 E-03 \quad 1.47 E-03 \quad 1.47 E-03 \quad 1.47 E-03 \quad 1.47 E-03 \quad 1.47 E-03 \quad 1.47 E-03$

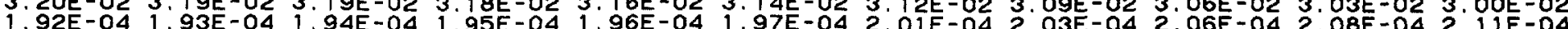
$4.29 E-03$ 4.28E-03 $4.27 E-03$ 4.26E-03 4.25E-03 4.23E-03 $4.20 E-034.18 E-034.14 E-034.11 E-0344.09 E-03$

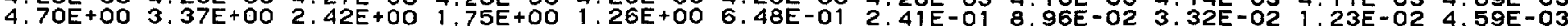

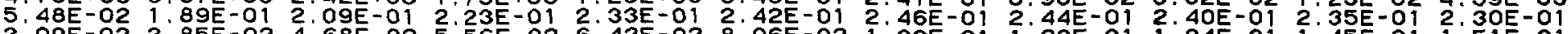
3. $09 \mathrm{E}-02$ 3.85E-02 4.68E-02 5.56E-02 6.42E-02 8.06E-02 1.03E-01 $1.20 \mathrm{E}-01$ 1.34E-01 $1.45 \mathrm{E}-011.51 \mathrm{E}-01$ 3. $01 \mathrm{E}+011.48 \mathrm{E}+01 \quad 7.34 \mathrm{E}+00 \quad 3.63 \mathrm{E}+00$ 1.79E+00 4.38E-01 5.29E-02 $6.38 \mathrm{E}-03$ 7.69E-04 9.33E-05 $1.12 \mathrm{E}-05$

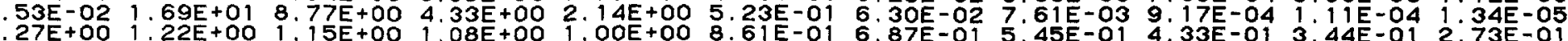

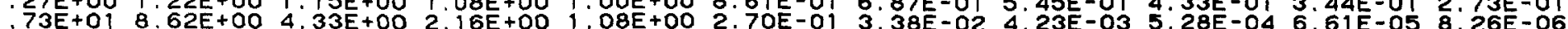
$15 E+021.13 E+001.12 E-021.10 E-041.08 E-061.05 E-101.00 E-16$ Q $-57 E-23-9.13 E-298.70 E-358.33 E-41$ $6.50 E+001.09 E+01 \quad 1.02 E+01 \quad 9.13 E+00 \quad 8.04 E+00 \quad 5.97 E+00 \quad 3.72 E+002.30 E+00 \quad 1.42 E+00 \quad 8.77 E-01 \quad 5.39 E-01$

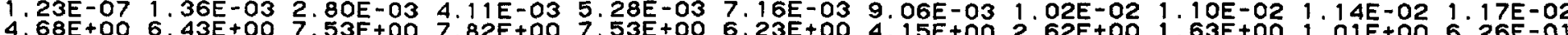
$4.68 E+006.43 E+007.53 E+007.82 E+007.53 E+006.23 E+004.15 E+002.62 E+00 \quad 1.63 E+001.01 E+006.26 E-01$

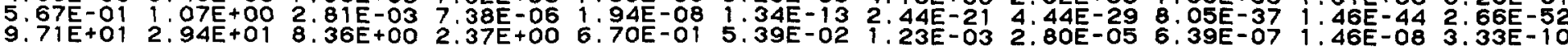


SMEKY

MRACTION OF REFRACTERIES PRESENT $=0.500$ RELAXATI ON LENGTH $=0.16$ GM SQCM $1,299 E-13$

DEBRIS DECAY FROM 1 TO 21 HOURS

ZERO TIME $1.00 E+00 \quad 2.00 E+00 \quad 3.00 E+00 \quad 4.00 E+00 \quad 6.00 E+00 \quad 9.00 E+00 \quad 1.20 E+01 \quad 1.50 E+01 \quad 1.80 E+01 \quad 2.10 E+01$

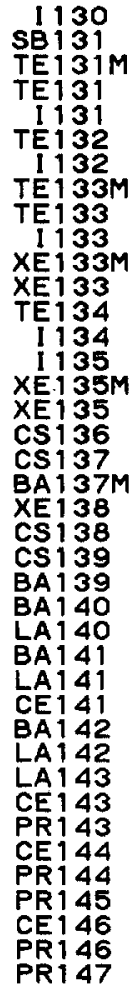

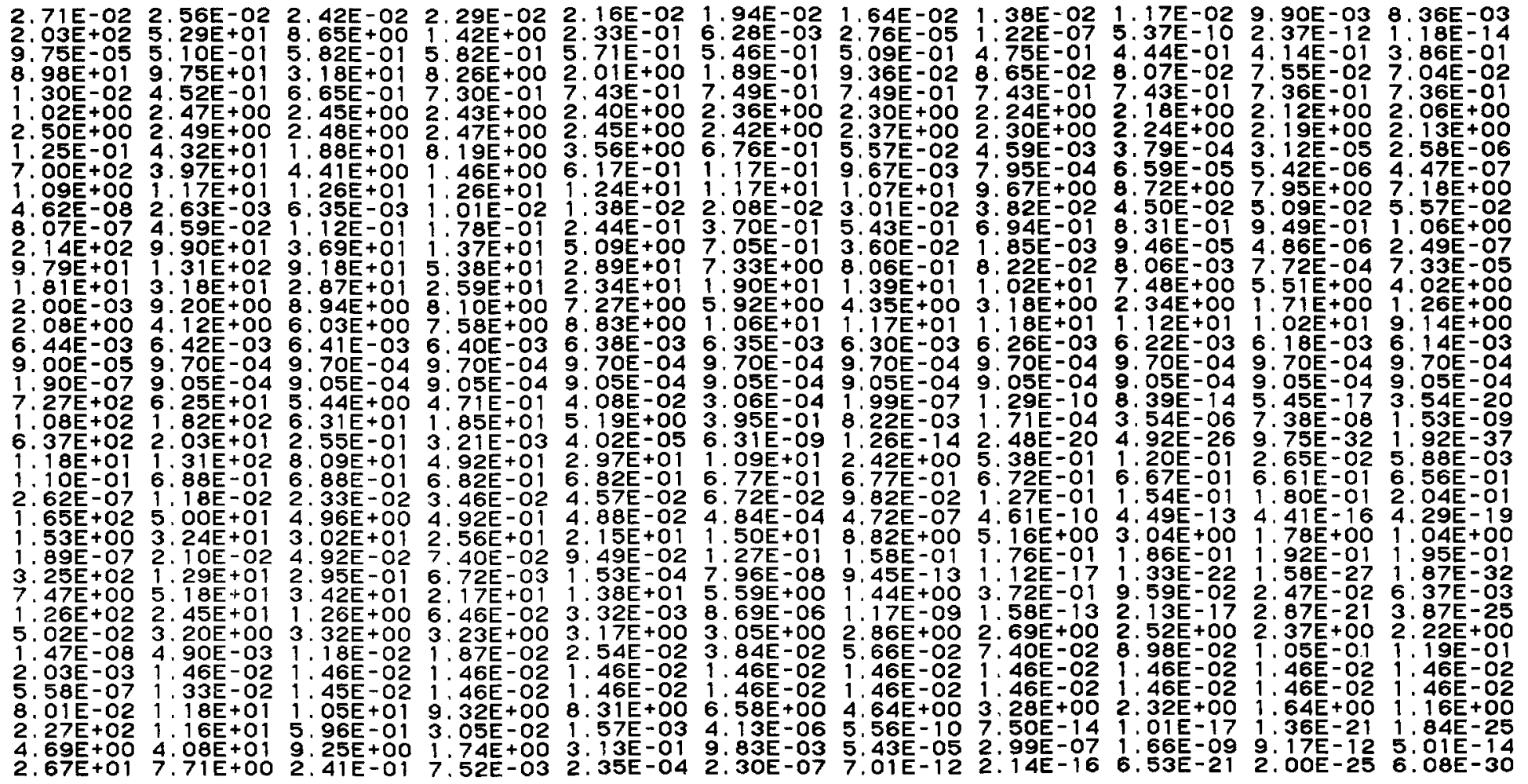


SMOKY MR/HR AT H+12 HOURS = MICROCURIES/SQ METER

PAGE

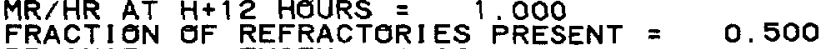

RELAXATION LENGTH $=0.16$ GMM SOCM $1.299 E-13$

DEBRIS DECAY FROM 1 TO 21 HOURS

ZERO TIME $1.00 E+00 \quad 2.00 E+00 \quad 3.00 E+00 \quad 4.00 E+00 \quad 6.00 E+00 \quad 9.00 E+001.20 E+01 \quad 1.50 E+01 \quad 1.80 E+012.10 E+01$

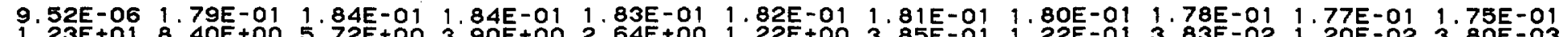

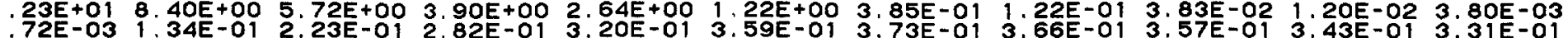

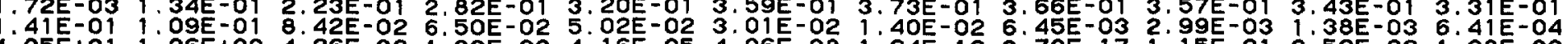

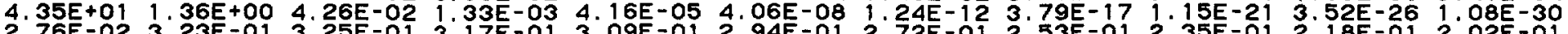

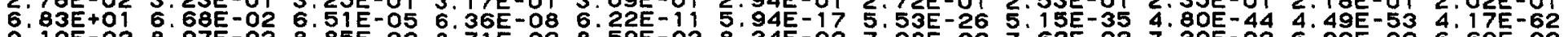
. 10 - 02 8.97E-02 8. 85E-02 8.71E-02 8.59E-02 8.34E-02 7.98E-02 7.62E-02 7.30 - 02 6.99E-02 $6.695-02$ 9.50E+00 1.56E+00 2.56E-01 4.18E-02 6.86E-03 1.85E-04 8.15E-07 3.59E-09 $1.58 E-117.05 \mathrm{E}-141.73 \mathrm{E}-16$ $\begin{array}{lllllll} & \end{array}$

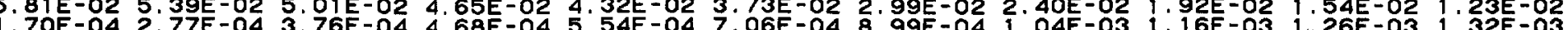

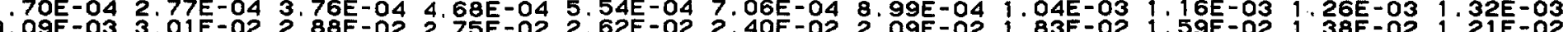
$2.15 E-018.71 E-02$ 3.52E-02 1.43E-02 5.77E-03 9.45E-04 6.30E-05 4.17E-06 2.77E-07 1.84E-08 $1.22 E-09$ $2.85 E-012.83 E-02$ 2.80E-03 2.79E-04 2.76E-05 2.72E-07 2.66E-10 2.60E-13 $2.53 E-162.48 E-192.42 E-22$

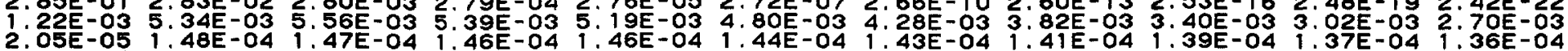

TOTAL

$9.96 E+03 \quad 2.22 E+03 \quad 8.85 E+02 \quad 5.40 E+02 \quad 3.93 E+02 \quad 2.61 E+02 \quad 1.76 E+02 \quad 1.33 E+02 \quad 1.06 E+02 \quad 8.67 E+01 \quad 7.32 E+01$ 
SMOKY

MICROCURIES/SQ METER

MR HR AT H+12 HOURS $=\overline{1}$ MIOOO

RELAXATION LENGTH $=0.16$ GM SQCM $1,299 E-13$

DEBRIS DECAY FROM 1 TO 300 DAYS

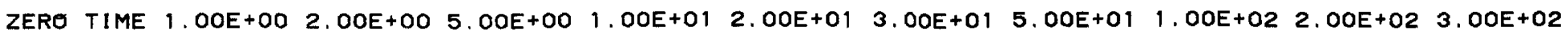

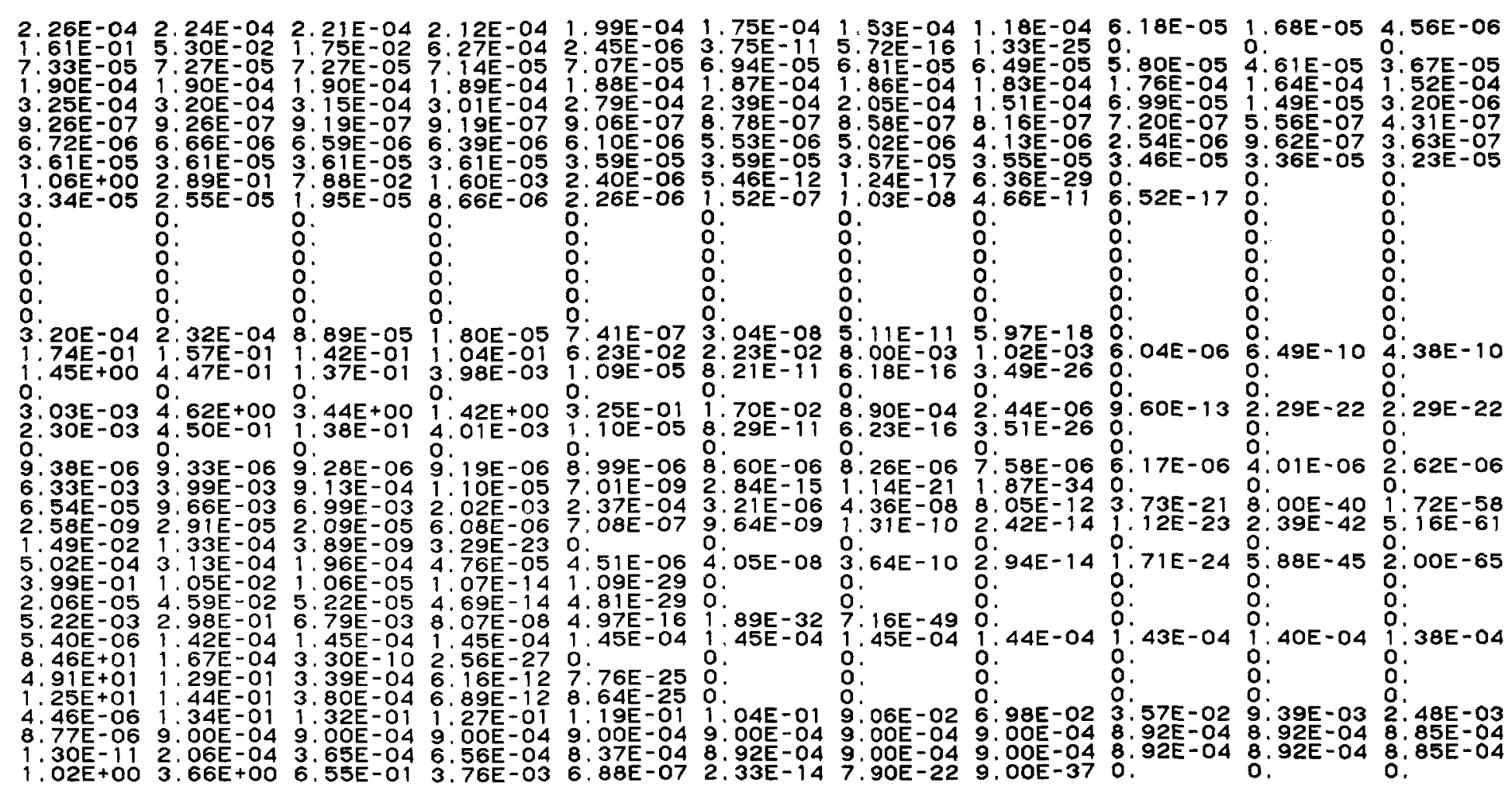


SMOKY MICROCURIES/SQ METER

PAgE $4-19$

MR/HR AT $H+12$ HOURS $=$ MICROCURIES/SQ METER

FRACTION OF REERACTORIES PRESENT $=0.500$
RELAXATION LENGTH $=0.16$ GM SQCM
BOMB FRACTI LN PER SQ. METER $=1.299 E-13$

DEBRIS DECAY FROM 1 TO 300 DAYS

ZERO TIME $1.00 E+002.00 E+00 \quad 5.00 E+001.00 E+01 \quad 2.00 E+01 \quad 3.00 E+01 \quad 5.00 E+01 \quad 1.00 E+02 \quad 2.00 E+02 \quad 3.00 E+02$

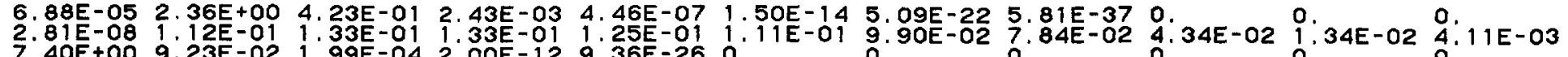

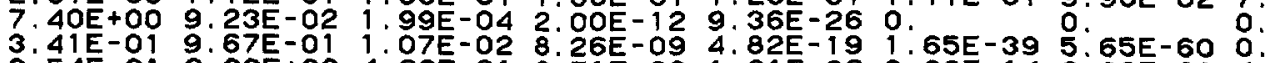

3.54E-01 2.39E+00 4.66E-01 3.51E-03 1.01E-06 8.33E-14 6.88E-21 $4.70 E-35: 0 \quad$ :. $\quad 0$.

$6.67 E-04$ 8.34E-02 8.25E-02 7.99E-02 7.58E-02 6.79E-02 6.11E-02 4.94E-02 2.90E-02 9.98E-03 3.42E-03 $1.42 E-112.82 E-045.15 E-04$ 9.83E-04 1.33E-03 1. $40 E-03$ 1.29E-03 1.05E-03 6. $14 E-042.12 E-04$ 7.29E-05

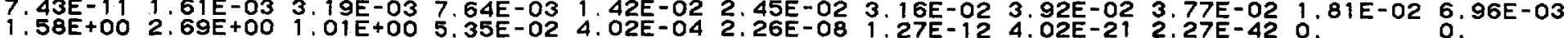

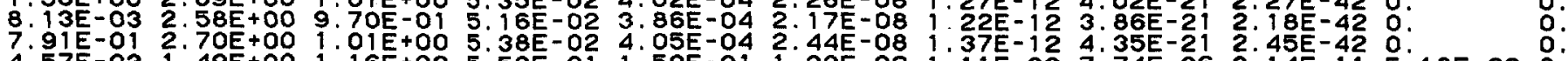

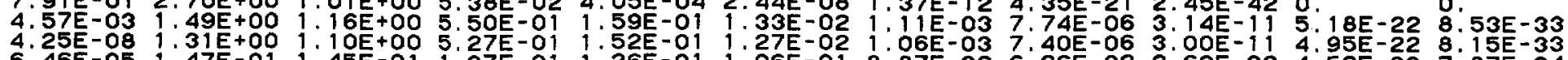

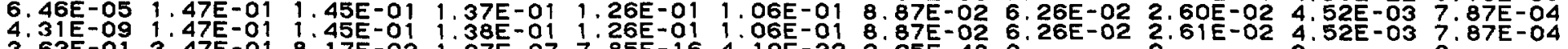
3.63E-01 $3.47 \mathrm{E}-018.17 \mathrm{E}-03$.

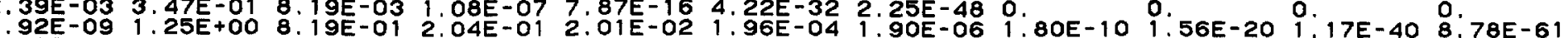

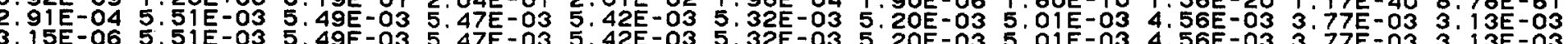
$3.15 E-065.51 E-035.49 E-035.47 E-035.42 E-035.32 E-035.203-035.01 E-034.56 E-03 \quad 3,77 E-03 \quad 3.13 E-03$

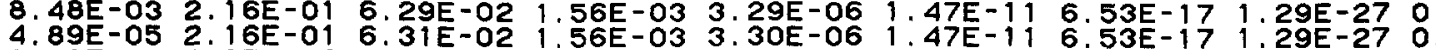
1.31E+00 6:35E-02 3.09E-03 3.54E-07 9.58E-14 6:99E-27 5:13E-40 0: 4.66E-01 5:13E-02 2:48E-03 2.85E-07 7.72E-14 5.62E-27 4:12E-40 : 3.70E-03 6.70E-02 3.26E-03 3.75E-07 1.01E-13 7:43E-27 5.43E-40 0

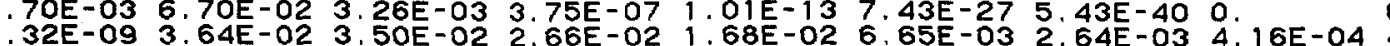
3.87E-01 1.76E-01 7.95E-02 7.38E-03 1.40E-04 5.09E-08 $1.85 \mathrm{E}-11$ 2. $43 \mathrm{E}-18 \mathrm{C}$

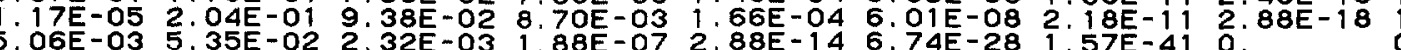

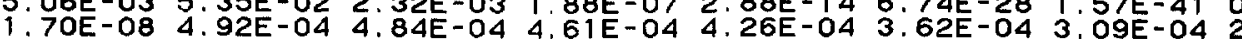

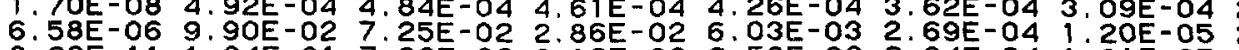

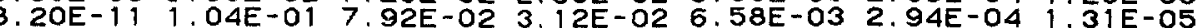
1.47E-01 3.20E-03 3.11E-06 2, 90E-15 2,58E-30 0. 7.04E-06 1.33E-02 1.54E-05 1.48E-14 $1.32 E-290$

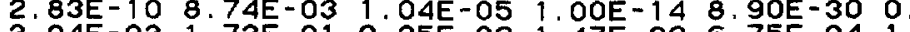
$\begin{array}{lllll} & \end{array}$ $\begin{array}{lll} & \end{array}$

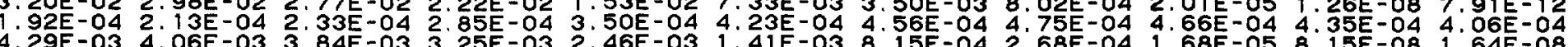

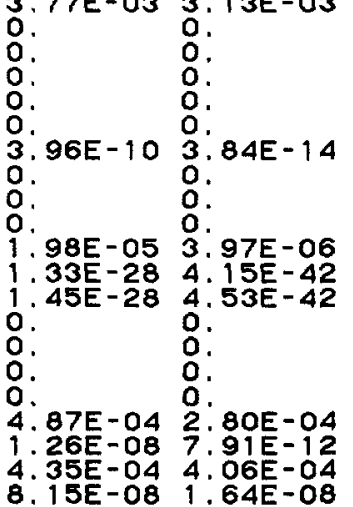


SMOKY AT H+12 HOURS = MI CROCURIES/SQ METER MR/HR AT H+12 HOURS = 12 1. OOO

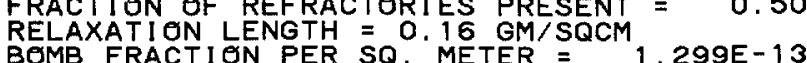

DEBRIS DECAY FROM 1 TO 300 DAYS

ZERO TIME 1. OOE+OO $2,00 E+00 \quad 5.00 E+00 \quad 1,00 E+012.00 E+01 \quad 3.00 E+01 \quad 5.00 E+01 \quad 1.00 E+022,00 E+02 \quad 3.00 E+02$

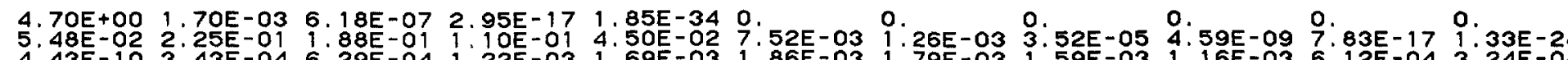

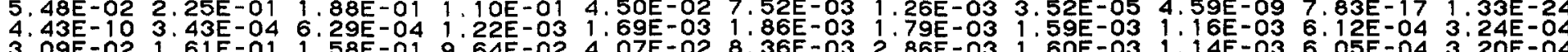

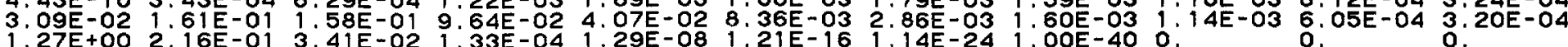

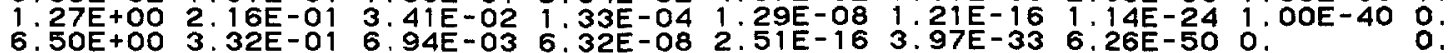

(1)

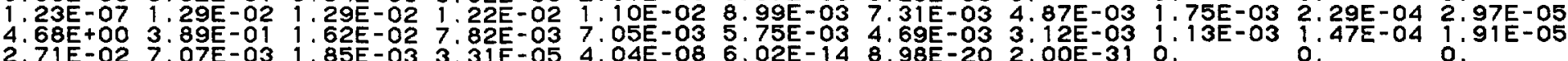

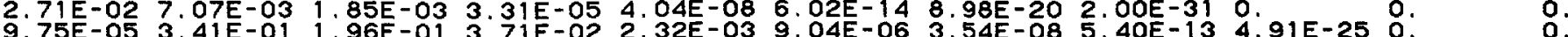
8.

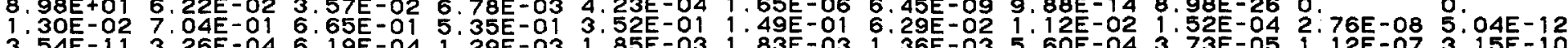

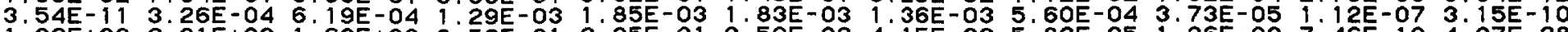
$1.02 E+00$
2

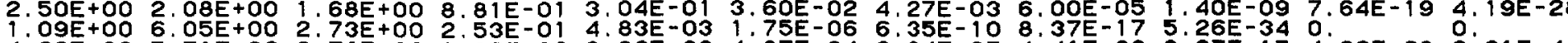

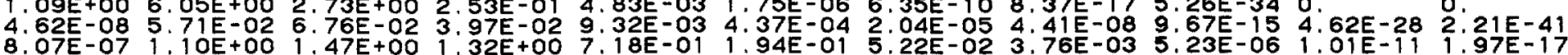

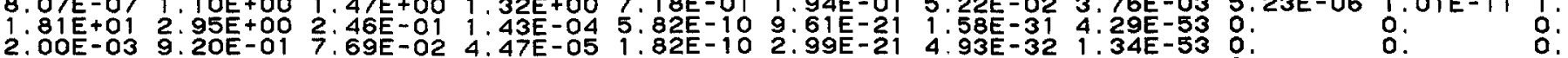

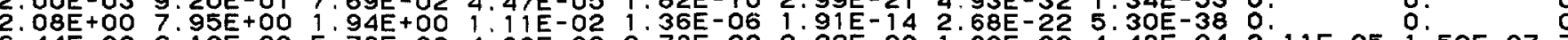
6.44E-03 6.10E-03 5.78E-03 4.93E-03 3.78E-03 2.22E-03 $1.30 E-03 \quad 4.48$ E-04 3.11E-05 1.50E-07 7.27E- 10 $9.00 E-05$ 9.70E-04 9.70E-04 9.70E-04 9.70E-04 9.70E-04 9.70E-04 9.70E-04 9.64E-04 9:59E-04 9:53E-04

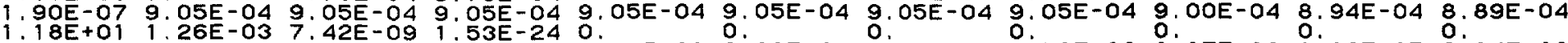
1.10E-01 6.51E-01 6.19E-01 5.25E-01 4.01E-01 2:33E-01 1.35E-01 4.59E-02 3.07E-03 1.36E-05 6.04E-08

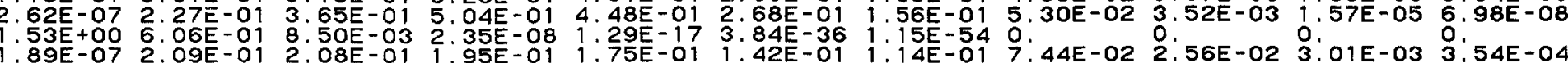

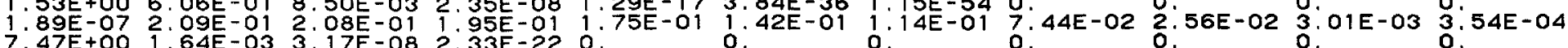

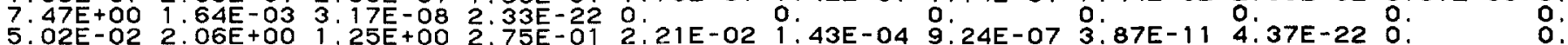

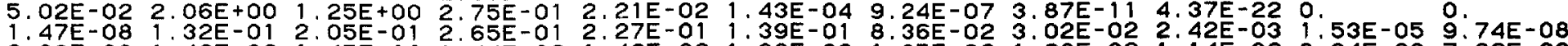

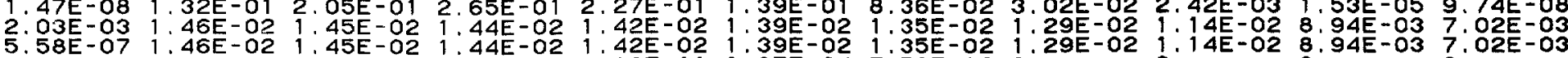

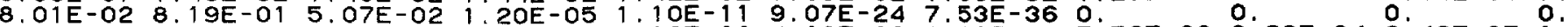

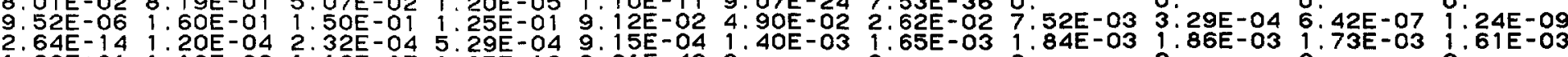

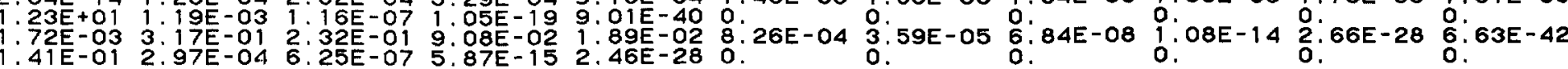
$1.41 E-01$ 2:.97E-04 6.25E-07 5.87E-15 2.46E-28 0 . 
SMOKY MICRGCURIES/SQ METER

MRA HR AT H+12 HOURS $=$ MT 1 MOOO OON $=0.500$

FRACTION OF REFRACTORIES PRESENT $=$
RELAXATION LENGTH $=0.16$ GM SQCM
BOMB FRACTION PER SQ. METER $=1.299 \mathrm{E}-13$

DEBRIS DECAY FROM 1 TO 300 DAYS

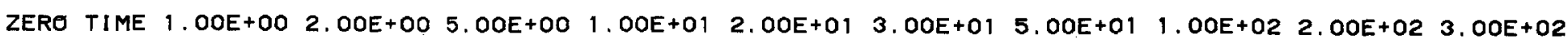

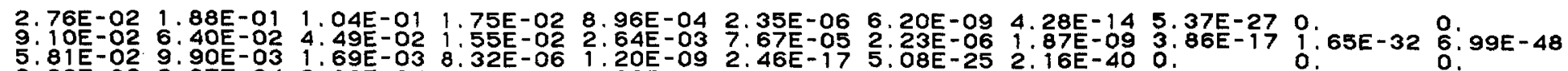

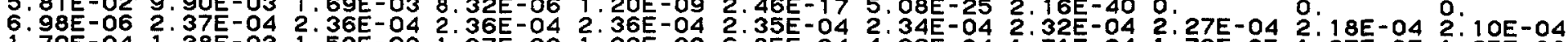

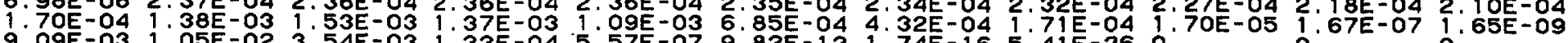

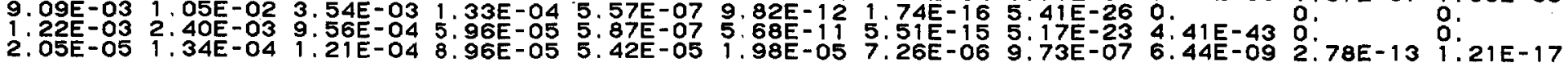

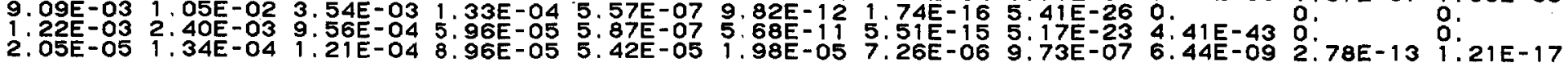

EU157

GD159

TOTAL

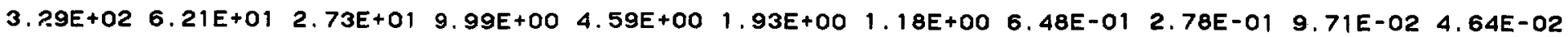


SMOKY AT H+12 HOURS $=$ MI CRECURIES/SQ METER

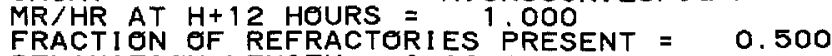

RELAXATION LENGTH $=0.16$ GM/SQCM

DEBRIS DECAY FROM 1 TO 50 YEARS

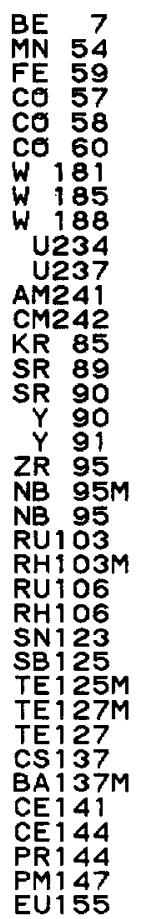

TOTAL
ZERO TIME 1. OOE+OO $1.50 E+00 \quad 2.00 E+00 \quad 3.50 E+00 \quad 5.00 E+00 \quad 7.00 E+00 \quad 1.00 E+01 \quad 2.00 E+01 \quad 3.50 E+01 \quad 5.00 E+01$

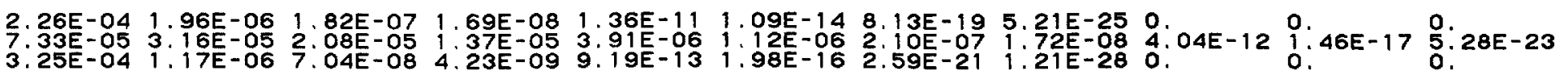

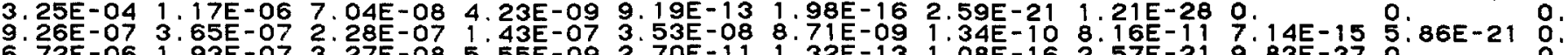
$6.72 E-061.93 E-073.27 E-085.55 E-092.70 E-111.32 E-13$ 1.08E-16 2.57E-21 9.83E-37 0.8 3.61E-05 3.16E-05 2.95E-05 2.76E-05 2.27E-05 1.86E-05 1.43E-05 9.64E-06 2.59E-06 3.59E-07 4.96E-08 0.

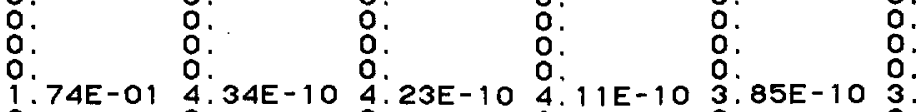

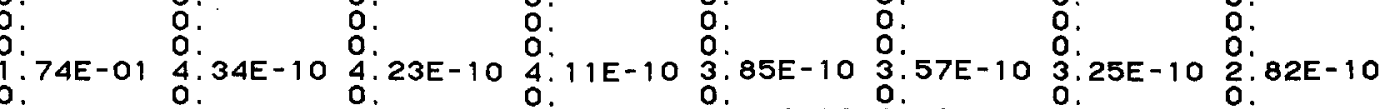
9.38E-06 1.99E-05 9.14E-07 4.20E-07 4.10E-08 3.99E-09 1.79E-10 0.

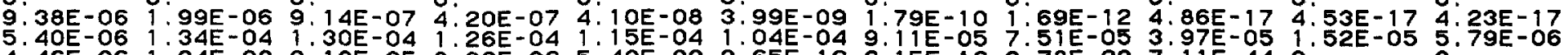

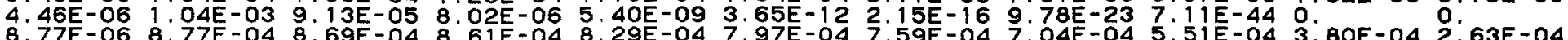

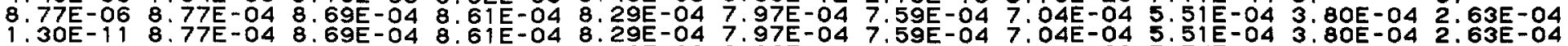

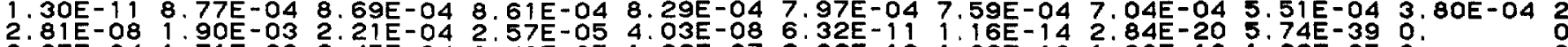
$6.67 \mathrm{E}-04$ 1.71E-03 2. 45E-04 3.48E-05 1.02E-07 2.96E-10 1.22E-13 1.03E-18 1.26E-35 0 $1.42 \mathrm{E}-11$ 3.63E-05 5.18E-06 7.40E-07 2.15E-09 6.26E-12 2.59E-15 2.19E-20 2.68E-37 0. $7.43 E-11$ 3.69E-03 5. 30E-04 7.55E-05 2.19E-07 6.35E-10 2.65E-13 2.24E-18 $2.74 E-350$. $6.46 \mathrm{E}-052.51 \mathrm{E}-041.03 \mathrm{E}-05$ 4.23E-07 2.88E-11 $1.98 \mathrm{E}-15$ 5.55E-21 2.61E-29 0. 4.31E-09 2.51E-04 1. 03E-05 4.23E-07 2.89E-11 1.98E-15 5.55E-21 2.61E-29 0.20 2.91E-04 2.77E-03 1. 96E-03 $1.39 E-034.94 E-04 \quad 1.75 E-044.42 E-055.58 E-06$ 5.63E-09 1. . $15 \mathrm{E}-062.77 \mathrm{E}-03$ 1.96E-03 2.57E-05 1.23E-06 法

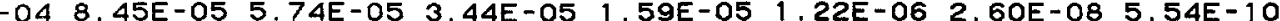

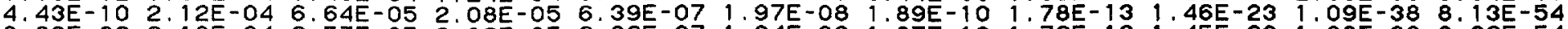
3.09E-02 2. 10 -04 6.57E-05 2.06E-05 6.32E-07 1.94E-08 $1.87 E-10 \quad 1.76 E-13 \quad 1.45 E-23 \quad 1.08 E-38$ 8.06E-54

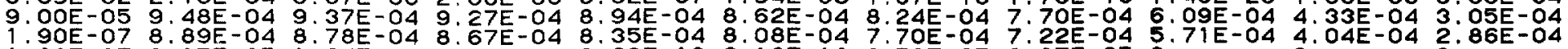

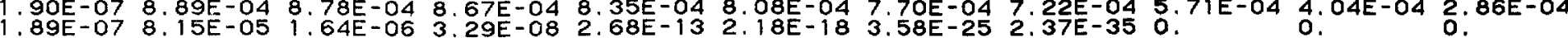

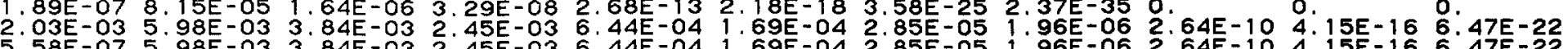

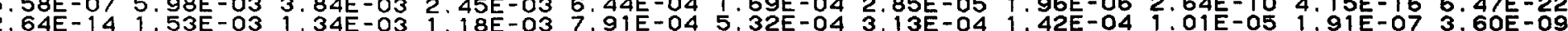

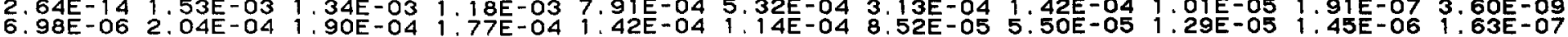
$\begin{array}{lllllllllll}2.09 E-01 & 3.32 E-02 & 1.87 E-02 & 1.34 E-02 & 7.03 E-03 & 4.92 E-03 & 3.88 E-03 & 3.25 E-03 & 2.35 E-03 & 1.61 E-03 & 1.12 E-03\end{array}$ 


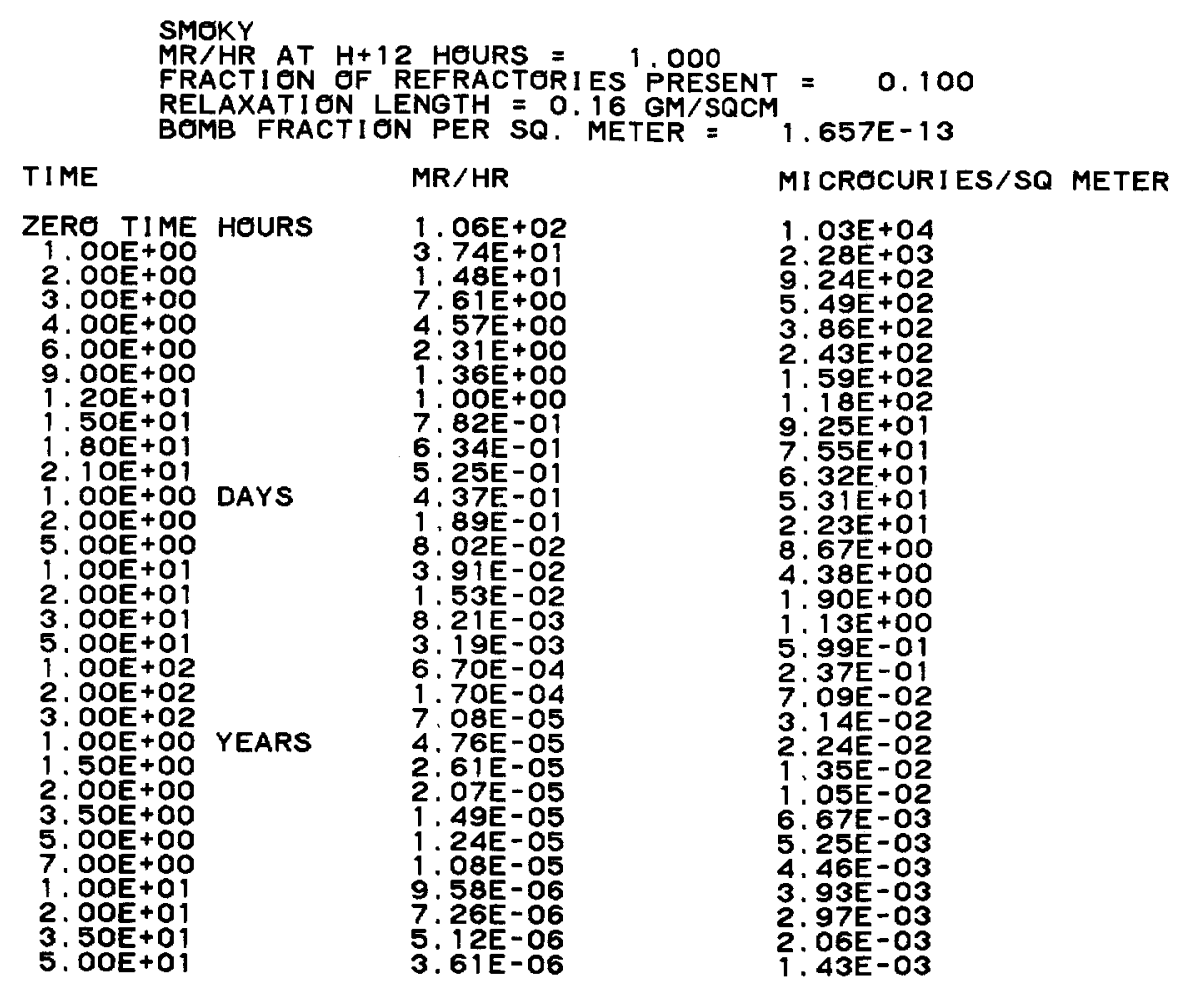

page $4-23$ 


\section{SMEKY AT H+12 HOURS $=$ MICROCURIES/SQ METER \\ MRAHR AT H+12 HOURS = MIORO 1 OOO}

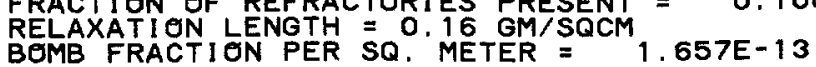

DEBRIS DECAY FROM 1 TO 21 HOURS

ZERO TIME $1.00 E+00 \quad 2.00 E+00 \quad 3.00 E+00 \quad 4.00 E+00 \quad 6.00 E+00 \quad 9.00 E+00 \quad 1.20 E+01 \quad 1.50 E+01 \quad 1.80 E+012.10 E+01$

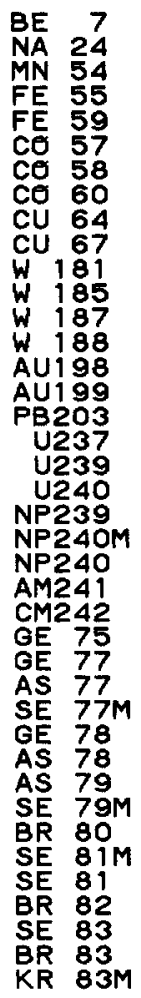

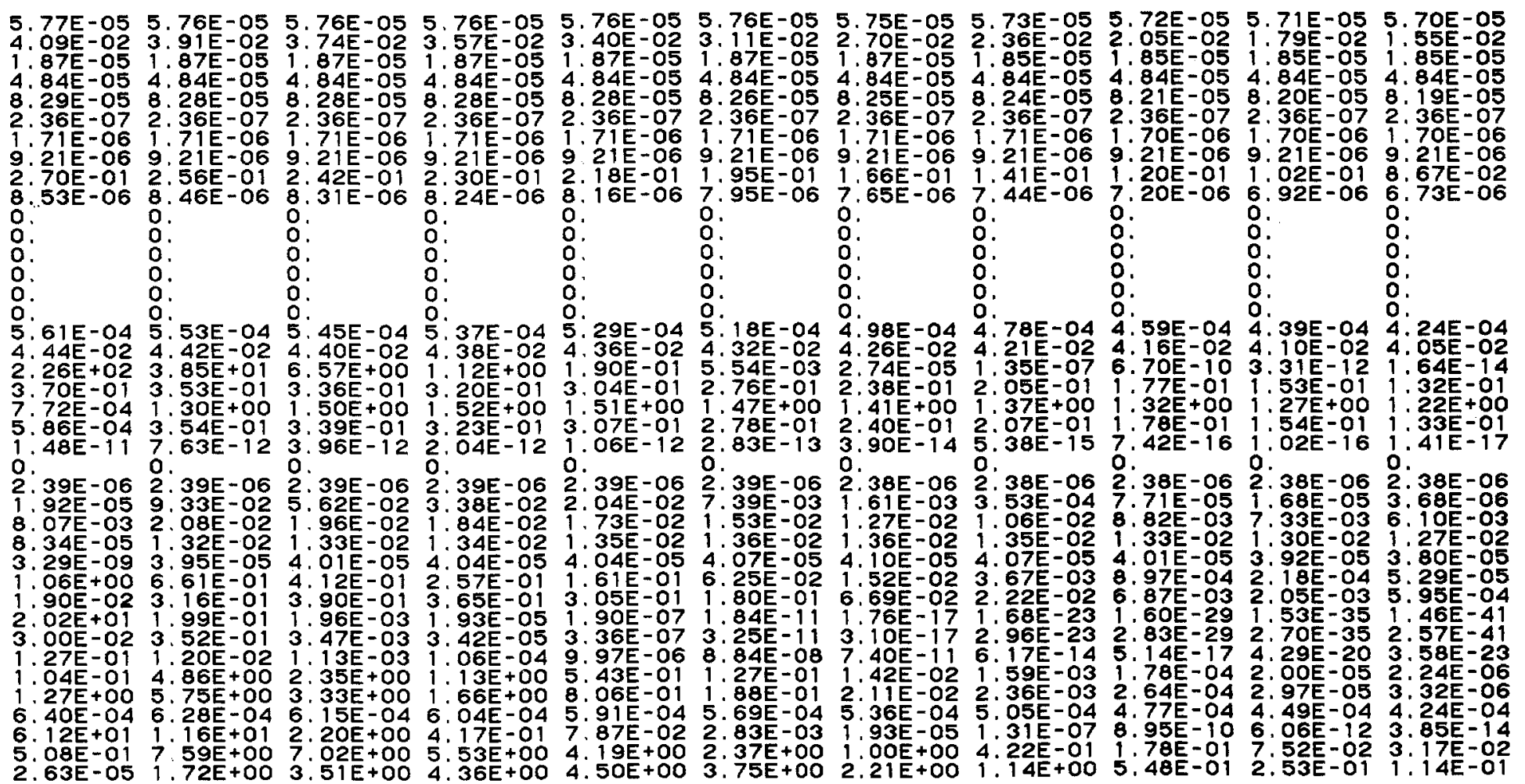


SMOKY MR/HR AT H+12 HOURS = MICROCURIES/SO METER RACTION OF REFRACTERIES PRESENT = 0.100
RELAXATION LENGTH $=0.16$ GMM SOCM
BOMB FRACTION PER SQ. METER $=1.657 E-13$

DEBRIS DECAY FROM 1 TO 21 HOURS

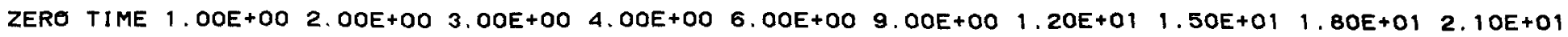

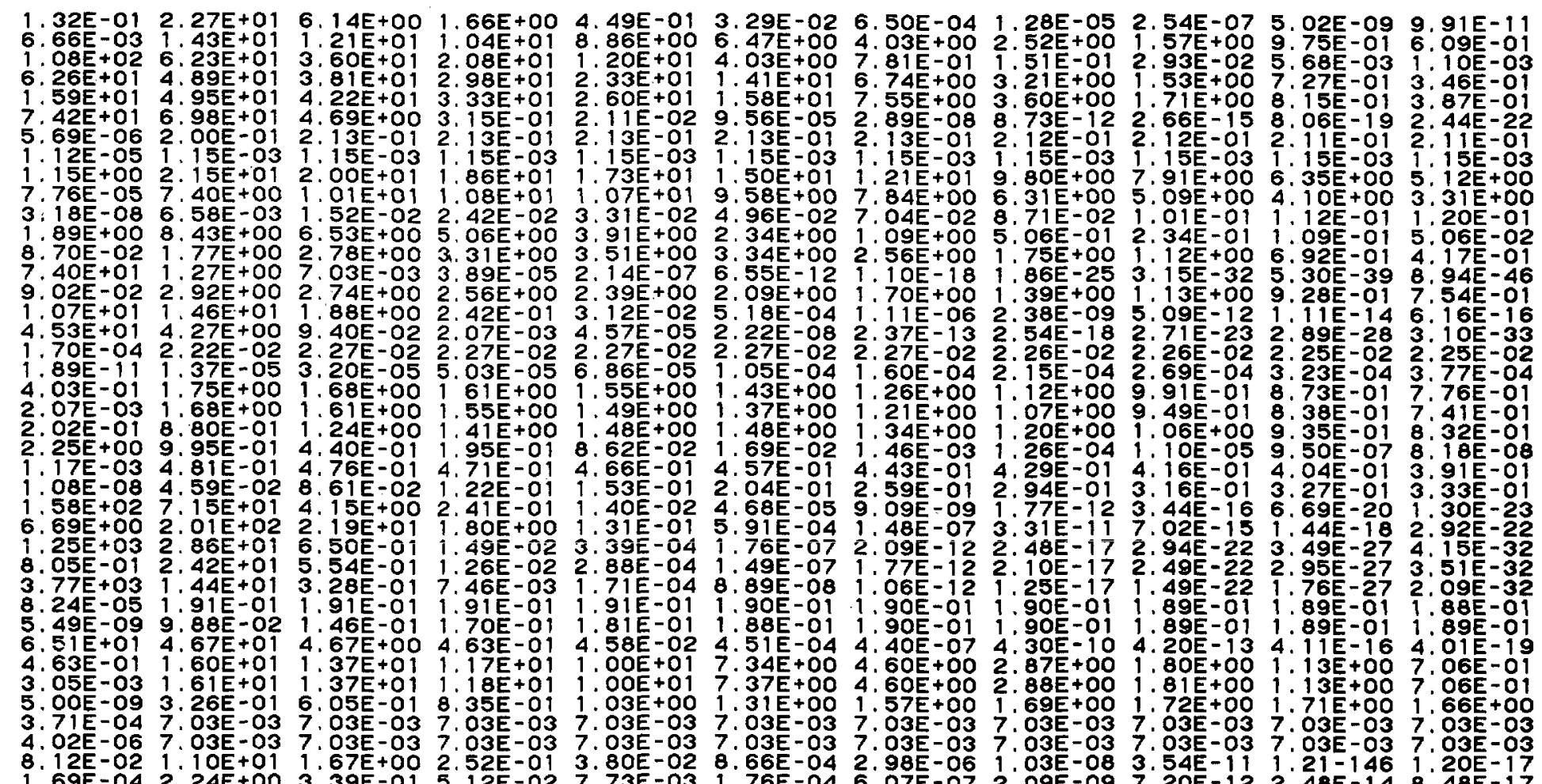


SMOKY

MICROCURIES/SQ METER

PD109 AGIO9M PDI111M AG 111 PDi112 AG 112 AG 113 AG 115 CD115M IN1 $15 \mathrm{M}$ CD117 IN1 179 CD118 IN118 IN1 $19 M$ N119 SN123M SN123 SN125

SB125

SN127

TE 127

SN1 128

SB 128

SN $129 M$

SB129

E 29

SB $130 M$
FRACTION

RELAXATION LENGTH $=0$. 16 GM/SQCM $1.657 E-13$

DEBRIS DECAY FROM 1 TO 21 HOURS

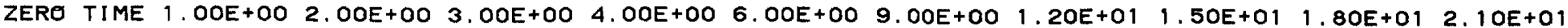

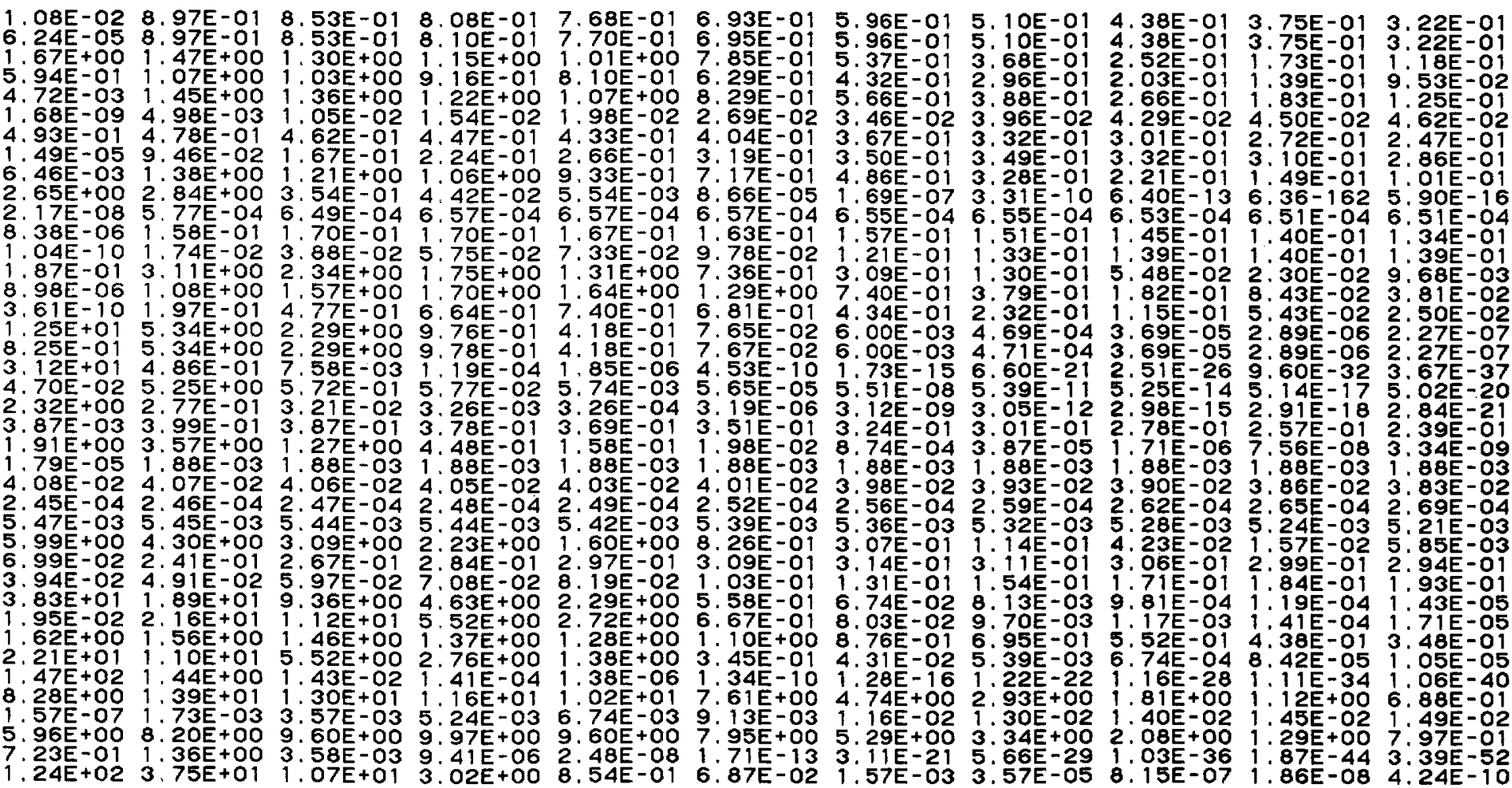


SMEKY

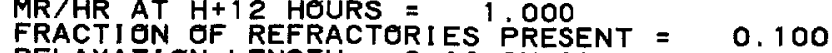

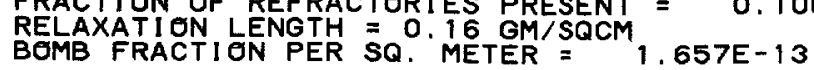

DEBRIS DECAY FROM 1 TO 21 HEURS

ZERO TIME $1.00 E+00 \quad 2.00 E+00 \quad 3.00 E+00 \quad 4.00 E+00 \quad 6.00 E+00 \quad 9.00 E+00 \quad 1.20 E+01 \quad 1.50 E+01 \quad 1.80 E+01 \quad 2.10 E+01$

SB 130

TEISIM

1131

TE1 132

TE $133 \mathrm{MM}$

TE133

XEI $33 \mathrm{M}$

$X$
$X E 133$
TE 134

$\begin{array}{lll}1 & 344 \\ 1 & 335\end{array}$

XE $135 \mathrm{M}$

XE 135

CS137

XE 138

CS1 38

BA 139

BA 40

BA 41

CA 141

BA 42

LA 142

CE143

CER 144

PR144

CEI 46

PRi46

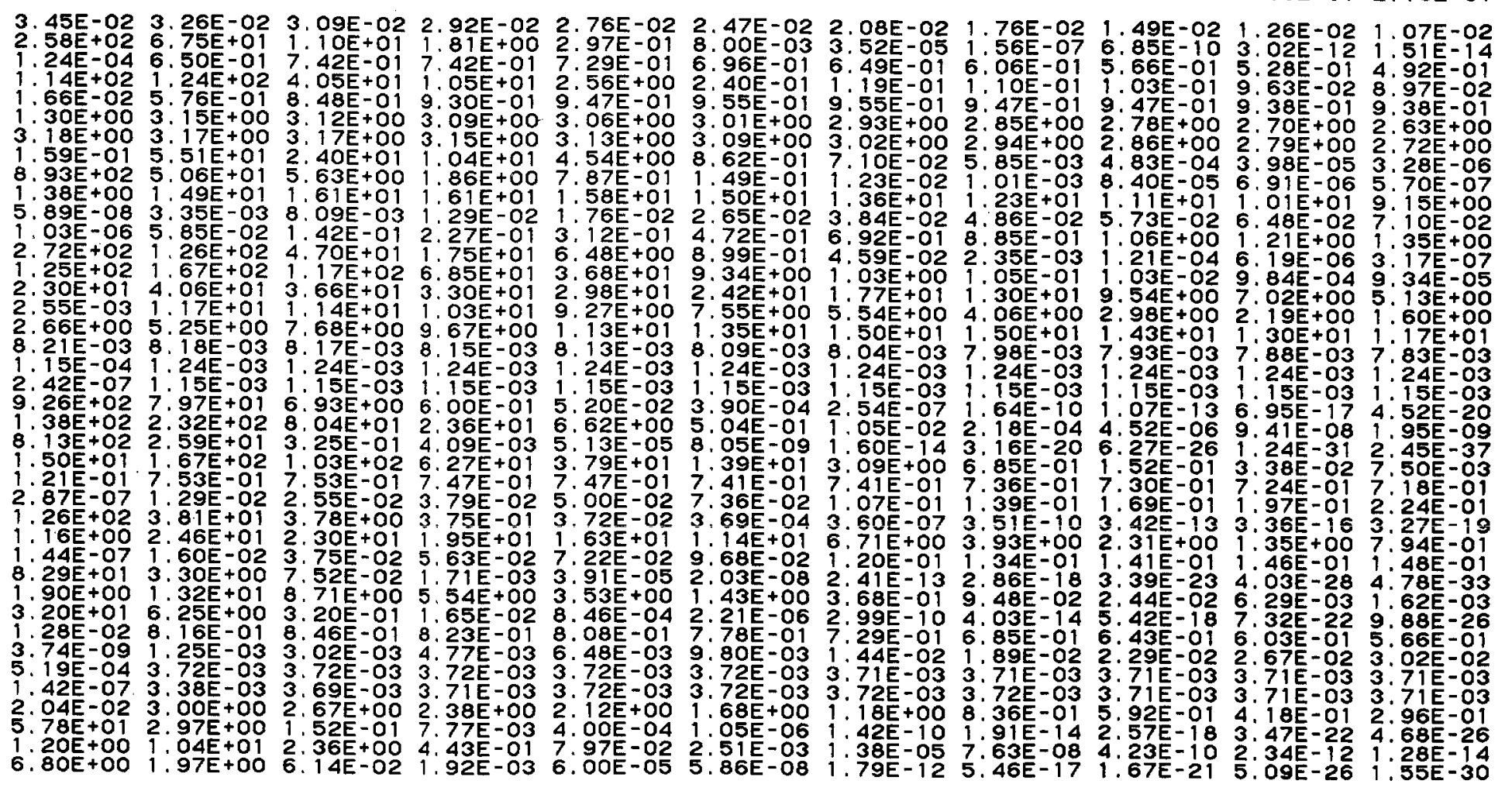


SMOKY MR/HR AT H+12 HOURS = MICTROCURIES/SO METER FRACTION OF REFRACTORIES PRESENT $=0.100$ RELAXATI ON LENOTH $=0.16$ GM/SQCM $1.657 E-13$

DEBRIS DECAY FROM 1 TO 21 HOURS

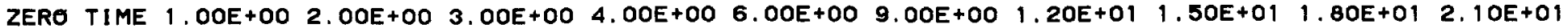
2. 43E-06 4.57E-02 4.69E-02 4.69E-02 4.67E-02 4.65E-02 4.62E-02 4.58E-02 4.54E-02 4.51E-02 4.47E-02 3.15E+OO 2.14E+OO $1.46 E+00$ 9.94E-01 6.72E-01 3.12E-01 9.82E-02 3.10E-02 9.76E-03 3.07E-03 9.70E-04 4.38E-04 3.43E-02 5.69E-02 7.20E-02 8.15E-02 9.16E-02 9.52E-02 9.34E-02 9.10E-02 8.75E-02 8.45E-02

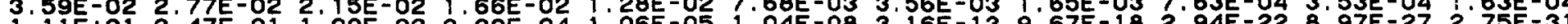
7.

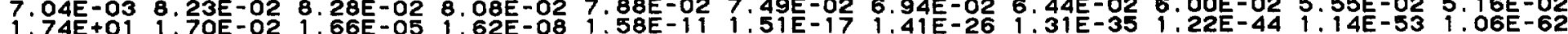
2.32E-02 2.29E-02 2.26E-02 2.22E-02 2.19E-02 2.13E-02 2.03E-02 1.94E-02 1.86E-02 $1.78 E-02 \quad 1.71 E-02$ 2. $42 E+00$ 3.98E-01 6.52E-02 1.07E-02 1.75E-03 4.71E-05 2.08E-07 9.1.4E-10 4.03E-12 $1.80 E-144.41 E-17$

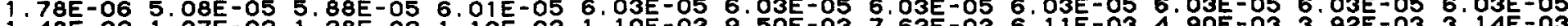

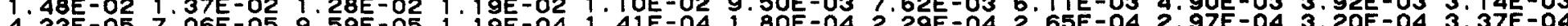

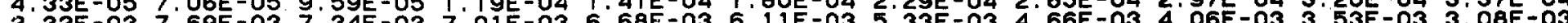

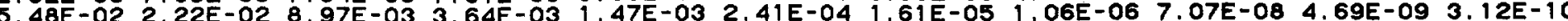
7.26E-02 $7.21 E-037.15 E-047.10 E-05$ 7.04E-06 $6.94 E-08$ 6.78E-11 $6.62 E-14 \quad 6.46 E-176.32 E-20 \quad 6.17 E-23$

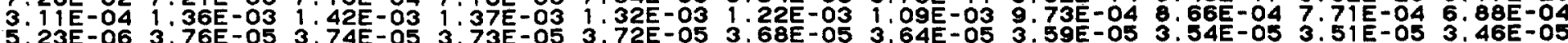

TOTAL

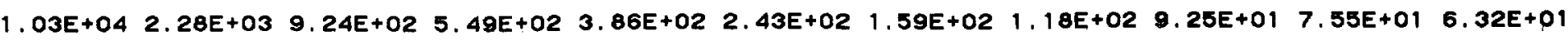


PAOE $\quad 4-29$

SMOKY

MR'HR AT H+12 HOURS = IOPCTION OF REFRACTORIES PRESENT a 0.100

RELAXATION LENGTH $=0.16$ GM/SOCM 1.657 E- 13

DEBRIS DECAY FROM 1 TO 300 DAYS

ZERO TIME 1. OOE+OO $2.00 E+00 \quad 5.00 E+00 \quad 1.00 E+01 \quad 2.00 E+01 \quad 3.00 E+01 \quad 5.00 E+01 \quad 1.00 E+02 \quad 2.00 E+02 \quad 3.00 E+02$ 
SMOKY AT H+12 HOURS MICROCURIES/SO METER MRAHR AT H+12 HOURS FRACTION OF REERACTORIES PROSENT = 0.100 ROLAXATION LENOTH $=0.16$ GM ISOCM $1.657 E-13$

DEBRIS DECAY FROM 1 TO 300 DAYS

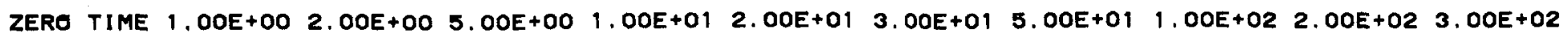

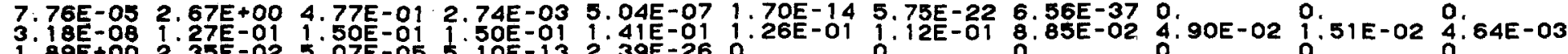

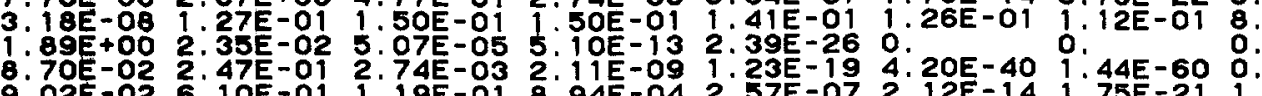

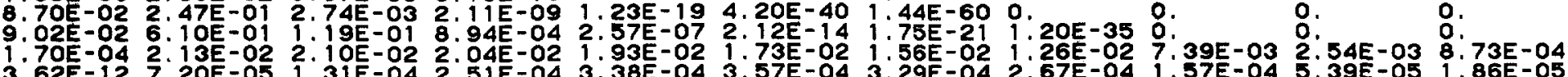

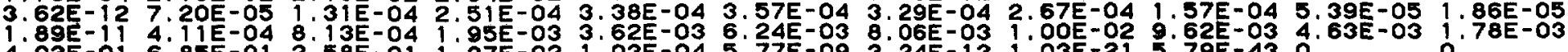

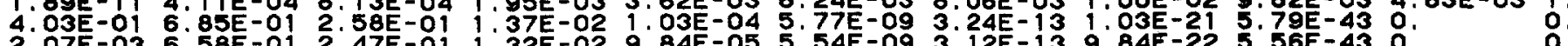

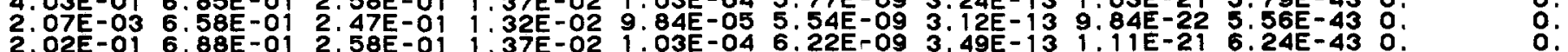

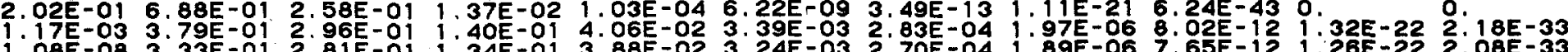

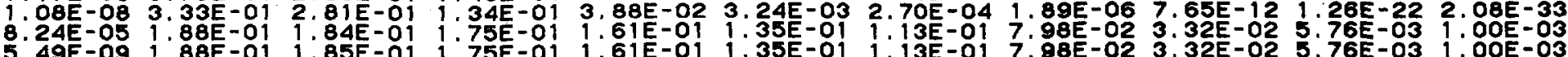

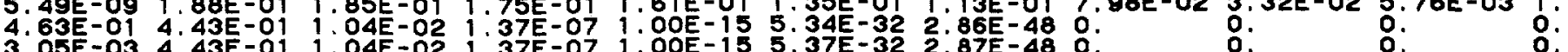

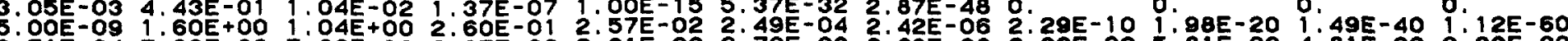

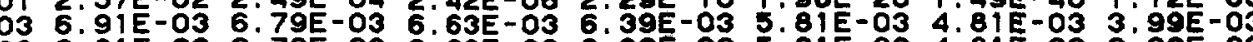
4. $02 E-06$ 7.03E-03 7.

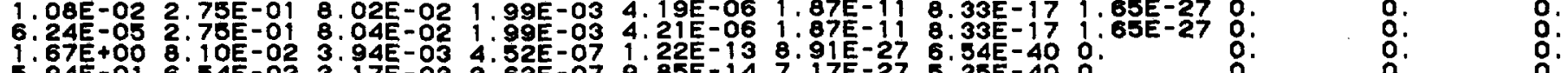

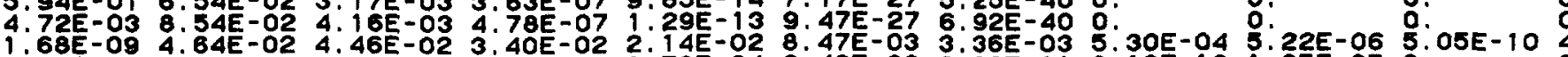

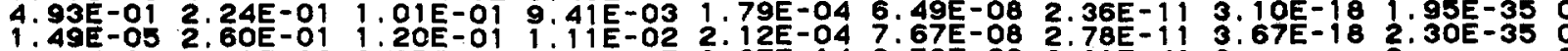

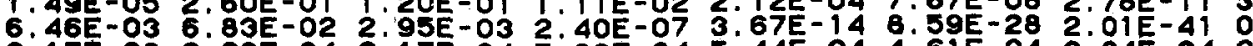

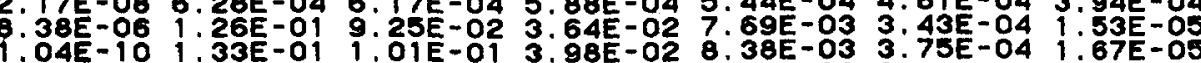
$1.87 E-014: 07 E-03 \quad 3.96 E-06$ 3.7OE-15 3.29E-30 O. 8.98E-06 1.69E-02 $1.96 E-051.69 E-141.68 E-290$. 法 $\begin{array}{ll} & \\ 0 & \end{array}$

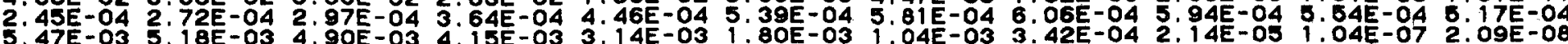

(2)

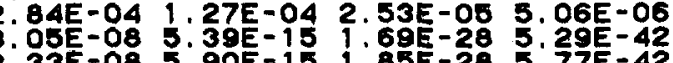
.85E-28 : ; 
SMOKY MICROCURIES/SO METER

ZERO TIME $1.00 E+002.00 E+00 \quad 5.00 E+001.00 E+01 \quad 2.00 E+01 \quad 3.00 E+01 \quad 5.00 E+01 \quad 1.00 E+02 \quad 2.00 E+02 \quad 3.00 E+02$ 5. $99 \mathrm{EE}+00$ 2.17E-03 $7.88 \mathrm{E}-07$ 3. $76 \mathrm{E}-172.36 \mathrm{E}-340$

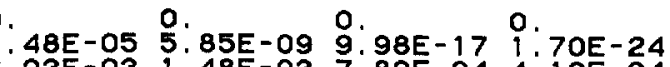

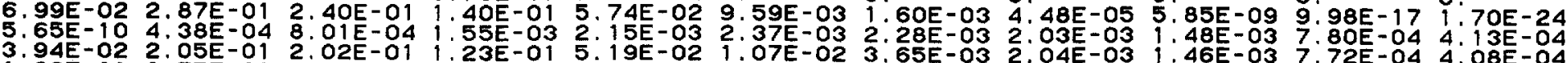

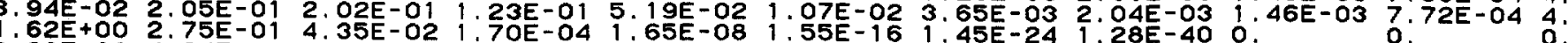

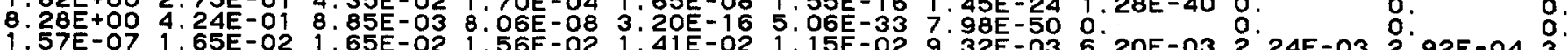

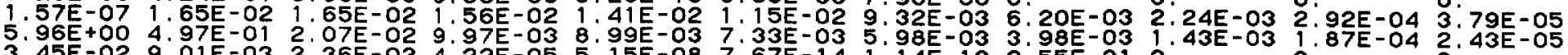

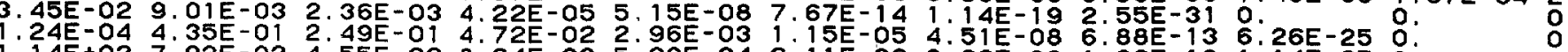
$1.14 E+02$ 7.93E-02 $4.55 E-02$ 8.64E-03 $5.39 E-042.11 E-06 \quad 8.22 E-09$ 1.26E-13 1.14E-25 0. $\quad 0$.

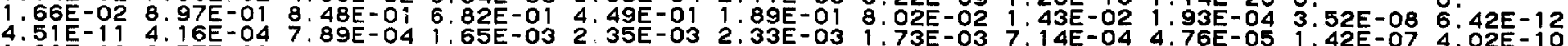

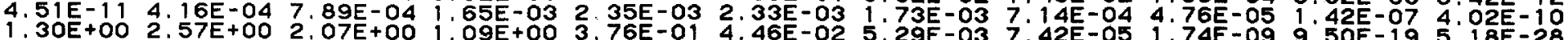

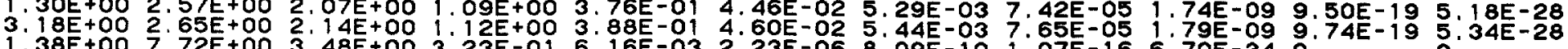
$.38 E+00$ 7.72E+00 3.48E+00 3.23E-01 6.16E-03 2.23E-06 8.09E-10 1.07E-16 6.70E-34 0.74E 0. .89E-08 7.28E-02 8.62E-02 5.06E-02 1.19E-02 5.58E-04 2.59E-05 5.63E-08 1.23E-14 5.89E-28 2.82E-41

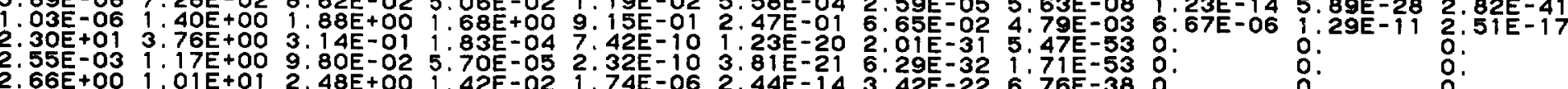

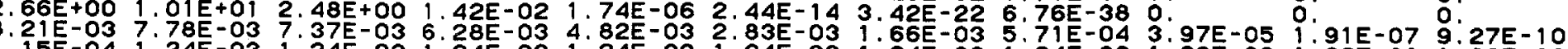

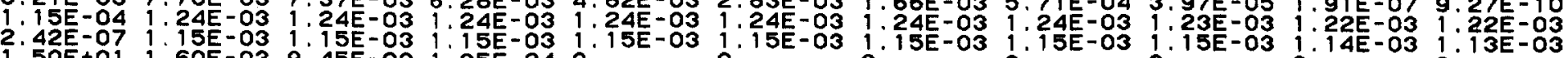
$50 E+01$ 1.60E-03 9.45E-09 1.95E-24 0.

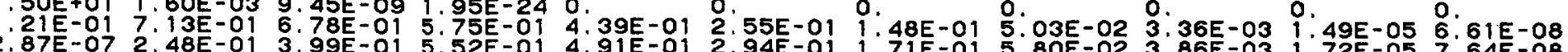
2.87E-07 2.48E-01 3.99E-01 5.52E-01 4.91E-01 2.94E-01 1.711E-01 5. 80E-02 3. 86E-03 . 72 -05 ?.64E-08

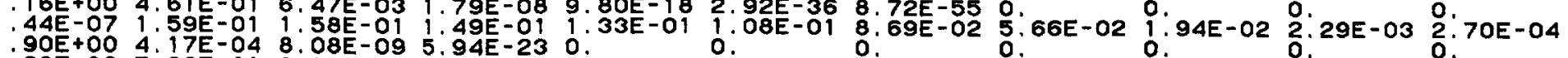

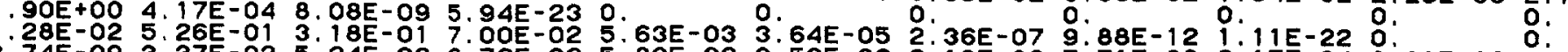

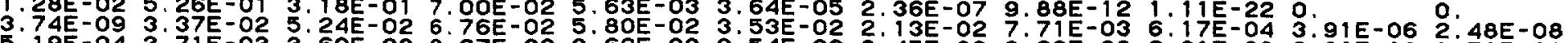
5.19E-04 3.71E-03 3.69E-03 3.67E-03 3.62E-03 3.54E-03 3. 45E-03 3.29E-03 2.91E-03 2.28E-03 $1.79 E-03$

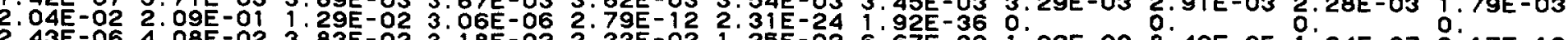

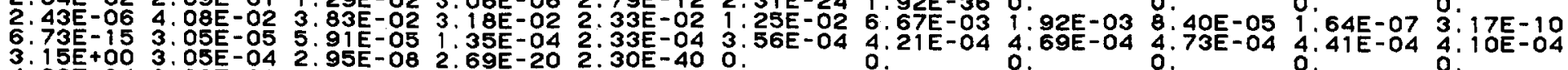

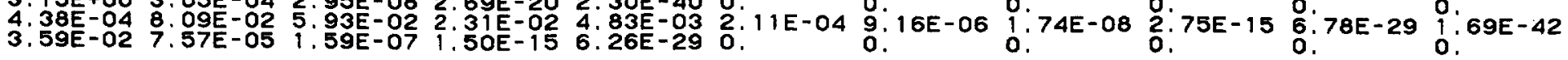


SMOKY

MI CRECURIES/SO METER

TRACT OF H+12 HOURS $=0.100$

RELAXATION LENGTH $=0$. 16 GM SQCM $1.657 E-13$

DEBRIS DECAY FROM 1 TO 300 DAYS

ZERO TIME $1.00 E+002.00 E+00 \quad 5.00 E+00 \quad 1.00 E+01 \quad 2.00 E+01 \quad 3.00 E+01 \quad 5.00 E+01 \quad 1.00 E+02 \quad 2.00 E+02 \quad 3.00 E+02$

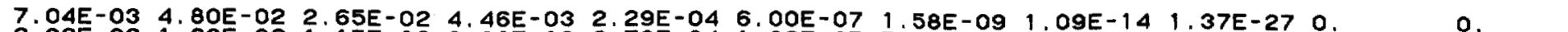

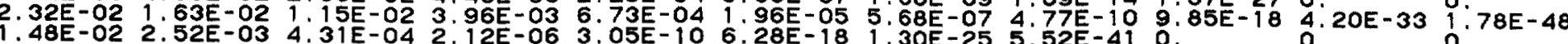
78E-06 6:03E-05 6.02E-05 6.02E-05 6.01E-05 5.98E-05 5.96E-05 5.91E-05 5.80E-05 5.57E-05 5.35E-05 $4.33 E-05$ 3.52E-04 3.90E-04 3.50E-04 2.78E-04 1.75E-04 1.10E-04 4.37E-05 4.33E-06 4.26E-08 4. $20 \mathrm{E}-10$ 2. $32 E-03$ 2.68E-03 $9.01 E-04$ 3.38E-05 $1.42 E-07$ 2.5OE-12 4.43E-17 1.38E-26 $0.13 E-430 . \quad 0$.

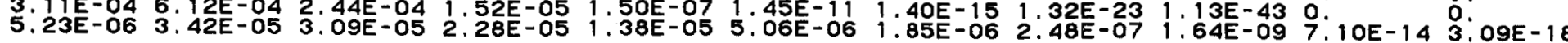

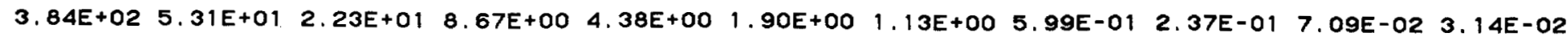

TOTAL 
SMOKY MICROCURIES/SQ METER FRACTION OF REFRACTORIES PRESENT $=0.100$ RELAXATION LENGTH $=0.16$ GMISOCM
BOMB FRACTI ON PER SO. METER $=1.657 E-13$

DEBRIS DECAY FROM 1 TO 50 YEARS

ZERO TIME $1.00 E+001.50 E+00 \quad 2.00 E+00 \quad 3.50 E+00 \quad 5.00 E+00 \quad 7.00 E+00 \quad 1.00 E+012.00 E+01 \quad 3.50 E+01 \quad 5.00 E+01$

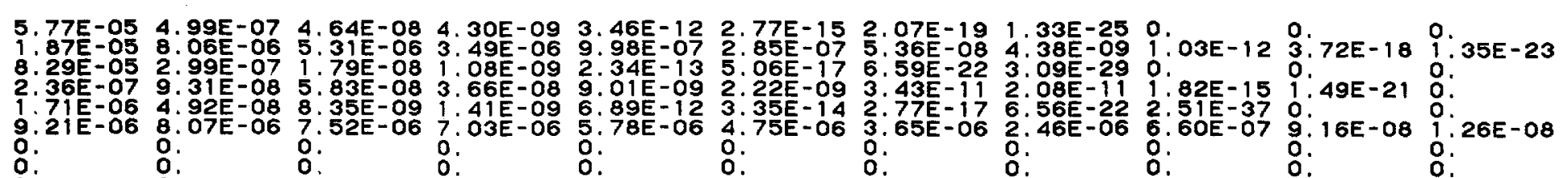

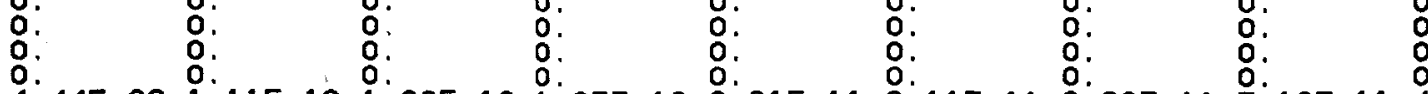

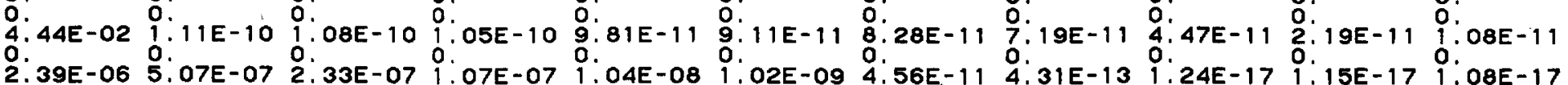

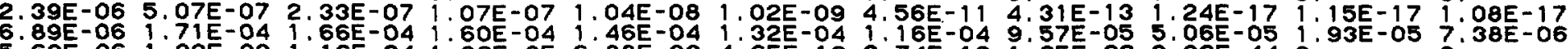

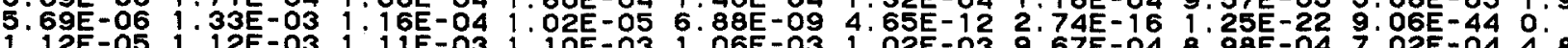

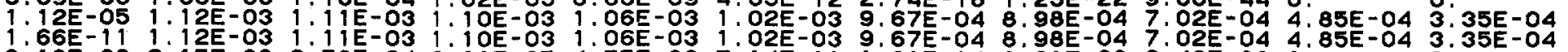
3. 18 -08 2.15E-03 2.50E-04 2.90E-05 4.55E-08 7.14E-11 1.31E-14 3.21E-20 6. $48 E-390$. $3.62 E-12$ 9.25E-06 $1.32 E-06$ 1.89E-07 $5.48 E-10$ 1:53E-11 3.12E-14 2.63E-19 $3.22 E-36:$ $1.89 E-11$ 9.40E-04 1.35E-04 1.92E-05 5.56E-08 1.62E-10 6.76E-14 5.711E-19 6.99E-36 0

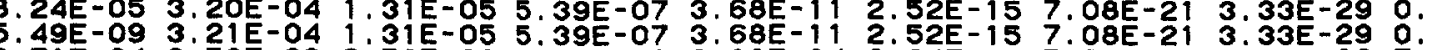
3.4 . 源

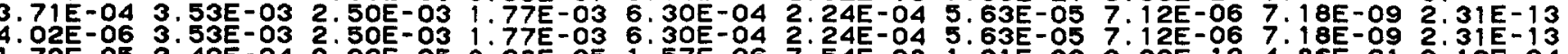

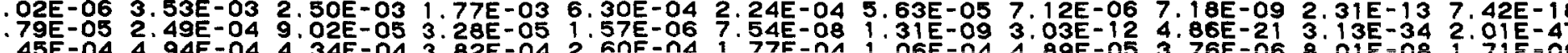

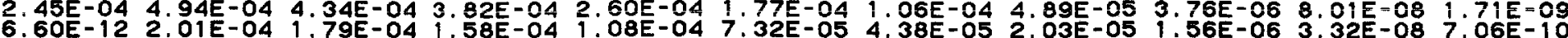

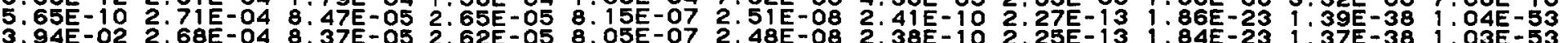

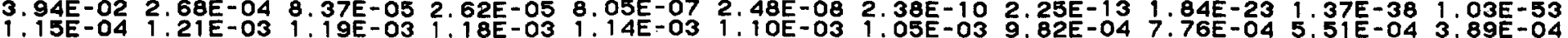
2. $42 E-07$ 1.13E-03 1.12E-03 1: $11 E-03$ 1:06E-03 $1: 03 E-03$ 9.82E-04 9.20E-04 7.28E-04 5. $15 E-04$ 3.65E-04

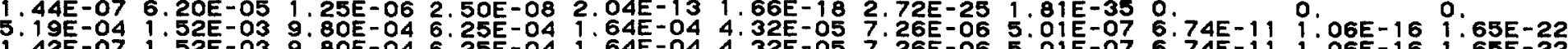

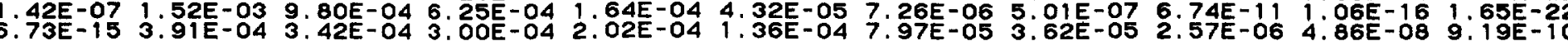

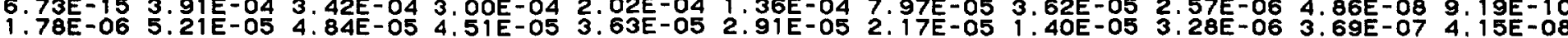

TOTAL

8.55E-02 2.24E-02 $1.35 E-02 \quad 1.05 E-02 \quad 6.67 E-03 \quad 5.25 E-03 \quad 4.46 E-03 \quad 3.93 E-03 \quad 2.97 E-03 \quad 2.06 E-03 \quad 1.43 E-03$ 


\section{APPENDIX 5}

SMOKY EVENT

$\begin{array}{lr}\qquad \begin{array}{ll}\mathrm{mR} / \mathrm{hr} \\ \text { Page }\end{array} \\ \text { Fraction of refractories present }=1.0 & 5-11 \\ \text { Fraction of refractories present }=0.5 & 5-21\end{array}$ 
DEBRIS DECAY FROM 1 TO 21 HOURS

ZERO TIME 1. OOE+OO 2. $2.00 E+00 \quad 3.00 E+00 \quad 4.00 E+00 \quad 6.00 E+00 \quad 9.00 E+00 \quad 1.20 E+01 \quad 1.50 E+01 \quad 1.80 E+012.10 E+01$ 
SMOKY

MR/HR

TRACT AT ON OF REFRACTORIES ${ }^{1}$ PRESENT $=1,000$

RELAXATION LENGTH $=0.16 \mathrm{GM} / \mathrm{SQCM}$

DEBRIS DECAY FROM 1 TO 21 HEURS

ZERO TIME $1.00 E+00 \quad 2.00 E+00 \quad 3.00 E+00 \quad 4.00 E+00 \quad 6.00 E+00 \quad 9.00 E+00 \quad 1.20 E+01 \quad 1.50 E+01 \quad 1.80 E+012.10 E+01$

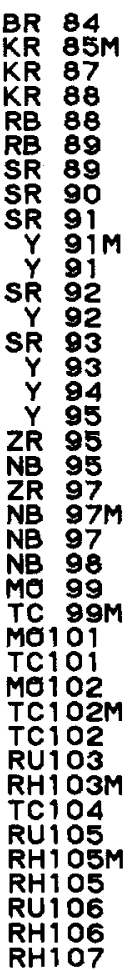

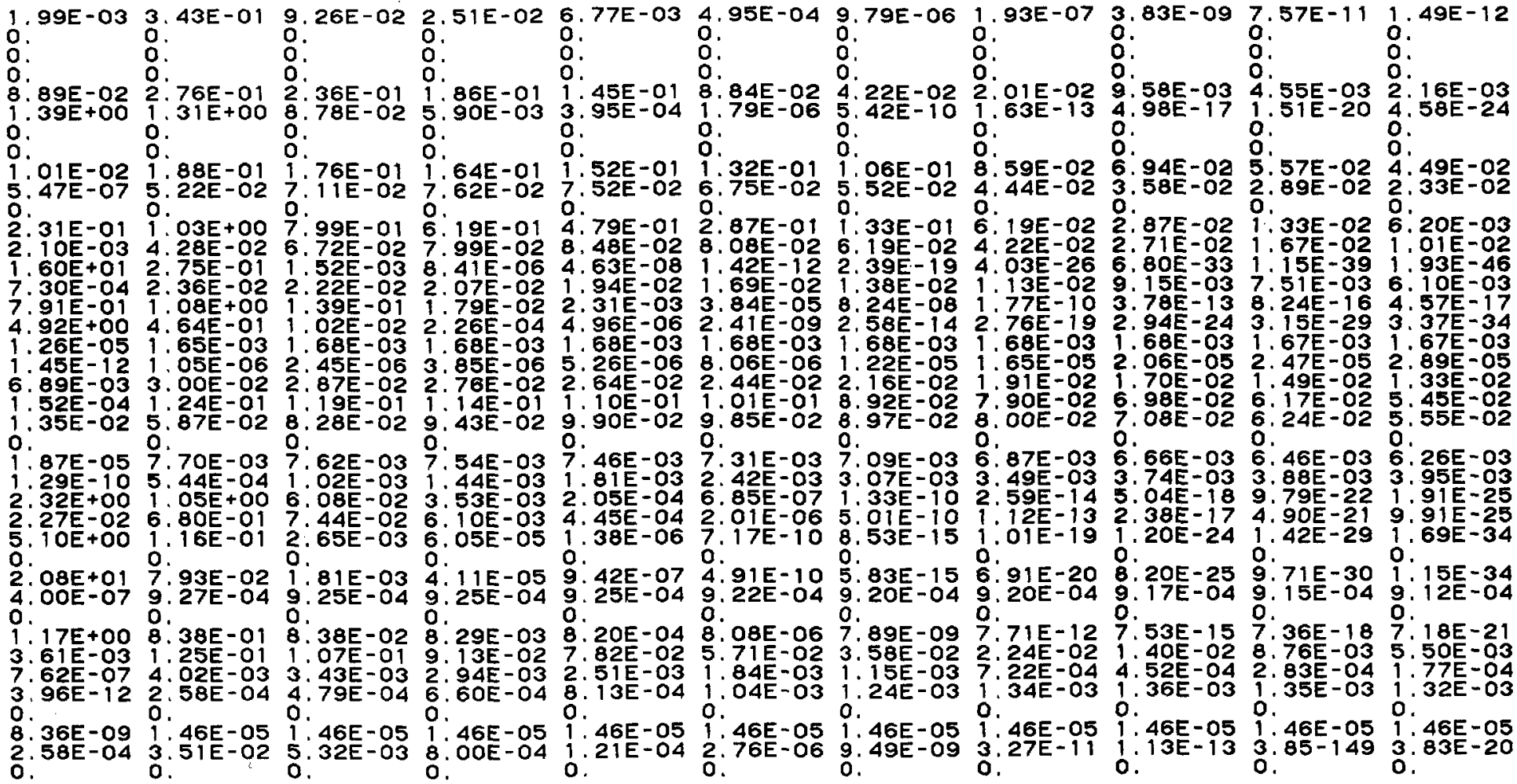

PDio7m 
SMEKY $M R / H R$

FRACTION OF REFRACTORIES PRESENT = 1.000

RELAXATION LENGTH $=0.16 \mathrm{GM} / \mathrm{SQCM}$ 1. O24E-13

DEBRIS DECAY FROM 1 TO 21 HOURS

ZERO TIME 1. OOE+OO 2. OOE+OO 3. OOE+OO $4.00 E+00 \quad 6.00 E+O 0 \quad 9.00 E+00 \quad 1.20 E+01 \quad 1.50 E+01 \quad 1.80 E+012.10 E+01$

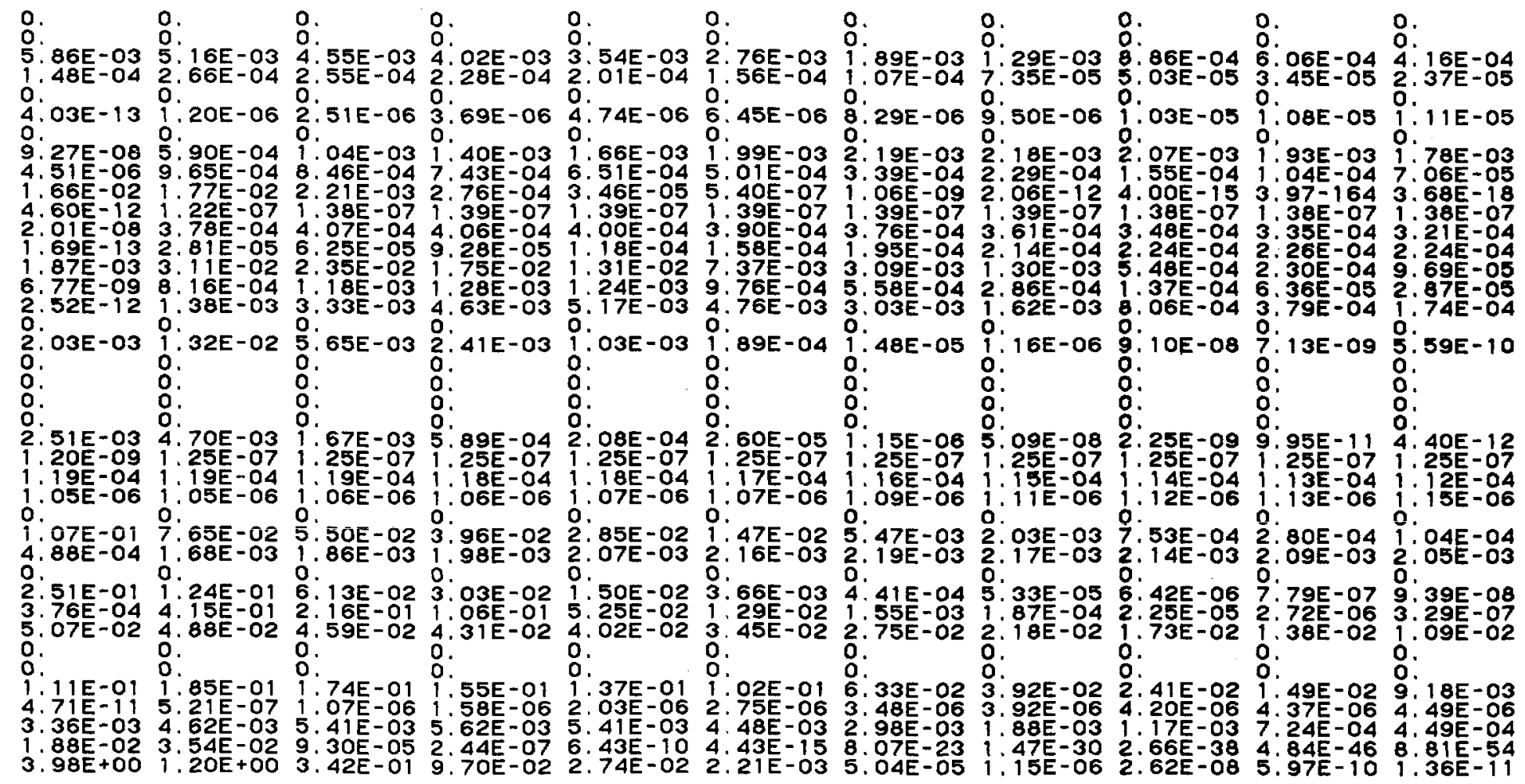


DEBRIS DECAY FROM 1 TO 21 HOURS

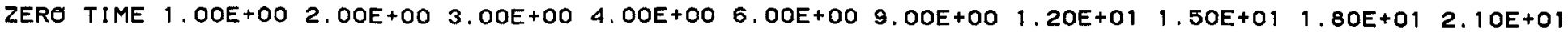

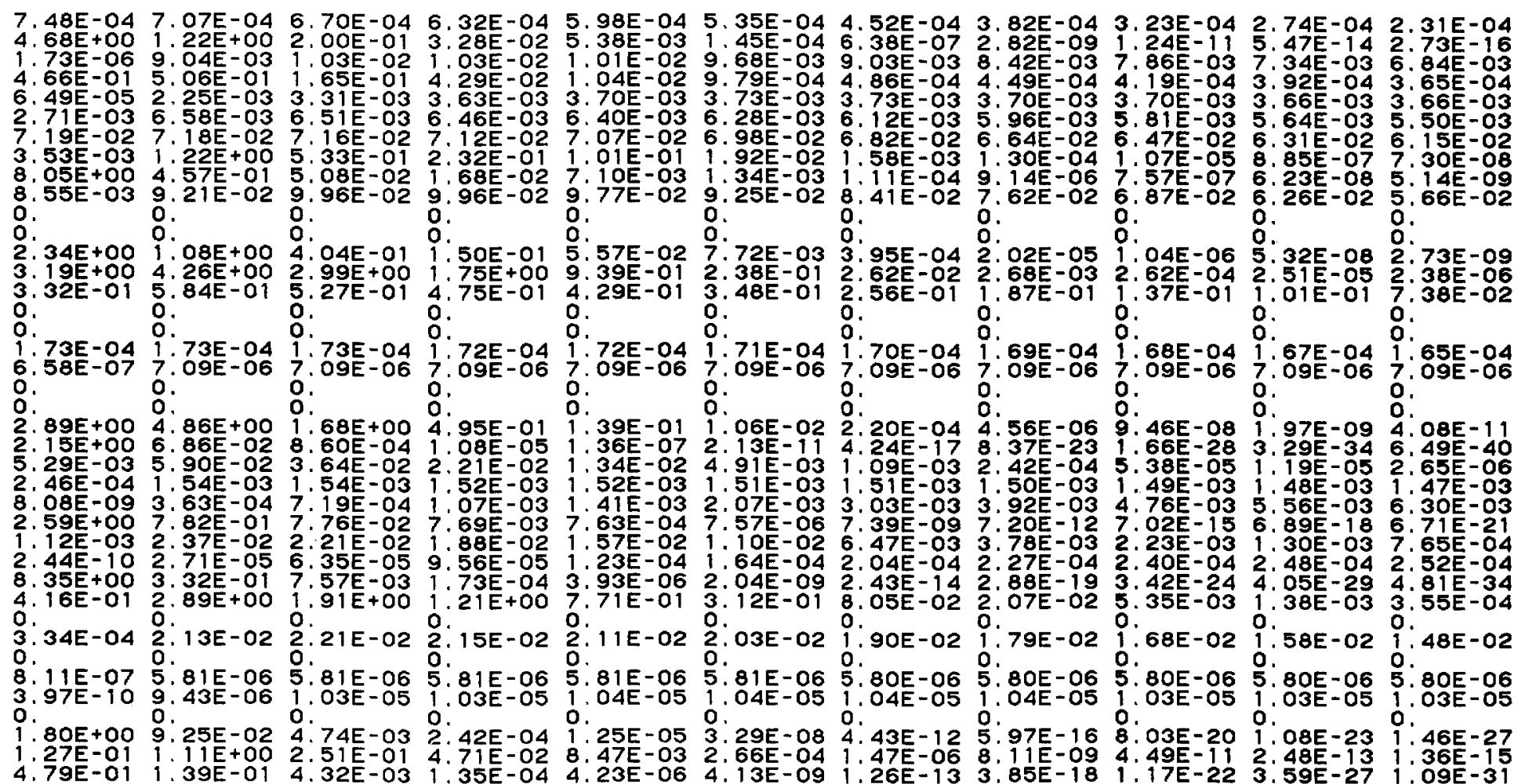




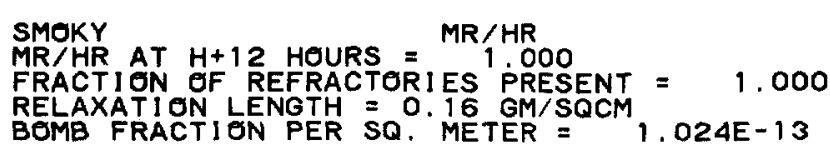

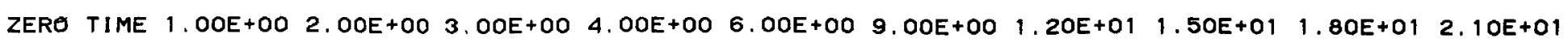

3.33E-08 6. $26 \mathrm{E}-04$ 6.44E-04 6.43E-04 6. 41E-04 6.38E-04 6.33E-04 6.28E-04 6.23E-04 6.18E-04 6.13E-04

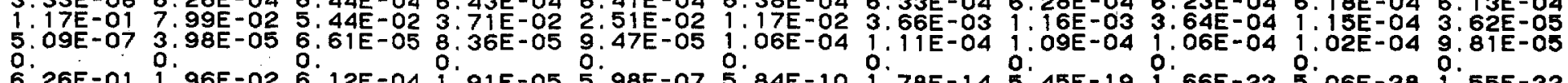

TOTAL $\begin{array}{lllllll} & \end{array}$ 1.050 1. $9.46 E-09$ 2.70E-07 3.12E-07 3.19E-07 3.20E-07.3.20E-07 3.20E-07 3.20E-07 3.20E-07 3.20E-07 3.

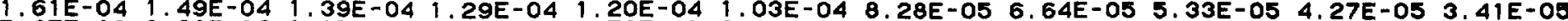
$5.27 E-06 \quad 8.60 E-06 \quad 1.17 E-05$ 1.45E-05 $1.72 E-05$ 2. $19 E-05$ 2.79E-05 3.23E-05 3.61E-05 3.90E-05 $4.11 E-05$ $5.56 E-05 \quad 1.84 E-04 \quad 1.76 E-04 \quad 1.68 E-04 \quad 1.60 E-04 \quad 1.46 E-04 \quad 1.28 E-04 \quad 1.12 E-04 \quad 9.72 E-05 \quad 8.46 E-05$ 7.38E-05

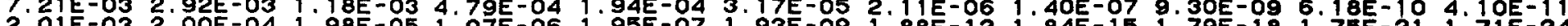

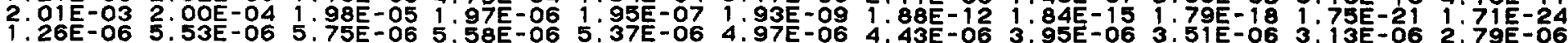

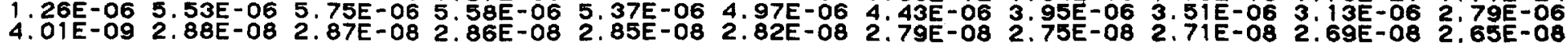

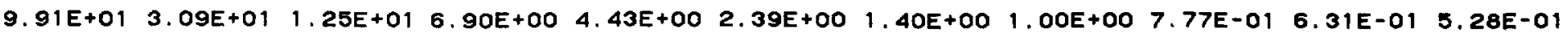


SMOKY $M$ MT $H+12$ HOURS $=$ MR/HR

FRACTION OF REFRACTORIES PRESENT $=1,000$

RELAXATION LENGTH E 0.16 GM SQCM $1.024 E-13$

DEBRIS DECAY FROM 1 TO 300 DAYS

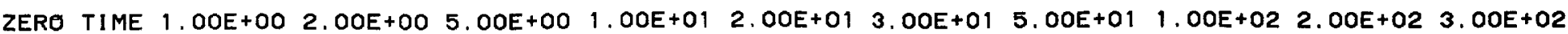
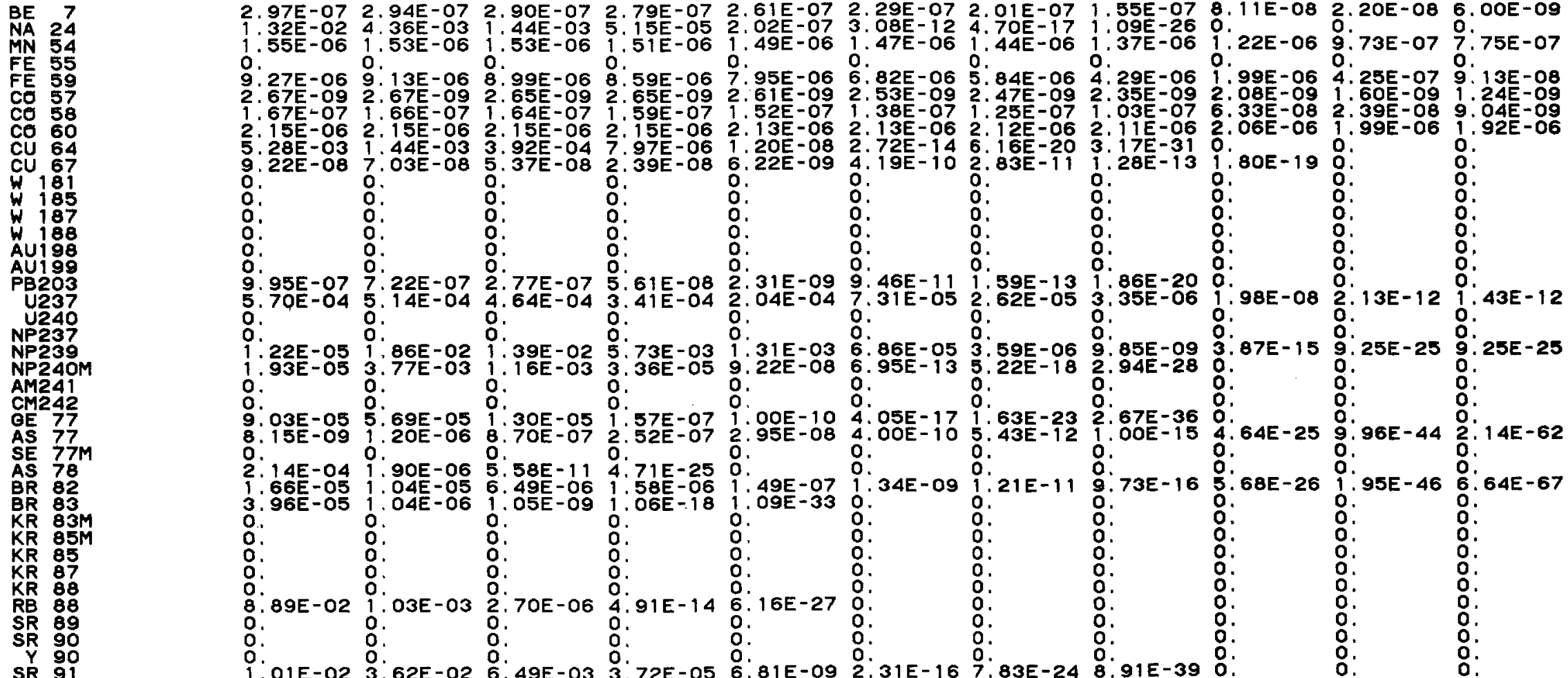
SMEKY AT H+12 HOURS $=$ MR/HR 1 MR

FRACTION OF REFRACTORIES PRESENT : 1.000

RELAXATI ON LENGTH $=0.16 \mathrm{GM} / \mathrm{SOCM}, 1.024 \mathrm{E}-13$

DEBRIS DECAY FROM 1 TO 300 DAYS

ZERO TIME 1. OOE+DO 2. $2.00 E+00 \quad 5.00 E+00 \quad 1.00 E+01 \quad 2.00 E+01 \quad 3.00 E+01 \quad 5.00 E+01 \quad 1.00 E+02 \quad 2.00 E+02 \quad 3.00 E+02$ 
SMOKY AT H+12 HOURS = MR/HR

FRACTION OF REFRACTORIES PRESENT $=1.000$

RELAXATION LENGTH $=0$. 16 GM/SQCM $1.024 E-13$

DEBRIS DECAY FROM 1 TO 300 DAYS

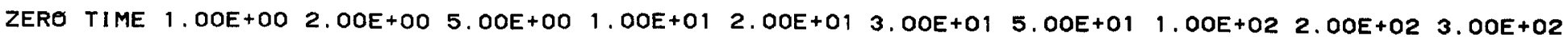

$1.07 E-013.86 E-05 \quad 1.40 E-08 \quad 6.70 E-19 \quad 4.20 E-36 \quad 0$.

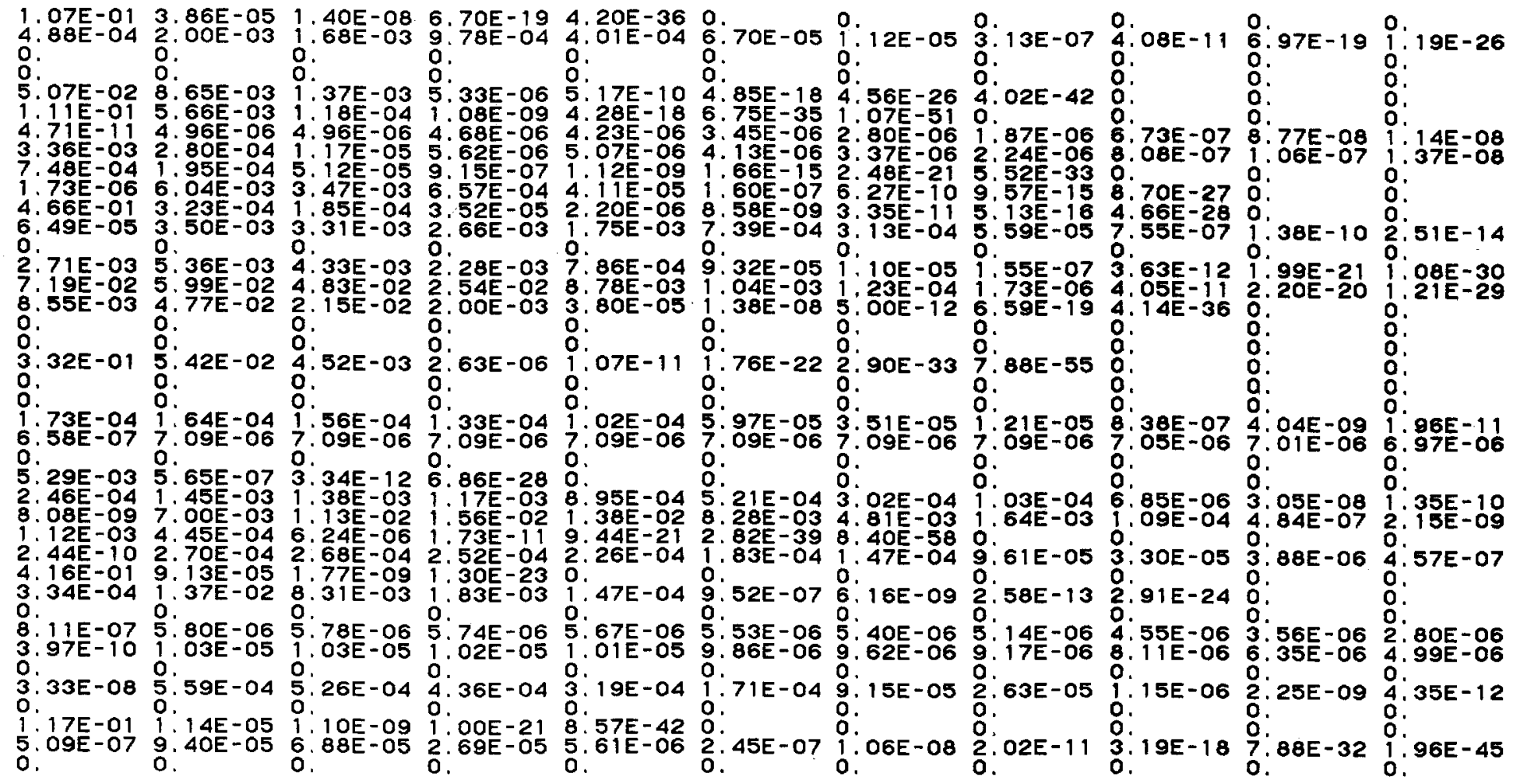

ND149

PM1 149

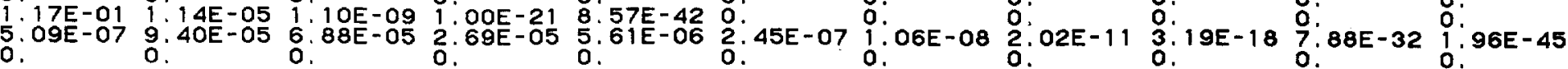


SMOKY

$M R / H R$

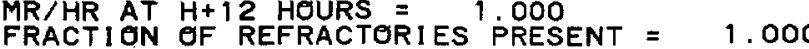

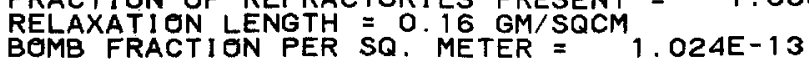

DEBRIS DECAY FROM 1 TO 300 DAYS

ZERO TIME 1. OOE+OO 2. $2.00 E+00 \quad 5.00 E+00 \quad 1.00 E+01 \quad 2.00 E+01 \quad 3.00 E+01 \quad 5.00 E+01 \quad 1.00 E+02 \quad 2.00 E+02 \quad 3.00 E+02$

$\begin{array}{llllllllllll}2.23 E-04 & 1.52 E-03 & 8.39 E-04 & 1.41 E-04 & 7.24 E-06 & 1.90 E-08 & 5.01 E-11 & 3.46 E-16 & 4.34 E-29 & 0.3 & \\ 1.22 E-04 & 8.55 E-05 & 6.00 E-05 & 2.07 E-05 & 3.52 E-06 & 1.03 E-07 & 2.98 E-09 & 2.50 E-12 & 5.16 E-20 & 2.20 E-35 & 9.34 E-51\end{array}$ $1.22 E-04$ 8.55E-05 6. 00E-05 2.07E-05 3.52E-06 1. 03E-07 2.98E-09 2.50E-12 5.16E-20 2.20E-35 9.34E-51

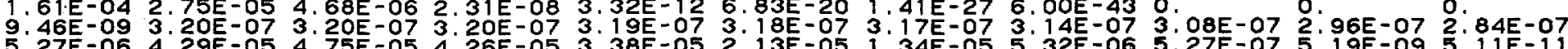

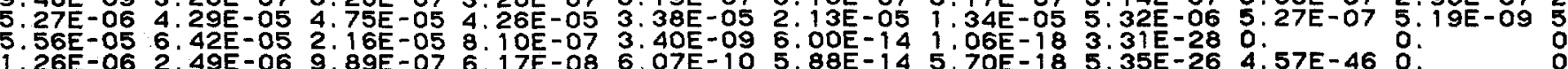

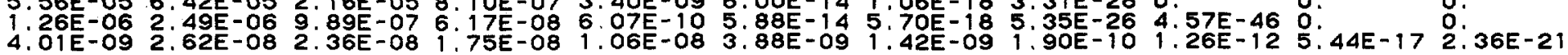

TD159

TOTAL

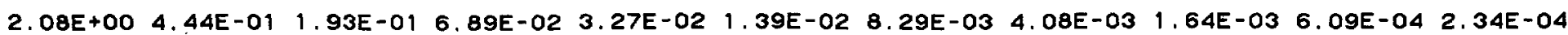




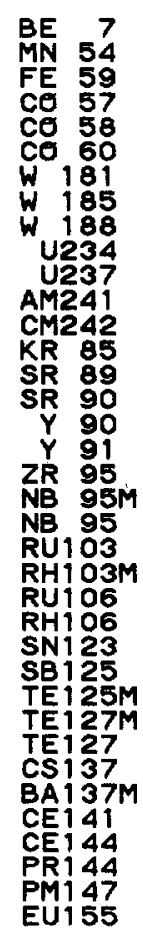

TOTAL

SMOKY

$M R / H R$

FRACTION OF REFRACTORIES PRESENT = 1.000

RELAXATION LENGTH $=0.16 \mathrm{GM} / \mathrm{SQCM}$, $1.024 \mathrm{E}-13$

DEBRIS DECAY FROM 1 TO 50 YEARS

ZERO TIME 1.OOE+OO 1.5OE+OO 2. $2.00 E+00 \quad 3.50 E+00 \quad 5.00 E+00 \quad 7.00 E+00 \quad 1.00 E+01 \quad 2.00 E+013.50 E+01 \quad 5.00 E+01$

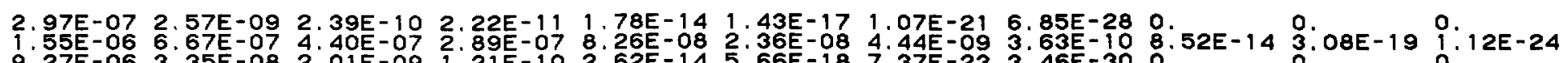

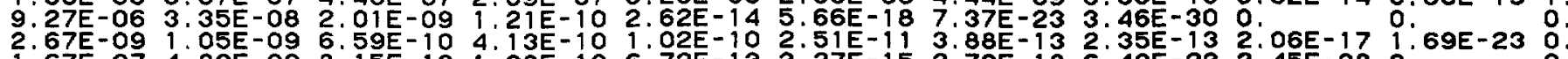

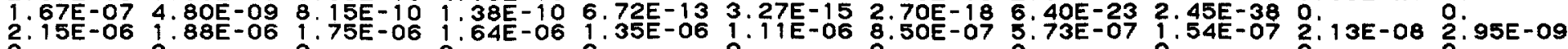

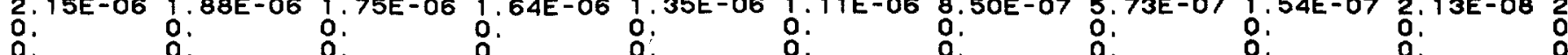

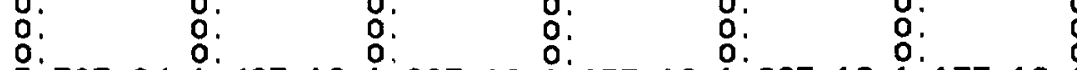

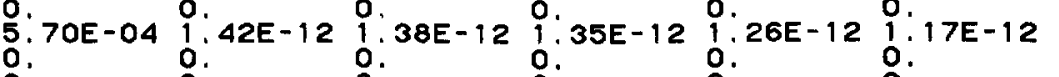

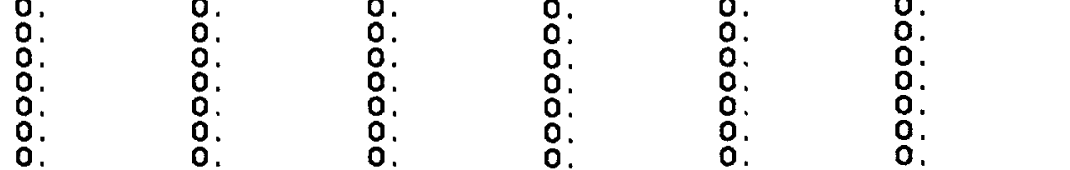
26E-05 3.24E-05 $4.62 E-00$ O. 1. $45 E-12$ 7. $20 \mathrm{E}-05$ O. $03 \mathrm{E}-05$ O. 4.

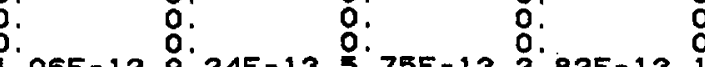
O6E-12 9: $24 E-13$ 5. $75 E-13$ 2. $82 E-13$ 1. $38 E-13$

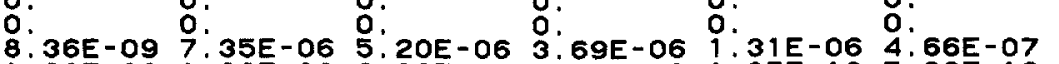

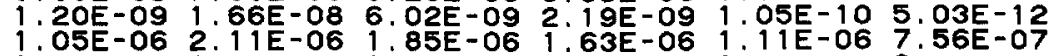
: O.

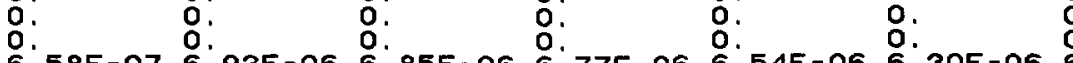

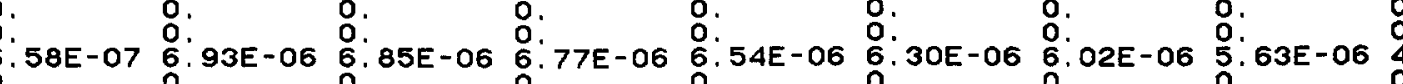

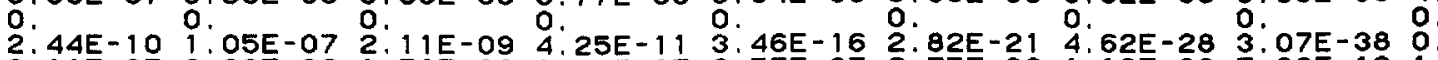

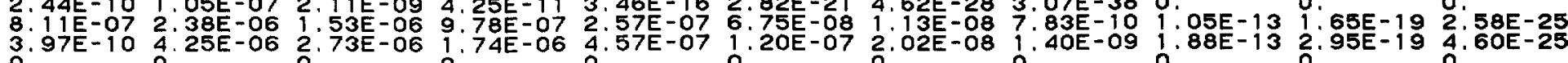

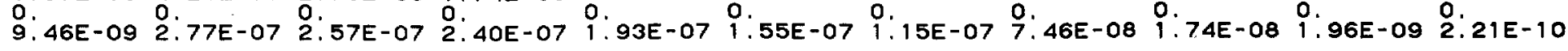
5.99E-04 1.32E-04 3.57E-05 1.91E-05 1.13E-05 8.99E-06 7.59E-06 6.50E-06 4.64E-06 3.19E-06 2.24E-06 
SMEKY MR H+12 HEURS MR/HR

FRACTION OF REFRACTORIES PRESENT $=0.500$

RELAXATION LENGTH $=0$. 16 GM SQCM $1.299 E-13$

DEBRIS DECAY FREM 1 TO 21 HOURS

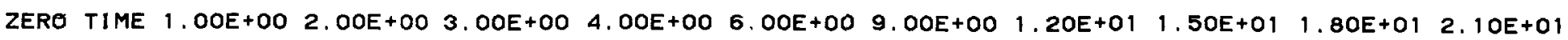
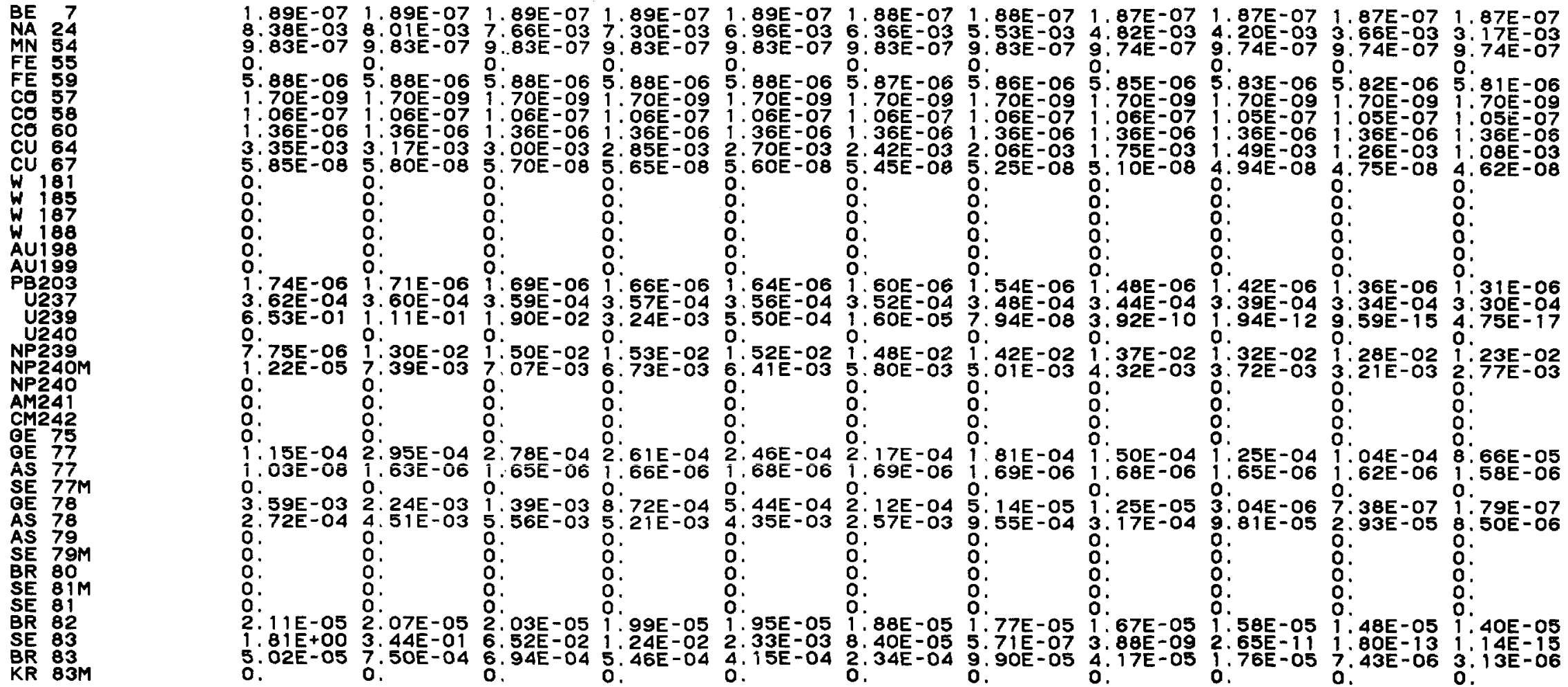
SMOKY

FRACTION OF REFRACTERIES PRESENT $=0.500$

RELAXATION LENGTH $=0.16$ GM/SQCM $1.299 E-13$

DEBRIS DECAY FROM 1 TO 21 HOURS

ZERO TIME 1.OOE+OO 2. OOE+OO 3. OOE+OO 4. OOE+OO $\quad 6.00 E+00 \quad 9.00 E+00 \quad 1.20 E+01 \quad 1.50 E+01 \quad 1.80 E+01 \quad 2.10 E+01$

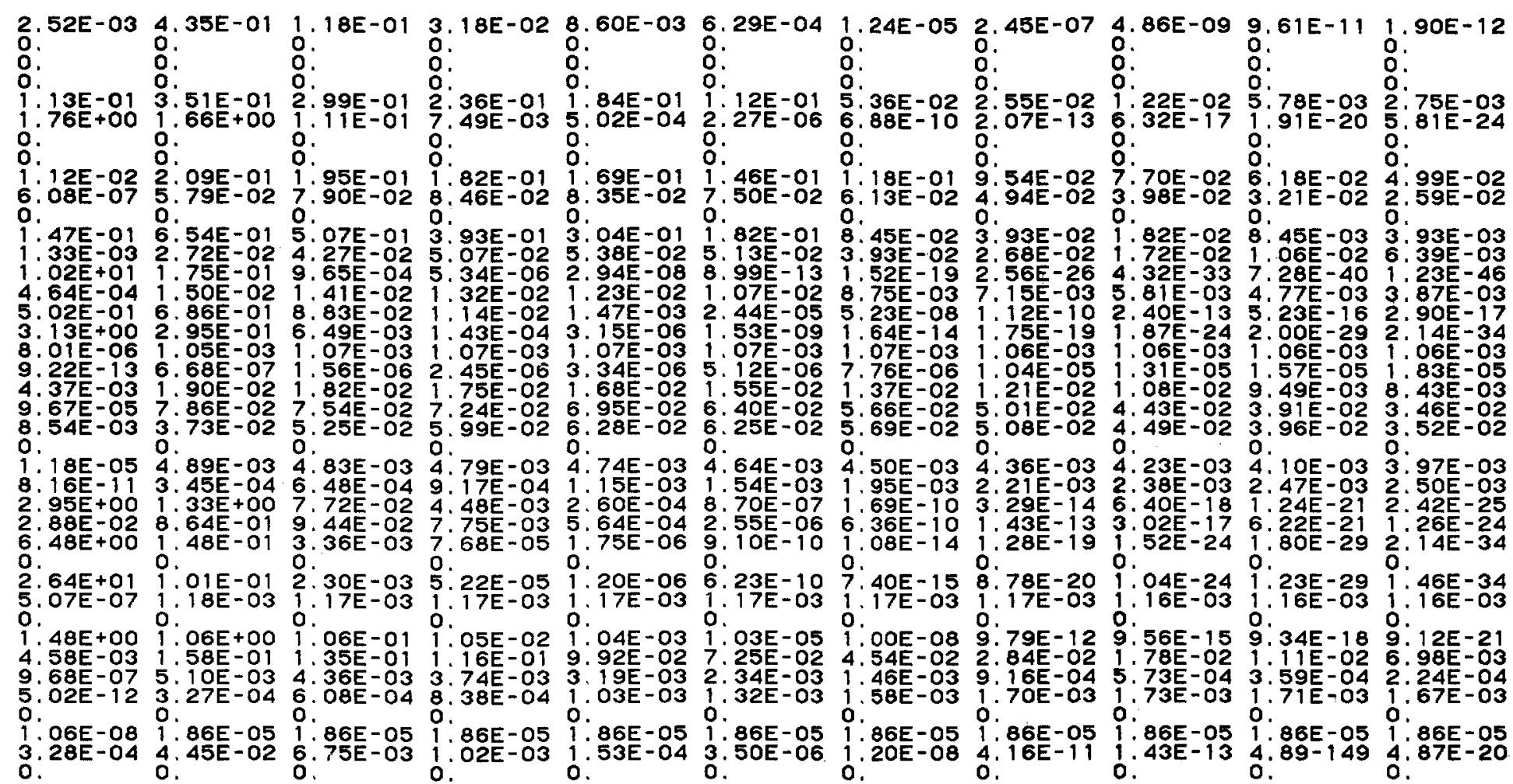


SMOKY

MR/HR

FRACTION OF REFRACTERIES PRESENT $=0.500$

RELAXATION LENGTH $=0.16$ GM SQ SQM $1.299 E-13$

DEBRIS DECAY FROM 1 TO 21 HEURS

ZERO TIME $1.00 E+00 \quad 2.00 E+00 \quad 3.00 E+00 \quad 4.00 E+00 \quad 6.00 E+00 \quad 9.00 E+00 \quad 1.20 E+01 \quad 1.50 E+01 \quad 1.80 E+012.10 E+01$

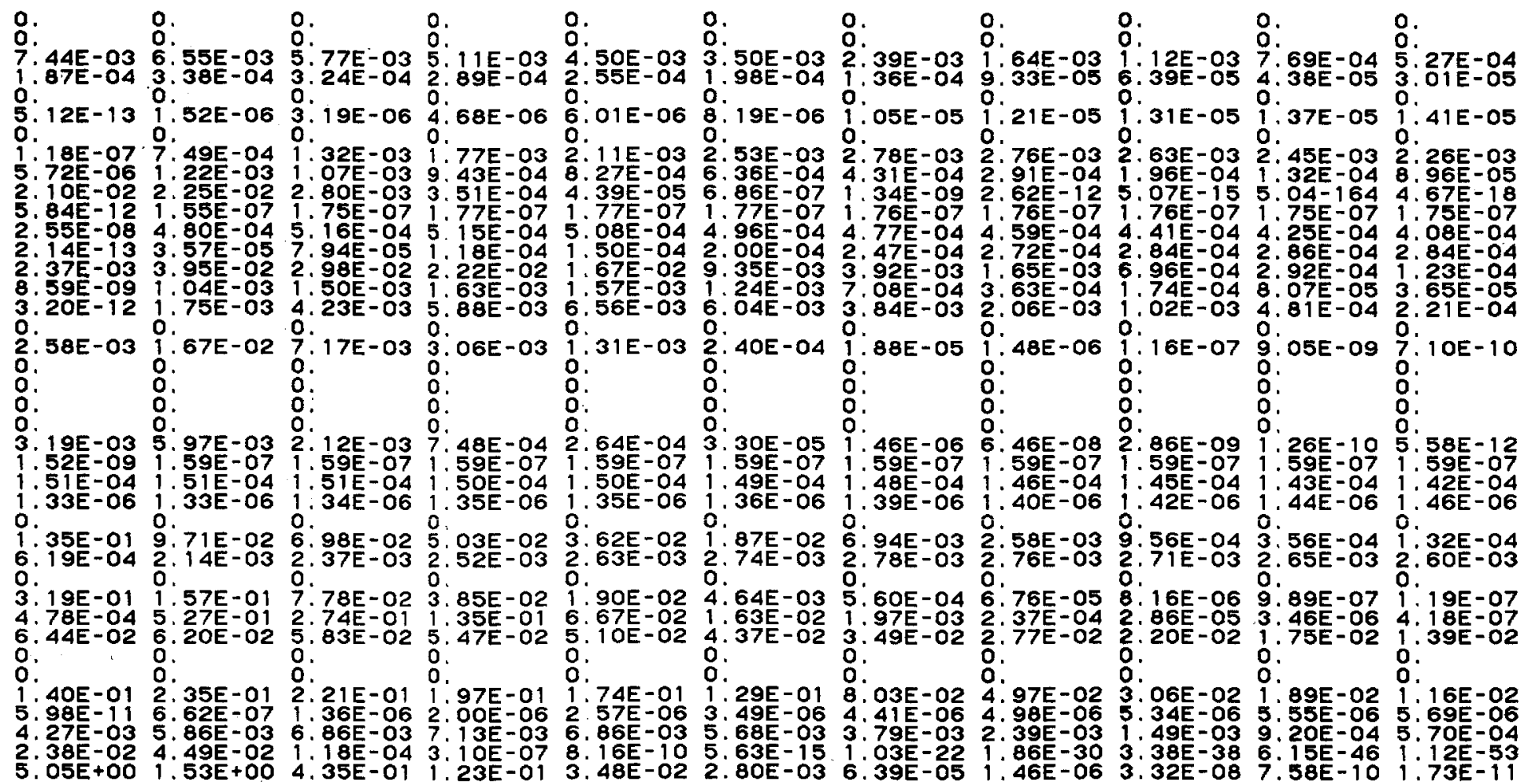


SMOKY

MACTION OF REFRACTORIES PRESENT $=0.500$

RELAXATION LENGTH $=0.16$ GM/SQCM

DEBRIS DECAY FROM 1 TO 21 HOURS

ZERO TIME 1. OOE+OO 2. OOE+OO $3.00 E+00 \quad 4.00 E+00 \quad 6.00 E+00 \quad 9.00 E+00 \quad 1.20 E+01 \quad 1.50 E+01 \quad 1.80 E+012.10 E+01$

9.49E-04 8.98E-04 8.5OE-04 8.03E-04 7.60E-04 6.80E-04 5.74E-04 4.86E-04 4.10E-04 3.47E-04 2.93E-04 5.94E+00 1.55E+00 2.54E-01 4.16E-02 6.83E-03 1.84E-04 8. 10E-07 3.58E-09 1.57E-11 6.94E-14 3.46E-16 5.91E-01 6.43E-01 2.31E-O2 $1.31 E-02$ 1.29E-02 1.2 $8.24 E-052.86 E-034.20 E-034.61 E-03$ 4.69E-03 $4.73 E-03$ 4.73E-03 4.69E-03 4.69E-03 $4.65 E-034.65 E-03$ $3.44 E-03 \quad 8.35 E-03 \quad 8.27 E-03$ 8.21E-03 8.12E-03 7.98E-03 7.77E-03 $7.56 E-03 \quad 7.37 E-037.16 E-03 \quad 6.98 E-03$ 9.13E-02 9.11E-02 9.09E-02 9.04E-02 8.98E-02 8.86E-02 8.66E-02 8.43E-02 8.21E-02 8.01E-02 $7.80 E-02$ 4.49E-03 $1.56 E+00$ 6.77E-01 2.95E-01 1.28E-01 2.44E-02 2.00E-03 1.65E-04 $1.36 E-051.12 E-06 \quad 9.27 E-08$

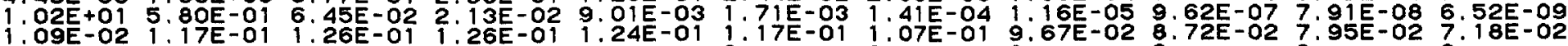

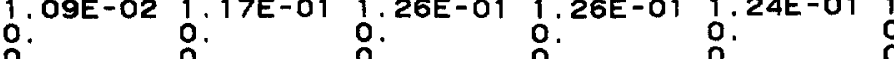

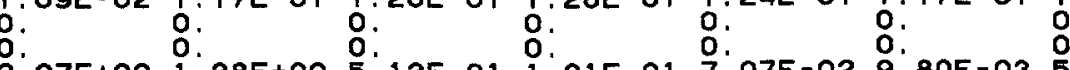
2.97E+00 1.38E+00 5.13E-01 1.91E-01 7.07E-02 9.80E-03 5.01E-04 2.57E-05 $1.31 E-06 \quad 6.75 E-08$ 3.46E-09

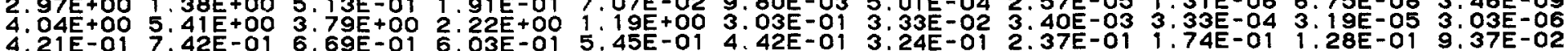

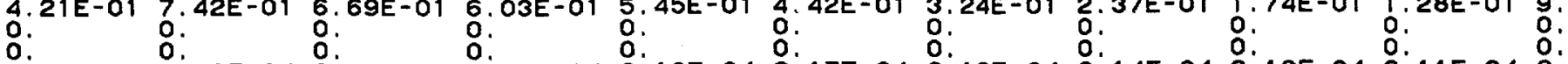

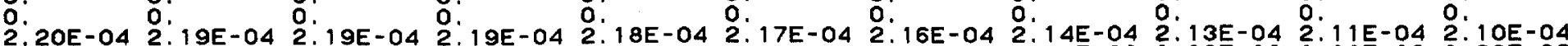

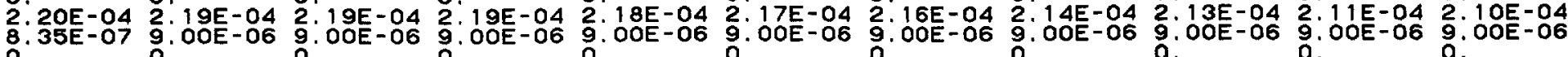

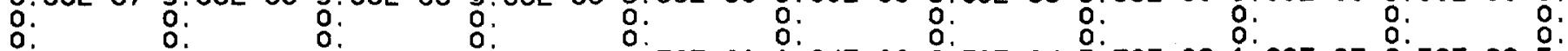

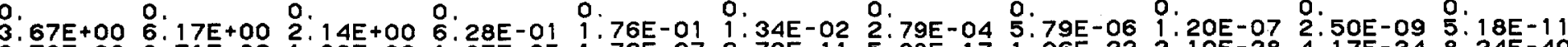

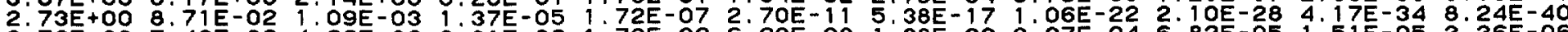

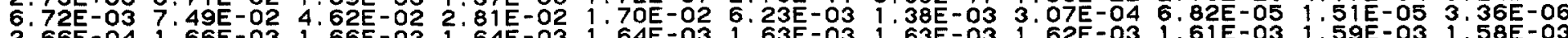

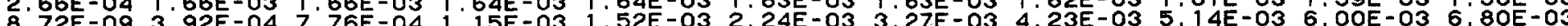
2. 72E $46 E-04$ 2. OOE-02 $1.87 E-021.58 E-021.33 E-029.31 E-035.46 E-033.19 E-031.88 E-031.10 E-036.46 E-04$ $\begin{array}{lllllllll} & \end{array}$

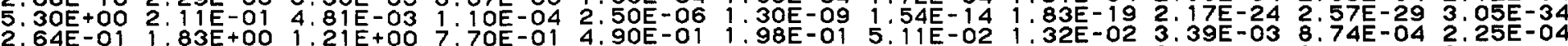

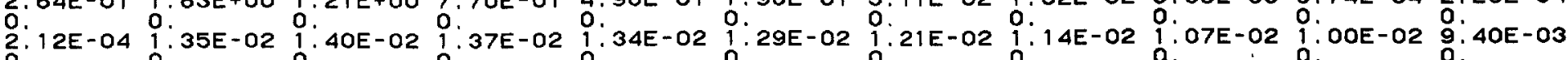
$5.15 E-07$
$5.69 E-06$
5

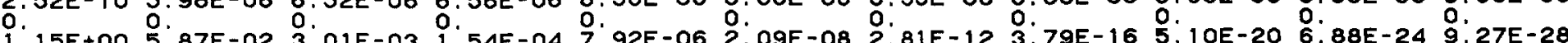

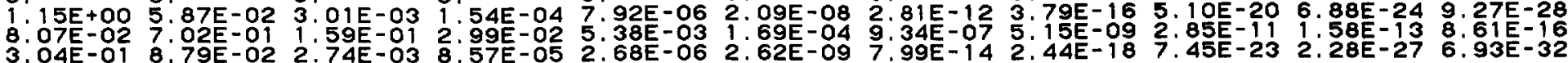


SMEKY AT H+12 HOURS MR/HR

FRACTION OF REFRACTORIES PRESENT $=0.500$

RELAXATION LENGTH $=0.16$ GM/SQCM $1299 E-13$

DEBRIS DECAY FROM I TO 21 HOURS

ZERE TIME 1. OOE+OO 2. $2.00 E+00 \quad 3.00 E+00 \quad 4.00 E+00 \quad 6.00 E+00 \quad 9.00 E+00 \quad 1.20 E+01 \quad 1.50 E+01 \quad 1.80 E+01 \quad 2.10 E+01$ 2.11E-08 3.98E-04 4.09E-04 4.08E-04 4.07E-04 4.05E-04 4.02E-04 3.99E-04 3.96E-04 3.93E-04 3.89E-04

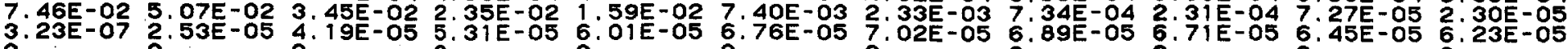

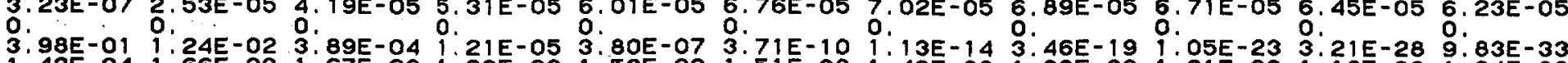

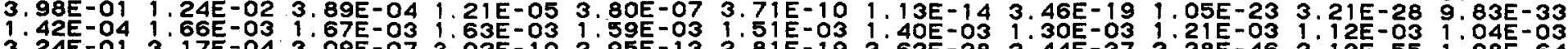

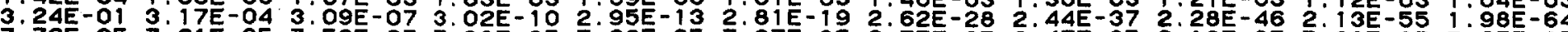
7.72E-05 7.61E-05 7.5OE-05 7.39E-05 7.29E-05 7.07E-05 6.77E-05 6.47E-05 6.19E-05 5.93E-05 5.67E-05

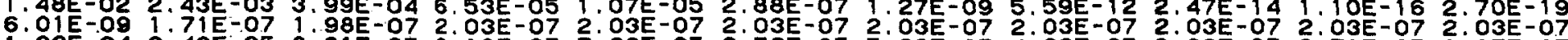
1.02E-04 9.49E-05 8.81E-05 8. $19 E-05$. 60 -05 6.56E-05 5.26E-05 4.22E-05 3.38E-05 2.71E-05 2.17E-05 3.34E-06 5.46E-06 7.41E-06 9.23E-06 1.09E-05 1.39E-05 1.77E-05 2.05E-05 2.29E-05 2.47E-05 2.61E-05 3.53E-05 1.17E-04 1. $12 E-041.07 E-041.02 E-04$ 9.3OE-05 8.12E-05 7.09E-05 6.17E-05 5.37E-05 4.69E-05 4.58E-03 1.85E-03 7.50E-04 3.04E-04 1.23E-04 2.01E-05 1.34E-06 8.89E-08 5.91E-09 3.92E-10 2.60E-11 $1.28 E-031.27 E-041.26 E-051.25 E-061.24 E-071.22 E-091.19 E-12 \quad 1.17 E-151.14 E-181.11 E-211.09 E-24$

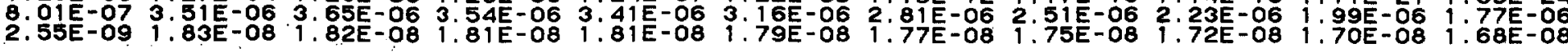

TOTAL

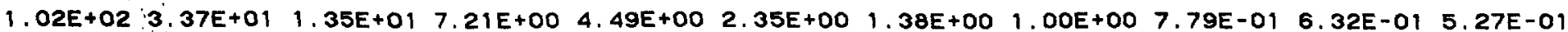


SMOKY

FRACTION OF REFRACTORIES PRESENT $=0.500$

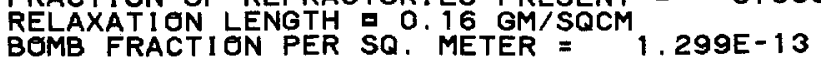

DEBRIS DECAY FROM 1 TO 300 DAYS

ZERO TIME $1.00 E+002.00 E+00 \quad 5.00 E+00 \quad 1.00 E+01 \quad 2.00 E+01 \quad 3.00 E+01 \quad 5.00 E+01 \quad 1.00 E+02 \quad 2.00 E+02 \quad 3.00 E+02$
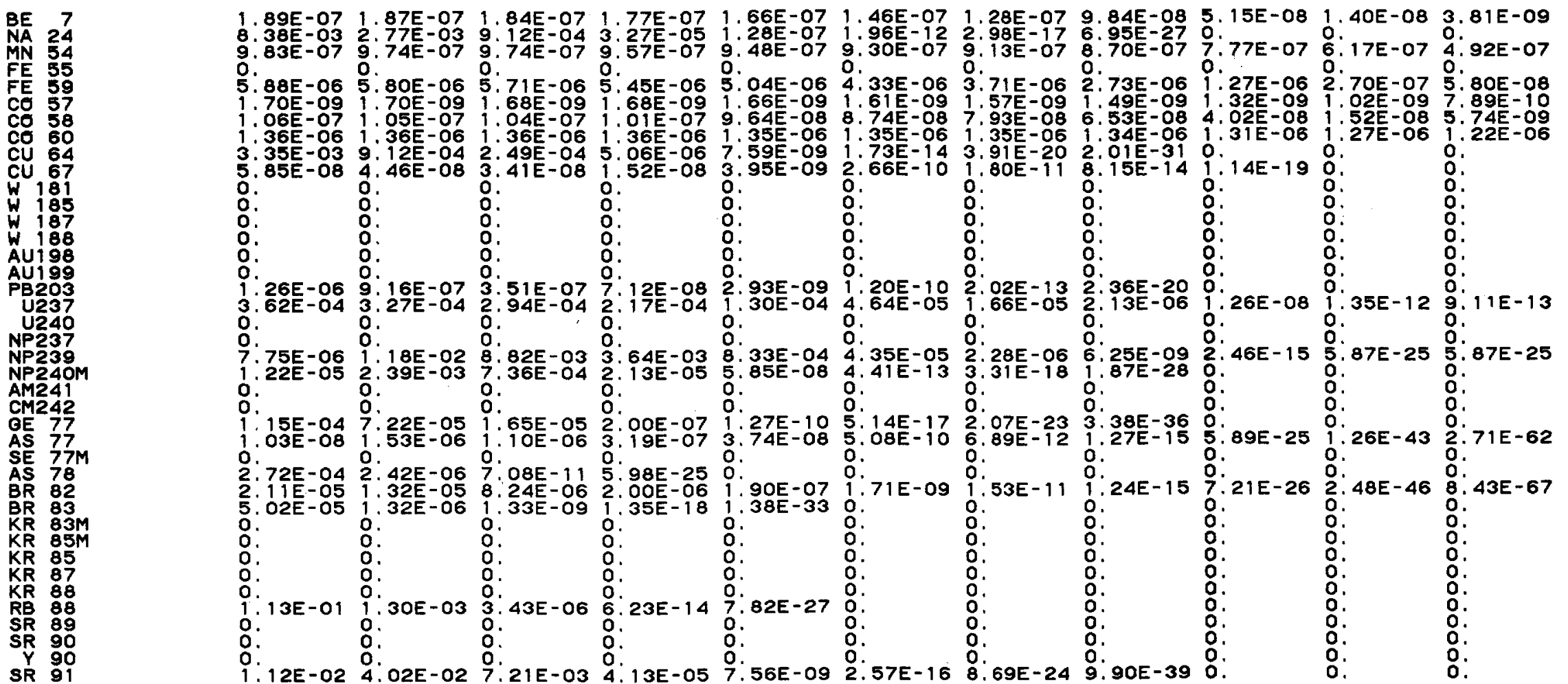
SMOKY $M$ AT $H+12$ HOURS $=$ MR/HR

MR/HR AT H+12 HOURS
FRACTION OF REFRACTERIES ${ }^{1}$ PRESENT 0000.500

BEMB FRACTI ON PER SQ. METER $=1,299 E-13$

DEBRIS DECAY FROM 1 TO 300 DAYS

ZERO TIME $1.00 E+002.00 E+00 \quad 5.00 E+00 \quad 1.00 E+01 \quad 2.00 E+01 \quad 3.00 E+01 \quad 5.00 E+01 \quad 1.00 E+02 \quad 2.00 E+02 \quad 3.00 E+02$

6. 08E-07 2.09E-02 3.74E-03 2.15E-05 3.94E-09 1.33E-16 4.50E-24 5.13E-39

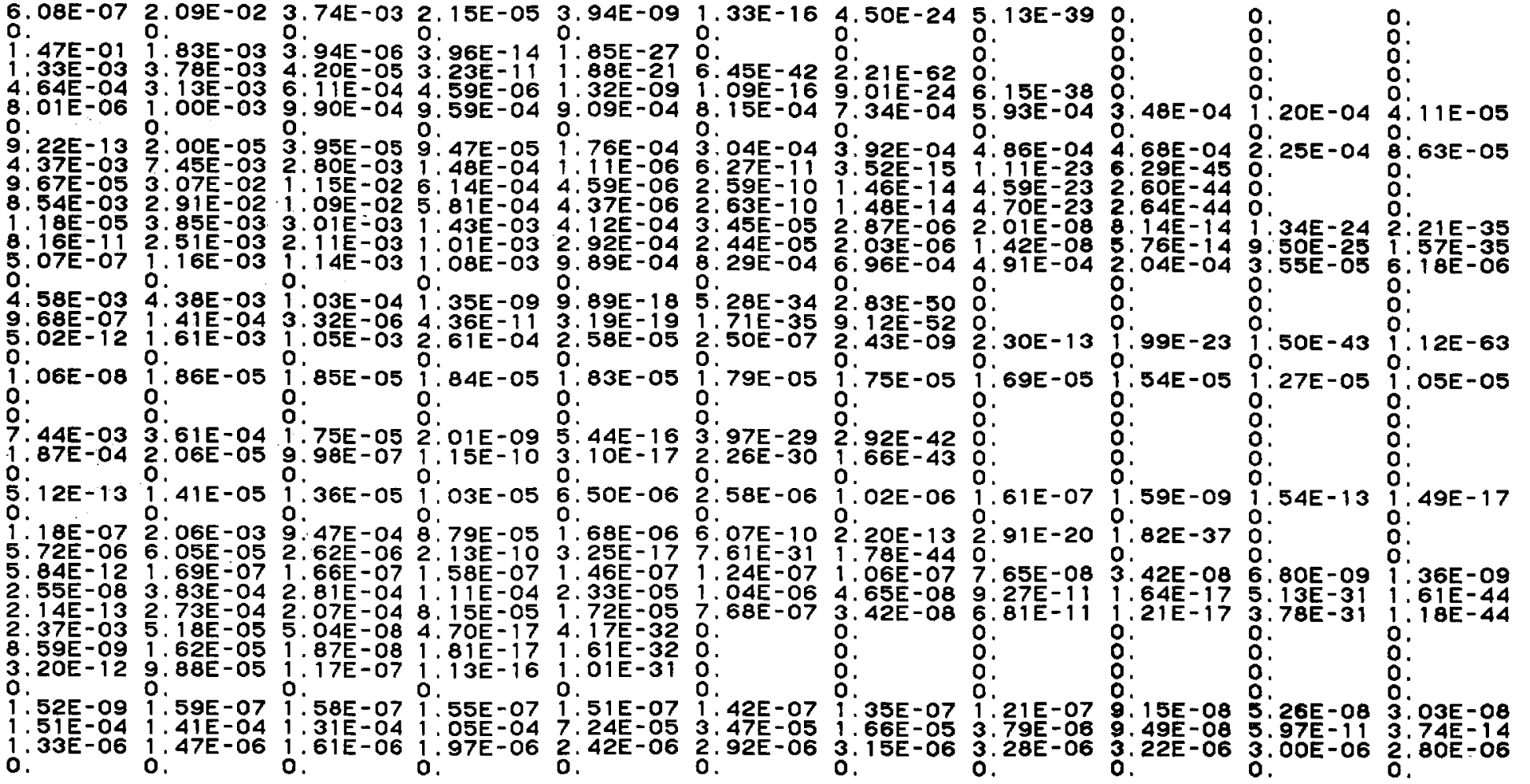


SMOKY

FRACTION OF REFRACTORIES PRESENT $=0.500$

RELAXAT ION LENGTH $=0.16$ GM/SQCM, $299 E-13$

DEBRIS DECAY FROM 1 TO 300 DAYS

ZERO TIME 1. $1.00 E+002.00 E+00 \quad 5.00 E+00 \quad 1.00 E+01 \quad 2.00 E+01 \quad 3.00 E+01 \quad 5.00 E+01 \quad 1.00 E+02 \quad 2.00 E+02 \quad 3.00 E+02$

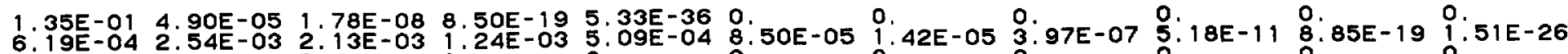
o. O.

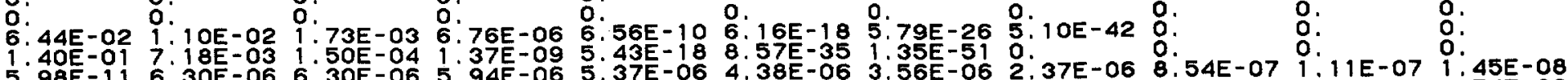
$\begin{array}{llllllll}1 & \end{array}$

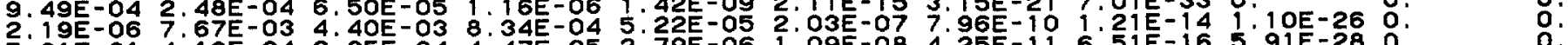

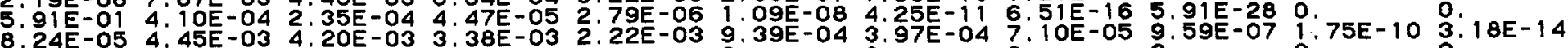

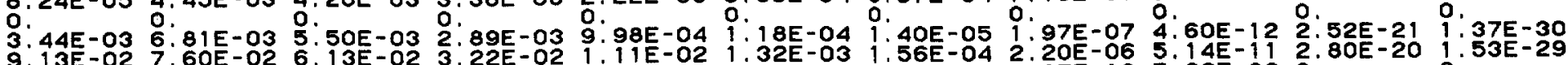

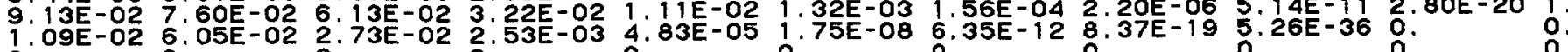

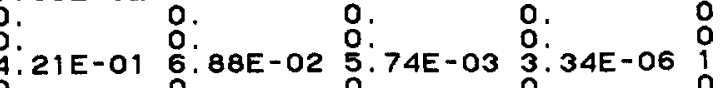
88E-02 . O: O. O. O. 2.20E-04 2.09E-04 1.98E-04 1.69E-04 1.29E-04 7.58E-05 4. 46E-05 8.35E-07 9.00E-06 9.00E-06 9. OOE-06 9: OOE-06

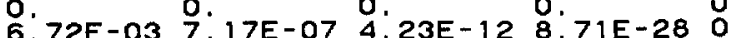
\begin{tabular}{lllll}
$2.66 \mathrm{E}-04$ & $1.57 \mathrm{E}-03$ & $1.49 \mathrm{E}-03$ & $1.27 \mathrm{E}-03$ & $9.65 \mathrm{E}-04$ \\
$8.72 \mathrm{E}-09$ & $7.55 \mathrm{E}-03$ & $1.21 \mathrm{E}-02$ & $1.68 \mathrm{E}-02$ & $1.49 \mathrm{E}-02$ \\
$9.46 \mathrm{E}-04$ & $3.75 \mathrm{E}-04$ & $5.26 \mathrm{E}-06$ & $1.46 \mathrm{E}-1$ & $1.97 \mathrm{E}-21$ \\
\hline
\end{tabular} 0. o.: $\quad$ o 


\section{SMEKY}

MR/HR AT $H+12$ HOURS = MR/HR

FRACTIN

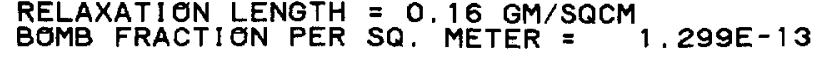

DEBRIS DECAY FREM 1 TO 300 DAYS

ZERO TIME 1. OOE+DO 2. $2.00 E+00 \quad 5.00 E+00 \quad 1.00 E+01 \quad 2.00 E+01 \quad 3.00 E+01 \quad 5.00 E+01 \quad 1.00 E+02 \quad 2.00 E+02 \quad 3.00 E+02$

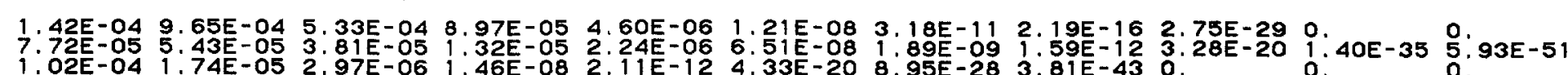

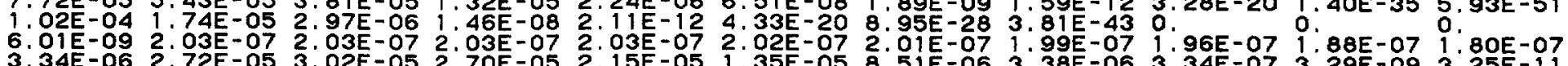
$3.34 E-06$
3.5

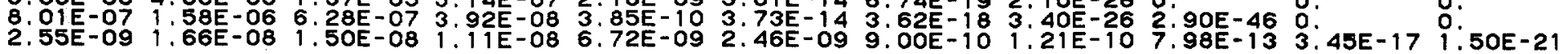
TOTAL

2. $12 E+00 \quad 4.41 E-01 \quad 1.91 E-01 \quad 7.38 E-02 \quad 3.55 E-02 \quad 1.45 E-02 \quad 8.25 E-03 \quad 3.69 E-03 \quad 1.22 E-03 \quad 4.18 E-04 \quad 1.63 E-04$ 
SMEKY

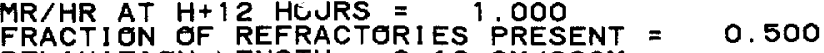

RELAXATION LENGTH $=0.16 \mathrm{GM} / \mathrm{SQCM}, 1.299 E-13$

DEBRIS DECAY FROM 1 TO 50 YEARS

ZERO TIME $1.00 E+00 \quad 1.50 E+00 \quad 2.00 E+00 \quad 3.50 E+00 \quad 5.00 E+00 \quad 7.00 E+00 \quad 1.00 E+01 \quad 2.00 E+01 \quad 3.50 E+01 \quad 5.00 E+01$

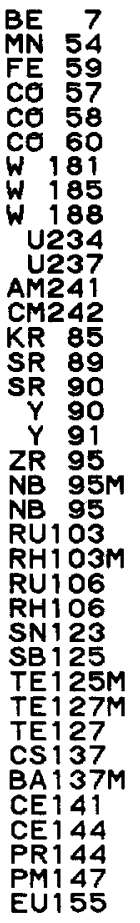

TOTAL

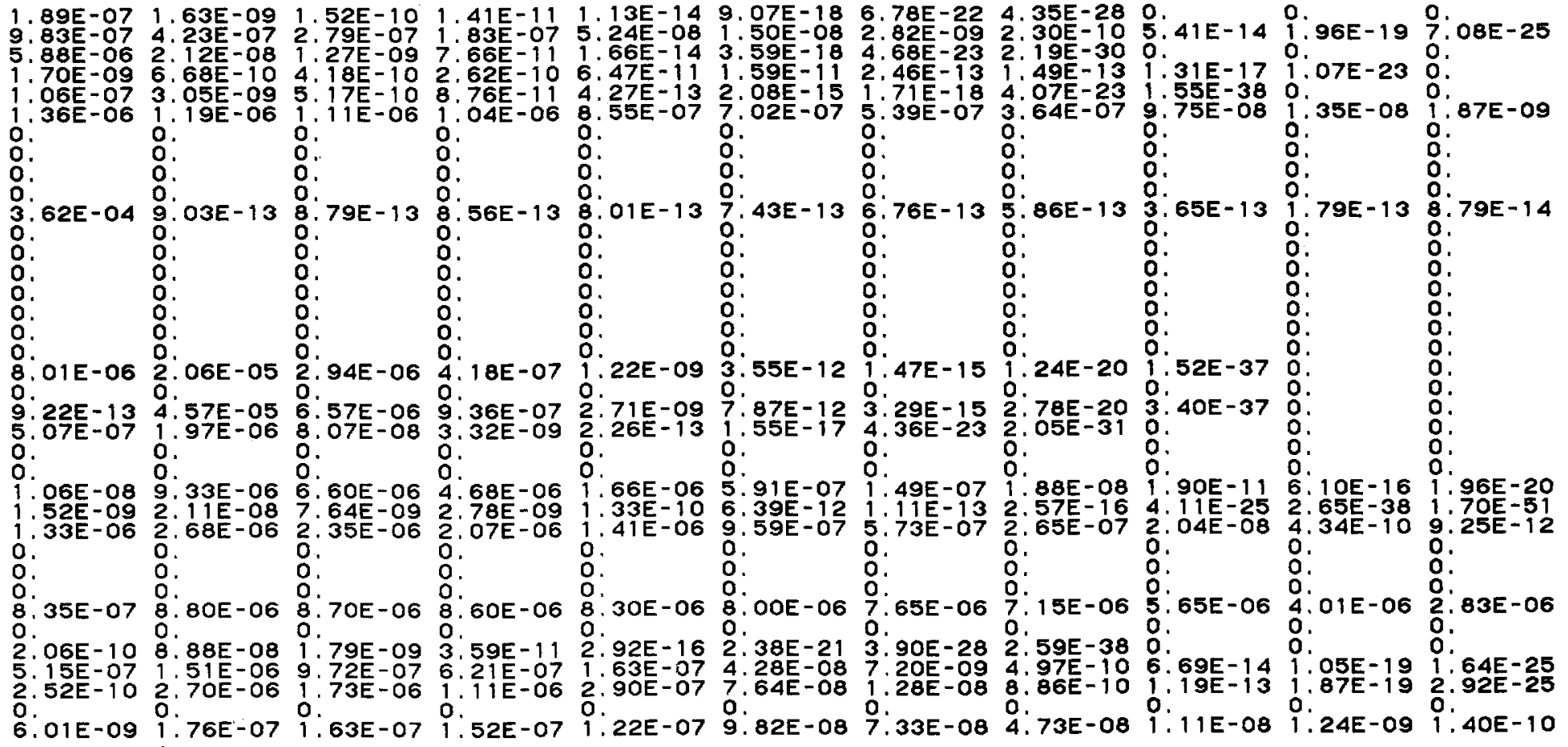

3.82E-04 9.52E-05 3.15E-05 1.98E-05 1.29E-05 $1.05 E-05 \quad 9.01 E-06 \quad 7.85 E-06 \quad 5.78 E-06 \quad 4.03 E-06 \quad 2.84 E-06$ 
SMOKY $M R / H R$ AT H+12 HOURS $=$ MR/HR

FRACTION OF REFRACTORIES PRESENT $=0.100$

RELAXATION LENOTH $=0.16$ GM/SQCM

DEBRIS DECAY FROM 1 TO 21 HOURS

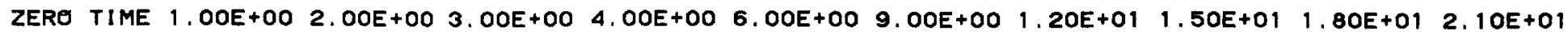


SMOKY

FR'HR AT H+12 HOURS = ${ }^{1}$ 'OOO

RELAXATION LENOTH $=0.16 \mathrm{GM} / \mathrm{SQCM}$
BOMB FRACT I ON PER SO. METER $=1.657 E-13$

DEBRIS DECAY FROM 1 TO 21 HOURS

ZERO TIME 1. OOE+OO 2. OOE+OO 3. OOE+OO 4. OOE+OO 6. OOE+O0 9.0OE+OO $1.20 E+01 \quad 1.50 E+01 \quad 1.80 E+012.10 E+01$

BR $84 \mathrm{M}$

KR $85 \mathrm{M}$

KR $8 B$

RB 89

SR 89

SR 91

Y $91 \mathrm{M}$

SR 92

SR 93

$\begin{array}{rl}Y & 93 \\ Y & 94 \\ Y & 95\end{array}$

$Y$ Y

NR 95

ZR 97

$\mathrm{NB} 97$

MO 99

MO 101

TCiol

Me102

TClO2

RU103

TC104

RH105M

RH105

RH106

RH107

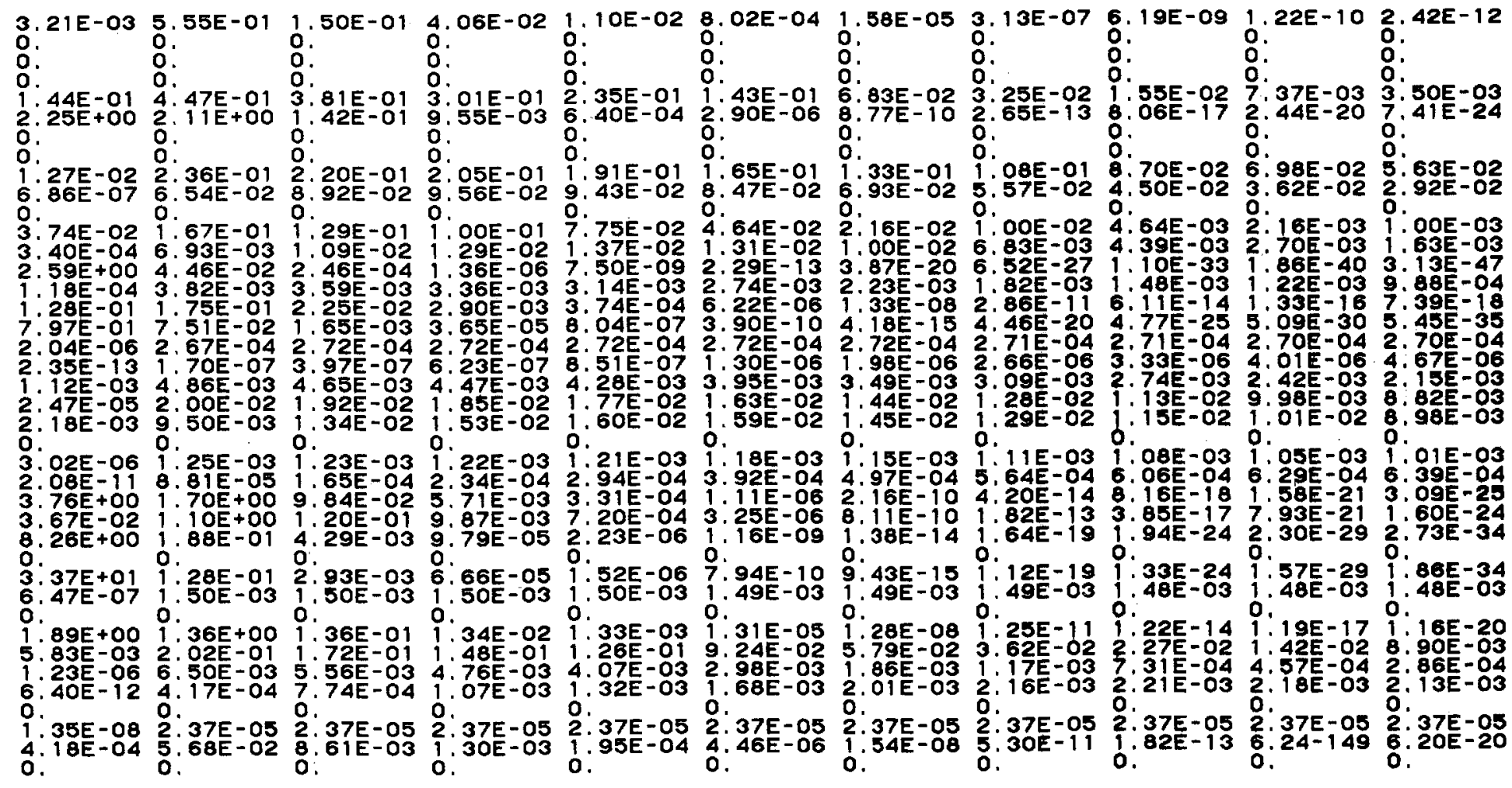


SMOKY

FRACTION OF REFRACTORIES PRESENT $=0.100$

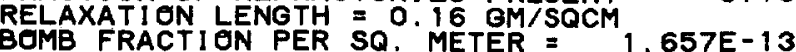

DEBRIS DECAY FROM 1 TO 21 HOURS

ZERO TIME $1.00 E+00 \quad 2.00 E+00 \quad 3.00 E+00 \quad 4.00 E+00 \quad 6.00 E+00 \quad 9.00 E+00 \quad 1.20 E+01 \quad 1.50 E+01 \quad 1.80 E+012.10 E+01$

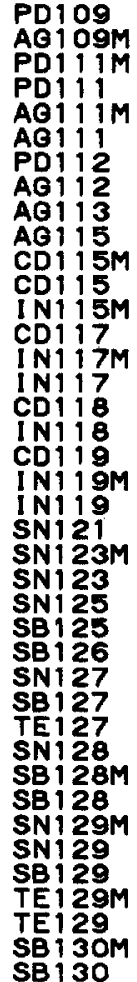

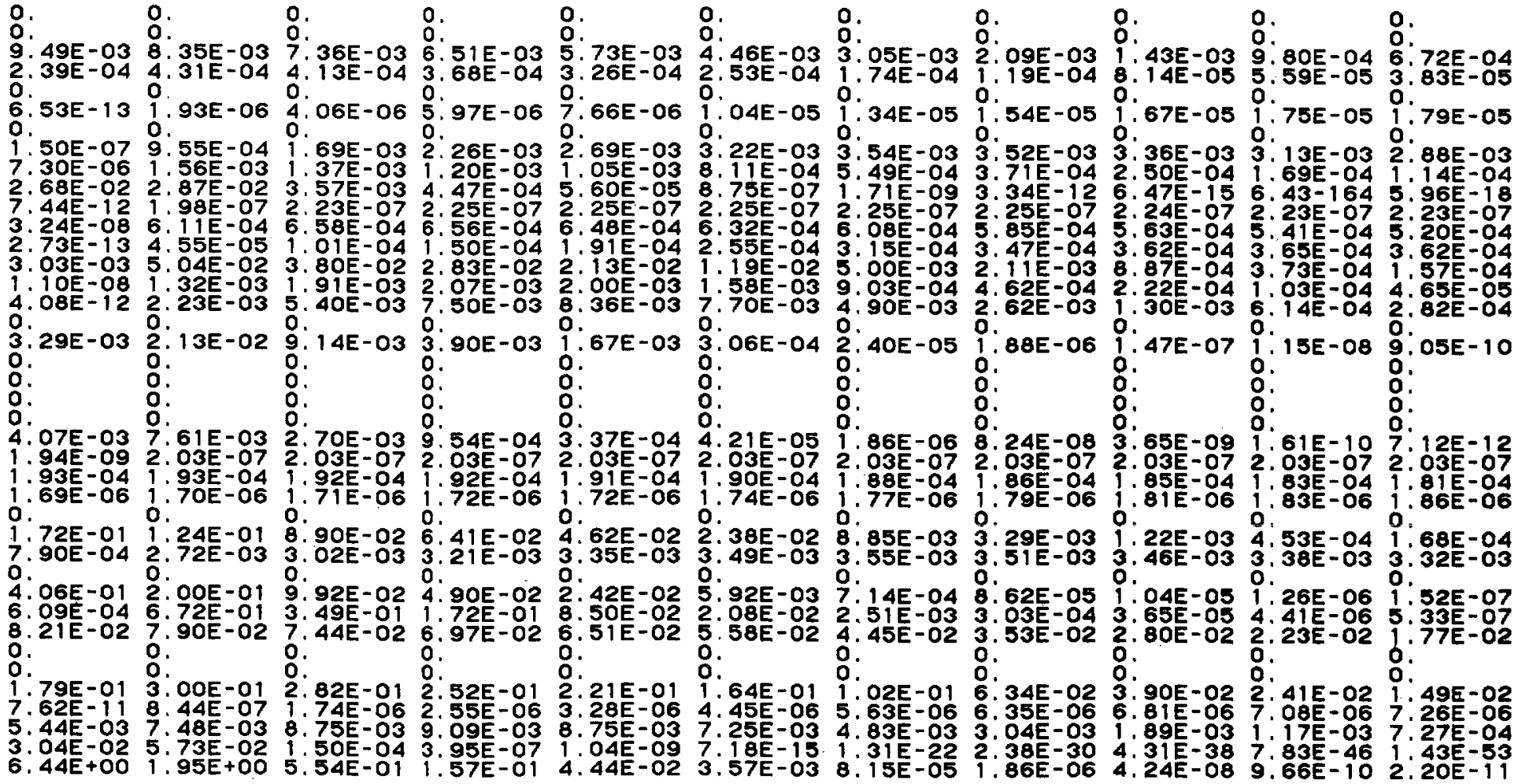




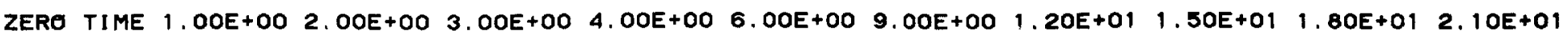

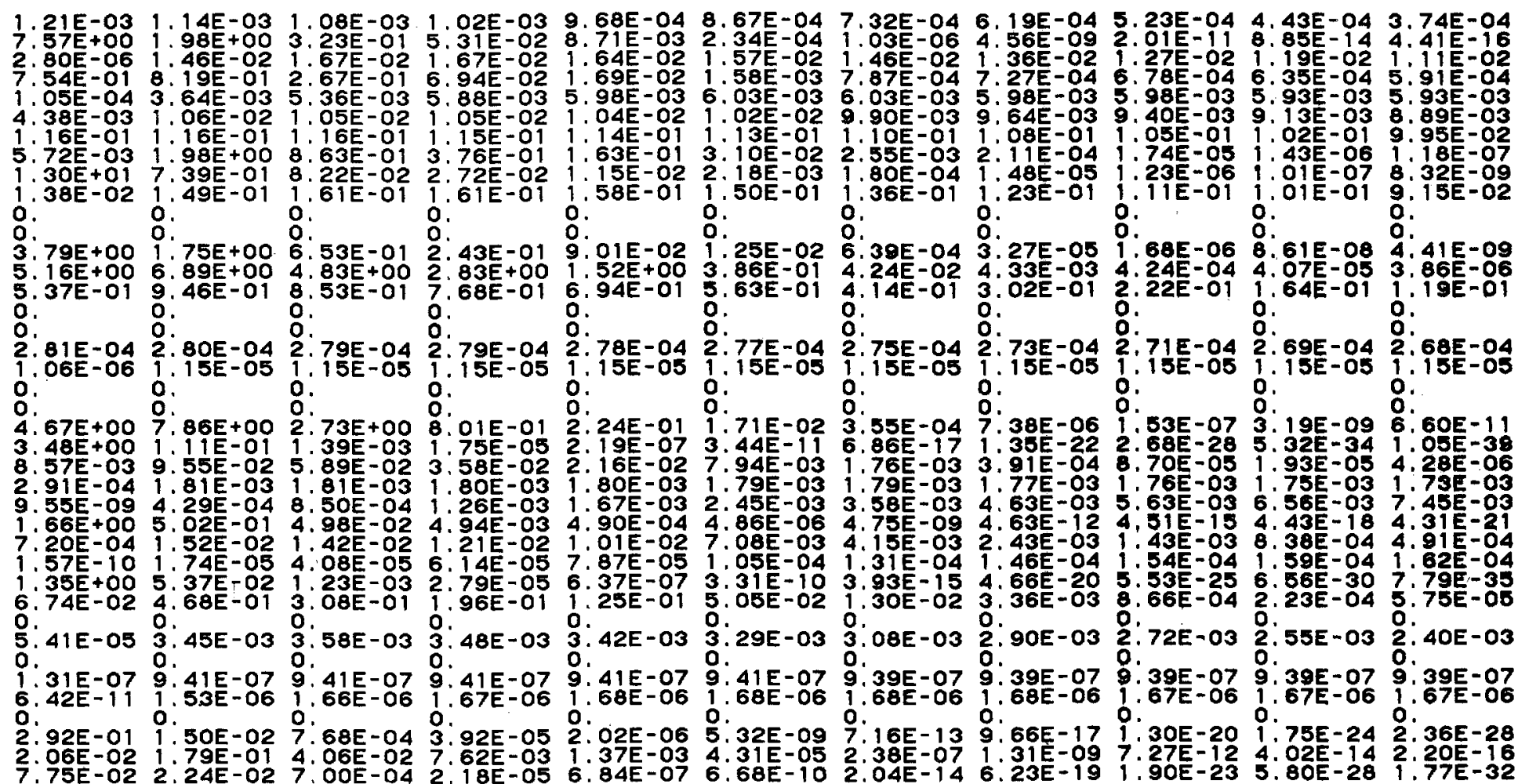

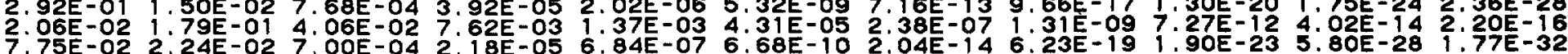


SMOKY

MR/HR AT H+12 HOURS $=$ MR/HR

RACTION HF RERACTORIES PRESENT $=0.100$

RELAXATION LENGTH $=0.16$ OM $/$ SOCM $1.657 E-13$

DEBRIS DECAY FROM 1 TO 21 HOURS

ZERO TIME 1. OOE+00 2. OOE+00 3. OOE+00 4.00E+00 6. OOE+00 9. OOE+00 $1.20 E+01 \quad 1.50 E+01 \quad 1.80 E+01 \quad 2.10 E+01$

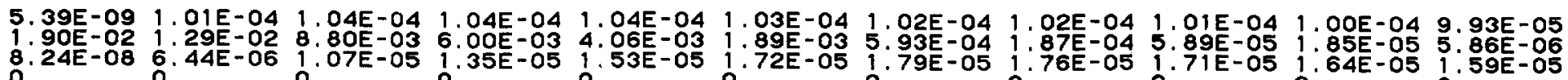

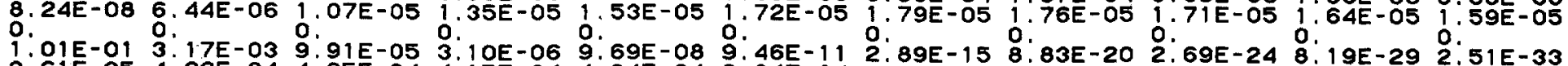
3.61E-05 4.22E-04 4.25E-04 3.10E-06 9.69E-08 $9.46 E-11$ 2.89E-15 8.83E-20 2.69E-24 8. $19 E-29$ 2.51E-33

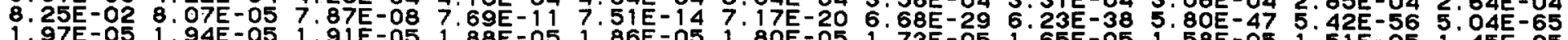

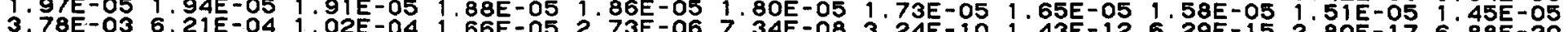

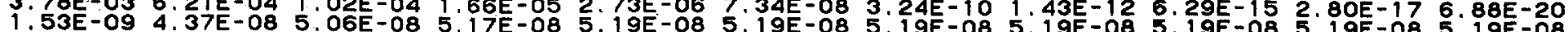

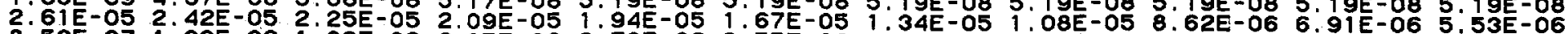
8. $53 E-07$ 1. $39 E-06$ 1.89E-06 $2.35 E-06 \quad 2.78 E-06$ 3.55E-06 4.51E-06 5.22E-06 5.85E-06 6.31E-06 6.64E-06

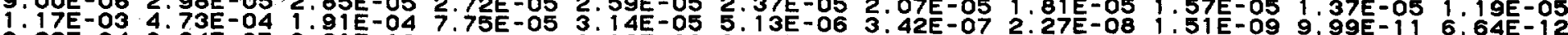

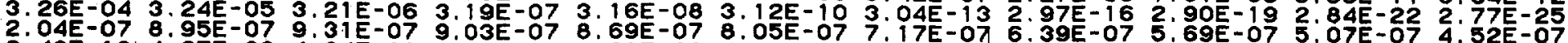
6.49E-10 4.67E-09 4.64E-09 4.62E-09 4.61E-09 4.56E-09 4:51E-09 4.35E-09 4.39E-09 4.07.35-09 4.29E-09

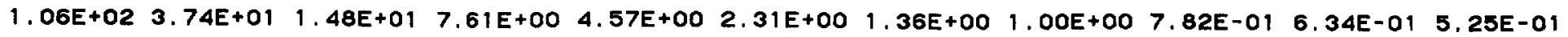




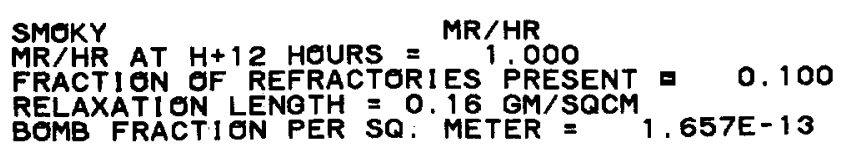

DEBRIS DECAY FROM 1 TO 300 DAYS

ZERO TIME 1. DOE+OO 2. $2.00 E+00 \quad 5.00 E+00 \quad 1.00 E+01 \quad 2.00 E+01 \quad 3.00 E+01 \quad 5.00 E+01 \quad 1.00 E+02 \quad 2.00 E+02 \quad 3.00 E+02$ 
SMOKY AT H+12 HOURS = MR/HR

FRACTION OF REFRACTORIES PRESENT $=0.100$

RELAXATION LENGTH $=0.16$ GM/SQCM $1.657 E-13$

DEBRIS DECAY FROM 1 TO 300 DAYS

ZERO TIME $1.00 E+00 \quad 2.00 E+00 \quad 5.00 E+00 \quad 1.00 E+01 \quad 2.00 E+01 \quad 3.00 E+01 \quad 5.00 E+01 \quad 1.00 E+02 \quad 2.00 E+02 \quad 3.00 E+02$

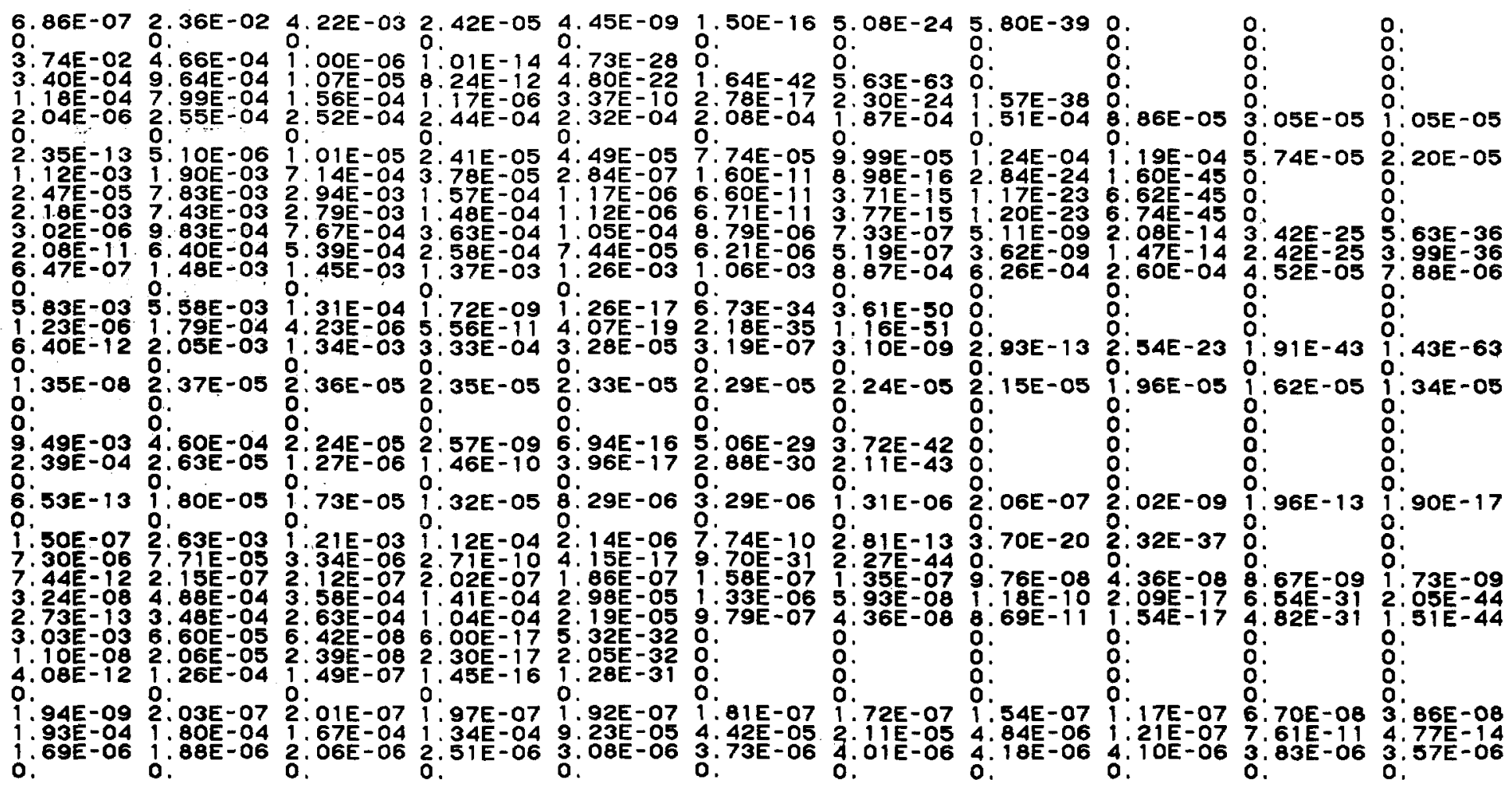


SMOKY AT H+12 HOURS MR/HR

FRACTION OF REFRACTORIES PRESENT = 0.100

RELAXATION LENGTH $=0.16 \mathrm{GM} / \mathrm{SQCM}$ 1.657E-13

DEBRIS DECAY FROM 1 TO 300 DAYS

ZERO TIME $1.00 E+00 \quad 2.00 E+00 \quad 5.00 E+00 \quad 1.00 E+01 \quad 2.00 E+01 \quad 3.00 E+01 \quad 5.00 E+01 \quad 1.00 E+02 \quad 2.00 E+02 \quad 3.00 E+02$

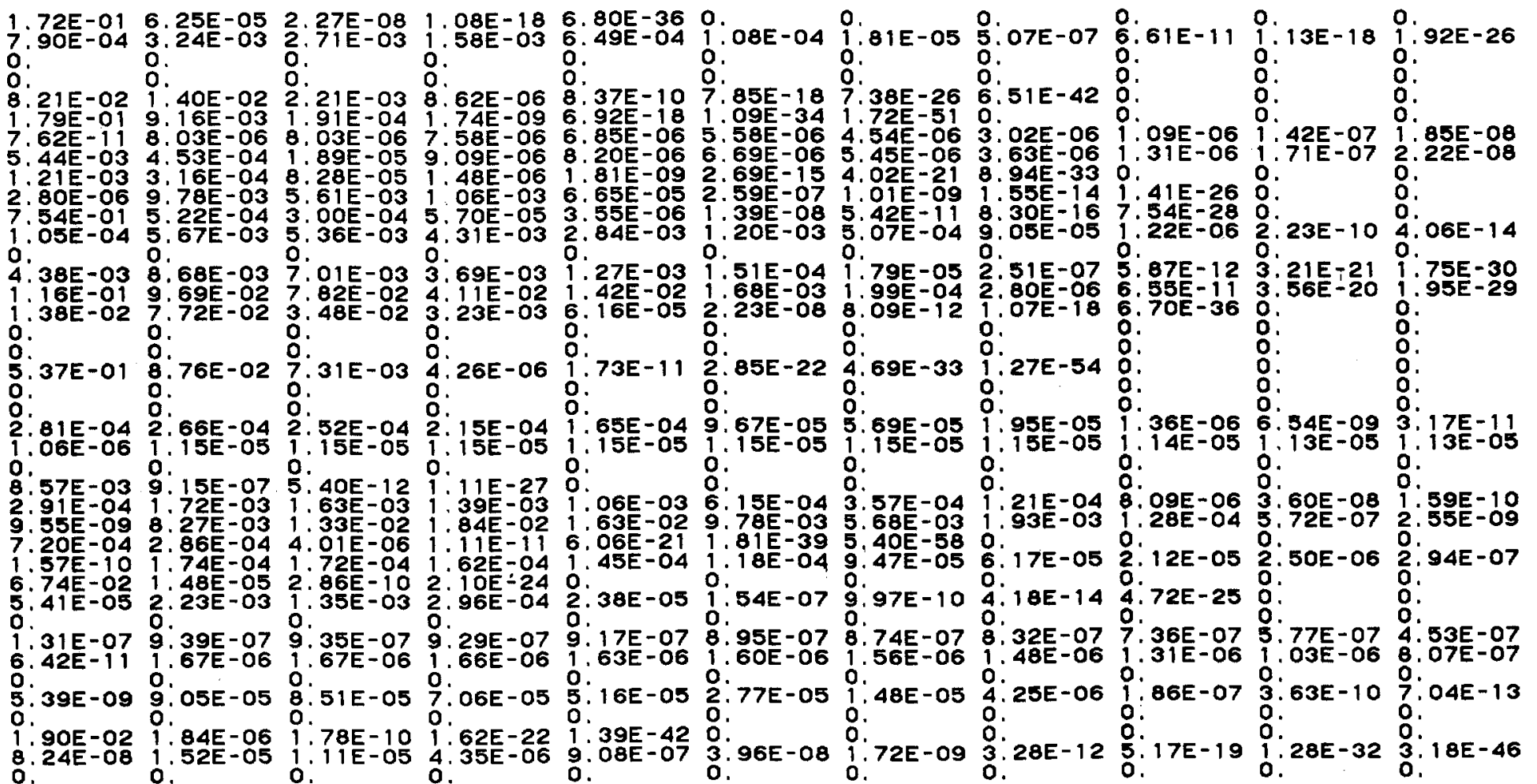


SMEKY AT H+12 HEURS $=$ MR/HR

PAGE $5=29$

MET 0.100

RELAXATION LENGTH $=0.16$ GM/SOCM $1,657 E-13$

DEBRIS DECAY FROM 1 TO 300 DAYS

ZERO TIME $1.00 E+002.00 E+00 \quad 5.00 E+00 \quad 1.00 E+01 \quad 2.00 E+01 \quad 3.00 E+01 \quad 5.00 E+01 \quad 1.00 E+02 \quad 2.00 E+02 \quad 3.00 E+02$

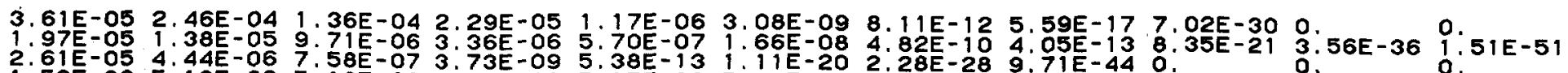

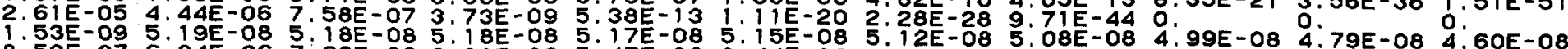

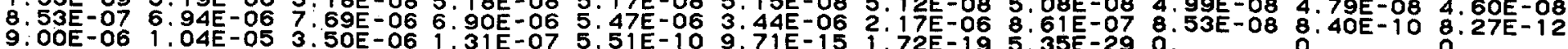
2. $04 \mathrm{E}-07$ 4.02E-07 1.60E-07 9.98E-09 9.83E-11 9.52E-15 $9.23 E-19$ 8.66E-27 7. 39E-47 0.

3. $83 E-22$

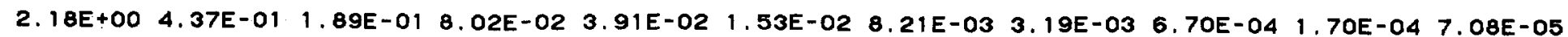


SMOKY MR/HR AT H+12 HOURS $=$ MR/HR

FRACTION OF REFRACTORIES PRESENT $=0.100$

RELAXATION LENGTH $=0.16$ GM/SOCM 1.657 - 13

DEBRIS DECAY FROM 1 TO 50 YEARS

ZERO TIME $1.00 E+00 \quad 1.50 E+00 \quad 2.00 E+00 \quad 3.50 E+00 \quad 5.00 E+00 \quad 7.00 E+00 \quad 1.00 E+01 \quad 2.00 E+01 \quad 3.50 E+01 \quad 5.00 E+01$

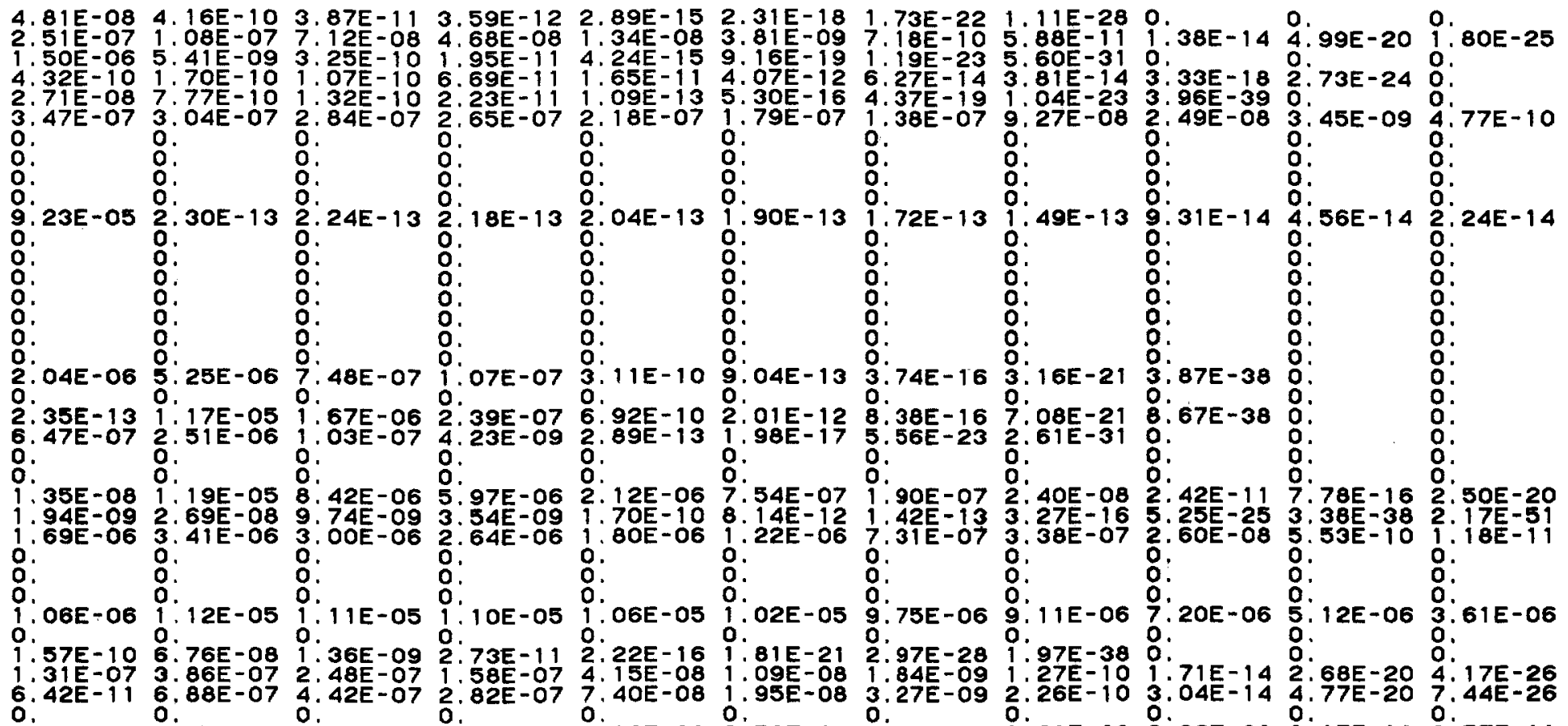

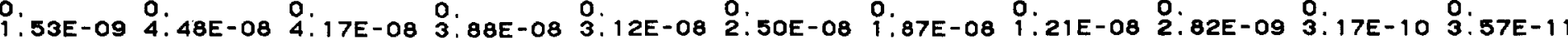

TOTAL

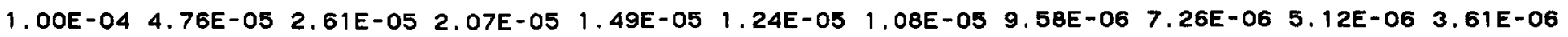




\section{APPENDIX 6}

SMOKY EVENT

Fit To Sum of Eleven Exponentials

The calculated values are fit to the function

$$
\sum_{i=1}^{11} a_{j} e^{-\lambda_{j} t}
$$

In the first table the $a_{j}$ 's are the variables and are designated by an odd number in the column under " $K$ "; the $i$ 's are fixed parameters so designated. The quality of fit to each point is shown in the second table.

$\begin{array}{ll}\text { Fraction refractories present }=1.0 & \frac{\text { Page }}{6-1} \\ \text { Fraction refractories present }=0.5 & 6-3 \\ \text { Fraction refractories present }=0.1 & 6-5\end{array}$


SMOKY

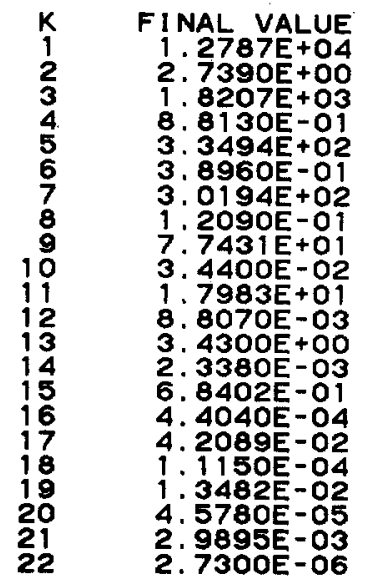

STND DEV

‥ $1354 \mathrm{E}+02$ 9. $8406 E+01$ 1. $9321 \mathrm{E}+01$ 4. $4923 E+00$ 8. 5791E-01 $3641 E-01$ 1. $8148 E-02$ . 7205E-03 $6210 E-04$ 3. $9613 E-05$ 0 .
TIME (HOURS) 2. OOOE + OO
3. OOOE+OO
OOOE+ +00
OOOE+OO
$500 E+01$
2. 1OOE + 1
$80 \mathrm{E}+01$
1. $200 E+02$
2. $400 \mathrm{E}+02$
$200 \mathrm{E}+02$
$200 E+03$
$400 \mathrm{O}+03$
$2 \mathrm{OOE}+03$
$320 E+04$
(3)
$380 E+04$
$140 E+04$
$750 \mathrm{E}+05$
3. $070 \mathrm{O}+05$
4. $380 \mathrm{O}+05$

CI/SO KM, 1.0 REFRACTORIES PRESENT

PAGE
FIXED PARAMETER

FIXED PARAMETER

FIXED PARAMETER

FIXED PARAMETER

FIXED PARAMETER

FIXED PARAMETER

FIXED PARAMETER

FIXED PARAMETER

FIXED PARAMETER

FIXED PARAMETER

FIXED PARAMETER
PERCENT

DEVIATION

-0.09
0.62

$-0.84$

-0.12

$-0.30$

$-0.21$

-0.23
0.52

-0.09

$-0.45$

$-1.50$

$-1.45$

3. 37

4.20

$-3.48$

$-1.54$

2.70

$-1.61$

1.07

8.

-0.62
-1.78
-2.13 
FIXED PARAMETER

FIXED PARAMETER

1. $1017 \mathrm{E}+00$

1. $6737 \mathrm{E}-01$

3. $6722 E-02$

6. $8870 \mathrm{E}-03$

1. 0543E-03

9. $4189 E-05$

6. 7005E-06

1.8761E-06

1. $2140 E-07$

FIXED PARAMETER

FIXED PARAMETER

FIXED PARAMETER

FIXED PARAMETER

FIXED PARAMETER

FIXED PARAMETER

FIXED PARAMETER

FIXED PARAMETER

FIXED PARAMETER
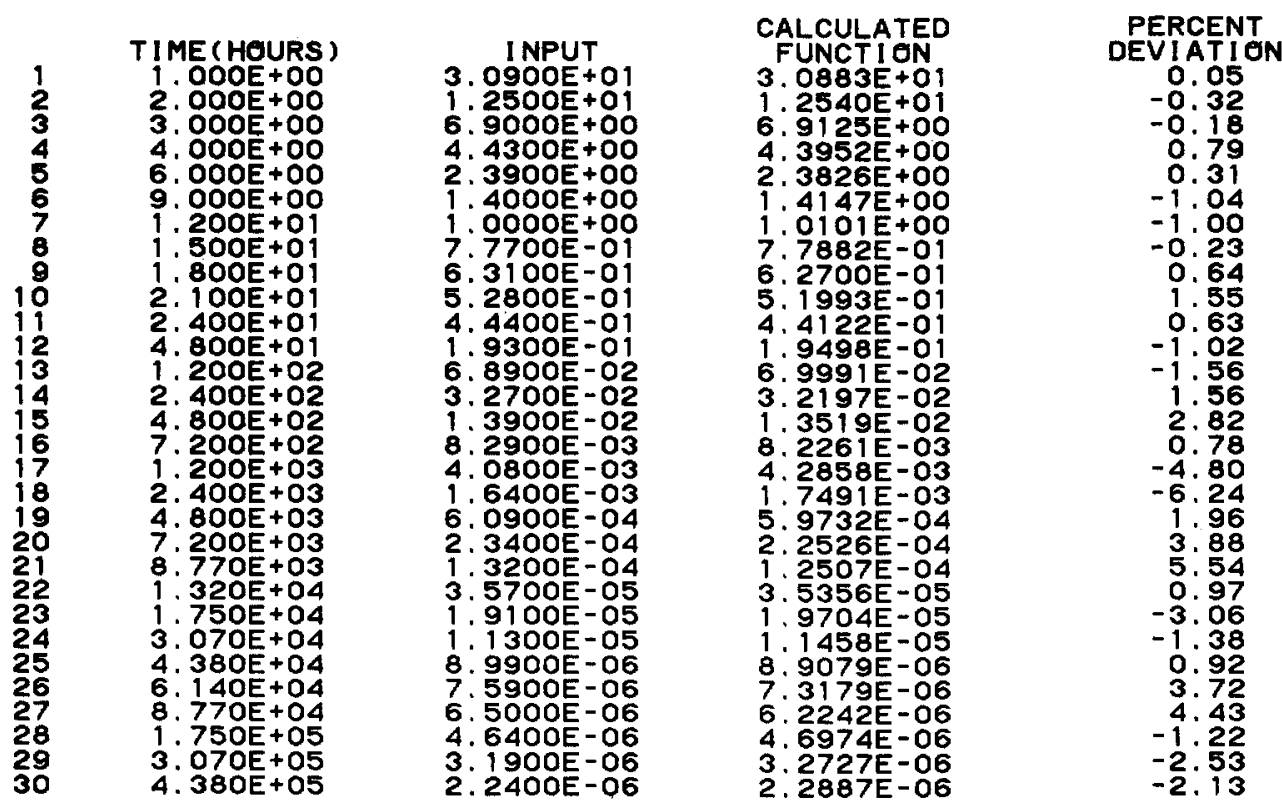
SMOKY

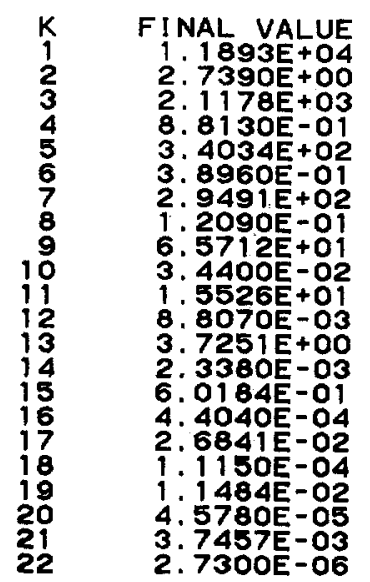

STND. DEV. 4. $0687 \mathrm{E}+02$

1. $2348 \mathrm{E}+02$

$3168 E+01$

5623E+00

$0524 E+00$

1. $6965 \mathrm{E}-01$

2. $1101 E-02$

o. $3425 E-03$

.0431E-03

6. $4566 \mathrm{E}-05$

:.

CI/SQ KM, 0.5 REFRACTORIES PRESENT

PAGE

FIXED PARAMETER

FIXED PARAMETER

FIXED PARAMETER

FIXED PARAMETER

FIXED PARAMETER

FIXED PARAMETER

FIXED PARAMETER

FIXED PARAMETER

FIXED PARAMETER

FIXED PARAMETER

FIXED PARAMETER

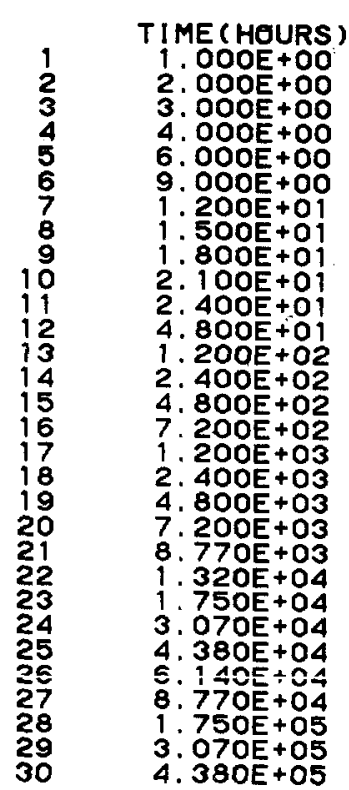

\begin{tabular}{|c|c|}
\hline 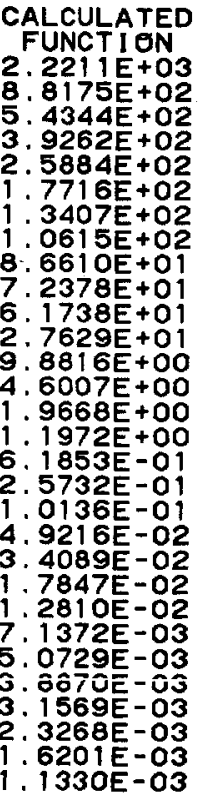 & $\begin{array}{c}\text { PERCENT } \\
\text { DEVIATION } \\
-0.05 \\
0.37 \\
-0.63 \\
0.10 \\
0.83 \\
-0.66 \\
-0.80 \\
-0.14 \\
0.10 \\
1.14 \\
0.59 \\
-1.19 \\
1.10 \\
-0.23 \\
-1.87 \\
-1.44 \\
4.77 \\
8.04 \\
-4.21 \\
-5.72 \\
-2.61 \\
4.78 \\
4.61 \\
-1.50 \\
-3.01 \\
-0.10 \\
2.95 \\
1.00 \\
-0.63 \\
-1.15\end{array}$ \\
\hline
\end{tabular}

$.1330 E-03$

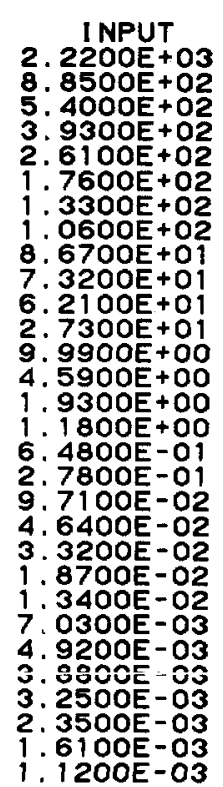


SMOKY

CI/SO KM, O.1 REFRACTORIES PRESENT

PAGE

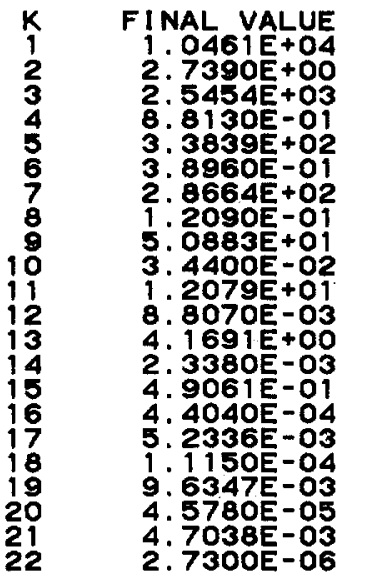

STND. DEV

6. $5219 E+02$

$1.8876 E+02$

3. $3117 E+01$

7. $6065 E+00$

1. $5556 \mathrm{E}+00$

2. $5699 \mathrm{E}-01$

2. $8208 E-02$

$48815 \mathrm{E}-03$

$1: 7415 E-03$

1. $3156 \mathrm{E}-04$

FIXED PARAMETER

FIXED PARAMETER

FIXED PARAMETER

FIXED PARAMETER

FIXED PARAMETER

FIXED PARAMETER

FIXED PARAMETER

FIXED PARAMETER

FIXED PARAMETER

FIXED PARAMETER

FIXED PARAMETER

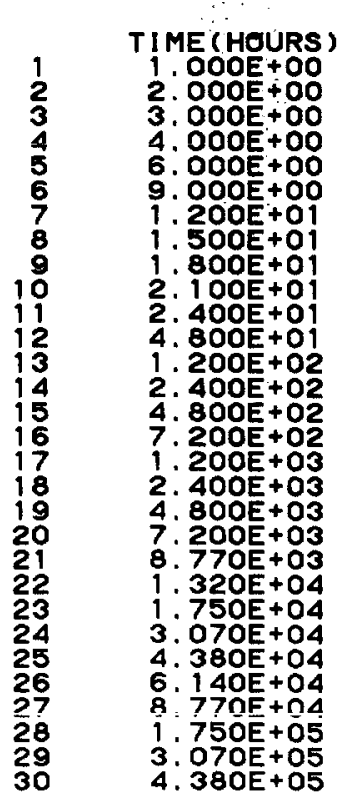

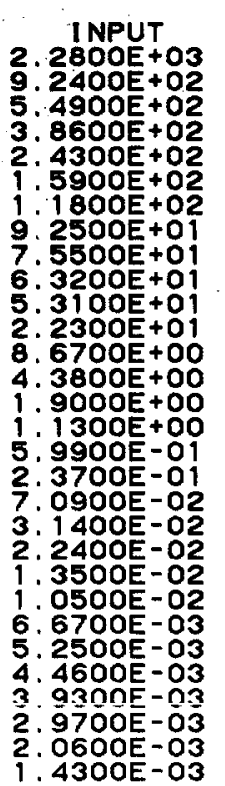

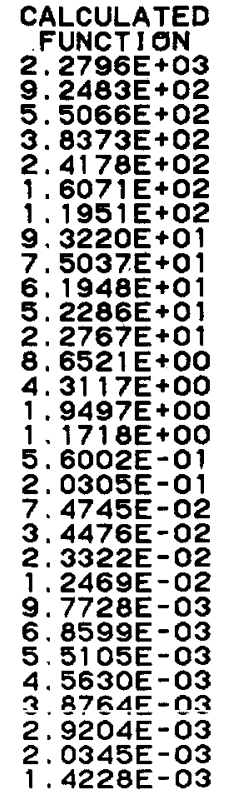

PERCENT
DEVIATION
0.02
-0.09
-0.30
0.59
0.50
-1.06
-1.26
-0.77
0.62
2.02
1.56
-2.05
0.21
1.58
-2.55
-3.57
6.96
16.72
-5.14
-8.92
-3.95
8.27
7.44
-2.77
-4.73
-2.26
1.38
1.70
1.25
0.51


PAOE

1
SMOKY

STND 3.63 $19 E+0$ i

5. $6574 E+00$

1. $3774 E+00$

2.0619E-01

4. $6227 E-02$

9. $2635 E-03$

$1.1691 \mathrm{E}-03$

0. 0204 E-05

7. $9412 \mathrm{E}-06$

2. 8689E-06

2. $2898 \mathrm{E}-07$

1. 1709 -O5

2.

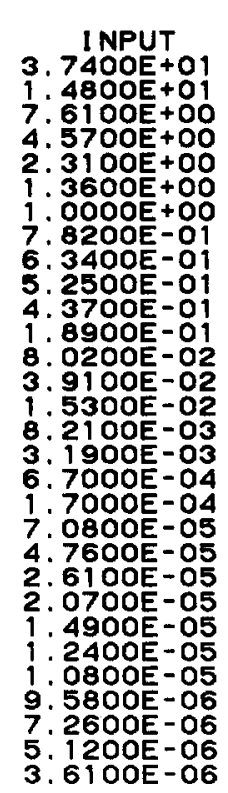

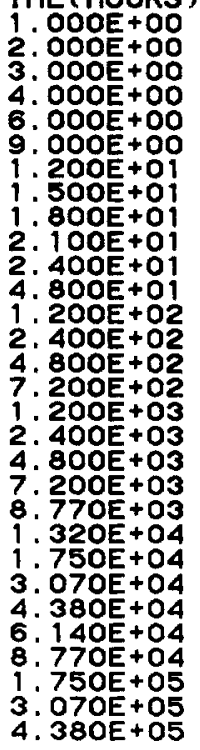

3. $6100 \mathrm{E}-06$
MR/HR, 0.1 REFRACTORIES PRESENT $0.16 \mathrm{GM} / \mathrm{SQCM}$
FIXED PARAMETER

FIXED PARAMETER

FIXED PARAMETER

FIXED PARAMETER

FIXED PARAMETER

FIXED PARAMETER

FIXED PARAMETER

FIXED PARAMETER

FIXED PARAMETER

FIXED PARAMETER

FIXED PARAMETER

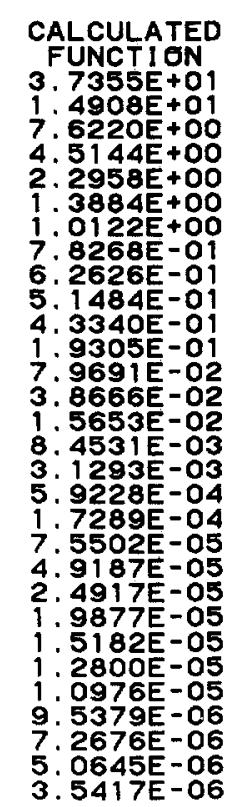

5. $0645 \mathrm{E}-06$

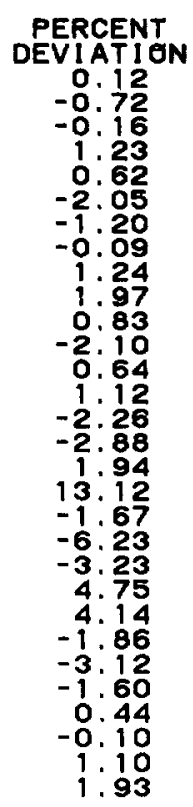




\section{APPENDIX 7}

ANNIE EVENT

Microcuries per Square Meter

Fraction of refractories present $=1.0$

$\underline{\text { Page }}$

Total $\mathrm{mR} / \mathrm{hr}$ and Total $\mu \mathrm{Ci} / \mathrm{m}^{2}$

$7-1$

Individual nuclide $\mu \mathrm{Ci} / \mathrm{m}^{2}$

$7-2$

Fraction of refractories present $=0.5$

Total $\mathrm{mR} / \mathrm{hr}$ and Total $\mu \mathrm{Ci} / \mathrm{m}^{2}$

Individual nuclide $\mu \mathrm{Ci} / \mathrm{m}^{2}$

Fraction of refractories present $=0.1$

Total $\mathrm{mR} / \mathrm{hr}$ and Total $\mu \mathrm{Ci} / \mathrm{m}^{2}$

Individual nuclide $\mu \mathrm{Ci} / \mathrm{m}^{2}$ 
ANNIE

MR/HR AT $H+12$ HOURS $=1,000$

FRACTION OF REFRACTORIES PRESENT $=1.000$

RELAXATION LENGTH $=0.16$ GM $/$ SOCM $2.873 E-13$

TIME

ZERO TIME HOURS

$1.00 E+O 0$

2. OOE+OO

3. OOE +00

OOE +00

$9 . \mathrm{OOE}+\mathrm{OO}$

$20 \mathrm{E}+\mathrm{O}$

$5 \mathrm{OE}+\mathrm{O}$

(1)

, 00 + +0 DAYS

2. 00 + +00

$\mathrm{OOE}+\infty \mathrm{O}$

. OOE+O 1

OOE+OI

. OOE+d

$.00 E+02$

2. OOE+

$.00 E+O O$ YEARS

. 50 +OO

$50 \mathrm{~T}+00$

. OOE +OO

$.00 \mathrm{E}+00$

1. OOE+O1

3. $50 \mathrm{E}+\mathrm{a}$

5. OOE+OI
MR/HR

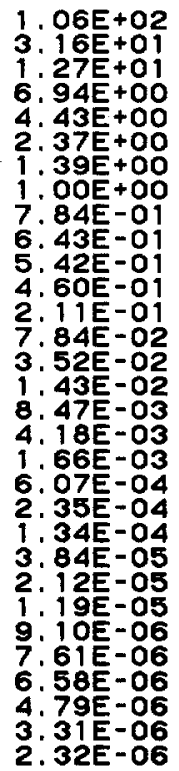

MICROCURIES/SO METER

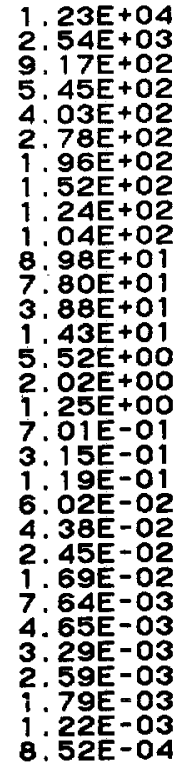


ANNIE AT H+12 HबURS = MICROCURIES/SQ METER

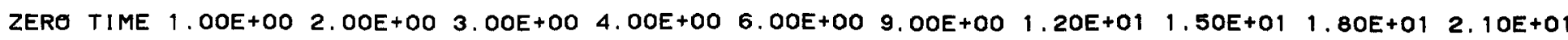

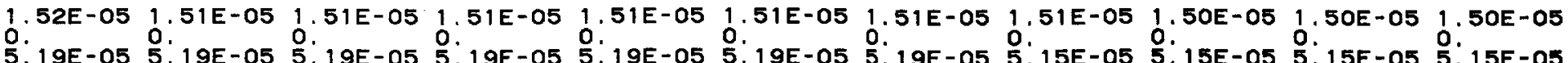

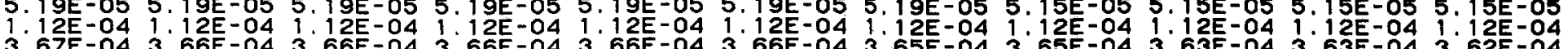

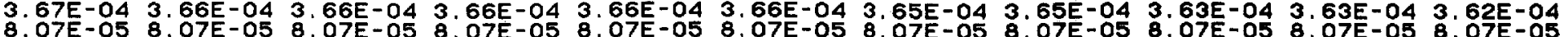
8.07 $7.13 E-04$ 7.13E-04 7:13E-04 7:13E-04 7.11E-0 $5.66 \mathrm{E}-05$ 5. $66 \mathrm{E}-05$ 5. $06 \mathrm{E}-05$ 5.66E-05 5.1 :

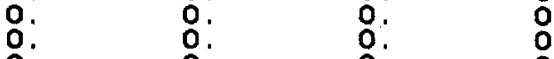

$\begin{array}{lll}0 . & 0 & 0 \\ 0 . & 0 & 0 \\ 0 & 0 & 0\end{array}$

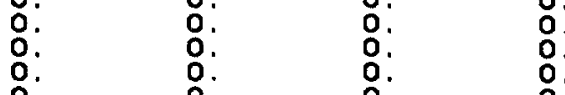

66E-01 $1.66 E-01$. $65 \mathrm{E}-01$

(65E-01 $34 E+035.70 \mathrm{E}+02,9.74 \mathrm{E}+01$

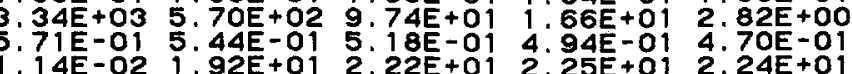
:

.65-05

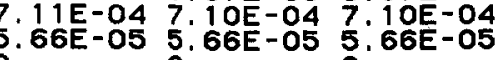

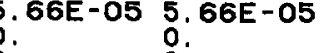

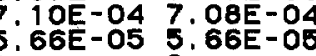
$7: 08 E-04$
$7: 07 \bar{E}-04$
$0.66-05$
0 . .74E-09 9:74E-09 9:74E-09 $9: 74$ E - 09 9:74E-09 $6.10 \mathrm{E}-06$ 2.96E-02 1.78E-02 $1.07 \mathrm{E}-02$ 6.45E-03

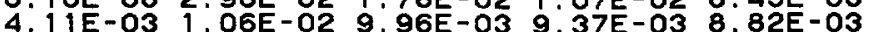

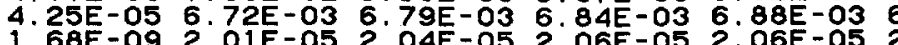

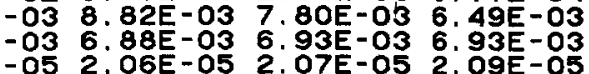

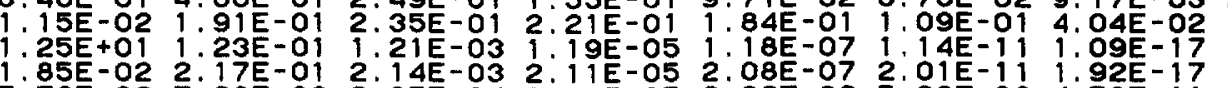

$7.72 E-02$ 7.28E-03 6.85E-04 6. 44E-05 6.06E-06 5.38E-08 4:50E-11 3 $\begin{array}{ll} & \end{array}$ 3.79E-04 3.72E-04 3.65E-04 3.58E-04 3.51E-04 3.37E-04 3.18E-04 3.00E-04 2.83E-04 2.66E-04 2.51E-04

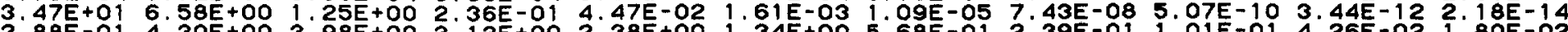

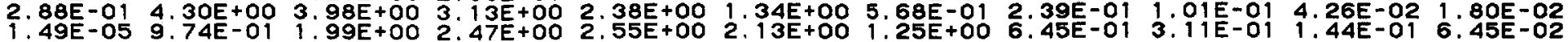


ANNIE MICROCURIES/SQ METER

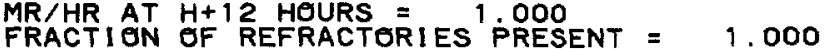
RELAXATION LENGTH $=0.16$ GM/SQCM
BOMB FRACTION PER SQ. METER $=2.873 E-13$

DEBRIS DECAY FROM 1 TO 21 HOURS

ZERO TIME 1. OOE+OO 2. OOE+OO 3. OOE+OO 4. $4.00 E+00 \quad 6.00 E+00 \quad 9.00 E+00 \quad 1.20 E+01 \quad 1.50 E+01 \quad 1.80 E+012.10 E+01$

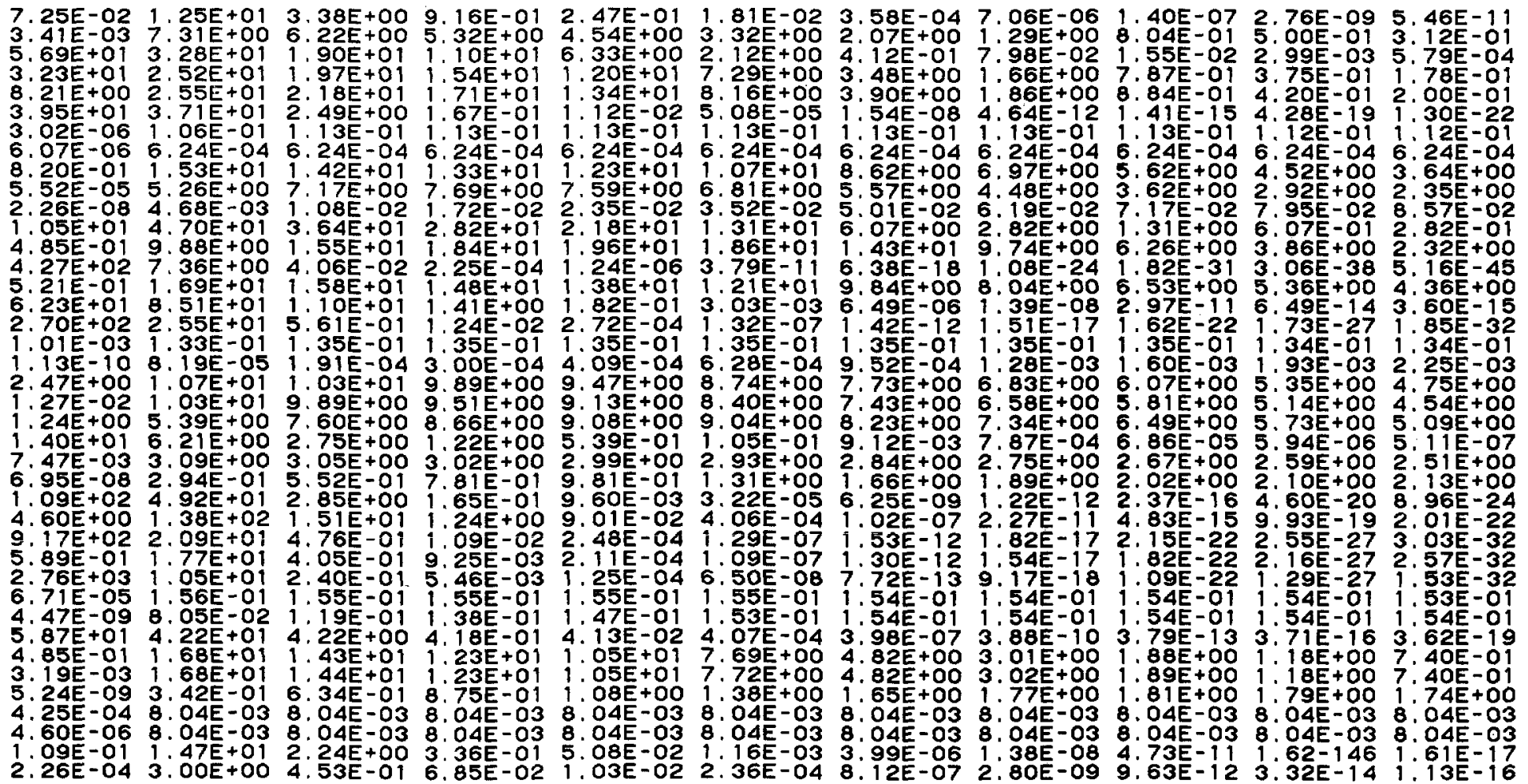


DEBRIS DECAY FROM 1 TO 21 HOURS

ZERO TIME 1. OOE+OO 2. $2.00 E+00 \quad 3.00 E+00 \quad 4.00 E+00 \quad 6.00 E+00 \quad 9.00 E+00 \quad 1.20 E+01 \quad 1.50 E+01 \quad 1.80 E+01 \quad 2.10 E+01$

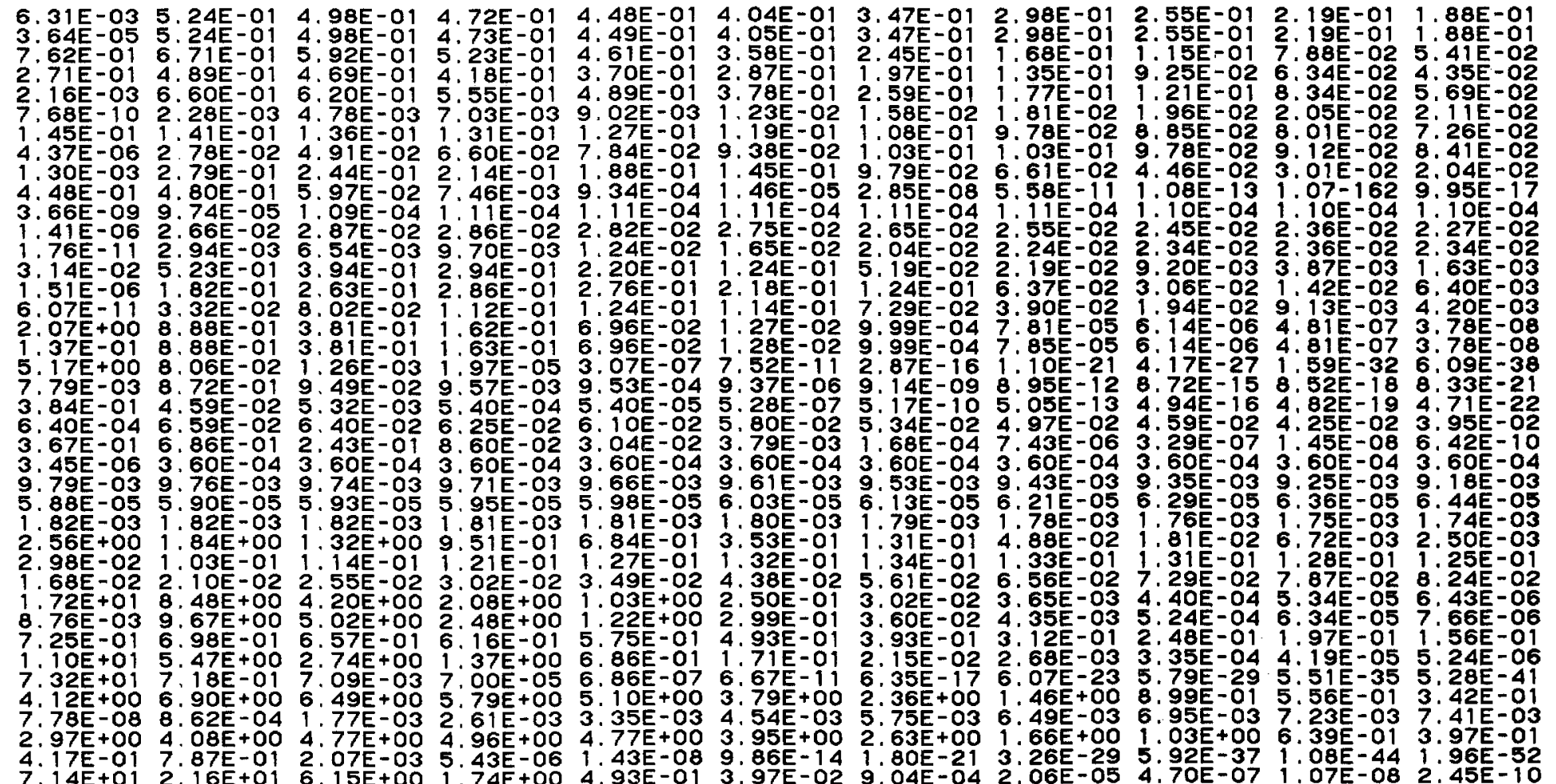




\section{ANNIE}

MICROCURIES/SO METER

PAGE

FRACTION OF REFRACTORIES PRESENT a 1.000 RELAXATION LENGTH $=0$. 16 GM/SQCM $2.873 E-13$

DEBRIS DECAY FROM 1 TO 21 HOURS

ZERO TIME 1. OOE+OO $2.00 E+00 \quad 3.00 E+00 \quad 4.00 E+00 \quad 6.00 E+00 \quad 9.00 E+00 \quad 1.20 E+01 \quad 1.50 E+01 \quad 1.80 E+01 \quad 2.10 E+01$ $\begin{array}{llllllllllll}1.99 E-02 & 1.88 E-02 & 1.78 E-02 & 1.68 E-02 & 1.59 E-02 & 1.42 E-02 & 1.20 E-02 & 1.02 E-02 & 8.59 E-03 & 7.28 E-03 & 6.15 E-03 \\ 1.65 E+02 & 4.31 E+01 & 7.04 E+00 & 1.016 E+00 & 1.90 E-01 & 5.11 E-03 & 2.25 E-05 & 9.94 E-08 & 4.37 E-10 & 1.93 E-12 & 9.62 E-1\end{array}$

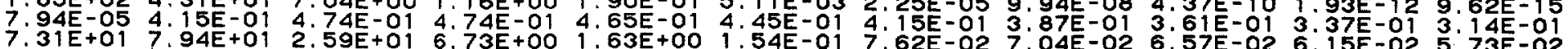
$1.06 E-023.68 E-01$ 5.41E-01 5.73E+0 1.63E-00 1.54E-01 7.62E-02 7.04E-02 6.57E-02 6. $15 E-02$ 5.73E-02

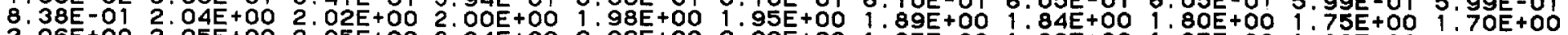
2. $06 E+002.05 E+002.05 E+002.04 E+002.02 E+002.00 E+001.95 E+001.90 E+00 \quad 1.85 E+00 \quad 1.80 E+00 \quad 1.76 E+00$ . $01 E-012.51 E+011.53 E+016.66 E+002.90 E+005.50 E-014.53 E-023.74 E-03 \quad 3.08 E-042.54 E-052.09 E-06$

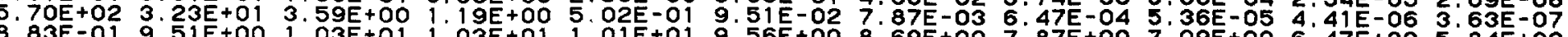

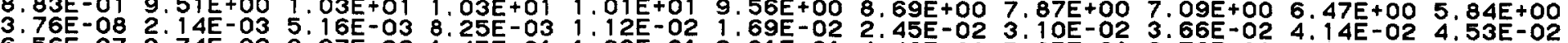
$6.56 E-073.74 E-02$ 9.07E-02 1.45E-01 1:99E-01 3.01E-01 4.42E-01 5.65E-01 6.76E-01 7.72E-01 8.59E-01

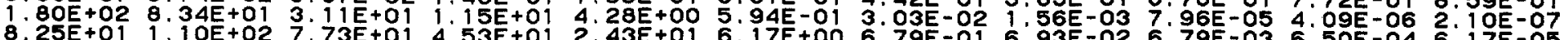

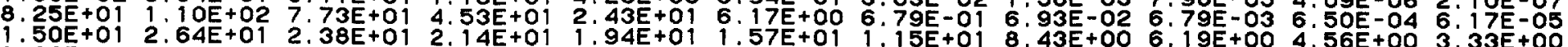
$.66 E-037.61 E+007.40 E+006.71 E+006.02 E+004.90 E+0$ $1.72 E+003.41 E+00 \quad 4.99 E+00 \quad 6.28 E+00 \quad 7.31 E+00 \quad 8.77 E+00 \quad 9.72 E+00$ 9.76E+00 9.29E+00 $8.47 E+007.57 E+00$

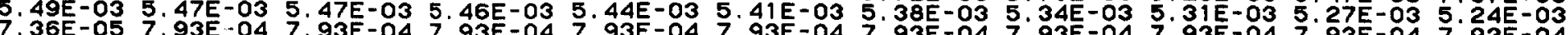

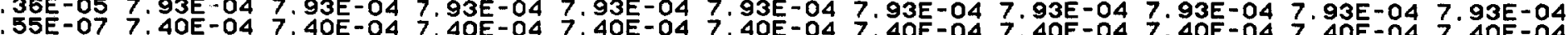

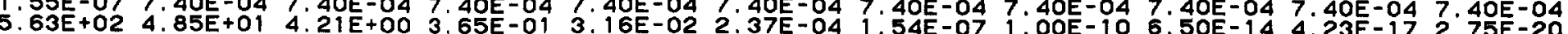
$8.38 E+011.41 E+02 \quad 4.89 E+01 \quad 1.44 E+01 \quad 4.03 E+003.06 E-016.37 E-03 \quad 1.32 E-042.75 E-065.72 E-081.18 E-20$

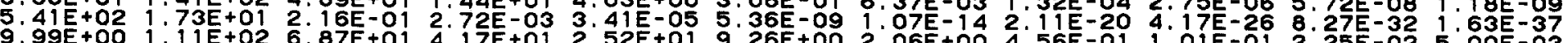

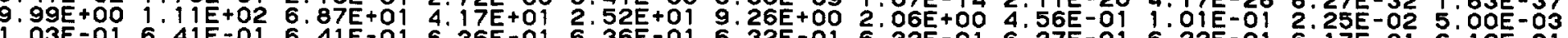

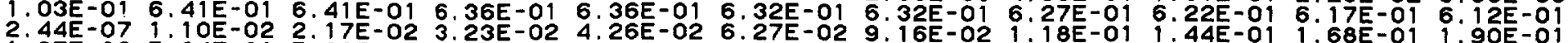

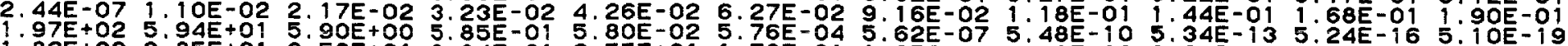

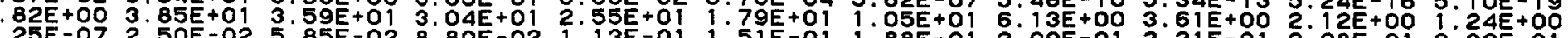
2.25E-07 2.50E-02 5.85E-02 8.80E-02 1. 13E-01 1.51E-01 1.88E-01 2.09E-01 2.21E-01 2.28E-01 $2.32 \mathrm{EE}-01$

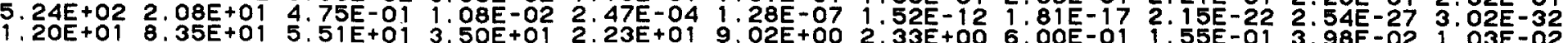
$.97 E+023.84 E+011.97 E+001.01 E-015.20 E-031.36 E-051.83 E-092.47 E-13 \quad 3.33 E-174.50 E-216.07 E-25$ 7.86E-02 5.01E+00 5.20E+00 5. $56 E+004.97 E+00$ 4.78E+00 $4.48 E+00$ 4.21E+00 $3.95 E+003.71 E+003.48 E+00$ 3.14E-03 2.65E-02 $.85 E-02$ 2.93E-02 3.98E-02 6.02E-02 8.88E-02 1.16E-01 $1.41 E-011.64 E-011.86 E-01$ $8.62 \mathrm{E}-072.05 \mathrm{E}-02$ 2.23E-02 2.25E-02 2.25E-02 2.25E-02 2.25E-02 2.25E-02 $2.25 \mathrm{E}-022.25 \mathrm{E}-022.25 \mathrm{E}-02$

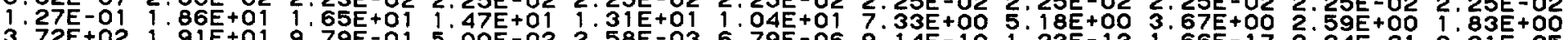
$3.72 E+02$ 1.91E+01 9.79E-01 5.00E-02 2.58E-03 6.79E-06 9.14E-10 1.23E-13 1.66E-17 2.24E-21 3.01E-25

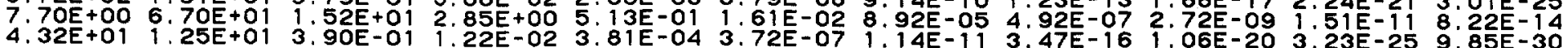




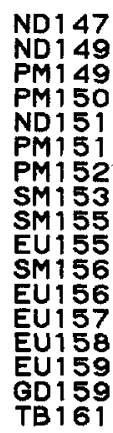

TOTAL
ANNIE MT $H+12$ HOURS = MI CROCURIES/SQ METER

ANNIE AT H+12 HOURS = MICROCURIES/SQ METER

FRACT I ON OF REFRACTORI ES PRESENT $=1.000$

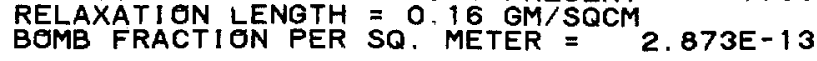

DEBRIS DECAY FROM 1 TO 21 HOURS

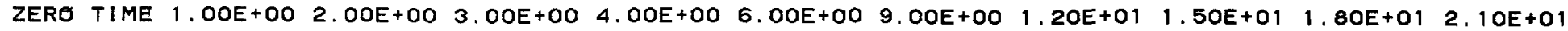

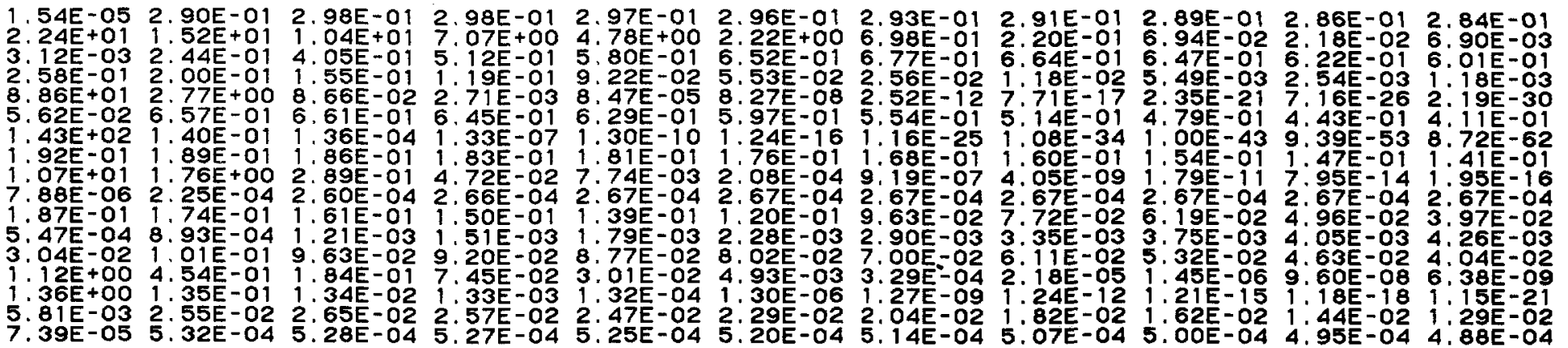

$\begin{array}{llllllll}1.23 E+04 & 2.54 E+03 \quad 9.17 E+02 & 5.45 E+02 & 4.03 E+02 & 2.78 E+02 & 1.96 E+02 & 1.52 E+02 & 1.24 E+02 \quad 1.04 E+02 \quad 8.98 E+01\end{array}$ 
FRACTION OF REFRACTORIES PRESENT $=1.000$ RELAXATION LENGTH $=0.16$ GM GMSSCM $2.873 E-13$

DEBRIS DECAY FROM 1 TO 300 DAYS

ZERO TIME 1. OOE+OO 2. OOE+OO $5.00 E+001.00 E+01 \quad 2.00 E+01 \quad 3.00 E+01 \quad 5.00 E+01 \quad 1.00 E+02 \quad 2.00 E+02 \quad 3.00 E+02$

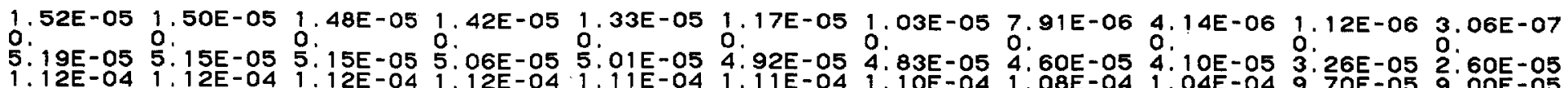
3.67E-04 3.61E-04 3.56E-04 3.40E-04 3.14E-04 2.70E-04 2.31E-04 1.70E-04 7.89E-05 1.68E-05 3.61E-06 8.07E-05 8.07E-05 8.01E-05 8.01E-05 7.89E-05 7.65E-05 7.47E-05 7.11E-05 6. 27E-05 4.85E-05 3.75E-05

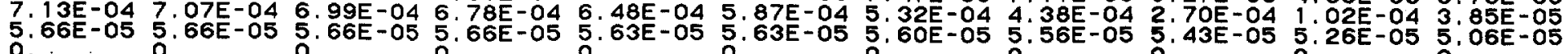

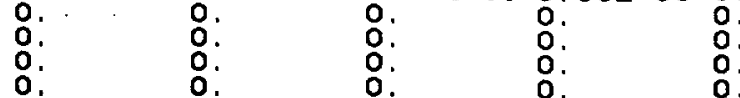

$\begin{array}{llll}0 & 0 & 0 & 0 \\ 0 & 0 & 0 & 0 \\ 0 & 0 & 0 & 0 \\ 0 & 0 & 0 & 0 \\ 0 & 0 & 0 & 0 \\ 0 & 0 & 0 & 0\end{array}$

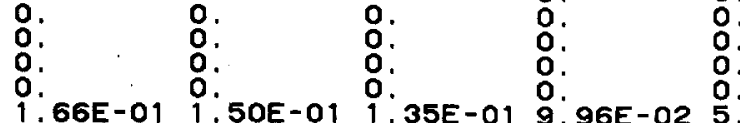

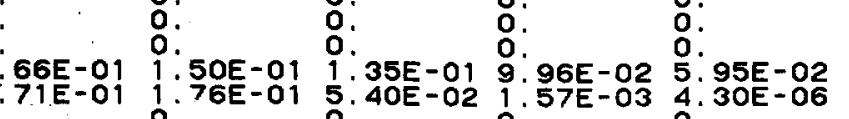

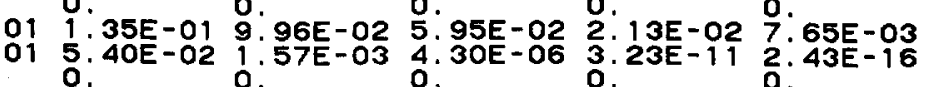

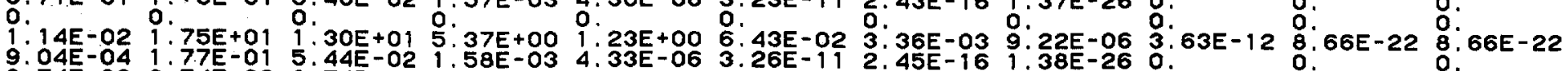

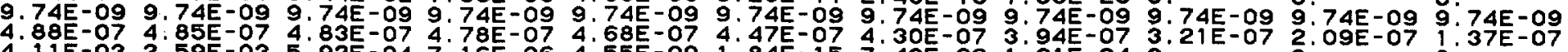
$4.11 \mathrm{E}-032.59$

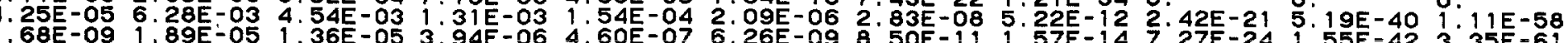
$1.15 E-021.021-04$ 3.00E-09 2.53E-23 0. 3.79E-04 2.37E-04 1:48E-04 3:60E-05 3.41E-06 3.:06E-08 2:.75E-10 2:22E-14 1:30E-24 4. $45 E-45$ 1:51E-65 . $49 \mathrm{E}-05$ 3.32E-02 3.77E-05 3.39E-14 3.48E-29 0

3.41E-03 1.95E-01 4.44E-03 5.27E-08 3.25E-16 1.24E-32 $4.68 E-49$ :. 源 .23E+01 8.5OE-02 $2: 23 E-04$ 4.05E-12 $5: 10 E-25:$ . 9.00E-12 $1.43 E-04$ 2.53E-04 4.54E-04 5.80E-04 6.18E-04 6.24E-04 6.24E-04 $6.18 E-04$ 6. $18 E-04$ G. $13 E-04$ 8.20E-01 2.94E+00 5.26E-01 3.02E-03 5.52E-07 1.87E-14 6. $35 E-22$ 7.22E-37 0. 
ANNIE AT HICROCURIES/SQ METER

FRACTION OF REFRACTORIES PRESENT - 1,000

RELAXATION LENGTH $=0.16$ GM SQCM $2.873 E-13$

DEBRIS DECAY FROM 1 TO 300 DAYS

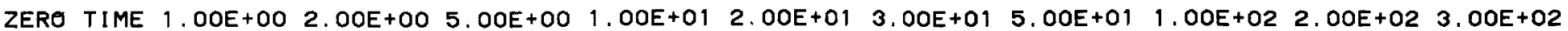

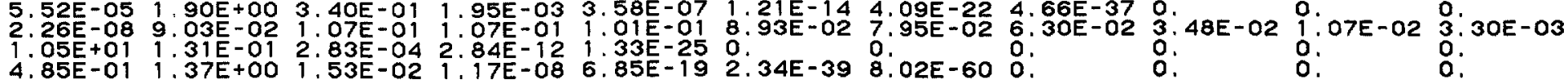

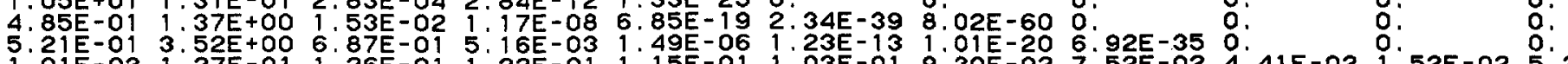

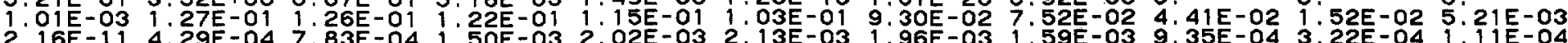
1.13E-10 2.45E-03 4:85E-03 1.16E-02 2.16E-02 3.73E-02 4.81E-02 5:96E-02 5.74E-02 2.76E-02 $1: 06$-02

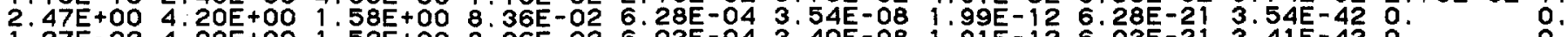

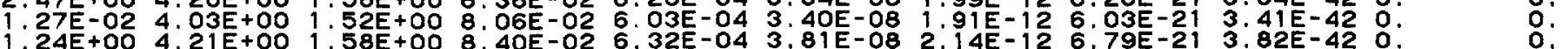

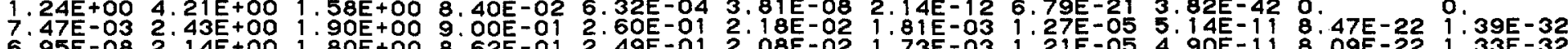

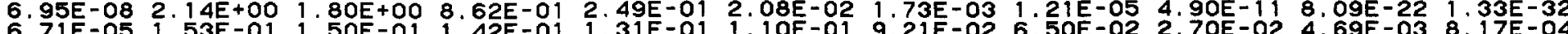

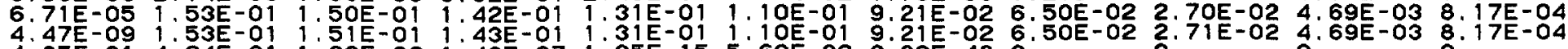

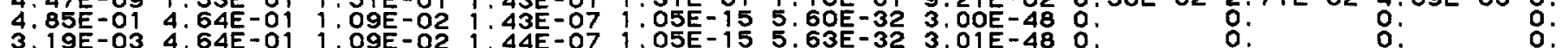

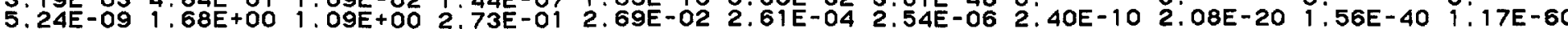

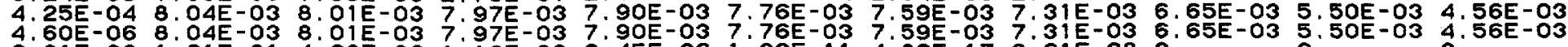
3.64E-05 1.61E-01 4.69E-02 1. 7.62E-01 3.70E-02 1.80E-03 2.06E-07 5.58E-14 4.07E-27 2.99E-40 0.

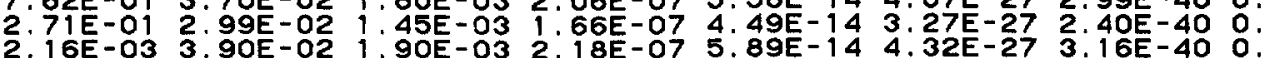
1.

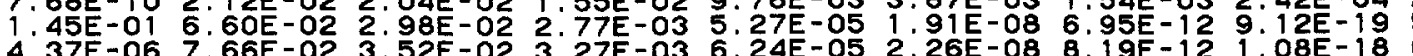
(1.37

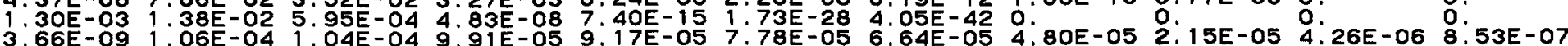

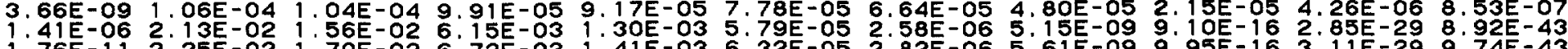
$1.76 \mathrm{E}-11$ 2.25E-02 1.70E-02 6.72E-03 1:41E-03 6.32E-05 2.82E-06 5:61E-09 9:95E-16 3.11E-29 9:74E-43 3.14E-02 6.85E-04 6.66E-07 6.22E-16 $5.52 \mathrm{E}-310$

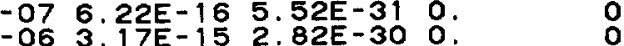
$6.07 E-111.87 E-03 \quad 2.22 E-06 \quad 2.15 E-15$ 1.91E-30 O

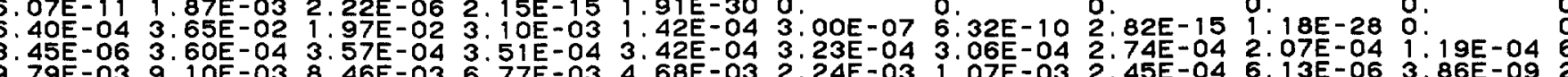

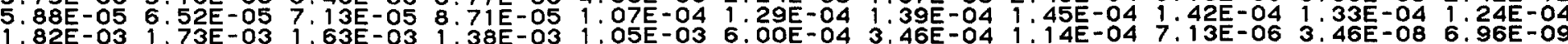


ANNIE

MICROCURIES/SQ METER

FRACTION OF OF REFACTORIES PRESENT $=1.000$

RELAXAT ION LENGTH $=0.16$ GM SQCM
BOMB FRACTION PER SQ. METER $=2.873 E-13$

DEBRIS DECAY FREM I TO 300 DAYS

ZERO TIME 1. OOE+OO $2.00 E+00 \quad 5.00 E+00 \quad 1.00 E+01 \quad 2.00 E+01 \quad 3.00 E+01 \quad 5.00 E+01 \quad 1.00 E+02 \quad 2.00 E+02 \quad 3.00 E+02$

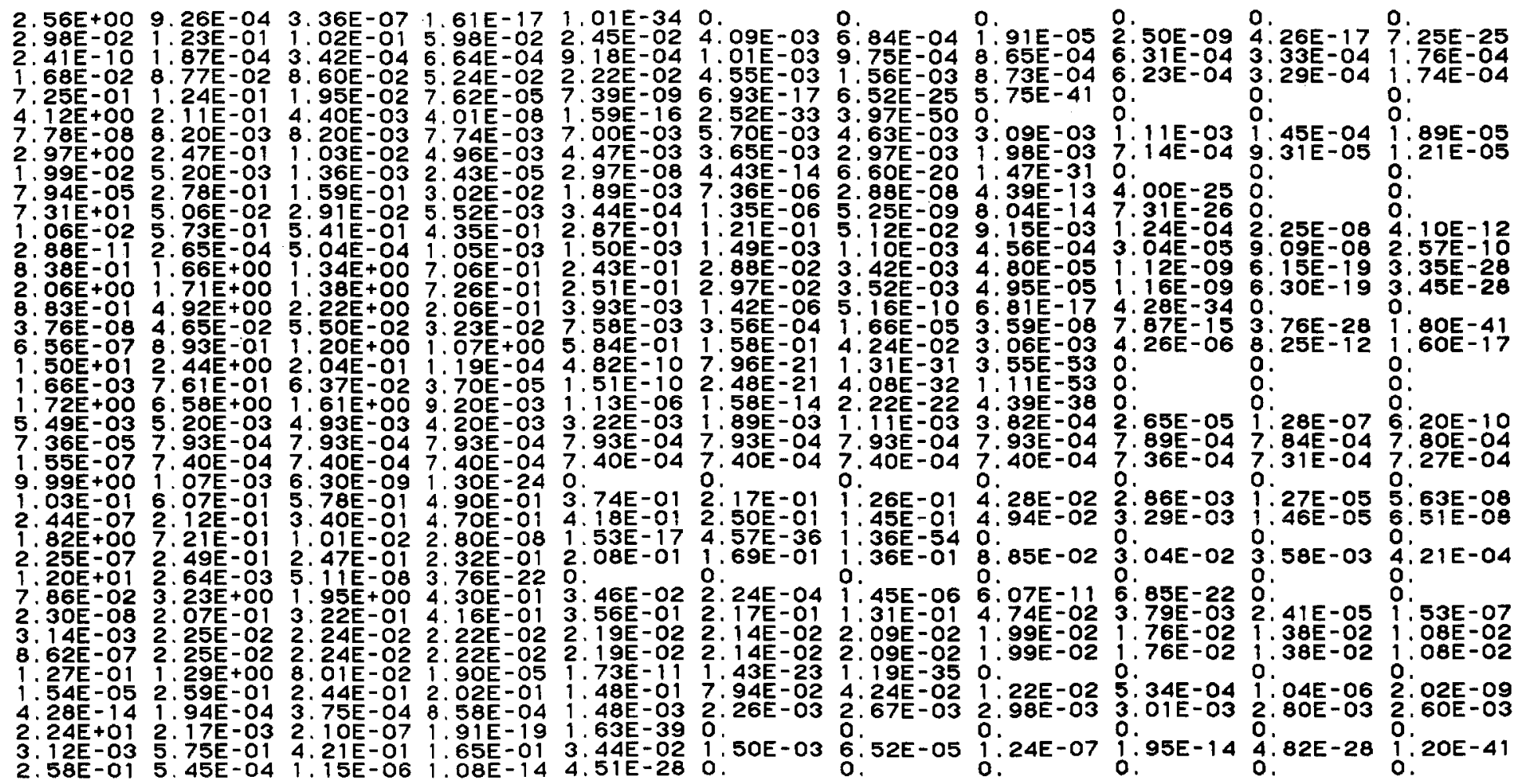


ANNIE AT H+12 HOURS MICROCURIES/SQ METER

MR/HR AT H+12 HOURS $=1$ MICROCURIES/SQ METER RELAXATION LENGTH $=$ O. 16 GM SQCM $2.873 E-13$

DEBRIS DECAY FROM 1 TO 300 DAYS

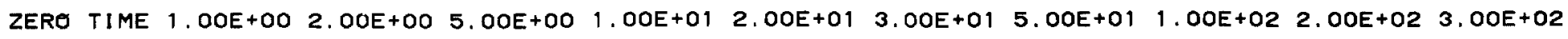

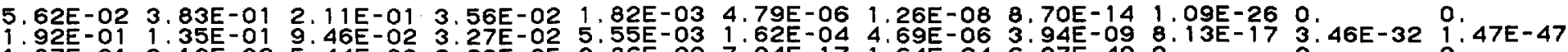

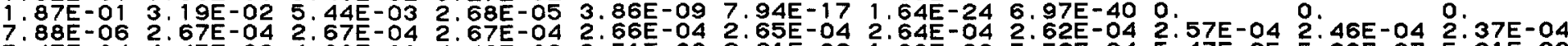

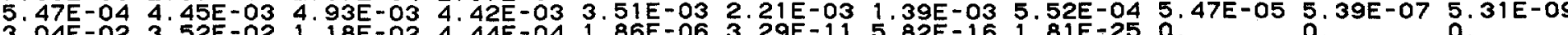

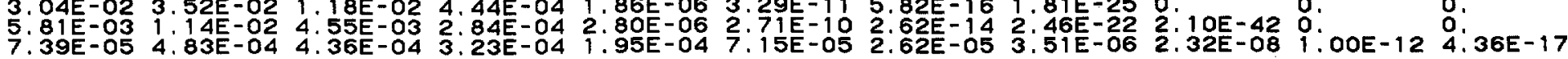

$2.67 E+02 \quad 7.80 E+01 \quad 3.88 E+01 \quad 1.43 E+01 \quad 5.52 E+00 \quad 2.02 E+00 \quad 1.25 E+007.01 E-01 \quad 3.15 E-01 \quad 1.19 E-01 \quad 6.02 E-02$

TOTAL

SM153

Eut 55

ODi59 
ANNIE AT H+12 HOURS MICROCURIES/SQ METER

FRACTION OF REFRACTORIES PRESENT $=1.000$

RELAXATION LENGTH $=0.16$ GMASSOCM $2.873 E-13$

DEBRIS DECAY FROM 1 TO 50 YEARS

ZERO TIME $1.00 E+001.50 E+00 \quad 2.00 E+00 \quad 3.50 E+00 \quad 5.00 E+00 \quad 7.00 E+00 \quad 1.00 E+01 \quad 2.00 E+01 \quad 3.50 E+01 \quad 5.00 E+01$

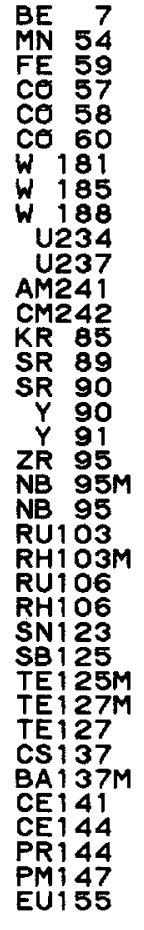

TOTAL

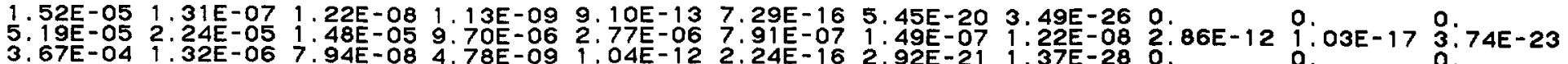
3.07E-05 3.18E-05 1.99E-05 1.25E-05 3.04E-12 2.24E-16 2.92E-21 1.37 E - 28

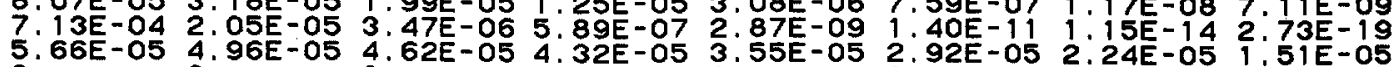

:.

O.: $\quad$ o: $\quad$ o.

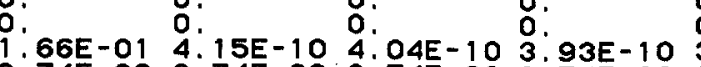

, $93 E-10$ 3. $68 E-10$ o.:

$4.88 E-071$. $03 E-074.75 E-082.19 E-08$ 2. 2 . $3 E-09$

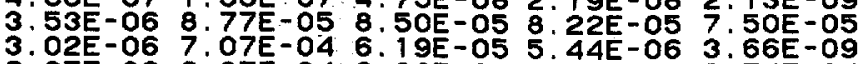

. 06 6. 67 - $04.62 E-045.96 E-04$

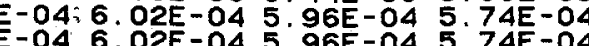

.

01E-03 2.61E-03 $3.72 E-045.30 E-05$ 1 $54 E-07$

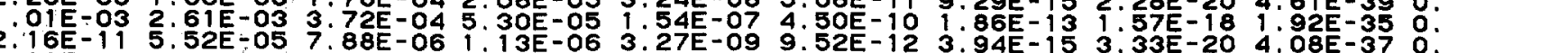

9.52E-12 3.94E- 15 3.33E-20 $4.08 E-37$.

$6.71 E-052.61 E-041.07 E-054.39 E-072.99 E-1122.05 E-15$ 5.77E-21 2.71E-29

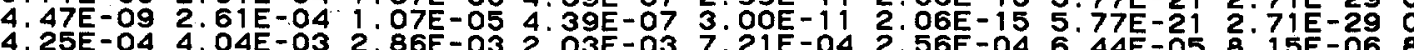

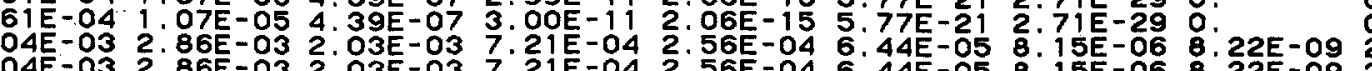

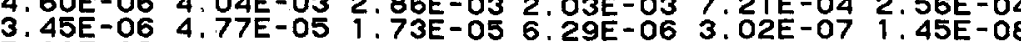

$5.88 E-051.18 E-04$ 1.04E-04 9. $15 E-05$ 6.24E-05 $4.24 E-05$

$1.58 \mathrm{E}-12$ 4.83E-05 $4.29 \mathrm{E}-05$ 3.78E-05 2.58E-05 $1.76 \mathrm{E}-05$

2.41E-10 1.16E-04 3.61E-05 1.13E-05 3.48E-07 1. 07E-08

$1.68 E-02$ 1. $14 \mathrm{E}-04$ 3.57E-05 $1.12 \mathrm{E}-05$ 3. $44 \mathrm{E}-07$ 1. $06 \mathrm{E}-08$

$7.36 \mathrm{E}-057.75 E-047.67 E-047.58 E-047.31 \mathrm{E}-04$

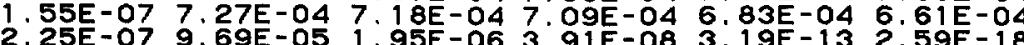

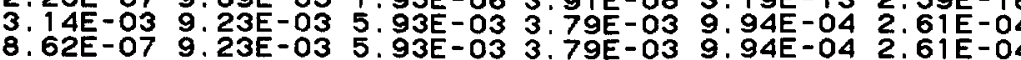

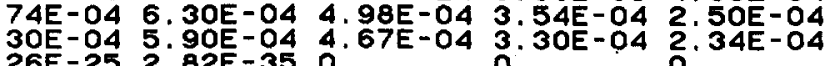

.5E-05 4.86E-06 3.73E-07 7.95E-09 1.69E-10

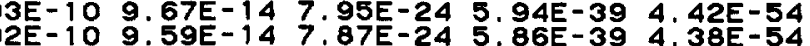
.

.

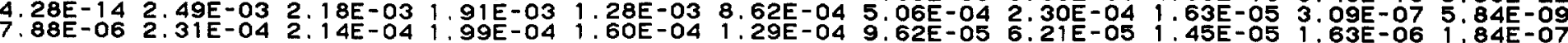

$\begin{array}{lllllllllll}1.89 E-01 & 4.38 E-02 & 2.45 E-02 & 1.69 E-02 & 7.64 E-03 & 4.65 E-03 & 3.29 E-03 & 2.59 E-03 & 1.79 E-03 & 1.22 E-03 & 8.52 E-04\end{array}$ 
ANNIE MRAHR AT H+12 HOURS $=1$ HO 1 OOO

$=0.500$

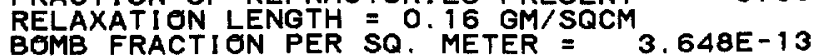

TIME

ZERO TIME HOURS

$1 . \mathrm{OOE}+\mathrm{OO}$

2. OOE +OO

$4.00 \mathrm{E}+\mathrm{OO}$

6. OOE +00

OOE+OO

$20 E+01$

$80 E+01$

OOE +OO DAYS

OOE +OO

OOE+OO

OOE+O

OOE+O

OOE + 01

OOE +02

.

OOE+OO YEARS

. $50 E+00$

DOE+OO

$50 E+00$

. $00 E+00$

OOE+

. $50 \mathrm{OE}+01$
MR/HR

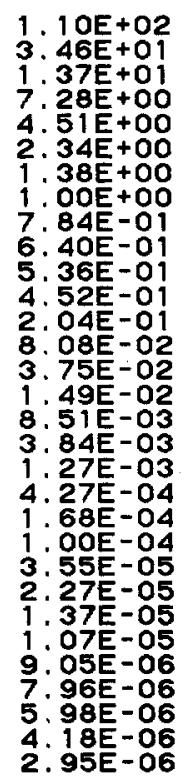

MICRECURIES/SQ METER

1. $20 E+04$
$2.47 E+03$
$9.25 E+02$
$5.47 E+02$
$3.95 E+02$
$2.62 E+02$
$1.80 E+02$
$1.38 E+02$
$1.11 E+02$
$9.28 E+01$
$7.93 E+01$
$6.82 E+01$
$3.25 E+01$
$1.22 E+01$
$5.12 E+00$
$2.00 E+00$
$1.22 E+00$
$6.72 E-01$
$2.88 E-01$
$1.011-01$
$4.99 E-02$
$3.65 E-02$
$2.12 E-02$
$1.52 E-02$
$7.50 E-03$
$4.93 E-03$
$3.73 E-03$
$3.09 E-03$
$2.25 E-03$
$1.55 E-03$
$1.08 E-03$ 


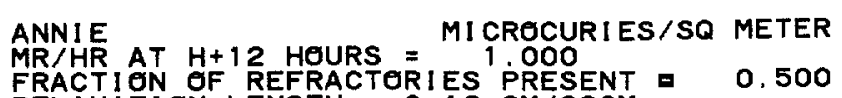
FRACTION OF REFRACTORIES PRESENT D 0.500 DEBRIS DECAY FREM 1 TO 21 HUURS

ZERE TIME $1.00 E+002.00 E+00 \quad 3.00 E+00 \quad 4.00 E+00 \quad 6.00 E+00 \quad 9.00 E+00 \quad 1.20 E+01 \quad 1.50 E+011.80 E+012.10 E+01$

\begin{tabular}{|c|}
\hline 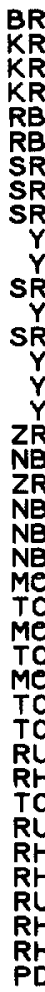 \\
\hline
\end{tabular}

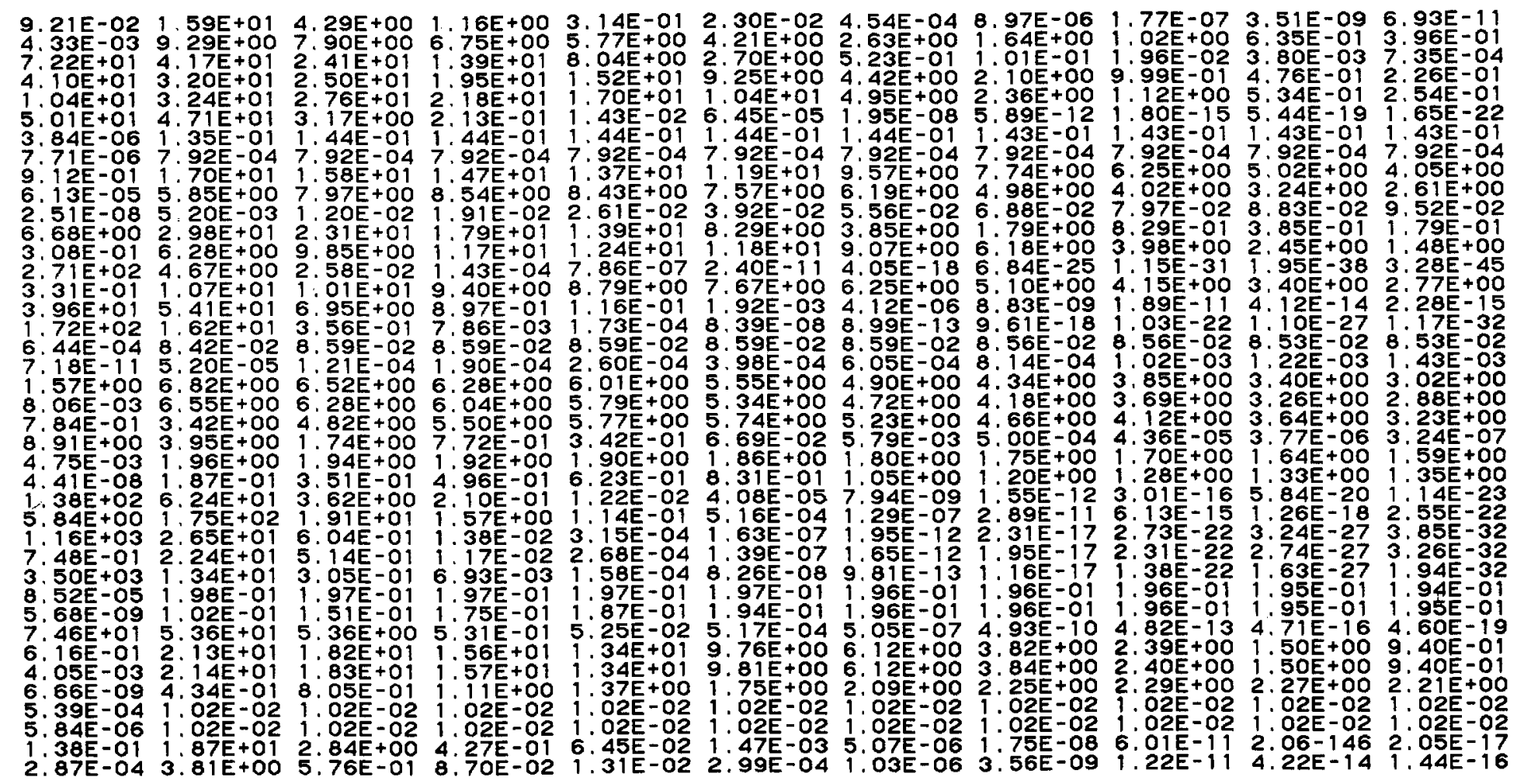


ANNIE MICROCURIES/SQ METER

DEBRIS DECAY FROM 1 TO 21 HOURS

ZERO TIME $1.00 E+00 \quad 2.00 E+00 \quad 3.00 E+00 \quad 4.00 E+00 \quad 6.00 E+00 \quad 9.00 E+00 \quad 1.20 E+01 \quad 1.50 E+01 \quad 1.80 E+012.10 E+01$

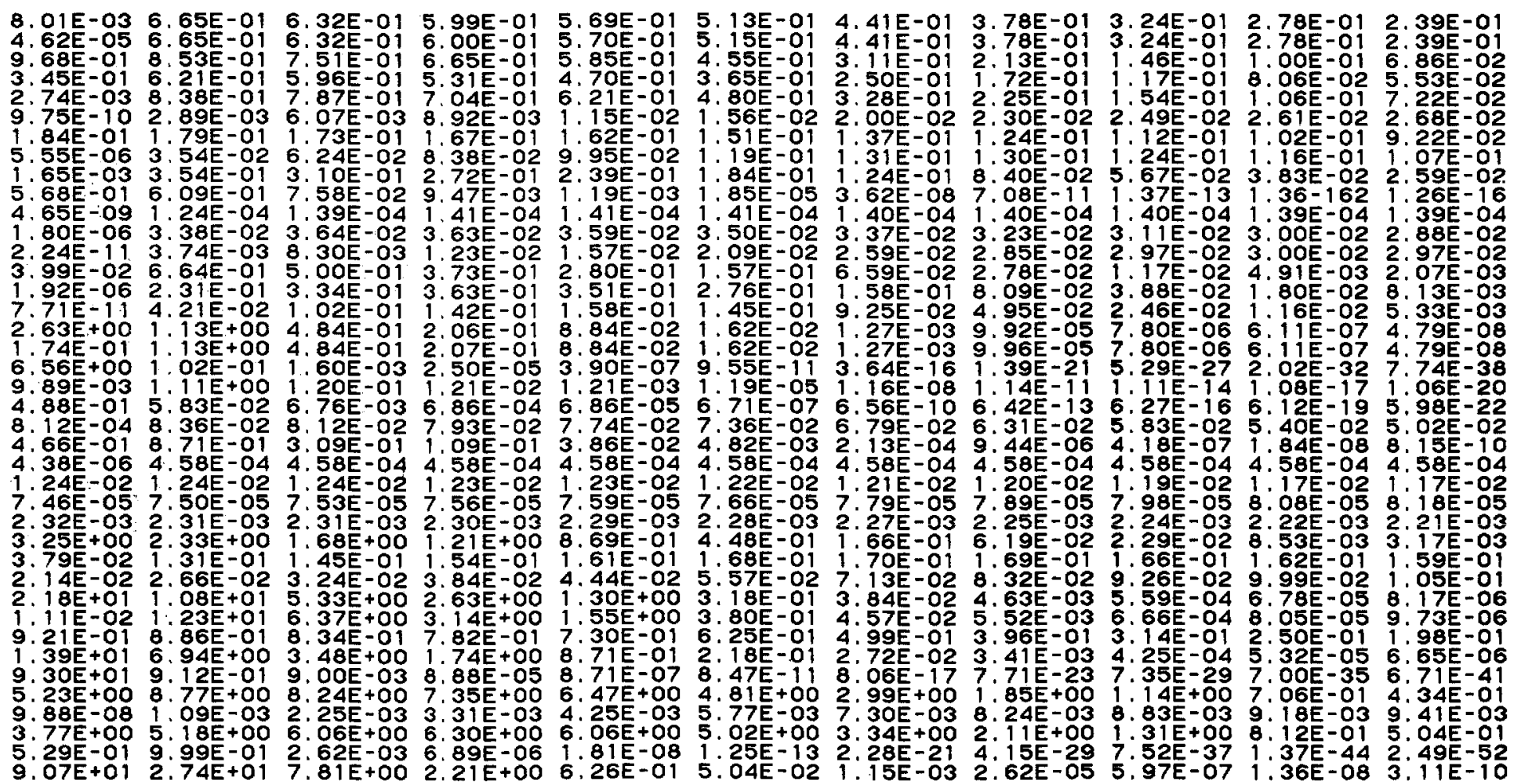


ANNIE MICROCURIES/SQ METER

MR/HR AT $\mathrm{H}+12$ HOURS $=$ MI 1 OOO FRACTION OF REFRACTORI ES PRESENT $=0.500$
RELAXATION LENGTH $=0.16$ GM/SQCM
BOMB FRACTION PER SQ. METER $=3.648 E-13$

BOMB FRACTION PER SQ. METER =

ZERO TIME 1. OOE+OO 2. OOE+OO 3. OOE+OO 4. OOE+OO 6.0OE+O0 9.00E+O0 1.20E+01 $1.50 E+01 \quad 1.80 E+012.10 E+01$

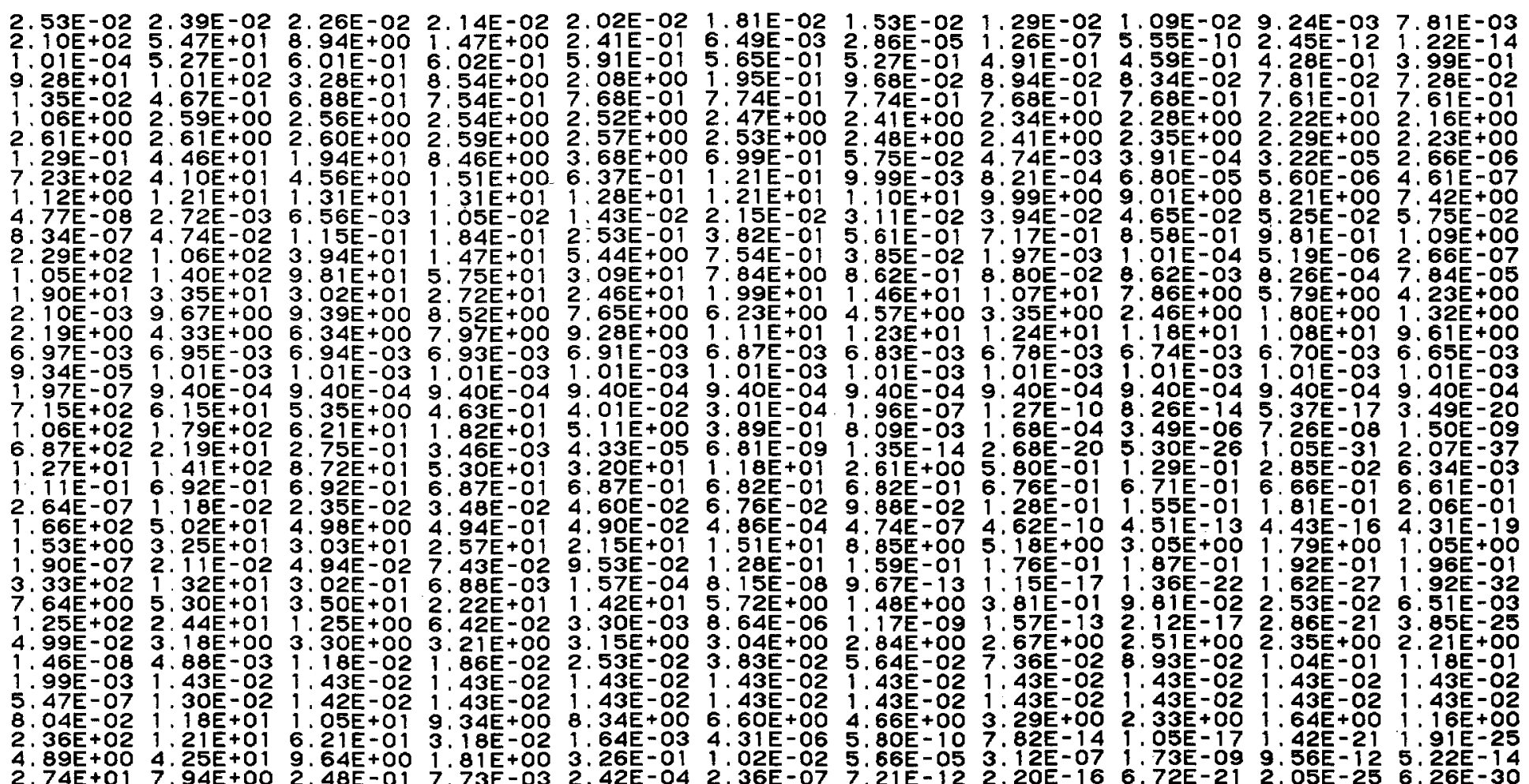


ANNI

MR H AR AT H+12 HOURS $=$ MT 1 OOO
FRACTION OF REFRACTORIES PRESENT - 0.500

OCURIES/SQ METER

PAgE $7-17$

RELAXATION LENGTH $=0.16$ GM GMOCM $3.648 E-13$

DEBRIS DECAY FROM 1 TO 21 HOURS

ZERO TIME 1.OOE+OO 2. OOE+OO 3. OOE+OO 4. OOE+OO $\quad 6.00 E+00 \quad 9.00 E+00 \quad 1.20 E+01 \quad 1,50 E+01 \quad 1.80 E+012.10 E+01$

9.79E-06 $1.84 E-01 \quad 1.89 E-01 \quad 1.89 E-01 \quad 1.89 E-01 \quad 1.88 E-01 \quad 1.86 E-01 \quad 1.85 E-01 \quad 1.83 E-01 \quad 1.82 E-01 \quad 1.80 E-01$ $.42 E+01$ 9.67E+00 6.58E+00 $4.49 E+003.04 E+001.41 E+004.43 E-011.40 E-014.41 E-02 \quad 1.39 E-02 \quad 4.38 E-03$ $.64 E-011.27 E-019.81 E-02$ 7.57E-02 5.86E-02 3.51E-02 1.63E-02 7.52E-03 3.49E-03 1.61E-03 7.47E-04

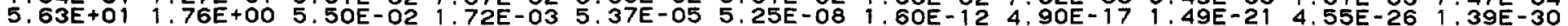
3.57E-02 4.17E-01 4.19E-01 4.09E-01 3.99E-01 3.79E-01 3.52E-01 3.27E-01 3.04E-01 2.81E-01 2.61E-01 $9.07 E+01$ 8.88E-02 8.65E-05 8.46E-08 8.26E-11 $7.89 E-17$ 7.34E-26 6.85E-35 6.38E-44 5.96E-53 $5.54 E-62$ 1.22E-01 1.20E-01 1. 18E-01 1.16E-01 1.15E-01 1.11E-01 1. 07E-01 1.02E-01 9.76E-02 9.35E-02 8.94E-02

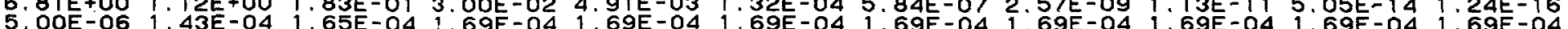

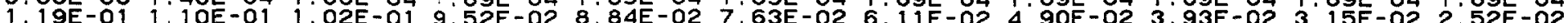
3.47E-04 5.67E-04 7.70E-04-9.58E-04 1.13E-03 1.45E-03 1.84E-03 2.13E-03 2.38E-03 2.57E-03 2.71E-03 1.93E-02 6.40E-02 6.11E-02 5.84E-02 5.57E-02 5.09E-02 4.44E-02 3.88E-02 3.38E-02 2.94E-02 2.57E-02

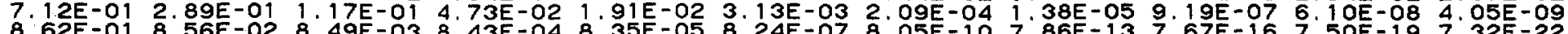
8.62E-01 8.56E-02 8.49E-03 8.43E-04 8.35E-05 8.24E-07 8.05E-10 7.86E-13 7.67E-16 7.5OE-19 7.32E-22

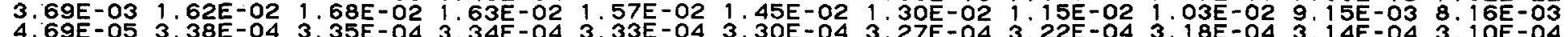


ANNIE

MRAHR AT H+12 HOURS = MIC 1 OOOO

BELAXATION LENGTH DO O. 16 GM SQCM $3.648 E-13$

DEBRIS DECAY FROM 1 TO 300 DAYS

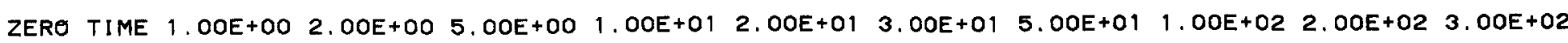
9. 63 E-06 9.51E-06 9.38E-06 9.03E-06 8. 46E-06 7.43E-06 6.52E-06 5.02E-06 2.63E-06 7.14E-07 1.94 E-07

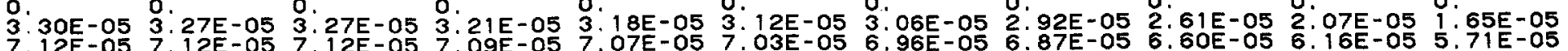

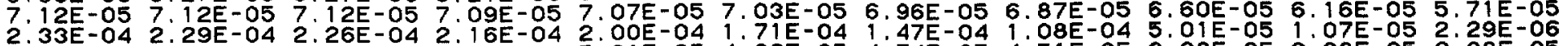

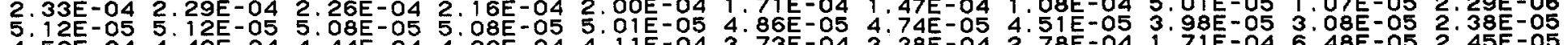

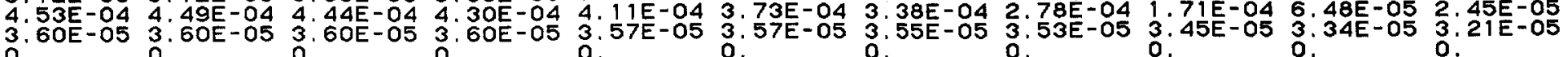

0 :

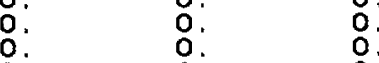

o. $\quad$ o: $\quad$ o: $\quad$ o:

o.:

0.:<smiles>COC(=O)C(C)(C)C</smiles>

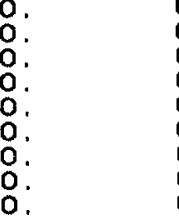

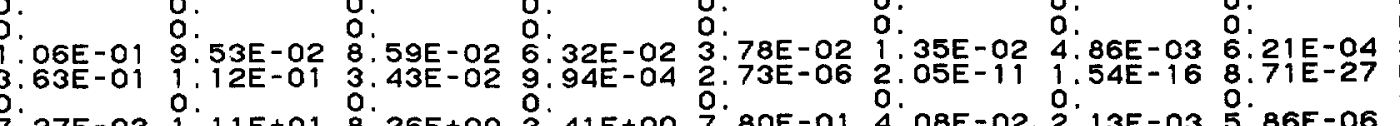
०.: $\quad$ : $\quad$ : $\quad$ : : $\quad$ : $: 0$

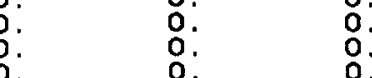

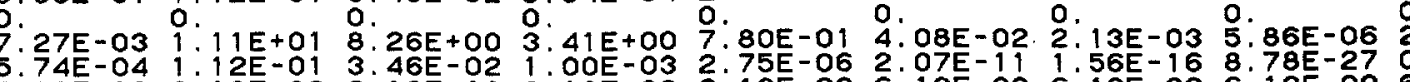

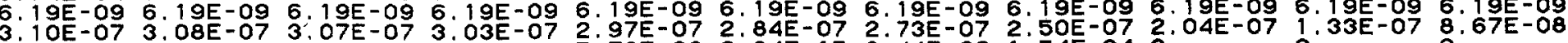

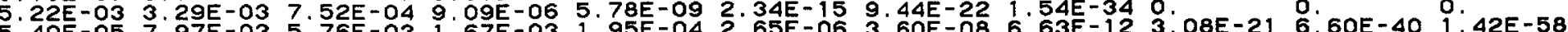

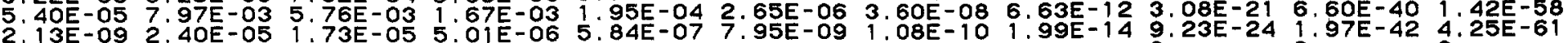

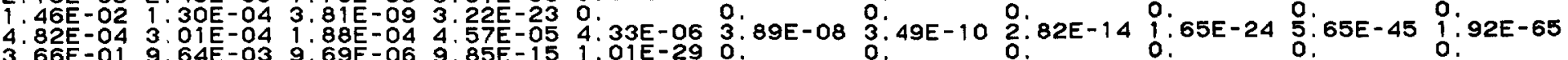

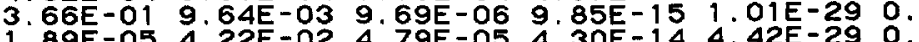
4.33E-03 2. $48 \mathrm{EE}-01$ 5.64E-03 $5.69 \mathrm{E}-08$ 4.13E-16 $1.57 \mathrm{E}-32$ 5.94E-49 (20E-04 $1.20 E-04$ $4.10 E+011.08 E-01 \quad 2.83 E-04 \quad 5.14 E-12 \quad 6.48 E-25$ $1.04 E+01 \quad 1.20 E-013.17 E-04 \quad 5.76 E-12 \quad 7.22 E-25 \quad 0$

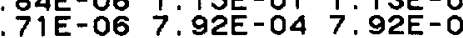
7. OSE-01 1.02E-01 8. 92E-02 7..80E-02 6.

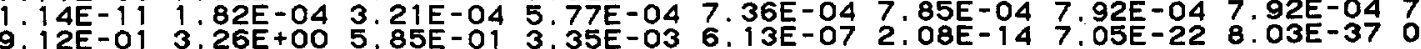

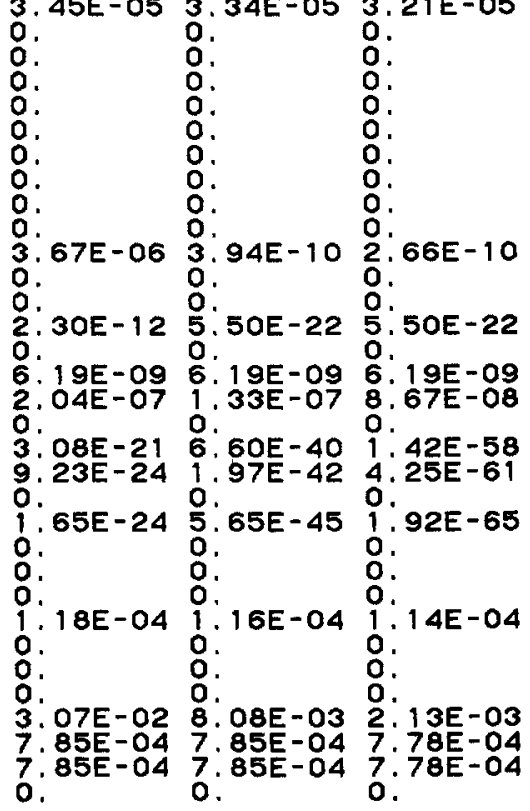


MNNIE AT H+12 HOURS = MICROCURIES/SQ METER

FRACTION OF REFRACTORIES PRESENT = 0.500

RELAXATION LENGTH $=0.16$ GM/SQCM $3.648 E-13$

DEBRIS DECAY FROM 1 TO 300 DAYS

ZERO TIME 1. OOE+OO 2.0OE+00 5. OOE+00 $1.00 E+01 \quad 2.00 E+01 \quad 3.00 E+01 \quad 5.00 E+01 \quad 1.00 E+02 \quad 2.00 E+023.00 E+02$ 


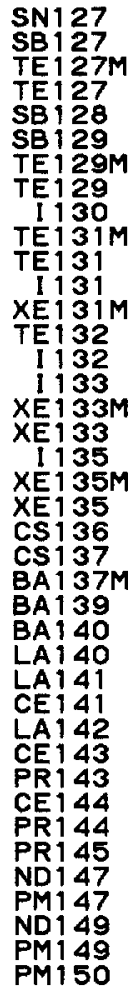

ANNIE

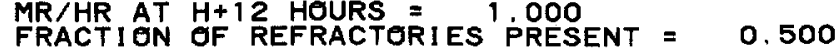
RELAXATION LENGTH $=0$. 16 GMISQCM $3.648 E-13$ DEBRIS DECAY FROM 1 TO 300 DAYS

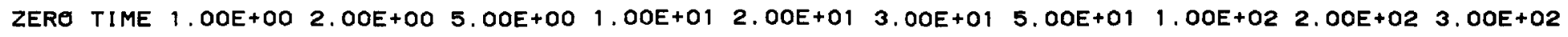

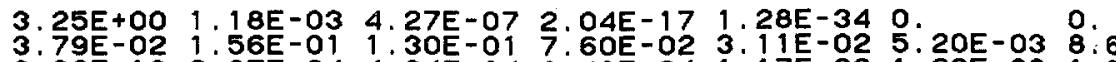

源

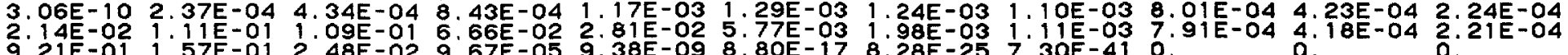

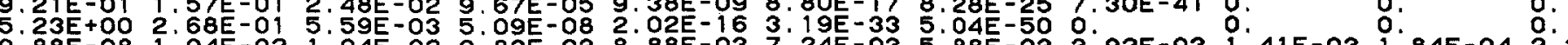
$\begin{array}{llll} & \end{array}$

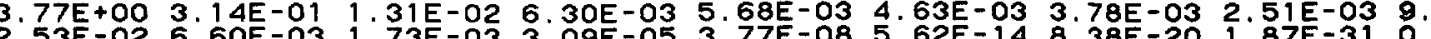

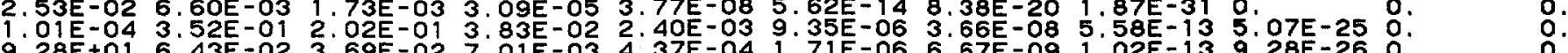
$.28 E+01$ 6. $43 E-02$ 3.69E-02 $7.01 E-03$ 4.37E-04 1.711E-06 6.67E-09 1.02E-13 9.28E-26 0.200. .35E-02 7.28E-01 6. 88 -01 5.53E-01 3.64E-01 1.54E-01 6.5OE-02 $1.16 E-02$ 1.57E-04 2.86E-08 $5.21 E-12$

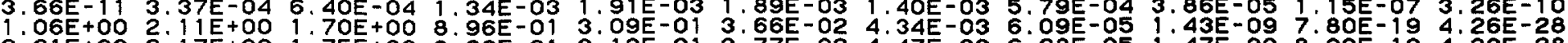
$2: 61 \mathrm{E}+00 \mathrm{2}$ : $17 \mathrm{E}+00$ 1:.75E+00 9.22E-01 3.19E-01 3.77E-02 4.47E-03 6.28E-05 1.47E-09 8.00E-19 4.39E-28

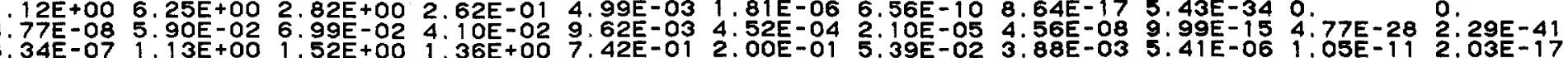

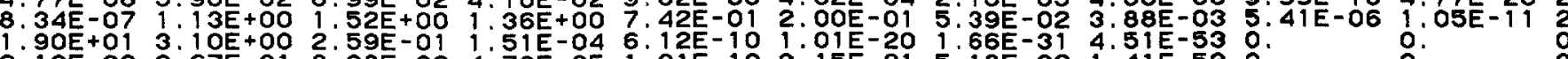

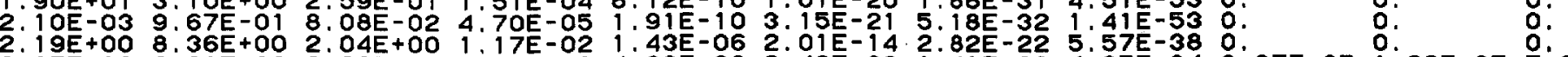

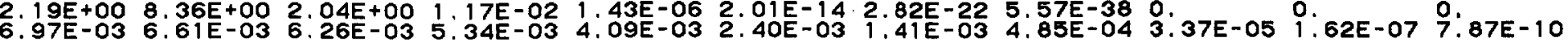

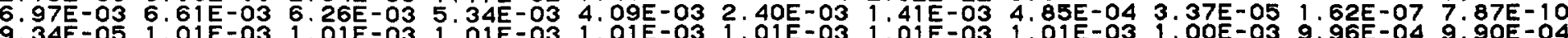
97E-07 9.4OE-04 9.4OE-04 9:4OE-04 9.4OE-04 9.4OE-04 9.4OE-04 9.4OE-04 9:34E-04 9:29E-04 9:23E-04

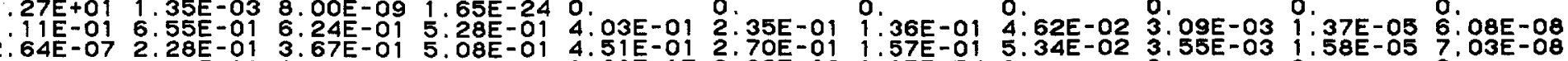

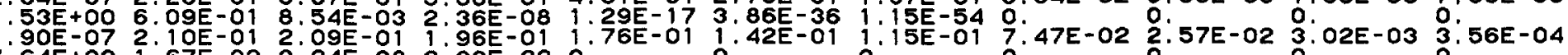

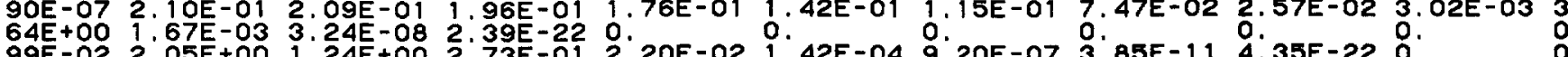

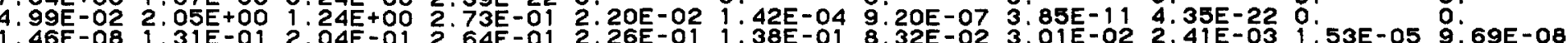
$\begin{array}{ll} & \end{array}$ $.47 E-07$ 1.43E-02 1:42E-02 1.41E-02 1.39E-02 1.36E-02 1.33E-02 1.26E-02 1:12E-02 8.76E-03 6.88E-03

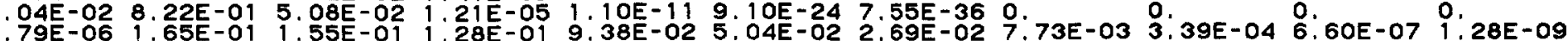

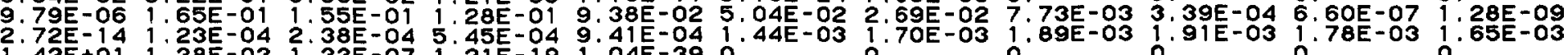

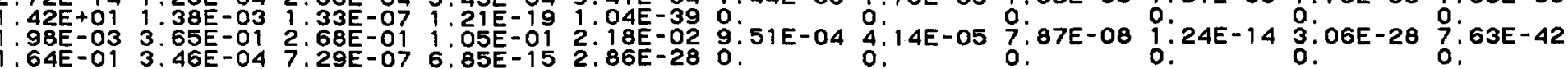


ANNIE MR/HR AT H+12 HOURS = MIC 1 ROCOORIIES/SQ METER MR/HR AT H+12 HOURS $=$ MRACTION OF REFRACTORIES PRESENT $=0.500$ RELAXATION LENGTH $=0.16$ GM/SQCM $=$
BOMB FRACTION PER SQ. METER $=3.648 E-13$

DEBRIS DECAY FROM 1 TO 300 DAYS

ZERO TIME $1.00 E+00 \quad 2.00 E+00 \quad 5.00 E+00 \quad 1.00 E+01 \quad 2.00 E+01 \quad 3.00 E+01 \quad 5.00 E+01 \quad 1.00 E+02 \quad 2.00 E+02 \quad 3.00 E+02$

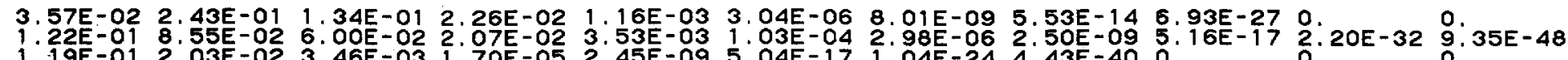

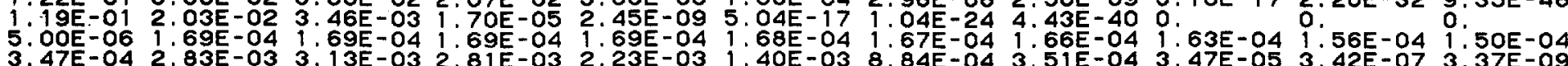

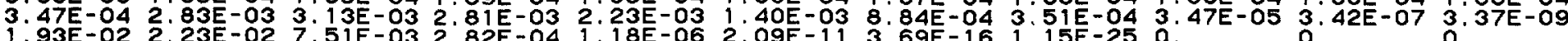

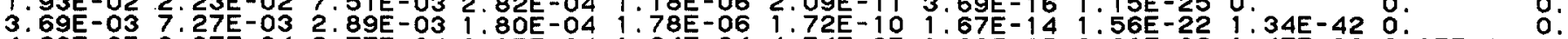

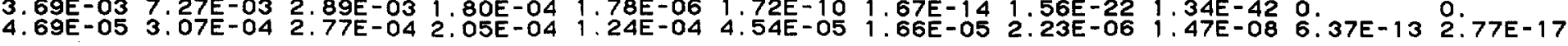

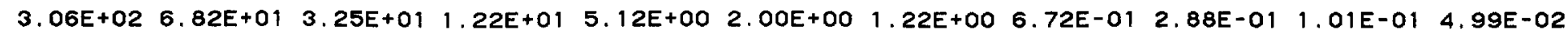

PAge $7-21$

TOTAL 
ANNIE MI CRECURIES/SQ METER FRACTION OF REFRACTORIES PRESENT $=0.500$ RELAXATI ON LENGTH $=0.16$ GM SOCM $3.648 E-13$

DEBRIS DECAY FROM 1 TO 50 YEARS

ZERO TIME $1.00 E+00 \quad 1.50 E+00 \quad 2.00 E+00 \quad 3.50 E+00 \quad 5.00 E+00 \quad 7.00 E+00 \quad 1.00 E+012.00 E+01 \quad 3.50 E+01 \quad 5.00 E+01$

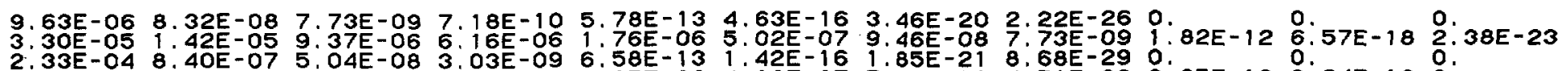

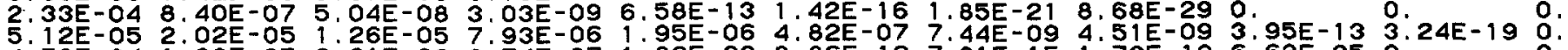

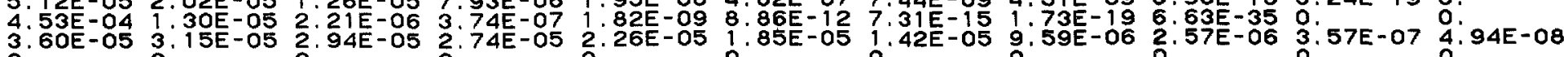
(3. $60 \mathrm{E}-05$

: : : 0 :

OSE-01 $2.63 E-10$ O. 2.63E-10 2.57E-10 2.50E-10 2. . $1.39 \mathrm{E}-08$

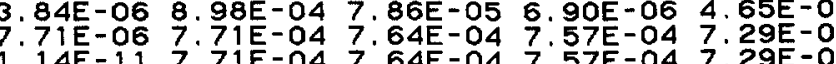
. 54 E 7.71E-04 7.64E-04 7.57E-04 7.29E-04 7:01E-04 6.67E-04 6.20E-04

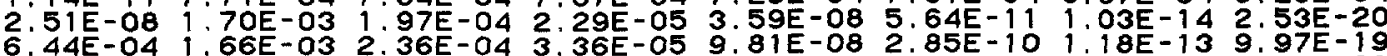
$1.37 E-113.50 E-05$ 5. 00E-06 7.15E-07 2.08E-09 6.05E-12 $2.50 E-15$ 2. 11 EE-20

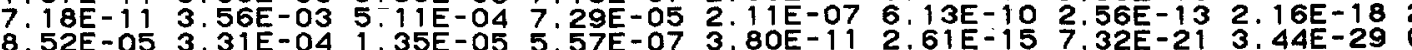

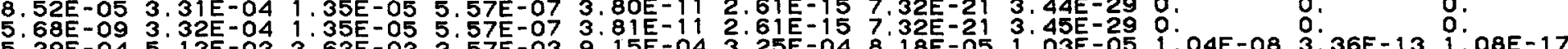

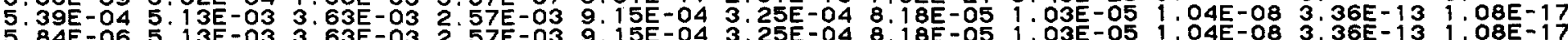
. 7. $46 E-05$ 1.50E-04 1 .32E-04 1 . $16 E-04$ 7.92E-05 5.39E-05 3.22E-05 1.49E-05 1. $15 E-06$ 2.44E-08 5. $19 E-10$ $\begin{array}{lllllll} & \end{array}$ 3.06E-10 1.47E-04 4.59E-05 1.44E-05 4.42E-07 1.36E-08 $1.31 E-10$ 1.23E-13 1.01E-23 7.54E-39 5.62E-54 2.14E-02 1.45E-04 4.54E-05 1.42E-05 4.36E-07 1.34E-08 1.29E-10 1.222-13 9.99E-24 7.44E-39 3.57 9.34E-05 9.85E-04 9.73E-04 9.62E-04 9.29E-04 8.95E-04 8.56E-04 8.00E-04 6.32E-04 4.49E-04 $3.17 E-04$

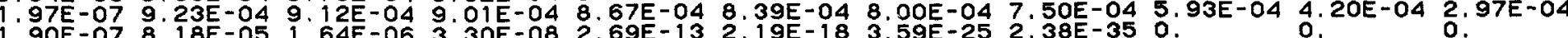

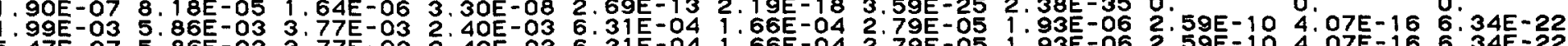
5.47E-07 5.86E-03 3.77E-03 2.40E-03 6.31E-04 1.66E-04 2.79E-05 1:93E-06 2.59E-10 4.07E-16 $6.34 E-22$

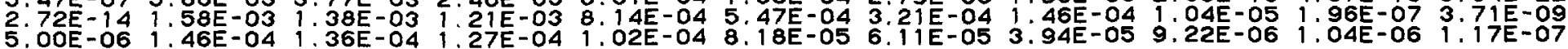

TOTAL

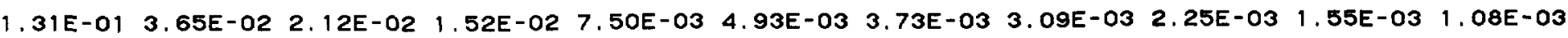




\author{
TIME \\ ZERO TIME HOURS
}

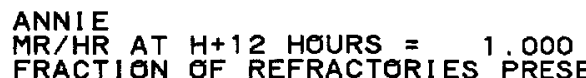
RELAXATION LENGTH $=0.16 \mathrm{GM} / \mathrm{SQCM}$ RELAXATI IN LENGTH $=0.16$ GM/SQCM
BOMB FRACTI ON PER SQ. METER $=$

$\mathrm{MR} / \mathrm{HR}$

4. $652 \mathrm{E}-13$

$1.16 E+O 2$

3. $83 E+01$

$7.5 \mathrm{OE}+01$

$4.61 E+\infty$
$2.31 E+\infty O$

$1.36 \mathrm{E}+00$

$1.00 E+00$

6.37E-01

5. $29 E-01$

4. $42 E-01$

8. $39 \mathrm{E}-02$

$4.05 \mathrm{E}-02$

$1.58 E-02$

3. $41 \mathrm{E}-03$

$1.94-04$

8. $19 E-05$

3. $18 \mathrm{E}-05$

2. $46 \mathrm{E}-05$

. $60 \mathrm{E}-05$

. $27 \mathrm{E}-05$

$.74 \mathrm{E}-06$

5. $32 \mathrm{E}-06$
MICROCURIES/SQ METER

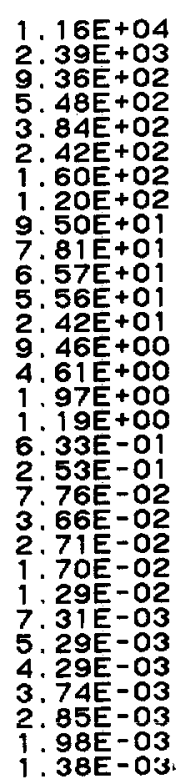


ANNIE AT H+12 HOURS - MICROCURIES/SQ METER

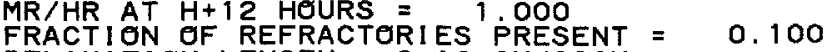

BEMAXATION LENGTH E O. 16 GM/SQCM $4.652 E-13$

DEBRIS DECAY FROM 1 TO 21 HOURS

ZERO TIME $1.00 E+00 \quad 2.00 E+00 \quad 3.00 E+00 \quad 4.00 E+00 \quad 6.00 E+00 \quad 9.00 E+00 \quad 1.20 E+01 \quad 1.50 E+01 \quad 1.80 E+01 \quad 2.10 E+01$

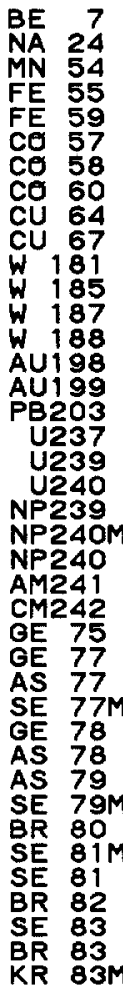

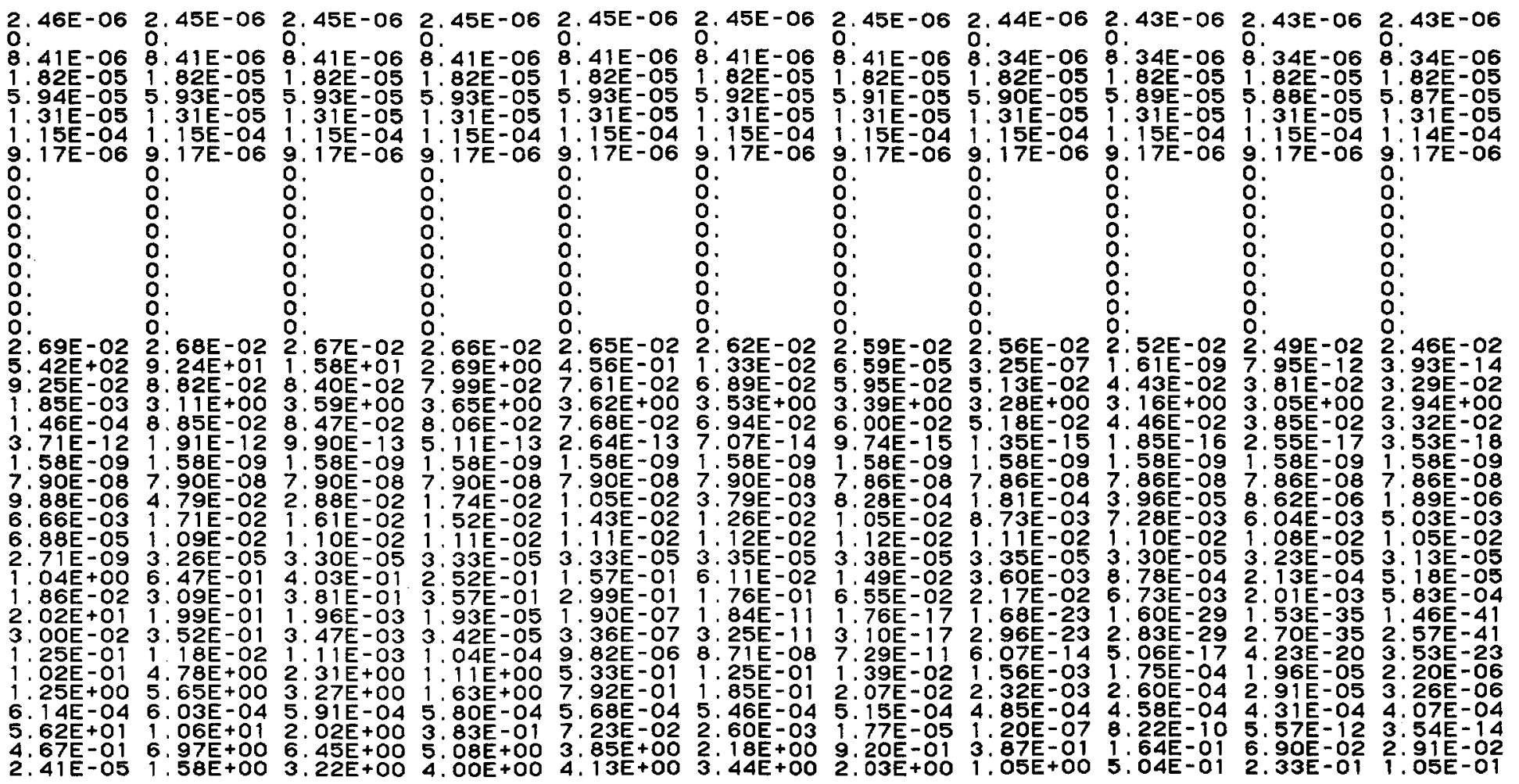


ANNIE AT H+12 HEURS MICRECURIES/SQ METER

MRAHR AT H+12 HOURS ME 1 OODO

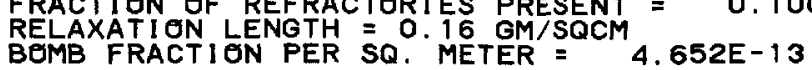

DEBRIS DECAY FREM 1 TO 21 HEURS

ZERO TIME $1.00 E+00 \quad 2.00 E+003.00 E+00 \quad 4.00 E+00 \quad 6.00 E+00 \quad 9.00 E+00 \quad 1,20 E+01 \quad 1.50 E+01 \quad 1,80 E+01 \quad 2.10 E+01$ 1. 17E-01 2. $03 E+015.48 E+001.48 E+00$ 4.01E-01 2. $93 E-02 \quad 5.79 E-04 \quad 1.14 E-05 \quad 2.26 E-07 \quad 4.48 E-09$ 8. $84 E-1$ 9:21E+01 5.31E+01 3:08E+01 1.78E+01 1:03E+01 3.44E+00 6.67E-01 1.29E-01 2:50E-02 4:85E-03 9:37E-04

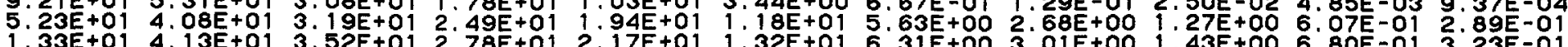

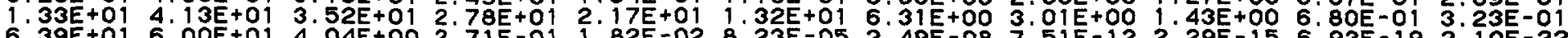

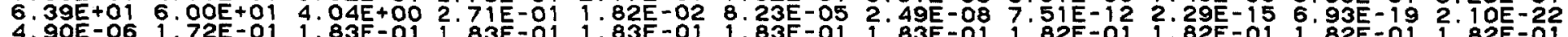

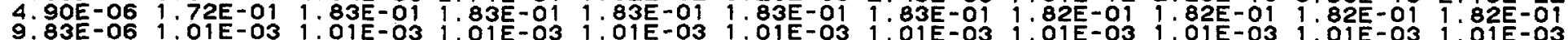

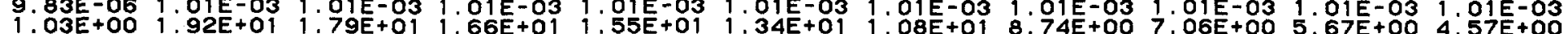

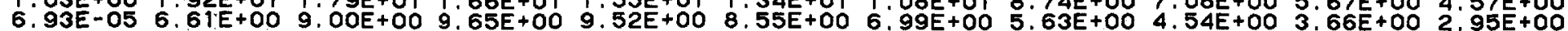
2.84E-08 5.87E-03 1.35E-02 2.16E-02 2.95E-02 4.42E-02 6.28E-02 7.77E-02 9:00E-02 9:97E-02 $1.07 E-01$ $1.70 E+007.61 E+005.89 E+004.56 E+003.53 E+002.12 E+00$ 9.82E-01 4.56E-01 2.12E-01 9.82E-02 4:57E-02

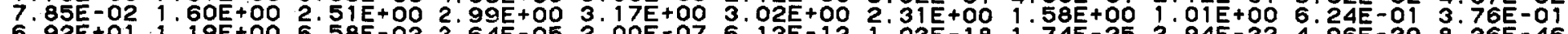

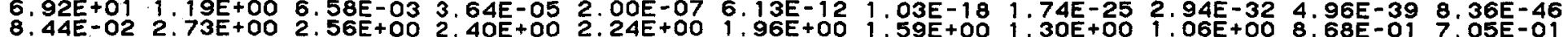

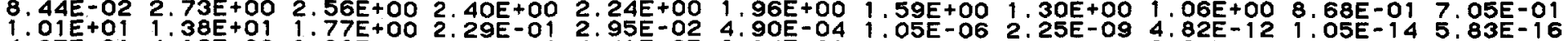

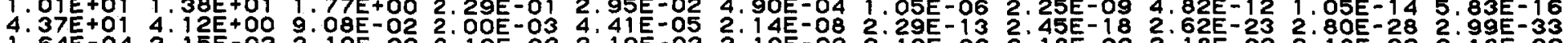
$1.64 E-042$. $15 E-02$ 2.19E-02 2.19E-02 2.19E-02 2.19E-02 2.19E-02 2.18E-02 2.18E-02 2.18E-02 2.18E-02 $1.02 E-041.54 E-042.08542 .59 E-043.12 E-043.64 E-04$

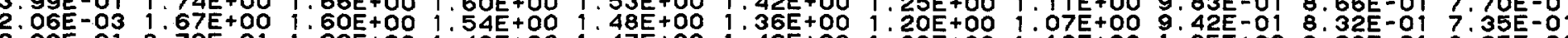

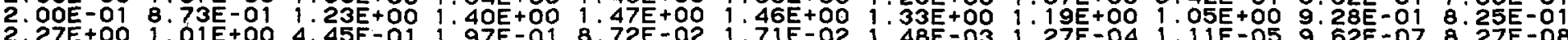

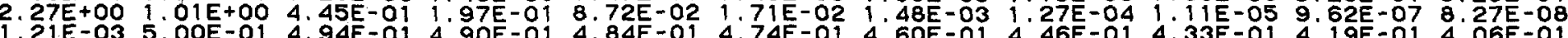

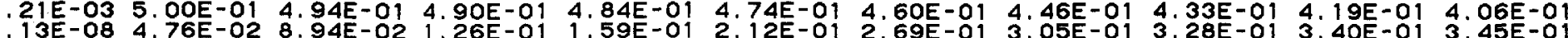

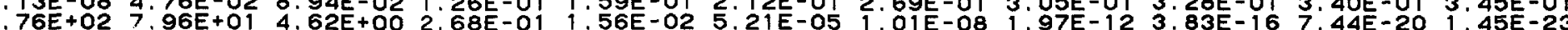

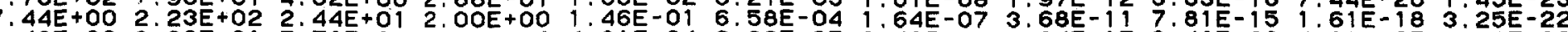

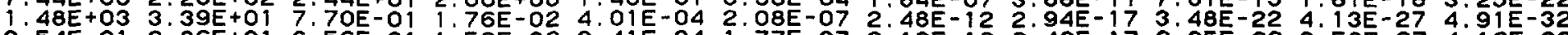
9.54E-01 2.86E+01 6.56E-01 1.50E-02 3.41E-04 1.77E-07 2.10E-12 2.49E-17 2.95E-22 3.50E-27 4.16E-32

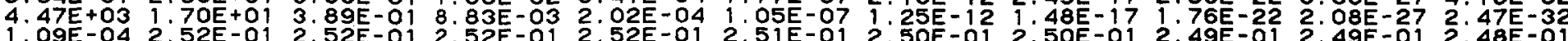
7.05E

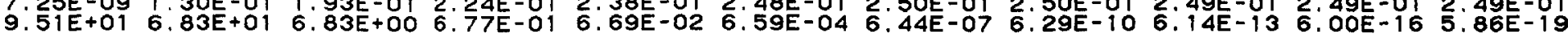

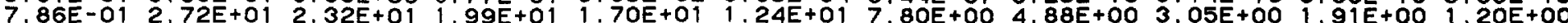

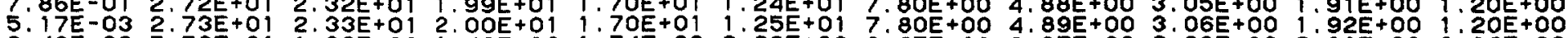
8. $49 E-095.53 E-012.03 E+001.42 E+00 \quad 1.74 E+002.23 E+002.67 E+002.87 E+002.93 E+002.90 E+002.82 E+00$

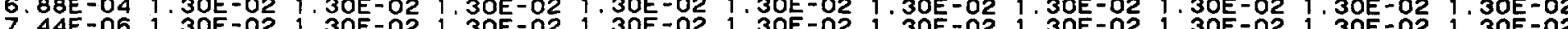

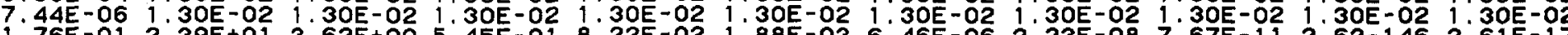
$\begin{array}{lllllllllll}1.76 E-01 & 2.39 E+01 & 3.62 E+00 & 5.45 E-01 & 8.22 E-02 & 1.88 E-03 & 6.46 E-06 & 2.23 E-08 & 7.67 E^{-11} & 2.62-146 & 2.61 E-17 \\ 3.65-04 & 4.86 E+00 & 7.34 E-01 & 1.11 E-01 & 1.67 E-02 & 3.82 E-04 & 1.31 E-06 & 4.53 E-09 & 1.56 E-11 & 5.38 E-14 & 1.84 E-16\end{array}$ 


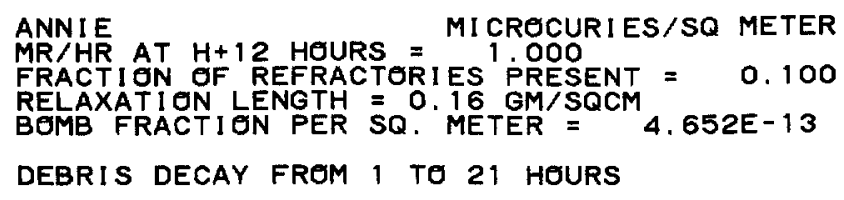

ZERO TIME $1.00 E+00 \quad 2.00 E+00 \quad 3.00 E+00 \quad 4.00 E+00 \quad 6.00 E+00 \quad 9.00 E+00 \quad 1.20 E+01 \quad 1.50 E+01 \quad 1.80 E+012.10 E+01$

PD109 AGIOgM PDII AGI11 PD1 12

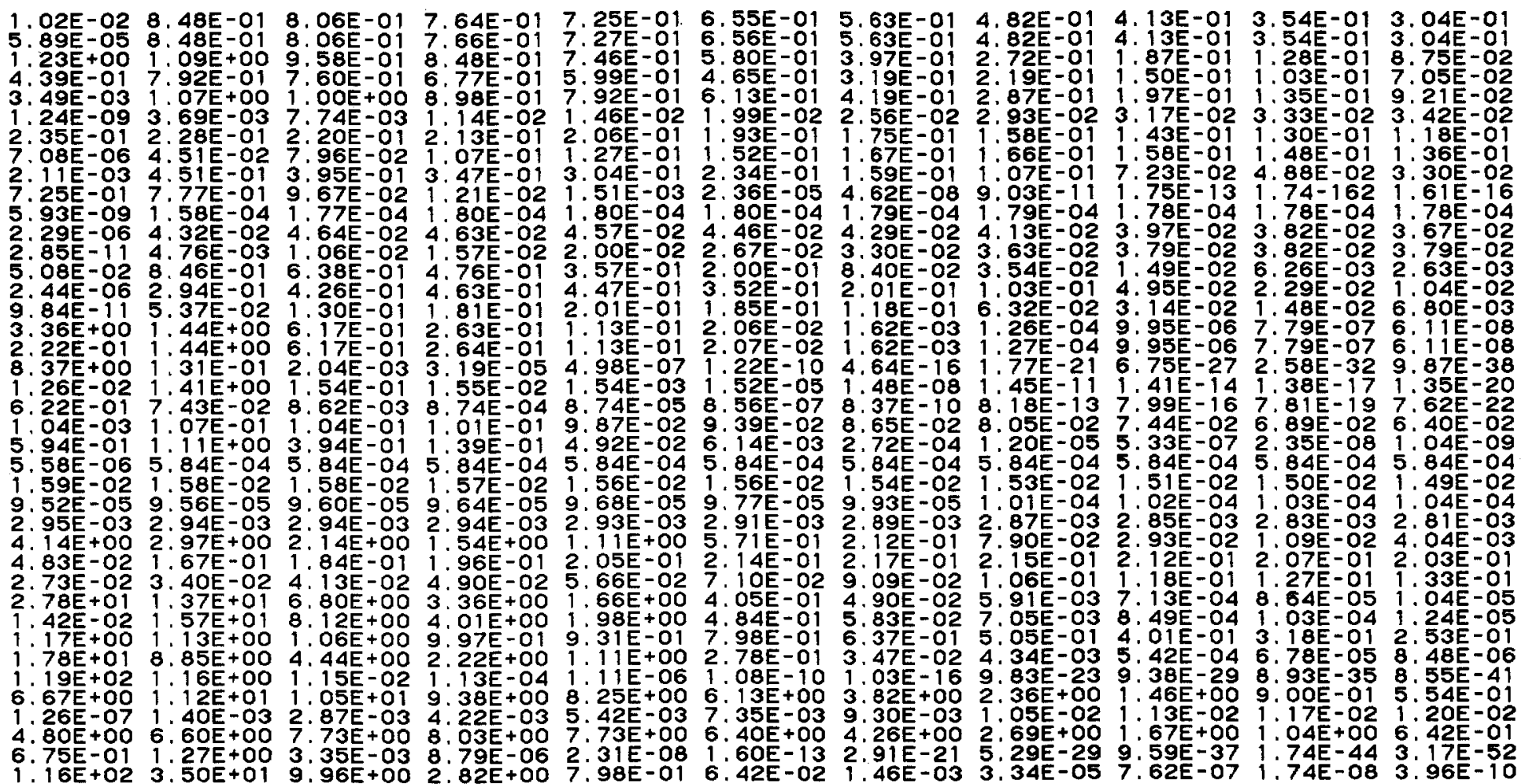


ANNIE AT HI CROCURIES/SQ METER

MR/HR AT H+12 HOURS = 1 FRACT OOO

FRACTION OF REFRACTORI ES PRESENT $=0.100$
RELAXATION LENGTH $=0.16$ GM/SQCM
BOMB FRACTION PER SQ. METER $=4.652 E-13$

PAGE

DEBRIS DECAY FROM 1 TO 21 HOURS

ZERO TIME $1.00 E+00 \quad 2.00 E+00 \quad 3.00 E+00 \quad 4.00 E+00 \quad 6.00 E+00 \quad 9.00 E+00 \quad 1.20 E+01 \quad 1.50 E+01 \quad 1.80 E+012.10 E+01$

SB 130

TEISIM

TEY 131

TE132

I 132

TE 133

XE133M

XE133

I 134

135
$\times E 135 M$

XE 135

CS137

BA137M

CS138

CS139

BA139

BA140

BA141

CA 141

BA 142

LA 142

LA143

PR 143

PR 144

PR 145

CE 146

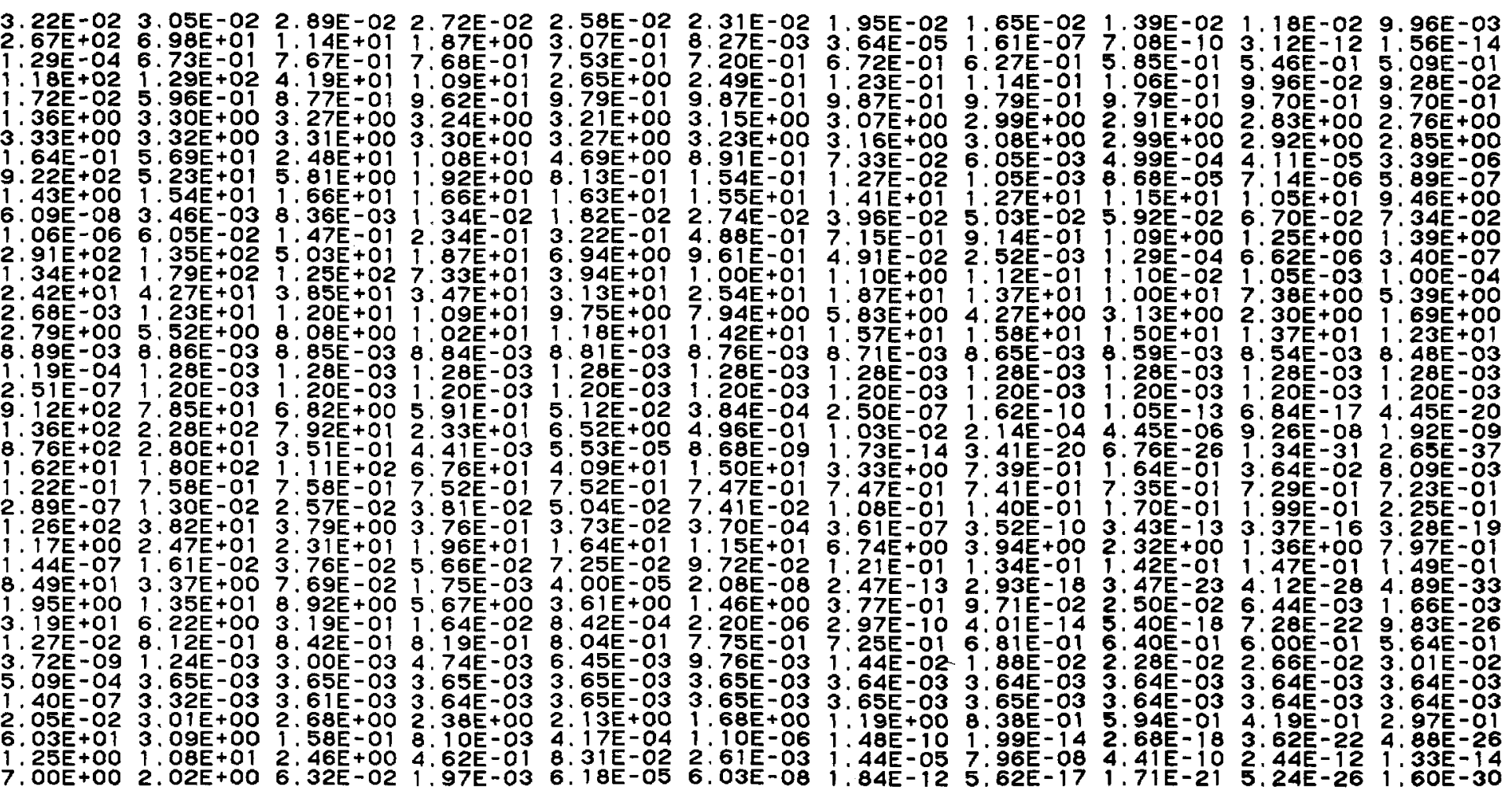


DEBRIS DECAY FROM 1 TO 21 HQURS

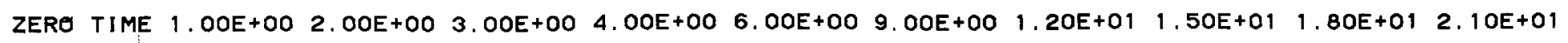
$2.50 E-06 \quad 4.70 E-02 \quad 4.83 E-02 \quad 4.82 E-02 \quad 4.81 E-02 \quad 4.79 E-02 \quad 4.75 E-02 \quad 4.71 E-02 \quad 4.68 E-02$ 4.64E-02 4.60E-02

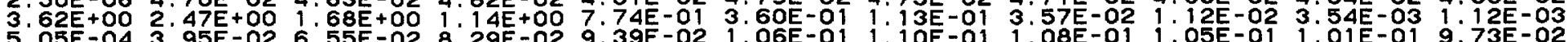

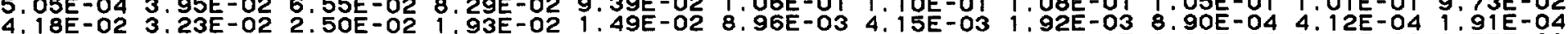

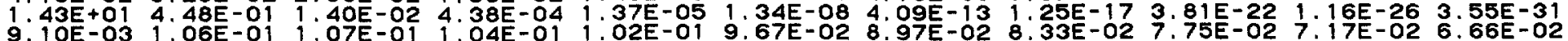

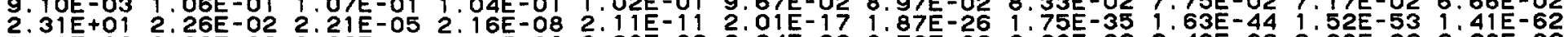

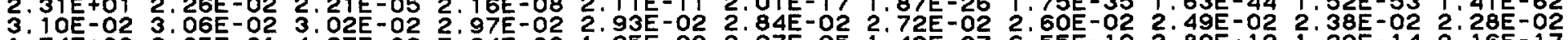
$1.74 E+002.85 E-014: 67 E-02$ 7:.64E-03 1.25E-03 3.37E-05 1:49E-07 6.55E-10 2.89E-12 1.29E-14 3. $16 E-17$ .28E-06 3.64E-05 4.21E-05 4:30E-05 4.32E-05 4. 32E-05 4. 32E-05 4. 32E-05 4. 32E-05 4.32E-05 $4.32 E-05$

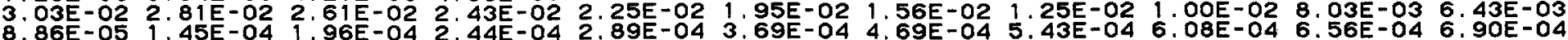

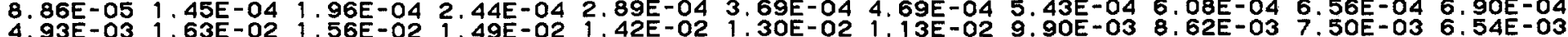

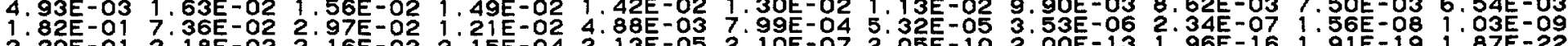

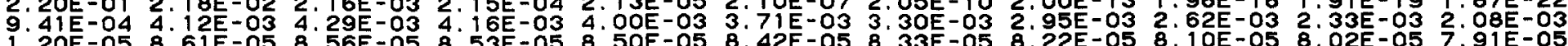

TOTAL

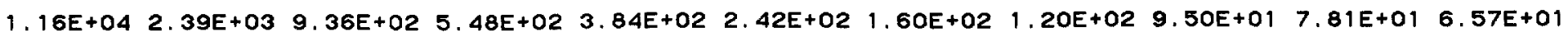


ANNIE MR/HR AT H+12 HOURS = MICROCURIES/SQ METER

MR/HR AT H+12 HOURS = MI 1 OOOO

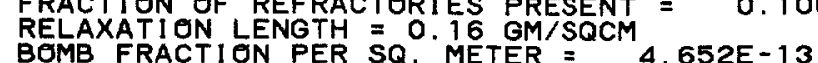

DEBRIS DECAY FROM 1 TO 300 DAYS

ZERO TIME 1. OOE+OO 2. OOE+OO $5.00 E+00 \quad 1.00 E+01 \quad 2.00 E+01 \quad 3.00 E+01 \quad 5.00 E+01 \quad 1.00 E+02 \quad 2.00 E+02 \quad 3.00 E+02$

2. 46E-06 2. 43E-06 2.39E-06 2.30E-06 2.16E-06 1. 89E-06 1.66E-06 1.28E-06 6.70E-07 1.82E-074.95E-08 8. 41 E-06 8.34E-06 8. 34E-06 8.1.9E-06 8.1 1E-06 7.96E-06 7.82E-06 7.44E-06 6.65E-06 5.28E-06 $4.21 E-06$

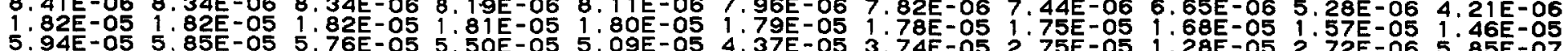

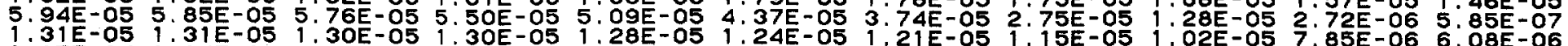

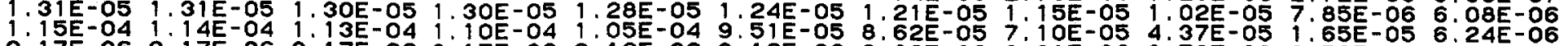

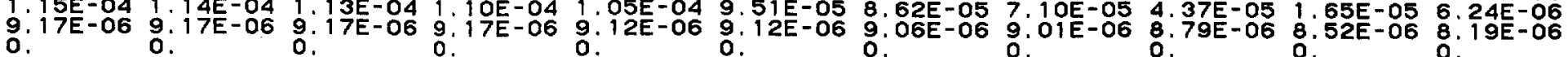

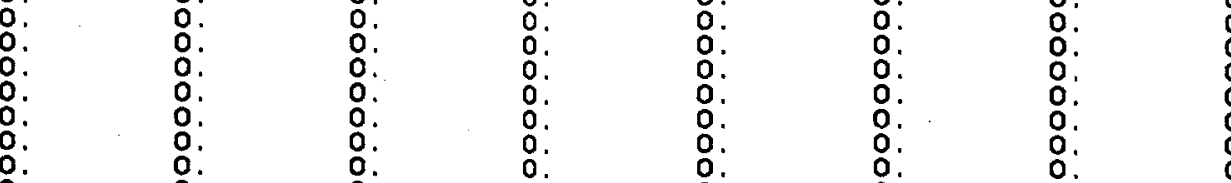

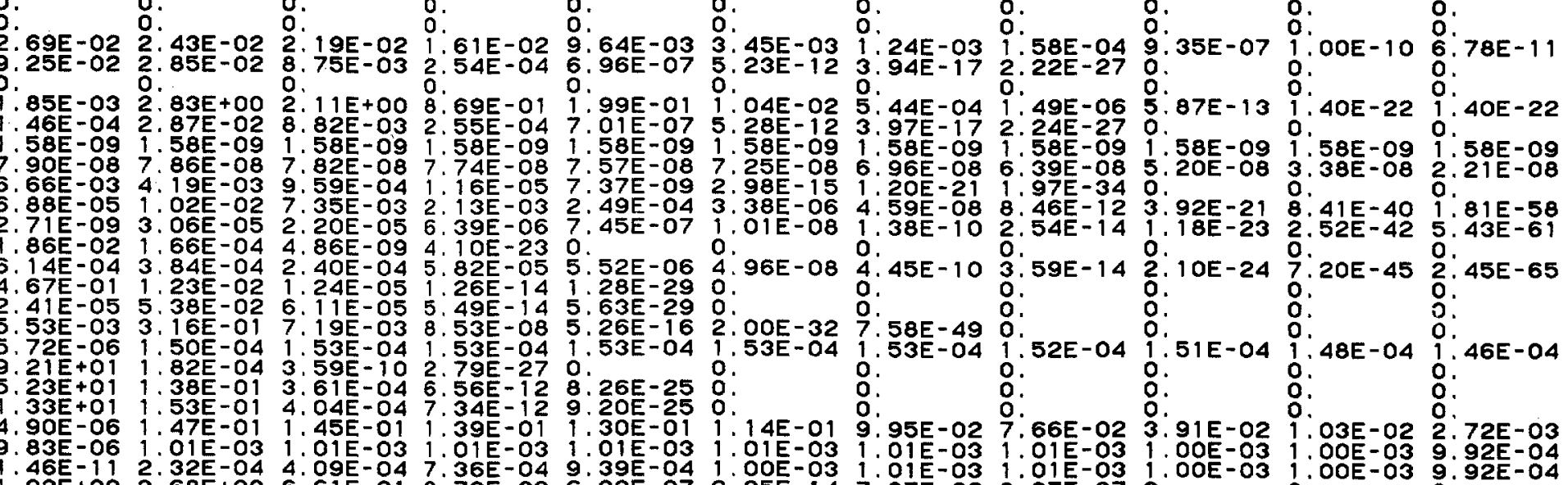

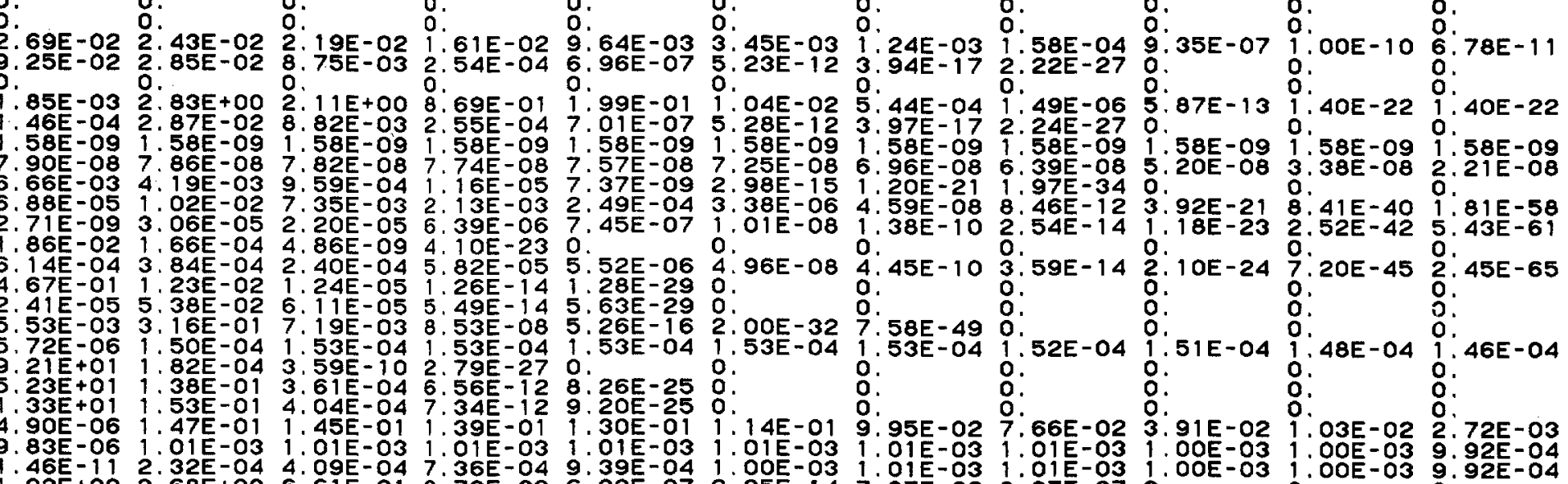

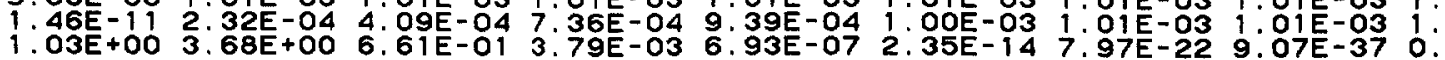




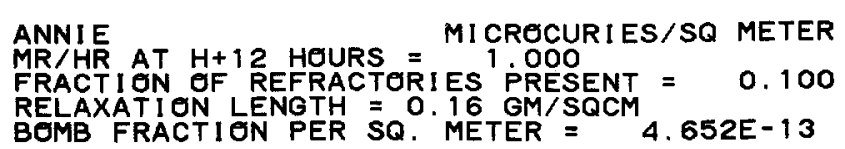

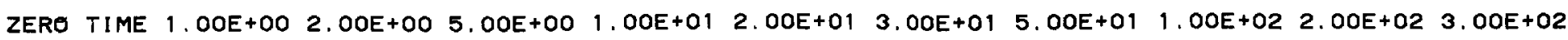

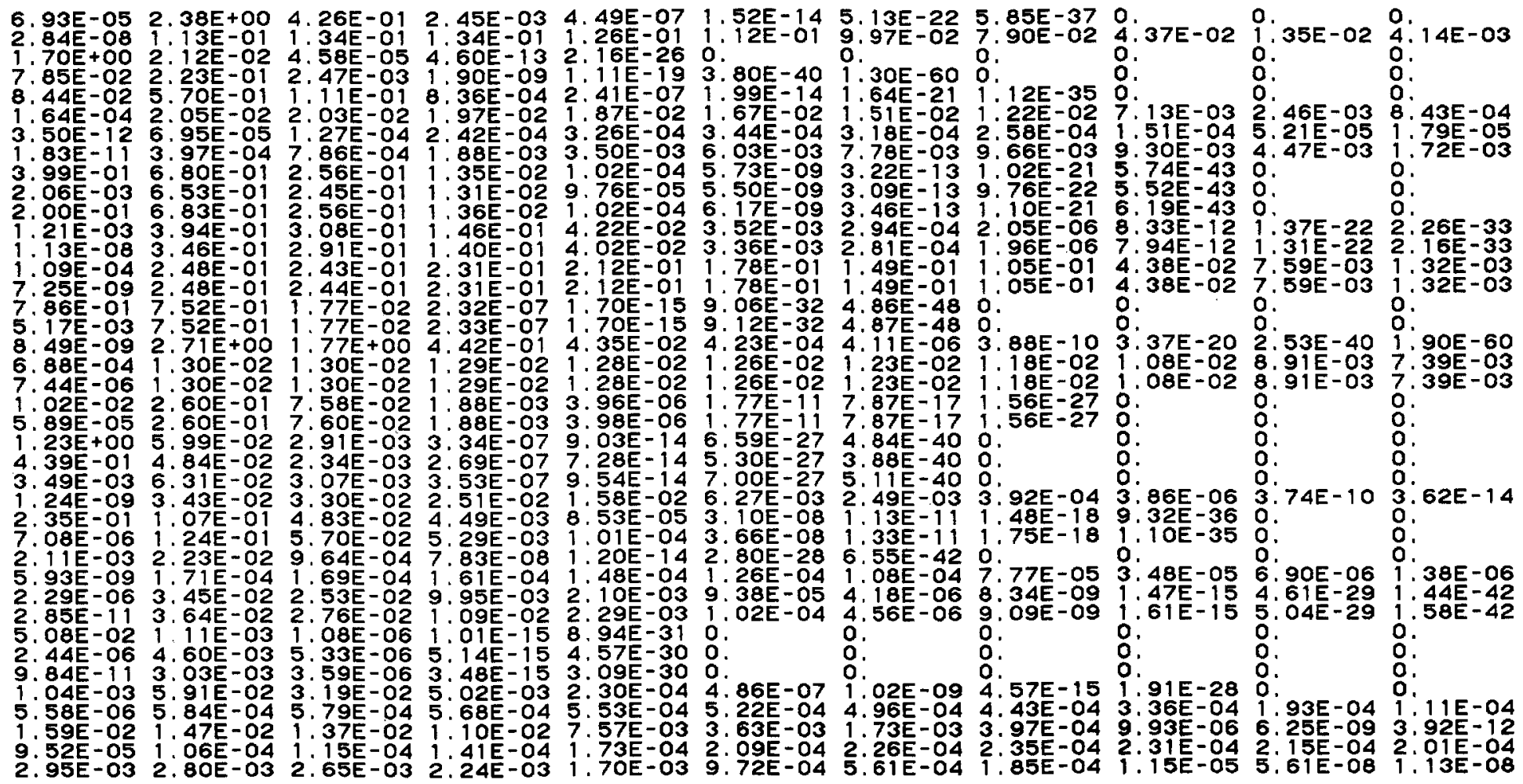


ANNIE MR/HR AT $\mathrm{H}+12$ HOURS = MIÇROCURIES/SQ METER

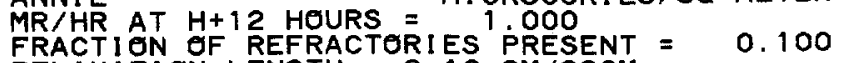

FRACTI IN OF REFRACTORI ES PRESENT $=0.100$
RELAXATION LENGTH $=0.16$ GM/SQCM
BOMB FRACT I ON PER SQ.

DEBRIS DECAY FROM 1 TO 300 DAYS

ZERO TIME $1.00 E+00 \quad 2.00 E+00 \quad 5.00 E+00 \quad 1.00 E+01 \quad 2.00 E+01 \quad 3.00 E+01 \quad 5.00 E+01 \quad 1.00 E+02 \quad 2.00 E+02 \quad 3.00 E+02$

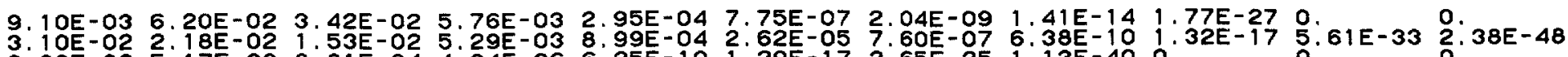

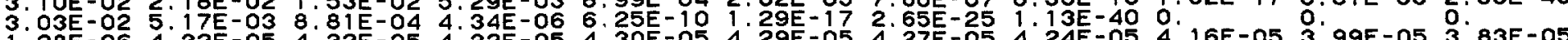

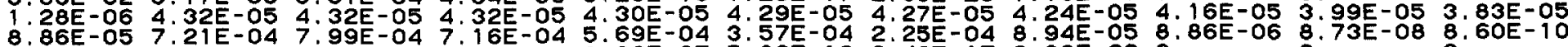

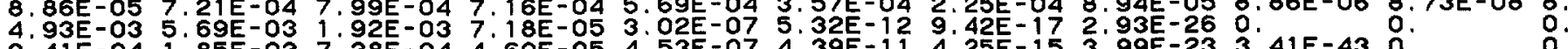

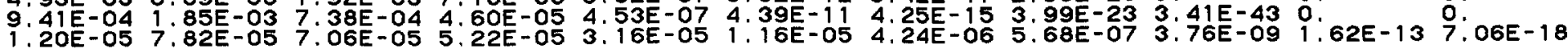

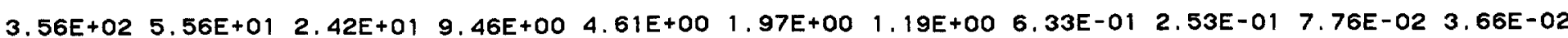

TOTAL 

MRACTIOT OF REFRACTERIES PRESENT $=0.100$ RELAXATION LENGTH $=0$. 16 GM SQCM
BOMB FRACTI ON PER SQ. METER $=4.652 E-13$

DEBRIS DECAY FROM 1 TO 50 YEARS

ZERO TIME $1.00 E+001.50 E+00 \quad 2.00 E+00 \quad 3.50 E+00 \quad 5.00 E+00 \quad 7.00 E+00 \quad 1.00 E+012.00 E+01 \quad 3.50 E+01 \quad 5.00 E+01$

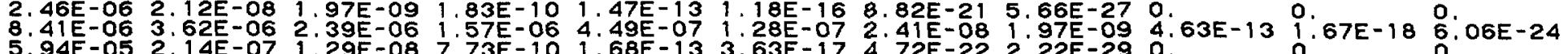

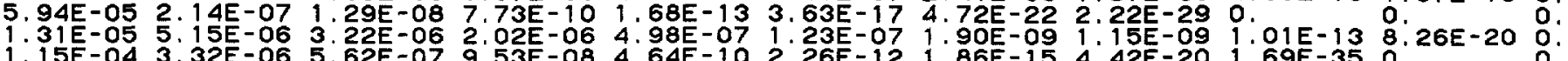

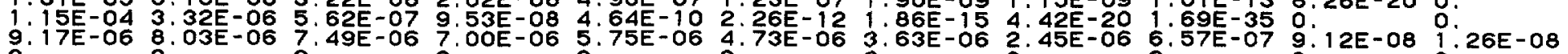

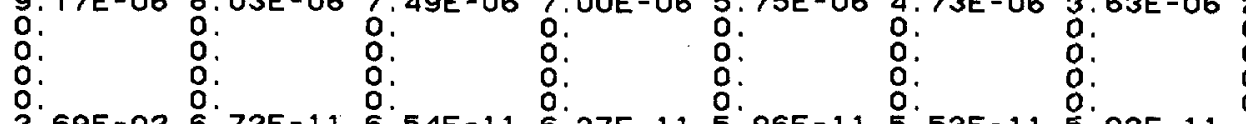

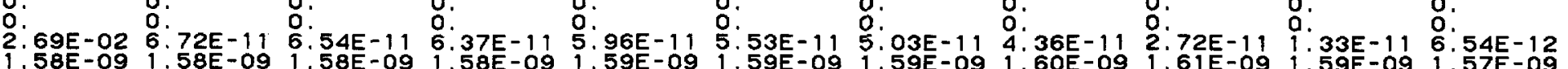

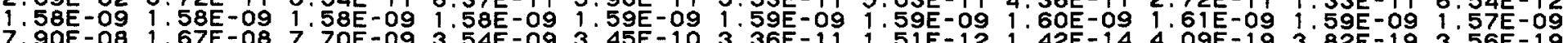
5.72E-06 1.42E-04 1.38E-04 1.33E-04 1.21E-04 1.10E-04 9.64E-05 7.95E-05 4.20E-05 $1.60 E_{-05} 6.13 E-06$

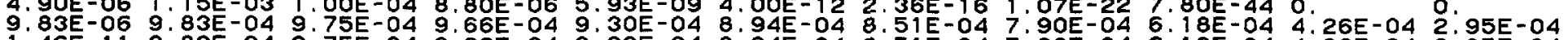
$1.46 E-11$ 9.83E-04 9.75E-04 9.66E-04 9.30E-04 8.94E-04 8.51E-04 7.90E-04 6. 18 E-04 4.26E-04 $2.95 \mathrm{E}-04$

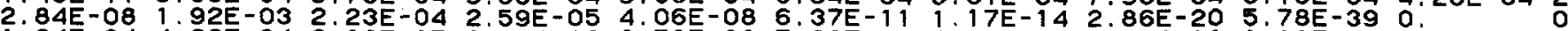

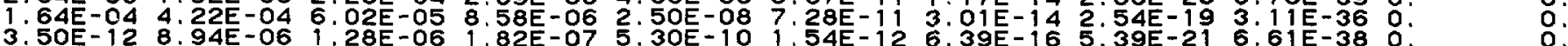

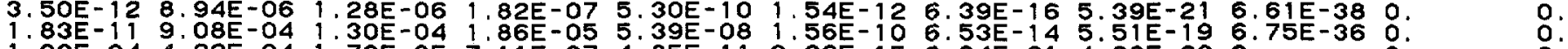

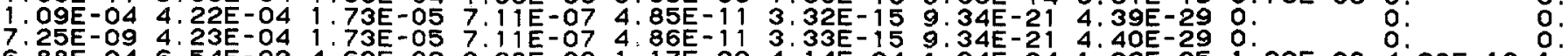

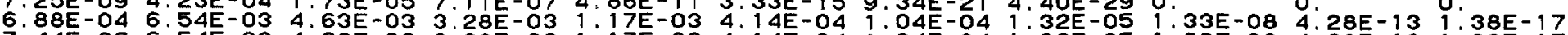

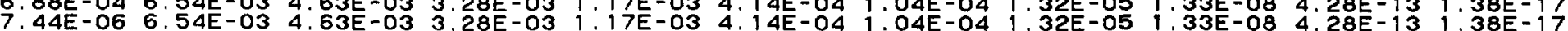

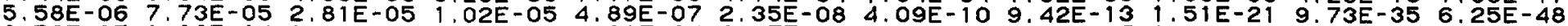
9.52E-05 1.92E-04 1.68E-04 1.48E-04 1.01E-04 6.87E-05 4.11E-05 1.90E-05 1.46E-06 3.11E-08 6.62E-10

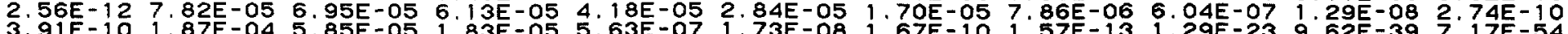

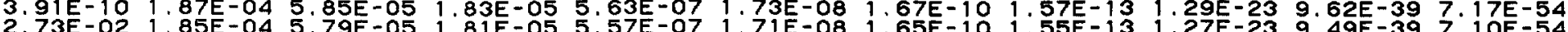

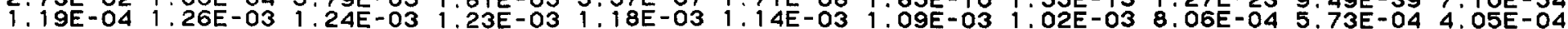

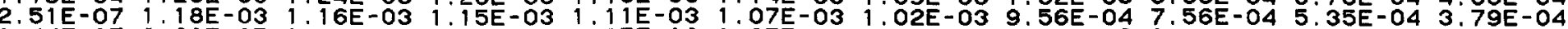

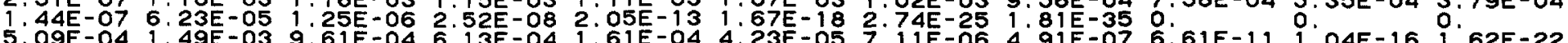

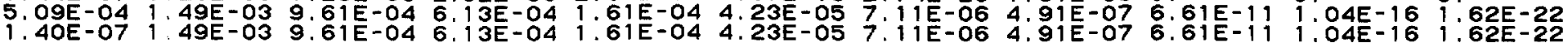

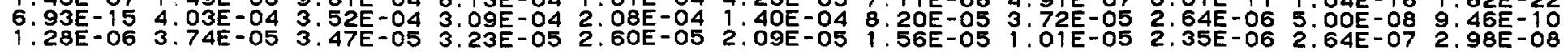


APPENDIX 8

\section{ANNIE EVENT}

$\mathrm{mR} / \mathrm{hr}$

$\begin{array}{lr}\text { Fraction of refractories present }=1.0 & \frac{\text { Page }}{8-1} \\ \text { Fraction of refractories present }=0.5 & 8-11 \\ \text { Fraction of refractories present }=0.1 & 8-21\end{array}$


DEBRIS DECAY FROM 1 TO 21 HOURS

ZERO TIME $1.00 E+00 \quad 2.00 E+00 \quad 3.00 E+00 \quad 4.00 E+00 \quad 6.00 E+00 \quad 9.00 E+001.20 E+01 \quad 1.50 E+01 \quad 1.80 E+012.10 E+01$

1. 27E-08 1. 26E-08 1.26E-08 1.26E-08 1.26E-08 1.26E-08 1.26E-08 1.26E-08 1.25E-08 1.25E-08 1. 25E-08

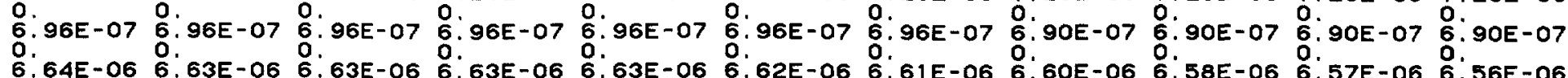

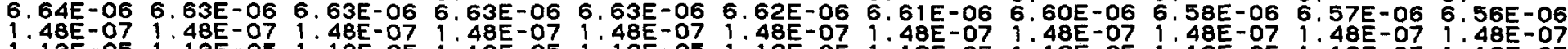

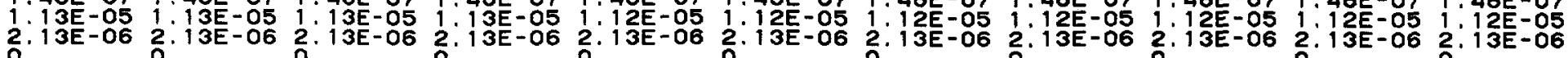
o: :.

::

:.

- 0 : 0 :. .46E-04 3. 44E-04 3. $43 E-04$ 3. $41 E-043.404-04$ 源

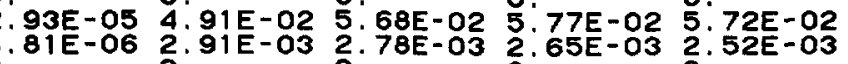

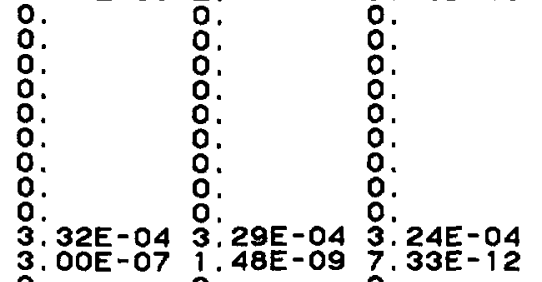
(1) $88 \mathrm{E}-12$ $88 E-12 \frac{2}{0} .88 E-12 \frac{0}{2} .88 E-12 \frac{0}{0}$ T.

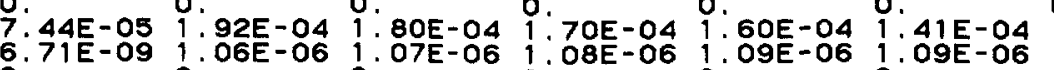

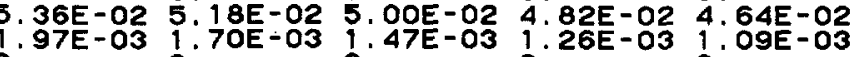
.71E-09 1.06E-06 1:.07E-06 1: O8E-06

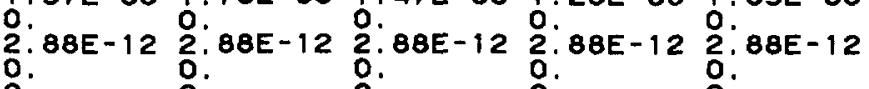
O. $76 \mathrm{E}-03$ O. $73 \mathrm{E}-03$ O. $07 \mathrm{E}-03 \mathrm{O} .72 \mathrm{E}-04 \mathrm{O} . \mathrm{O}$ 17 -04 9.76E-05 8. $13 E-05$ 5. $75 E-05$ 5:.62E-05

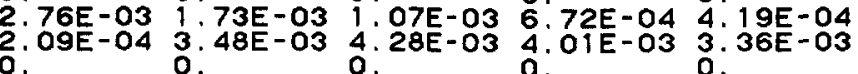
:: :. 0.
$:$
0
0

60E-05 1.57E-05 O. $54 E-05$ O.: 3. $63 \mathrm{E}-05$ .09E-06 $1.09 \mathrm{O}-06$

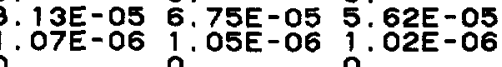
O. O2E-04 3
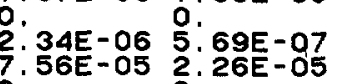

O. $38 \mathrm{E}-07$ . 44 - 44 7. 05 26E-05 6.55E-06 o. :: 26E-05 : $19 \mathrm{O}^{-05}$ :: $\quad:$ $12 \mathrm{E}-\mathrm{OS}$ :
$1: 06 E-05$
$8.26 E-16$
$27 E-06$

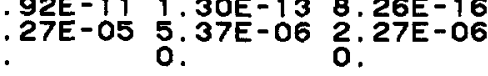




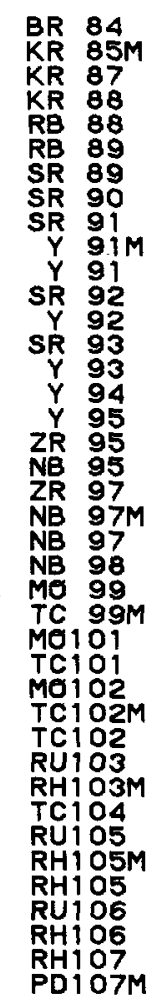

ANNIE $M R / H R$

FRACTION OF REFRACTERIES PRESENT a 1.000

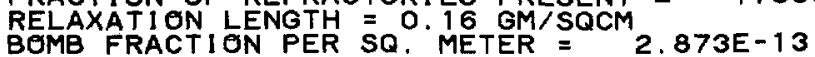

DEBRIS DECAY FROM 1 TO 21 HEURS

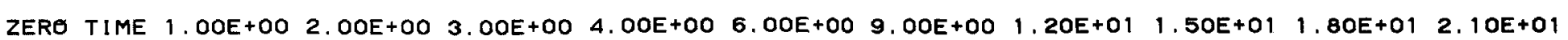

1.77E-03 3.06E-01 8.25E-02 2.24E-02 6. 04E-03 4. 42E-04 8.73E-06 1.72E-07 3.41E-09 6.74E-11 1.33E-12

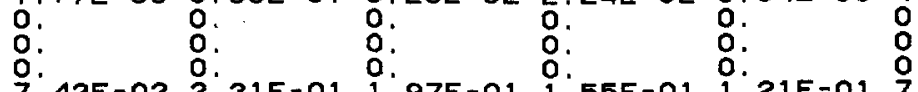

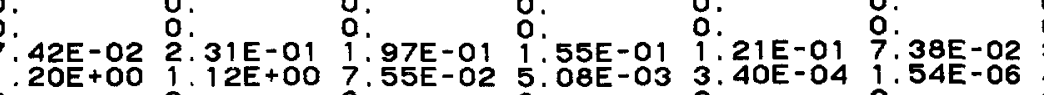

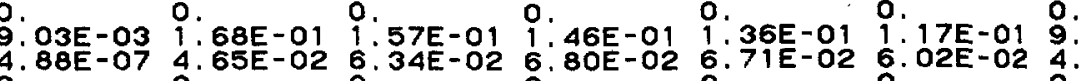

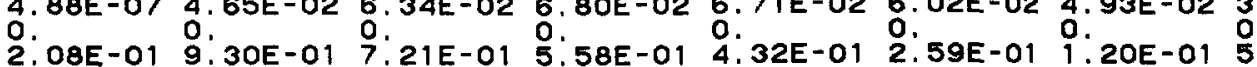

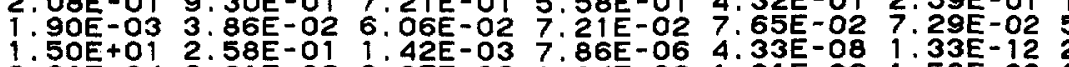

(.SOE

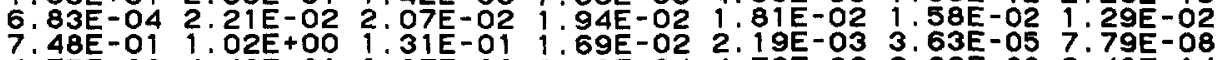

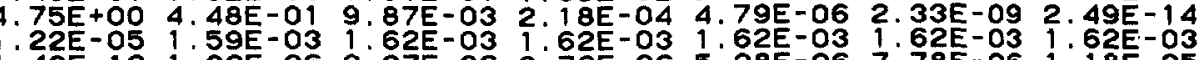

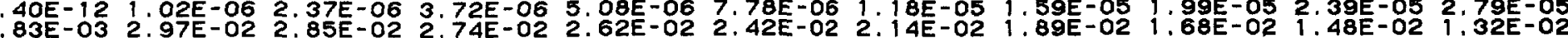

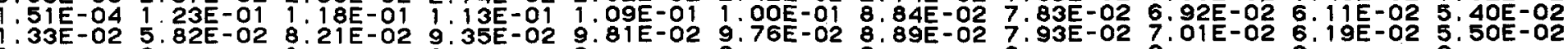

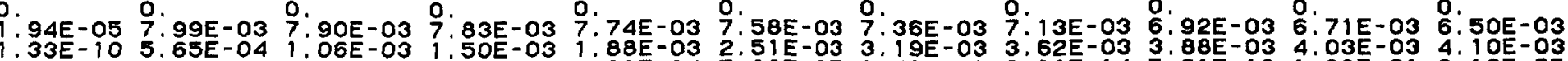
1.

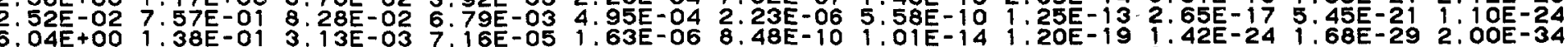

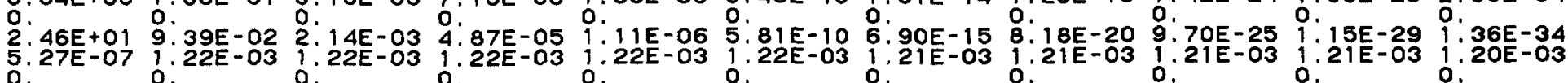

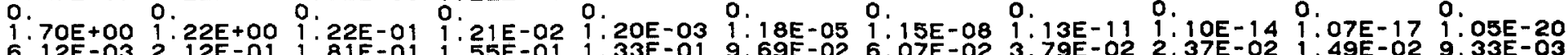
源

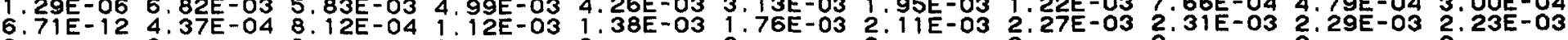

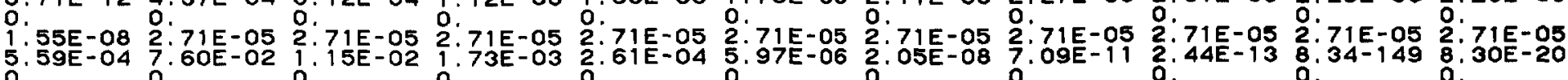


ANNIE MR/HR AT H+12 HOURS $=$ MR/HR 1,000

FRACTION OF REFRACTORIES PRESENT $=1,000$

RELAXATION LENGTH $=0.16$ GM/SOCM $2.873 E-13$

DEBRIS DECAY FROM 1 TO 21 HOURS

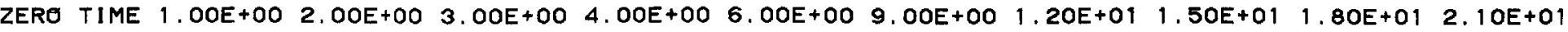

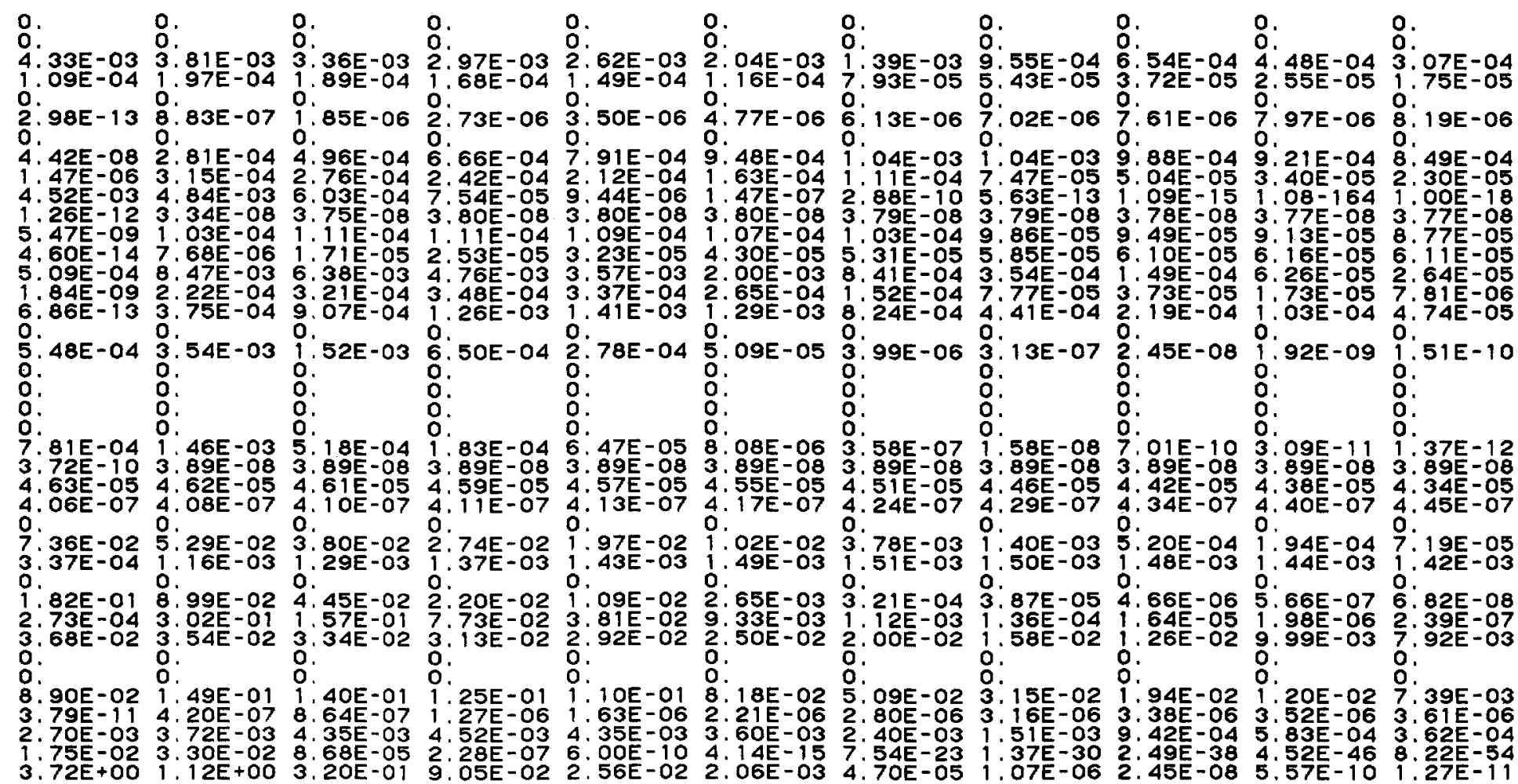


ANNIE MR $/ H+12$ HOURS $=$ MR/HR

FRACTION OF REFRACTORIES PRESENT $=1.000$

RELAXATION LENGTH =0. 16 GM/SQCM $2.873 E-13$

DEBRIS DECAY FROM 1 TO 21 HEURS

ZERO TIME 1.OOE+OO 2. $2.00 E+00 \quad 3.00 E+00 \quad 4.00 E+00 \quad 6.00 E+00 \quad 9.00 E+00 \quad 1.20 E+01 \quad 1.50 E+01 \quad 1.80 E+01 \quad 2.10 E+01$

sisi3i

TE131M

131

TE132

TE133M

$I 133$
$\times E 133 M$

XE133

TE 134

XE135

XE135M

CS 136

CS137

XE138

CS138

CS139

BA139

BA 140

LA1 41

BE1 41

LA 142

CA143

PRi43

CEI 44

PR144

PR145

CE1 46
PR1 46
PR1 47

6. 98E-04 6.6OE-04 6.25E-04 5.90E-04 5.59E-04 5. O0E-04 4.22E-04 3.57E-04 3.02E-04 2.56E-04 2.16E-04

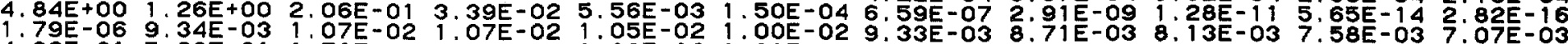
$4.82 E-01$ 5.23E-01 1.70E-01 4.43E-02 1.08E-02 1.01E-03 5. 02E-04 4.64E-04 4.33E-04 4.05E-04 3.78E-03 6.71E-05 2.33E-03 3.42E-03 3.75E-03 3.82E-03 3.85E-03 3.85E-03 3.82E-03 3.82E-03 3.79E-03 3.79E-03 2.83E-03 $6.88 E-03$ 6.82E-03 $6.76 E-03$ 6.69E-03 6.57E-03 6.40E-03 6.23E-03 6.08E-03 $5.91 E-035.75 E-03$

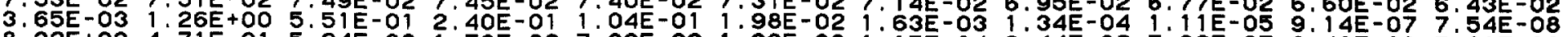
$8.32 E+00$ 4.71E-01 5.24E-02 1.73E-02 7.33E-03 1.39E-03 1.15E-04 9.44E-06 7.82E-07 6.43E-08 5.31E-09 8.83E-03 9.51E-02 1.03E-01 1.03E-01 1.01E-01 9.56E-02 8.69E-02 $7.87 E-02$ 7.82E-02 6.43E-08 5.31E-09

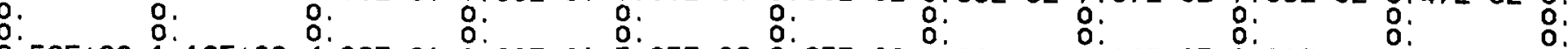

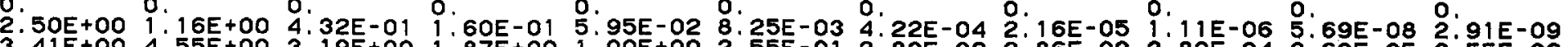

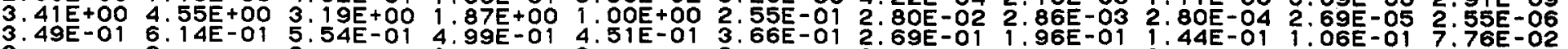
O.

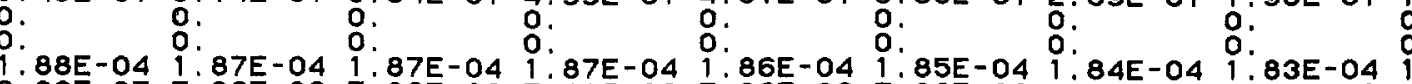

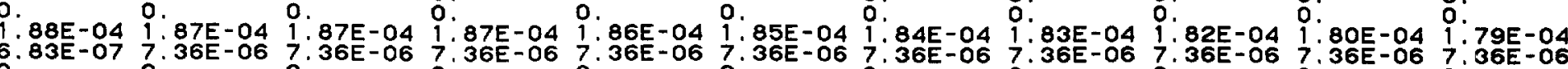

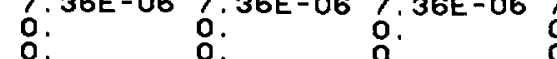

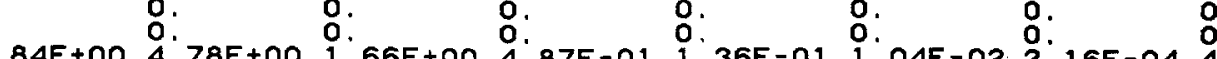

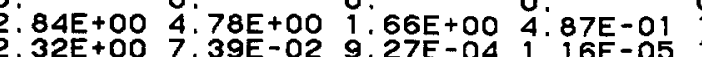

$5.71 E-03$ 6. 36E-02 $3.92 E-02$ 2.38E-02

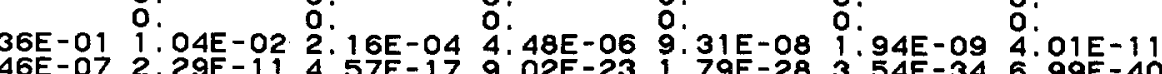
2.48E-04 1.55E-03 1.55E-03 1.53E-03 1.53E-03 1.52E-03 1.52E-03 $1.51 E-03$ 1.5OE-03 $1.49 E-03 \quad 1.47 E-03$ $2.60 E+00 \quad 7.85 E-01 \quad 7.79 E-02 \quad 7.72 E-03 \quad 7.66 E-04 \quad 7.60 E-06 \quad 7.41 E-097.23 E-12 \quad 7.04 E-15 \quad 6.92 E-18$ 6.74E-21

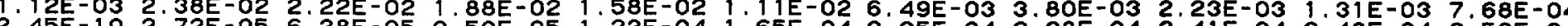
2. $45 E-1002.72 E-056.38 E-05$ 9.59E-05 1.23E-04 1.65E-04 2.05E-04 2.28E-04 2.41E-04 2.48

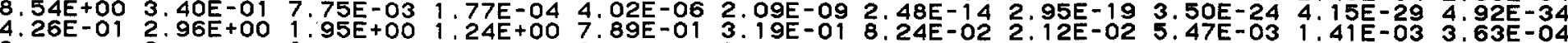

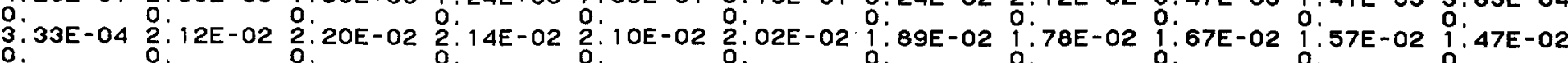

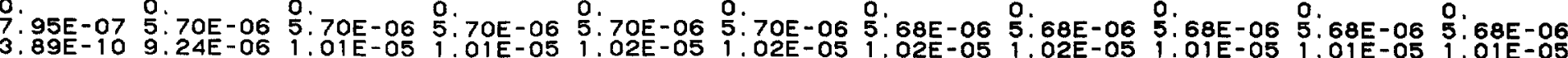

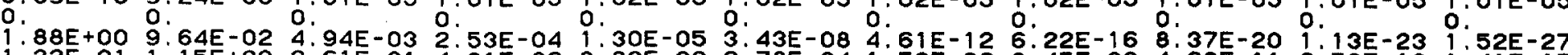

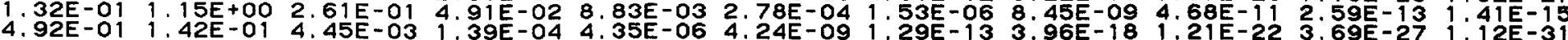


ANNIE AT H+12 HOURS $=$ MR/HR

MRACTION OF REFRACTERIES PRESENT $=1.000$

RELAXATION LENGTH $=0.116$ GM SQCM
BOMB FRACTION PER SQ. METER $=2.873 E-13$

DEBRIS DECAY FROM 1 TO 21 HOURS

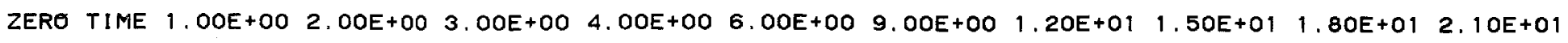

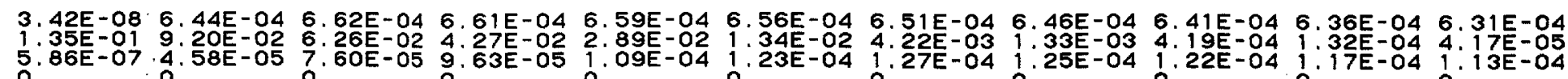

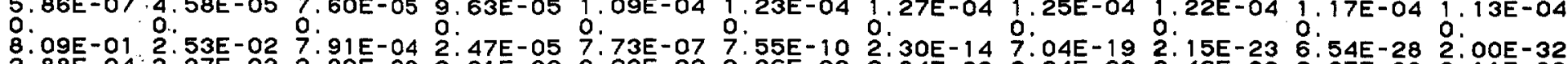

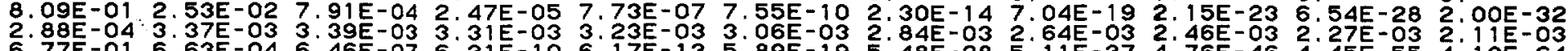

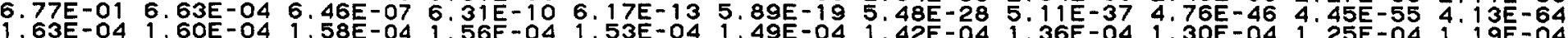
1.67E-02 2:75E-03 4.50E-04 7.36E-05 1.21E-05 3.25E-07 1:43E-09 6.31E-12 2:78E-14 1:24E-16 3:05E-19

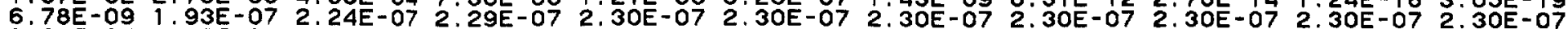
3.29E-04 3.06E-04 2.84E-04 2.64E-04 2.45E-04 2.11E-04 $1.69 E-04$ 1.36E-04 1.09E-04 8.73E-05 $6.98 E-05$

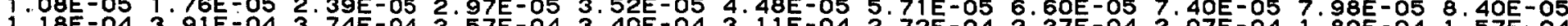

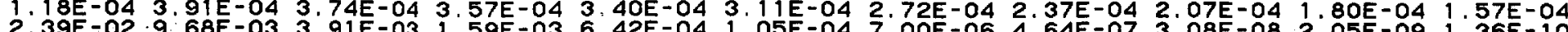
2.

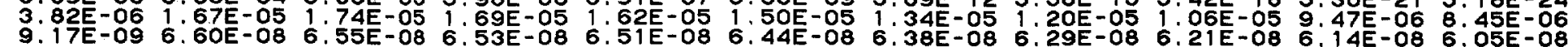

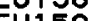

TB159

TOTAL

$\begin{array}{llllllll}1.06 E+02 & 3.16 E+01 & 1.27 E+01 & 6.94 E+00 & 4.43 E+00 & 2.37 E+00 & 1.39 E+00 & 1.00 E+00 \quad 7.84 E-01 \quad 6.43 E-01 \quad 5.42 E-01\end{array}$ 


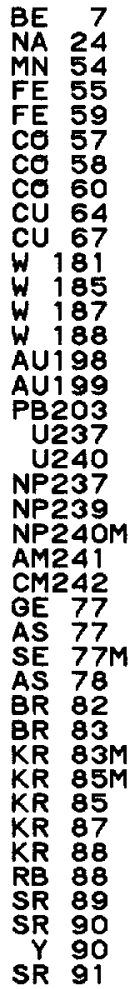

ANNIE MR/HR AT H+12 HOURS $=$ MR/HR

FRACTION OF REFRACTORIES PRESENT $=1.000$

RELAXATI ON LENGTH $=0.16$ GM SOCM

$2.873 E-13$

DEBRIS DECAY FROM 1 TO 300 DAYS

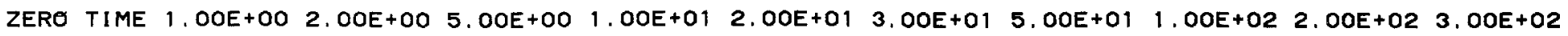
1.27E-08 1.25E-08 1.23E-08 1.19E-08 1.11E-08 9.76E-09 8.57E-09 6.59E-09 3.45E-09 9.37E-10 2.55E-10

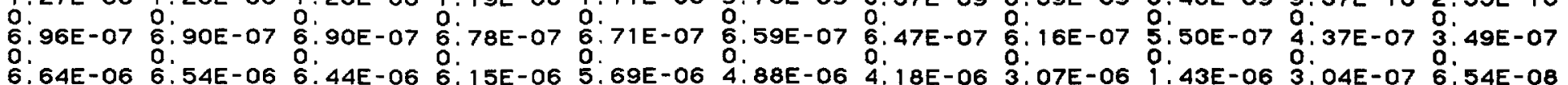

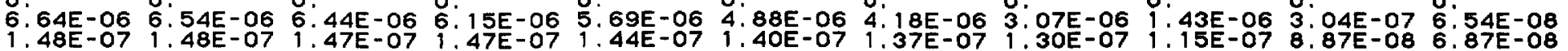

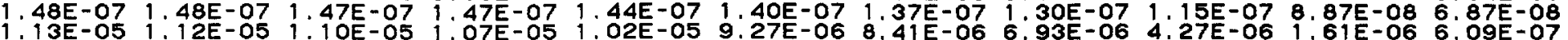
2:13E-06 2:13E-06 2:13E-06 2:13E-06 2.12E-06 2.12E-06 2.11E-06 2.10E-06 2.27E-06 1:.98E-06 $1.91 \mathrm{E}-06$

$\begin{array}{llll}0: & 0 & 0 & 0 \\ 0: & 0 & 0 & 0 \\ 0 & 0 & 0 & 0\end{array}$

:.

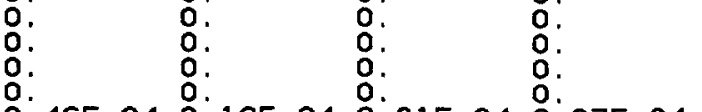

3. $46 \mathrm{E}-04$ 3. $12 \mathrm{E}-04$ 2.81E-04 2:07E-04

.

$93 \mathrm{E}-05$.

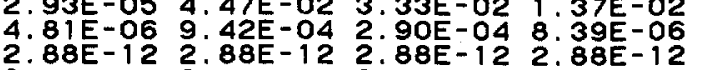

$\begin{array}{llll} & \\ 2 & & \end{array}$

7.44E-05 4.69E-05 1.07E-05 1. 30E-07 8.24E-11 3.34E-17 1.35E-23 $2.20 E-36$

2.09E-04 $1.86 E-06$ 5: $46 E-11$ 0. $61 E-250$

0 . 0.

a.

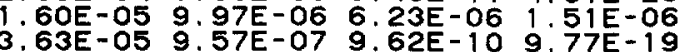

$\begin{array}{llll}0 & 0 \\ 0 & 0 & 0 & 0 \\ 0 & 0 & 0 & 0 \\ 0 & 0 & 0 & 0\end{array}$

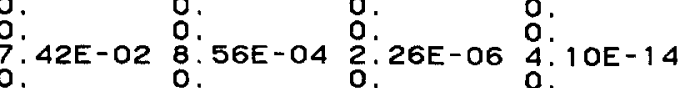

0

O. $03 E-03$ O.

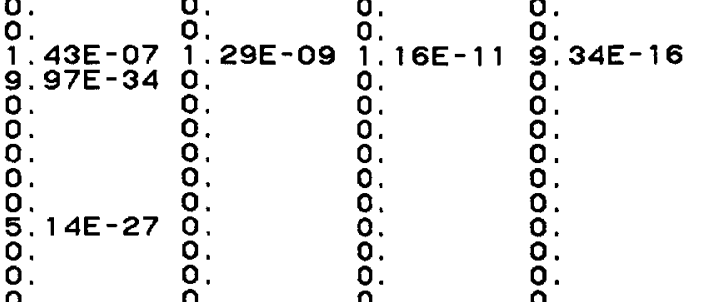

0
0
0
0
0
0
0
0
1
0
0
9
0
0
0
0
0
0
5
0
0
0
0
0
0
0
0

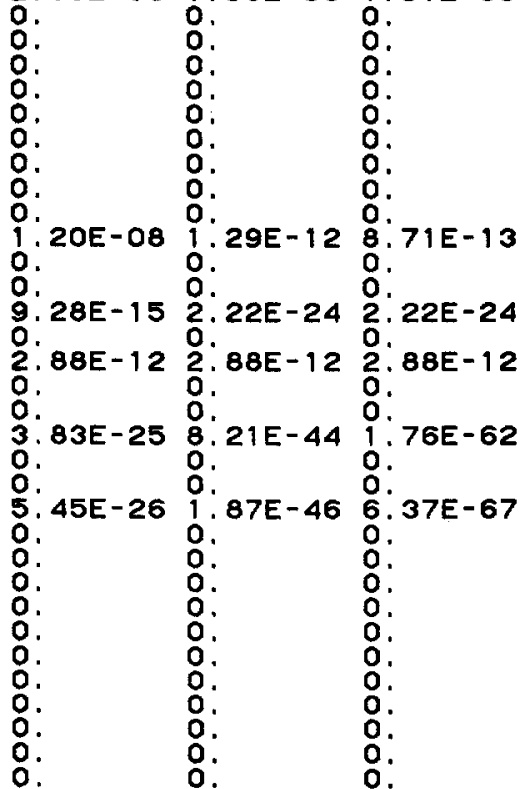


ANNIE

$M R / H R$

MRAHR AT H+12 HOURS $=$ MR 1 OODO

RELAXATION LENGTH $=0.16$ GM SQCM $=1.000$

DEBRIS DECAY FROM 1 TO 300 DAYS

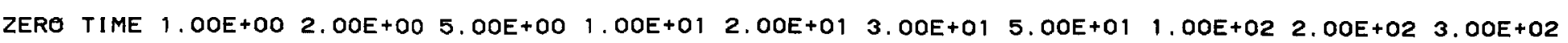

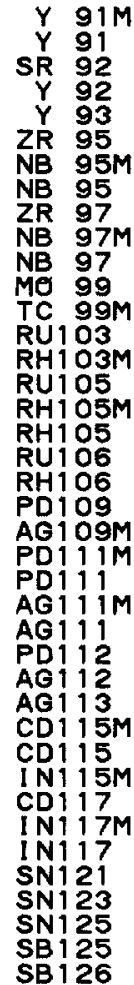

4. 88E-07 1.68E-02 3.00E-03 1.72E-05 3.17E-09 1.07E-16 3.61E-24 4.12E-39 0.

2. 08 -01 $2.60 E-03 \quad 5.60 E-065.63 E-14$ 2.64E-27:

$1.90 E-03$ 5.38E-03 5.97E-05 4.59E-11 2.68E-21 9.16E-42 3.14E-62

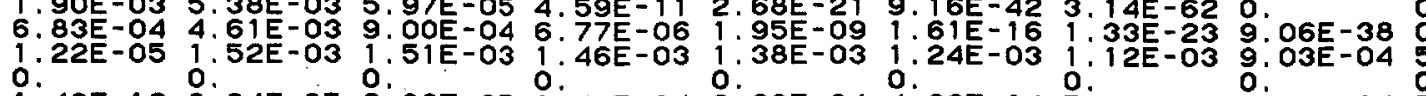

6. $40 \mathrm{E}$

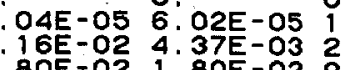

$44 E-04$ (24E-03 1.

(1)

. $33 \mathrm{E}-02$ 4.55E-02 1.71E-02 9.08E-04 6.83E-06 $4.11 \mathrm{E}-10$ 2.31E-14 $7.33 \mathrm{E}-23 \quad 4.13 \mathrm{E}-44$

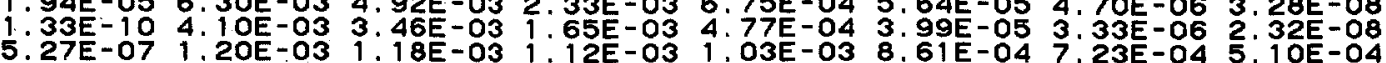

5.27E-07 1. $20 \mathrm{E}-03$ 1. $18 \mathrm{E}-03$ 1:12E-03 1.03E-03 8:61E-04 ?:23E-04 5.

$\begin{array}{llllllll}6.12 E-03 & 5.85 E-03 & 1.37 E-04 & 1.81 E-09 & 1.32 E-17 & 7.05 E-34 & 3.78 E-50 & 0 \\ 1.29 E-06 & 1.88 E-04 & 4.43 E-06 & 5.83 E-11 & 4.26 E-19 & 2.28 E-35 & 1.22 E-51 & 0\end{array}$

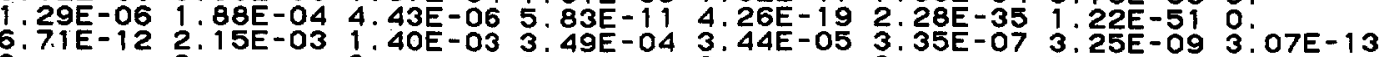

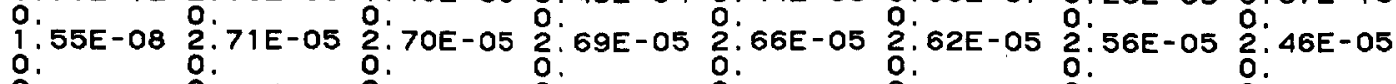

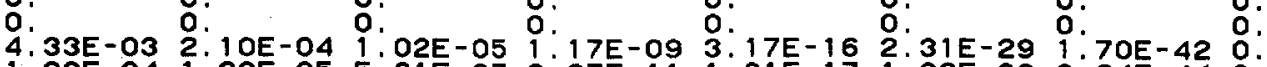

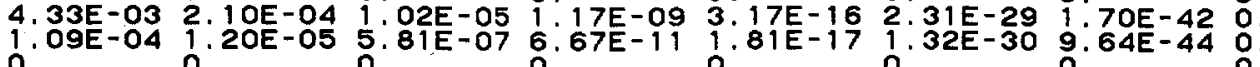

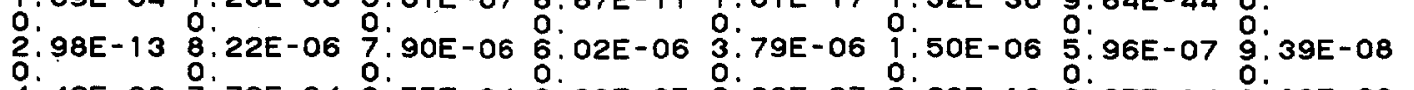

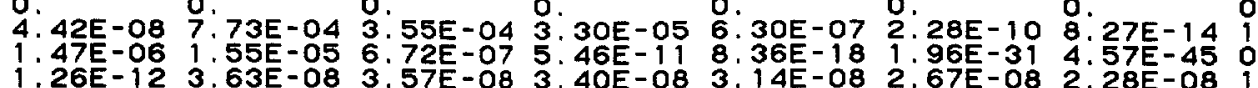

$1.26 E-12$ 3.63E-08 3.57E-08 3.40E-08 3.14E-08 2.67E-08 2.28E-08 $1.65 E-08$

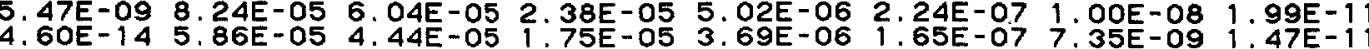

.09E-04 1.11E-05 1.08E-08 1.01E-17 8.94E-33 :. O. O

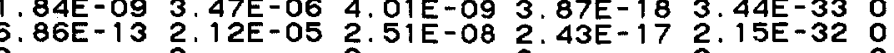

O.

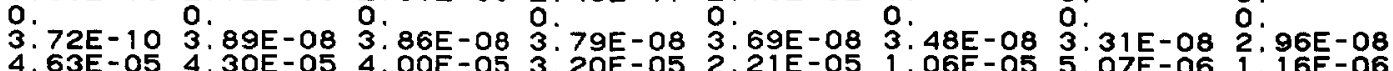

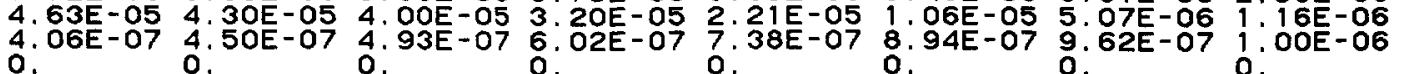

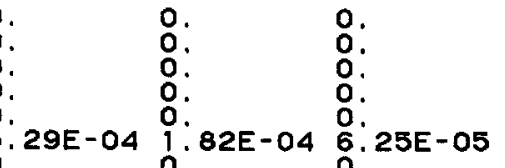
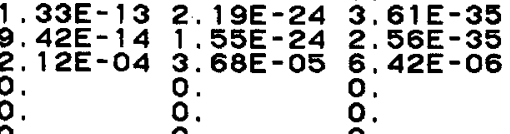

. $660-23$ :.
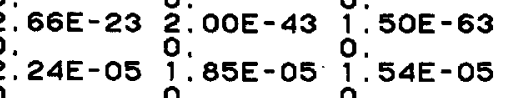

O.

::

$24 E-10$ 8. $95 E-14$ 8. $68 E-18$

$.84 E-38$ : :

(36)-09 $\begin{array}{lll}3.5 E-18 & 1: 46 E-09 & 2.92 E-10 \\ 10 E-31 & 3.45 E-45\end{array}$ :

$\begin{array}{ll}0 & 0 \\ 2.24 E-08 & 1: 29 E-08\end{array}$ .84E-07 $9: 18 E-07$ 8.57E-07 
ANNIE

MR/HR

FRACTI ON OF OF REFACTORIES PRESENT $=1.000$

RELAXATI ON LENGTH $=0.16$ GM/SQCM $2.873 E-13$

DEBRIS DECAY FROM 1 TO 300 DAYS

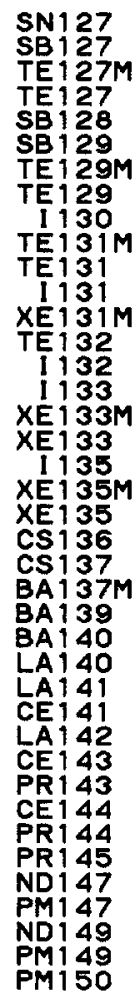

ZERO TIME 1. OOE+OO $2.00 E+00 \quad 5.00 E+00 \quad 1.00 E+01 \quad 2.00 E+01 \quad 3.00 E+01 \quad 5.00 E+01 \quad 1.00 E+02 \quad 2.00 E+02 \quad 3.00 E+02$

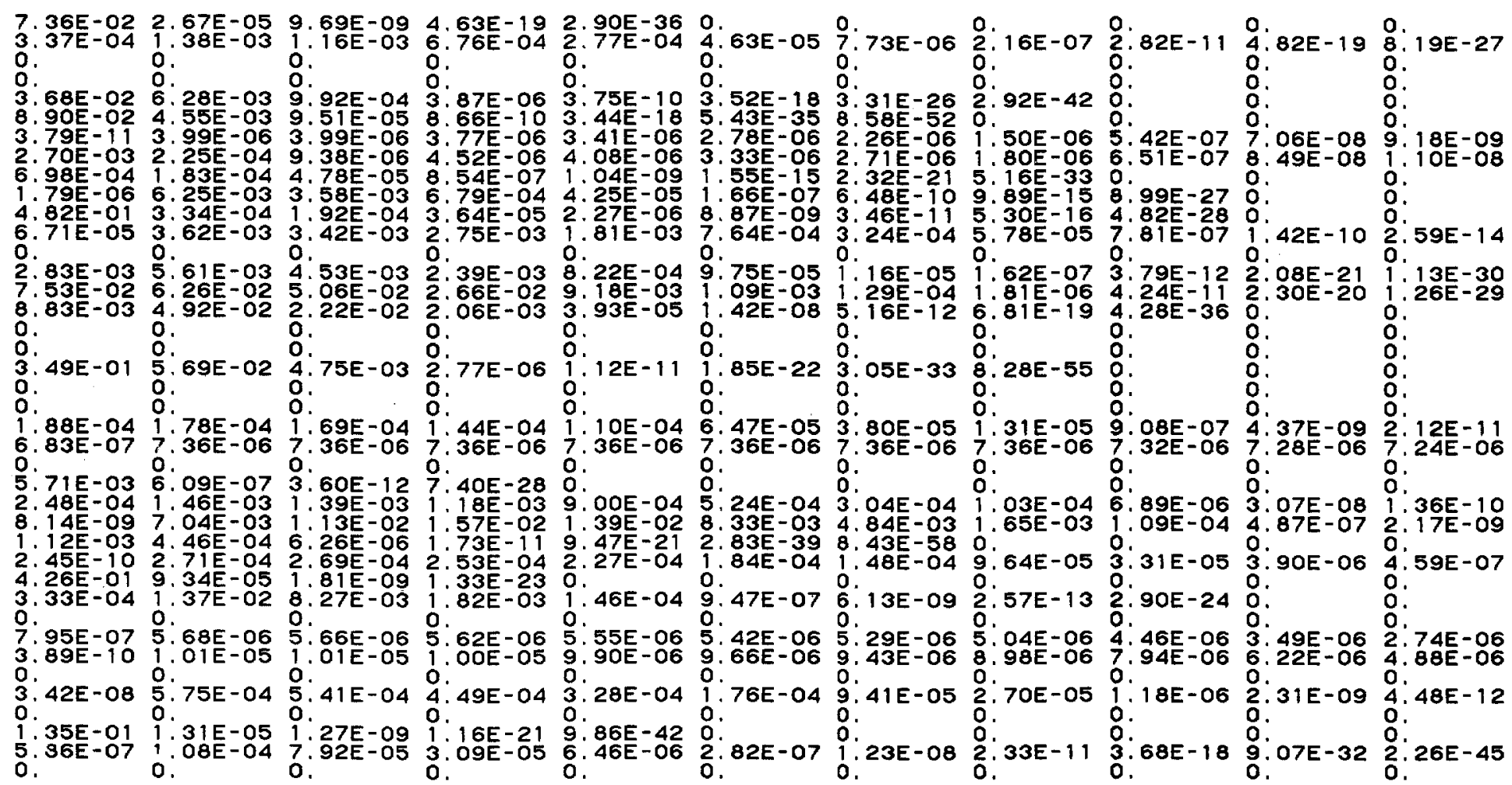


ANNIE

MR/HR

MRACTIOT H+12 HOURS $=$ MF TERT POO

BELAXATION LENGTH $=$ O. 16 GM SQCM $2.873 E-13$

DEBRIS DECAY FROM 1 TO 300 DAYS

ZERO TIME $1.00 E+002.00 E+00 \quad 5.00 E+00 \quad 1.00 E+01 \quad 2.00 E+01 \quad 3.00 E+01 \quad 5.00 E+01 \quad 1.00 E+02 \quad 2.00 E+02 \quad 3.00 E+02$

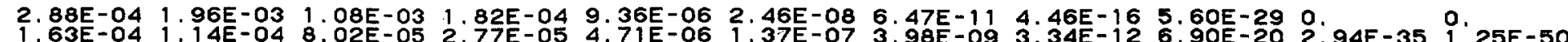

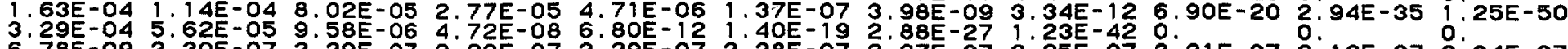

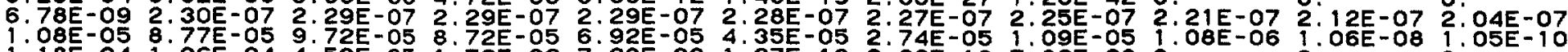

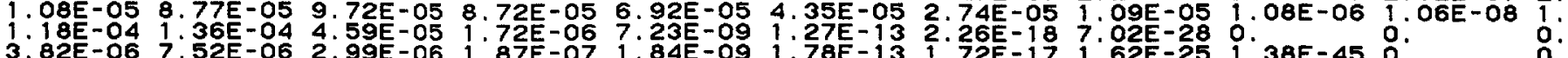

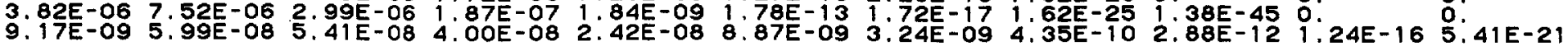




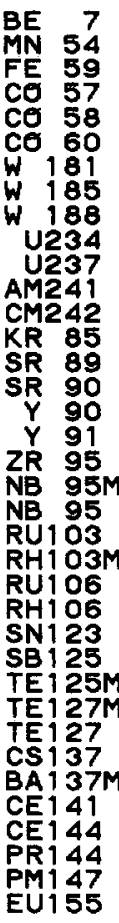

TOTAL
ANNIE

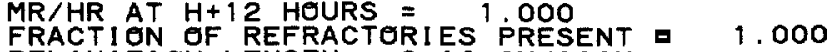

RELAXATION LENGTH $=0.16$ GM SOCM

BEMB FRACTI ON PER SQ. METER $=2.873 E-13$

DEBRIS DECAY FROM 1 TO 50 YEARS

ZERO TIME 1.OOE+OO 1.5OE+OO 2. OOE+OO 3.5OE+OO $5.00 E+00 \quad 7.00 E+O 0 \quad 1.00 E+01 \quad 2.00 E+01 \quad 3.50 E+01 \quad 5.00 E+01$

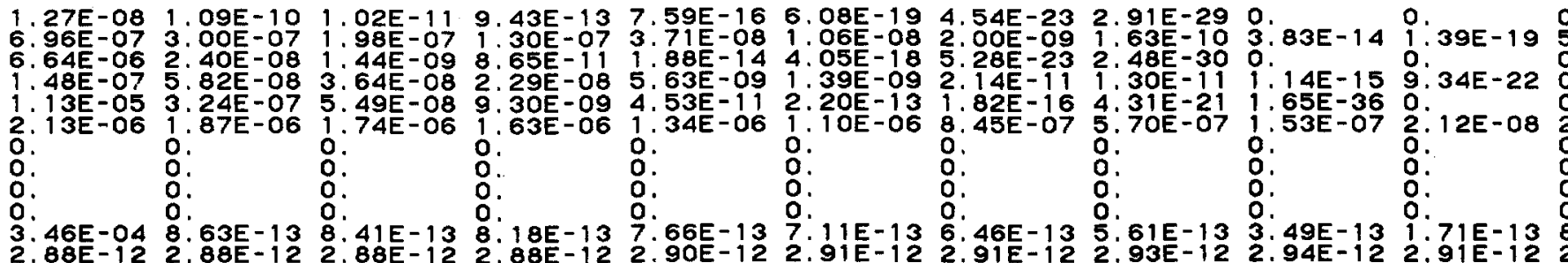

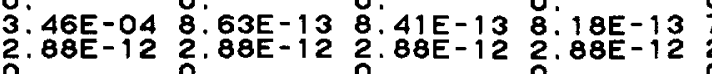

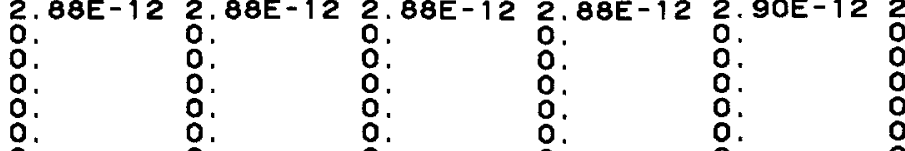

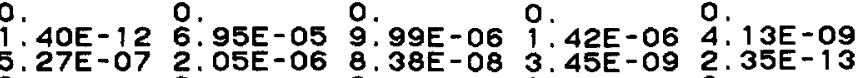

O.

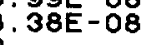

55E-08 O. $36 \mathrm{E}-05$ O. $63 \mathrm{E}-06$ O. $33 \mathrm{E}-0 \mathrm{O}^{\circ}$

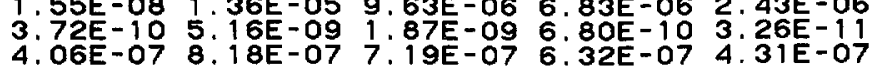
$4.06 E-07$

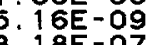

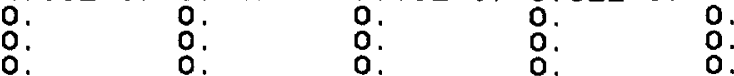

6. 83E-07 7.20E-06 7..11E-06 7.03E-06 6. 06 EE-06

:.:

39E- 12 2. 23E- 15

. 2OE-11 $5.00 E-15$ 4. $22 E-20$

. $61 \mathrm{E}-174.53 \mathrm{E}-232.13 \mathrm{E}-31$

O.

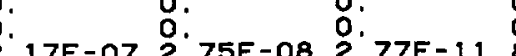

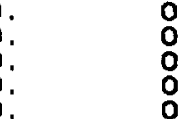

0
0
0
0

.

:

$01 E-25$

$93 E-09$ .

$41 E-14$
$87 E-12$

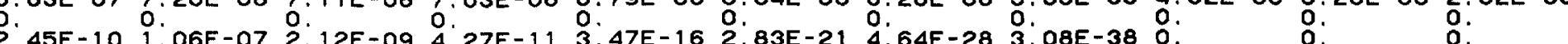

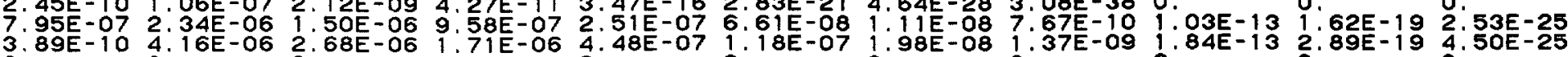
6.78E-09 1.98E-07 1.84E-07 1.72E-07 1. 38E-07 1. 11E-07 8.27E-08 5.34E-08 $1.25 E-08$ 1. $40 E-09$ 1.58E-10

3.81E-04 1.34E-04 3.84E-05 2.12E-05 1.19E-05 9.10E-06 7.61E-06 6.58E-06 4.79E-06 3.31E-06 2.32E-06 
ANNIE MR/HR AT $H+12$ HOURS $=$ MR/HR

FRACTION OF REFRACTORIES PRESENT $=0.500$

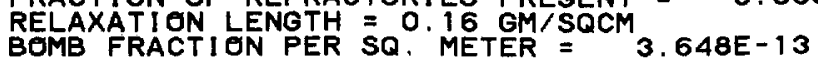

DEBRIS DECAY FROM 1 TO 21 HEURS

ZERO TIME $1.00 E+00 \quad 2.00 E+00 \quad 3.00 E+00 \quad 4.00 E+00 \quad 6.00 E+00 \quad 9.00 E+00 \quad 1.20 E+01 \quad 1.50 E+01 \quad 1.80 E+012.10 E+01$

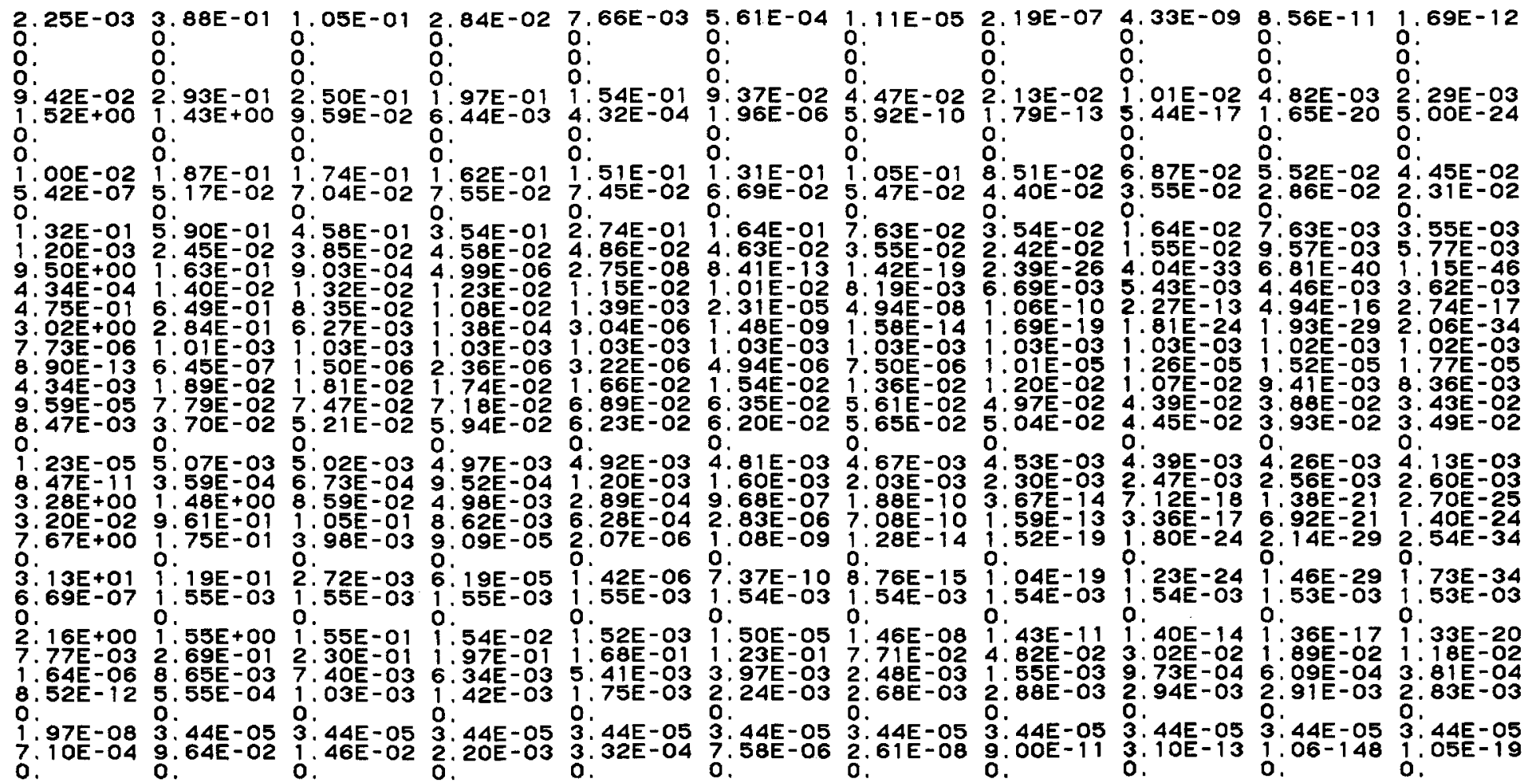




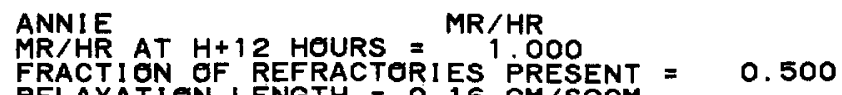

FRACTION OF RERACTORI ES PRESENT $=0.500$
RELAXATION LENGTH $=0.16$ GM SQCM
BOMB FRACTION PER SQ. METER $=38 \mathrm{3}-13$

DEBRIS DECAY FROM 1 TO 21 HOURS

ZERO TIME $1.00 E+00 \quad 2.00 E+00 \quad 3.00 E+00 \quad 4.00 E+00 \quad 6.00 E+00 \quad 9.00 E+00 \quad 1.20 E+01 \quad 1.50 E+01 \quad 1.80 E+012.10 E+01$

SB 130

TEI3IM

TEI31

I 132

TE 1333

1133

XE133

TE 34

1135
$\times E$
$\times 135 M$

XE

CSI36

BA137M

XE138

CS139

BA 40

BAI41

CA 141

BAi 42

LA 423

CEI 43

CER 144

PR 45

CE 1460

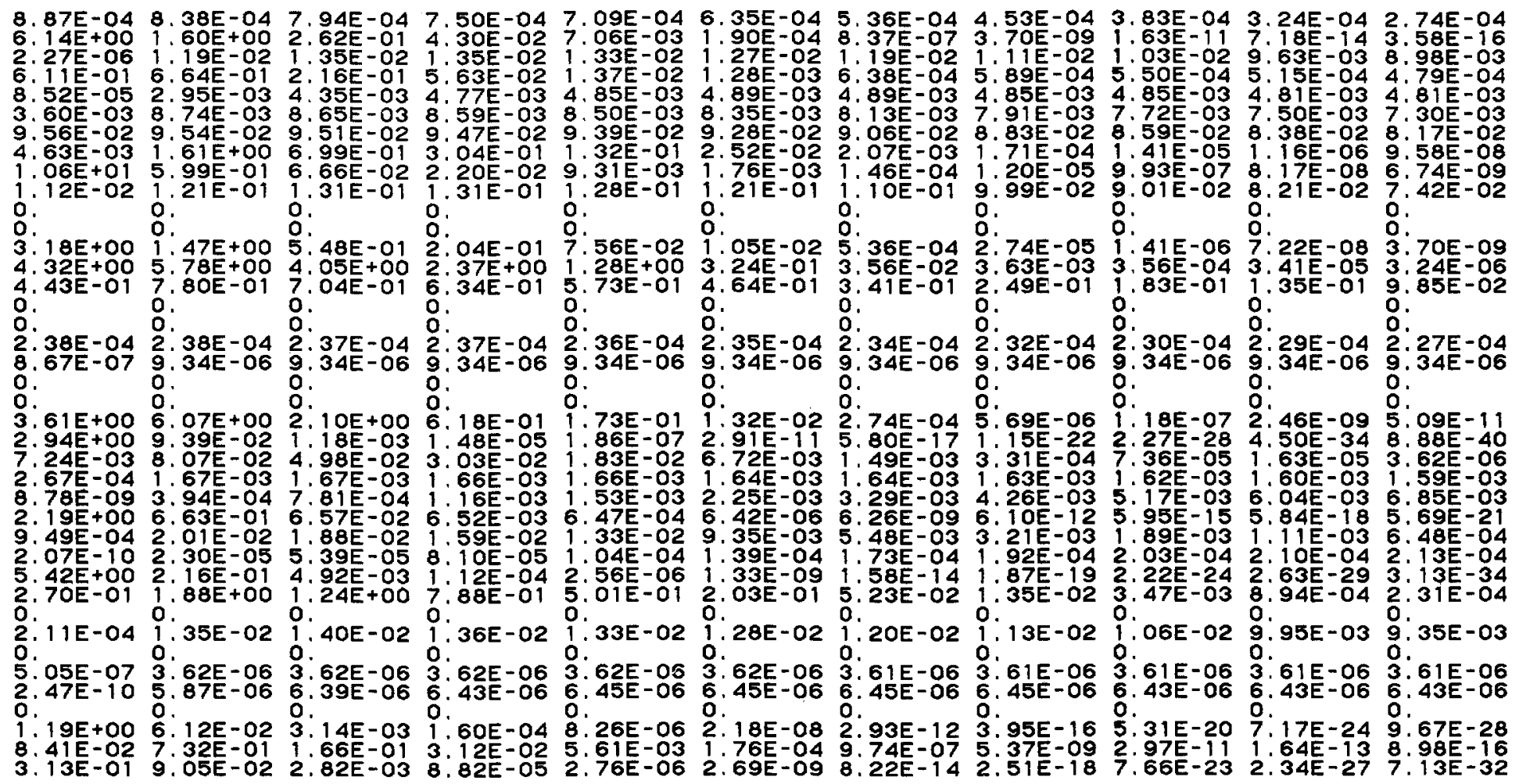

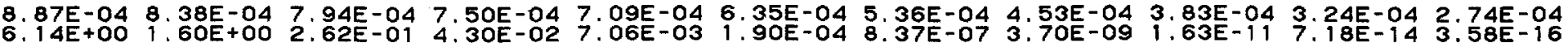

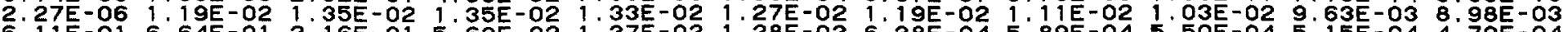
$9.56 E-02$ 9 (1.

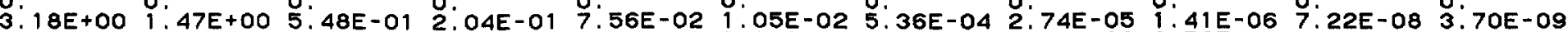
4. 32E+00 5.78E+00 4.05E+00 2.37E+00 1.28E+00 3.24E-01 3.56E-02 3.63E-03 3.56E-04 3.41E-05 3.24E-06 2.38E-04 2.38E-04 2.37E-04 2. $94 \mathrm{E}+0 \mathrm{O}$

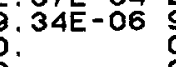

2.07E

2.7OE-01 $1.88 E+001.24 E+007.88 E-015.01 E-01$ 5. $45 \mathrm{E}-07$ 3. $32 \mathrm{E}-06$ 3.62E-06 $3.62 \mathrm{E}-06$

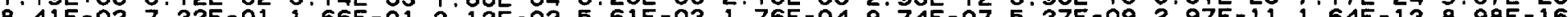
3.13E-01 9.05E-02 $2.82 E-03$ 8.82E-05 2.76E-06 2.69E-09 8.22E-14 2.51E-18 $7.66 E-232.34 E-277.13 E-32$ 
ANNIE $M$ MT H+12 HOURS $=$ MR/HR

FRACTION OF REFRACTORIES PRESENT $=0.500$

REIAXAT LN LENGTH

BOMB FRACTION PER SQ. METER $=3.648 E-13$

DEBRIS DECAY FROM 1 TO 21 HOURS

ZERO TIME $1.00 E+00 \quad 2.00 E+00 \quad 3.00 E+00 \quad 4.00 E+00 \quad 6.00 E+00 \quad 9.00 E+00 \quad 1.20 E+01 \quad 1.50 E+01 \quad 1.80 E+01 \quad 2.10 E+01$

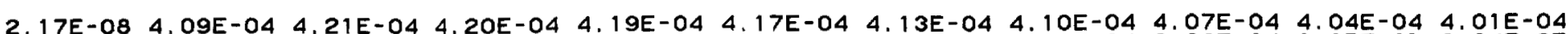

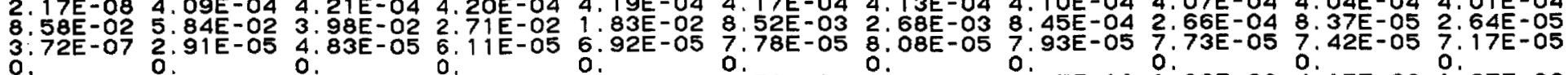

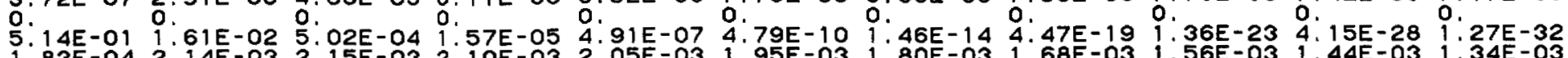

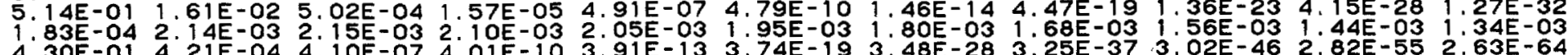

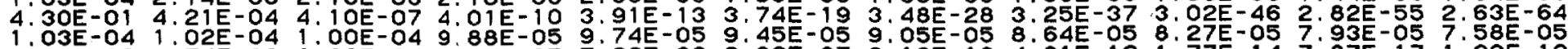

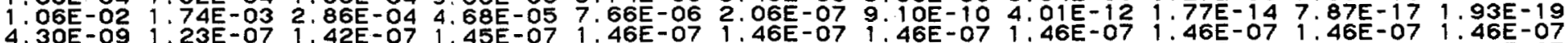

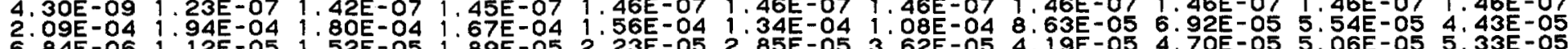
6.84E.06 192

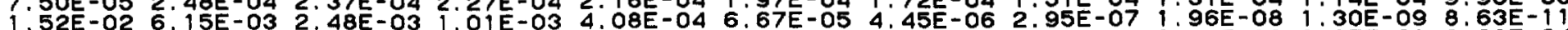
3.87E-03 3.84E-04 3.81E-05 3.78E-06 3.75E-07 3.70E-09 3.61E-12 3.53E-15 3. 44E-18 3.37E-21 3. 29E-24

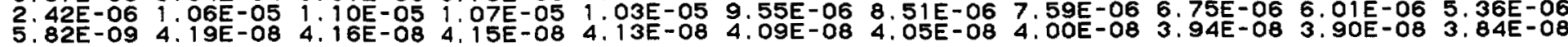

TOTAL

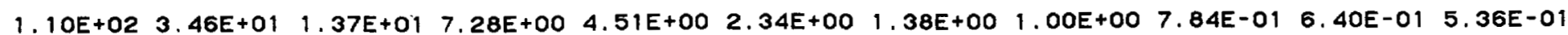


DEBRIS DECAY FROM 1 TO 300 DAYS

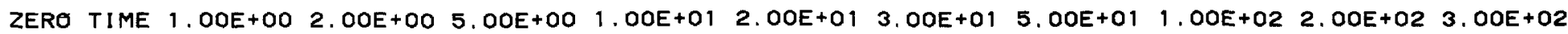

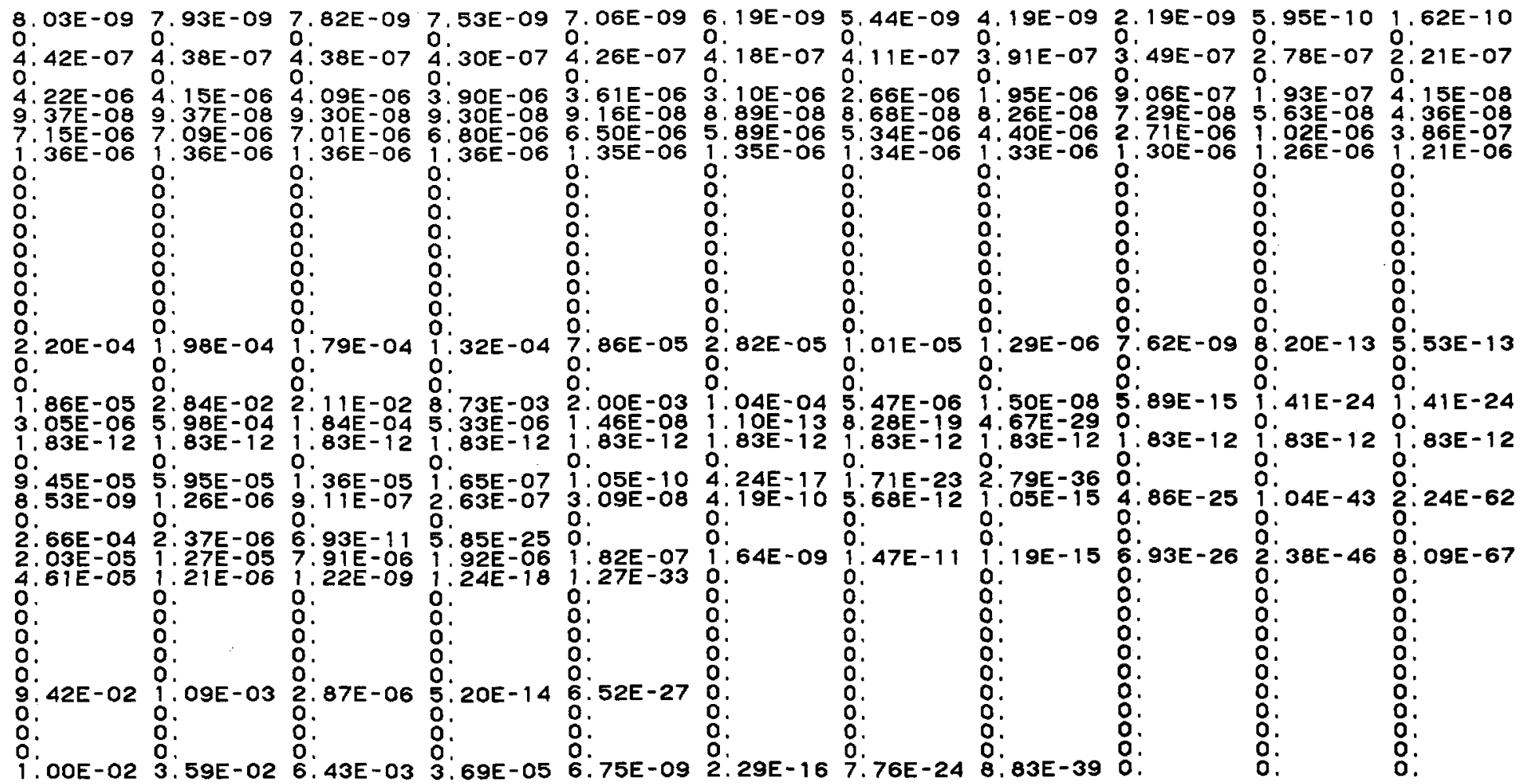


ANNIE AT H+12 HOURS $=$ MR/HR 1 HRO

FRACTION OF REFRACTERIES PRESENT $=0.500$

RELAXATI ON LENGTH $=0.16 \mathrm{GM} / \mathrm{SQCM}$

DEBRIS DECAY FROM 1 TO 300 DAYS

ZERO TIME $1.00 E+00 \quad 2.00 E+00 \quad 5.00 E+00 \quad 1.00 E+01 \quad 2.00 E+01 \quad 3.00 E+01 \quad 5.00 E+01 \quad 1.00 E+02 \quad 2.00 E+02 \quad 3.00 E+02$

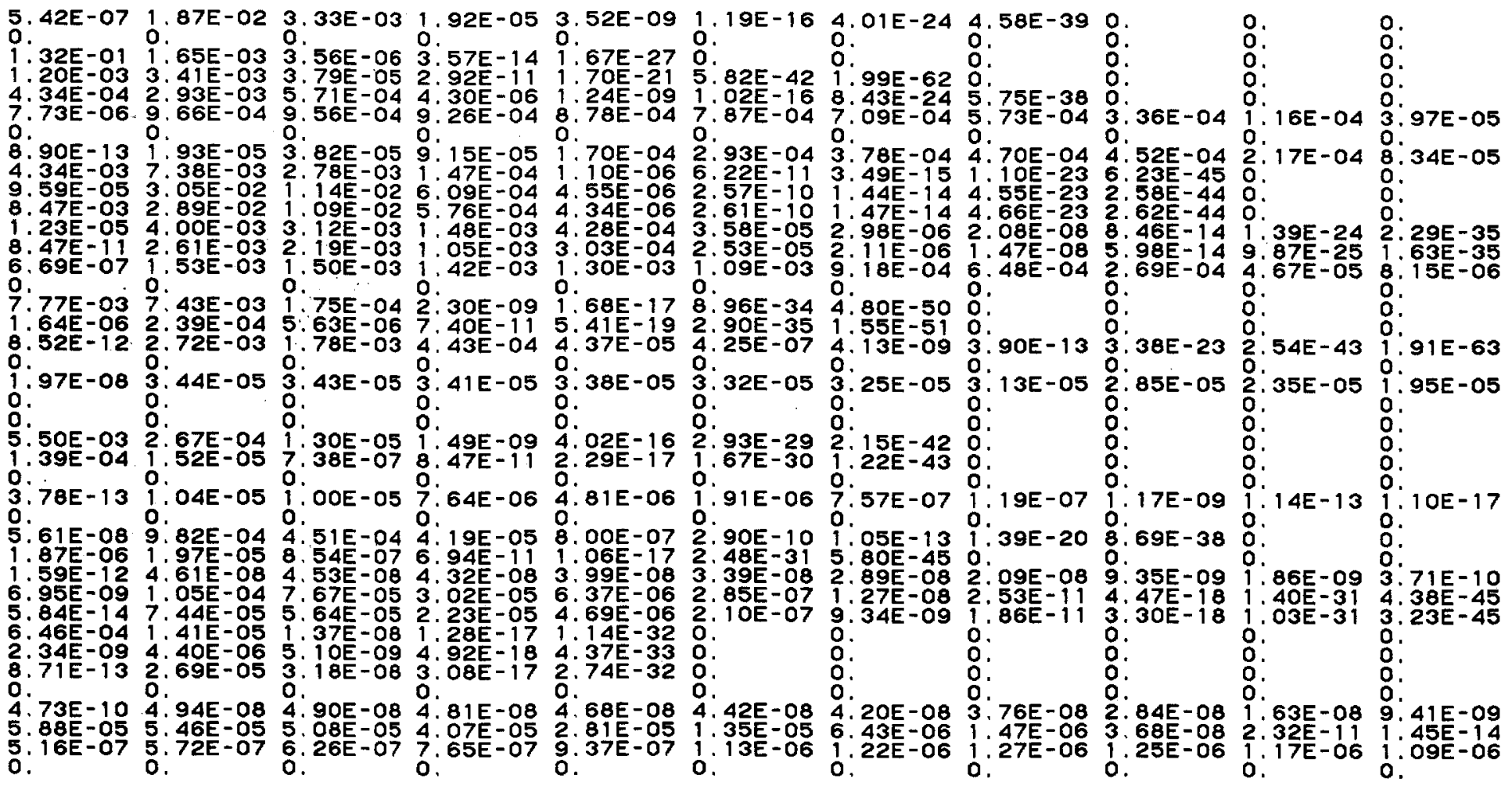


ANNIE MR

FRACTION OF REFRACTORIES PRESENT $=0.500$

RELAXATION LENGTH $=0.16$ GM SQCM $3.648 E-13$

DEBRIS DECAY FROM 1 TO 300 DAYS

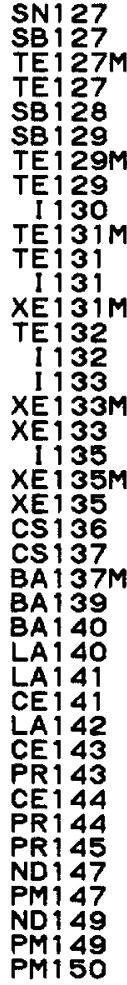

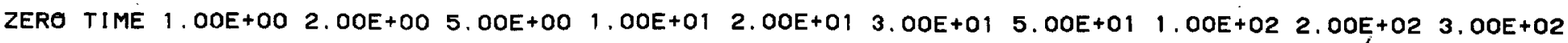

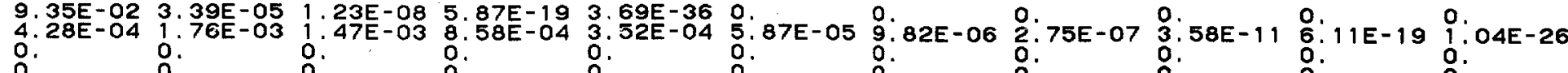

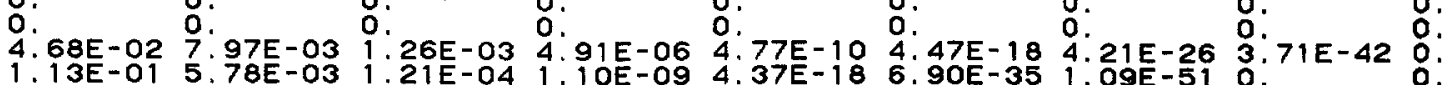
$4.81 E-11$ 5.07E-06 5.07E-06 4.79E-06 4.33E-06 3.52E-06 2.87E-06 $1.91 E-06$. $5.88 E-07$

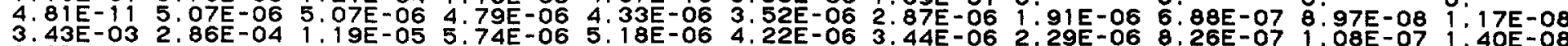

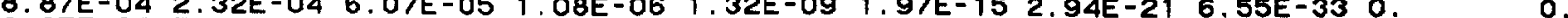
2.27E-06 7.93E-03 4.55E-03 $8.62 E-04 \quad 5.39 E-05$ 2. $10 E-07 \quad 8,23 E-10$ 1.26E-14 $1.14 E-26 \quad 0$.

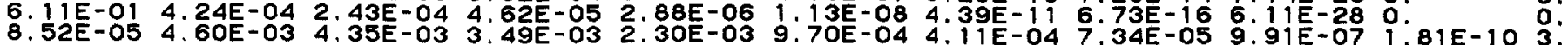
$\begin{array}{lll} & \end{array}$

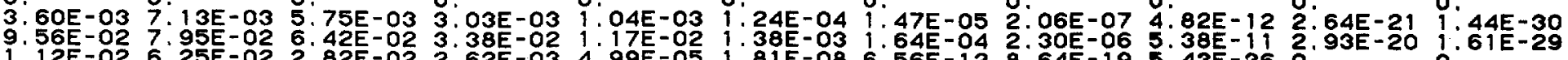
$1.12 E-02$ 6.25E-02 2.82E-02 2.62E-03 4.99E-05 1.81E-08 6.56E-12 8.64E-19 5:43E-36 0.

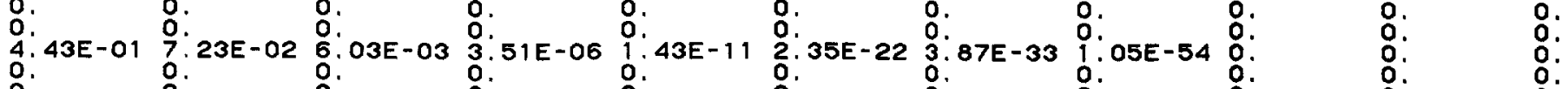

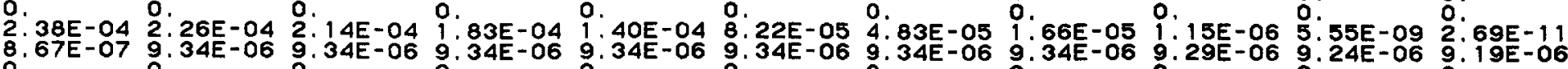

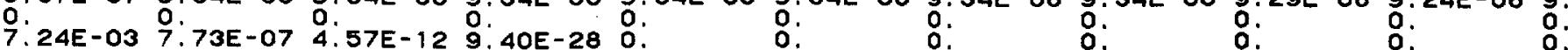

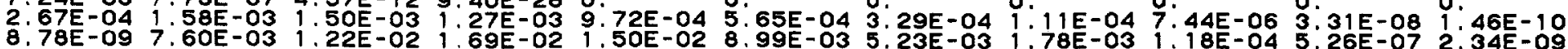

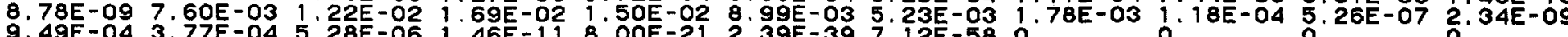
2.07E-10 2.29E-04 2.27E-04 2:14E-04 1.92E-04 1.55E-04 1:25E-04 8.1 $14 E-05$ 2. $80 E-05$ 3. $29 E-06$ 3. $88 E-07$ 2.11E-04 8.68E-03 5:25E-03 1. 16 E-03 9:30E-05 5.01E-07 3.89E-09 1.63E-13 1.84E-24 0.

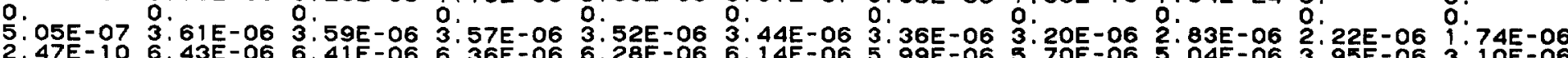

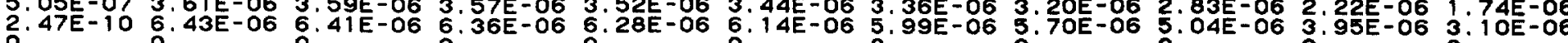

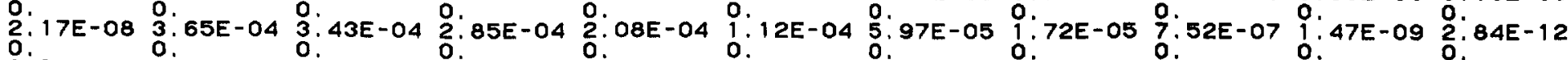

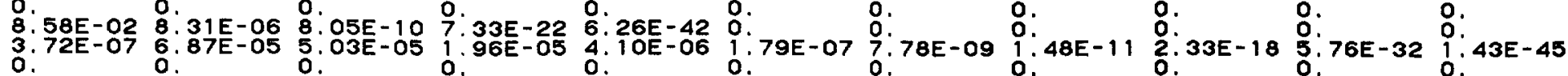


ANNIE MR HR H+12 HOURS $=$ MR/HR

FRACTI ON EF REFRACTORIES PRESENT $=0.500$

RELAXATION LENGTH BO. 16 GM/SQCM $3.648 E-13$

DEBRIS DECAY FROM 1 TO 300 DAYS

ZERO TIME $1.00 E+002.00 E+00 \quad 5.00 E+00 \quad 1.00 E+01 \quad 2.00 E+01 \quad 3.00 E+01 \quad 5.00 E+01 \quad 1.00 E+02 \quad 2.00 E+02 \quad 3.00 E+02$

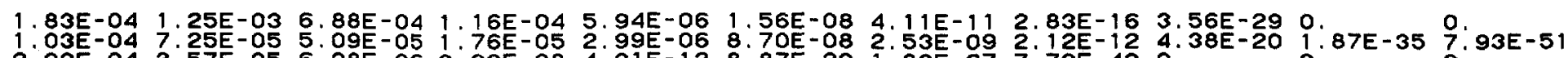

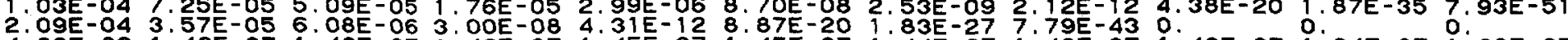

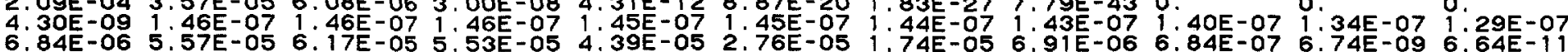
$7.50 E-05$ 8.66E-05 2.91E-05 1.09E-06 4.59E-09 8.09E-14 1:43E-18 4.46E-28 0

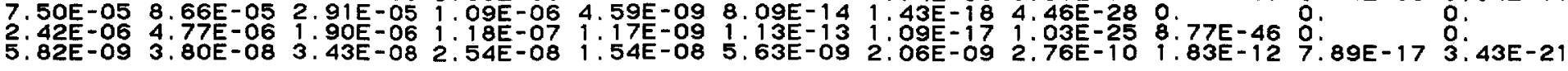
2.05E+00 4.52E-01 2.04E-01 8.08E-02 3.75E-02 1.49 E-02 8.51E-03 3.84E-03 $1.27 E-03 \quad 4.27 E-04 \quad 1.68 E-04$ 


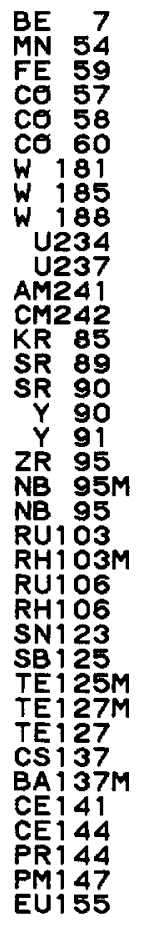

TOTAL
ANNIE MR/HR AT H+12 HOURS $=$ MR/HR 1 HOO

FRACTION OF REFRACTORIES PRESENT $=0.500$

RELAXATION LENGTH $=0.16$ GM/SQCM $3.648 E-13$

DEBRIS DECAY FREM 1 TO 50 YEARS

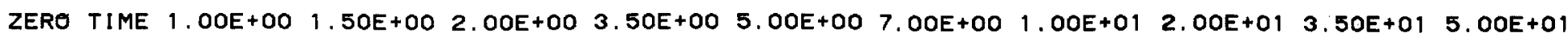

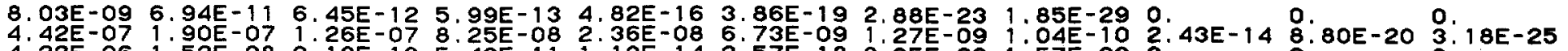

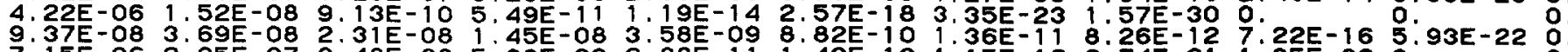

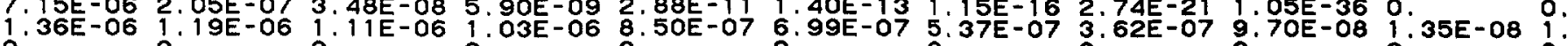

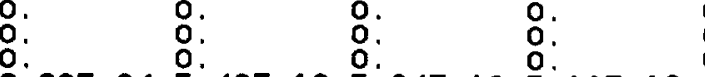

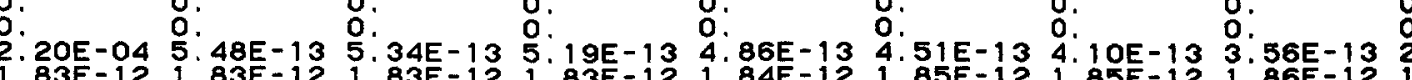
$1.83 E-12$ 1. $83 E-12$ 1. $83 E-12$ 1.83E-12 $1.84 E-12$ o.<smiles>[O-][O+]=[Ge]O</smiles>

:. 7.73E-06 . 04E-07

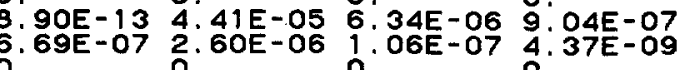
$.97 E-08 \quad 1.73 E-05 \quad 1.22 E-05 \quad 8.67 E-06$ 0. $85 E-121.85 E-12$ l. $86 E-121: 87 E-12$ o.

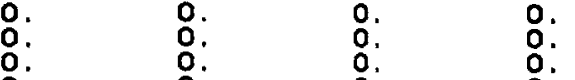
18 E-09 3 . $42 E-12$ l. $42 E-15$ l. $20 E-20$

0. 0. $42 E-12$ 1. $42 E-15$ ?. $20 E-20$

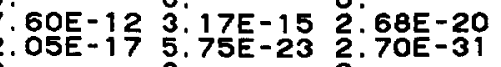
O. O. O. O. O. 35E-08 $1: 86 E-09$

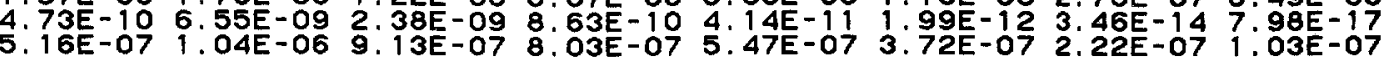
:.

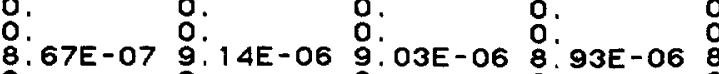
(. .

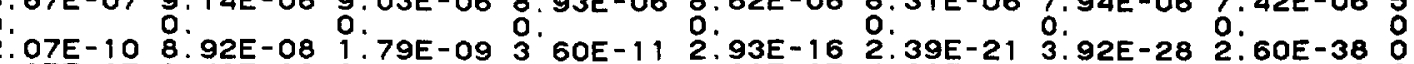
2.47E-10 2.64E-06 1.70E-06 1.08E-06 2.85E-07 7.40E-08 7.06E-09 4.87E-10 6.56E-14 $1.03 E-19$ 1.60E-25 4.30E-09 1.26E-07 1. 17E-07 1.09E-07 8.76E-08 7.03E-08 5.25E-08 3.39E-08 7.93E-09 8.91E-10 1.00E-10 2. 43E-04 1.00E-04 3.55E-05 2.27E-05 1.37E-05 1.07E-05 9.05E-06 7.96E-06 5.98E-06 4.18E-06 2.95E-06 
ANNIE MR/HR AT H+12 HOURS $=\begin{aligned} & \text { MR/HR } \\ & 1.000\end{aligned}$

FRACTION OF REFRACTORIES PRESENT $=0.100$

RELAXATION LENGTH $=0.16$ GM SQCM $_{4.6525-13}$

DEBRIS DECAY FROM 1 TO 21 HOURS

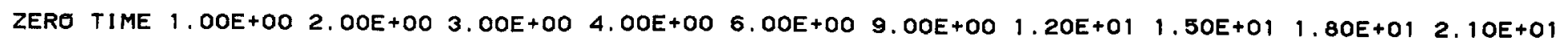

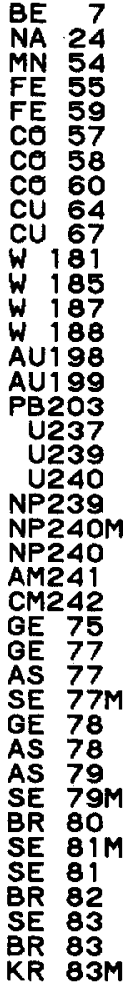

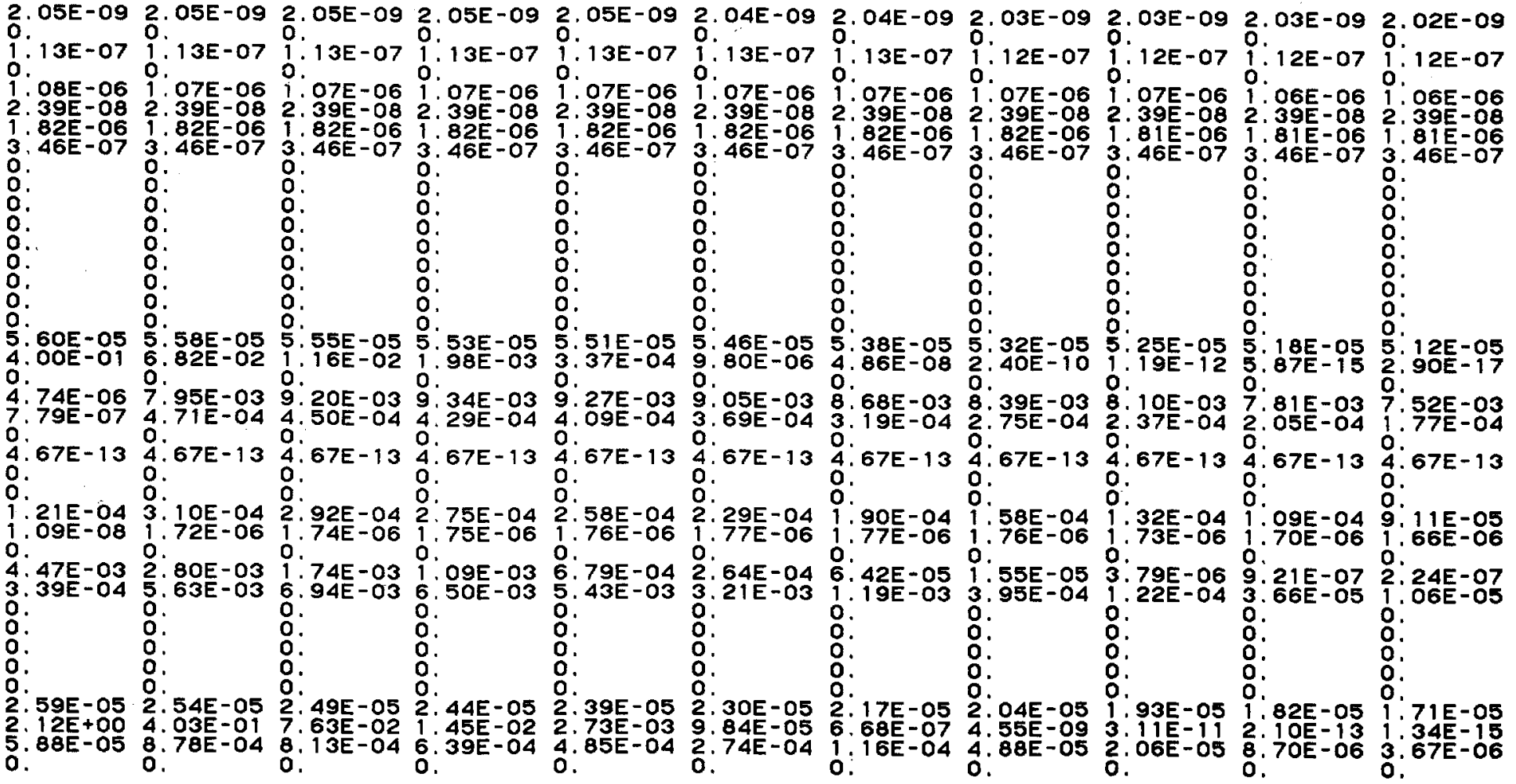


ANNIE

FRACTION OF REFRACTORIES PRESENT $=0.100$

RELAXATION LENGTH $=0.16$ GM/SQCM

DEBRIS DECAY FROM 1 TO 21 HOURS

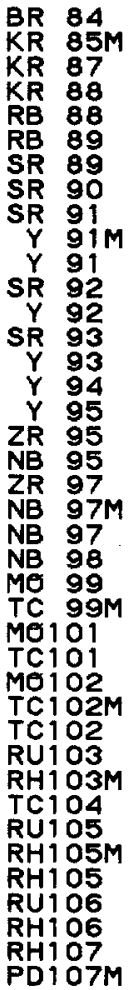

ZERO TIME $1.00 E+00 \quad 2.00 E+00 \quad 3.00 E+00 \quad 4.00 E+00 \quad 6.00 E+00 \quad 9.00 E+00 \quad 1.20 E+01 \quad 1.50 E+01 \quad 1.80 E+01 \quad 2.10 E+01$ 2.87E-03 4.95E-01 1.34E-01 3.62E-02 9.77E-03 7.15E-04 1.41E-05 2.79E-07 5.52E-09 1.09E-10 2.16E-12 $0:$ 2OE-01 O. $73 E-01$ O: 1.

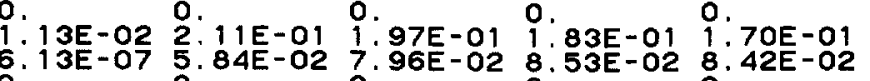
3. $37 E-02$ 1.51E-01 1.:17E-01 $9.04 E-02$ \%.00E-02 0 .

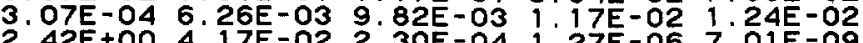
2. $1.21 \mathrm{E}-01$ 1. $65 \mathrm{E}-01$ 2.13E-02 $2.74 \mathrm{E}-03$ 3. $34 \mathrm{E}-04$ (1) (.

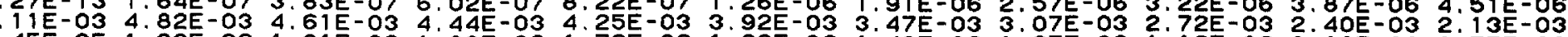
2.45E-05 1.9E-02 1.91E-02 1.83E-02 1.76E-O2 1.62E-02 1.43E-02 1.27E-02 1. 12E-02 9:90E-03 $8.75 E-03$

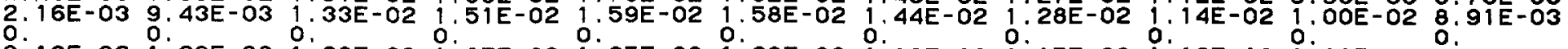

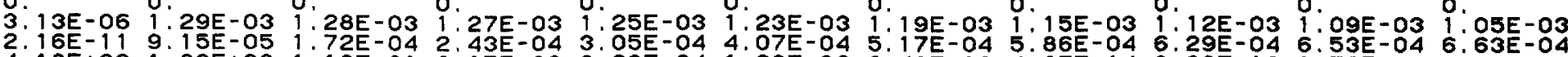

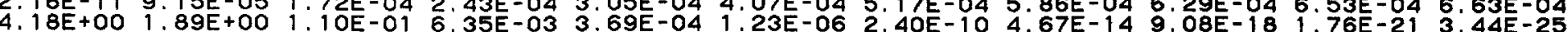

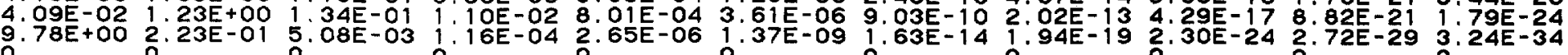

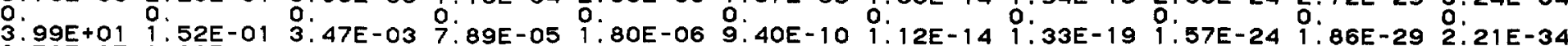

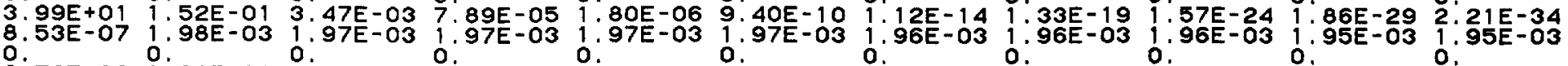

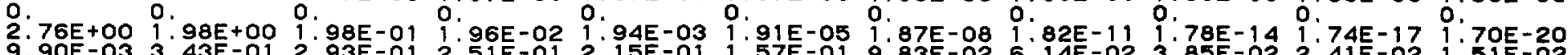

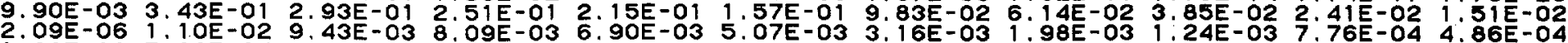

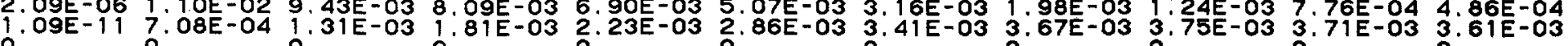

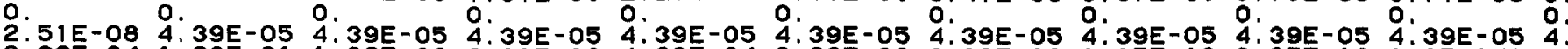

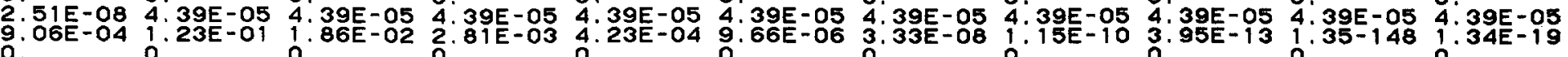


ANNIE MR/HR

FRACTION OF REFRACTORIES PRESENT $=0.100$

RELAXATION LENGTH $=0.16$ GM/SQCM

$4.652 E-13$

DEBRIS DECAY FROM 1 TO 21 HOURS

ZERO TIME $1.00 E+00 \quad 2.00 E+00 \quad 3.00 E+00 \quad 4.00 E+00 \quad 6.00 E+00 \quad 9.00 E+00 \quad 1.20 E+01 \quad 1.50 E+01 \quad 1.80 E+01 \quad 2.10 E+01$

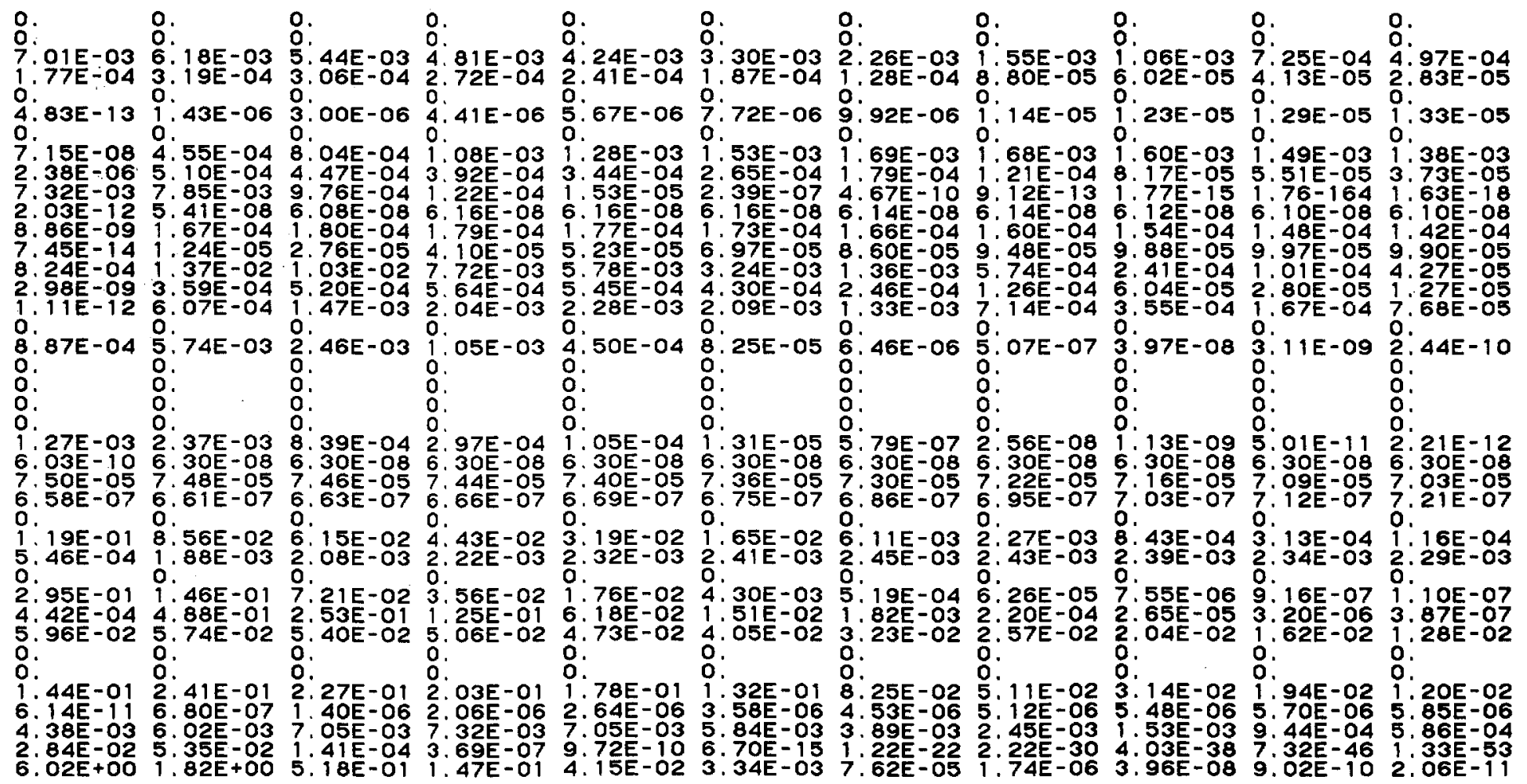


ANNIE

MR/HR

FRACT ION OF REFRACTORIES PRESENT $=0.100$

RELAXATI ON LENGTH $=0.16 \mathrm{GM} / \mathrm{SQCM}$ $4.652 \mathrm{E}-13$

DEBRIS DECAY FROM 1 TO 21 HOURS

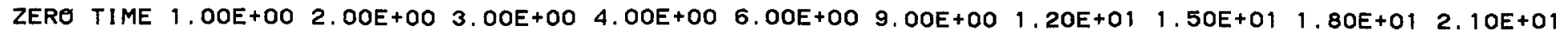

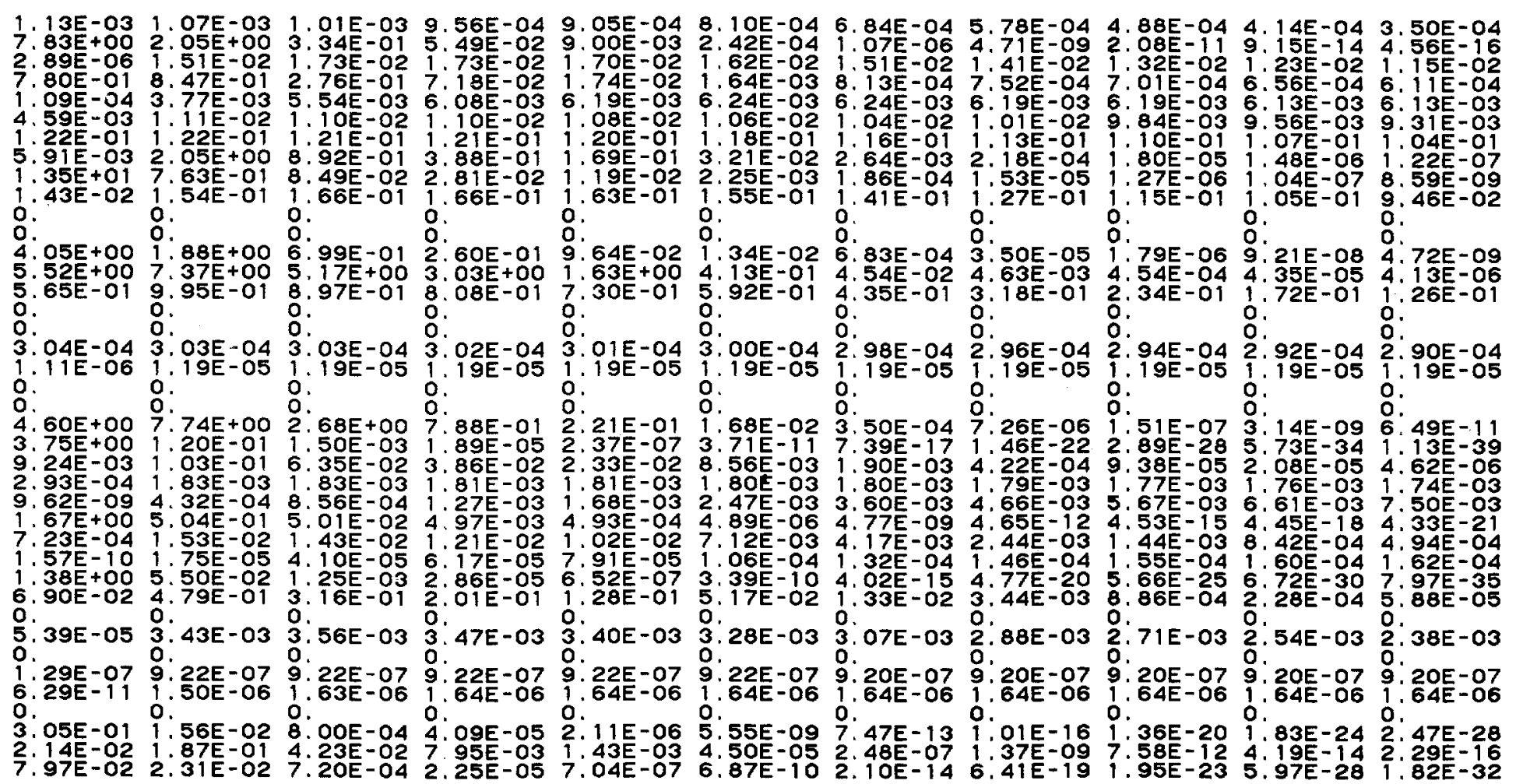


MR/HR AT $H+12$ HOURS $=$ MR/HR

PRACTAT H+12 HOURS MRHR PRESENT $0 \quad 0.100$

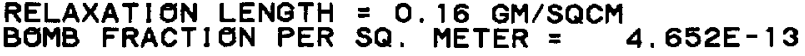

DEBRIS DECAY FROM 1 TO 21 HOURS

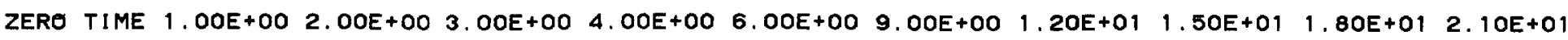

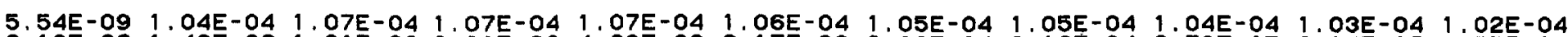

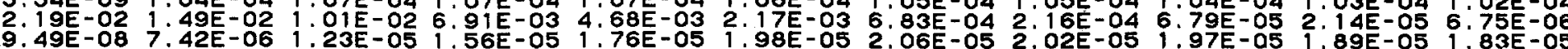

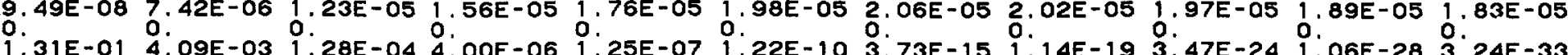


ANNIE MR/HR AT $\mathrm{H}+12$ HOURS $=$ MR/HR

FRACTION OF REFRACTORIES PRESENT $=0.100$

RELAXATION LENGTH $=0.16$ GM/SQCM ${ }_{4,652 E-13}$

DEBRIS DECAY FROM 1 TO 300 DAYS

ZERO TIME $1.00 E+002.00 E+00 \quad 5.00 E+001.00 E+01 \quad 2.00 E+01 \quad 3.00 E+01 \quad 5.00 E+01 \quad 1.00 E+02 \quad 2.00 E+02 \quad 3.00 E+02$

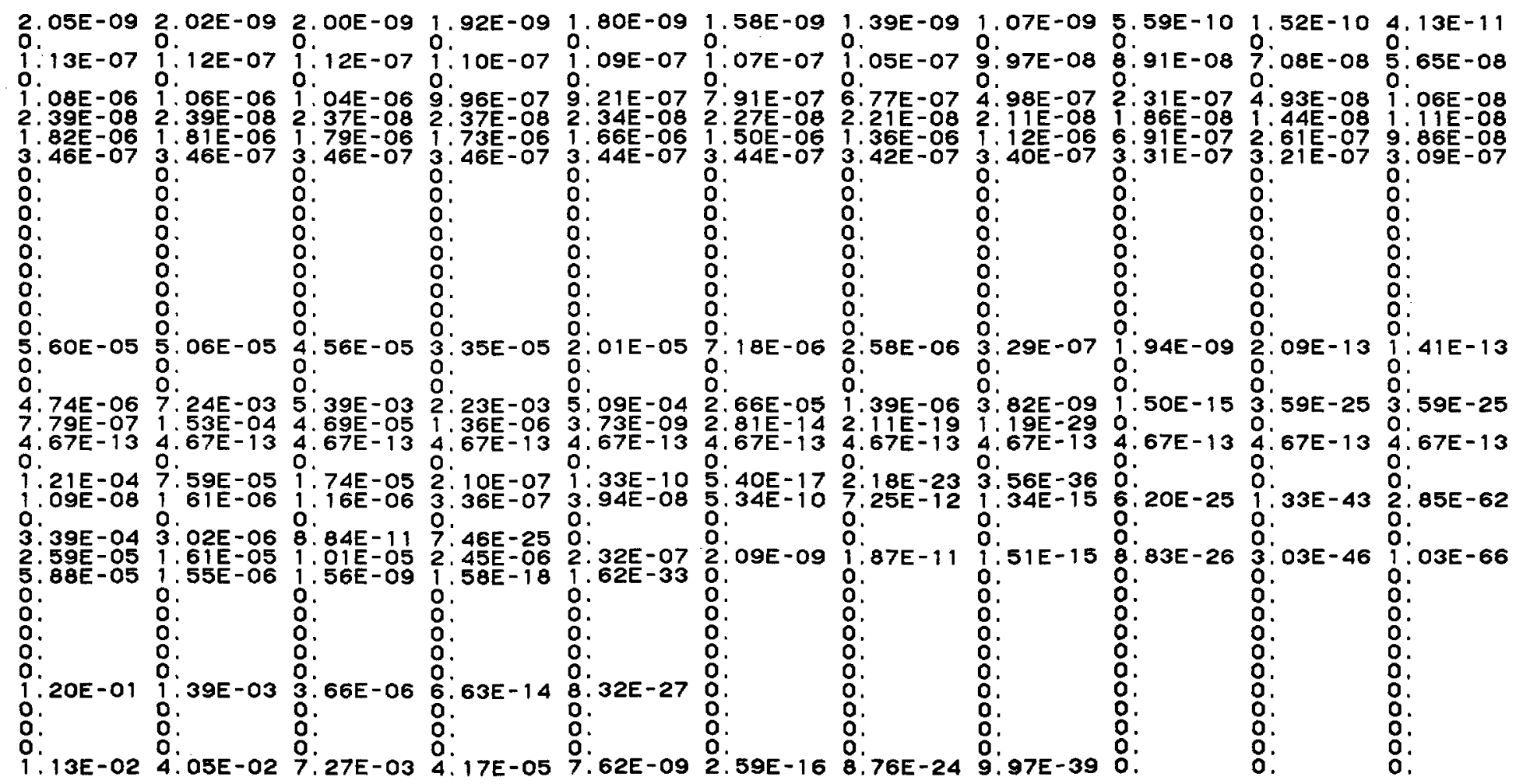


ANNIE AT H+12 HOURS $=$ MR/HR

FRACTION OF REFRACTORIES PRESENT $=0.100$

RELAXATION LENGTH $=0.16$ GM $=0$ SQM $4.652 E-13$

DEBRIS DECAY FROM 1 TO 300 DAYS

ZERO TIME $1.00 E+00 \quad 2.00 E+00 \quad 5.00 E+00 \quad 1.00 E+01 \quad 2.00 E+01 \quad 3.00 E+01 \quad 5.00 E+01 \quad 1.00 E+02 \quad 2.00 E+02 \quad 3.00 E+02$
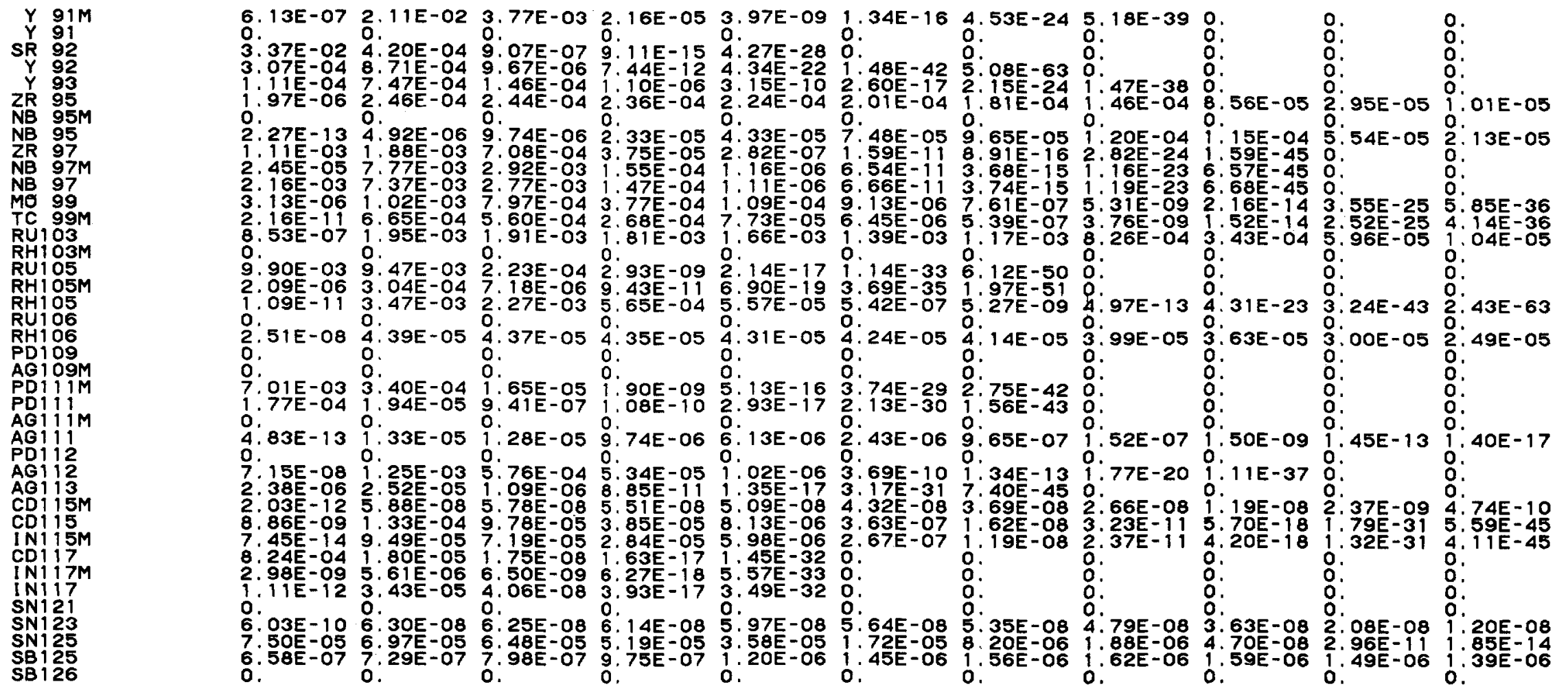
ANNIE MT $H+12$ HOURS $=$ MR/HR

FRACTION OF REFRACTORIES PRESENT $=0.100$

RELAXATION LENGTH $=0.16$ GM

DEBRIS DECAY FROM 1 TO 300 DAYS

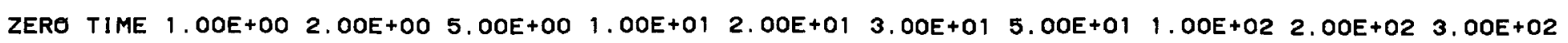

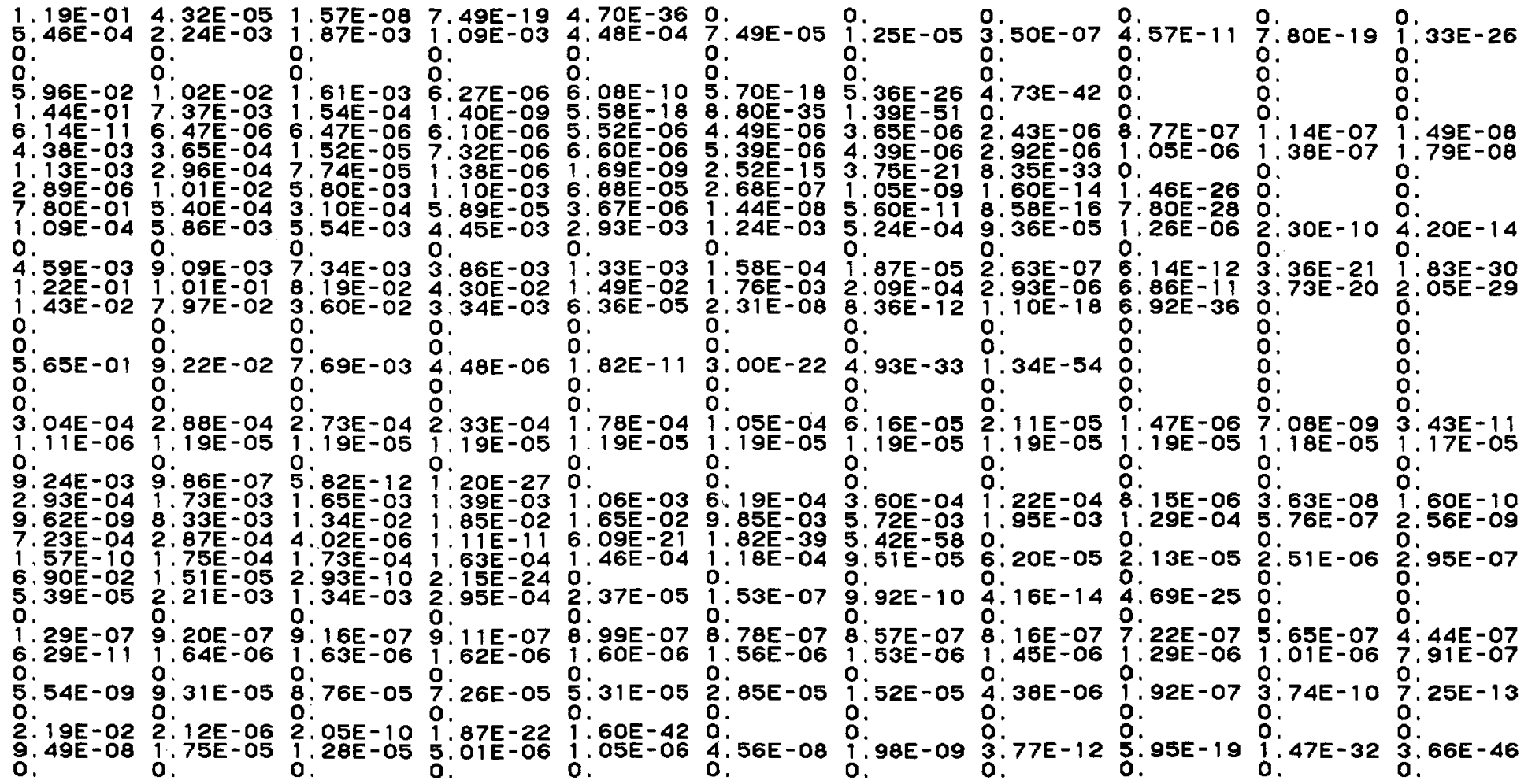


ANNIE

MR/HR

FRACTION OF REFRACTORIES PRESENT $=0.100$ RELAXATION LENGTH $=0.16$ GM/SQCM

DEBRIS DECAY FROM 1 TO 300 DAYS

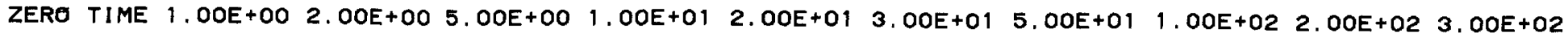

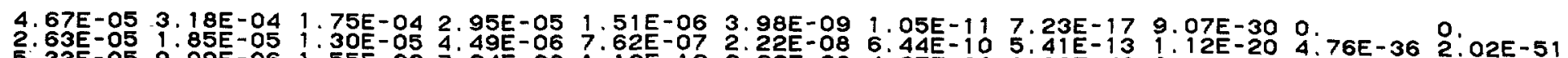
1.33E-05 9.09E-06 1.55E-06 7.64E-09 $1.10 E-12$ 2.26E-20 $4.67 \mathrm{EE}-28$ 1.99E-43 0. 0.0. (

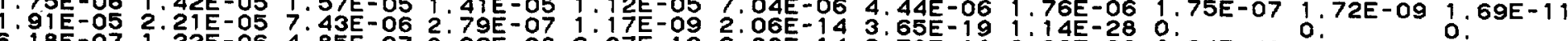

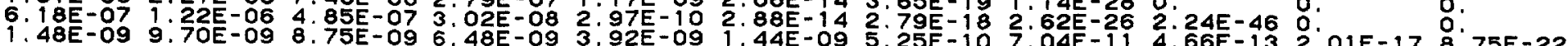

TOTAL

2. $10 E+00 \quad 4.42 E-01 \quad 1.95 E-01 \quad 8.39 E-02 \quad 4.05 E-02 \quad 1.58 E-02 \quad 8.55 E-03 \quad 3.41 E-03 \quad 7.61 E-04 \quad 1.94 E-048.19 E-05$ 
DEBRIS DECAY FROM 1 TO 50 YEARS

ZERO TIME $1.00 E+00 \quad 1.50 E+00 \quad 2.00 E+00 \quad 3.50 E+00 \quad 5.00 E+00 \quad 7.00 E+00 \quad 1.00 E+01 \quad 2.00 E+01 \quad 3.50 E+01 \quad 5.00 E+01$

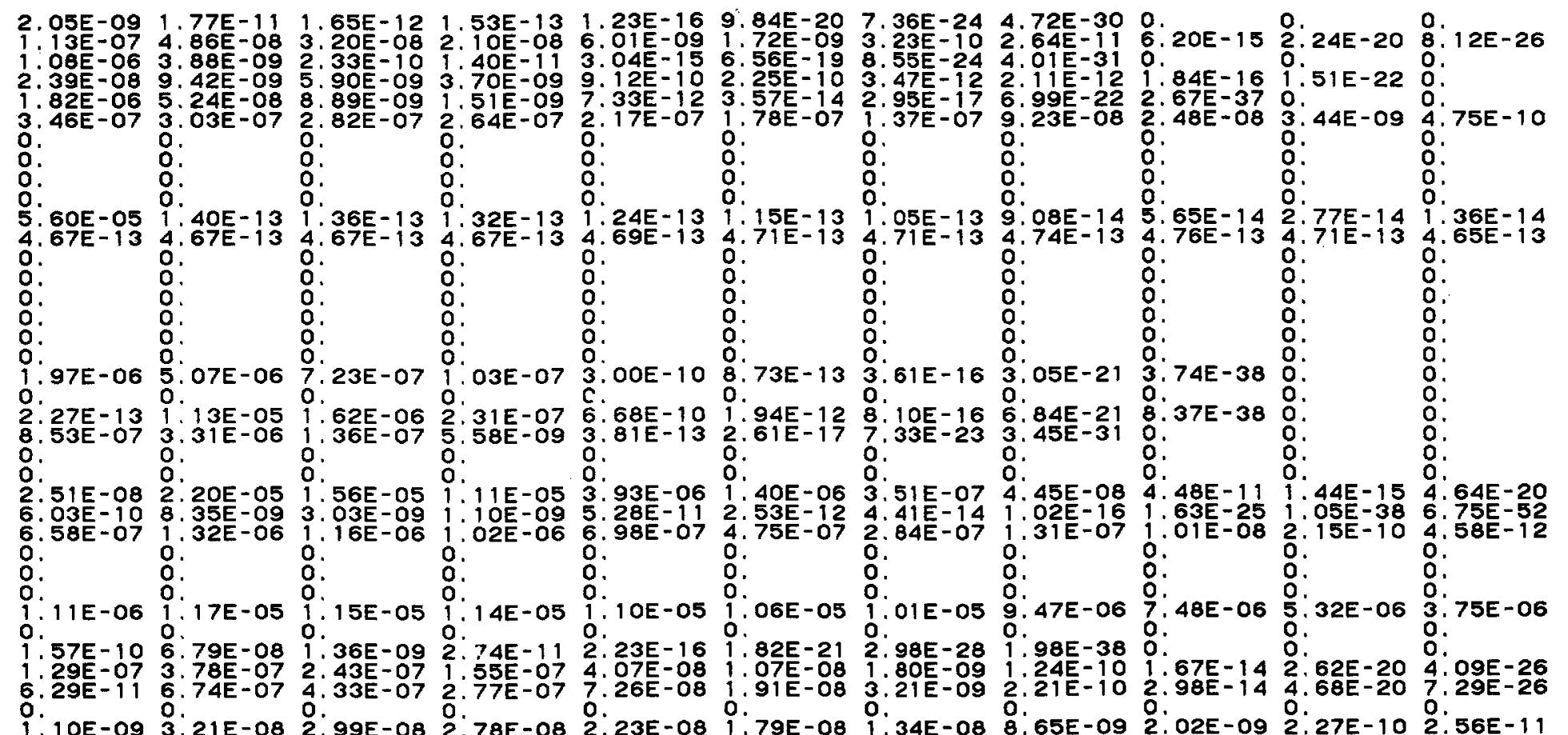

TOTAL 
APPENDIX 9

ANNIE EVENT

Fit To Sum of Eleven Exponentials

The calculated values are fit to the function

$$
\sum_{i=1}^{11} a_{i} e^{-\lambda_{j} t}
$$

In the first table the $a_{j}$ 's are the variables and are designated by an odd number in the column under " $K$ "; the $i$ 's are fixed parameters so designated. The quality of fit to each point is shown in the second table.

Page

Fraction refractories present $=1.0$

$9-1$

Fraction refractories present $=0.5$

$9-3$

Fraction refractories present $=0.1$

$9-5$ 
ANNIE

CI/SO KM, 1.0 REFRACTORIES PRESENT

PAGE

\begin{tabular}{|c|}
\hline 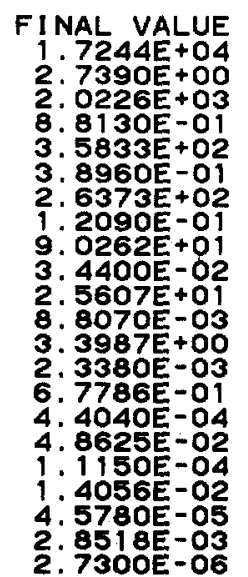 \\
\hline
\end{tabular}

STND DEV.

4. $3437 E+02$

1. $3741 \mathrm{E}+02$

2. $8367 E+01$

6. $8489 E+00$

1. $3290 E+00$

i: $8519 \mathrm{E}-01$

2. 4332E-O2

3. $6623 \mathrm{E}-03$

O. $9803 E-04$

$4.9658 \mathrm{E}-05$

FIXED PARAMETER

FIXED PARAMETER

FIXED PARAMETER

FIXED PARAMETER

FIXED PARAMETER

FIXED PARAMETER

FIXED PARAMETER

FIXED PARAMETER

FIXED PARAMETER

FIXED PARAMETER

FIXED PARAMETER
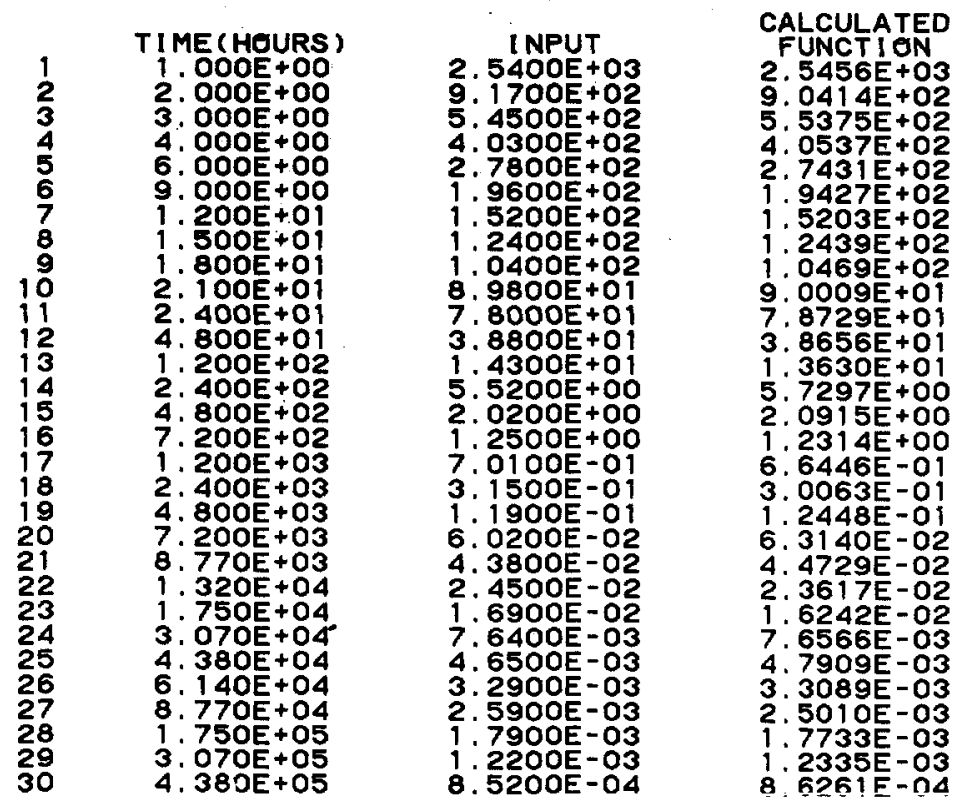

PERCENT

$-0.22$

$1: 42$
$-1: 58$

-1.58
-0.59

1.34
0.89

$-0.02$

$-0.66$

$-0.23$

-0.93
0.37

4.92
-3.66

$-3.42$

5.50

$-4.40$

-4.66
-2.08

-2.08
3.74

4.05
-0.22

-2.94
-0.57

-0.57
3.56

-1.94
-1.23 
PAgE

ANNIE

F INAL VALUE $1.2011 \mathrm{E}+02$ 4. $1194 \mathrm{E}+01$ . $3730 \mathrm{OE}+\mathrm{O}$

3. $8960 \mathrm{E}-01$

. $595 E+00$

2O9OE-O

4. $7289 \mathrm{E}-01$

.3556E-01

$8.8070 \mathrm{E}-03$

2. $5513 \mathrm{E}-\mathrm{O} 2$

$4.5926 \mathrm{E}-03$

4. $4040 \mathrm{E}-04$

4. $1436 E-05$

$1.3970 \mathrm{E}-05$

7. $7792 \mathrm{E}-06$

2.7300E-06

\section{.}

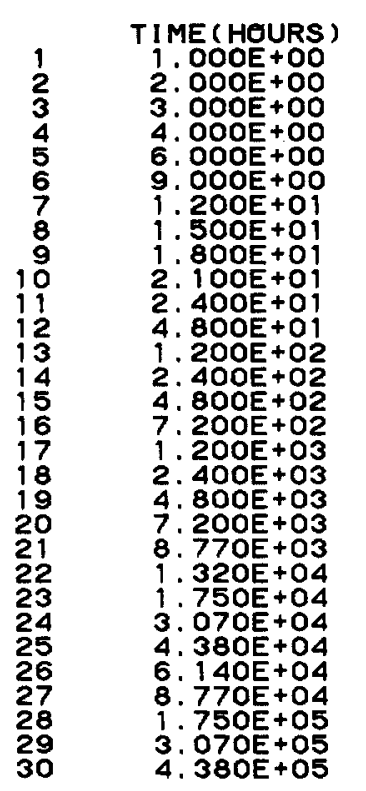

MR/HR, 1.0 REFRACTORIES PRESENT $0.16 \mathrm{GM} / \mathrm{SQCM}$ STND DEV. 2. $9251 \mathrm{E}+00$ 7. 5572E- 01 1. $1796 \mathrm{E}-01$ 2. $6496 \mathrm{E}-02$ 5. $0761 E-03$ 7. 3202E-04 6. 4576E- 05 4. $7514 E-06$ 1. $3102 E-06$ 8. 4495E-08 o:

FIXED PARAMETER FIXED PARAMETER FIXED PARAMETER FIXED PARAMETER FIXED PARAMETER FIXED PARAMETER FIXED PARAMETER FIXED PARAMETER FIXED PARAMETER FIXED PARAMETER FIXED PARAMETER

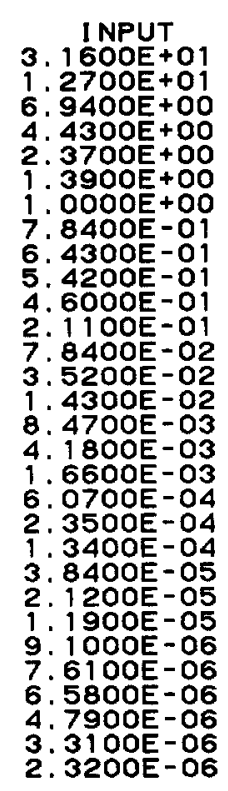

\begin{tabular}{|c|c|}
\hline 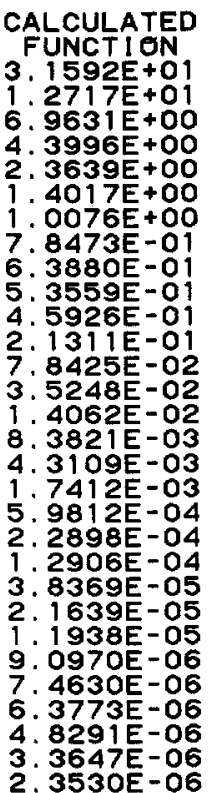 & $\begin{array}{r}\text { PERCENT } \\
\text { DEVVIAT1ON } \\
0.03 \\
-0.13 \\
-0.33 \\
-0.33 \\
0.69 \\
0.26 \\
-0.83 \\
-0.85 \\
-0.09 \\
-0.06 \\
1.66 \\
1.20 \\
-0.15 \\
-0.99 \\
-0.03 \\
-0.14 \\
1.69 \\
1.05 \\
-3.04 \\
-4.67 \\
1.49 \\
2.63 \\
3.82 \\
0.08 \\
-2.03 \\
-0.31 \\
0.03 \\
1.97 \\
3.18 \\
-0.81 \\
-1.63 \\
-1.40\end{array}$ \\
\hline
\end{tabular}


ANNIE

CI/SQ KM, 0.5 REFRACTORIES PRESENT

PAGE

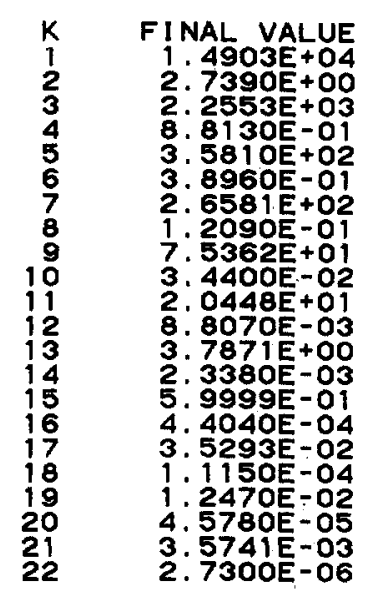

STND. DEV

5. $1526 \mathrm{E}+02$

1. $5779 E+02$

3. $0952 E+01$

7. $3873 E+00$

1. $4653 E+00$

1633E-01

$7002 E-02$

$4.2902 \mathrm{E}-03$

1.2938E-03

?. $5650 E-05$

FI XED PARAMETER

FI XED PARAMETER

FIXED PARAMETER

FIXED PARAMETER

FIXED PARAMETER

FIXED PARAMETER

FIXED PARAMETER

FIXED PARAMETER

FIXED PARAMETER

FIXED PARAMETER

FIXED PARAMETER

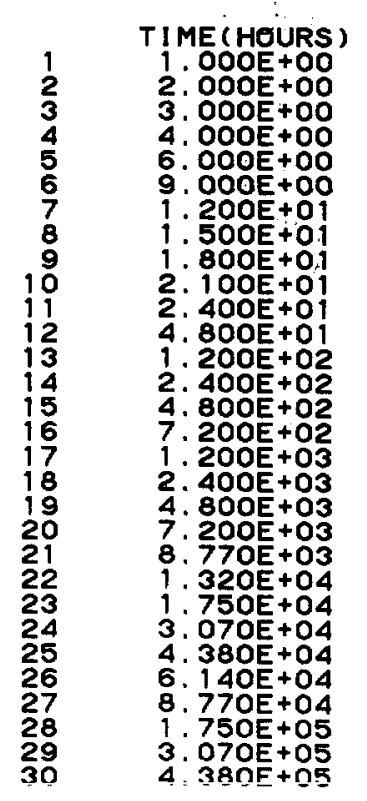

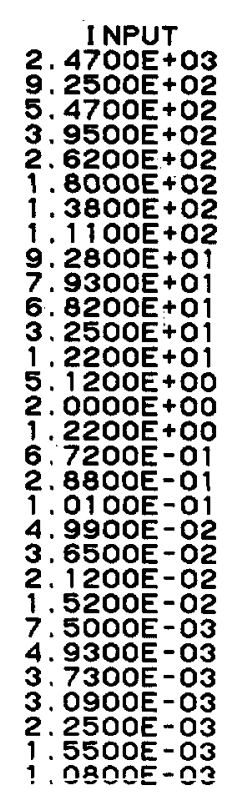

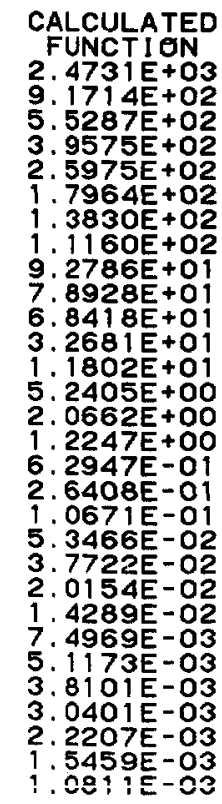

PERCENT
DEVIATION

-0.13

-0.86

$-0.19$

0.87

0.20
-0.22

$-0.53$

0.02

$-0.32$

-0.55
3.37

$-2.30$

$-3.20$

$-0.38$

6.76
-5.06
-5.35

-5.35
-6.67

$-3.24$

6. 38

$-3.6$

-3.66
-2.10

1. 64

0.26
-0.10 


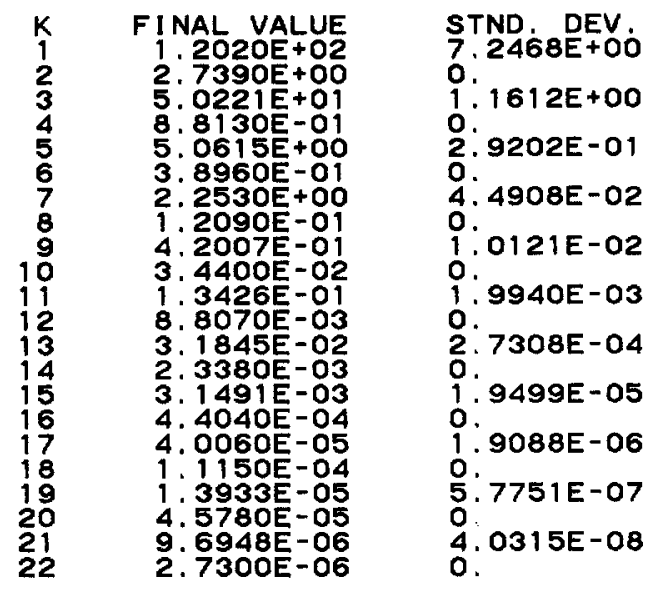

MR/HR, 0.5 REFRACTERIES PRESENT $0.16 \mathrm{GM} / \mathrm{SOCM}$

FIXED PARAMETER

FIXED PARAMETER

FIXED PARAMETER

FIXED PARAMETER

FIXED PARAMETER

FIXED PARAMETER

FIXED PARAMETER

FIXED PARAMETER

FIXED PARAMETER

FIXED PARAMETER

FIXED PARAMETER
PAGE

\begin{tabular}{|c|c|}
\hline $\begin{array}{l}\text { CALCULATED } \\
\text { FUNCTION } \\
3.4571 E+O 1 \\
1.3770 E+01 \\
7.2872 E+00 \\
4.4657 E+00 \\
2.3369 E+00 \\
1.3955 E+00 \\
1.0095 E+O O \\
7.8451 E-01 \\
6.3467 E-01 \\
5.2837 E-01 \\
4.5016 E-01 \\
2.0697 E-01 \\
8.0539 E-02 \\
3.7393 E-02 \\
1.4936 E-02 \\
8.5053 E-03 \\
3.8434 E-03 \\
1.2635 E-03 \\
4.2493 E-04 \\
1.6963 E-04 \\
1.0005 E-04 \\
3.5568 E-05 \\
2.2604 E-05 \\
1.3643 E-05 \\
1.0781 E-05 \\
9.0793 E-06 \\
7.8843 E-06 \\
6.0171 E-06 \\
4.1933 E-06 \\
2.9325 E-06\end{array}$ & $\begin{array}{r}\text { PERCENT } \\
\text { DEVIATION } \\
\text { O. } 08 \\
-0.51 \\
-0.10 \\
0.99 \\
0.13 \\
-1.19 \\
-0.94 \\
-0.07 \\
0.84 \\
1.44 \\
0.41 \\
-1.43 \\
0.32 \\
0.29 \\
-0.24 \\
0.06 \\
-0.09 \\
0.51 \\
0.49 \\
-0.96 \\
-0.05 \\
-0.19 \\
0.42 \\
0.42 \\
-0.75 \\
-0.32 \\
0.96 \\
-0.62 \\
-0.32 \\
0.60\end{array}$ \\
\hline
\end{tabular}
2. OOOE +OO
3. 000 + +00
. OOOE + +00
. OOOE +OO
500 +o 1
$800 E+01$
2. $100 E+01$
$4.800 E+01$
$200 E+02$
$800 E+02$
$7.200 E+02$
$.400 E+03$
4. $800 E+03$
7. $200 E+03$
. $320 E+04$
O70E+04
$140 E+04$
$750 \mathrm{O}+05$
4. $380 E+05$

I NPUT

3. $4600 \mathrm{E}+01$

7.3700 + +1

. $5100 E+00$

$3800 \mathrm{E}+\mathrm{OO}$

OOOOE+OO

.8400E-O1

$3600 E-01$

. $5200 \mathrm{E}-\mathrm{O} 1$

O800E-02

$7500 \mathrm{E}-02$

$4900 E-02$

$8400 \mathrm{E}-03$

2700 - 03

4

$5500 E-05$

$3700 E-05$

O5OOE-06

$.9600 E-06$

$4.1800 E-06$
$2.9500 E-06$ 

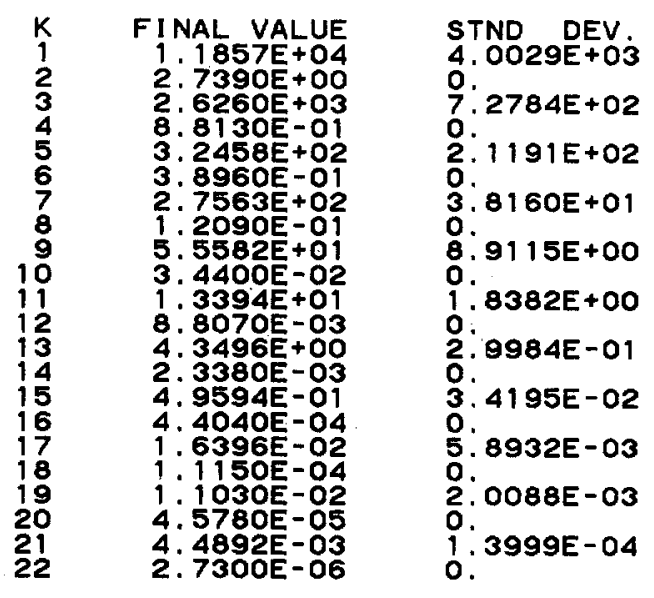

FIXED PARAMETER

FIXED PARAMETER

FIXED PARAMETER

FIXED PARAMETER

FIXED PARAMETER

FIXED PARAMETER

FIXED PARAMETER

FIXED PARAMETER

FIXED PARAMETER

FIXED PARAMETER

FIXED PARAMETER
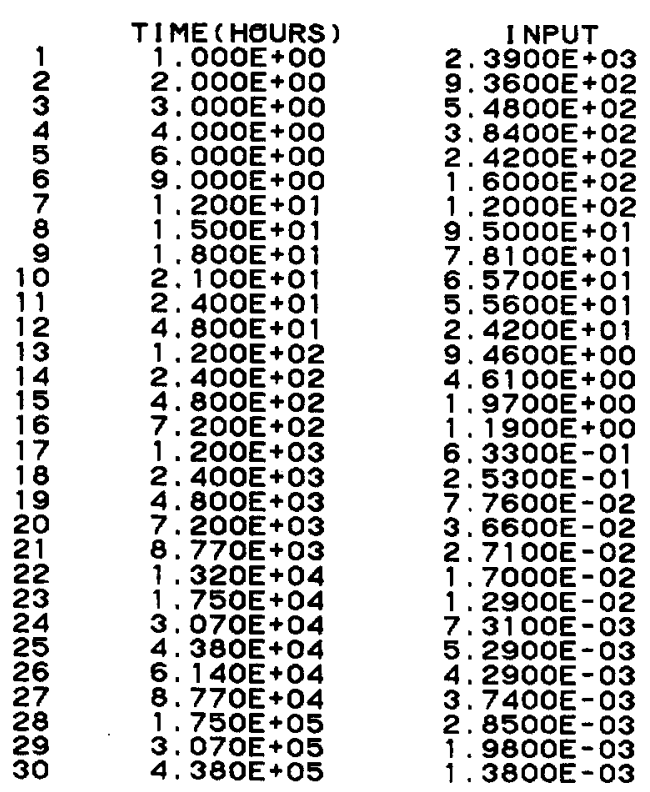

CALCULATED
FUNCTION
2.3901 I +O3
$9.3538 E+02$
$5.5053 E+02$
$3.8199 E+02$
$2.4079 E+02$
$1.6147 E+02$
$1.2128 E+02$
$9.5533 E+01$
$7.7617 E+01$
$6.4639 E+01$
$5.4990 E+01$
$2.4675 E+01$
$9.3386 E+00$
$4.5917 E+00$
$2.0437 E+00$
$1.2230 E+00$
$5.8498 E-01$
$2.1514 E-01$
$8.2835 E-02$
$4.0494 E-02$
$2.8357 E-02$
$1.5602 E-02$
$1.1783 E-02$
$7.3688 E-03$
$5.5924 E-03$
$4.4773 E-03$
$3.7334 E-03$
$2.7878 E-03$
$1.9417 E-03$
$1.3579 E-03$

DEVRCENT

$-0.00$

$-0.46$

-53
0.50

-0.91
-0.92

$-1.06$

$-0.56$

1.64

$-1: 11$
$-1: 93$

1.30

1.040
-3.61
-3

$17: 60$

-6.32
-9.62

$-4.43$

9.98
-9.80

$-5.81$

$-4.18$

0.18

2.23 
Apri1 22, 1981

T0: Distribution

SUBJECT: ERRATA, UCID-18870

The following pages are in error:

$\begin{array}{lll}4-2 & 4-13 & 4-24 \\ 4-7 & 4-18 & 4-29 \\ 4-11 & 4-22 & 4-33\end{array}$

Errata sheets are attached.

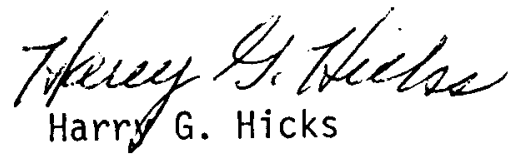


TIME

ZERO TIME HOURS

$1 . O O E+O O$

5. OOE +OO

$3.00 E+00$
$4.00 E+0 O$

$6.00 \mathrm{OE}+\mathrm{OO}$

$9.0 O E+O O$

$.50 \mathrm{OE}+\mathrm{O} 1$

$1.80 E+01$

$1.00 E+\infty O$ DAYS

2. $00 \mathrm{OE}+\mathrm{OO}$

$5.00 \mathrm{E}+00$

$1.00 E$
2.001

2.
$3.00 E+01$
DO

$5.00 \mathrm{O}+01$

$1.00 E+02$

$3.00 \mathrm{O}+02$

1.00 +OO YEARS

$1.50 E+00$

2. $00 \mathrm{OOO}$

$3.50 E+00$

$5.00 E+O 0$

1.OOE

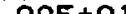

5. $00 \mathrm{OE}+01$
MR/HR

9. $91 E+91$

$25 \mathrm{E}+0$

$6.90 \mathrm{E}+0 \mathrm{O}$

4. $43 E+00$

2. $39 E+\infty 0$

$1.00 E+00$

7.77-ㅣ

$5.28 \mathrm{E}-01$

4. $44 E-01$

$6.89 \mathrm{E}-02$

$3.27 \mathrm{E}-02$

$1.39 \mathrm{E}-02$

$8.29 E-03$
$4.085-93$

$1.64 \mathrm{E}-03$

. $09 E-04$

1. $34 \mathrm{E}-04$

3. 5 TE-05

$1.91 E-05$

$1.13 E-05$
$8.99 E-06$

7. $5 \mathrm{SE}-06$

4.. 64 E -06

2., $24 \mathrm{E}-06$
MI CROCURIES/SO METER

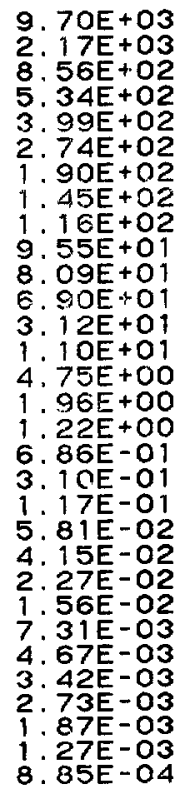


DEERIS DECAY FROM 1 TO 21 HEURS

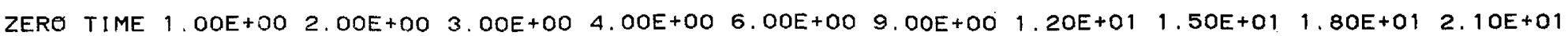

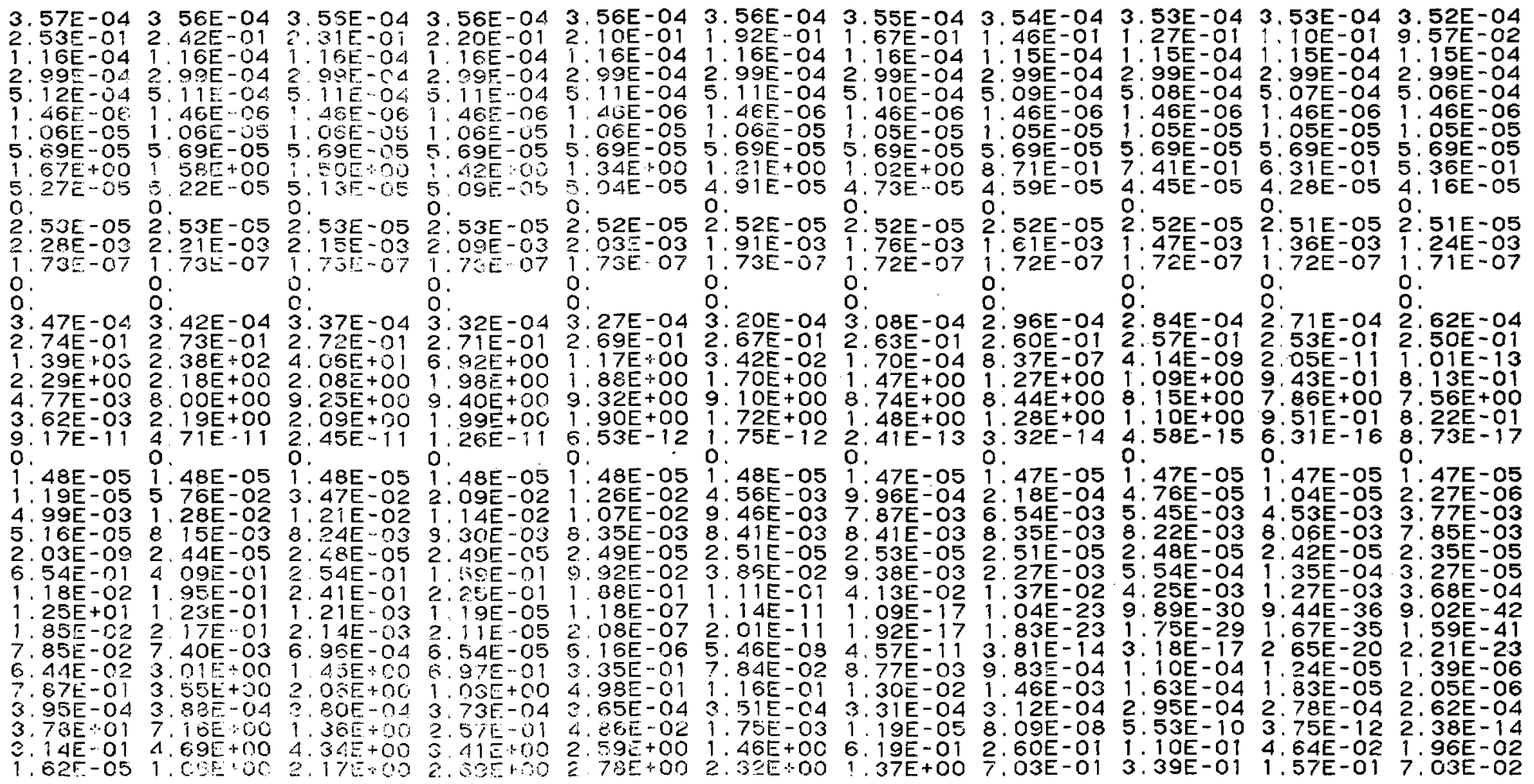


FRACTION OF REFRACTORIES PRESENT $=1.000$

RELAYATION LENGTH $=0.16 \mathrm{GM} / \mathrm{SQCM}, 1.024 E-13$

DEBRIS DECAY FROM 1 TO SOO DAYS

BE

MA 24

FE 59

Co 58

cu 60

CU 67

W1 81

W187

W188

AU198

PB203

4240

NP237

NP240M

AM2 41

CM242

AS 77

SE 77M

AS 78

$B R 83$

KR $83 \mathrm{M}$

KR 85

KR 87

KR 88

$\begin{array}{ll}\text { RE } & 88 \\ \text { SR } & 89\end{array}$

SR 90

SR 91

ZERO TIME 1 OOE+OO $2.00 E+O 05$ OOE+OO $1.00 E+01 \quad 2.00 E+01 \quad 3.00 E+01 \quad 5.00 E+01 \quad 1.00 E+02 \quad 2.00 E+02 \quad 3.00 E+02$ 3.57E-04 3.52E-04 3.48E-04 3.35E-04 3.13E-04 2.75E-04 2.42E-04 1.86E-04 9.73E-05 2.64E-05 $7.19 E-06$

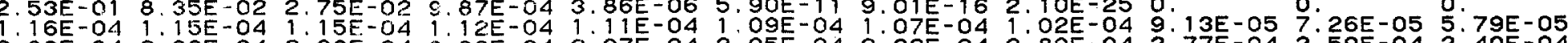
2.99E-04 2.99E-04 2.99E-04 2. $28 E-04 \quad 2.97 E-04 \quad 2.95 E-04 \quad 2.92 E-04 \quad 2.89 E-042.77 E-042.59 E-042.40 E-04$

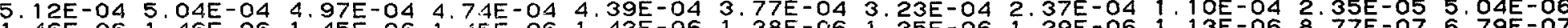

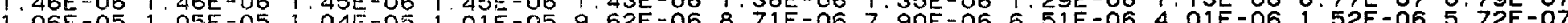
5.69E-05 5.69E-05 5.69E-05 5.69E-05 5.66E-05 5.66E-05 5.63E-05 5.59E-05 5.46E-05 5.29E-05 5.09E-05

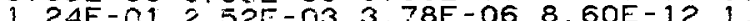
$5.27 E-05$ 4.02E-05 3.07E-05 1.36E-05 3.55E-06 2.40E-07 1.62E-08 7.34E-11 1.03E-16 0

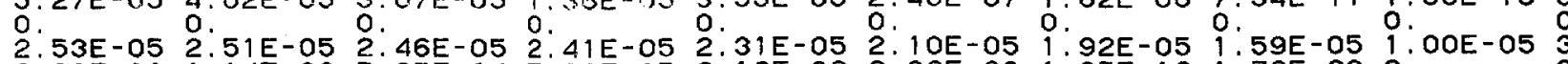

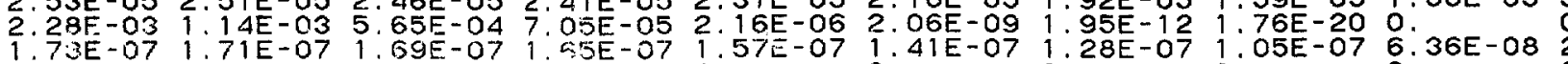

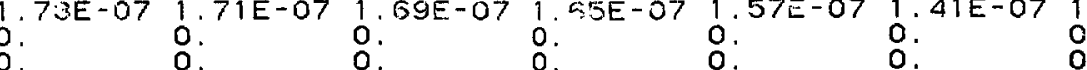
C. 0.

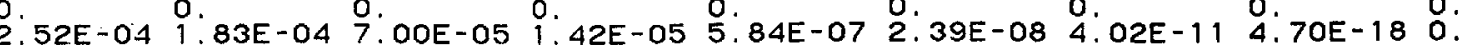
.

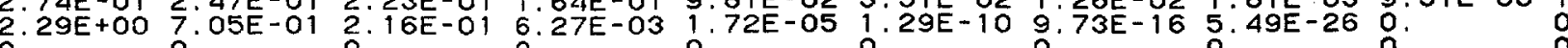

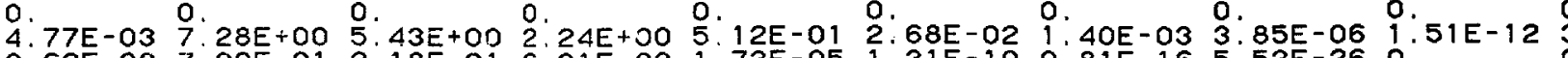

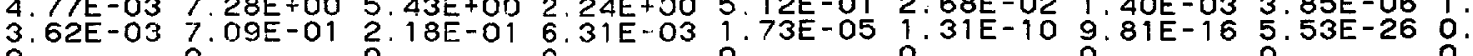

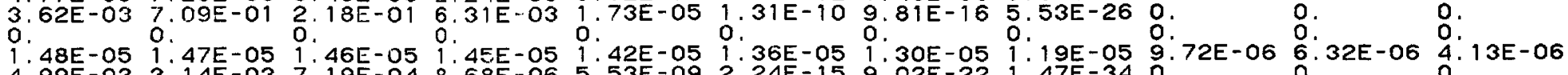

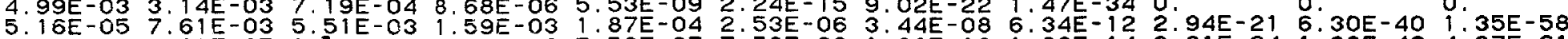

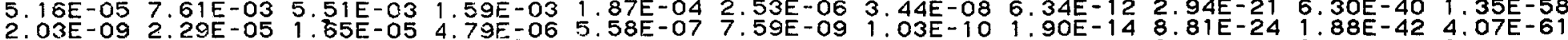

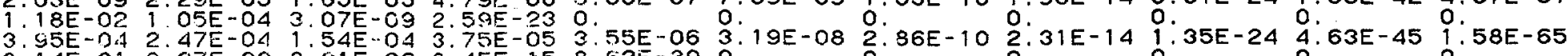
15 8. $625-30$ o. Q 14 EE-01 8 .

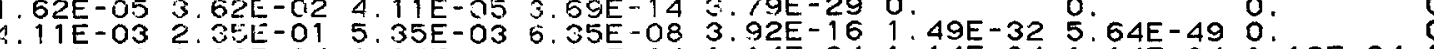
4.

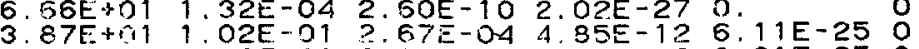
O. $1.13 E-012.99-045.43 E-12$

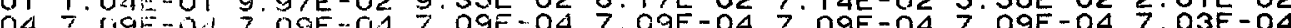

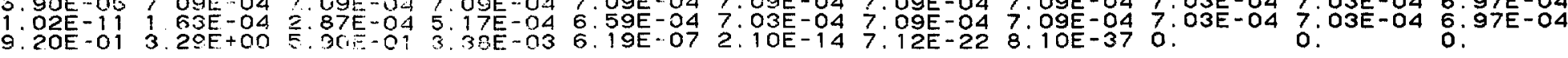
: $\quad 0$. . $12 \mathrm{E}-04 \quad 10 \mathrm{1}-04 \quad 108 \mathrm{E}-04$ O. . :. 
DEBRIS DECAY FROM 1 TO 300 DAYS

ZERO TIME 1. $1.00 E+00 \quad 2.00 E+00 \quad 5.00 E+00 \quad 1.00 E+01 \quad 2.00 E+01 \quad 3.00 E+01 \quad 5.00 E+01 \quad 1.00 E+02 \quad 2.00 E+02 \quad 3.00 E+02$

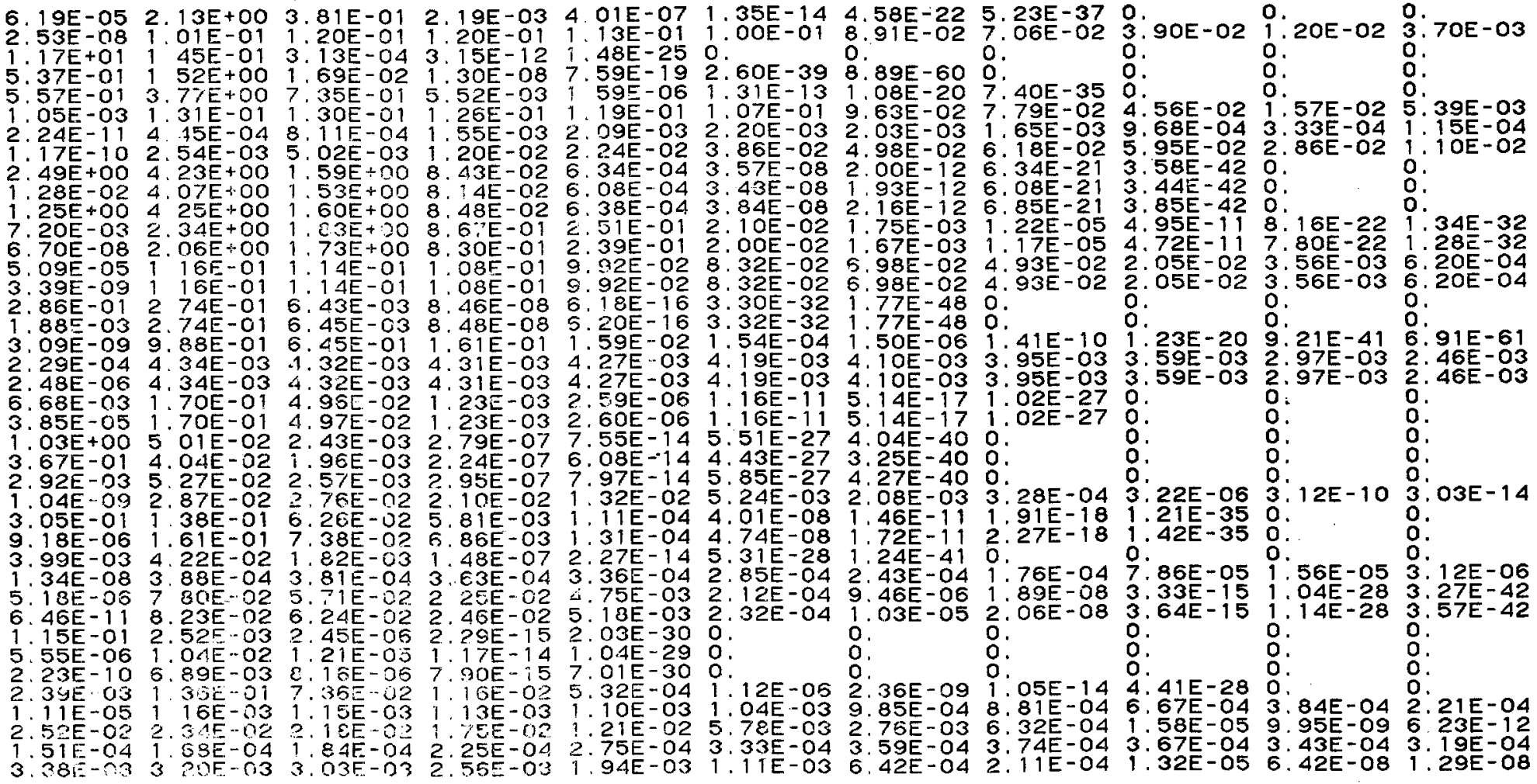


FRACTIOT H+12 HOURS D RELAXATION LENGTH DO 0.16 GM ISQCM

DEBRIS DECAY FROM 1 TO 50 YEARS

ZERO TIME 1. OOE+00 1.5OE+00 $2.00 E+00 \quad 3.50 E+00 \quad 5.00 E+00 \quad 7.00 E+001.00 E+012.00 E+01 \quad 3.50 E+01 \quad 5.00 E+01$

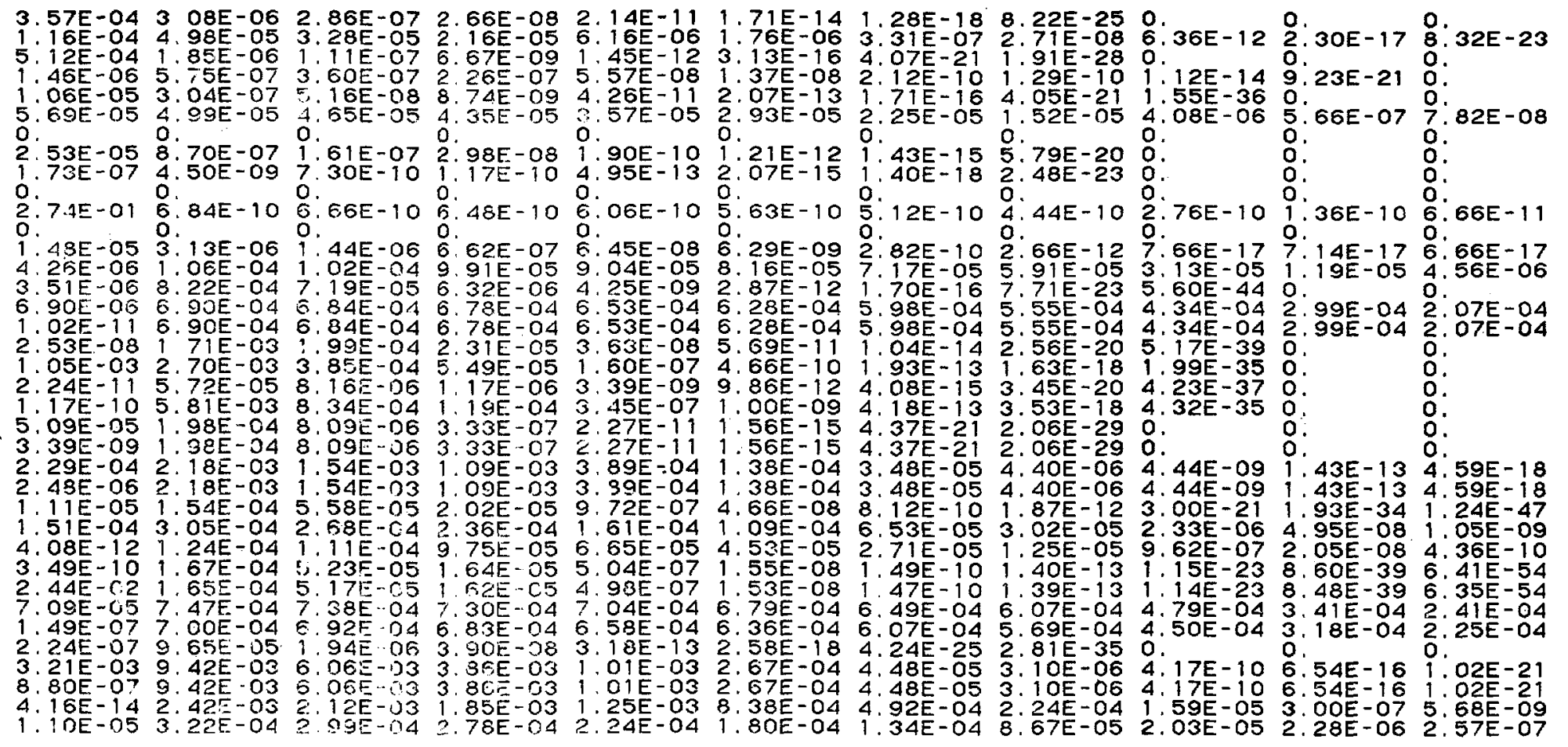

3. 04E-01 4.15E-02 2.27E-02 1.56E-02 $7.31 E-03 \quad 4.67 E-03 \quad 3.42 E-03 \quad 2.73 E-03 \quad 1.87 E-03 \quad 1.27 E-03 \quad 8.85 E-04$ 
FRACTION OF REFRACTERIES PRESENT $=0.500$

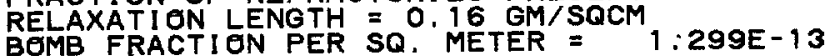

TIME

ZERO TIME HOURS

$1.00 E+00$

3. $00 \mathrm{OE}+00$

A. 00 E + +00

6. $\mathrm{OOE}+\mathrm{OO}$

$1.20 \mathrm{O}+01$

$1.50 \mathrm{OE}+\mathrm{O} 1$

$1.00 \mathrm{O}+00$ DAYS

2. $00 \mathrm{OE}+\infty \mathrm{O}$

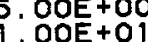

2. $00 \mathrm{E}+01$

3. OOE+01

$1.00 \mathrm{OE}+\mathrm{O} 2$

2. OOE +02

1.00 +OO YEARS

2. $O O E+O O$

$3.50 E+O 0$

. $00 \mathrm{OE}+\mathrm{OO}$

$1 . \mathrm{OOE}+01$

2. $00 \mathrm{E}+01$

5. $00 \mathrm{OE}+01$
$M R / H R$

MICROCURIES/SQ METER

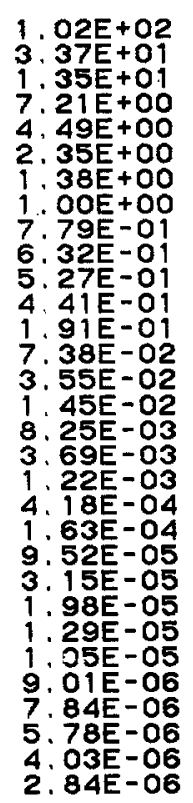

9. $96 \mathrm{E}+03$

$8.85 \mathrm{E}+02$

5. $40 \mathrm{E}+02$

2. $61 E+02$

$.76 E+02$

$.33 \mathrm{E}+02$

$8.67 \mathrm{E}+01$

6 . $1 E+01$

2. $73 E+01$

$4.59 \mathrm{E}+00$

$1.93 E+00$

1. $18 E+00$

6. $48 E-01$

2.78E-O1

9. $71 \mathrm{E}-02$

3. $32 \mathrm{E}-02$

$1.87 \mathrm{~B}-02$

$7.03 E-03$

. $92 \mathrm{E}-03$

3. $88 \mathrm{E}-03$

$3.25 \mathrm{E}-03$

1. 1 I IE-03 
SMOKY MR/HR AT H+12 HOURS = MICROCURIES/SO METER

MRACR AT H+12 HOURS = 1 I OOO

RELAXATION LENGTH $=0.16$ GM/SQCM $1.2995-13$

DEBRIS DECAY FROM I TO 21 HOURS

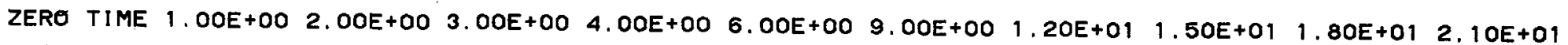

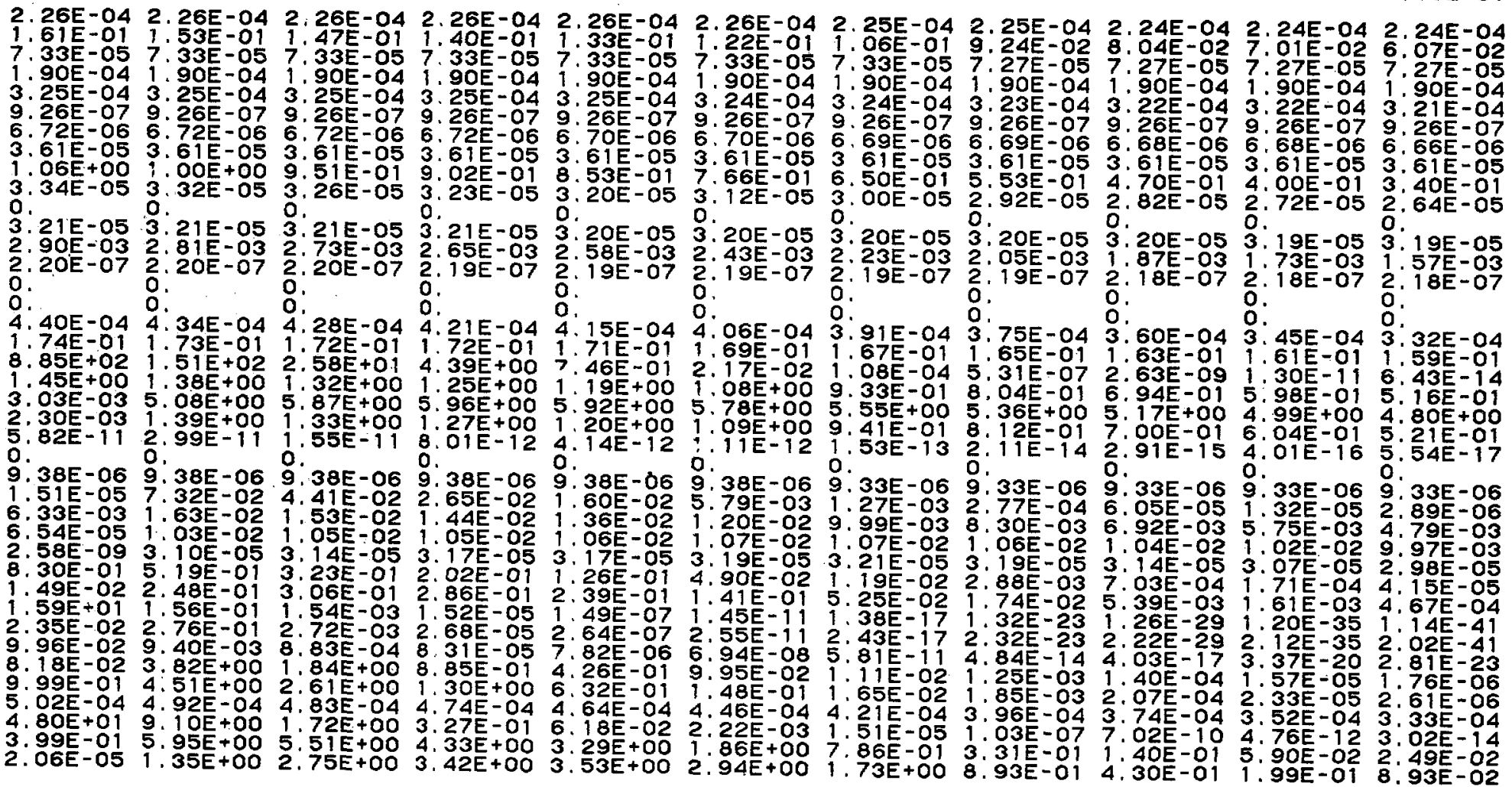


FRACTI ON OF REFRACTORIES PRESENT $=0.500$ RELAXATION LENGTH $=0.16 \mathrm{GM} / \mathrm{SQCM}$
BOMB FRACTI ONN PER SQ. METER $=1 ; 299 \mathrm{E}-13$

DEBRIS DECAY FROM 1 TO 21 HOURS

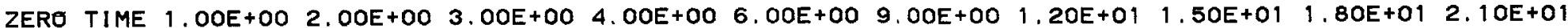

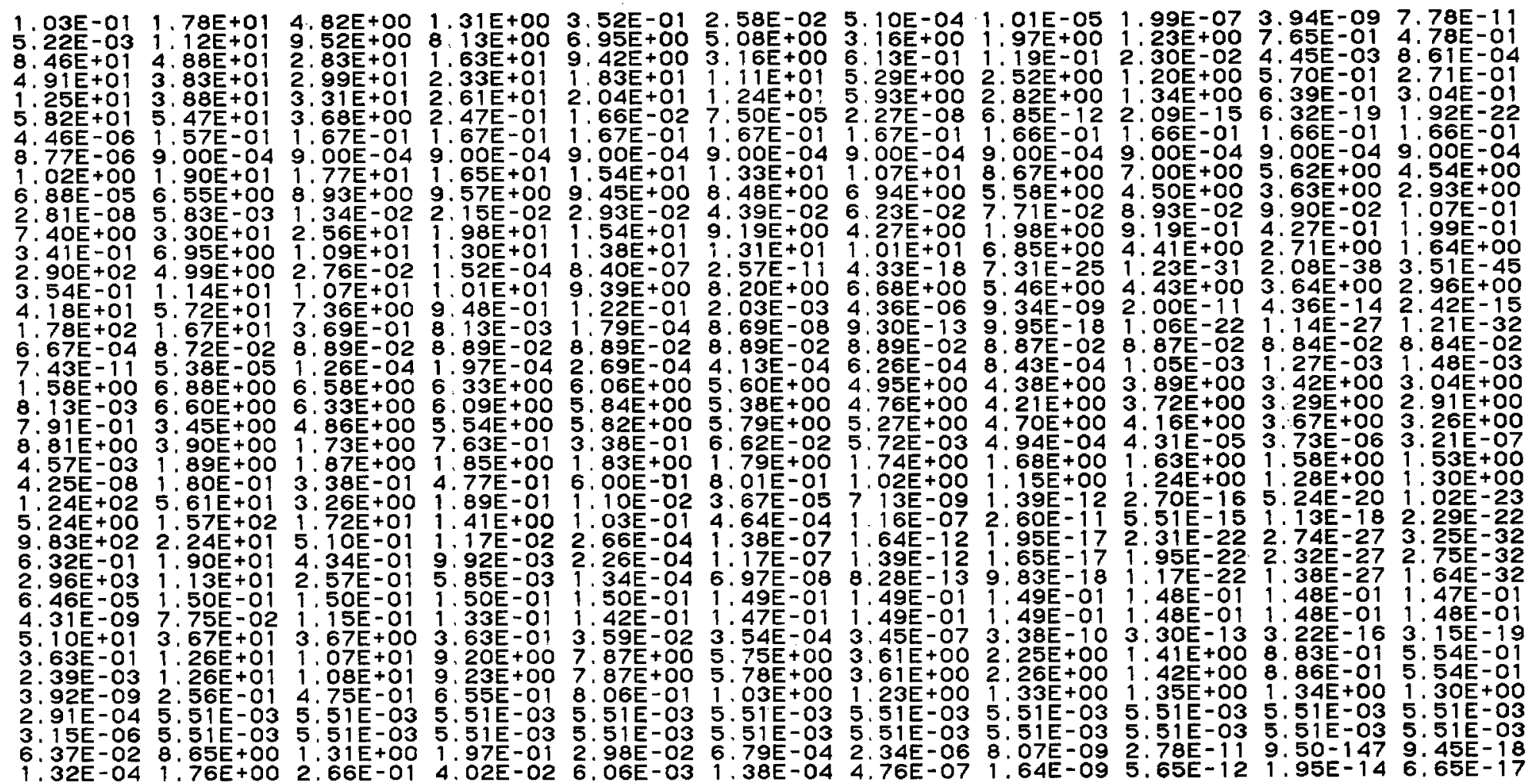



SMEKY MR/HR AT H+12 HOURS = MI CRRCURIES/SO METER

FRACTI ON OF REFRACTORI ES PRESENT $=0.500$

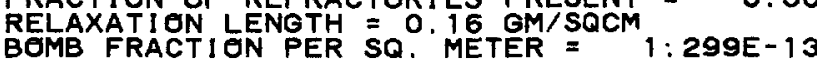

DEBRIS DECAY FROM 1 TO 21 HUURS

ZERO TIME 1. OOE+OO 2. OOE+OO $3.00 E+00 \quad 4.00 E+00 \quad 6.00 E+00 \quad 9.00 E+00 \quad 1.20 E+01 \quad 1.50 E+01 \quad 1.80 E+012.10 E+01$

$-01,75 \mathrm{E}-01$

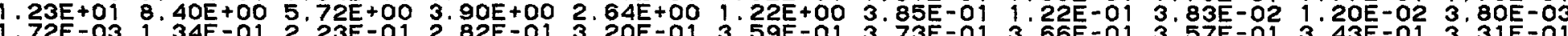

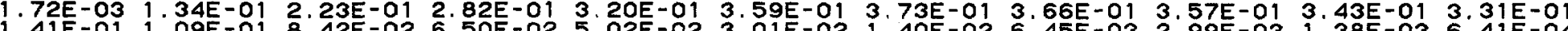

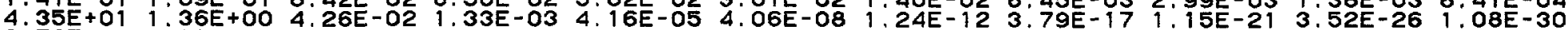
$2.76 E-023$ 23E-01 3. 25E-01 3 17E-01 3 O $6.83 E+016.68 E-026.51 E-056.36 E-086.22 E-115.94 E-175.53 E-265$ 15E-35 $4.80 E-444.48 E-534.17 E-62$ 9.10E-02 8.97E-02 8.85E-02 8.71E-02 8.59E-02 8.34E-02 7.98E-02 7.62E-02 7.30E-02 6.99E-02 6.69E-02 $9.50 E+001.56 E+002.56 E-014.18 E-02 \quad 6.86 E-03 \quad: 85 E-04 \quad 8.15 E-07$ 3.59E-09 $1.58 E-117.05 E-14 \quad 1.73 E-16$ 6.98E-06 1.99E-04 2.31E-04 2.36E-04 2, 37E-04 2.37E-04 2.37E-04 2.37E-04 2.37E-04 2.37E-04 2.37E-04 $5.81 E-025.39 E-025.01 E-024.65 E-02$ 4.32E-02 3.73E-02 2.99E-02 2. 50 - 52 - $1.92 E-021.54 E-02 \quad 1.23 E-02$ $1.70 E-042.77 E-043.76 E-044.68 E-045.54 E-04 \quad 7.06 E-04 \quad 8.99 E-04 \quad 1.04 E-03 \quad 1.16 E-03 \quad 1.26 E-03 \quad 1.32 E-03$

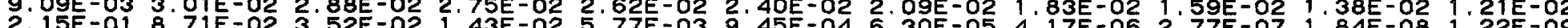
$2.85 E-012.83 E-022.80 E-032.79 E-042.76 E-052.42 E-072.305$

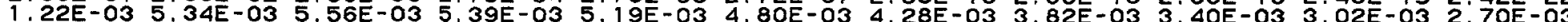
$2.05 E-05$ 1.48E-04 $1.47 E-04$ 1.46E-04 $1.46 E-04$ 1.44E-04 $1.43 E-04$ 1.41E-04 $1.39 E-04$ 1.37E-04 $1.36 E-04$

TOTAL

$9.96 E+03 \quad 2.22 E+03 \quad 8.85 E+02 \quad 5.40 E+02 \quad 3.93 E+02 \quad 2.61 E+02 \quad 1.76 E+02 \quad 1.33 E+02 \quad 1.06 E+02 \quad 8.67 E+01 \quad 7.32 E+01$ 
FRACTION OF REFRACTERIES PRESENT $=0.500$

RELAXATION LENGTH $=0.16$ GM'SOCM $=0.500$

DEBRIS DECAY FROM 1 TO 300 DAYS

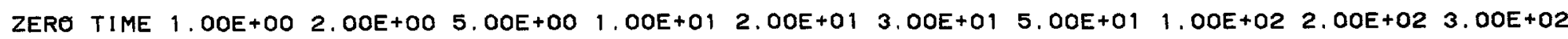

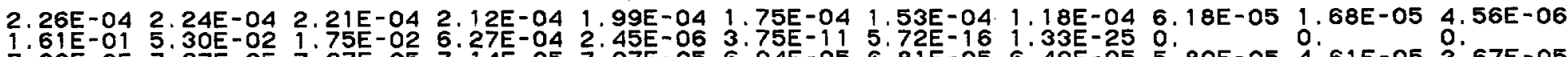
$7.33 E-05$ 7.27E-05 7.27E-05 7.14E-05 7.07E-05 6.94E-05 6.81E-05 6.49E-05 5.80E-05 4.61E-05 3.67E-05

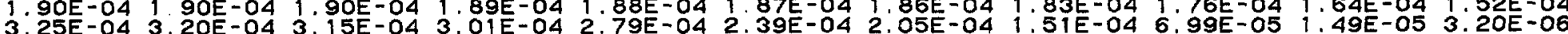
9.26E-07 9.26E-07 9. 19E-07 9.19E-07 9.06E-07 8.78E-07 8.58E-07 8. $16 E-07$ 7. 20E-07 5.56E-07 4.31E-07 6.7256 .067 . 3.61E-05 3.61E-05 3.61E-05 3.61E-05 3.59E-05 3.59E-05 3.57E-05 3.55E-05 3. 46E-05 3. 36E-05 3. 23E-05

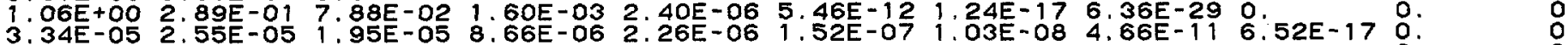

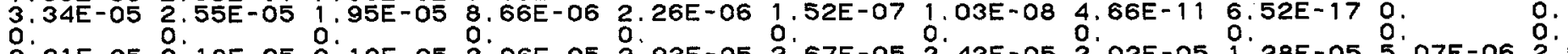
3.21E-05 3.18E-05 3.13E-05 3.06E-05 2.93E-05 2.67E-05 2.43E-05 2.02E-05 1.28E-05 5.07E-06 2.01E-06

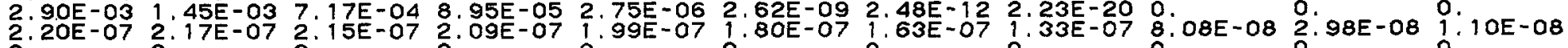

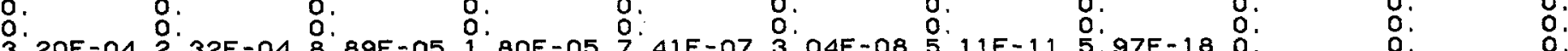

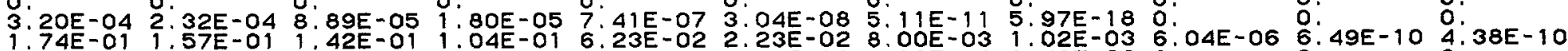

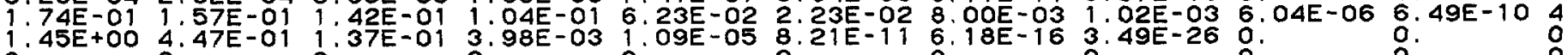

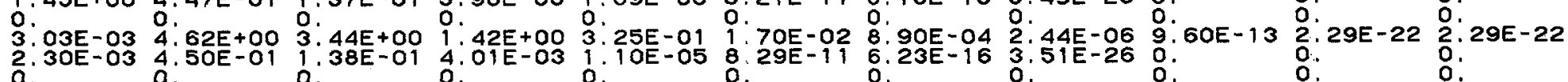
9.38E-06 9. 33E-06 9:28E-06 9.1 $19 E-06$ 8.99E-06 8.60E-06 8.26E-06 7.58E-06 5.17E-06 4.01E-06 2.62E-06

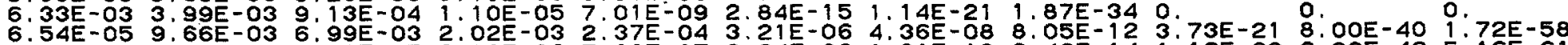
2.58E-09 2.91E-05 2.09E-05 6.08E-06 7.08E-07 9.64E-09 1.31E-10 2.42E-14 1:12E-23 2:39E-42 5:16E-51

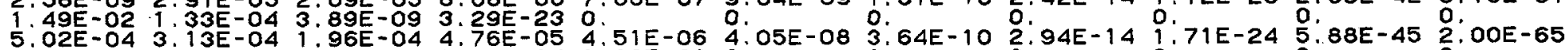

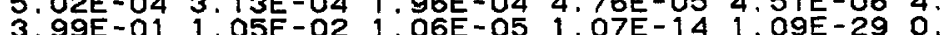
2. 06 - 05 4.59E-02 5 . $22 \mathrm{E}-054.69 \mathrm{E}-144.81 \mathrm{E}-29$ : .22E-03 2.98E-01 6.79E-03 $8.06 E-08$ 4.97E-16 $1.89 E-32$ 7. $16 E-49$

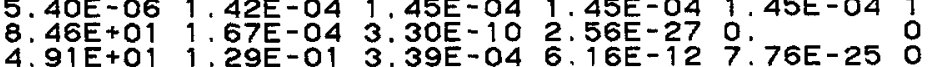

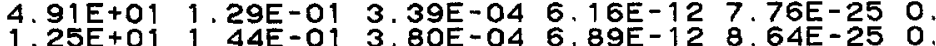
$44 E-04$ O. . . $43 E-04$ ?: $40 E-04$ : $38 E-04$

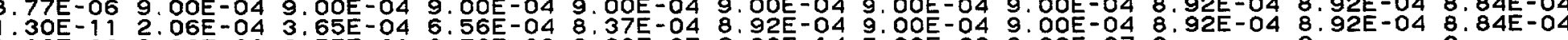

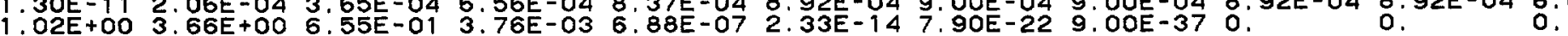


SMEKY

MI CROCURIES/SQ METER

FRACTION OF RERURS

RELAXTION LENGTH $=0.16$ G GM SSCCM $1: 2992-13$

DEBRIS DECAY FROM 1 TO 300 DAYS

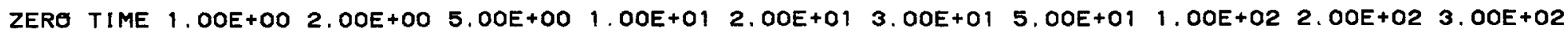

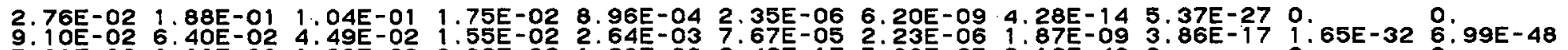
5:81E-02 9: 90E-03 1.69E-03 8.32E-06 1.20E-09 2.46E-17 5: 08E-25 2.16E-40 O.

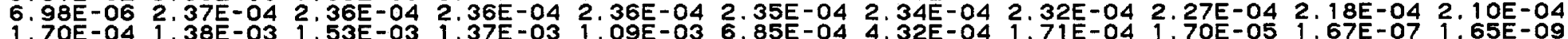

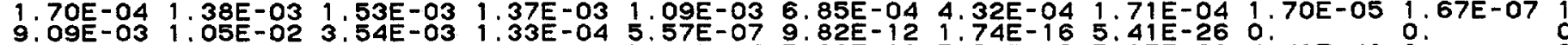

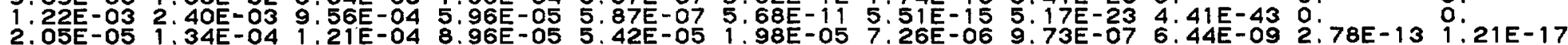

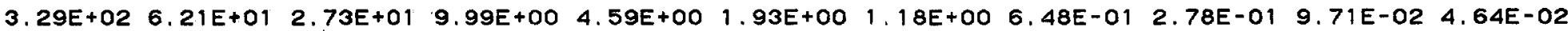

PAGE
PM151

SM156

U156

GD 159

TB161

TOTAL 


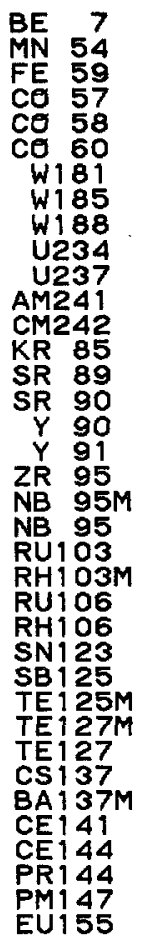

TOTAL
SMOKY MRAHR AT H+12 HOURS $=$ MEST 1 POOO RELAXATION LENGTH $=0.116$ GM SQCM : $299 \mathrm{~S}-13$

DEBRIS DECAY FROM 1 TO 50 YEARS

ZERO TIME $1.00 E+00 \quad 1.50 E+00 \quad 2.00 E+00 \quad 3.50 E+00 \quad 5.00 E+00 \quad 7.00 E+00 \quad 1.00 E+01 \quad 2.00 E+01 \quad 3.50 E+01 \quad 5.00 E+01$

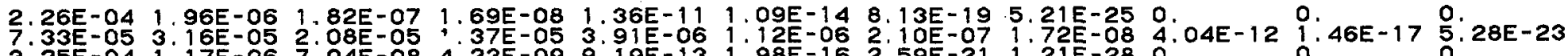

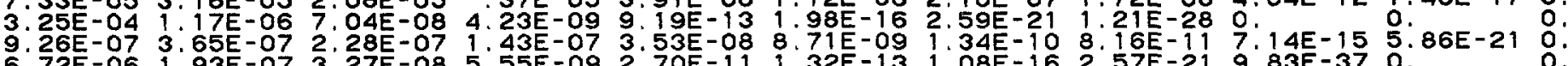
$\begin{array}{lllllll}6.72 E-06 & 1.93 E-07 & 3 & 27 E-08 & 5 & 55 E-09 & 2\end{array}$

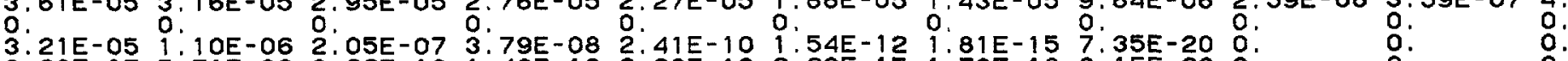

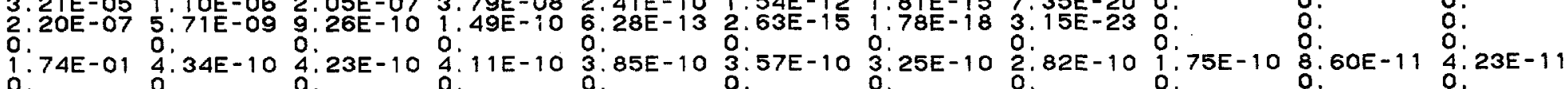

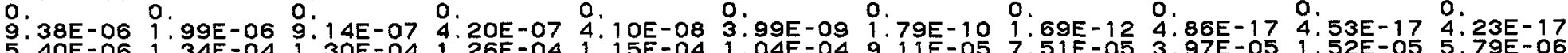

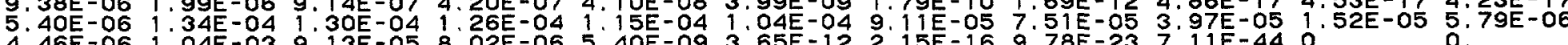

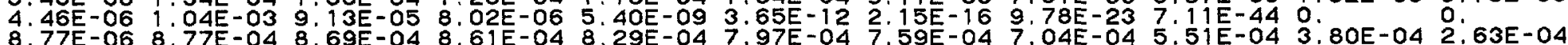
$1.30 E-11$ 8.77E-04 8.69E-04 8.61E-04 8.29E-04 7.97E-04 7.59E-04 7.04E-04 5.51E-04 3.80E-04 $2.63 E-04$

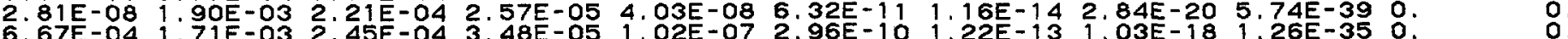

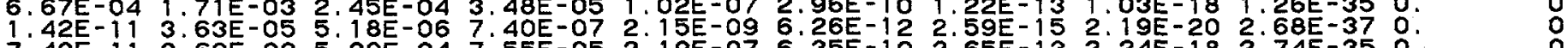

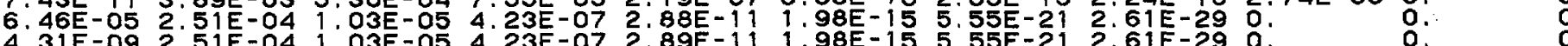

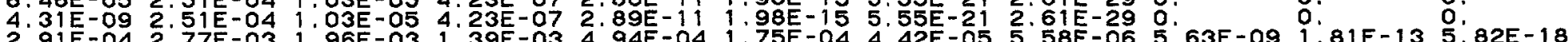

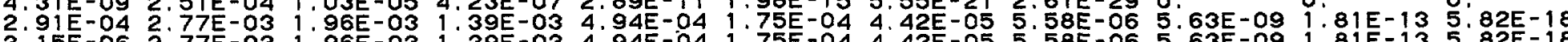
3. $15 E-062.77 E-03 \quad 1.96 E-03 \quad 1.39 E-03 \quad 4.94 E-04 \quad 1.75 E-0444.42 E-05$ 5.58E-06 5.63E-09 $1.81 E-135.82 E-18$

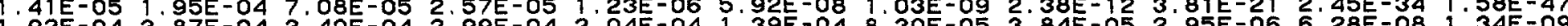

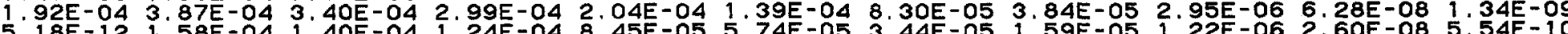
4.43E-10 2:12E-04 6.64E-05 2.08E-05 6:39E-07 1:97E-08 1.89E-10 1:78E-13 1.46E-23 1:09E-38 $8.13 E-54$ 3.0050 1.90E-07 8.89E-04 8.78E-04 8.67E-04 8.35E-04 8.02E-04 7 7.70E-04 7.22E-04 5.71E-04 4.04E-04 2.86E-04

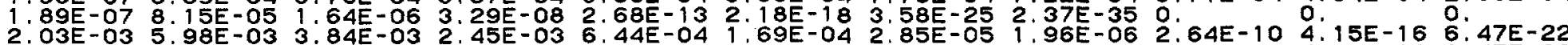

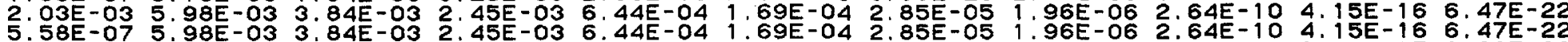

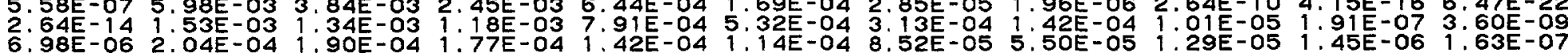

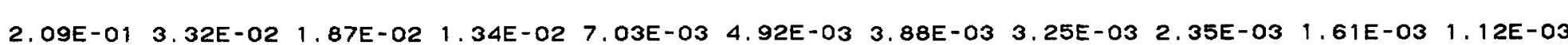


MR/HR AT $H+12$ HOURS $=1.000$

$N T=0.100$

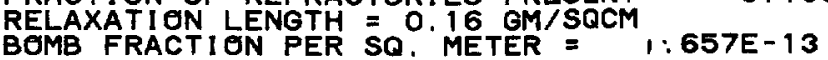

TIME

ZERO TIME HOURS

$1.00 E+O 0$

2. $3.00 \mathrm{OE}+\mathrm{OO}$

$4.00 E+\infty 0$

$9.00+00$

$1 \cdot 2 \mathrm{OE}+\mathrm{O} 1$

1.80E+01

1. 00 + +0 DAYS

$2.00{ }^{2}+\infty 0$

. OOE+O

2. 00 + +01

3. $\mathrm{OOE}+01$

5. $\mathrm{OOE}+01$

$2,00 E^{\circ}+02$

1.00 +OO YEARS

$1,50 \mathrm{E}+00$

SOE+O

3. 50 E + 00

5. $00 \mathrm{OE}+00$

$7.00 E+00$

$1.00 E+01$

2. $00 \mathrm{E}+01$

5. $00 \mathrm{E}+01$
MR/HR

$1.06 E+02$
$3.74 E+01$
$1.48 E+01$
$7.611+00$
$4.57 E+00$
$2.31 E+00$
$1.36 E+00$
$1.00 E+00$
$7.82 E-01$
$6.34 E-01$
$5.25 E-01$
$4.37 E-01$
$1.89 E-01$
$8.02 E-02$
$3.91 E-02$
$1.53 E-02$
$8.21 E-03$
$3.19 E-03$
$6.70 E-04$
$1.70 E-04$
$7.08 E-05$
$4.76 E-05$
$2.611-05$
$2.07 E-05$
$1.49 E-05$
$1.24 E-05$
$1.08 E-05$
$9.58 E-06$
$7.265-06$
$5.12 E-06$
$3.61 E-06$

MICROCURIES/SQ METER

1. $03 E+04$
$2.28 E+03$
$9.24 E+02$
$5.49 E+02$
$3.86 E+02$
$2.43 E+02$
$1.59 E+02$
$1.196+02$
$9.25 E+01$
$7.55 E+01$
$6.32 E+01$
$5.31 E+01$
$2.23 E+01$
$8.67 E+00$
$4.38+00$
$1.90 E+00$
$1.13 E+00$
$5.99 E-01$
$2.37 E-01$
$7.09 E-02$
$3.14 E-02$
$2.24 E-02$
$1.35 E-02$
$1.05 E-02$
$6.67 E-03$
$5.25 E-03$
$4.46 E-03$
$3.93 E-03$
$2.97 E-03$
$2.06-03$
$1.43 E-03$ 
MR/HR AT H+12 HOURS = RELAXATION LENGTH $=0.16 \mathrm{GM} / \mathrm{SQCM} / 1: 657 \mathrm{E}-13$

DEBRIS DECAY FROM 1 TO 21 HOURS

ZERO TIME 1, OOE+OO 2. OOE+OO 3. OOE+OO 4. OOE+OO 6. OOE+O0 9. OOE+OO 1.20E+01 $1.50 E+01 \quad 1.80 E+012.10 E+01$

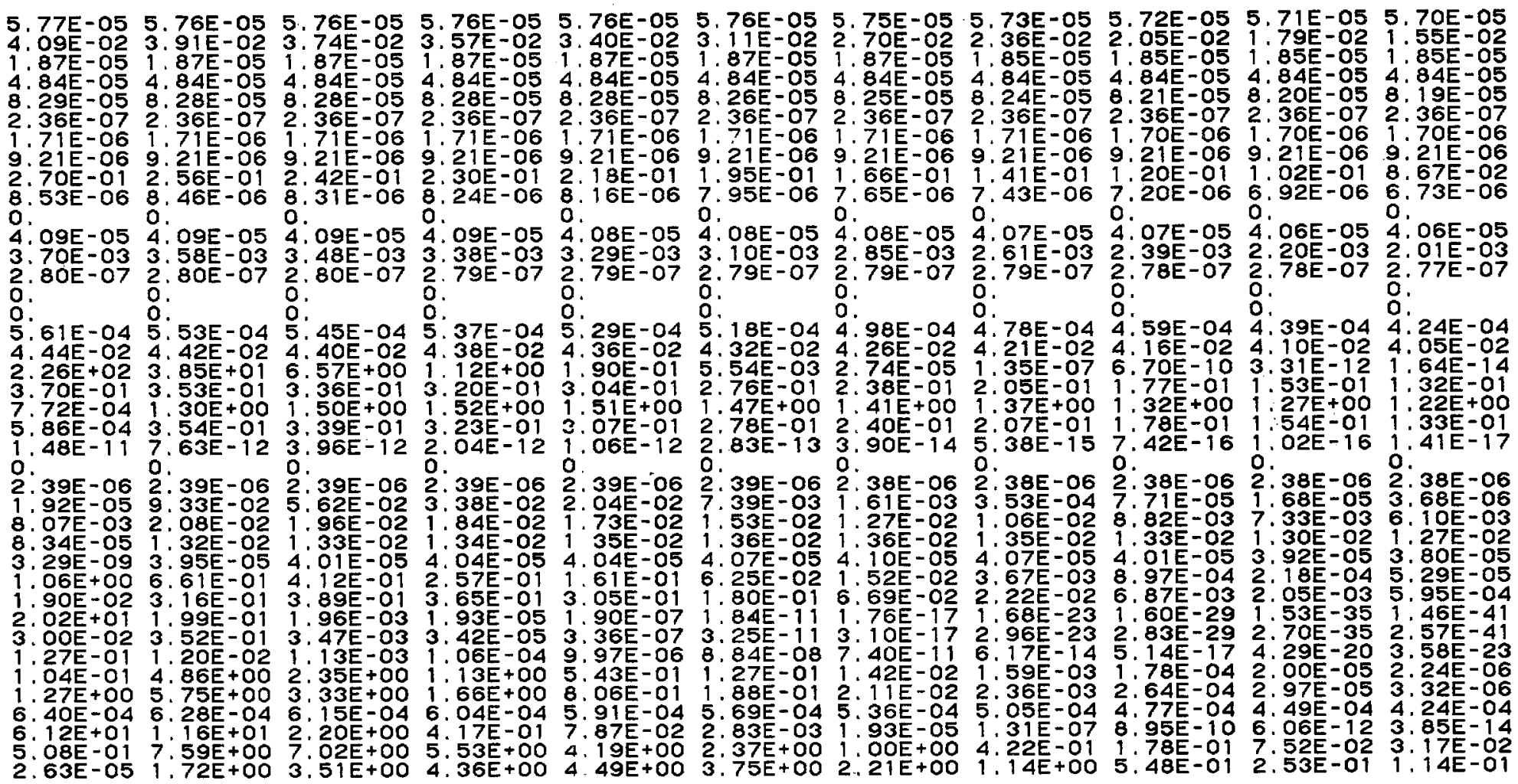


MR HR AT H+12 HOURS = M RELAXATION LENGTH $=0.16$ GM/SQCM $1: 657 E-13$

DEBRIS DECAY FROM 1 TO 300 DAYS

ZERO TIME $1.00 E+00$ 2. OOE+OO $5.00 E+001.00 E+012$ OOE+013 OOE+01 $5.00 E+011$ OOE+O2 2 OOE+O2 3 OOE+O2

NA 24

MA 24

FE 55

ce 57

CO 60

CU 64

W181

W187

$W 188$
AU198

AUI 99

$\mathrm{PB} 203$

U240
NP237

NP239

AM241

CM242

AE 77

SE $77 \mathrm{M}$

$\begin{array}{ll}\mathrm{AR} & 82 \\ \mathrm{BR} & 83 \\ \mathrm{~K} & 83\end{array}$

KR 83M

KR 85M

KR 87

KR 88

RR 88

SR 90

SR 91

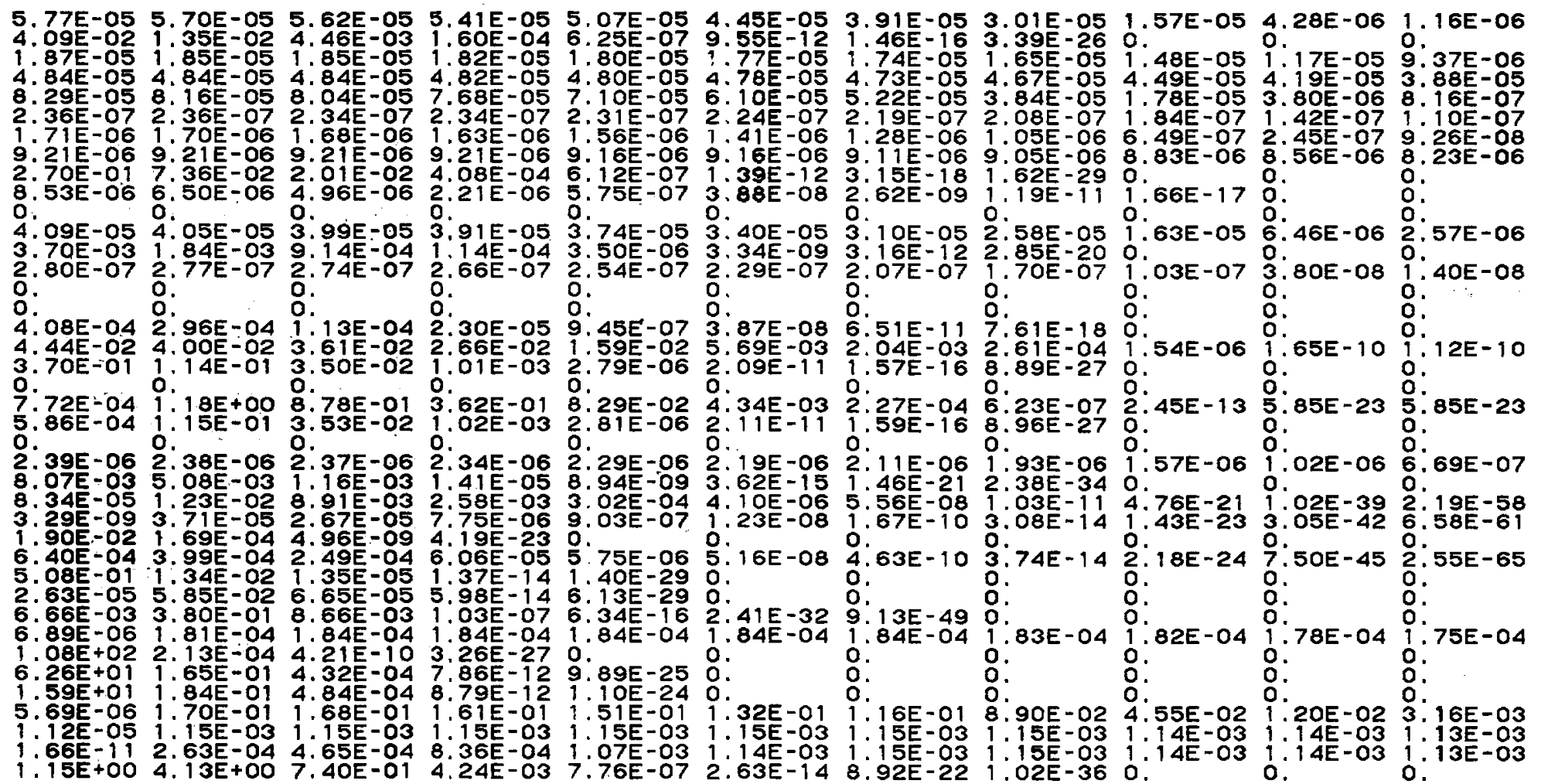


MICRGCURIES/SQ METER

MR/HR AT H+12 HOURS =

DEBRIS DECAY FROM 1 TO 300 DAYS

ZERO TIME 1, OOE+OO $2.00 E+00 \quad 5.00 E+00 \quad 1.00 E+01 \quad 2.00 E+01 \quad 3.00 E+01 \quad 5.00 E+01 \quad 1.00 E+02 \quad 2.00 E+02 \quad 3.00 E+02$

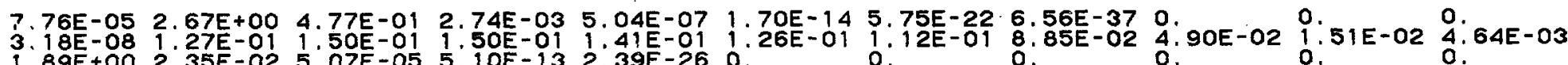
$1.89 E+002.35 E-025.07 E-05$ 5. $20 \mathrm{E}-132.39 \mathrm{E}-26$. (1)

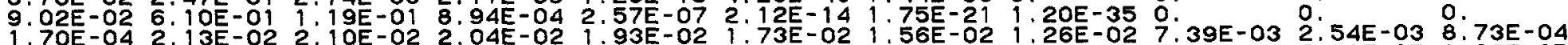
-04 3.29E-04 2.67E-04 1.57E-04 5.39E-05 1.86E-05 $1.89 E-11$ 4.11E-04 8.13E-04 1.95E-03 3.62E-03 6.24E-03 8.06E-03 1.00E-02 9.62E-03 4.63E-03 $1.78 E-03$ 4.03E-01 6.85E-01 2.58E-01 $1.37 E-02$ 1.03E-04 5.77E-09 3.24E-13 $1.03 E-215.79 E-430$ 2.07E-03 6.58E-01 2.47E-O1 1.32-02 9.84E-04 5.24E-09 $3.49 E-131.11 E-216.24 E-430$. $\begin{array}{lllllll} & \end{array}$

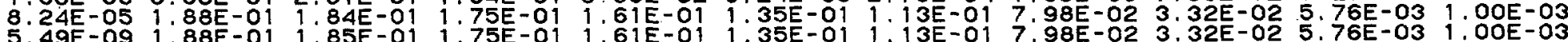

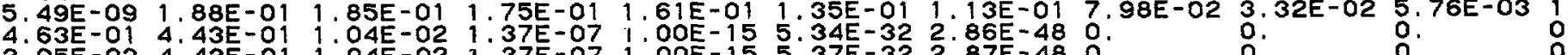
3.05E-03 4.43E-01 1.04E-02 1.37E-07 1.00E- 15 5.37E-32 2.87E-48 0. $4.02 E-06$ 7.03E-03 7.00E-03 $6.97 E-03$ 6.91E-03 $6.79 E-03$. $63 E-03$ - $39 E-035.81 E-034.81 E-03$ 3.99E-03

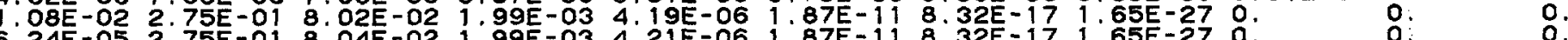

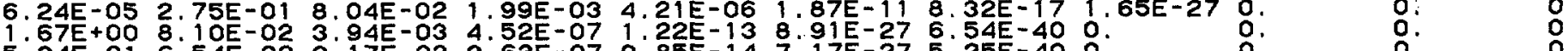
$5.94 \mathrm{E}-01$ 6.54E-02 3.17E-03 3.63E-07 9.85E-14 7.17E-27 5.25E-40 .68E-09 4.64E-02 4.46E-02 3.40E-02 2.14E-02 8.47E-03 3.36E-03 $5.30 E-04$ $1.49 E-05$ 2.60E-01 1 . 20E-01 1.11E-02 2:12E-04 7.67E-08 2.78E-11 3.67E-18 :. : . $46 E-03 \quad 6.83 E-02$ 2.95E-03 2.40E-07 3.67E- $148.59 E-28 \quad 2.01 E-41$ $8.38 \mathrm{E}-06$ 1.26E-01 9.25E-02 3.64E-02 7.69E-03 $3.43 \mathrm{E}-041$ 04E-10 1.33E-01 1.01E-01 3.98E-02 8.,38E-03 3.75E-04 1.67E-05 $1.87 \mathrm{E}-01$ 4.07E-03 3.96E-06 3.70E-15 3.29E-30 0. $8.98 E-06 \quad 1.69 E-02 \quad 1.96 E-051.89 E-14$ 1.68E-29 0. $28.61 E-041.82 E-0633$

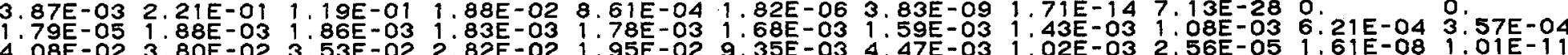

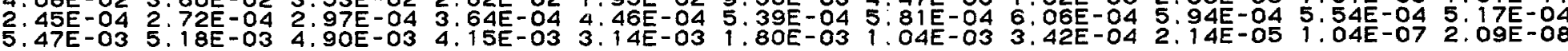

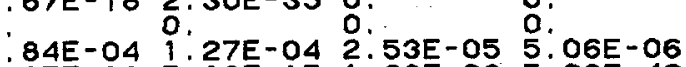
$.84 E-041.27 E-042.53 E-055.06 E-06$

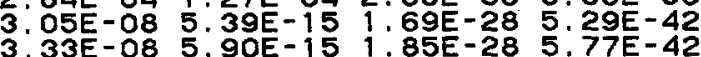


SMOKY FRACT AT H+12 HOURS OF REFRACTORIES PRESENT RELAXATI GN LENGTH $=0.16 \mathrm{GM} / \mathrm{SQCM}=1.657 \mathrm{E}-13$

DEBRIS DECAY FROM 1 TO 50 YEARS

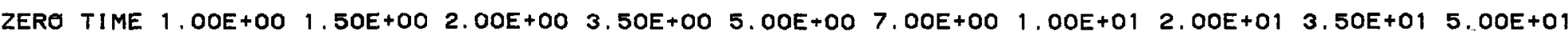

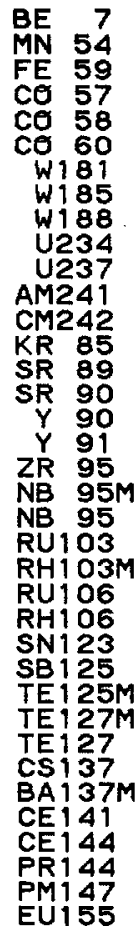

TOTAL

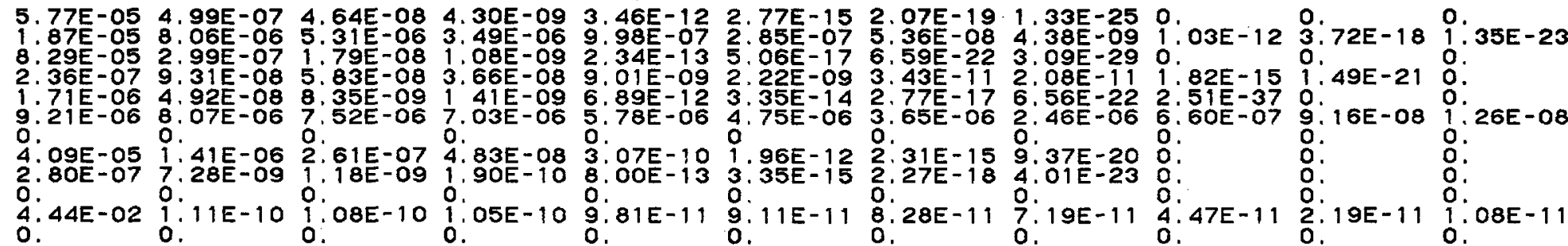

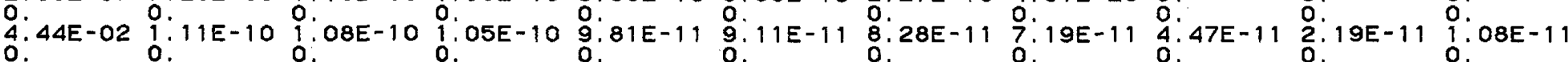
2.39E-06 5.07E-07 2.33E-07 1.07E-07 1.04E-08 1.02E-09 4.56E-11 4.31E-13 1.24E-17 $1.15 E-17$ 1.08E-17

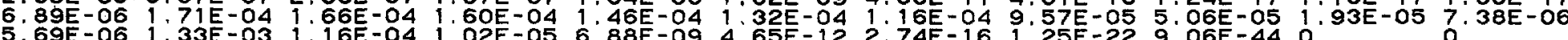

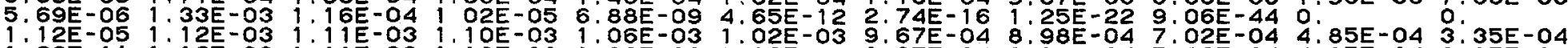

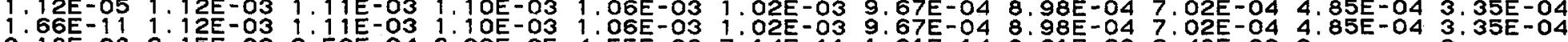
3.18E-08 2.15E-03 2.50E-04 2.90E-05 4.55E-08 7.14E-19 1.31E-14 3.21E-20 6.48E-39 0. $1.70 E-044.37 E-04 \quad 6.24 E-058.88 E-06$ 2.59E-08 7.53E-11 3.12E-14 2.63E-19 3.22E-36 0.

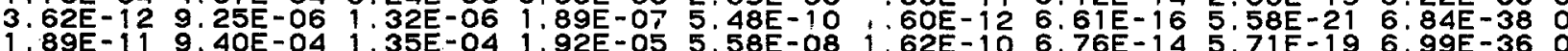
$1.89 E-11$ 9.40E-04 1.35E-04 1.92E-05 5.58E-08 1.62E-10 6.76E-14 5.71E-19 6.90 . 24E-05 3.20E-04 1.31E-05 5.39E-07 3.68E-11 2.52E-15 7.08E-21 3.33E-29 0. 5.49E-09 3.21E-04 1.31E-05 5.39E-07 3.68E-11 2.52E-15 7.08E-21 3.33E-29 0.

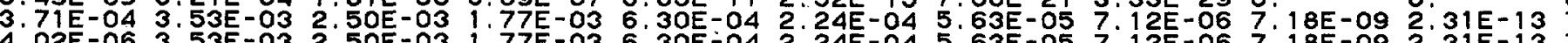
源 .60E-12 2.01E-04 1.79E-04 1.58E-04 1 $5.65 E-10$ 2.71E-04 8.47E-05 2.65E-05 8. 15E-07 2.51E-08 2.41E- 10 2.27E-13 $1.86 E-231.39 E-381.04 E-53$ 3.94E-02 2.68E-04 8.37E-05 2.62E-05 8.05E-07 2.48E-08 2.38E-10 2.25E-13 1.84E-23 $1.37 \mathrm{E}-38 \quad 1.03 E-53$ 1.15E-04 1.21E-03 1.19E-03 1.18E-03 1.14E-03 1.10E-03 $1.05 E-039.82 E-047.76 E-045.51 E-043.89 E-04$

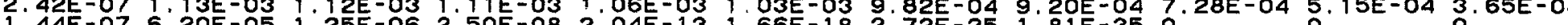

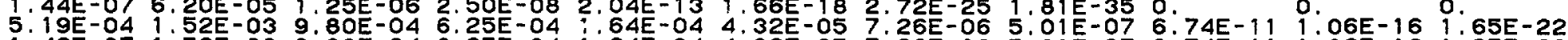
$1.42 E-071.52 E-03$ 9.8OE-04 6. 25E-04 1.64E-04 4.32E-05 7.26E-06 5.01E-07 6.74E-11 $1.06 E-16 \quad 1.65 E-22$

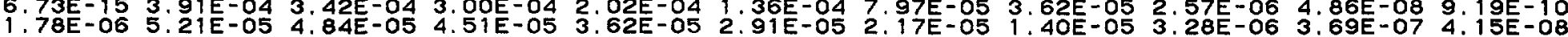

8.56E-02 2.24E-02 $1.35 E-02 \quad 1.05 E-02 \quad 6.67 E-03 \quad 5.25 E-03 \quad 4.46 E-03 \quad 3.93 E-03 \quad 2.97 E-03 \quad 2.06 E-03 \quad 1.43 E-03$ 


\section{DISCLAIMER}

This document was prepared as an account of work sponsored by an agency of the United States Government. Neither the United States Government nor the University of California nor any of their employees, makes any warranty, express or implied, or assumes any legal liability or responsibility for the accuracy, completeness, or usefulness of any information, apparatus, product, or process disclosed, or represents that its use would not infringe privately owned rights. Reference herein to any specific commercial products, process, or service by trade name, trademark, manufacturer, or otherwise, does not necessarily constitute or imply its endorsement, recommendation, or favoring by the United States Government or the University of California. The views and opinions of authors expressed herein do not necessarily state or reflect those of the United States Government thereof, and shall not be used for advertising or product endorsement purposes.

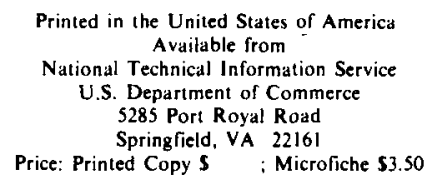

\begin{tabular}{lrrrr} 
Page Range & $\begin{array}{c}\text { Domestic } \\
\text { Price }\end{array}$ & Page Range & \multicolumn{1}{c}{$\begin{array}{c}\text { Domestic } \\
\text { Price }\end{array}$} \\
\cline { 5 - 5 } $001-025$ & $\$ 5.00$ & $326-350$ & $\$ 18.00$ \\
$026-050$ & 6.00 & $351-375$ & 19.00 \\
$051-075$ & 7.00 & $376-400$ & 20.00 \\
$076-100$ & 8.00 & $401-425$ & 21.00 \\
$101-125$ & 9.00 & $426-450$ & 22.00 \\
$126-150$ & 10.00 & $451-475$ & 23.00 \\
$151-175$ & 11.00 & $476-500$ & 24.00 \\
$176-200$ & 12.00 & $501-525$ & 25.00 \\
$201-225$ & 13.00 & $526-550$ & 26.00 \\
$226-250$ & 14.00 & $551-525$ & 27.00 \\
$251-275$ & 15.00 & $526-550$ & 28.00 \\
$276-300$ & 16.00 & $601-401$ & \\
$301-325$ & 17.00 & &
\end{tabular}

$1^{\text {Add }} 2.00$ for each additional 25 page increment from 601 pages up. 